


\section{MISCELLANEA ON ENCEPHALOPATHIES - A SECOND LOOK}

Edited by Radu Tanasescu 


\section{Contributors}

Radu Tanasescu, Laura Dumitrescu, Carmen Dragos, Dimela Luca, Alexandra Oprisan, Catalina Coclitu, Oana Simionescu, Lorena Cojocaru, Marius Stan, Andreea Carasca, Andreea Gitman, Adela Chiru, Marina Ticmeanu, Brendan McGuire, Daniela Benedeto-Stojanov, Dragan Stojanov, Pedro Moreno Pimentel-Coelho, Rosalia MendezOtero, Marcelo Felippe Santiago, Yuji Odagaki, Guillermo Bodega, Isabel Suárez, Benjamín Fernández, Praveen Sharma, Martin Safo, Roberto Contestabile, Verne Schirch, Martino Di Salvo, Mohini Ghatge, Sayaly Karve, Lore Lapeire, Anne Sieben, Simon Van Belle, Patrick Santens, Marshall Orloff, Claudia Zwingmann, Pilvi Ilves, Olguín, Mireia Moragas Garrido, Jordi Gascón-Bayarri, Niels Hansen, Peter Paul De Deyn, Annemie Van Dijck, Wendy Van Daele

\section{(c) The Editor(s) and the Author(s) 2012}

The moral rights of the and the author(s) have been asserted.

All rights to the book as a whole are reserved by INTECH. The book as a whole (compilation) cannot be reproduced, distributed or used for commercial or non-commercial purposes without INTECH's written permission. Enquiries concerning the use of the book should be directed to INTECH rights and permissions department (permissions@intechopen.com).

Violations are liable to prosecution under the governing Copyright Law.

\section{(cc) BY}

Individual chapters of this publication are distributed under the terms of the Creative Commons Attribution 3.0 Unported License which permits commercial use, distribution and reproduction of the individual chapters, provided the original author(s) and source publication are appropriately acknowledged. If so indicated, certain images may not be included under the Creative Commons license. In such cases users will need to obtain permission from the license holder to reproduce the material. More details and guidelines concerning content reuse and adaptation can be foundat http://www.intechopen.com/copyright-policy.html.

\section{Notice}

Statements and opinions expressed in the chapters are these of the individual contributors and not necessarily those of the editors or publisher. No responsibility is accepted for the accuracy of information contained in the published chapters. The publisher assumes no responsibility for any damage or injury to persons or property arising out of the use of any materials, instructions, methods or ideas contained in the book.

First published in Croatia, 2012 by INTECH d.o.o.

eBook (PDF) Published by IN TECH d.o.o.

Place and year of publication of eBook (PDF): Rijeka, 2019.

IntechOpen is the global imprint of IN TECH d.o.o.

Printed in Croatia

Legal deposit, Croatia: National and University Library in Zagreb

Additional hard and PDF copies can be obtained from orders@intechopen.com

Miscellanea on Encephalopathies - A Second Look

Edited by Radu Tanasescu

p. cm.

ISBN 978-953-51-0558-9

eBook (PDF) ISBN 978-953-51-5281-1 


\section{We are IntechOpen, \\ the world's leading publisher of Open Access books}

Built by scientists, for scientists

\section{$4,100+$}

Open access books available

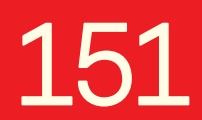

Countries delivered to
$116,000+$

International authors and editors
$120 \mathrm{M}+$

Downloads

Our authors are among the

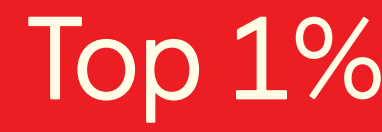

most cited scientists

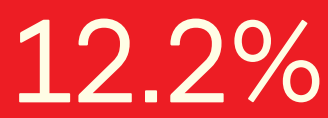

Contributors from top 500 universities

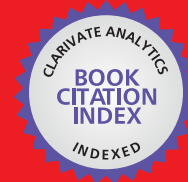

WEB OF SCIENCE ${ }^{\mathrm{TM}}$

Selection of our books indexed in the Book Citation Index in Web of Science ${ }^{\mathrm{TM}}$ Core Collection (BKCI)

Interested in publishing with us?

Contact book.department@intechopen.com

Numbers displayed above are based on latest data collected.

For more information visit www.intechopen.com

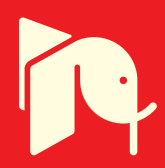





\section{Meet the editor}

Dr Radu Tanasescu is Senior Lecturer in the Department of Neurology, "Carol Davila "University of Medicine and Pharmacy, Colentina Hospital - Bucharest, Romania. Dr Tanasescu fulfilled his clinical training in France (2001-2003, Central Hospital Nancy). He pursued training in neurophysiology (University of Bristol, UK) and immunology of multiple sclerosis (Division of Clinical Neurology University Hospital, Nottingham, UK). In 2010 and 2011, he was awarded by the European Neurological Society and the European Federation of Neurological Societies with two scientific Fellowship research grants for projects studying advanced imaging techniques and stem cell therapy in multiple sclerosis (University of Nottingham, UK). He conducted the firsts studies in Romania on neurological manifestations associated with antiphospholipid antibodies, being awarded with a PhD degree in 2007. He is author of more than 100 published scientific contributions and was contributor to 6 medical books. As a member of EFNS scientific Panels of Neuroimmunology and Neurotoxicology he co-authored two Guideline Papers of the EFNS. Dr Tanasescu's area of expertise covers clinical neuroimmunology, multiple sclerosis, neurology of systemic diseases and stroke. 



\section{Contents}

\section{Preface XIII}

Chapter 1 Minimal Hepatic Encephalopathy (MHE) 1

Daniela Benedeto-Stojanov and Dragan Stojanov

Chapter 2 Uremic Encephalopathy 23

Annemie Van Dijck, Wendy Van Daele and Peter Paul De Deyn

Chapter 3 Drug-Induced Encephalopathy 39

Niels Hansen

Chapter 4 Sonographic Changes

in Hypoxic-Ischaemic Encephalopathy 61

Pilvi llves

Chapter 5 Neoplasm Related Encephalopathies 91

Lore Lapeire, Anne Sieben, Patrick Santens and Simon Van Belle

Chapter 6 Hepatic Encephalopathy 121

Jeffrey E. Juneau and Brendan M. McGuire

Chapter 7 Hypoxic Encephalopathy 149

Mireia Moragas Garrido and Jordi Gascón Bayarri

Chapter 8 Encephalopathy Associated with Psychotropic Drug Therapy 167

Yuji Odagaki

Chapter 9 The Use of Microdialysis in the Study of Encephalopathies 199

Liliana Carmona-Aparicio, Liliana Rivera-Espinosa and Hugo Juárez-Olguín

Chapter 10 Portal-Systemic Encephalopathy in Emergency Treatment of Cirrhosis and Bleeding Esophageal Varices 225 Marshall J. Orloff 
Chapter 11 Future Perspectives for the Treatment of Neonatal Hypoxic-Ischemic Encephalopathy 243

Pedro M. Pimentel-Coelho, Marcelo F. Santiago

and Rosalia Mendez-Otero

Chapter 12 Molecular Defects of Vitamin $\mathbf{B}_{6}$ Metabolism Associated with Neonatal Epileptic Encephalopathy 267 Mohini S. Ghatge, Martino L. Di Salvo, Roberto Contestabile, Dorothy N. Eseonu, Sayali Karve, Verne Schirch and Martin K. Safo

Chapter 13 Disaccharides in the Treatment

of Hepatic Encephalopathy in Patients with Cirrhosis 291

Praveen Sharma

Chapter 14 Dopaminergic Dysfunction

in Experimental Hepatic Encephalopathy 309

Isabel Suárez, Guillermo Bodega and Benjamín Fernández

Chapter 15 Wernicke's Encephalopathy 327

Radu Tanasescu, Laura Dumitrescu, Carmen Dragos, Dimela Luca, Alexandra Oprisan, Catalina Coclitu, Oana Simionescu,

Lorena Cojocaru, Marius Stan, Andreea Carasca, Andreea Gitman, Adela Chiru and Marina Ticmeanu

Chapter 16 L-carnitine in Hyperammonemia and Hepatic Encephalopathy 365

Jane Missler and Claudia Zwingmann 

"There is only one good, knowledge, and one evil, ignorance" Socrates 


\section{Preface}

The brain is an exceptionally complex structure anatomically, physiologically and pharmacologically. A vast spectrum of perturbations can impact its function. 'Encephalopathy' is a non-specific term for any diffuse disease of the brain altering brain function or structure. Etymologically the word has Greek roots (i.e. 'encephalo' and 'pathy') that translate as 'suffering of the brain'. However, under this 'umbrella' are grouped a plethora of brain disturbances with various etiologies including infectious, metabolic, toxic or immune conditions.

Nevertheless, the medical concept of encephalopathy is slightly more restrictively used, commonly designating only non-focal, structural or only functional, more or less reversible or progressive diseases of the brain disrespectful of their cause. Though one may argue the need for a more rigid definition, this would not serve my purpose of drawing attention to the vast spectrum of diseases grouped under the concept of encephalopaties and at the same time to the great variety of etiologies, mechanisms and clinical expressions of this diseases. Therefore, as already understood by the reader of the previous book 'Miscellanea on Encephalopathies' one may and should not aspire to an exhaustive coverage on the subject. In the same spirit of the previous book and guided by the same need of completing the ever changing kaleidoscopic mosaic of the spectrum of encephalopathies by putting together more or less detailed pieces written by authors with expertise in the chosen field, I propose to you 'Miscellanea on Encephalopathies: a Second Look'. As the previous book, the present one addresses not only neurologists, but also fellow physicians of various specialties and medical researchers. I take great honor in thanking the colleagues that contributed to the developing of this book by sharing their experience and knowledge. Along with them I hope that this second attempt to group aspects related to different encephalopathies adds important information to the one offered by the previous book and I put great hope in the future collaborations that will help us to build more comprehensive and up to date view on this broad topic.

Radu Tanasescu

University of Medicine and Pharmacy "Carol Davila" Bucharest, Department of Neurology, Colentina Hospital,

Romania 



\title{
Minimal Hepatic Encephalopathy (MHE)
}

\author{
Daniela Benedeto-Stojanov and Dragan Stojanov \\ Faculty of Medicine, University of Nis \\ Serbia
}

\section{Introduction}

Hepatic encephalopathy (HE) reflects a spectrum of neuropsychiatric abnormalities seen in patients with liver dysfunction after exclusion of other known brain disease.

Classification of hepatic encephalopathy is based on the clinical setting in which symptoms occur (Table 1). ${ }^{1}$ The encephalopathy of acute liver failure shares clinical characteristics with that of cirrhosis, but also exhibits unique features. In cirrhosis, three major syndromes can be present:

1. Episodic (precipitant-induced) encephalopathy, commonly seen in the hospital setting, where a superimposed event is a key factor.

2. Persistent (chronic) encephalopathy, seen with extensive portal-systemic shunts and after portocaval shunt surgery or placement of transjugular intrahepatic portosystemic shunt (TIPS).

3. Minimal (subclinical) encephalopathy reflects alterations in cognitive function in patients who clinically exhibit a normal mental state.

\begin{tabular}{llll}
\hline & $\begin{array}{l}\text { Hepatic } \\
\text { failure }\end{array}$ & $\begin{array}{l}\text { Extrahepatic } \\
\text { portal-systemic } \\
\text { shunting }\end{array}$ & Special features \\
\hline Acute liver failure & Maximal & Absent & $\begin{array}{l}\text { Development of brain oedema and } \\
\text { intracranial hypertension } \\
\text { Cirrhosis }\end{array}$ \\
$\begin{array}{l}\text { Episodic } \\
\text { encephalopathy }\end{array}$ & Variable & Variable & $\begin{array}{l}\text { Lowrade cerebral oedema without } \\
\text { overt signs of intracranial hypertension }\end{array}$ \\
$\begin{array}{l}\text { Persistent } \\
\text { encephalopathy } \\
\text { Minimal } \\
\text { encephalopathy }\end{array}$ & Variable & Genarally large & $\begin{array}{l}\text { Most often seen after portocaval } \\
\text { surgery or TIPS }\end{array}$ \\
\hline
\end{tabular}

TIPS, transjugular intrahepatic portosystemic shunt.

Table 1. Classification of hepatic encephalopathy. 
Minimal hepatic encephalopathy (MHE), the mildest form of HE, is characterized by subtle motor and cognitive deficits, and impairs health-related quality of life (HRQOL). ${ }^{2}$

Cirrhotic patients with MHE have a normal neurological and mental status by the standards of clinical examination, yet demonstrate quantifiable neuropsychological defects. ${ }^{3}$ The term MHE refers to the subtle changes in cognitive function, electrophysiological parameters, cerebral neurochemical/neurotransmitter homeostasis, cerebral blood flow, metabolism, and fluid homeostasis that can be observed in patients with cirrhosis who have no clinical evidence of hepatic encephalopathy. ${ }^{4}$

MHE has been described previously using several different names, such as, early, lowgrade, latent or subclinical HE to identify patients with subtle cognitive function abnormalities.

These subtle neurocognitive abnormalities primarily affect attention, speed of information processing, and motor abilities and coordination that are not recognizable on standard neurological examination. These neurocognitive abnormalities are independent of sleep dysfunction or problems with overall intelligence. . $^{5}$

It has been well-described that MHE has a subtle but negative impact on a patient's spatial skills, motor skills, the ability to perform complex tasks such as driving, and even quality of life. ${ }^{3-5}$ MHE predicts the development of overt HE and is associated with poor survival ${ }^{2}$. Its negative impact on daily living, among other reasons, has led some authors to suggest that the failure to diagnose this condition could be classified as a medical error.,, 10

\section{Epidemiology}

There are no accurate data on the incidence of HE. HE is classified as: overt hepatic encephalopathy (OHE) and MHE.

OHE occurs in $30 \%$ to $45 \%$ of cirrhotic patients ${ }^{11}$ and in $10 \%$ to $50 \%$ of patients with TIPS 12 and can be clinically diagnosed.

The true prevalence of MHE in patients with portal hypertension is inknown. MHE has been diagnosed in patients with liver cirrhosis and in patients with noncirrhotic portal hypertension. The prevalence of MHE has been reported in as many as $20 \%-84 \%$ of cirrhotics, depending on which methods or tools are used and fixed diagnostic cut-offs. 2,3 Large variations in the prevalence of MHE are related to prior episode of OHE, severity of liver disease, age, preasence of esophageal varices, and surgical porto-systemic shunts. Patients who develop MHE are older, more often have alcohol as etiology of cirrhosis, have history of overt HE in the past, have more severe liver disease, and more often have esophagogastric varices. 2,13

\section{Pathogenesis}

Despite much scientific research, the exact pathophysiological mechanisms leading to HE are not clearly understood. The most widely accepted theory of the pathogenesis of HE is that nitrogenous substances derived from the gut adversely affect the cerebral function. 


\subsection{Ammonia}

There are various explanations why liver dysfunction or portosystemic shunting might lead to encephalopathy. In healthy subjects, intestinal neurotoxins, such as ammonia, manganase and the benzodiazepine-GABA system generated by gut bacteria from food, are transported by the portal vein to the liver, where $80-90 \%$ is metabolized and/or excreted immediately. In all subtypes of hepatic encephalopathy this process is impaired, either because the hepatocytes are incapable of metabolizing the neurotoxins or because portal venous blood bypasses the liver through collateral circulation or a medically constructed shunt. Neurotoxins accumulate in the systemic circulation. Ammonia plays a key role in the pathogenesis of HE. The small molecules of ammonia cross the blood-brain barrier and are absorbed and metabolized by astrocytes, population of cells in the brain that constitutes $30 \%$ of the cerebral cortex. Alzheimer type II astrocytes are the only cells containing glutamine synthetase that metabolize ammonia. It is hypothesed that glutamine synthesis within the astrocytes causes brain swelling.14,15 Astrocytes also provide physical and nutritional support for neurons, maintain the integrity of the blood-brain barrier and regulate cerebral blood flow. 16 Ammonia also modulates glutamate neurotransmission and induces neurosteroid production in neurons, leading to a positive modulatory effect on the gammaaminobutyric acid-A receptor. ${ }^{17}$ The precise molecular mechanism(s) responsible for neurological alteration in HE are not known. HE is associated with alterations in the expression of astrocytic and neuronal genes that code for various proteins that play a critical role in central nervous system function including maintenance of cell volume and neurotransmission. ${ }^{14}$

The pathogenesis of MHE is similar to that of HE. ${ }^{18-22}$ An increase in brain glutamine and brain water is pathophysiological change associated with deterioration in neuropsychological performance. Alterations in cerebral blood flow and glucose metabolism induced by ammonia are associated with a significant decrease of glucose utilization by various cortical regions that are involved in cognitive functions. ${ }^{21}$ The cerebral metabolic rate for ammonia and the permeability-surface area product for ammonia are significantly higher in patients with MHE. ${ }^{21}$ The increased permeability-surface area product of the blood-brain barrier permits ammonia to diffuse across the blood-brain barrier into the brain more freely than normal. This may cause ammonia-induced encephalopathy even though arterial ammonia levels are normal or near normal.

Cognitive deficits observed in patients with noncirrhotic portal hypertension have also been linked to ammonia. ${ }^{18}$ Patients with noncirrhotic portal hypertension, such as extrahepatic portal venous obstruction, exhibited abnormalities in the results of neuropsychological tests, oral glutamine challenge test, and magnetic resonance (MR) imaging and spectroscopy similar to those described in HE associated with cirrhosis. ${ }^{22}$

Other waste products implicated in hepatic encephalopathy include mercaptans (substances containing a thiol group), short-chain fatty acids and phenol.23

\subsection{Serotonin}

Serotonin, a neurotransmitter with widespread distribution in the CNS, is important for the regulation of sleep, circadian rhythmicity and locomotion. Changes in the synthesis, metabolism, storage and release of neuronal serotonin in HE suggest a serotonergic synaptic 
deficit. Serotonin metabolism is exquisitely and selectively sensitive to the degree of portosystemic shunting and hyperammonaemia, suggesting a role for serotonin in early neuropsychiatric symptoms of HE. ${ }^{24}$

\subsection{Branched-chain amino acids (BCAA) and false neurotransmitters}

An imbalans between aromatic aminoacids (AAA) (phenylalanine, tryptophan and tyrosine) and branched-chain amino acids (BCAA)(leucine, isoleucine and valine) has been described in patients with severe liver dysfunction. AAA and BCAA share a common transport mechanism into the CNS. AS a consequence of increased concentration of AAA, neuronal levels may be raised leading to the production of false neurotransmitters (octopamide and phenylethanolamide) ${ }^{25}$ with subsequent development of HE. ${ }^{26}$

\subsection{Zink}

Zinc is a substrate of urea cycle enzymes. It may be depleted in patients with cirrhosis. Zinc supplementation increases the activity of ornithine transcarbamalyse increasing excretion of ammonia ions. There is conflicting clinical data regarding zinc supplementation in the management of HE. ${ }^{27,28,29}$

\subsection{Manganese}

Manganese is a neurotoxin that accumulates in the brains of patients with cirrhosis and portosystemic shunts. 30,31 Levels of manganase correlate with hyperintensity of nucleus pallidus seen on MR brain scans of patients with cirrhosis. These patients may also demonstrate extrapyramidal signs, suggesting that altered homeostasis of manganese and other minerals could be responsible for the cognitive deficits associated with liver cirrhosis.

\subsection{Systemic inflammatory response}

Iinflammatory response may be important factor that may contribute to the development of MHE and its progression to OHE. Inflammation elsewhere in the body may precipitate encephalopathy through the action of cytokines and bacterial lipopolysaccharide on astrocytes. ${ }^{32} \mathrm{~A}$ recent study reported that severity of MHE was independent of severity of liver disease and levels of blood ammonia but markers of inflammation (higher neutrophil counts, C-reactive protein levels, and interleukin-6 levels) were significantly higher in those with MHE compared to those without MHE.33 Same authors showed that induced hyperammonemia resulted in significantly greater deterioration in psychometric tests in cirrhotic patients who had an ongoing infection compared with those in whom the infection had resolved. ${ }^{34}$ These two studies suggest that inflammation plays a synergistic role with ammonia in producing and modulating MHE.

\subsection{Intestinal flora}

Intestinal flora and endotoxins are another link between inflamation, ammonia and MHE. Imbalance of intestinal flora among cirrhotics compared to normal healthy controls has been demonstrated in the study of Zhao et al. ${ }^{35}$ They found increase in the counts of aerobes (such as Enterobacter and Enterococcus) and anaerobes (such as Clostridium) and a decrease in 
the count of Bifidobacterium. The severity of imbalance in intestinal flora matched the degree of liver dysfunction. Liu et al. ${ }^{36}$ reported that cirrhotic patients with MHE had substantial derangements in the gut microecology, with significant fecal overgrowth of potentially pathogenic Escherichia coli and Staphylococcus species. Treatment with synbiotics significantly increased the fecal content of non-urease-producing Lactobacillus species at the expense of these other bacterial species. Such modulation of gut flora was associated with a significant reduction in blood ammonia levels and reversal of MHE in 50\% of patients. Synbiotic treatment was also associated with a significant reduction in endotoxemia. The CTP functional class improved in nearly $50 \%$ of the patients.

\section{Clinical characteristics}

OHE is traditionally classified into four grades according to the West Haven criteria (Table 2). ${ }^{6}$

\begin{tabular}{ll}
\hline Grade 0 & Lack of detectable changes in personality or behavior \\
Grade 1 & No asterixis \\
& Trivial lack of awareness \\
& Euphoria or anxiety \\
& Shortened attention span \\
& Impaired performance of addition \\
& Asterixis may be present \\
& Lethargy or apathy \\
Grade 2 & Minimal disorientation for time or place \\
& Subtle personality change \\
& Inappropriate behavior, slurred speech \\
& Impaired performance of subtraction \\
& Asterixis is present \\
& Somnolence to semi-stupor, but responsive to verbal stimuli \\
Grade 3 & Confusion \\
& Gross disorientation \\
Grade 4 & Asterixis is usually absent \\
& Coma (unresponsive to verbal or noxious stimuli) \\
\hline
\end{tabular}

Adapted from Mullen KD6

Table 2. West Haven criteria for semiquantitative grading of mental state

Patients with MHE have a normal neurological examination; however they may still be symptomatic. Symptoms relate to disturbances in sleep, memory, attention, concentration and other areas of cognition. ${ }^{37,38}$ A classic sign of HE is a sleep disturbance. On a sleep questionnaire, disturbance is seen in $47 \%$ of cirrhotics compared to $4.5 \%$ of controls. ${ }^{37} \mathrm{~A}$ higher frequency of sleep disturbance in cirrhotic patients with MHE has been confirmed in studies using HRQOL questionaires. ${ }^{39,40}$ Sleep disturbance in cirrhosis is not associated with cognitive impairment; thus it may not truly be an MHE symptom. Unsatisfactory sleep is associated with higher scores for depression and anxiety, raising the possibility that the effects of chronic disease may underlie the pathogenesis of sleep disturbance. ${ }^{41}$ Disturbances in cirrhotics may also be related to abnormalities of circadian rhythm. 
Defective memory may be a signe of MHE. Patients with MHE have impaired short- and long-term memory. ${ }^{38}$ This impairment is predominantly related to deficits in attention and visual perception. Memory deficit of MHE seems to comprise short-term but not long-term memory impairment. This can be described as an encoding defect, in which memory recall (or retrieval) is intact.

Several cognitive statements (i.e. complaints), have predictive value for MHE, including impaired psychomotor performance ('I have difficulty doing handwork; I am not working at all'); impaired sleep or rest ('I spend much of the day lying down in order to rest'); decreased attention ('I am confused and start several actions at a time'); and poor memory ('I forget a lot; for example, things that happened recently, where I put things, etc.').41

\section{Health-related quality of life}

\subsection{Effect of MHE on daily functioning}

MHE adversely affects HRQOL. Cognitive impairment in MHE mainly affects complex activities involving attention, information processing and psychomotor skills such as driving a car, planning a trip, etc. whereas basic activities of daily life, such as shopping, dressing, personal hygiene, etc. are preserved. $39,42,43$ Patients with MHE had a significant impairment of daily functioning, such as social interaction, alertness, emotional behavior, sleep, work, home management, recreation and pastimes compared with cirrhotic patients who did not have MHE. ${ }^{39,42}$ Treatment with lactulose improved both cognitive functions and HRQOL; improvement in the latter was linked to improvement in cognitive function. ${ }^{39}$

\subsection{Effect of MHE on driving}

MHE adversely affects driving skills. Patients with MHE have higher rates of traffic violations and motor vehicle accidents. ${ }^{41}$ Schomerus et al. ${ }^{44}$ were the first to demonstrate a negative effect of psychomotor deficits in patients with MHE on driving fitness. Similar results were reported by Watanabe et al. ${ }^{45}$ Wein et al. ${ }^{46}$ found that the fitness to drive a car was impaired in cirrhotic patients with MHE using a standarizad 90-minute on-road driving test. Increased risk of automobile accidents was related to a decline in cognitive function. 47 Impairment in attention and speed of mental processing adversely affects an individual's ability to react to unexpected traffic conditions. Patients with MHE have higher rates of traffic violations and motor vehicle accidents. ${ }^{47-50}$ Patients with MHE also had impaired navigation skills. ${ }^{51}$ Navigation, required for safe driving, is a complex process that depends on functioning working memory, attention, and speed of mental processing; impairment in navigation skills correlated with impairment in response inhibition and attention.

\section{Diagnosis of MHE}

The absence of clinical evidence of hepatic encephalopathy is key to the diagnosis of MHE and can only be determined by a detailed assessment of the patient history and a comprehensive neurological assessment of consciousness, cognitive, and motor function.

Various tools have been evaluated for the diagnosis of MHE and include the neuropsychological tests, computerized tests, short neuropsychological and computerized test batteries and neurophysiological tests. Regional cerebral blood flow changes, ${ }^{2}$ and 
magnetic resonance imaging and spectroscopy,53 though useful for understanding pathogenic mechanisms, are currently not considered of diagnostic value.

\subsection{Neuropsychological tests}

Neuropsychological testing is an established methodology for quantifying cognitive impairment due to various forms of encephalopathy, including low-grade or minimal hepatic encephalopathy. Neropsychological tests directly measure cognitive functions that are directly relevant to activities of daily living. They have been applied for the diagnosis of $\mathrm{HE}$ for more than 50 years. In the fifties, measures like the construction or reproduction of a fivepointed star (Fig.1.) or a coil and handwriting have been used for the diagnosis of HE. Someties even more complex figures were presented to the patients to be reproducted. Although all tests were able to depict an increase or decrease of the constructional ability of a patients it was extremely difficult to quantify the test result. In general, psychometric tests have to fulfill the following criteria: they have to be objective, reliable, valid and sensitive. ${ }^{54}$

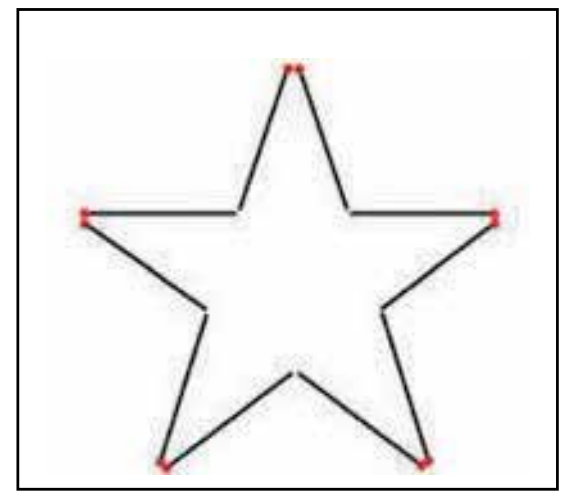

Fig. 1. Star construction test

The neuropsychological features of MHE point to a disorder of executive functioning, particularly selective attention, visuospatial abilities and fine motor skills . Although these domains are most commonly implicated in MHE, impairments of memory have also been reported. 55,56

The attentional impairments in MHE are observed on a variety of measures. These include measures of cognitive processing speed involving psychomotor responding, such as the Number Connection test A (NCT- A), the Number Connection test B (NCT- B), block design test (BDT), the Digit Symbol test (DST), Line drawing test, Circle dotting, Serial-dotting test (SDOT), Figure connection test. Impairments on measures of cognitive processing speed and response inhibition that do not require a motor response have also been reported (e.g. with verbal fluency tasks and measures such asthe Stroop test). ${ }^{57-60}$ Visuospatial impairments have been primarily reported on block design tasks ${ }^{39,61-63}$ (which also include a motor/practic component), but also on more pure measures of visuospatial perception, such as line orientation or the Hooper test. ${ }^{64,65}$ Fine motor skill impairments have been noted on measures such as the grooved pegboard task, 57,58 and on line tracing tasks (the latter also involve visuospatial abilities).66,67 
Psychometric test batteries that shall be used for the diagnosis of MHE ought to examine exactly the fields of cognition: visual perception, visuo-spatial orientation, visual construction, concentration, attention and memory. ${ }^{29}$

The small number of neuropsychological tests represent the cerebral disfunction of MHE. This were: the Number Connection test A (NCT- A), the Number Connection test B (NCTB), block design test (BDT), the Digit Symbol test (DST), Line drawing test, Circle dotting. Time-tested with well recognized clinical significance, established.

The number connection test (NCT) is the most widely used test in the psychometric assessment of cirrhotic patients. It was found to be capable of detecting mild mental dysfunction in cirrhotic patients.

The NCT-A (Fig.2) is a test of visuo-spatial orientation and psychomotor speed. The subject is shown a sheet of paper with 25 numbered circles which are randomly spread over the paper. The task is to connect the circles from 1-25 as quick as possible. Test result is the time needed by the subject including error correction time. ${ }^{68}$

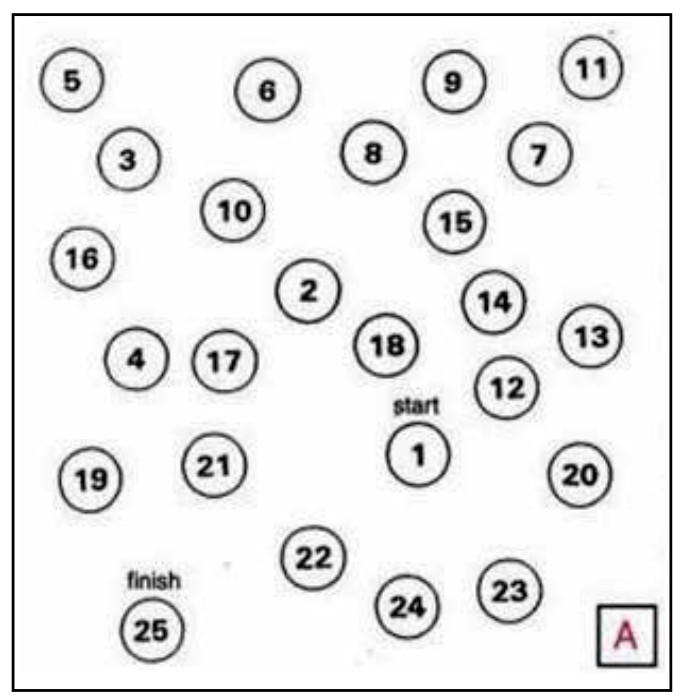

Fig. 2. Number Connection test A (NCT-A) ${ }^{68}$

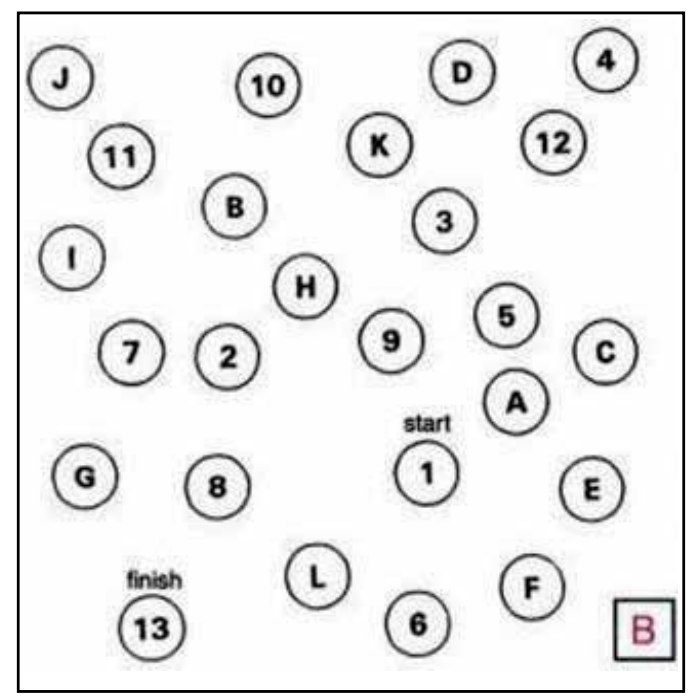

Fig. 3. Number Connection test B (NCT- B $)^{68}$

The NCT-B (Fig.3) is quite similar.The circles include the numbers from 1-13 and the letters from A-L. The subjects are asked to connect numbers and letters in alternating manner, that means go from 1-A-2-B-3-C and so on. Test result is the time needed including error correction time. Besides visuo-spatial orientation and psychomotor speed this test is appropriate to study the ability to shift attention. 68

The Block Design Test (BDT) is a test of visuo-spatial and motor skills (Fig.4). The task is to take 6- 9 blocks that have all white sides, all red sides, and red and white sides and arrange them according to a pattern formed by examiner or shown on a card. Scored for speed and accuracy. 69 


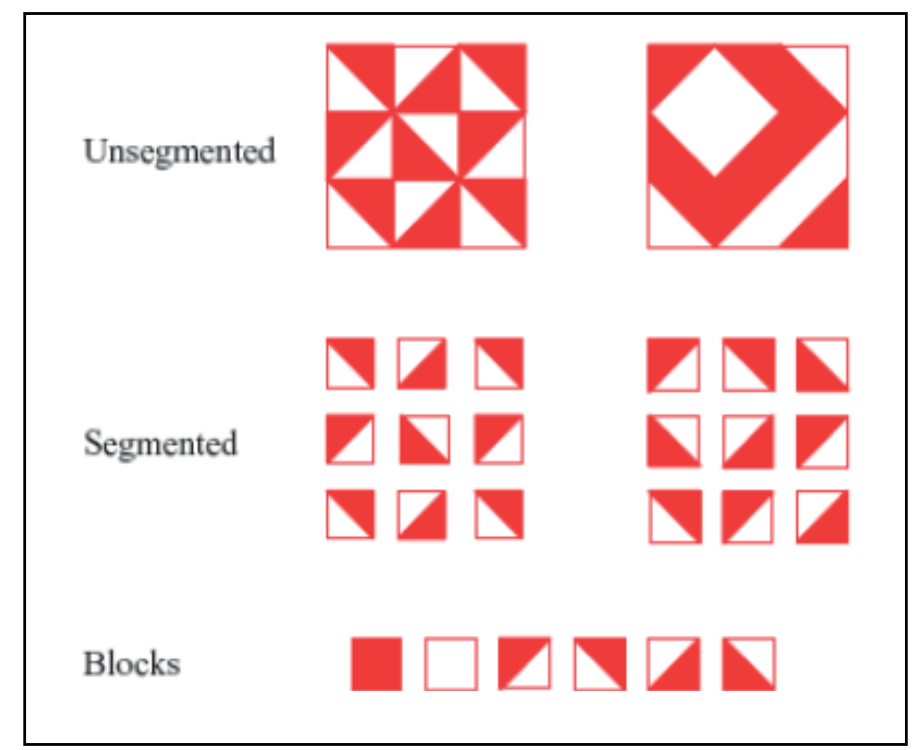

Fig. 4. The Block Design Test (BDT) ${ }^{69}$

The Digit Symbol test (DST) (Fig. 5) - the subject is given a series of double-boxes with a number given in the upper part. The task is to draw a symbol pertinent to this number into the lower part of the boxes. Nine fixed pairs of numbers and symbols are given at the top of the test sheet. Test result is the number of boxes correctly filled within 90 seconds. Pathological test results indicate a deficit in visuo-constructive abilities, especially. ${ }^{70}$

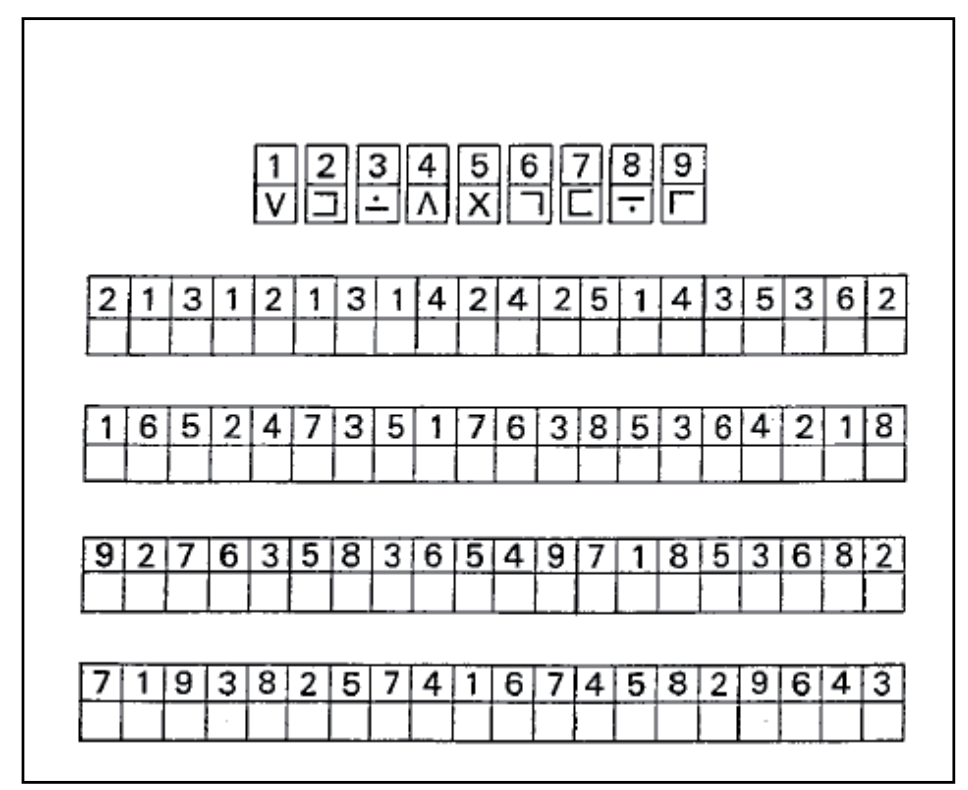

Fig. 5. The Digit Symbol test (DST) 70 
The line drawing test (Fig. 6) is a test of motor speed and accuracy. The patients have to follow the route of this labyrinth without crossing or even touching the borderlines. ${ }^{70}$

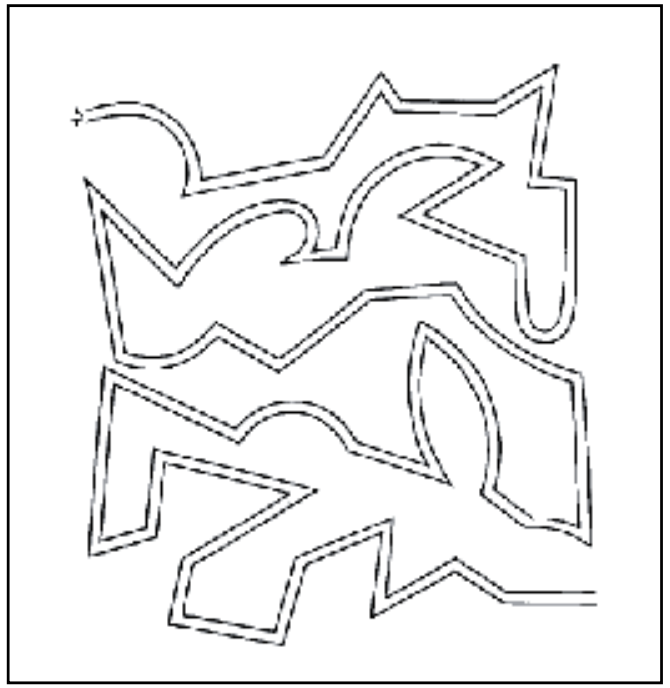

Fig. 6. The line drawing test

For the assessment of the test result the whole route is devided into small sections (Fig.7) and each touching or crossing the border in a section is counted. The number of mistakes and the time needed to go through the labyrinth, both, are test results. ${ }^{70}$

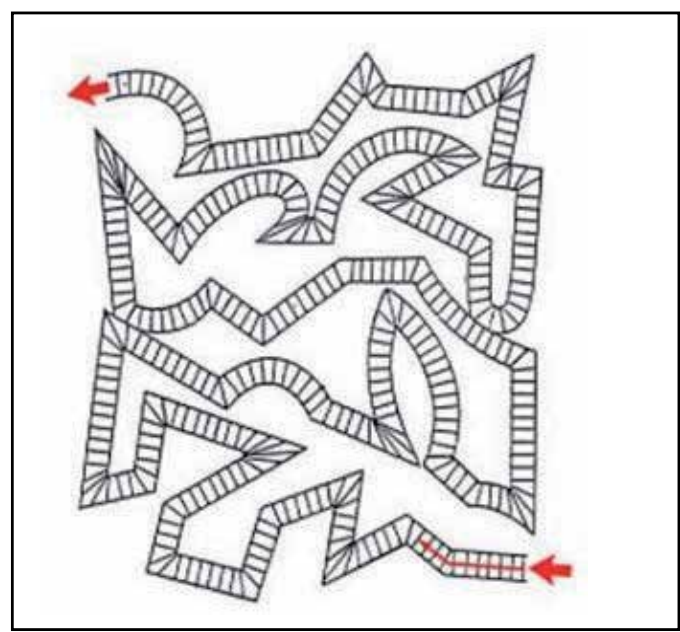

Fig. 7. Division of the whole route of the line drawing test for the assessment of the errors.

The circle dotting test (Fig.8) is the most simple test of the battery. It is a test of pure motor speed. The subjects are asked to put a dot in each of the 100 circles given on the sheet, after they have prepared by dotting the 20 circles at the top of the sheet, first. Test result is the time needed. 


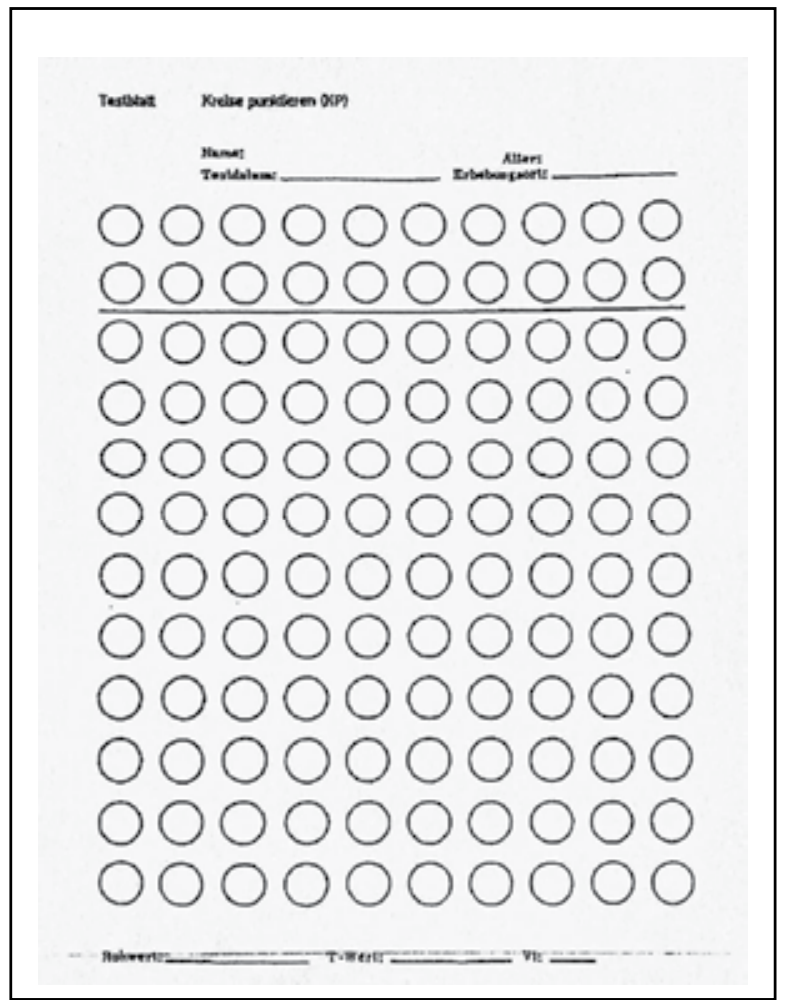

Fig. 8. The circle dotting test

The Working Party recommends that the diagnosis of MHE requires a normal mental status examination and impairment in the performance of at least two of the following tests: NCTA, or figure connection test-A (FCT-A), NCT-B, BDP, DST.41

In 2009, the Commission on Neuropsychological Assessment of Hepatic Encephalopathy concluded that neuropsychological test batteries aimed at measuring multiple domains of cognitive function are generally more reliable than single tests, and tend to be more strongly correlated with functional status.71 Both the Repeatable Battery for the Assessment of Neuropsychological Status (RBANS) ${ }^{72}$ and PSE-Syndrom-Test ${ }^{73}$ may be used for this purpose.

The PSE-Syndrom-Test, developed in Germany and validated in several other European countries, incorporates older assessment tools such as NCT-A and NCT-B, the line-tracing test (LTT), the serial-dotting test (SDT), and DST.

The psychometric hepatic encephalopathy score (PHES) is a standardized test battery including NCT-A and B, the line-tracing test for time $(t)$ and error $(e)$, the serial-dotting test, and the digit symbol test. This battery examines many of the abnormalities seen in patients with MHE, including motor speed and accuracy, visuo-spatial orientation, visual perception, visual construction, attention, concentration, and, to a lesser extent, memory. PHES has a prognostic value for the occurrence of overt HE and mortality in cirrhotic patients. ${ }^{74,75}$ 
The RBANS contains measures of verbal and visual anterograde memory, working memory, cognitive processing speed, language (including semantic fluency) and visuospatial function (line orientation and figure copy). It is a portable pencil-and-paper test that requires a

folding stimulus booklet and paper record form to administer. Administration time is approximately 20-25 min. ${ }^{71}$ In the study of Sorrel et al. ${ }^{76}$ RBANS scores were strongly correlated with liver disease as measured by the model for end-stage liver disease staging. Scores on the RBANS also predicted disability independently of liver disease severity in this study.

Use of either the RBANS or the PSE-Syndrom-Test is recommended for diagnosing and monitoring minimal hepatic encephalopathy. The choice of which battery to use should be based upon the availability of local translations and normative data. ${ }^{71}$

\subsection{Neurophysiological tests}

Quantitative neurophysiologic tools include Simple electroencephalography (EEG), evoked potentials (auditory, visual, Somatosensory) and P300 (type of auditory evoked potential).

Changes in EEG/evoked responses are non-specific.

The major finding on EEG is a general decrease in wave frequency and an increase in wave amplitude. First, socalled theta-waves with a frequency between 4 and 7 cps occur, then these theta waves predominate and are committed by delta waves with a frequency of 1-3 cps. Preterminally there is a loss of wave amplitude and a flattening of the curve. These abnormalities may be found even in cirrhotics without clinical signs of encephalopathy. There is no close correlation between the grade of HE and the degree of EEG abnormalities..$^{70}$ The sensitivity of the EEG for the diagnosis of subclinical HE is limited compared to psychometric tests. ${ }^{70}$ The EEG is useful for follow-up examinations, predominantly.

Among EEG variations, the most sensitive test is computer-assisted analysis, including the mean dominant EEG frequency and the power of a particular rhythm. ${ }^{77,78}$ Quantified-EEG has a prognostic value for occurrence of bouts of overt $\mathrm{HE}$ and mortality in cirrhotic patients. ${ }^{78}$

Evoked potentials are subdivided into the group of exogenous evoked potentials and endogenous evoked potentials. The exogenous evoked potentials like the flash or checkerboard visual evoked potentials (VEP), brainstem auditory evoked potentials (BAEP) and somatosensory evoked potentials are used to examine the function of sensory pathways. The endogenous evoked potentials are measures of cognitive function. In the only study that compares the different exogenous evoked potentials for their diagnostic ensitivity in hepatic encephalopathy, the BAEP were the most sensitive measure for the diagnosis of HE. ${ }^{79}$

Among evoked responses, the P300 peak obtained in an auditory oddball paradigm is the most sensitive test.80-83 These tests can supplement neurological or neuropsychiatric examination. It has been demonstrated that there was a greater likelihood of development of overt HE in cirrhotic patients with abnormal P300 event-related potential latencies and NCT than in patients with no such abnormality. ${ }^{80}$ 
Neurophysiological tests can be used during follow up to demonstrate change in a patient's condition. Their major limitations are: (i) need for specialized equipment and technical expertise for evaluation and interpretation; and (ii) inability to perform these tests in an outpatient clinic. ${ }^{41}$

The changes observed in cerebral blood flow and metabolism in SPET, PET, and ${ }^{1} \mathrm{H}$ and ${ }^{31} \mathrm{P}$ MRS studies reflect the pathogenic process that underlies the condition rather than providing diagnostic information. Similarly, the morphological brain abnormalities identified in this population, including mild brain oedema, hyperintensity of the globus pallidus and other subcortical nuclei observed in cerebral MR studies, and the central and cortical atrophy observed in neural imaging studies, are unlikely to have diagnostic utility. 4

\subsection{Computerized tests}

Computerized psychometric tests measuring both the reaction time and the accuracy of performing tasks requiring psychomotor speed, attention, short-term memory, or choice ability.

Critical flicker frequency (CFF) tests the ability of a patient to perceive flickering and its fusion threshold. The CFF threshold measures visual discrimination and general arousal. ${ }^{84}$ CFF is a simple, reliable and accurate method for the diagnosis of MHE. The technique shows little dependence on age, education or training. ${ }^{75,85}$

Inhibitory control test (ICT) is a computerized test of attention and response inhibition that has been used to characterize attention deficit disorder, schizophrenia and traumatic brain injury. ICT has been validated for the diagnosis of MHE in USA and found to be reliable and sensitive for detection as well as follow-up of patients with MHE. 86

\subsection{Magnetic resonance imaging and spectroscopy}

Magnetic resonance imaging (MRI) identified the morphological brain abnormalities in the population of patients with cirrhosis, including mild brain oedema, hyperintensity of the globus pallidus and other subcortical nuclei, and central and cortical atrophy. High-signal abnormalities on T1-weighted images in the globus pallidum have been observed in cirrhotic patients, even without clinical evidence of HE. Deposition of manganese is regarded as the most likely explanation of this high-signal abnormality. ${ }^{87}$ There is no direct correlation between pallidal hyperintensity and grade of encephalopathy. ${ }^{88}$ Basal ganglia T1-weighted signal intensity and manganese accumulation appear to be related to the underlying degree of portal-systemic shunting rather than directly to neuropsychiatric impairment. 90 Hyperintense globus pallidus on MRI is common in patients with liver cirrhosis and also occurs in patients with noncirrhotic portal hypertension.

Magnetic resonance spectroscopy (MRS) shows a decrease in myo-inositol/creatine and choline/creatine ratios in the white matter with an increase in the Glx (glutamine and glutamate) concentration in the basal ganglia in patients with MHE.91,92 Liver transplantation as well as lactulose therapy have been shown to reverse these changes at 4 weeks and later after transplantation. ${ }^{91}$ However, the ability of MRS to differentiate between cirrhotic patients without HE and those with MHE has not been conclusively shown. ${ }^{41}$

Diffusion-weighted imaging allows assessment of intracellular and extracellular water content in the brain, which helps in differentiating cytotoxic from vasogenic edema. ${ }^{93}$ 
Diffusion tensor imaging has revealed that mean diffusivity, a measure of water movement across cell membranes, is significantly higher in patients with MHE in the regions of the corpus callosum, internal capsules, caudate nuclei and occipital white matter. Increase in mean diffusivity indicates the presence of interstitial brain edema. Mean diffusivity values increase as the grade of HE increases, suggesting that brain edema present in patients with HE may contribute to its pathogenesis. ${ }^{94}$ Mean diffusivity values decreased significantly and there was a corresponding improvement in neuropsychological test scores in patients with MHE after three weeks of lactulose therapy. ${ }^{94} \mathrm{MR}$ imaging techniques therefore complement neuropsychological evaluation of MHE.41

\section{Diagnostic criteria for MHE}

The diagnostic criteria for MHE have not been standardized but rest on careful patient history and physical examination, normal mental status examination, demonstration of abnormalities in cognition and/or neurophysiological function, and exclusion of concomitant neurological disorders.

No consensus on diagnostic criteria or diagnostic tests has been established.

- Cirrhotic patients

- Without clinical signs of encephalopathy

- Perform worse in psychometric tests when compared with healthy controls

- Affects an estimated $60 \%(50 \% \text { to } 80 \%)^{*}$ of patients with cirrhosis

- Cerebral dysfunction has a major impact on patients' daily living

- The presence of a disease that can cause MHE, such as, cirrhosis and/or the presence of a portalsystemic shunt

- normal mental status on clinical examination

- demonstration of abnormalities of cognition and/or neurophysiological variables

- exclusion of concomitant neurological disorders.

The INASL Working Party recommends that all patients with cirrhosis be screened for the presence of MHE using a standard battery of psychometric tests, PHES, CFF or ICT, depending upon the availability of tests and their validation for local populations from different parts of the world (Fig. 1). Patients whose index psychometric or computerized test results do not indicate pathology should be screened every 6-12 months.

It has been shown conclusively that cognitive functions improve with therapy for MHE.3,62-67 Such therapy may improve HRQOL of patients with MHE3,67 and delay the development of HE.68 Hence all patients with liver cirrhosis should be subjected to testing for MHE. Special attention should be given to those who have cognitive symptoms and high-risk groups such as active drivers, patients handling heavy machines or reporting decline in work performance.

\section{Natural history}

\subsection{Development of overt hepatic encephalopathy}

Patients with MHE may improve, remain unchanged or deteriorate and develop overt HE over a long-term follow-up. 
The frequency of MHE increases as the severity of liver disease increases.4,13-16,18,22 In view of a high frequency of MHE in patients with liver disease, it is important to understand its impact on future clinical outcomes, such as occurrence of overt HE, quality of life and survival, and to determine whether treatment of MHE can induce improvements in these outcomes.

Several studies that looked at the frequency of development of overt HE in cirrhotic patients found that those with MHE developed overt HE more often during follow up than those without MHE (Table 4).4,15,17,20,48,88,89 In addition, some studies have shown an increased risk of death in patients with liver cirrhosis and MHE compared to those without MHE (Table 4).20,22,88 However, patients with MHE had poorer liver function than those without MHE in these studies, making it difficult to ascribe the poor outcome to the presence of MHE. Das et al.4 studied the relationship of progression of MHE to overt HE in relation to the severity of liver dysfunction and found that the rate of progression to overt HE was much higher in patients with MHE and a CTP score $>6$ than in those with MHE and a CTP score _ 6 . Amodio et al.88 found that the presence of MHE and that of liver dysfunction were both associated with mortality on univariate analysis; however, on multivariate analysis, liver functional status was the only independent predictor of mortality. In another study, progression of MHE to overt HE was associated with abnormal response to oral glutamine challenge, which in turn was associated with poor liver function.90 Furthermore, MHE in patients with preserved liver function but large portalsystemic shunts (congenital shunts, non-cirrhotic portal hypertension and cirrhosis with preserved liver function) appears to have a good outcome, even though these data are based on a small number of patients.10 Thus, it appears that the higher risk of overt HE or death in patients with MHE may not be related to MHE per se but to the poorer liver function in patients with MHE.

\section{Survival}

Current data suggest that patients with MHE tend to have more frequent episodes of overt $\mathrm{HE}$ and poorer survival than in those without MHE, and indicate that patients with MHE have a more advanced liver disease. Child-Turcotte-Pugh score and PHES were associated with a poor prognosis

\section{Treatment}

Ammonia plays a key role in the pathogenesis of MHE Empiric therapy is based on the principle of reducing the production and absorption of ammonia in the gut-a number of agents are beneficial for this purpose.

\subsection{Nutritional interventions}

The European Society for Parenteral and Enteral Nutrition recommended, in 2006, that patients with cirrhosis must eat at least $1.2 \mathrm{~g} / \mathrm{kg}$ of protein daily. They also recommended that the diet of patients with cirrhosis should be supplemented with branched-chain amino acids (BCAAs) and vegetable protein once HE has developed. Vegetable-based protein is better tolerated by patients with cirrhosis than meat-based protein . 


\subsection{Pharmacological therapy}

Non-absorbable disaccharides include lactulose and lactitol. Treatment for MHE may be initiated with lactulose; patients should receive 30-60 mL of lactulose in two or three divided doses so that they pass two to three semi-soft stools per day. Although the appropriate duration of therapy for MHE is unsettled, at least three studies suggest that treatment may be advised for 3-6 months. $(3,67,95)$

Lactulose decreases blood ammonia levels, and improves psychometric performance and HRQOL (Table 5).3,59,62,64,67,91-95 Using cerebral diffusion tensor imaging, Kale et al.59 showed that interstitial brain edema observed in patients with MHE resolves after treatment for 3 weeks with lactulose in parallel with improvements in neuropsychiatric performance.

Prasad et al.3 studied the effect of treatment of MHE with lactulose on psychometric performance (measured by NCT, FCT-A, FCT-B, picture completion and block-design tests) and HRQOL (measured by Sickness Impact Profile [SIP]). Patients with MHE showed significant impairment in 11 scales of the SIP, the psychosocial and physical subscores, and in the total SIP. Patients received $30-60 \mathrm{~mL}$ of lactulose in two or three divided doses so that the patient passed two to three semi-soft stools per day. Following lactulose therapy for 3 months, both psychometric performance and HRQOL improved; MHE reversed in $64.5 \%$ of treated patients compared with $6.7 \%$ in the no-treatment group $(P<0.0001)$. Significant improvement was found in five (emotional behavior, ambulation, mobility, sleep/rest and recreation and pastimes) of the 12 scales of the SIP and in the total psychosocial and physical sub-scores in the treated patients compared with the untreated patients. Improvement in HRQOL was linked to improvement in cognitive function. A recent study that compared lactulose, a probiotic and LOLA with no treatment, confirmed these findings.67 Lactulose or lactitol, both non-absorbable, synthetic disaccharides with multiple effects on gut flora, are regarded as intestinal prebiotics.96 Dietary addition of lactulose can exert a bifidogenic effect accompanied by a favorable effect on colonic $\mathrm{NH}_{3}$ metabolism.97 Ameta-analysis of randomized trials of lactulose versus placebo or no intervention in treatment of patients with MHE showed that the treatment with lactulose was associated with improvement in psychometric (cognitive) performance. 35

Branched-chain amino acids, flumazenil, L-ornithine L-aspartate, acetyl L-carnitine, and probiotics/synbiotics. A majority of these attempts were aimed at reducing blood ammonia level, and most studies have shown improvement in psychometric measurements, ammonia levels, cerebral edema and health-related quality of life (HRQoL)

\subsection{Prebiotics, probiotics or synbiotics}

Prebiotics, probiotics or synbiotics (probiotics and fermentable fiber) are effective in treating patients with MHE,63-67 and can also be used as long-term therapy. Liu et al.65 showed that modulation of gut microecology and acidification of gut lumen in patients with liver cirrhosis and MHE by treatment with synbiotics resulted in increased fecal content of nonurease-producing Lactobacillus species, whereas the number of urease-producing pathogenic Escherichia coli and Staphylococcal species decreased. This effect persisted for 14 days after cessation of supplementation. It was associated with a significant reduction in blood ammonia and endotoxin levels and reversal ofMHEin nearly $50 \%$ of the patients. The severity of liver disease, as assessed according to CTP class, also improved in nearly $50 \%$ of 
the patients. In a recent randomized control trial, supplementation with probiotic yogurt resulted in a significant reversal ofMHEin the group receiving yogurt compared

to no treatment.63 Treatment with a probiotic preparation also improves HROQL.67 Prebiotics, probiotics or synbiotics are efficacious in the treatment of HE by decreasing bacterial urease activity, $\mathrm{pH}$ in the gut lumen, ammonia absorption and total ammonia in the portal blood, and by improving nutritional status of gut epithelium resulting in decreasing intestinal permeability. In addition, they help ameliorate the inflammation and oxidative stress in the hepatocytes, leading to increased hepatic clearance of ammonia.98 These mechanisms may be additive or synergistic in treating MHE. Probiotics may represent a safe, effective, long-term therapy for MHE and may be an alternative to lactulose.

\subsection{L-ornithine-L-aspartate}

Clinical studies evaluating the role of LOLA in the treatment of MHE did not show its effectiveness; however, these studies were small and underpowered. A recent study that compared lactulose, a probiotic and LOLA with no treatment, however, showed that LOLA is as effective as lactulose or a probiotic preparation in improving psychometric performance and HRQOL.67 Larger prospective studies are warranted to evaluate the role of LOLA before it can be recommended for the treatment of MHE.

\subsection{Antibiotics}

The role of antibiotics in MHE has not been evaluated. Prospective studies with poorly absorbed antibiotics are required to evaluate their efficacy in improving MHE.

Antibiotics: Rifaximin, Neomycin

\section{References}

[1] Häussinger D, Blei AT.Portal hypertension and its complications. In : Rodes J, Benhamou JP, Blei AT, Reichen J, Rizzetto M, eds. Textbook of hepatology. From basic science to clinical practice, third edition. Oxford: Blackwell Publishing ; 2007: 623-760

[2] Dhiman RK, Chawla YK. Minimal hepatic encephalopathy. Indian J Gastroenterol 2009; 28:5-16

[3] Prasad S, Dhiman RK, Duseja A, Chawla YK, Sharma A, Agarwal R. Lactulose improves cognitive functions and health-related quality of life in patients with cirrhosis who have minimal hepatic encephalopathy. Hepatology 2007; 45: 549-59

[4] Tan HH, Lee GH, Thia KTJ, Ng HS, Chow WC, Lui HF. Minimal hepatic encephalopathy runs a fluctuating course: results from a three-year prospective cohort follow-up study. Singapore Med J 2009; 50(3): 255-260

[5] Amodio P, Montagnese S, Gatta A , Morgan MY. Characteristics of minimal hepatic encephalopathy. Metabolic Brain Disease 2004; 19: 253-267

[6] Arguedas MR, DeLawrence TG, McGuire BM. Influence of hepatic encephalopathy on health-related quality of life in patients with cirrhosis. Dig Dis Sci 2003; 48: 16221626

[7] Mullen KD. Review of the final report of the 1998 Working Party on definition, nomenclature and diagnosis of hepatic encephalopathy. Aliment Pharmacol Ther 2007;25:11-16 
[8] Kharbanda PS, Saraswat VA, Dhiman RK. Minimal hepatic encephalopathy: diagnosis by neurophchological and neurophysiological methods. Indian J Gastroenterol 2003; 22: 537-541

[9] Ortiz M, Jacas C, Cordoba J. Minimal hepatic encephalopathy: diagnosis, clinical signifi cance and recommendations. J Hepatol 2005;42: 45-53

[10] Lockwood AH. “What's in a name?" Improving the care ofcirrhotics. J Hepatol 2000; 32: 859-861

[11] Quero Guillén JC, Groeneweg M, Jiménez Sáenz M, Schalm SW, Herrerías Gutiérrez JM. Is it medical error if we do not screen cirrhotic patients for minimal hepatic encephalopathy? Rev Esp Enferm Dig 2002; 94: 544-557

[12] Amodio P, Del Piccolo F, Petteno E, et al. Prevalence and prognostic value of quantified electroencephalogram (EEG) alterations in cirrhotic patients. J Hepatol 2001; 35: 37-45

[13] Boyer TD, Haskal ZJ, American Association for the Study of Liver Diseases. The role of transjugular intrahepatic portosystemic shunt in the management of portal hypertension. Hepatology 2005; 41: 386-400

[14] Kurmi R, Reddy K, Dhiman RK, et al. Psychometric hepatic encephalopathy score, critical flicker frequency and p300 event-related potential for the diagnosis of minimal hepatic encephalopathy: Evidence that psychometric hepatic encephalopathy score is enough. Indian J Gastroenterol 2008; 27: S1

[15] Butterworth RF. Pathophysiology of hepatic encephalopathy: a new look at ammonia. Metab Brain Dis 2002; 17: 221-227

[16] Vaquero J, Chung C, Blei AT. Brain edema in acute liver failure. A window to the pathogenesis of hepatic encephalopathy. Ann Hepatol 2003; 2: 12-22

[17] Takano T, Tian GF, Peng W, et al. Astrocyte-mediated control of cerebral blood fl ow. Nat Neurosci 2006; 9: 260-270

[18] Ahboucha S, Butterworth RF. The neurosteroid system: implication in the pathophysiology of hepatic encephalopathy. Neurochem Int 2008; 52: 575-587

[19] Sharma P, Sharma BC, Puri V, Sarin SK. Minimal hepatic encephalopathy in patients with extrahepatic portal vein obstruction. Am J Gastroenterol 2008;103: 1406-1412

[20] Balata S, Damink SW, Ferguson K, et al. Induced hyperammonemia alters neuropsychology, brain MR spectroscopy and magnetization transfer in cirrhosis. Hepatology 2003; 37: 931-939

[21] Cordoba J, Alonso J, Rovira A, et al. The development of low-grade cerebral edema in cirrhosis is supported by the evolution of (1)H-magnetic resonance abnormalities after liver transplantation. J Hepatol 2001; 35: 598-604

[22] Lockwood AH, Yap EW, Wong WH. Cerebral ammonia metabolism in patients with severe liver disease and minimal HE. J Cereb Blood Flow Metab 1991; 11:337-341

[23] Kale RA, Gupta RK, Saraswat VA, et al. Demonstration of interstitial cerebral edema with diffusion tensor MR imaging in type $C$ hepatic encephalopathy. Hepatology 2006; 43: 698-706

[24] Chung RT, Podolsky DK2005. Cirrhosis and its complications. In Kasper DL, Braunwald E, Fauci AS,eds. Harrison's Principles of Internal Medicine, 16th edition. New York, NY: McGraw-Hill;2005: 1858-69

[25] Lozeva-Thomas V. Serotonin brain circuits with a focus on hepatic encephalopathy. Metab Brain Dis 2004; 19:413-20

[26] Capocaccia L, Cangiano C, Cascino A, Calcaterra V,Cardelli P, Rossi FF. Influence of phenylethanolamine on octopamine plasma determination in hepatic encephalopathy. Clin Chim Acta 1979; 93:371-6. 
[27] Fischer JE, Rosen HM, Ebeid AM, James JH, Keane JM,Soeters PB. The effect of normalization of plasma amino acids on hepatic encephalopathy in man. Surgery 1976;80:77-91.

[28] Yoshida Y, Higashi T, Nouso K, Nakatsukasa H, Nakamura SI, Watanabe A, et al. Effects of zinc deficiency/zinc supplementation on ammonia metabolism in patients with decompensated liver cirrhosis. Acta Med Okayama 2001; 55:349-55.

[29] Marchesini G, Fabbri A, Bianchi G, Brizi M, Zoli M. Zinc supplementation and amino acid-nitrogen metabolism in patients with advanced cirrhosis. Hepatology 1996; 23:1084-92.

[30] Cash WJ, McConville P, McDermott E, McCormick PA, Callender ME, McDougall NI. Current concepts in the assessment and treatment of hepatic encephalopathy. QJM 2010;103: 9-16

[31] Das K, Singh P, Chawla Y, Duseja A, Dhiman RK, Suri S. Magnetic resonance imaging of brain in patients with cirrhotic and non-cirrhotic portal hypertension. Dig Dis Sci 2008; 53: 2793-2798

[32] Rama Rao KV, Reddy PV, Hazell AS, Norenberg MD. Manganese induces cell swelling in cultured astrocytes. Neurotoxicology 2007; 28:807-812

[33] Sundaram V, Shaikh OS. Hepatic encephalopathy: pathophysiology and emerging therapies. Med Clin North Am 2009;3: 819-36

[34] Shawcross DL, Wright G, Olde Damink SW, Jalan R. Role of ammonia and inflammation in minimal hepatic encephalopathy. Metab Brain Dis 2007; 22: 125138

[35] Shawcross DL, Davies NA, Williams R, Jalan R. Systemic inflammatory response exacerbates the neuropsychological effects of induced hyperammonemia in cirrhosis. J Hepatol 2004; 40: 247-254.

[36] Zhao HY, Wang HJ, Zhi LU, Zhen XU. Intestinal microflora in patients with liver cirrhosis. Chin. J. Dig. 2004; 5: 64-7.

[37] Liu Q, Duon ZP, Ha DK et al. Synbiotic modulation of gut flora:effect on minimal hepatic encephalopathy in patients with cirrhosis.Heptology 2004; 39: 1441-9.

[38] Cordoba J, Cabrera J, Lataif L, Pener P, Zee P, Blei AT. High prevalence of sleep disturbances in cirrhosis. Hepatology 1998; 27: 339-45.

[39] Weissenborn K, Heidenreich S, Giewekemeyer K, Ruckert N, Hecker H. Memory function in early hepatic encephalopathy. J.Hepatol. 2003; 39: 320-5.

[40] Prasad S, Dhiman RK, Duseja A, Chawla YK, Sharma A, Agarwal R. Lactulose improves cognitive functions and health-related quality of life in patients with cirrhosis who have minimal hepatic encephalopathy. Hepatology 2007; 45: 549-59.

[41] Goeneweg M, Moerland W, Quero JC. Screening of subclinical hepatic encephalopathy. J. Hepatology 2000; 32: 748-53.

[42] Dhiman R, Saraswat V, Sharma B, et al. Minimal hepatic encephalopathy: Consensus statement of a working party of Indian National Association for study of the liver. Journal of Gastroenterology and Hepatology 2010;25: 1029-41.

[43] Groeneweg M, Quero JC, De Bruijn I et al. Subclinical hepatic encephalopathy impairs daily functioning. Hepatology 1998; 28:45-9.

[44] Schomerus H, Hamster W. Quality of life in cirrhotics with minimal hepatic encephalopathy. Metab. Brain. Dis. 2001; 16:37-41.

[45] Schomerus H, Hamster W, Blunck H, Reinhard U, Mayer K, Dolle W. Latent portasystemic encephalopathy. I. Nature of cerebral functional defects and their effect on fitness to drive. Dig. Dis. Sci.1981; 26: 622-30. 
[46] Watanabe A, Tuchida T, Yata Y, Kuwabara Y. Evaluation of neuropsychological function in patients with liver cirrhosis with special reference to their driving ability. Metab. Brain. Dis. 1995;10: 239-48.

[47] Wein C, Koch H, Popp B, Oehler G, Schauder P. Minimal hepatic encephalopathy impairs fitness to drive. Hepatology 2004; 39:739-45.

[48] Marotolli RA, Cooney LM, Wagner S, Doucette J, Tinetti ME. Predictors of automobile crashes and moving violations among elderly drivers. Ann. Intern. Med. 1994; 121: 842-6.

[49] Bajaj JS, Hafeezullah M, Hoffmann RG, Saeian K. Minimal hepatic encephalopathy: a vehicle for accidents and traffic violations. Am. J. Gastroenterol. 2007; 102: 1903-9.

[50] Bajaj JS, Ananthakrishnan AN, McGinley EL, Hoffmann RG, Brasel KJ. Deleterious effect of cirrhosis on outcomes after motor vehicle crashes using the nationwide inpatient sample. Am. J. Gastroenterol. 2008; 103: 1674-81.

[51] Bajaj JS, Saeian K, Schubert CM et al. Minimal hepaticencephalopathy is associated with motor vehicle crashes: the reality beyond the driving test. Hepatology 2009; 50: $1175-83$.

[52] Bajaj JS, Hafeezullah M, Hoffmann RG et al. Navigation skill impairment: Another dimension of the driving difficulties in minimal hepatic encephalopathy. Hepatology 2008; 47: 596-604.

[53] Venktaramarao SH, Mittal, Prabhakar S, Dhiman RK. Brain perfusion single photon emission computed tomography (SPECT) abnormalities in patients with minimal hepatic encephalopathy (abstract). J Gastroenterol Hepatol 2008; 23 : A62

[54] Grover VP, Dresner MA, Forton DM, et al. Current and future applications of magnetic resonance imaging and spectroscopy of the brain in hepatic encephalopathy. World J Gastroenterol 2006; 12: 2969-2978.

[55] Weissenborn K. Diagnosis of subclinical hepatic encephalopathy. Med Sci Monit 1999; 5: 568-575.

[56] Bahceci F, Yildirim B, Karincaoglu M, Dogan I, Sipahi B. Memory impairment in patients with cirrhosis. J Natl Med Assoc 2005; 97:213-6.

[57] Meyer T, Eshelman A, Abouljoud M. Neuropsychological changes in a large sample of liver transplant candidates. Transplant Proc 2006; 38: 3559-60.

[58] McCrea M, Cordoba J, Vessey G, Blei AT, Randolph C. Neuropsychological characterization and detection of subclinical hepatic encephalopathy. Arch Neurol 1996; 53: 758-63.

[59] Tarter RE, Van Thiel DH, Arria AM, Carra J, Moss H. Impact of cirrhosis on the neuropsychological test performance of alcoholics. Alcohol Clin Exp Res 1988; 12: 619-21.

[60] Binesh N, Huda A, Thomas MA, et al. Hepatic encephalopathy: a neurochemical, neuroanatomical, and neuropsychological study. J Appl Clin Med Phys 2006; 7: 8696.

[61] Mattarozzi K, Stracciari A, Vignatelli L, et al. Minimal hepatic encephalopathy: longitudinal effects of liver transplantation. Arch Neurol 2004; 61: 242-7.

[62] Gitlin N, Lewis DC, Hinkley L. The diagnosis and prevalence of subclinical hepatic encephalopathy in apparently healthy, ambulant, non-shunted patients with cirrhosis. J Hepatol 1986; 3: 75-82.

[63] Sood GK, Sarin SK, Mahaptra J, Broor SL. Comparative efficacy of psychometric tests in detection of subclinical hepatic encephalopathy in nonalcoholic cirrhotics: search for a rational approach. Am J Gastroenterol 1989; 84: 156-9. 
[64] Tarter RE, Hegedus AM, Van Thiel DH, et al. Nonalcoholic cirrhosis associated with neuropsychological dysfunction in the absence of overt evidence of hepatic encephalopathy. Gastroenterology 1984; 86: 1421-7.

[65] Binesh N, Huda A, Thomas MA, et al. Hepatic encephalopathy: a neurochemical, neuroanatomical, and neuropsychological study. J Appl Clin Med Phys 2006; 7: 86-96.

[66] Mittal VV, Sharma P, Sharma BC, Sarin S. Treatment of minimal hepatic encephalopathy: A randomised controlled trial comparing lactulose, probiotics and l-ornithine l-aspartate with placebo. Hepatology 2009; 50: 471A

[67] Minguez B, Garcia-Pagan JC, Bosch J, et al. Noncirrhotic portal vein thrombosis exhibits neuropsychological and MR changes consistent with minimal hepatic encephalopathy. Hepatology 2006;43: 707-14.

[68] Schomerus H, Hamster W. Neuropsychological aspects of portalsystemic encephalopathy. Metab Brain Dis 1998; 13: 361-77.

[69] Kugler CF, Petter J, Taghavy A, et al. Dynamics of cognitive brain dysfunction in patients with cirrhotic liver disease: an event-related P300 potential perspective. Electroencephalogr Clin Neurophysiol 1994; 91: 33-41.

[70] Conn HO: Trailmaking and Number Connection Tests in the Assessment of Mental State in Portal Systemic Encephalopathy. Dig Dis, 1977; 22(6): 541-50.

[71] Loguercio C, Del Vecchio-Blanco C, Coltorti M: Psychometric Tests and Latent PortalSystemic Encephalopathy. Brit J Clin Pract 1984; 38: 407-11.

[72] Weissenborn K. Diagnosis of subclinical hepatic encephapolaphy. Med Sci Monit 1999; 5:568-575.

[73] Randolph C, Hilsabeck R, Kato A, et al. Neuropsychological assessment of hepatic encephalopathy: ISHEN practice guidelines. Liver International 2009;629-35.

[74] Randolph C. The Repeatable Battery for the Assessment of Neuropsychological Status (RBANS). San Antonio: The Psychological Corporation, 1998.

[75] Schomerus H, Weissenborn K, Hamster W, Ruckert N, Hecker H. PSE-Syndrom-Test. Frankfurt: Swets \& Zeitlinger B.V., 1999.

[76] Dhiman RK, Kurmi R, Thumburu KK et al. Diagnosis and prognostic significance of minimal hepatic encephalopathy in patients with cirrhosis of liver. Dig. Dis. Sci. 2010; 55:2381-90.

[77] Romero-Gómez M, Córdoba J, Jover R et al. Value of the critical flicker frequency in patients with minimal hepatic encephalopathy.Hepatology 2007; 45: 879-85.

[78] Sorrell JH, Zolnikov BJ, Sharma A, Jinnai I. Cognitive impairment in people diagnosed with end-stage liver disease evaluated for liver transplantation. Psychiatry Clin Neurosci 2006; 60: 174-81.

[79] Amodio P, Quero JC, Del Piccolo F, Gatta A, Schalm SW. Diagnostic tools for the detection of subclinical hepatic encephalopathy: comparison of standard and computerized psychometric tests with spectral-EEG. Metab. Brain. Dis. 1996; 11:315-27.

[80] Amodio P, Del Piccolo F, Pettenò E et al. Prevalence and prognostic value of quantified electroencephalogram (EEG) alterations in cirrhotic patients. J. Hepatol. 2001; 35: 37-45.

[81] Mehndiratta MM, Sood GK, Sarin SK, Gupta M: Comparative evaluation of visual, somatosensory, and auditory evoked potentials in the detection of subclinical hepatic encephalopathy in patients with nonalcoholic cirrhosis. Am J Gastroenterol 1990; 85: 799-803

[82] Saxena N, Bhatia M, Joshi YK, Garg PK, Dwivedi SN, Tandon RK. Electrophysiological and neuropsychological tests for the diagnosis of subclinical hepatic encephalopathy and prediction of overt encephalopathy. Liver. 2002; 22: 190-7. 
[83] Amodio P, Valenti P, Del Piccolo F et al. P300 latency for the diagnosis of minimal hepatic encephalopathy: evidence that spectral EEG analysis and psychometric tests are enough. Dig. Liver. Dis. 2005; 37: 861-8.

[84] Amodio P, Gatta A. Neurophysiological investigation of hepatic encephalopathy. Metab. Brain. Dis. 2005; 20: 369-79.

[85] Saxena N, Bhatia M, Joshi YK et al. Auditory P300 event-related potentials and number connection test for evaluation of subclinical hepatic encephalopathy in patients with cirrhosis of the liver: a follow-up study. J. Gastroenterol. Hepatol. 2001; 16: 322-7.

[86] Kircheis G, Wettstein M, Timmermann L et al. Critical flicker frequency for quantification of low-grade hepatic encephalopathy. Hepatology 2002; 35: 357-66.

[87] Sharma P, Sharma BC, Puri V, Sarin SK. Critical fl icker frequency: diagnostic tool for minimal hepatic encephalopathy. J Hepatol 2007;47:67-73.

[88] Bajaj JS, Hafeezullah M, Franco J et al. Inhibitory control test for the diagnosis of minimal hepatic encephalopathy. Gastroenterology 2008; 135: 1591-600.

[89] Binesh N, Huda A, Bugbee $\mathrm{M}$ et al. Adding another spectraldimension to $1 \mathrm{H}$ magnetic resonance spectroscopy of hepatic encephalopathy. J. Magn. Reson. Imaging. 2005; 21:398-405.

[90] Weissenborn K, Ehrenheim C, Hori A, Kubicka S, Manns MP.Pallidal lesions in patients with liver cirrhosis: clinical and MRI evaluation. Metab. Brain. Dis. 1995; 10: 219-31.

[91] Rose C, Butterworth RF, Zayed J et al. Manganese deposition in basal ganglia structures results from both portal-systemic shunting and liver dysfunction. Gastroenterology 1999; 117: 640-4.

[92] Yadav SK, Srivastava A, Srivastava A et al. Encephalopathy assessment in children with extra-hepatic portal vein obstruction with MR, psychometry and critical flicker frequency. J. Hepatol. 2010; 216: 683-91.

[93] Naegele T, Grodd W, Viebahn R et al. MR imaging and (1)H spectroscopy of brain metabolites in hepatic encephalopathy: time-course of renormalization after liver transplantation. Radiology 2000; 52: 348-54.

[94] Nie YQ, Zeng Z, Li YY, Sha WH, Ping L, Dai SJ. Long-term efficacy of lactulose in patients with subclinical hepatic encephalopathy. Zhonghua. Nei. Ke. Za. Zhi. 2003; 42: 261-263

[95] Weissenborn K, Ahl B, Fischer-Wasels D et al. Correlations between magnetic resonance spectroscopy alterations and cerebral ammonia and glucose metabolism in cirrhotic patients with and without hepatic encephalopathy. Gut. 2007; 56: 1736-42.

[96] Lodi R, Tonon C, Stracciari A et al. Diffusion MRI shows increased water apparent diffusion coefficient in the brains of cirrhotics. Neurology 2004; 62: 762-6.

[97] Kale RA, Gupta RK, Saraswat VA et al. Demonstration of interstitial cerebral edema with diffusion tensor MR imaging in type $C$ hepatic encephalopathy. Hepatology 2006; 43: 698-706.

[98] Caron M-J et al. Brain 2006; 129: 1789-1802

[99] Ferenci P, Lockwood A, Mullen K, Tarter R, Weissenborn K, Blei AT. Hepatic encephalopathy - definition, nomenclature, diagnosis, and quantification: fi nal report of the working party at the 11th World Congresses of Gastroenterology, Vienna, 1998. Hepatology 2002; 35: 716-721

[100] Das A, Dhiman RK, Saraswat VA, Naik SR. Prevalence and natural history of subclinical hepatic encephalopathy in cirrhosis. J Gastroenterol Hepatol 2001; 16: 531-535 


\title{
Uremic Encephalopathy
}

\author{
Annemie Van Dijck, Wendy Van Daele and Peter Paul De Deyn \\ University of Antwerp \\ Belgium
}

\section{Introduction}

Patients with renal failure often have signs and symptoms related to fluid and electrolyte disturbances, anemia, malnutrition, bone disease and gastrointestinal problems. One of those complications is the uremic encephalopathy. With the introduction of dialysis and renal transplantation, the incidence and severity of uremic encephalopathy have declined, but many patients fail to fully respond to dialytic therapy. In patients with renal failure, encephalopathy is a common problem that may be caused by uremia, thiamine deficiency, dialysis, transplant rejection, hypertension, fluid and electrolyte disturbances or drug toxicity (Mahoney \& Arieff, 1982). In this chapter, the symptoms, pathophysiology and treatment of uremic encephalopathy will be discussed. Other neurological complications of renal failure are not discussed in this chapter, but were recently reviewed (Brouns \& De Deyn, 2004).

\section{Clinical presentation}

Uremic encephalopathy may accompany any form of severe acute or chronic renal failure. The clinical features appear to be related to the rate of development of renal failure. In patients with acute renal failure the symptoms are generally more pronounced and progress more rapidly than in patients with chronic renal failure (Aminoff, 1995; Burn \& Bates, 1998).

The symptoms begin insidiously and are often not noticed by the patients but by their family members or caregivers. Most encephalopathies are reversible, making prompt recognition and treatment important. After hemodialysis, significant improvement of uremic encephalopathy occurs, but the level of azotemia correlates poorly with the degree of neurological dysfunction (Burn \& Bates, 1998).

\subsection{Mental status}

Encephalopathy is a global cerebral dysfunction, often in the absence of primary structural brain disease. Nevertheless, in some contexts it can also lead to permanent brain injury, while in other cases it is reversible. It can be due to direct injury to the brain, or illness remote from the brain. In medical terms it can refer to a wide variety of brain disorders with very different etiologies, prognoses and implications.

Uremic encephalopathy usually presents with alterations in mental status fluctuating from mild sensorial clouding to delirium and coma. Impaired attention can be tested by simple 
bedside tasks such as serial subtraction or naming months of the year in reverse. Other common findings include a disturbed sleep-wake cycle, decreased alertness, hypervigilance, hallucinations, sensory misperceptions, impaired memory and disorientation. The thought process is often disorganized and conversation is confused. Apathy, fatigue, irritability and inattentiveness are usually the initial symptoms while confusion, disturbances of sensory perception, hallucinations and stupor appear later. The level of alertness reflects the severity of the encephalopathy, coma being the most serious stage (Chen, 1996; Earnest, 1993).

\subsection{Associated symptoms}

Besides the alterations in mental status, other associated symptoms are often present. Clouding of the sensorium is almost always associated with mild diffuse weakness and a variety of motor disturbances. Tremor is common, but other involuntary movements such as fasciculations, multifocal myoclonus, chorea, asterixis or seizures are seen in patients at various times. Tremor is usually coarse and irregular at a rate of 8-10 Hz. Asterixis or flapping tremor is a dramatic problem, with jerking movements arising from lapses of posture holding, as of the outstretched hands. It is almost always bilateral. Unilateral asterixis suggests an occult structural lesion. Multifocal myoclonus is characterized by sudden, non-rhythmic, gross muscle twitching, particularly involving the face and the proximal muscles (Chen, 1996).

Besides the general symptom complex of encephalopathy, headache, focal motor signs and the "Uremic twitch convulsive" syndrome can be observed (Aminoff, 1995; De Deyn et al., 1992b). Focal neurological signs such as hemiparesis, dysarthria, visual abnormalities or reflex asymmetry tend to be transient and alternate from side to side (Bolton, 1990). Other common associated symptoms include uremic polyneuropathy, pruritus -often leading to self induced skin lesions-, and restless-legs syndrome. All these signs fluctuate from day to day or sometimes from hour to hour (Aminoff, 1995).

\section{Diagnostic investigations}

A laboratory investigation for encephalopathy includes a complete blood count, electrolyte panel and examination of glucose, urea, creatinine, liver enzymes and ammonia. No laboratory value, including specific evaluations of the renal function, correlates well with clinical symptoms and signs of uremia. Lumbar puncture often reveals elevated protein and occasionally a mild pleocytosis. A lumbar puncture is primarily performed to exclude an infectious cause of encephalopathy. CT or MRI of the head are only indicated when focal signs are present on physical examination and to exclude the presence of a subdural hematoma, ischemic stroke or hydrocephalus. Electroencephalographic (EEG) findings in uremic encephalopathy are non-specific but correlate with clinical symptoms and, therefore, may be of diagnostic value. In addition, it can be useful to exclude other causes of confusion such as infection or structural abnormalities. The most common EEG finding is a generalized slowing of the normal background. Intermittent frontal rhythmic theta activity and paroxysmal, bilateral, high voltage delta waves are also frequent.. Sometimes bilateral spike-waves complexes or triphasic waves in the frontal regions are found (Fig. 1). Convulsions are often a late stage manifestation of chronic renal failure. Seizures are usually generalized tonic-clonic convulsions. Nevertheless, focal motor seizures are not uncommon. Epilepsia partialis continua may occur without generalized seizures (Brenner, 1985). 
In patients with neurologically asymptomatic chronic renal disease, impaired cognitive processing can be disclosed by event-related potentials. Increase in P3 latency and decrease in P3 amplitude is found (Aminoff, 1995; Burn \& Bates, 1998).

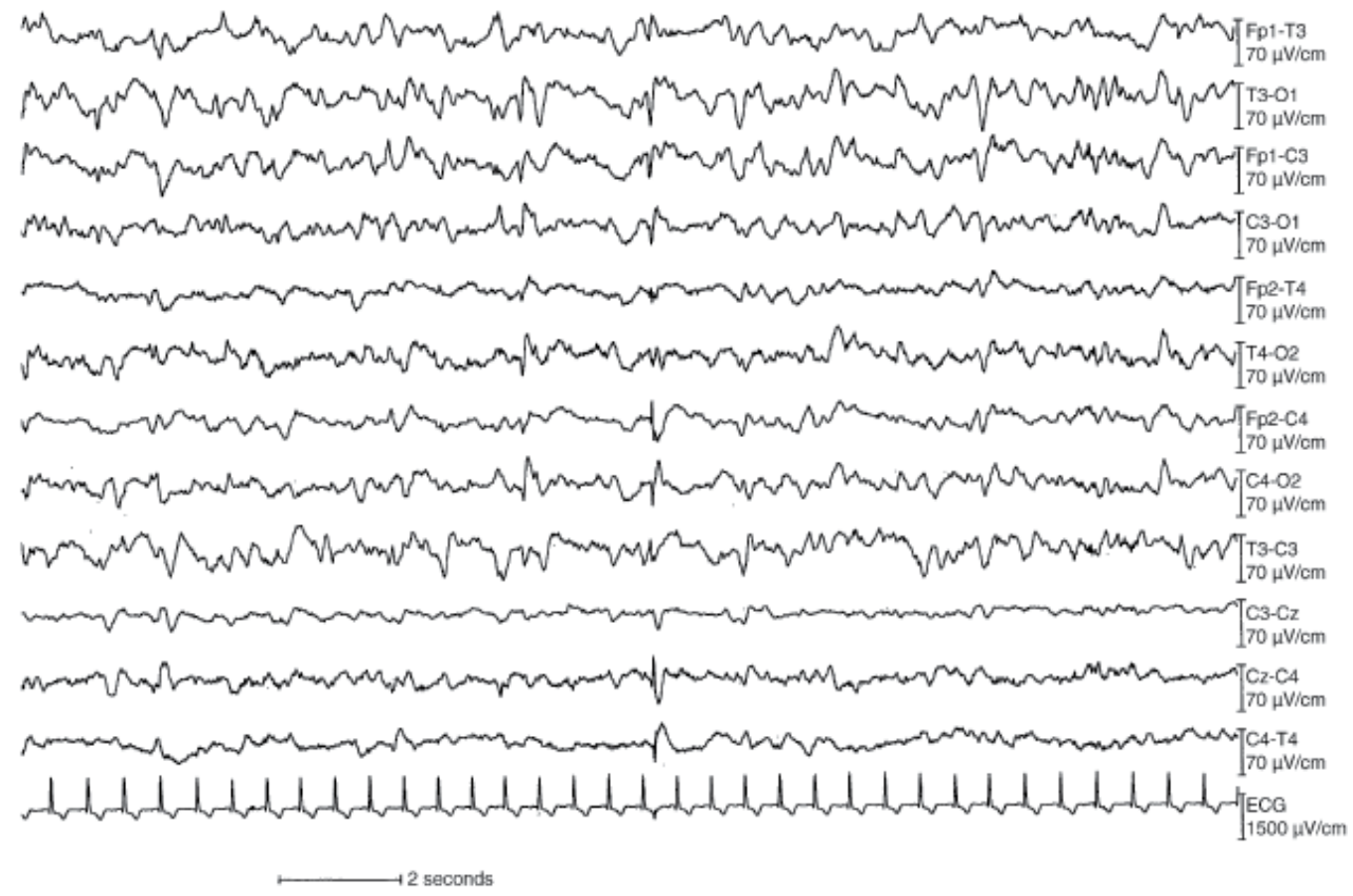

Fig. 1. Electroencephalographic findings in a patient with uremic encephalopathy, showing generalized slowing with an excess of delta and theta waves and bilateral spikes.

\section{Pathophysiology}

All forms of acute toxic-metabolic encephalopathy interfere with the function of the ascending reticular activating system and its projections to cerebral cortex, leading to impairment of arousal and awareness (Plum, 1982). The neurophysiologic mechanisms of encephalopathy include interruption of polysynaptic pathways and altered excitatoryinhibitory amino acid balance. Accumulation of metabolites, hormonal disturbance, disturbance of the intermediary metabolism and imbalance in excitatory and inhibitory neurotransmitters have been identified as contributing factors.

\subsection{Uremic toxins}

Renal failure results in accumulation of numerous organic substances that possibly act as uremic neurotoxins, but no single metabolite has been identified as the sole cause of uremia (Vanholder et al., 2003b). Symptoms are usually alleviated by dialysis or successful renal transplantation. Accumulation of urea, guanidino compounds, uric acid, hippuric acid, various amino acids, polypeptides, polyamines, phenols and conjugates of phenol, phenolic and indolic acids, acetone, glucuronic acid, carnitine, myoinositol, sulphates and phosphates has been reported in the literature (Enomoto et al., 2003; Topczewska-Bruns et al., 2002). 
By some sources, uremic retention solutes are subdivided into three major classes: 1) small solutes (<500 Da) with no known protein binding; 2) solutes with known or likely protein binding and 3) middle molecules ( $\geq 500 \mathrm{Da}$ ). This classification is based on the characteristics that potentially influence their removal pattern during dialysis. Concentrations of 90 uremic solutes and ratios between mean uremic and normal concentration were reported by Vanholder et al. (2003a). Their meta-analysis illustrates the complexity of uremic retention. Not all solutes are retained to the same extend, and their retention is often not in correlation with the current markers, urea and creatinine. This is due to their molecular weight, protein binding, and/or multicompartmental behavior (Vanholder et al., 2001; Vanholder \& De Smet, 1999). In addition, a high concentration is not necessarily related to a strong biologic activity. For example, the two molecules with the highest concentration (urea and creatinine) are known for their relatively limited biologic activity (Vanholder et al., 2001; Vanholder \& De Smet, 1999). This indicates that removal strategies should be designed in such a way that not only the standard molecules, but also other molecules that might be important in the deterioration of the clinical condition, can be removed efficiently.

Urea has been used as a marker of uremic retention and removal for several years (Gotch \& Sargent, 1985), and its removal is directly correlated with patient survival (Owen, Jr. et al., 1993). Nevertheless, there are very few studies demonstrating a direct biologic impact of urea at currently encountered uremic concentrations (Vanholder \& De Smet, 1999), and those studies show an impact that not necessarily concentrates on key organic functions in the biochemical/biological status of the human body. When urea was added to the dialysate during a period of several months at concentrations largely exceeding those currently encountered in dialyzed uremics, uremic symptomatology was not consistently altered over the entire study period (Johnson et al., 1972), again suggesting that urea by itself is not very important in the development of uremic toxicity. It is difficult to explain the apparent paradox between the validity of urea as a marker and its presumed lack of toxicity. Of note, urea removal seems to be related as a surrogate marker only indirectly to survival, and not to quality of life. One possibility to consider is that urea removal by itself does not affect survival, but that it is representative for the removal of one or more other solutes with a more consistent impact. One such potential culprit is potassium, another small-water soluble compound known to substantially affect dialytic survival (Bleyer et al., 1999). Another possibility is that, together with urea, other uremic solutes antagonizing its toxic impact are retained (Lee et al., 1991). Finally, urea might be at the origin of other, more toxic moieties, such as some of the guanidines or carbamylation products (De Deyn et al., 2003; De Deyn et al., 2009; Stim et al., 1995; Vanholder \& De Smet, 1999) .

Other metabolic disturbances which may or may not be correlated with the intensity of cerebral dysfunction are acidosis, hyponatremia, hyperkalemia, hypocalcemia, hypermagnesemia, hyperhydration and dehydration (Bierman, 1970). However, the clinical features of uremic encephalopathy do not correlate precisely with any single laboratory change. On the other hand, symptoms are usually alleviated by dialysis and are generally relieved almost entirely after successful renal transplantation (Brenner et al., 1982; Raskin \& Fishman, 1976; Teschan \& Arieff, 1985).

\subsection{Guanidino compounds}

Among the guanidino compounds, guanidinosuccinic acid, methylguanidine, guanidine and creatinine were found to be highly increased in serum, cerebrospinal fluid and brain of 
uremic patients (De Deyn et al., 2001). It is postulated that these compounds may contribute to the epileptic and cognitive symptoms accompanying uremic encephalopathy (D'Hooge et al., 1992b; D'Hooge et al., 1992a; Pan et al., 1996). Activation of the excitatory N-methyl-daspartate (NMDA) receptors and concomitant inhibition of inhibitory $\gamma$-aminobutyric acid $(\mathrm{GABA})_{\mathrm{A}}$-ergic neurotransmission have been proposed as underlying mechanisms (De Deyn et al., 2001). This will be further explained in paragraphs 4.2.1 and 4.2.2.

In addition, transketolase is a thiamine-dependent enzyme of the pentose phosphate pathway that is found predominantly in the myelinated structures of the nervous system and has been reported to have a critical role in the maintenance of axon-cylinder myelin sheats (Dreyfus, 1965; Yonezawa \& Iwanami, 1966). This enzyme was shown to be significantly inhibited by plasma, cerebrospinal fluid and low molecular weight $(<500$ dalton) dialysate fractions obtained from patients with uremia (Sterzel et al., 1971). It is also of interest that in uremic subjects, transketolase activity of erythrocytes was found to be below normal but increasing following dialytic therapy. Guanidinosuccinic acid was capable of reproducing this inhibition which might underlie demyelinative changes contributing to both central and peripheral nervous system changes in chronic uremia (Lonergan et al., 1971). Moreover, other guanidino compounds, such as guanidine and methylguanidine, have been shown in vivo, in experimental animals, to induce clinical alterations comparable to those observed in uremia. Methylguanidine induced a syndrome similar to the uremic encephalopathy including epilepsy and symptoms similar to the uremic "twitch-convulsive" syndrome (Giovannetti et al., 1969; Matsumoto et al., 1976; Minot \& Dodd, 1933; Mori, 1987). In decreasing potency, guanidinosuccinic acid, methylguanidine, guanidine and creatinine inhibited responses to GABA and glycine on mouse neurons in cell culture (De Deyn \& Macdonald, 1990). The same order of epileptogenic potency was found for these uremic guanidine compounds in behavioral studies (D'Hooge et al., 1992b). Guanidinosuccinic acid brain concentrations in this chemical model of epilepsy were comparable to the levels observed in uremic brain (De Deyn et al., 1992a). The effects on inhibitory neurotransmission might, in combination with the other effects exerted by these toxins, underlie the pathogenesis of the myoclonus and epilepsy. Moreover, guanidinosuccinic acid was shown to inhibit excitatory synaptic transmission in CA1 region of rat hippocampal slices; this is an effect that might contribute to the cognitive symptomatology presenting in uremic encephalopathy (D'Hooge et al., 1991).

Guanidino compounds are produced as a result of protein and amino acid metabolism. Specific guanidino compounds were found to accumulate in biologic fluids and tissues of uremic patients. Their levels have been determined in serum, urine and cerebrospinal fluid of non-dialyzed and dialyzed renal insufficient patients. Four highly increased compounds are creatinine, guanidine, guanidinosuccinic acid and methylguanidine. In addition, accumulation of asymmetric (ADMA) and symmetric (SDMA) dimethylarginine was reported. In the case of guanidinosuccinic acid, increased cerebrospinal fluid concentrations of severely uremic patients were found to be as high as 350 times the mean concentration in controls.

In addition, guanidino compounds are found to stimulate leukocytes, with methylguanidine and guanidinoacetic acid significantly enhancing the lipopolysaccharide-stimulated production of tumor necrosis factor- $\alpha$ by normal monocytes (Glorieux et al., 2004) and SDMA enhancing the monocytic burst via store-operated calcium influx (Schepers et al., 2009). In addition, guanidino compounds also modify albumin structure in such way that 
they decrease the protein binding of homocystein (Perna et al., 2004). The resulting free active homocysteine consequently contributes to cardiovascular damage.

Impaired cognition and epileptic symptomatology are the most typical manifestations of uremic encephalopathy. However, it is not entirely clear which of the putative uremic toxins are responsible for these central nervous system complications in uremia. Probably, the complications are due to the combined effects of different neurotoxic compounds. Guanidino compounds may play an important role in the etiology of uremic encephalopathy and they might contribute to the hyperexcitability of the uremic brain. A possible mechanism is described in the next paragraph.

\subsubsection{Effects of uremic guanidino compounds on amino acid receptors}

The four most increased uremic guanidino compounds induced clonic-tonic convulsions in adult mice (D'Hooge et al., 1992b). Guanidinosuccinic acid and methylguanidine were markedly more potent convulsants than guanidine and creatinine. Brain concentrations corresponding with the intraperitoneal CD50 (convulsive dose in 50\%) of these convulsants were $1328 \mathrm{nmol} / \mathrm{g}$ tissue for creatinine, $209 \mathrm{nmol} / \mathrm{g}$ tissue for guanidine, $56 \mathrm{nmol} / \mathrm{g}$ tissue for guanidinosuccinic acid, and $94 \mathrm{nmol} / \mathrm{g}$ tissue for methylguanidine. Apparently, brain creatinine and guanidinosuccinic acid concentrations, corresponding with intraperitoneal doses that induce clonic convulsions in mice, are similar to the concentrations found in brain of uremic patients. However, creatinine only induced myoclonic jerking and slight convulsions, whereas guanidinosuccinic acid induced vigorous generalized clonic and tonic convulsions. The convulsive concentrations in mice of guanidine and methylguanidine were higher than those found in uremic brain. Guanidinosuccinic acid is highly increased in uremic serum, cerebrospinal fluid, and brain. This compound was shown by our group to be an experimental convulsant (D'Hooge et al., 1992b; D'Hooge et al., 1992a). In addition, it appears to be the uremic guanidino compound most likely to play an important role in the etiology of the hyperexcitability of uremic brain. The compound induced clonic and tonic convulsions as well as epileptiform electrocorticographic discharges in adult mice (D'Hooge et al., 1992a).

Neuroexcitatory effects of these compounds might be due to their actions at inhibitory and excitatory amino acid receptors. The four uremic guanidino compounds blocked GABAand glycine-evoked depolarization in mouse spinal cord neurons in primary dissociated cell cultures (De Deyn \& Macdonald, 1990). Guanidinosuccinic acid was shown to be the most potent compound, whereas methylguanidine, guanidine, and creatinine (in decreasing order) blocked GABA and glycine responses less potently. It was suggested that the uremic guanidino compounds might be blocking the $\mathrm{GABA}_{\mathrm{A}}$ and glycine receptor-associated chloride channel (De Deyn et al., 1991). Later studies using the patch clamp technique suggested that guanidinosuccinic acid, methylguanidine, and creatinine may rather act as competitive antagonists at the transmitter recognition site of the $\mathrm{GABA}_{\mathrm{A}}$ receptor $\left(\mathrm{D}^{\prime}\right.$ Hooge et al., 1999). Depending on the clamping potential, GABA-evoked outward or inward whole-cell currents, which were blocked by the $\mathrm{GABA}_{\mathrm{A}}$ receptor antagonist bicuculline. Guanidinosuccinic acid, methylguanidine, and creatinine dose-dependently block these GABA-evoked whole-cell currents (D'Hooge et al., 1999). Guanidinosuccinic acid was shown to be more potent than methylguanidine or creatinine, but all three blocked inward as well as outward GABA-evoked current. The $\mathrm{GABA}_{\mathrm{A}}$ and glycine receptor antagonism that was shown in in vitro experiments, might underlie the convulsive action of the uremic 
guanidino compounds in vivo and might contribute to the epileptic symptomatology in uremia. However, in the case of guanidinosuccinic acid induced clonic convulsions, antiepileptic drugs like diazepam or phenobarbital did not or only slightly attenuate these convulsions (D'Hooge et al., 1992a; D'Hooge et al., 1993). Competitive and noncompetitive NMDA receptor antagonists, on the other hand, did effectively block these convulsions (D'Hooge et al., 1993). Also, guanidinosuccinic acid potentiated NMDA- but not glutamateor kainate- induced convulsions. These findings suggested that, in addition to the blockade of GABAergic inhibition, NMDA receptors were somehow involved in the guanidinosuccinic acid induced convulsions. The hypothetical activation of NMDA receptor by guanidinosuccinic acid was first corroborated by Reynolds and Rothermund (Reynolds \& Rothermund, 1992). They found that creatinine, guanidine and methylguanidine blocked the NMDA receptor-associated ionophore in a similar manner to magnesium, but that guanidinosuccinic acid was able to enhance $\left[{ }^{3} \mathrm{H}\right]$ dizocilpine binding to rat brain membranes, and increase intracellular $\left[\mathrm{Ca}^{2+}\right]$ in rat forebrain neurons. Both latter effects are indicative of agonist actions of guanidinosuccinic acid at the NMDA receptor. We found behavioral and electrophysiological evidence that guanidinosuccinic acid (but not methylguanidine) acts as a selective agonist at NMDA-type excitatory amino acid receptors in a similar manner to the structurally related L-aspartate (D'Hooge et al., 1996). Guanidinosuccinic acid was shown to abolish the excitatory postsynaptic potential recorded from CA1 region in rat hippocampal slices (D'Hooge et al., 1991; D'Hooge et al., 1996). The inhibition of this effect by a selective NMDA receptor antagonist indicated that this was probably due to NMDA receptormediated depolarization of hippocampal neurons (D'Hooge et al., 1996). Pan et al. (Pan et al., 1996) demonstrated that intrahippocampal injection of guanidinosuccinic acid in rats induces epileptiform electrographic discharges, and leads to hippocampal damage, which could be blocked by treatment with the NMDA receptor antagonist ketamine. It is indeed well established that the application of NMDA agonists, even in amounts that are not immediately toxic, induce neurodegeneration. Excessive calcium influx through NMDA receptor-associated ion channels leads to loss of mitochondrial and nuclear function, activation of proteases and other calcium-dependent enzymes, and ultimate excitotoxic cell death. The effect of intrahippocampal guanidinosuccinic acid injection on both (cognitive) behavior and hippocampal volume in mice was investigated as well (Torremans et al., 2005). A significant dose-dependent effect of intrahippocampal injection of guanidinosuccinic acid on cognitive performance, activity, and social exploratory behavior was observed. Volume of hippocampal cornu ammonis region decreased significantly and dose-dependently after guanidinosuccinic acid injection. Systemic guanidinosuccinic acid injection increased cGMP concentration in hippocampal formation. Knowledge of neurotoxic effects and mechanisms of action of guanidinosuccinic acid and other uremic retention solutes could help in the development of more efficient treatment of uremic patients.

\subsubsection{Hypothetical mechanism of neuroexcitation by uremic guanidino compounds}

Based on the results summarized above, a hypothetical mechanism for the action of uremic guanidino compounds on glutamatergic transmission in the central nervous system was proposed by De Deyn et al. (De Deyn et al., 2009) (Fig. 2). A simplified model of the Schaffer collateral-pyramidal cell synapse in the CA1 region of the rodent hippocampus was used (Collingridge \& Lester, 1989). In a changed form, the proposed mechanism might also apply to other glutamatergic pathways. The mechanism could explain the neuroexcitatory and 
convulsant actions of guanidinosuccinic acid (and other uremic guanidino compounds) in experimental animals, but it might also link uremic guanidino compounds to uremiaassociated epileptic symptomatology. In CA1 region, fast synaptic events are carried by two kinds of ionotropic excitatory amino acid receptors: a-amino-3-hydroxyl-5-methyl-4isoxazole-propionate (AMPA) and NMDA receptors (Collingridge \& Lester, 1989). Both receptor types react to endogenously released L-glutamate. During low-frequency transmission, the NMDA receptor- associated ionic channel is voltage dependently blocked by $\mathrm{Mg}^{2+}$. Low-frequency activation of AMPA receptors does elicit $\mathrm{Na}^{+}$influx, but this depolarizing current does not provide sufficient membrane depolarization to reduce the $\mathrm{Mg}^{2+}$ block on the NMDA receptor. GABAergic interneurons mediate powerful feedforward as well as feedback synaptic inhibition. Endogenously released GABA binds to GABA receptors, activating the ligand-gated ionic channel of the receptor, and eliciting hyperpolarizing chloride influx.

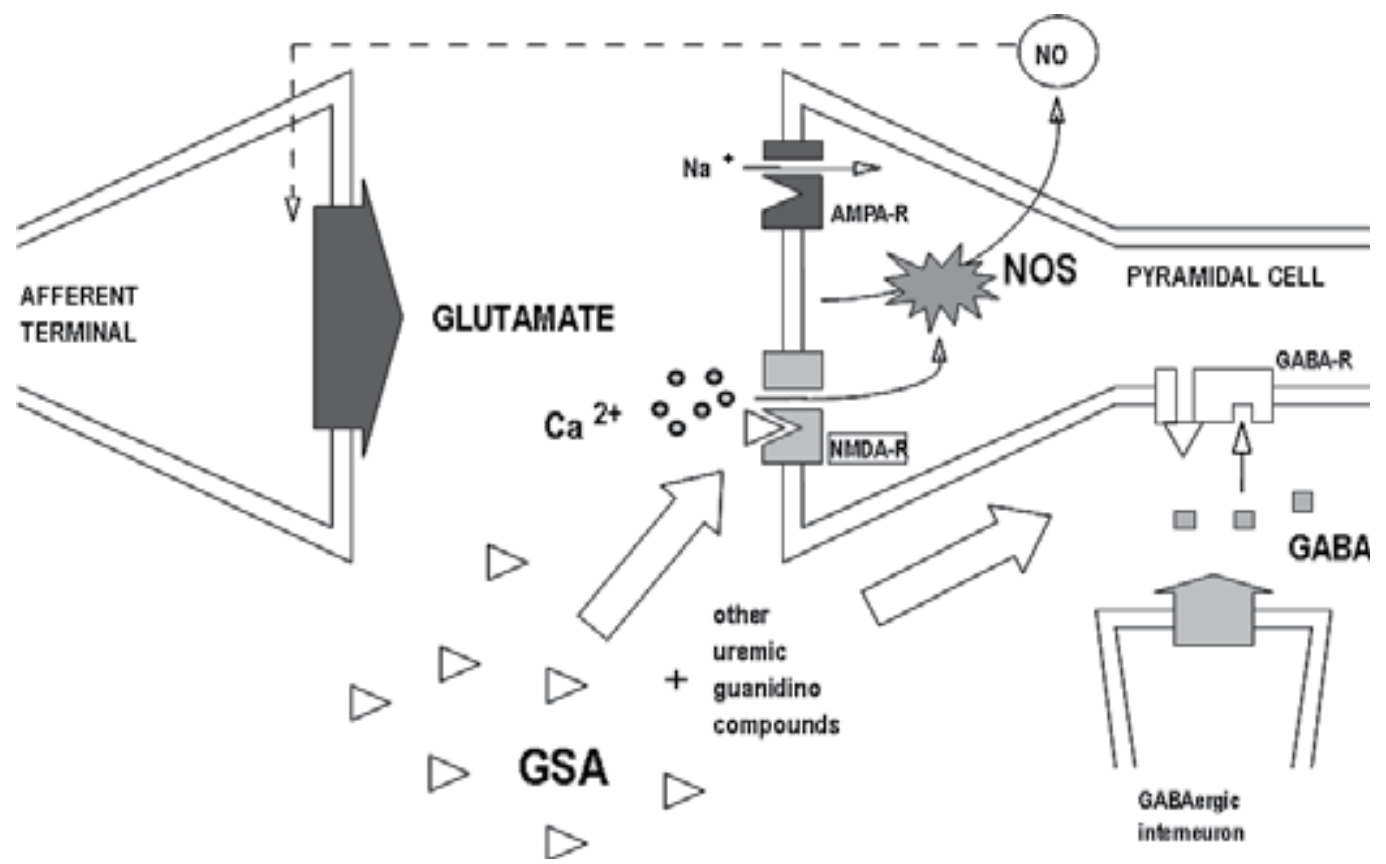

Fig. 2. Excitatory effects of uremic guanidino compounds. Hypothetical mechanism of action of guanidinosuccinic acid on synaptic transmission in rat hippocampal CA1 region. During low-frequency transmission, the excitatory neurotransmitter L-glutamate is released by the afferent terminal and binds to AMPA and NMDA receptors (NMDA-R). GABAergic interneurons provide synaptic inhibition through activation of $G_{A B A}$ receptors (GABA-R) and chloride influx. Due to insufficient membrane depolarization, the voltage dependent $\mathrm{Mg}^{2+}$ block on the NMDA-R is not lifted. However, in the presence of increased guanidinosuccinic acid levels, blocked $\mathrm{GABA}_{\mathrm{A}}$ receptors, and depolarizing effects of other uremic guanidino compounds, the $\mathrm{Mg}^{2+}$ block may be lifted from the NMDA-R. Activation of NMDA-Rs elicits $\mathrm{Ca}^{2+}$ influx through NMDA-R ionophores and activation of $\mathrm{Ca}^{2+}$ triggered events such as activation of nitric oxide synthase (NOS) leading to nitric oxide (NO) production and increased glutamate release presynaptically (De Deyn et al., 2009). 
According to the proposed mechanism (Fig. 2), guanidinosuccinic acid evokes activation of NMDA receptors in conjunction with blockade of $\mathrm{GABA}_{\mathrm{A}}$ receptor ionophores. Under these conditions, the pyramidal cells might be sufficiently depolarized to reduce the $\mathrm{Mg}^{2+}$ block on NMDA receptors. Activation of NMDA receptors elicits $\mathrm{Ca}^{2+}$ influx, potentially causing calcium-mediated neurotoxicity. Production of nitric oxide through calcium dependent activation of nitric oxide synthase could be one of the mechanisms involved in the sustained excitatory activity following guanidinosuccinic acid application. As already mentioned, Pan et al. (Pan et al., 1996) have shown NMDA receptor-mediated hippocampal damage following intrahippocampal injection of guanidinosuccinic acid in rats. In uremic brain, the depolarizing effects of guanidino compounds and other uremic toxins might enhance the effect of guanidinosuccinic acid. The joint presence of increased levels of uremic guanidino compounds could increase the block on $\mathrm{GABA}_{\mathrm{A}}$ receptors since it has been shown that, e.g., co-application of guanidine and methylguanidine results in a significantly larger inhibition of GABA responses than when either of these guanidino compounds were applied alone (De Deyn \& Macdonald, 1990). Moreover, guanidino compounds were shown to have other neurotoxic effects, which might also lead to neuronal depolarization (Mori, 1987). One such effect is the inhibition of brain $\mathrm{Na}^{+} / \mathrm{K}^{+}$-ATPase by methylguanidine (Yokoi et al., 1984).

\subsection{Energy metabolism}

Besides toxins, evidence indicates that energy metabolism might play a role. Experimental animal studies and in vitro tests demonstrated disturbances of intermediary metabolism. In the brain of rats with acute renal failure, creatine phosphate, adenosine triphosphate and glucose levels are increased in the presence of decreased adenosine monophosphate, adenosine diphosphate and lactate levels. Thus, the uremic brain in experimental uremia appears to use less adenosine triphosphate and to produce less aadenosine diphosphate, adenosine monoposphate and lactate. These changes are associated with a decrease in both brain metabolic rate and cerebral oxygen consumption (Mahoney et al., 1984; Van den Noort et al., 1968) and are consistent with a generalized decrease in brain energy use. Moreover, an inhibition of cerebral sodium-potassium-ATPase was shown in experimental uremic animals (Burn \& Bates, 1998; Minkoff et al., 1972). This could correlate with the elevation in intracellular sodium and could therefore be associated with some of the aspects of cerebral dysfunction, particularly with seizure activity. More recent studies on metabolically active and purified brain synaptosomes showed that both the sodium potassium adenosine triphosphate pump and several calcium pumps are altered in uremic rats (Fraser et al., 1985a; Fraser et al., 1985b).

\subsection{Hormonal disturbances}

The role of hormonal disturbances in the genesis of the uremic syndrome should be considered as well. Blood levels of many hormones such as parathyroid hormone, insulin, growth hormone, glucagon, thyrotropin, prolactin, luteinizing hormone and gastrin are elevated in patients with uremia. One of the major hormonal imbalances in uremia is the rise in the levels of parathyroid hormone. The possible pathophysiological role of parathyroid hormone in the development of nervous system complications in uremia has been considerably discussed (Heath et al., 1980; Slatopolsky et al., 1980). Parathyroid hormone appears to produce some of the central nervous system changes of uremia in 
healthy dogs (Guisado et al., 1975). Previously parathyroidectomised rats, subjected to bilateral uretral ligation, were protected against the uremia-induced alterations of somatosensory evoked potentials (Kanda et al., 1990). In humans, parathyroid hormone produced central nervous system effects, even in the absence of renal failure (Cogan et al., 1978). The mechanisms by which parathyroid hormone might impair central nervous system function are not completely understood. However, the increased calcium content in diverse tissues, among which brain, in patients with uremia and secondary hyperparathyroidism suggests that parathyroid hormone may somehow facilitate the entry of calcium in these tissues (Akmal et al., 1984; Burn \& Bates, 1998; Massry, 1985). Since calcium is an essential mediator of neurotransmitter release and plays an important role in intracellular metabolic and enzymatic processes, alterations of brain calcium, may possibly disrupt cerebral function by interfering with any of these processes (Rasmussen, 1986).

Brain edema and alterations in water transport have also been implicated (Arieff et al., 1973; Arieff \& Massry, 1974). Decreased brain energy demand, free amino acid changes, and blood-brain barrier derangement have been shown to be involved in both acute and chronic uremic encephalopathy (Jeppsson et al., 1982; Kikuchi et al., 1983; Mahoney et al., 1984). In a mouse model for acute kidney injury, it was demonstrated that pyknotic neuronal cells were significantly increased in region CA1 of the hippocampus. In addition, acute kidney injury resulted in significant increases in levels of the chemokines keratinocyte-derived chemoattractant and G-CSF in the brain at $24 \mathrm{~h}$ after ischemia. On the other hand, brain water content during acute kidney injury was not increased or even decreased, while an increase in microvascular permeability in the brain was observed (Liu et al., 2008).

\section{Treatment}

\subsection{Dialytic treatment}

Acute uremic encephalopathy reverses with hemodialysis or peritoneal dialysis, although a lag time of 1 to 2 days is usually required before mental status clears. Subtle cognitive difficulties may persist even after dialysis. A disadvantage of dialysis is its non-specificity and the fact that it removes also essential compounds. In addition, lipophilic compounds, which may be responsible at least in part for functional alterations in uremia, are inadequately removed by dialysis. Also, renal transplant can be considered a treatment. However, uremic encephalopathy can complicate renal transplant.

\subsection{Non-dialytic treatment}

Removal of uremic toxins are also influenced by intestinal intake and preservation of the renal function. Intestinal uptake can be reduced by influencing dietary uptake or by oral administration of absorbents. Approaches that have been shown to result in decrease in concentration include a low protein diet, administration of prebiotics such as resistant starch (Birkett, 1996) or probiotics such as bifidobacterium (Taki, 2005). Preservation of residual renal function may also be an important manner to pursue additional removal of retention solutes.

Acute renal failure induces brain mitochondrial dysfunction. Administration of the antioxidants $\mathrm{N}$-acetylcysteine and deferoxamine was able to prevent the inhibition of 
mitochondrial respiratory chain complexes I and IV (Barbosa et al., 2010). Therefore, it can be speculated that excessive reactive species generation might contribute to the neuropathology associated with acute renal failure. Creatine kinase was inhibited in prefrontal cortex, cerebral cortex and hippocampus in an animal model of acute renal failure. In this way, diminished creatine kinase might be involved in the cognitive impairment in patients with uremic encephalopathy. The inhibition of creatine kinase was prevented by antioxidants. It was speculated that oxidative stress might be involved in the mechanism of creatine kinase activity inhibition (Di-Pietro et al., 2008). In addition, increased malondialdehyde and diminished glutathione levels in brain of rats submitted to a model of chronic renal failure (Sener et al., 2007).

\section{Dialysis disequilibrium}

Dialysis disequilibrium syndrome occurs in patients receiving hemodialysis. The symptoms include headache, nausea, emesis, blurred vision, muscular twitching, disorientation, delirium, hypertension, tremors and seizures. The condition tends to be self-limited and subsides over several hours. It is attributed to a reverse urea effect. Urea is cleared more slowly from the brain than from the blood, an effect that causes an osmotic gradient leading to the net flow into the brain and to transient cerebral edema (Bucurescu, 2008).

\section{Dialysis encephalopathy}

Some patients undergoing long-term dialysis develop dialysis encephalopathy or dialysis dementia. This is a subacute, progressive and often fatal disease. It is believed to be a part of a multiple system disease that includes encephalopathy, osteomalacic bone disease, proximal myopathy and anemia. Other symptoms include dysarthria, aparaxia, personality changes, psychosis, myoclonus, seizures and finally dementia. In most cases, the condition progress to death in six months (Brouns, 2004).

\section{Conclusion}

In spite of the introduction of different dialytic procedures during the last decades, the neurological complications of uremia, although declined, remain manifold and sometimes serious. Although onset of uremic encephalopathy is often insidious, early recognition is very important as it comes to treatment. The different symptoms to be looked for are reviewed in this chapter. Urea is often used as a marker of dialytic efficiency, but has limited biological activity. Therefore, in the future, removal strategies should be designed in such a way that not only the standard molecules, but also other molecules that might be important in the deterioration of the clinical condition, can be removed efficiently. In contrast, the guanidino compounds are of great biological relevance. Those molecules have been shown to have neuroexcitatory effects and lead to convulsions. Activation of the excitatory Nmethyl-d-aspartate (NMDA) receptors and concomitant inhibition of inhibitory $\mathrm{\gamma}^{-}$ aminobutyric acid $(\mathrm{GABA})_{\mathrm{A}}$-ergic neurotransmission have been proposed as underlying mechanisms. In this chapter, putative action mechanisms are enlightened but those pathways remain to be corroborated. Knowledge of neurotoxic effects and mechanisms of action of guanidinosuccinic acid and other uremic retention solutes could add to the limited treatment options of uremic patients. 


\section{References}

Akmal, M., Goldstein, D.A., Multani, S., Massry, S.G. (1984). Role of uremia, brain calcium, and parathyroid hormone on changes in electroencephalogram in chronic renal failure. Am. J. Physiol Vol. 246, No.5 Pt 2 F575-F579

Aminoff, M.J. (1995). Neurological dysfunction and kidney disease, In: Neurology and general medicine, N.H.Raskin (Ed.), pp. 303-319, Churchill Livingstone, Philadelphia.

Arieff, A.I., Massry, S.G. (1974). Calcium metabolism of brain in acute renal failure. Effects of uremia, hemodialysis, and parathyroid hormone. J. Clin. Invest Vol. 53, No.2 387392

Arieff, A.I., Massry, S.G., Barrientos, A., Kleeman, C.R. (1973). Brain water and electrolyte metabolism in uremia: effects of slow and rapid hemodialysis. Kidney Int. Vol. 4, No.3 177-187

Barbosa, P.R., Cardoso, M.R., Daufenbach, J.F., Goncalves, C.L., Machado, R.A., Roza, C.A., Scaini, G., Rezin, G.T., Schuck, P.F., Dal-Pizzol, F., Streck, E.L. (2010). Inhibition of mitochondrial respiratory chain in the brain of rats after renal ischemia is prevented by $\mathrm{N}$-acetylcysteine and deferoxamine. Metab Brain Dis. Vol. 25, No.2 219-225

Bierman, E.L. (1970). Abnormalities of carbohydrate and lipid metabolism in uremia. Arch. Intern. Med. Vol. 126, No.5 790-792

Birket A., Muir J., Phillips J., Jones G. O'Dea K. (1996) Resistant starch lowers fecal concentrations of ammonia en phenols in humans. Am. J. Clin. Nutr. 63; 766

Bleyer, A.J., Russell, G.B., Satko, S.G. (1999). Sudden and cardiac death rates in hemodialysis patients. Kidney Int. Vol. 55, No.4 1553-1559

Bolton C.F., Young G.B. (1990) Uremic encephalopathy. In: Neurological complications of renal disease Bolton C.F., Young G.B. 44 Buttersworth

Brenner R.P. (1985) The electroencephalogram in altered states of consciousness. Neurol Clin, 3, 615

Brenner, B.M., Meyer, T.W., Hostetter, T.H. (1982). Dietary protein intake and the progressive nature of kidney disease: the role of hemodynamically mediated glomerular injury in the pathogenesis of progressive glomerular sclerosis in aging, renal ablation, and intrinsic renal disease. N. Engl. J. Med. Vol. 307, No.11 652-659

Brouns R, De Deyn PP. (2004). Neurological complications in renal failure: a review. Clin. Neurol. Neurosurg. Vol. 107, No.1 1-16

Bucurescu G. (2008) Uremic encephalopathy. E-medicine

Burn, D.J., Bates, D. (1998). Neurology and the kidney. J. Neurol. Neurosurg. Psychiatry Vol. 65, No.6 810-821

Cogan, M.G., Covey, C.M., Arieff, A.I., Wisniewski, A., Clark, O.H., Lazarowitz, V., Leach, W. (1978). Central nervous system manifestations of hyperparathyroidism. Am. J. Med. Vol. 65, No.6 963-970

Collingridge, G.L., Lester, R.A. (1989). Excitatory amino acid receptors in the vertebrate central nervous system. Pharmacol. Rev. Vol. 41, No.2 143-210

Chen R. (1996) Metaolic encephalopathies. In: Baillere's clinical neurology Bolton C.F., Young G.B. p. 577 Balliere Tindall

D'Hooge, R., De Deyn, P.P., Van de Vijver, G., Antoons, G., Raes, A., Van Bogaert, P.P. (1999). Uraemic guanidino compounds inhibit gamma-aminobutyric acid-evoked whole cell currents in mouse spinal cord neurones. Neurosci. Lett. Vol. 265, No.2 83-86 
D'Hooge, R., Manil, J., Colin, F., De Deyn, P.P. (1991). Guanidinosuccinic acid inhibits excitatory synaptic transmission in CA1 region of rat hippocampal slices. Ann. Neurol. Vol. 30, No.4 622-623

D'Hooge, R., Pei, Y.Q., De Deyn, P.P. (1993). N-methyl-D-aspartate receptors contribute to guanidinosuccinate-induced convulsions in mice. Neurosci. Lett. Vol. 157, No.2 123126

D'Hooge, R., Pei, Y.Q., Manil, J., De Deyn, P.P. (1992a). The uremic guanidino compound guanidinosuccinic acid induces behavioral convulsions and concomitant epileptiform electrocorticographic discharges in mice. Brain Res. Vol. 598, No.1-2 316-320

D'Hooge, R., Pei, Y.Q., Marescau, B., De Deyn, P.P. (1992b). Convulsive action and toxicity of uremic guanidino compounds: behavioral assessment and relation to brain concentration in adult mice. J. Neurol. Sci. Vol. 112, No.1-2 96-105

D'Hooge, R., Raes, A., Lebrun, P., Diltoer, M., Van Bogaert, P.P., Manil, J., Colin, F., De Deyn, P.P. (1996). N-methyl-D-aspartate receptor activation by guanidinosuccinate but not by methylguanidine: behavioural and electrophysiological evidence. Neuropharmacology Vol. 35, No.4 433-440

De Deyn, P.P., D'Hooge, R., Marescau, B., Pei, Y.Q. (1992a). Chemical models of epilepsy with some reference to their applicability in the development of anticonvulsants. Epilepsy Res. Vol. 12, No.2 87-110

De Deyn, P.P., D'Hooge, R., Van Bogaert, P.P., Marescau, B. (2001). Endogenous guanidino compounds as uremic neurotoxins. Kidney Int. Suppl Vol. 78, S77-S83

De Deyn, P.P., Macdonald, R.L. (1990). Guanidino compounds that are increased in cerebrospinal fluid and brain of uremic patients inhibit GABA and glycine responses on mouse neurons in cell culture. Ann. Neurol. Vol. 28, No.5 627-633

De Deyn, P.P., Marescau, B., Macdonald, R.L. (1991). Guanidino compounds that are increased in hyperargininemia inhibit GABA and glycine responses on mouse neurons in cell culture. Epilepsy Res. Vol. 8, No.2 134-141

De Deyn, P.P., Saxena, V.K., Abts, H., Borggreve, F., D'Hooge, R., Marescau, B., Crols, R. (1992b). Clinical and pathophysiological aspects of neurological complications in renal failure. Acta Neurol. Belg. Vol. 92, No.4 191-206

De Deyn, P.P., Vanholder, R., Eloot, S., Glorieux, G. (2009). Guanidino compounds as uremic (neuro)toxins. Semin. Dial. Vol. 22, No.4 340-345

De Deyn PP, Vanholder R, D'Hooge R. (2003). Nitric oxide in uremia: effects of several potentially toxic guanidino compounds. Kidney Int Suppl. Vol. 84 S25-8.

Di-Pietro, P.B., Dias, M.L., Scaini, G., Burigo, M., Constantino, L., Machado, R.A., Dal-Pizzol, F., Streck, E.L. (2008). Inhibition of brain creatine kinase activity after renal ischemia is attenuated by $\mathrm{N}$-acetylcysteine and deferoxamine administration. Neurosci. Lett. Vol. 434, No.1 139-143

Dreyfus, P. (1965). The regional distribution of transketolase in the normal and the thiamine deficient nervous system. J. Neuropathol. Exp. Neurol. Vol. 24, 119-129

Earnest M.P. (1993) Metabolic encephalopathies and coma from medical causes. In: Management of the acutely ill neurological patient. Churchill livingstone 1

Enomoto, A., Takeda, M., Taki, K., Takayama, F., Noshiro, R., Niwa, T., Endou, H. (2003). Interactions of human organic anion as well as cation transporters with indoxyl sulfate. Eur. J. Pharmacol. Vol. 466, No.1-2 13-20 
Fraser, C.L., Sarnacki, P., Arieff, A.I. (1985a). Abnormal sodium transport in synaptosomes from brain of uremic rats. J. Clin. Invest Vol. 75, No.6 2014-2023

Fraser, C.L., Sarnacki, P., Arieff, A.I. (1985b). Calcium transport abnormality in uremic rat brain synaptosomes. J. Clin. Invest Vol. 76, No.5 1789-1795

Giovannetti, S., Biagini, M., Balestri, P.L., Navalesi, R., Giagnoni, P., De, M.A., Ferro-Milone, P., Perfetti, C. (1969). Uraemia-like syndrome in dogs chronically intoxicated with methylguanidine and creatinine. Clin. Sci. Vol. 36, No.3 445-452

Glorieux, G.L., Dhondt, A.W., Jacobs, P., Van Langeraert, J., Lameire, N.H., De Deyn, P.P., Vanholder, R.C. (2004). In vitro study of the potential role of guanidines in leukocyte functions related to atherogenesis and infection. Kidney Int. Vol. 65, No.6 2184-2192

Gotch, F.A., Sargent, J.A. (1985). A mechanistic analysis of the National Cooperative Dialysis Study (NCDS). Kidney Int. Vol. 28, No.3 526-534

Guisado, R., Arieff, A.I., Massry, S.G., Lazarowitz, V., Kerian, A. (1975). Changes in the electroencephalogram in acute uremia. Effects of parathyroid hormone and brain electrolytes. J. Clin. Invest Vol. 55, No.4 738-745

Heath, H., Hodgson, S.F., Kennedy, M.A. (1980). Primary hyperparathyroidism. Incidence, morbidity, and potential economic impact in a community. N. Engl. J. Med. Vol. 302, No.4 189-193

Jeppsson, B., Freund, H.R., Gimmon, Z., James, J.H., von Meyenfeldt, M.F., Fischer, J.E. (1982). Blood-brain barrier derangement in uremic encephalopathy. Surgery Vol. 92, No.1 30-35

Johnson, W.J., Hagge, W.W., Wagoner, R.D., Dinapoli, R.P., Rosevear, J.W. (1972). Effects of urea loading in patients with far-advanced renal failure. Mayo Clin. Proc. Vol. 47, No.1 21-29

Kanda, F., Jinnai, K., Tada, K., Fujita, T. (1990). Somatosensory evoked potentials in acute renal failure: effect of parathyroidectomy. Kidney Int. Vol. 38, No.6 1085-1088

Kikuchi, S., Matsumoto, H., Ito, M. (1983). Free amino acid changes in the cerebral cortex of experimental uremic rat. Neurochem. Res. Vol. 8, No.3 313-318

Lee, J.A., Lee, H.A., Sadler, P.J. (1991). Uraemia: is urea more important than we think? Lancet Vol. 338, No.8780 1438-1440

Liu, M., Liang, Y., Chigurupati, S., Lathia, J.D., Pletnikov, M., Sun, Z., Crow, M., Ross, C.A., Mattson, M.P., Rabb, H. (2008). Acute kidney injury leads to inflammation and functional changes in the brain. J. Am. Soc. Nephrol. Vol. 19, No.7 1360-1370

Lonergan, E.T., Semar, M., Sterzel, R.B., Treser, G., Needle, M.A., Voyles, L., Lange, K. (1971). Erythrocyte transketolase activity in dialyzed patients. A reversible metabolic lesion of uremia. N. Engl. J. Med. Vol. 284, No.25 1399-1403

Mahoney, C.A., Arieff, A.I. (1982). Uremic encephalopathies: clinical, biochemical, and experimental features. Am. J. Kidney Dis. Vol. 2, No.3 324-336

Mahoney, C.A., Sarnacki, P., Arieff, A.I. (1984). Uremic encephalopathy: role of brain energy metabolism. Am. J. Physiol Vol. 247, No.3 Pt 2 F527-F532

Massry, S.G. (1985). Current status of the role of parathyroid hormone in uremic toxicity. Contrib. Nephrol. Vol. 49, 1-11

Matsumoto, M., Kobayashi, K., Kishikawa, H., Mori, A. (1976). Convulsive activity of methylguanidine in cat and rabbits. IRCS Med. Sci. Vol. 4, 65 
Minkoff, L., Gaertner, G., Darab, M., Mercier, C., Levin, M.L. (1972). Inhibition of brain sodium-potassium ATPase in uremic rats. J. Lab Clin. Med. Vol. 80, No.1 71-78

Minot, A.S., Dodd, K. (1933). Guanidine intoxication: a complicating factor in certain clinical conditions in children. Am. J. Dis. Child Vol. 43, No.3 522-542

Mori, A. (1987). Biochemistry and neurotoxicology of guanidino compounds. History and recent advances. Pavlov. J. Biol. Sci. Vol. 22, No.3 85-94

Owen, W.F., Jr., Lew, N.L., Liu, Y., Lowrie, E.G., Lazarus, J.M. (1993). The urea reduction ratio and serum albumin concentration as predictors of mortality in patients undergoing hemodialysis. N. Engl. J. Med. Vol. 329, No.14 1001-1006

Pan, J.C., Pei, Y.Q., An, L., Lai, L., D'Hooge, R., De Deyn, P.P. (1996). Epileptiform activity and hippocampal damage produced by intrahippocampal injection of guanidinosuccinic acid in rat. Neurosci. Lett. Vol. 209, No.2 121-124

Perna, A.F., Ingrosso, D., Satta, E., Lombardi, C., Galletti, P., D'Aniello, A., De Santo, N.G. (2004). Plasma protein aspartyl damage is increased in hemodialysis patients: studies on causes and consequences. J. Am. Soc. Nephrol. Vol. 15, No.10 2747-2754

Plum F., Posner J.B. (1982) The diagnosis of stupor and coma. FA Davis company p.177

Raskin, N.H., Fishman, R.A. (1976). Neurologic disorders in renal failure (first of two parts). N. Engl. J. Med. Vol. 294, No.3 143-148

Rasmussen, H. (1986). The calcium messenger system (1). N. Engl. J. Med. Vol. 314, No.17 1094-1101

Reynolds, I.J. and Rothermund, K. (1992). Multiple modes of NMDA receptor regulation by guanidines, In: Guanidino compounds in biology and medicine, P.P.De Deyn, B.Marescau, V.Stalon, I.A.Qureshi (Eds.), pp. 441-448, John Libbey, London.

Schepers, E., Glorieux, G., Dhondt, A., Leybaert, L., Vanholder, R. (2009). Role of symmetric dimethylarginine in vascular damage by increasing ROS via store-operated calcium influx in monocytes. Nephrol. Dial. Transplant. Vol. 24, No.5 1429-1435

Sener, G., Sakarcan, A., Sehirli, O., Eksioglu-Demiralp, E., Sener, E., Ercan, F., Gedik, N., Yegen, B.C. (2007). Chronic renal failure-induced multiple-organ injury in rats is alleviated by the selective CysLT1 receptor antagonist montelukast. Prostaglandins Other Lipid Mediat. Vol. 83, No.4 257-267

Slatopolsky, E., Martin, K., Hruska, K. (1980). Parathyroid hormone metabolism and its potential as a uremic toxin. Am. J. Physiol Vol. 239, No.1 F1-12

Sterzel, R.B., Semar, M., Lonergan, E.T., Treser, G., Lange, K. (1971). Relationship of nervous tissue transketolase to the neuropathy in chronic uremia. J. Clin. Invest Vol. 50, No.11 2295-2304

Stim, J., Shaykh, M., Anwar, F., Ansari, A., Arruda, J.A., Dunea, G. (1995). Factors determining hemoglobin carbamylation in renal failure. Kidney Int. Vol. 48, No.5 1605-1610

Taki K., Takayama F., Niwa T. (2005) Beneficial effects of bifidobacteria in a gastroresistant searnless capsule on hyperhomocysteinemie in hemodialysis patients. J. ren. nutr. $15 ; 77$

Teschan, P.E. and Arieff, A.I. (1985). Uremic and dialysis encephalopathies, In: Cerebral energy metabolism and metabolic encephalopathy, D.W.McCandless (Ed.), pp. 263-286, Plenum Press, New York. 
Topczewska-Bruns, J., Pawlak, D., Chabielska, E., Tankiewicz, A., Buczko, W. (2002). Increased levels of 3-hydroxykynurenine in different brain regions of rats with chronic renal insufficiency. Brain Res. Bull. Vol. 58, No.4 423-428

Torremans, A., Marescau, B., Van, D.D., Van, G.C., Van, M.F., Van Bogaert, P.P., D'Hooge, R., de, V.J., De Deyn, P.P. (2005). GSA: behavioral, histological, electrophysiological and neurochemical effects. Physiol Behav. Vol. 84, No.2 251-264

Van den Noort, S., Eckel, R.E., Brine, K., Hrdlicka, J.T. (1968). Brain metabolism in uremic and adenosine-infused rats. J. Clin. Invest Vol. 47, No.9 2133-2142

Vanholder, R., Argiles, A., Baurmeister, U., Brunet, P., Clark, W., Cohen, G., De Deyn, P.P., Deppisch, R., scamps-Latscha, B., Henle, T., Jorres, A., Massy, Z.A., Rodriguez, M., Stegmayr, B., Stenvinkel, P., Wratten, M.L. (2001). Uremic toxicity: present state of the art. Int. J. Artif. Organs Vol. 24, No.10 695-725

Vanholder, R., De Smet, R. (1999). Pathophysiologic effects of uremic retention solutes. J. Am. Soc. Nephrol. Vol. 10, No.8 1815-1823

Vanholder, R., De Smet, R., Glorieux, G., Argiles, A., Baurmeister, U., Brunet, P., Clark, W., Cohen, G., De Deyn, P.P., Deppisch, R., Descamps-Latscha, B., Henle, T., Jorres, A., Lemke, H.D., Massy, Z.A., Passlick-Deetjen, J., Rodriguez, M., Stegmayr, B., Stenvinkel, P., Tetta, C., Wanner, C., Zidek, W. (2003a). Review on uremic toxins: classification, concentration, and interindividual variability. Kidney Int. Vol. 63, No.5 1934-1943

Vanholder, R., De, S.R., Glorieux, G., Argiles, A., Baurmeister, U., Brunet, P., Clark, W., Cohen, G., De Deyn, P.P., Deppisch, R., scamps-Latscha, B., Henle, T., Jorres, A., Lemke, H.D., Massy, Z.A., Passlick-Deetjen, J., Rodriguez, M., Stegmayr, B., Stenvinkel, P., Tetta, C., Wanner, C., Zidek, W. (2003b). Review on uremic toxins: classification, concentration, and interindividual variability. Kidney Int. Vol. 63, No.5 1934-1943

Yokoi, I., Toma, J., Mori, A. (1984). The effect of homoarginine on the EEG of rats. Neurochem. Pathol. Vol. 2, No.4 295-300

Yonezawa, T., Iwanami, H. (1966). An experimental study of thiamine deficiency in nervous tissue, using tissue culture technics. J. Neuropathol. Exp. Neurol. Vol. 25, No.3 362372 


\title{
Drug-Induced Encephalopathy
}

\author{
Niels Hansen \\ Julius-Maximilians University Würzburg, \\ Department of Neurology \\ Germany
}

\section{Introduction}

Drug-induced encephalopathy is a disease entity often caused by impaired cerebral metabolism that is not attributed to structural brain lesions. However, some drug-induced encephalopathies can develop structural lesions and share other underlying pathophysiological mechanisms (table 2). Leading symptoms are acute or chronic disturbances of consciousness, brain function and personality changes with concomitant neurological symptoms such as asterixis, myoclonias, paresis or seizures (see table 3). Isoniazid-induced encephalopathy was one of the earliest descriptions of a drug-induced encephalopathy (Adams \& White, 1965). Clinical symptoms depend on the type and severity of the drug-evoked encephalopathy. A well-described and frequently-reported drug-induced encephalopathy is valproic acid encephalopathy, first described in the late 1970s. This acute encephalopathy was characterized by altered behaviour, worsening seizure control and confusion. After a reduction in the valproate acid dose, the patient's symptoms resolved completely (Chadwick et al., 1979). Encephalopathies have been reported after consumption of several types of drugs as depicted below (table 1).

\section{Drugs}

\subsection{Analgesics and anaesthesia}

Drug-induced encephalopathy was reported after morphine administered intrathecally and the use of propofol (Eran \& Barak, 2009). Morphine has been described as having induced an encephalopathy characterized by a myoclonus, motor dysfunction, or vertigo (Goundrey, 1990; Kakinohana et al., 2003). Spinal anaesthesia with hyperbaric bupivacaine lead to an encephalopathy that developed a few days after the drug's administration (Ho \& Chan, 2007). A drug abuse-evoked encephalopathy was also reported after ketamine and gamma hydroxybutyrate (Virmani et al., 2010). Toxic encephalopathy has been described after intake of an acetaminophen overdose (Brusilow \& Cooper, 2011).

\subsection{Antibiotics}

Drug-induced encephalopathy can occur after an intake of cefepime and metronidazole (Kim et al., 2011; Lin et al., 2011). The incidence of metronidazole-induced encephalopathy is unknown. Several studies addressed reversible brain changes caused by metronidazole 
induced-encephalopathy (Ahmed et al., 1995), and bilateral, symmetric brain abnormalities have been observed in patients (Ahmed et al., 1995; Kim et al., 2011). Ceftriaxone induced a reversible encephalopathy in an patient treated for a urinary tract infection (RanconAlbuquerque et al., 2009). That encephalopathy was completely reversible. An early-onset encephalopathy a day and a half after linezolid therapy occurred in a male, thus clinicians must be aware of the potential of linezolid-induced encephalopathy, particularly in patients presenting risk factors (Fletcher et al., 2010). Clarithromycin has also induced an encephalopathy in adults characterized by symptoms appearing 1-10 days after drug intake and displaying clinical features ranging from delirium to non-convulsive status epilepticus (Bandettin di Poggio et al., 2011). Chinolones like ciprofloxacin and gemifloxacin are also reported to induce an encephalopathy (Rfidah et al., 1995; Barrett MJ 2009). Cephalosporine is reported to evoke an encephalopathy associated with a variety of electroencephalografic manifestations (Grill \& Magati, 2008). Other cephalosporines such as cefuroxime, ceftazidime and cefazoline can result in an encephalopathy as well (Herishanu et al. 1988, Jackson et al., 1992; Ortiz et al., 1991). Cefoperazone is a cephalosporine that can cause a reversible encephalopathy characterized by triphasic waves in electroencephalography (Pro et al., 2011). Also penicillin-based antibiotics like penicillin itself, piperacillin and pivmecillinam caused an encephalopathy (Park-Matsumoto et al., 1996; Conway et al., 1968; Lokrantz et al., 2004).

\subsection{Antiviral agents}

Antiviral agents have seldom been reported to have induced an encephalopathy. As described in one case report, aciclovir can cause an encephalopathy. That patient had normal blood levels of aciclovir, and his renal function was normal (Delluc et al., 2004).

\subsection{Antidepressants}

Antidepressants can also result in an encephalopathy. The drug amitriptyline can cause an encephalopathy appearing as a neuroleptic malignant syndrome or a serotonin syndrome spectrum disorder (Miyaoka \& Kamijama, 1995).

\subsection{Anticonvulsants}

The following anticonvulsants have been reported to induce a drug-induced encephalopathy: carbamazepine, gabapentin, levetiracetame, lamotrigine, phenytoine, primidone, topiramate, valproic acid and vigabatrine (Engel et al., 1971; Bauer \& Elger, 1993; Hennessy \& Miles, 1996; Garcia-Pastor et al., 2000; Sechi et al., 2004; Siniscalchi et al., 2004; Cheung et al., 2005; Horvath et al., 2005; Bauer, 2008). The most studied encephalopathy is valproic acid encephalopathy, which was first reported in epileptic and later psychiatric patients (Duarte et al., 1993; Settle, 1995). Valproic acid encephalopathy is often reversible after a week; prolonged time courses have been rare (Bauer \& Elger, 1993). Antiepileptic drug-induced encephalopathies represent a seldom, but important side effect of antiepileptic drug therapy. There is an estimated $2 \%$ incidence of combined topiramate, valproate acid- induced hyperammonemic encephalopathy (Cheung et al., 2005). An average age of 38.6 years has been reported in a long-term study of valproic acid-induced encephalopathy (Gerstner et al., 2007). 


\subsection{Antineoplastic drugs and chemotherapeutics}

Capecitabine is an antineoplastic drug replacing 5-Fluouracil in clinical practice. This drug can result in an encephalopathy with seizures even if a conventional dosis is used. No correlation was found between the encephalopathy development and a dihydropyridimidine dehydrogenase mutation (Fantini et al., 2010). Carmofur, a 5fluorouracil derivative that induced a subacute leukencephalopathy, revealed an unsteady gait and dementia (Kuzuhara et al., 1987). A rare complication associated with cisplatin therapy is an encephalopathy with or without seizures (Steeghs et al., 2003). In particular, high doses of ifofosfamide can induce an encephalopathy. Ifofosfamide can result in myoclonus-encephalopathy syndrome (Savica et al., 2011). A cohort study revealed a prevalence of $10-40 \%$ of this drug-evoked encephalopathy. Female sex, low total albumin and haemoglobulin levels, as well as obesity appear to be risk factors associated with a ifofosfamide-evoked encephalopathy (Sweiss et al., 2008). There are few reports of the CNS toxicity of paclitaxel. Seizures have been reported in two patients. Little or no blood brain barrier penetration were the result of confusion and word-finding difficulties, and the encephalopathy resolved itself (Perry \& Warner, 1996). Vincristine is known to be an agent that may lead to consecutive sensory and motor dysfunction and eventually fatal myeloencephalopathy (Fawaz al, 1992). Cyclosporine encephalopathy has also been reported (Kwon et al., 2008). Methotrexate has rarely led to an acute encephalopathy, its incidence is $0.8 \%$ in leukaemia or lymphoma and $4.5 \%$ in osteosarcoma or malignant fibrous histiocytoma (Inaba et al., 2008).

\subsection{Immunosuppressants}

An encephalopathy occurred after tacrolimus administration and improved after the drug was discontinued. It clinically depicted a right-sided hemiplegia with responsible lesions on diffusion-tensor imaging and diffusion-tensor tractography of the white matter tract (Kim et al., 2011). Sorafenib was reported to induce an encephalopathy in a patient with hepatocellular carcinoma (Dogan et al., 2010). Furthermore, a posterior, reversible leukoencephalopathy syndrome was observed after an infusion of infliximab (Zamvar et al., 2009).

\subsection{Immunomodulators}

Intravenous immunoglobulins (IVIG) can induce an acute encephalopathy probably caused by a cytotoxic brain oedema (Wada et al., 2005).

\subsection{Neuroleptics and lithium}

Lithium can to lead to an encephalopathy characterized by seizures, choreiforme as well as parkinsonian movements with cerebellar signs. Three risk factors contribute to lithium toxicity: a nephrogenic diabetes insipidus, age over 50 years, and thyroid dysfunction (Smith et al., 2003). Haloperidol can evoke an encephalopathy characterised by an electroencephalography (EEG) with characteristics of toxic encephalopathy (Maxa et al., 1997). The combination of lithium-risperidone induces an reversible encephalopathy (Boora \& Hyatt, 2008). Two patients presented a prolonged postictal encephalopathy with clozapine-induced seizures (Karper et al., 1992) which lasted 63-72 hours and caused electroencephalographic abnormalities. 


\begin{tabular}{|c|c|c|c|}
\hline Drug & Dosage/d & Outcome after drug discontinuation & Reference \\
\hline aciclovir & $\begin{array}{l}\text { standard } \\
\text { dosis }\end{array}$ & symptoms reversed after $72 \mathrm{~h}$ & Delluc et al., 2004 \\
\hline capecitabine & $2000 \mathrm{mg} / \mathrm{m}^{2}$ & improvement of symptoms & Fantini et al., 2010 \\
\hline carbamazepine & $1200 \mathrm{mg}$ & symptoms and EEG normal after $2 \mathrm{w}$ & Horvath et al., 2005 \\
\hline ceftazidime & $4 \mathrm{~g}$ & symptoms resolved after a few days & Jackson et al., 1992 \\
\hline cefoperazone & $2 g$ & EEG and symptoms normal after $36 \mathrm{~h}$ & Pro et al., 2011 \\
\hline dexamethasone & $4 \mathrm{mg}$ p.o. & neuroimaging improvement after $4 \mathrm{~d}$ & Irvin et al., 2007 \\
\hline $\begin{array}{l}\text { Duodopa } \\
\text { +entacapone }\end{array}$ & $\begin{array}{l}1200 \mathrm{mg} / \mathrm{d} \\
76 \mathrm{mg} / \mathrm{h}\end{array}$ & $\begin{array}{l}100 \% \text { mental status recovery after } \\
48 \mathrm{~h}\end{array}$ & Manca et al., 2009 \\
\hline gabapentin & $900 \mathrm{mg}$ & symptoms normalized after $4 \mathrm{w}$ & Sechi et al., 2004 \\
\hline gemifloxacin & $320 \mathrm{mg}$ & full recovery $2 d$ later & Barrett MJ 2009 \\
\hline isoretinoin & $80 \mathrm{mg}$ & full recovery $24 \mathrm{~h}$ later & Wong et al., 2010 \\
\hline IVIG & $1000 \mathrm{mg}$ & full recovery after $11 d$ & Wada et al., 2005 \\
\hline lamotrigine & $400 \mathrm{mg}$ & symptoms normalized after $4 \mathrm{w}$ & Sechi et al., 2004 \\
\hline levetiracetame & $3000 \mathrm{mg}$ & symptoms normalized & Bauer, 2008 \\
\hline lithium & $400 \mathrm{mg}$ & symptoms resolved after $1 \mathrm{w}$ & Smith et al., 2003 \\
\hline metronidazole & $45.5 \mathrm{~g}$ & symptoms improved within $6.7 \mathrm{~d}$ & Kim et al., 2011 \\
\hline morphine & $0,5 \mathrm{mg}$ & recovery after $10 \mathrm{~d}$ & Barak, 2009 \\
\hline odansetron & $4 \mathrm{mg}$ & full recovery of symptoms & Ritter et al., 2003 \\
\hline oxcarbazepine & $1800 \mathrm{mg}$ & $\begin{array}{l}\text { EEG and symptoms normalized after } \\
\text { 20d }\end{array}$ & $\begin{array}{l}\text { Siniscalchi et al., } \\
2004\end{array}$ \\
\hline penicillin & $\begin{array}{l}\text { i.v. } 60 \text { Mega } \\
\text { Units }\end{array}$ & patient died & $\begin{array}{l}\text { Conway et al., } \\
1968\end{array}$ \\
\hline phenytoine & $300 \mathrm{mg}$ & symptoms and EEG nor & Engel et al., 1971 \\
\hline pivmecillinam & $600 \mathrm{mg}$ & fast symptom recovery & $\begin{array}{l}\text { Lokrantz et al., } \\
2004\end{array}$ \\
\hline primidone & $600 \mathrm{mg}$ & $\begin{array}{l}\text { symptoms and EEG normalized after } \\
2 \mathrm{w}\end{array}$ & Katano et al., 2002 \\
\hline propofol & $150 \mathrm{mg}$ & recovery after $10 \mathrm{~d}$ & Eran \& Barak, 2009 \\
\hline sorafenib & $400 \mathrm{mg} / 2 \mathrm{x}$ & all symptoms resolved after $5 \mathrm{~d}$ & Dogan et al., 2010 \\
\hline tacrolimus & $0.1 \mathrm{mg} / \mathrm{kg} / \mathrm{d}$ & $\begin{array}{l}\text { imaging abnormalities normalized } \\
\text { after } 4 \mathrm{~m}\end{array}$ & Kim et al., 2011 \\
\hline topiramate & $1400 \mathrm{mg}$ & $\begin{array}{l}\text { EEG and symptoms normalized after } \\
7 \mathrm{~d}\end{array}$ & $\begin{array}{l}\text { Cheung et al., } \\
2005\end{array}$ \\
\hline valproic acid & $2400 \mathrm{mg}$ & improvement in EEG and symptoms & $\begin{array}{l}\text { Bauer \& Elger, } \\
1993\end{array}$ \\
\hline vigabatrine & $3000 \mathrm{mg}$ & improvement in $2 \mathrm{w}$ & $\begin{array}{l}\text { Garcia-Pastore et } \\
\text { al., } 2000\end{array}$ \\
\hline
\end{tabular}

$\mathrm{d}=$ days, EEG = Electroencephalography, $\mathrm{h}=$ hours, IVIG intravenous immunoglobulines, $\mathrm{m}=\mathrm{month}$, $\mathrm{w}=$ weeks, p.o. $=$ per os

Table 1. Drug-induced encephalopathy- clinics and outcome (not all drugs mentioned in the text are depicted in the table) 


\subsection{Other classes of drugs}

There are several other classes of drugs that can result in a drug-induced encephalopathy. For instance, baclofen [a derivative of gamma-aminobutyric acid (GABA)] caused an encephalopathy with severe electroencephalographic abnormalities (Kumar et al., 2010). Another class of drugs such as duodopa (a combination of levodopa and carbidopa) has induced a reversible encephalopathy in Parkinson's disease. An intermittent multifocal myoclonus was observed, and neurologic examination revealed a flaccid tetraparesis (Manca et al., 2009). An inadvertent injection of gadolinium (solutions of chelated organic gadolinium complexes) can result in grand mal seizures and mental changes due to an encephalopathy (Kapoor et al., 2010). This gadolinium encephalopathy probably occurred due to the inadvertent simultaneous entry of gadolinium and blood into the subarachnoid space. This case highlights the importance of using only a small amount of gadolinium. Agents like isoretinoin (medication in the therapy of Acne) may induce an encephalopathy with myoclonic jerks and confusion (Wong et al., 2010). Ondansetron (a 5-HT 3 receptor antagonist used mainly as an antiemetic drug) can produce a multifocal encephalopathy depicted by a transient pyramidal and extrapyramidal dysfunction with Babinski signs, oculogyric crisis, oromandibular and limb dystonia. The symptoms resolved after hours. Anaesthesiologists must take special care when administering ondansetrone therapy because of this rare complication and the severe clinical manifestation reflecting transient structural brain damage that however results in a full resolution of neurological symptoms. Sulfasalazine is a drug used primarily as an anti-inflammatory agent in the treatment of inflammatory bowel disease and rheumatoid arthritis. It has caused an encephalopathy characterised by cerebrospinal fluid with a high protein level (Mut et al., 2008).

\section{Pathophysiological mechanisms}

The underlying causes of a drug-induced encephalopathy are not yet fully understood. Several mechanisms of drug-induced encephalopathy are discussed below (table 3).

\subsection{Cytotoxic and neurotoxic effects}

There are several pharmaceutical cytotoxic and neurotoxic side effects that can cause an encephalopathy. A rise in the glutamine and glutamate complex peak in MR spectroscopy suggests for example an exitotoxic injury in the neurons and astrocytes in an acute IVIGinduced encephalopathy (Wada et al., 2005), and it is one possible mechanism inducing neurotoxicity.

\subsection{Electrolytic disturbances}

There are electrolytic disturbances such as a hypo-or hypernatremia that can promote druginduced encephalopathy. Hyponatremia may be a side effect of drugs such as oxcarbazepine or diuretics. Severe hyponatremia is commonly caused by the syndrome of inappropriate antidiuresis (SIADH), which can also be induced by drugs like cyclophosphamide, vincristine, vinblastine, thiothixene, thioridazine, haloperidol, monoamine oxidase inhibitors, tricyclic antidepressants, selective serotonin reuptake inhibitors and bromocripitine (Esposito et al., 2011). Hypopotassemia plays a role in the pathogenesis of convulsions and the high rate of mortality in theophylline encephalopathy (Suarez Ortega et al., 1995). 


\subsection{Hepatic enzyme interactions and hyperammonemia}

Valproic acid can inhibit differential enzymes of the urea cycle, inducing a hyperammonemia (Sechi et al 2004, Treem et al., 1994). Moreover, there is the potential of damage on an enzymatic level that can lead to hyperammonemia: (1) carbamylphosphat synthetase-, (2) ornithin-transcarbamylase-, (3) N-acetylglutamat-synthetase-, argininosuccinat-synthetase- and (5) arginino-succinat-lyase deficiency. As the incidence of these enzyme defects is low, valproic-acid encephalopathy very seldom has a hereditary cause. A high level of ammoniac can lead to hepatic necrosis in addition to encephalopathy. Hyperammonemia can be induced by the drugs depicted in table 2. Recent study data indicate that the hyperammonemia observed in patients under valproic-acid treatment is based on the direct inhibition of hepatic N-acetylglutamate synthase activity by valproylCoA (Aires et al., 2011). Hyperammonemia can induce an encephalopathy by inhibiting the glutamate uptake by astrocytes, thus provoking neuronal damage and cerebral oedema (Blindauer et al., 1998). Moreover, elevated extracellular glutamate reduces the size of the astroctyes, thereby inhibiting their function. The reduced synthesis of glutathione causes the neurons and glia cells to become more vulnerable to oxidative stress (Verotti et al., 2002). Finally, the over-production of glutamine leads to a swelling of the astrocytes followed by cerebral oedema and even higher cerebral pressure (Noremberg, 1996).

\begin{tabular}{|l|l|}
\hline Drug & Reference \\
\hline 5-Fluouracil & Advani \& Fakih et al., 2011 \\
\hline Acetazolamide & Kim et al., 2007 \\
\hline Carbamazepine & Adams et al., 2009 \\
\hline Haloperidol & Rubenstein et al., 1990 \\
\hline Lamotrigine & Fan et al., 2008 \\
\hline Primidone & Katano et al., 2002 \\
\hline Valproate acid & Aires et al., 2011 \\
\hline Zonisamide & Shaikh et al., 2009 \\
\hline
\end{tabular}

Table 2. Drugs inducing hyperammonemia

An acute intermittent porphyria as one form of acute hepatic porphyria can present as a diffuse encephalopathy (Maramattom et al., 2005). Additionally, a mouse model has demonstrated that griseofulvin induces a hepatic porphyria characterized by psychiatric behavior sometimes observed in drug-induced encephalopathies (Satoh et al., 2008).

Drugs such as barbiturates, bernegride, chloramphenicol, chlordiazepoxide, chloroquine, chlorpropamide, danazol, diazepam, ergot preparations, estrogens, ethanol excess, griseofulvin, halothane, hydantoins, imipramine, ketamine, meprobamate, methyldopa, methyprylon, methsuximide, nikethamide, oral contraceptives, pentazocine, phensuximide, phenylbutazone, progestogens, pyrazinamide, pyrazolone derivatives, sulfonamides, theophylline derivatives, tolbutamide, troxidone and valproic acid (Bonkowsky et al., 1982) have been reported to exacerbate acute porphyrias. These drugs should thus be administered with caution in patients with an encephalopathy associated with porphyria. A further mechanism leading to an encephalopathy based on an increase in neuronal P450 CYP2E1 activity is induced by acetaminophen in an animal model (Posadas et al., 2010). Posadas et al. showed that acetoaminophen can result in a concentration-dependent neuronal apoptosis on rat cortical neurons through a mitochondrial-mediated mechanism 
that includes cytochrome c release and caspase 3 activation (Posadas et al., 2010). Surprisingly, the neurotoxic action by acetoaminophen in rats is below those required to induce hepatotoxicity.

\subsection{Effects on cerebral receptors}

Effects on cerebral receptors play a important role as underlying pathomechanisms in druginduced encephalopathy. The neurotoxicty in metronidazole encephalopathy is based on the RNA (Bradley et al., 1977) and DNA binding of intermediate metabolites of metronidazole (Wright \& Tyler, 2003), modulating inhibitory GABA receptors in the cerebellar and vestibular systems (Evans et al., 2003).

Interaction with the GABA receptor plays a role in the intrinsic toxic effects of valproic acid encephalopathy (Miyazaki et al., 1988). Topiramate can induce a direct toxic effect on the central nervous system (CNS). Combined therapy with valproat acid produces this effect by reducing the metabolism of topiramate due to the interaction of valproic acid with the cytochrome-P450 effect. Gabapentin may cause a reversible encephalopathy clinically characterised by an asterixis. One candidate mechanism this encephalopathy is the agonistic interaction of gabapentin on cerebral GABA receptors in conjunction with increased inhibitory action (Fink et al., 2002). Cephalosporine-induced encephalopathy seems to involve GABA A receptor inhibition (Grill \& Magati et al., 2008).

\subsection{Metabolic effects}

Severe diseases or malnutrition have a reduction in glucuron acid as a consequence. It is thus possible to inhibit the glucoronidation of valproate acid, resulting in a higher cumulative concentration of valproic acid, lamotrigine and oxcarbazepine in blood levels.

\subsection{Vasogenic and cytotoxic brain oedema}

Vasogenic and cytotoxic brain oedema as an underlying mechanism of a drug-induced encephalopathy is widespread. Metronidazole encephalopathy is probably caused by vasogenic and cytotoxic brain oedema. Most of the lesions in metronidazole encephalopathy correspond to areas of vasogenic oedema according to diffusion weighted imaging. Some lesions are located in the corpus callosum and correspond to cytotoxic oedema. Cytotoxic oedema is also a candidate mechanism in IVIG-induced encephalopathy. An intramyelinic oedema in the myelin sheath was observed in IVIG-induced encephalopathy (Wada et al., 2005). Many drug-induced encephalopathies share in common a posterior reversible leukoencephalopathy syndrome (PRES) possibly due to vasogenic oedema.

\subsection{Posterior reversible leukoencephalopathy syndrome}

The PRES has been described after the intake of immunosuppressants such as tacrolimus, cyclosporine or in association with acute hypertensive encephalopathy and eclampsia (Hinchey et al., 1996). It is characterised by capillary-leak syndrome in the brain caused by hypertension, liquid retention, immunosuppressants, and chemotherapeutics affecting the vascular endothelium. Clinical symptoms are headache, vomiting, confusion, seizures, cortical blindness and other visual symptoms. Neuroimaging reveals bilateral signal alterations in the posterior white mater suggesting oedema. 


\begin{tabular}{|l|l|l|}
\hline Candidate Mechanism & Drugs & Reference \\
\hline cytotoxic brain edema & IVIG, metronidazole & $\begin{array}{l}\text { Kim et al., 2011; } \\
\text { Wada et al., 2005 }\end{array}$ \\
\hline $\begin{array}{l}\text { effect on cerebral } \\
\text { receptors }\end{array}$ & methotrexate & Sasazaki et al., 1992 \\
\hline electrolytic disturbance & theophylline & Suarez Ortega et al., 1995 \\
\hline $\begin{array}{l}\text { hepatic enzyme } \\
\text { interactions }\end{array}$ & valproic acid & Bauer \& Elger,1993 \\
\hline hypoalbuminia & ifofosfamide & Sweiss et al., 2008 \\
\hline metabolic effects & $\begin{array}{l}\text { lamotrigine, oxcarbazepine, } \\
\text { valproic acid, }\end{array}$ & $\begin{array}{l}\text { Bauer \& Elger 1993, } \\
\text { Hennessy \& Miles, 1996 }\end{array}$ \\
\hline neurotoxic effect & IVIG & Wada et al., 2005 \\
\hline $\begin{array}{l}\text { posterior reversible } \\
\text { leukoencephalopathy } \\
\text { syndrome }\end{array}$ & $\begin{array}{l}\text { dexametha-sone, } \\
\text { tacrolimus, }\end{array}$ & $\begin{array}{l}\text { Kim et al., 2011; } \\
\text { Irvin et al., 2007; } \\
\text { Zhang, 2011 }\end{array}$ \\
\hline vasogenic brain edema & metronidazole & Kim et al., 2011 \\
\hline
\end{tabular}

Table 3. Drug-induced encephalopathy-pathophysiological mechanisms

\section{Pathological studies}

Mild gliosis of the white matter and ischemic lesions in the temporal area were observed in a patient's postmortem analysis (Steeghs et al., 2003). Pathological-anatomic studies showed changes in the cerebellum and temporal lobe of predominantly the pyramidal and purkinje cells in rats after chronic administration of valproate acid (Sobaniek-Lotowska, 2003). Those studies reported damage to the hippocampal astrocytes and neocortex. All these abnormalities seemed to disappear three months after discontinuation of the drug.

\section{Genetic susceptibility}

A further factor contributing to the development of a drug-induced encephalopathy is genetic susceptibility. The individual's genetic patrimony including ethnicity and gender influences the susceptibility to the risk of a drug-induced encephalopathy. Any genetic polymorphism may influence the metabolism, excretion or action of the drug depending on single or multiple genes or by changes in gene expression (Dodd et al., 2004). For instance, some mutations can promote development of an encephalopathy, i.e. a mutation in ETHE1, a mitochondrial matrix sulphur dioxygenase causing an ethlymalonic encephalopathy (Viscomi et al., 2010). In a patient with the rare missense variant methionine synthetase c.2756A $>G$ (D919G), a methotrexate encephalopathy was observed probably due to a modified effect of methotrexate on homocysteine metabolism (Linnebank et al., 2007). A recent clinical study showed that the genetic polymorphism of the human thymidylate synthetase gene contributes to 5fluorouracil-associated hyperammonemic encephalopathy. A GABA A receptor modification caused by knockout of the taurine transporter resulted in striatal disinhibition in mice. This animal study demonstrates that a genetic defect ending up in a lack of taurine partly explains the pathophysiology of a hepatic encephalopathy (Sergeeva et al., 2007). Mitochondrial dysfunction underlies different types of encephalopathy, for example, mitochondrial myopathy, encephalopathy, lactic acidosis, and stroke-like episodes (MELAS). As an example, the mutation of mitochondrial DNA (mtDNA) G13513A encoding the ND5 subunit of 
respiratory chain complex 1 causes mitochondrial encephalopathy with lactic acidosis (Wang et al. 2008). Therefore, supplementation with the mitochondrial respiratory chain cofactor coenzyme Q10 has been demonstrated to advance recuperation following heroin-induced encephalopathy (Gacouin et al., 2003).

\section{Epidemiology}

The epidemiology of ifofosfamide encephalopathy is well known. Ifofosfamide encephalopathy occurred in $31.2 \%$ of patients with soft tissue and bone sarcomas $(17 / 61)$ treated with ifofosfamide, and in $13.6 \%$ of ifofosfamide treatment courses. A history of cisplatin was identified as a potential risk factor for the development of an ifofosfamideinduced encephalopathy (Tajino et al., 2010). Furthermore, a dose of ifofosfamide at $>9 \mathrm{~g} / \mathrm{m}^{2}$ is a further risk factor of ifofosfamide-induced encephalopathy (Tajino et al., 2010). In other previous studies, risk factors such as large tumors in the female pelvic cavity (Meanwell et al., 1986), poor performance status (Antman et al., 1989), hypoalbuminemia (Merimsky et al., 1992), high serum creatinine level (Antmann et al., 1990) and low iron bicarbonate level (Antmann et al., 1989) were identified as risk factors for ifofosfamide-induced encephalopathy. It remains controversial as to whether there are risk factors of ifofosfamideinduced encephalopathy, as another study showed no risk factors associated with this encephalopathy and that each patient has his own predisposition (Rieger et al. 2004). Alcoholism was identified as a risk factor in linezolide-induced encephalopathy (Fletcher et al., 2010). Renal failure and previous central nervous system disease may predispose to ceftriaxone- and cefepime-induced encephalopathy (Roncon- Albuquerque et al., 2009; Garces et al. 2008). Dialysis may be a risk factor in isoniazide-induced encephalopathy (Cheung et al. 1993). There are common risk factors affecting the neuronal health for different types of drug-induced encephalopathies such as environmental toxins, infectious diseases, traumatic events, brain tumors, brain ischemia, age (state of health, disease), nutritional deficiencies and intolerances, and even poverty (Virmani et al. 2010).

In conclusion, not everyone develops an encephalopathy after taking a certain drug, but those individuals who are at risk (see above) - although the extent and nature of the risk are often unknown - may be more apt to develop an encephalopathy.

Patients with metronidazole encephalopathy showed a mean age of 61 years (49-71 years) (Kim et al., 2007), whereas those with clarithromycin encephalopathy exhibited an average age of 51 years (19-87 years) (Bandetti di Poggio et al., 2011). These data are based on case series, and there are no long-term clinical studies addressing the epidemiology of druginduced encephalopathies. In a cohort study of 19 patients, 8 patients $(42 \%)$ developed an ifofosfamide induced encephalopathy (Sweiss et al. 2008). The exact prevalence of a druginduced encephalopathy is unknown, as case series with calculated epidemiologic data are rare. Furthermore, there are no studies larger in scale examining the specific age of a druginduced encephalopathy. The average age for valproic-acid encephalopathy was 38.6 years in a long-term study (Gerstner et al. 2007).

Toxic encephalopathies are accompanied by high blood levels of the suspected drug, whereas drug-induced encephalopathies often reveal therapeutic blood levels of the drug. Thus we know of no dose-dependent effect of drugs that induce an encephalopathy. The symptoms can develop from within hours until a month after taking the drug. 


\section{Basic clinical features}

The clinical spectrum of symptoms can result in slight disturbances of the mental state up to severely damaged consciousness (table 4). Transient acute encephalopathy has been observed in 3-15\% of cancer patients after methotrexate therapy (Rubnitz et al. 1998). Chronic encephalopathy develops slowly, may progress, and can permanently impair neurological function. A drug-induced encephalopathy may reveal a varied spectrum of psychiatric symptoms, i.e. hallucinations (Sorafenib; Dogan et al., 2010), psychotic state (vigabatrine; Garcia-Pastore et al., 2000), depression (sodium valproate, Connacher et al., 1987) and neuropsychologic symptoms like reduced psychomotor speed and impaired working memory (levetiracetame, valproic acid; Bauer et al., 2008). The development of psychiatric symptoms may be acute, subacute or chronic.

\begin{tabular}{|c|c|c|}
\hline Clinics & Drugs & Reference \\
\hline abnormal sensation & metronidazole & Wada et al., 2005 \\
\hline $\begin{array}{l}\text { aggravation of preexisting } \\
\text { neurological deficits }\end{array}$ & $\begin{array}{l}\text { carbamazepine, gabapentine, } \\
\text { levetiracetame, lamotrigine, } \\
\text { oxcarbazepine, primidone, } \\
\text { topiramate }\end{array}$ & $\begin{array}{l}\text { Bauer, 2008; Hennessy \& } \\
\text { Miles, 1996, Horvath et al., } \\
\text { 2005; Katano et al., 2002; } \\
\text { Latour et al., 2004; } \\
\text { Siniscalchi et al., 2004; } \\
\text { Sechi et al., } 2004\end{array}$ \\
\hline $\begin{array}{l}\text { altered consciousness, } \\
\text { concentration }\end{array}$ & $\begin{array}{l}\text { capecitabine, gabapentine, } \\
\text { IVIG, lithium, valproic acid }\end{array}$ & $\begin{array}{l}\text { Bauer \& Elger 1993; Fantini } \\
\text { et al., 2010; Katano et al., } \\
\text { 2002; Sechi et al., } 2004\end{array}$ \\
\hline anisocoria, diplopia & dexamethasone & Irvin et al., 2007 \\
\hline aphasia & carbamazepine, topiramate & $\begin{array}{l}\text { Horvath et al., 2005, Latour } \\
\text { et al., } 2004\end{array}$ \\
\hline ataxia, apraxia & $\begin{array}{l}\text { capecitabine, carbamazepine, } \\
\text { phenytoine, topiramate, } \\
\text { valproic acid }\end{array}$ & $\begin{array}{l}\text { Fantini et al., 2010, Horvath } \\
\text { et al., } 2005\end{array}$ \\
\hline $\begin{array}{l}\text { choreiform movements, } \\
\text { athetosis }\end{array}$ & tiagabine, trimetazidine, & $\begin{array}{l}\text { Sivet et al., 2008; } \\
\text { Tombini et al., } 2006\end{array}$ \\
\hline delirium, coma & carmofur & Kuzuhara et al., 1987 \\
\hline dementia, memory loss & $\begin{array}{l}\text { carbamazepine, carmofur, } \\
\text { gabapentine, levetiracetame }\end{array}$ & $\begin{array}{l}\text { Bauer, 2008; Horvath et al., } \\
\text { 2005, Kuzuhara et al., 1987; } \\
\text { Sechi et al., } 2004\end{array}$ \\
\hline dysathria & $\begin{array}{l}\text { metronidazole, lithium, } \\
\text { tacrolimus, }\end{array}$ & $\begin{array}{l}\text { Smith et al., 2003; } \\
\text { Wada et al., 2005, }\end{array}$ \\
\hline gait disturbance & $\begin{array}{l}\text { carmofur, sorafenib, } \\
\text { trimetazidine, }\end{array}$ & $\begin{array}{l}\text { Dogan et al., 2010; } \\
\text { Kuzuhara et al., 1987; Sivet } \\
\text { et al., } 2008\end{array}$ \\
\hline headache & paclitaxel & Perry \& Warner, 1996 \\
\hline myoclonias & $\begin{array}{l}\text { carbamazepine, } \\
\text { levetiracetame, odansetrone, } \\
\text { vigabatrine, lithium }\end{array}$ & $\begin{array}{l}\text { Bauer, 2008; Garcia-Pastor } \\
\text { et al., 2000; Horvath et al., } \\
\text { 2005, Ritter et al., 2003; } \\
\text { Smith et al., } 2003\end{array}$ \\
\hline
\end{tabular}




\begin{tabular}{|l|l|l|}
\hline $\begin{array}{l}\text { oculogyric crisis, } \\
\text { oromandibular and } \\
\text { limb dystonia }\end{array}$ & odansetrone & Ritter et al., 2003 \\
\hline parkinsonism & carbamazepine & Horvath et al., 2005 \\
\hline psychiatric symptoms & $\begin{array}{l}\text { valproic acid, sorafenib, } \\
\text { levetiracetame, vigabatrine }\end{array}$ & $\begin{array}{l}\text { Dogan et al.2010; Garcia- } \\
\text { Pastor et al., 2000; } \\
\text { Bauer, 2008 }\end{array}$ \\
\hline ptosis & dexamethasone & Irvin et al., 2007 \\
\hline seizures & $\begin{array}{l}\text { cisplatin, cyclosporine, } \\
\text { gadolinium levetiracetame, } \\
\text { valproic acid, vigabatrine }\end{array}$ & $\begin{array}{l}\text { Bauer, 2008; Bauer \& Elger, } \\
\text { Garcia-Pastor et al., 2000; } \\
\text { Kapoor et al., 2010; Steeghs } \\
\text { et al., 2003; }\end{array}$ \\
\hline $\begin{array}{l}\text { sleep disturbance, } \\
\text { hypersomnia, insomnia }\end{array}$ & $\begin{array}{l}\text { capecitabine, carbamazepine, } \\
\text { topiramate }\end{array}$ & $\begin{array}{l}\text { Cheung et al., 2005, Fantini } \\
\text { et al., 2010, Horvath et al., } \\
\text { 2005, Cheung et al., 2005 }\end{array}$ \\
\hline stupor, agitated state & morphine & Eran \& Barak, , 2009 \\
\hline tremor & tacrolimus & Kim et al., 2011 \\
\hline vertigo & sorafenib, valproic acid & $\begin{array}{l}\text { Bauer \& Elger, 1993; Dogan } \\
\text { et al., 2010 }\end{array}$ \\
\hline $\begin{array}{l}\text { visual symptoms, } \\
\text { nystagmus }\end{array}$ & $\begin{array}{l}\text { metronidazole, phenytoine, } \\
\text { primidone, trimetazidine, } \\
\text { sorafenib, topiramate }\end{array}$ & $\begin{array}{l}\text { Wada et al., 2005; } \\
\text { Dogan et al., 2010; } \\
\text { Engel, 1971, } \\
\text { Katano et al., 2002, } \\
\text { Latour et al., 2004; } \\
\text { Sivet et al., 2008; }\end{array}$ \\
\hline vomiting, nausea & Bauer \& Elger, 1993 \\
\hline
\end{tabular}

Table 4. Drug-induced encephalopathy- Clinics

\section{Diagnostics}

\subsection{Electrophysiologic studies}

EEG has often revealed signs of encephalopathy. The main characteristics are a diffuse, unusually mild to heavy general changes (Horvath et al., 2005, Tombini et al., 2006). Triphasic waves with intermittent frontal delta activity are sometimes observed (Gallmetzer et al., 2004; Rancon-Albuquerque et al., 2009). Focal and generalised slow waves, and generalised or focal epileptic spike-wave complexes have also been seen. Once the responsible drug is discontinued, the encephalopathy with general slowing and epileptic discharges resolve after days or weeks, sometimes after months (Bauer \& Elger, 1993; Latour et al., 2004).

\subsection{Laboratory investigations}

The effective concentration of the drug in sera is often within the normal range in patients with valproic acid-induced encephalopathy (Bauer \& Elger, 1993), whereas higher concentration of the drugs were noted in carbamazepine (Neumann et al. 1994) and lamotrigine encephalopathy (Hennesy \& Miles, 1996). When clinical signs and symptoms of 
a drug-induced encephalopathy are present, relevant clinical routine tests for natremia, ammonemia or glycemia should always be performed to identify the reason for the encephalopathy and develop a treatment strategy. A hypoglycemic encephalopathy can be detected by measuring the blood glucose level, thereby differentiating it from drug-induced encephalopathies. The clinical spectrum of hypoglycemic encephalopathy ranges from simple neurological deficits and mental changes to severe coma and death (Lo et al., 2006). A specific lesion pattern is frequently detected in hypoglycemic encephalopathy, often affecting the cerebral cortex, basal ganglia, hippocampus, splenium and bilateral internal capsula (Aoki et al., 2004; Chan et al., 2003; Terakawa et al., 2007; Cho et al., 2006). This selective vulnerability in hypoglycemic encephalopathy may be associated with the extent to which the metabolism necessary to conserve the function of brain structures and neuronal integrity has been compromised (Lee et al., 2011).

\subsection{Imaging patterns}

Cerebral atrophy has been observed in valproic-acid encephalopathy, especially in chronic encephalopathy as some authors have described in cranial computertomography (CT) and magnetic resonance imaging (MRI) (Baganz et al., 1994; Papazian et al., 1995). Lacunar lesions were found in gabapentin-induced encephalopathy (Sechi et al., 2004). Metronidazole-induced encephalopathy induced bilateral symmetric T2-hyperintense lesions in the cerebellar dentate nucleus, midbrain, dorsal pons, medulla, and splenium of the corpus callosum. Except for the corpus callosum, all lesions were irreversible. The lesions are often bilateral and symmetric. High signal intensity in T2-weighted images appeared, but the signal alterations did not demonstrate contrast enhancement and were reversible after drug discontinuation. Dexamethasone encephalopathy in MRI resulted in diffuse cortical and subcortical white matter lesions in symmetric bilateral distribution involving predominantly occipital areas, the cerebellum and focal areas of bilateral thalami not evident in the T1-weighted images characteristic of PRES. In cyclosporine-induced encephalopathy, the lesions show vasogenic oedema apparently in diffusion coefficient maps (Bartynski et al., 2007). Some imaging pattern are shown in table 5.

\begin{tabular}{|l|l|l|}
\hline Anatomic lesion pattern & Drugs & Reference \\
\hline $\begin{array}{l}\text { bilateral temporal } \\
\text { periventricular white-matter } \\
\text { lesions }\end{array}$ & sulfasalazine & Mut et al., 2008 \\
\hline centrum semi-oval atrophy & tacrolimus & Kim et al., 2011 \\
\hline cerebellum & metronidazole & Kim et al., 2011 \\
\hline corpus callosum & metronidazole & Kim et al., 2011 \\
\hline $\begin{array}{l}\text { cortical and subcortical parietal, } \\
\text { occipital and frontal white } \\
\text { matter lesions }\end{array}$ & dexamethasone, IVIG & $\begin{array}{l}\text { Irvin et al., 2007; Wada et } \\
\text { al., 2005 }\end{array}$ \\
\hline $\begin{array}{l}\text { deep white matter lesions } \\
\text { midbrain, pons, medulla lesions }\end{array}$ & metronidazole & $\begin{array}{l}\text { Fantini et al., 2010; Perry \& } \\
\text { Warner, 1996 }\end{array}$ \\
\hline
\end{tabular}

Table 5. Neuroimaging of drug-induced encephalopathies 


\section{Differential diagnosis}

The most important differential diagnosis of metronidazole-induced encephalopathy is Wernicke encephalopathy. In the early stages of the disease, the two entities may be confounded because they can produce similar clinical features. In metronidazole-induced encephalopathy (unlike Wernicke encephalopathy), lesions of cerebellar dentate nuclei are supported by pathological studies (Troncoso et al., 1981). Further differential diagnoses are acute infectious encephalitis and demyelinating disease including Marchiafava-Bignami disease. Other types of encephalopathies must be differentiated from drug-induced encephalopathies such as hepatic, heavy metal, uremic, septic, and mitochondrial encephalopathy.

\section{Therapeutic options}

Therapy consists in the immediate discontinuation of the suspected drug when first signs of encephalopathy appear. A complete reversal of symptoms should take place soon after drug discontinuation. The atrophy in valproate-induced encephalopathy can also be reversed in individual cases. The administration of L-carnitine makes therapeutic sense in cases of carnitine deficiency (Kelley, 1994). Intravenous carnitine was recently shown to be useful in the treating hyperammonemic encephalopathy (Bøhmer \& Hoymork, 2010). Moreover, the treatment of a valproate-induced encephalopathy via haemodialysis has succeeded (Tsai \& Chen, 2008). Short-term hemodialysis often helps to reverse the symptoms in cefepime encephalopathy (Lin et al., 2011). Drug-induced encephalopathy often has a good prognosis. Methylen blue is an therapeutic option for an ifosfamideinduced encephalopathy (Patel, 2006). A drug-induced encephalopathy can sometimes be prevented by adjusting the dosage and monitoring serum concentrations of the suspected drug. Normally the blood level of the suspected drug is within the therapeutic range, so that treating an overdose would make no sense. Only in particular situations are certain measures to treat overdoses necessary, i.e. gastric lavage, activated charcoal, hemodialysis, hyperhydration or forced diuresis.

\section{Conclusions}

Several drugs can induce drug-induced encephalopathies. They seldom occur in clinical practice, but are important pharmacological side effects. Drug-induced encephalopathies are a key differential diagnosis when a disturbance of the consciousness is initially unclear. The effective levels of the drug in the blood often fall within the reference and not in the toxic range. Effective therapy consists in immediately discontinuing the drug.

\section{References}

Adams P \& White C (1965). Isoniazid-induced encephalopathy. Lancet, Vol.1, No. 7387, (March 1965), pp. 680-682, PMID 14258550.

Adams EN, Marks A \& Lizer MH (2009). Carbamazepine-induced hyperammonemia. Am J Health Syst Pharm, Vol. 66, No.16, (August 2009), pp. 1468-70, PMID 19667003. 
Advani PP \& Fakih MG (2011). 5-FU-induced hyperammonemic encephalopathy in a case of metastatic rectal adenocarcinoid successfully rechallenged with the fluoropyrimidine analog, capecitabine. Anticancer Res, Vol. 31, No.1, (January 2011), pp. 335-8, PMID 21273620.

Ahmed A, Loes DJ \& Bressler EL (1995). Reversible resonance imaging findings in metronidazole-induced encephalopathy. Neurology, Vol.45, No.3 (Pt 1), (March 1995), pp. 588-589, PMID7898724.

Aires CC, Van Cruchten A, Ijlst L \& de Almeida IT, Duran M, Wanders RJ \& Silva MF (2011). New insights on the mechanisms of valproate-induced hyperammonemia: inhibition of hepatic N-acetylglutamate synthase activity by valproyl-CoA. J Hepatol. Vol. 55, No.2, pp. (August 2011) 426-34, PMID 21147182.

Aoki T, Sato T, Hasegawa K, Ishizaki R \& Saiki M (2004). Reversible hyperintensity lesion on diffusion-weighted MRI in hypoglycemic coma. Neurology, Vol.63, No.2, (July 2004), pp.392-393, PMID 15277649.

Antmann KH, Elias A \& Ryan L (1990). Ifofosfamide and mesna: response and toxicity at standard- and high-dose schedules. Semin Oncol, Vol.17, No. 68, (April 1990), pp. 72-5, PMID 2110386.

Antmann KH, Ryan L, Elias A, Sherman D \& Grier HE (1989). Response to ifofosfamide and mesna: 124 previously treated patients with metastatic or resectable sarcoma. J Clin Oncol, Vol. 7, No.1, (January), pp. 126-131, PMID 2491883.

Baganz MD \& Dross PE (1994). Valproic acid induced hyperammonemic encephalopathy. Am J Neuroradiol, Vol. 15, No.9, (October 1994), pp. 1779-1781, PMID 7847228.

Bandettin di Poggio M, Anfosso S, Andenino D, Primavera A (2011). Clarithromycininduced neurotoxicity in adults. J Clin Neurosci, Vol.18, No.3, (March 2011), pp. 313318. PMID 21269833.

Barret MJ \& Login IS (2009). Gemifloxacin associated neurotoxicity presenting as encephalopathy. The Annals of Pharmacotherapy, Vol.43, No.4, (April 2009), pp. 782284. PMID 19276313.

Bartynski WS \& Boardman JF (2007). Distinct imaging patterns and lesion distribution in posterior reversible encephalopathy syndrome. Am J Neuroradiol, Vol.28, No.7, (August 2007), pp. 1320-1327, PMID17698535.

Bauer JC \& Elger CE (1993). Die akute Valproinsäure-Enzephalopathie. Akt Neurol, Vol.20: , No.1, pp. 16-21.

Bauer J (2008). Encephalopathy induced by levetiracetam added to valproate. Acta Neurol Scand, Vol. 117, No.5, (May 2008), pp. 374-376, PMID 18081909.

Blindauer KA, Harrington C, Morris 3rd GL \& Ho KC et al. (1998). Fulminant progression of demyelinating disease after valproate-induced encephalopathy. Neurology, Vol. 51, No. 1, (July 1998), pp. 292-295, PMID 9674826

Bøhmer T, Bøen A \& Høymork SC (2010). Valproic-induced hyperamonemic encephalopathy, rapidly improved by i.v. carnitine and glucose/thiamine. Scand J Gastroenterol, Vol.45, No. 6, (June 2010), pp. 762-763, PMID 20163200

Boora K, Xu J \& Hyatt J. (2008). Encephalopathy with combined lithium-risperidone administration. Acta Psychiatr Scand, Vol.117, No. 5, (May 2008), pp. 394-395, PMID 18331580 . 
Bonkowsky HL (1982). Porphyrin and heme metabolism and the porphyrias. In Zakim D, Boyer TD, Eds: Hepatology: A Textbook of Liver Disease. Saunders, Philadelphia, 1982.

Bradley WG, Karllson IJ \& Rassol CG (1977). Metronidazole neuropathy. BMJ, Vol. 2, No. 6087, (September 1977), pp. 610-611, PMID 198056.

Brusilow SW \& Cooper AJ (2011). Encephalopathy in acute liver failure resulting from acetaminophen intoxication: New observations with potential therapy. Crit Care Med. (23 June 2001) Epub ahead of print, PMID 21705899.

Chadwick DW, Cumming WJ, Livingstone I \& Cartlidge NE. (1979). Acute intoxication with sodium valproate. Ann Neurol, Vol.-6, No.6, (December 1979), pp.552-553, PMID119482.

Cheung E, Wong V \& Fung CW (2005). Topiramate-valproate-induced hyperammonemic encephalopathy syndrome: case report. J Child Neurol, Vol. 20, No. 2, (February 2005), pp.157-160, PMID 15794187.

Chan R, Erbay S, Oljeski S, Thaler D \& Bhadelia R (2003). Case report: hypoglycemia and diffusion-weighted imaging. J Comput Assist Tomogr, Vol.27, No.3, (May-June 2003), pp. 420-43, PMID 12794609.

Cheung WC, Lo CY, Lo WK, Ip M \& Cheng IK (1993). Isoniazid induced encephalopathy in dialysis patients. Tuber Lung Dis, Vol.74, No.2, (April 1993), pp. 136-9, PMID 8324207.

Cho SJ, MinnYK \& Kwon KH (2006). Severe hypoglycemia and vulnerability of the brain. Arch Neurol, Vol. 63, No. 1, (January 2006), p. 138, PMID 16401748.

Connacher AA, Macnab MS, Moody JP \& Jung RT (1987). Fatality due to massive overdose of sodium valproate. Scott Med J, Vol. 32, No.3, (June 1987), pp. 85-6, PMID3114876.

Conway N, Beck E \& Somerville J (1968). Penicillin encephalopathy. Postgrad Med J,Vol. 44, No.518, (December 1968), pp. 891-897,PMID 5738046.

Delluc A, M. Y., Latour P \& Goas JY (2004). Encephalopathy and acute renal failure during acyclovir treatment. Rev Neurol (Paris), Vol.160, No. 6-7, (July 2004), pp.704- 706, PMID 15247861.

Dodd PR, Foley PF, Buckley ST, Eckert AL \& Innes DJ (2004). Genes and gene expression in the brain of the alcoholic. Addict Behav, Vol. 29, No. 7, (September 2004), pp. 12951309, PMID15345266.

Dogan E, Aksoy S, Arslan C, Dede DS \& Altungdag K (2010). Probable sorafenib-induced reversible encephalopathy in a patient with hepatocellular carcinoma. Med Oncol, Vol. 27, No.4, (December 2010), pp.1436-1437, PMID20012235.

Duarte J, Macias S \& Coria F (1993). Valproate induced coma: case report and literature review. Ann Pharmacother, Vol. 27, No. 5, (May 1993), pp. 582-583, PMID 8347908.

Dzudie A, Boissoannat P, Roussoulieres A, Cakmak, Mosbah K, Bejui FT, Obadia JF \& Sebbag L (2009). Cyclosporine-related posterior reversible encephalopathy syndrome after heart transplantation: should we withdraw ord reduce cyclcosporine?: case reports. Tranplant Proc, Vol. 41, No. 2, (March 2009), pp. 716720, PMID 19328965.

Engel, Cruz ME \& Shapiro B (1971). Phenytoin encephalopathy? Lancet, Vol. 2, No. 7728, (October 1971), pp. 824-825, PMID 4106649. 
Eran A \& Barak M (2009). Posterior reversible encephalopathy syndrome after combined general and spinal anesthesia with intrathecal morphine. Anesth Analg, Vol. 108, No. 2, (February 2009),pp. 609-612, PMID 19151296.

Esposito P, Piotti G, Bianzina S, Malul Y \& Dal Canton A (2011). The syndrome of inappropriate antidiuresis: pathophysiology, clinical management and new therapeutic options. Nephron Clin Pract, Vol.119, No.1, (Epub June 15 2011), pp.6273, PMID 21677440.

Evans J, Levesque D, Knowles K, Longshore R \& Plummer S (2003). Diazepam as a treatment for metronidazole toxicosis in dogs. J Vet Int Med, Vol. 17, No. 3, (MayJun 2003), pp. 304-310, PMID 12774970.

Fan CC, Huang MC \& Liu HC (2008). Lamotrigine might potentiate valproic acid-induced hyperammonemic encephalopathy. Neuropsychopharmacol Biol Psychiatry, Vol. 32, No. 7, (October 2008), pp. 1747-8, PMID 18602440.

Fantini M, Gianni L, Tassinari D, Nicoletti S, Possenti C, Drudi F, Sintini M, Bagli L, Tamburini E \&Ravaioli A. (2010). Toxic encephalopathy in elderly patients during treatment with capecitabine: literature review and a case report. J Oncol Pharm Practice (epub ahead of print) Oct 6 , PMID 20926454.

Fawaz al IM (1992). Fatal myeloencephalopathy due to intrathecal vincristine administration. Ann Trop Paediatr, Vol. 12, No.3, pp.339-342, PMID 1280054.

Fink K, Dooley DJ, Meder WP, Suman-Chauhan N, Duffy S, Clusmann H \& Göthert M (2002). Inhibition of neuronal Ca $2+$ influx by gabapentin and pregabalin in the human neocortex. Neuropharmacology, Vol. 42, No. 2, (February 2002), pp. 229-236, PMID 11804619.

Fletcher J, A. L., Feucht EC \& Curtis JM (2010). Early onset probable linezolid induced encephalopathy. J Neurol, Vol.257, No. 3, (March 2010), pp- 433-435, PMID19888623.

Gacouin A, Lavoue S, Signouret T, Person A, Dinard MD, Shpak N \& Thomas R (2003). Reversible spongiform leucoencephalopathy after inhalation of heated heroin. Intensive Care Med, Vol. 29, No.6, (June 2003), pp. 1012-5, PMID 12637976.

Gallmetzer P, Leutmezer F \& Baumgartner C (2004). Postictal paresis in focal epilepsiesincidence, duration and causes: a video-EEG monitoring study. Neurology, Vol. 62, No.12, (June 2004), pp.2160-2164, PMID 15210875.

Garcia-Pastor A, Garcia-Zarza E \& Peraita Adrados R (2010). Acute encephalopathy and myoclonic status induced by vigabatrine monotherapy. Neurologia, Vol. 15, No.8, (October 2010), pp. 370-374, PMID 11143506.

Gerstner T, Büsing D, Longin E, Kasper JM, Klostermann W, Hebing B, Hanefeld F, Eckel U, Hoffmann R, Bettendorf U, Weidner B, Wiemer-Kruel A, Brockmann K, Neumann FW, Sandrieser T, Wolff M \& König S. (2007). Valproat-assozierte Enzephalopathie19 neue Fälle in Deutschland zwischen 1994 und 2003-eine Nebenwirkung nicht nur bei Kindern. J Neurol Neurochir Psychiatr, Vol. 8, No.3, pp.10-15.

Goundrey J (1990). Vertigo after epidural morphine. Can J Anesth, Vol. 37, No. 7, (October 1990), pp. 804-805, PMID 2225300.

Grill MF \& Magati R (2008). Cephalosporin-induced neurotoxicity: clinical manifestations, potential pathogenic mechanisms, and the role of electroencephalografic monitoring. Ann Pharmacother, Vol. 42, No. 12, (December 2008), pp.-1843-1850, PMID19033476. 
Hennessy MJ \& Miles CM (1996). Lamotrigine encephalopathy. Lancet, Vol. 347, No. 9006, pp.974-975, PMID 8598790.

Herishanu YO, Zlotnik M, Mostoslavsky M, Podgaietski M, Frisher S \& Wirguin I (1998). Cefuroxime-induced encephalopathy. Neurology, Vol. 50, No.6, (June 1998), pp.1873-5, PMID 9633748.

Hinchey J, Chaves C, Appignani B, Breen J, Pao L, Wang A, Pessin MS, Lamy C, Mas JL \& Caplan LR (1996). A reversible posterior leukoencephalopathy syndrome. $N$ Engl J Med, Vol. 334, No. 8, (February 1996), pp.494-500, PMID 8559202.

Ho CM \& Chan KH (2007). Posterior reversible encephalopathy syndrome with vasospasm in a postpartum woman after postdural puncture headache following spinal anesthesia (case report). Anesth Analg, Vol. 105, No.3, (September 2007), pp.770-772, PMID 17717238.

Horvath J, Coeytaux A, Jallon P, Landis T, Temperli P \& Burkhard PR (2005). Carbamazepine encephalopathy masquerading as Creutzfeld-Jakob disease. Neurology, Vol. 65, No.4, (August 2005), pp. 650-651, PMID16116141.

Inaba H, Khan RB, Laningham FH, Crews KR, Pui CH \& Daw NC. (2008). Clinical and radiological characteristics of methotrexate-induced acute encephalopathy in pediatric patients with cancer. Ann Oncol., Vol. 19, No. 1, (19 (1): 178-184, PMID 17947226.

Irvin W, MacDonald G, Smith JK \& Kim WY (2007). Dexamethasone-induce posterior reversible encephalopathy syndrome. J Clin Oncol, Vol.25, No.17, (June 2007), pp. 2484-2486, PMID17557962.

Jackson GD \& Berkovis SF (1992). Ceftazidime encephalopathy: absence status and toxic hallucinations. J Neurol Neurosurg Psychiatry, Vol.55, No.4 (April 1992), pp. 333-334, PMID 583528.

Kakinohana M, Marsala M, Carter C, Davison JK \& Yaksh TL. (2003). Neuroaxial morphine may trigger transient motor dysfunction after a non injurious interval of spinal cord ischemia: a clinical and experimental study. Anesthesiology, Vol.98, No.4, (April 2003), pp. 862-870, PMID12657847.

Kapoor R, Liu J, Devasenapathy A \& Gordin V (2010). Gadolinium encephalopathy after intrathecal gadolinium injection. Pain Physician, Vol.13, No.5, (September-October 2010), pp.231-236, PMID20859323.

Karper LP, Salloway S, Seibyl JP \& Krystal JH (1992). Prolonged postictal encephalopathy in two patients with clozapine-induced seizures. J Neuropsychiatry Clin Neurosci, Vol. 4, No 4 : (Fall 1992), pp. 454-457, PMID1422175.

Katano H, Fukishimuga T, Karasawa K, Sugiyama N, Ohkura A \& Kamiya K (2002). Primidone induced hyperammonemic encephalopathy in a patient with cerebral astrocytoma. J Clin Neurosci, Vol. 9, No. 1, (January 2002), pp. 79-81, PMID: 11749025.

Kelley RI (1994). The role of carnitine supplementation in valproic acid therapy. Pediatrics, Vol. 93, No.(6 Pt1), (June 1994), pp.891-892, PMID 8190571.

Kim E, Na DG, Kim EY, Kim JH, Son KR \& Chang KH (2007). MR imaging of metronidazole-induced encephalopathy: lesion distribution and diffusion-weighted imaging findings. AJNR Am J Neuroradiol, Vol. 28, No.9, (October 2007), pp. 1652-8, PMID 17885234. 
Kim H, Kim Y, Kim SR, Park IS \& Jo KW (2011). Metronidazole-induced encephalopathy in a patient with infectious colitis: a case report. J Med Case Reports, Vol. 5, (February 2011), pp. 63, PMID 21320332.

Kim JM, Ryu WS, Hwang YH \& Kim JS (2007). Aggravation of ataxia due to acetazolamide induced hyperammonaemia in episodic ataxia. J Neurol Neurosurg Psychiatry, Vol. 78, No.7, (July 2007), pp. 771-2. PMID17575025.

Kim MU, Kim SY, Son SM \& Park YH (2011). A case ot tacrolimus-induced encephalopathy after kidney transplantation. Korean J Pediatr, Vol. 54, No. 1, (January 2011), pp.4044, PMID 21359060.

Kim SR, Park CH, Park S, Park JO, Lee J \& Lee SY (2010). Genetic polymorphisms associated with 5-Fluorouracil-induced neurotoxicity. Chemotherapy, Vol.56, No.4, (Epub 13 August 2010), pp. 313-317, PMID 20714149.

Kumar G, Sahava K, Goyal MK, Sivaraman M \& Sahota PK (2010). Electroencephalographic abormalities in baclofen-induced encephalopathy. J Clin Neurosic, Vol. 17, No. 12, (December 2010), pp.1594, PMID20833050.

Kuzuhara S, Ohkoshi N, Kanemaru K, Hashimoto H, Nakanishi T \& Toyokura Y (1987). Subacute leucoencephalopathy induced by carmofur, a 5-fluorouracil derivative. J Neurol, Vol. 234, No. 6, pp.-365-370, PMID3309192.

Kwon HM, Kim HK, Cho J, Hong YH \& Nam H (2008). Cycloserine-induced encephalopathy: evidence on brain MRI. Eur J Neurol , Vol. 15, No. 7, (July 2008), pp.e60-61, PMID18484995.

Latour P, Biraben A, Polard E, Bentué-Ferrer D, Beauplet A, Tribut O \& Allain H(2004). Drug-induced encephalopathy in six epileptic patients: topiramate? valproate? or both? Hum Psychopharmacol, Vol.19 , No.3, (April 2004), pp.-193-203, PMID15079854.

Lee SH, Kang CD, Kim SS, Tae WS, Lee SY, Kim SH \&Koh SH (2010). Lateralization of hypoglycemic encephalopathy: evidence of a mechanism of selective vulnerability. J Clin Neurol, Vol.6, No.2, (June 2010), pp. 104-108, PMID 20607051.

Lin CJ, Chen SP, Wang SJ \& Fuh JL (2011). Cefepime-related encephalopathy in peritoneal dialysis patients. J Chin Med Assoc, Vol. 74, No. 2, (February 2011), pp.87-90, PMID2135408

Linnebank M, Malessa S, Moskau S, Semmler A, Pels H, Klockgether T \& Schlegel U (2007). Acute methotrexate-induced encephalopathy- causal relation to homozygous allelic state for MTX c.2756 A>G (D919G). J Chemother, Vo. 19, No.4, (August 2007), pp. 455-7, PMID 17855192.

Lo T, Tan AC, Umapathi T \&Lim CC (2006). Diffusion-weighted MR imaging in early diagnosis and prognosis of hypoglycemia. AJNR Am J Neuroradiol, Vol. 27, No.6, (June-July 2006), pp.1222-1224, PMID 16775268.

Lokrantz CM, Eriksson B, Rosén I \& Asztely F (2004). Hyperammonemic encephalopathy induced by a combination of valproate and pivmecillinam. Acta Neurol Scand, Vol. 109, No.4, (April 2004), pp. 297-301, PMID 15016014.

Maramattom BV, Zaldivar RA, Glynn MS, Eggers SD \& Wijdicks EFM (2005). Acute intermittent porphyria presenting as a diffuse encephalopathy, Vol.57, No.4, (April 2005), pp. 581-584, PMID 15786449. 
Manca D, Cossu G, Murgia D, Molari A, Ferrigna P, Marcia E \& Melis M (2009). Reversibility of encephalopathy and axonal neuropathy during in Parkinson's disease during Duodopa therapy. Mov Disord, Vol. 24, No. 15, pp. 2293-2294, PMID19795477.

Maxa JL, Taleghani A, Ogu CC \& Tanzi M (1997). Possible toxic encephalopathy following high-dose intravenous haloperidol. Ann Pharmacother, Vol. 31, No. 15, (November 1997), pp.736, PMID 19795477.

Meanwell CA, Blake AE, Kelly KA, Honigsberger L \& Blackledge G (1986). Prediction of ifofosfamide /mesna associated encephalopathy. Eur J Cancer Clin Oncol, Vol.22, No.7, (July 1986), pp.815-819, PMID 3095121.

Merimsky O, Reider-Grosswasser I, Wigler N \& Chaitchik S (1992). Encephalopathy in ifofosfamide-treated patients. Acta Neurol Scand, Vol.86, No.5, (November 1992), pp.521-525, PMID1481635.

Miyaoka H \& Kamijama K (1995). Encephalopathy during amitriptyline therapy: are neuroleptic malignant syndrome and serotonin syndrome spectrum disorders? Int Clin Psychopharmacol, Vol. 10, No. 4, (November 1995), pp.265-267, PMID8748050.

Miyazaki C, Kamijima K \& Ichikawa M. (1988). Effect on sodium valproate (VPA)- induction cerebral amino acids: mechanism of gammaaminobutyric acid (GABA) elevation and possible causal relation of VPA-induced encephalopathy and glutamine level. Chem Pharm Bull, Vol.36, No. 9, (September 1988), pp. 3589-3594, PMID3149216.

Morales Odia Y, Jinka M \& Ziai WC (2010). Severe leukencephalopathy following acute oxycodone intoxication. Neurocrit Care, Vol. 13, No. 1 (August 2010), pp. 93-97, PMID 22440598.

Mut SE, Kutlu G, Ucler S, Erdal A \& Inan LE (2008). Reversible encephalopathy due to sulfasalazine. Clin Neuropharmacol, Vol. 31, No. 6, pp. 6 368-371, PMID19050416.

Noremberg MD (1996). Astrocytic-ammonia interactions in hepatic encephalopathy. Semin Liver Dis, Vol. 16, No. 3, (August 1996), pp.245-253, PMID8989810.

Ortiz A, Martin-Llonch N, Garron MP, Alberola ML, Caramelo C \& Ortiz-Gonzalez A (1991). Cefazolin-induced encephalopathy in uremic patients. Rev Infect Dis, Vol.13, No.4, (July-August 1991), pp. 772-3, PMID 1925303.

Park-Matsumoto YC \& Tazawa T (1996). Piperacillin-induced encephalopathy. J Neurol Sci, Vol. 1, No.140, (September 1996), pp. 1-2, PMID 8866141.

Patel N (2006). Methylene blue for management of Ifosfamide-induced encephalopathy. Ann Pharmacother, Vol. 40, No.2, (February 2006), pp.299-303, PMID16391008.

Papazian O, Canizales E, Alfonso I, Archila R, Duchowny M \& Aicardi J (1995). Reversible dementia and apparent brain atrophy during valproate therapy. Ann Neurol, Vol. 38, No. 4,

(October 1995), pp.687-691, PMID 7574471.

Perry JR \& Warner E (1996). Transient encephalopathy after paclitaxel (Taxol) infusion. Neurology, Vol. 46, No.1596-159, (June 1996), pp. 1596-9, PMID8649555.

Posadas I, Santos P, Blanco A, Munoz-Fernandez M \& Cena V (2010). Acetaminophen induces apoptosis in rat cortical neurons. PloS ONE, Vol.5, No.12, e15360, PMID 21170329. 
Pro S, Randi F, Pulitano P, Vicenzini E \& Mecarelli O (2011) Reversible encephalopathy induced by cefoperazone: a case report monitored with EEG. Neurol Sci, Vol.32, No.3, (June 2011), pp. 465-467, PMID 20927561.

Rancon-Albuquerque R JR, Pires I, Martins K, Real R, Sousa G \& van Hafe P (2009). Ceftriaxone-induced reversible encephalopathy in a patient treated for a urinary tract infection. Noth J Med, Vol. 67, No. 2, (February 2009), pp. 72-75, PMID19299850.

Rfidah El, Findlay CA \& Beattie TJ (1995). Reversible encephalopathy after intravenous ciprofloxacin therapy. Pediatr Nephrol, Vol. 9, No. 2, (April 1995), pp. 250-251, PMID 7794728.

Rieger C, Fiegl M, Tischer J, Ostermann H \& Schiel X (2004). Incidence and severity of ifosfamide-induced encephalopathy. Anticancer Drugs, Vol.15, No.4, (April 2004), pp. 347-50, PMID 15057138.

Ritter MJ, Goodman BP, Sprung J \& Wijdicks EF (2003). Ondansetrone-induced multifocal encephalopathy. Mayo Clin Proc, Vol. 78, No.9, (September 2003), pp. 1150, PMID12962170.

Rubenstein JL, Johnston K, Elliott GR \& Brusilow SW (1990). Haloperidol-induced hyperammonaemia in a child with citrullinaemia. J Inherit Metab Dis, Vol.13, No. 5, pp. 754-5, PMID 2246861.

Sasazaki Y, Asami K \&Utsumi J (1992). Transient subacute encephalopathy induced by highdose methotrexate treatment in children with acute lymphoblastic leukemia and malignant lymphoma. Gan To Kagaku Ryoho, Vol. 19, No. 11, (September 1992), pp. 1851-1857,PMID1519928.

Savica R, Rabinstein A \& Josephs KA (2011). Ifosfamide associated myoclonusencephalopathy syndrome. J Neurol, Mar 12. [Epub ahead of print], PMID 21399985

Sechi G, Murgia B, Sau G, Peddone L, Tirotto A, Barrocu M \& Rosati G (2004). Asterixis and toxic encephalopathy induced by gabapentin. Prog Neuropsychopharmacol Biol Psychiatry, Vol. 28, No. January 2004: 195-199, PMID14687874.

Sergeeva OA, Fleischer W, Chepkova AN, Warskulat U, Häussinger D, Siebler M \& Haas HL (2007). GABA A receptor modification in taurine transporter knockout mice causes striatal disinhibition. J Physiol, Vol. 585, No. 2, (December 2007), pp. 539548, PMID 17962336.

Settle, EC (1995). Valproic acid -associated encephalopathy with coma. Am J Psychiatry, Vol.152, No.8, (August 1995), pp.1236-1237, PMID 7625438.

Shaikh AY, Muranjan MN, Gogtay NJ \& Lahiri KR (2009). Possible mechanism for zonisamide-induced hyperammonemia in a child with citrullinemia type 1. Indian J Med Sci, Vol. 63, No. 5, (May 2009), pp. 203-6, PMID 19584493.

Siniscalchi A, Mancuso F, Scornaienghi D, Ferreri G \& De Sarro G (2004). Acute encephalopathy induced by oxcarbazepine and furosemide. Ann Pharmacother, Vol. 38, No. 3, (March 2004), pp.509-510, PMID 14970374.

Sivet J, de la Gastine B, Mosquet B, Lescure P, Boutemy J, Le Boisselier R \& Coquerel A (2008). Trimetazidine-induced encephalopathy with choreiform disorders: a case report. Rev Med Interne, Vol. 29, No.6, (June 2008), pp. 512-515, PMID 18206269.

Smith D, Keane P, Donovan J, Malone K \& McKenna TJ (2003). Lithium encephalopathy. J R Soc Med, Vol.96, No. 12, pp.590-591, PMID 14645609. 
Steeghs N, de Jongh JF, Sillevis Smitt PA \& van den Bent MJ (2003). Cisplatin-induced encephalopathy and seizures. Anticancer Drugs, Vol. 14, No. 6, (July 2003), pp. 443446, PMID12853886.

Sobaniek-Lotowska ME (2003). Ultrastructure of astrocytes in the cortex of the hippocampal gyrus and in the neocortex of the temporal lobe in experimental valproate encephalopathy and after withdrawal. Int J Exp Pathol, Vol. 84, No. 3, (June 2003), pp. 115-125, PMID12974941.

Suarez Ortega S, Rodriguez Perdomoe E, Parrilla DJ, Ayalan Galan E, Artiles Vizcaino J \& Melado Sanchez P (1995). Encephalopathy, convulsions and hypopotassemia in theophylline poisoning: a case analysis. Arch Bronchopneumol, Vol._31, No. 7, (August-September 1995), pp. 368-370, PMID8777534.

Sweiss KI, Beri R \& Shord SS (2008). Encephalopathy after high-dose ifosfamide: a retrospective cohort study and review of the literature. Drug Saf, Vol. 31, No. 11, pp. 989-996, PMID 18840018.

Tajino T, Kikuchi SI, Yamada H, Takeda A \& Konno SI (2010). Ifofosfamide encephalopathy associated with chemotherapy for musculoskeletal sarcomas: incidence, severity, and risk factors. J Orthop Sci, Vol. 15, No.1, (January 2010), pp.104-111, PMID 20151259.

Terakawa Y, Tsuyuguchi N, Nunomura K, Murayama N, Fujishige M, Yamamura A Nakagawa T \& Hashi K (2007). Reversible diffusion-weighted imaging changes in the splenium of the corpus callosum and internal capsule associated with hypoglycemia-case report. Neurol Med Chir (Tokyo),Vol. 47, No.10, (October 2007), pp. 486-488, PMID 17965569.

Tombini M, P. L., Pasarelli F et al. (2006). Transient athetosis induced by tiagabine. Epilepsia, Vol. 47, No. 4, (April 2006), pp.799-800, PMID 16650148.

Troncoso JC, J. M., Hess KM, et al. (1981). Model of Wernicke's encephalopathy. Arch Neurol, Vol. 38, No. 6, (June 1981), pp. 350-354, PMID 7236062.

Tsai MF \& Chen CY (2008). Valproate-induced hyperammonemic encephalopathy treated by hemodialysis. Ren Fail, Vol. 30, No. 8, pp. 822-824, PMID 18791959.

Virmani A, Ali S \& Zbigniew KB (2010). Neuroprotective strategies in drug abuse-evoked encephalopathy. Annals of the New York Academy of Sciences, Vol. 1199, ( June 2010), pp.52-68, PMID20633109.

Viscomi C, Burlina AB, Dweikat I, Savoiardo M, Lamperti C, Hildebrandt T, Tiranti V \& Zeviani M (2010). Combined treatment with oral metronidazole and $\mathrm{N}$ acetylcysteine is effective in ethylmalonic encephalopathy. Nature Medicine, Vol. 16, No.8, (August 2010), pp. 869-871, PMID 20657580.

Wada A, Yoshida R, Oda K, Fukuba E, Uchida E \& Kitagaki H. (2005). Acute encephalopathy associated with intravenous immunoglobulin therapy. AJNR, Vol. 26, No. 9, (October 2005), pp. 2311-2315, PMID 162119838.

Wang SB, Weng WC, Lee NC, Hwu WL, Fan PC \& Lee WT (2008). Mutation of mitochondrial DNAG13513A presenting with Leigh syndrome, Wolff-ParkinsonWhite syndrome and cardiomyopathy. Pediatr Neonatol, Vol. 49, No.4, (August 2008), pp.145-149, PMID 1905492.

Wong A, Williams M \& Gibb W (2010). Isotretinoin-induced encephalopathy. J Dermatolog Treat, Vol. 21, No. 6, (November 2010), pp. 361-362, PMID20059367. 
Wright KH \& Tyler J. (2005). Recognizing metronidazole toxicity in dogs. Vet Med , Vol. 98: pp. 410-418.

Zamvar V, Sugarman I, Tawfik RF, Macmullen-Price J \& Puntis JW (2009 ). Posterior reversible encephalopathy syndrome following infliximab infusion. J Pediatr Gastroenterol Nutr, Vol.-48, No. 1, (January 2009), pp. 102-5, PMID19172132.

Zhang HL (2011). Tacrolimus leukoencephalopathy- it is posterior reversible encephalopathy syndrome? Pediatr Neurol, Vol._44, No. 3, (March 2011), pp. 236, PMID 21310344. 


\title{
Sonographic Changes in Hypoxic-Ischaemic Encephalopathy
}

\author{
Pilvi Ilves \\ University of Tartu \\ Estonia
}

\section{Introduction}

Hypoxic-ischaemic encephalopathy (HIE) in a full-term infant is a clinically defined syndrome of the disturbed neurologic function in the earliest days after birth in infancy, manifested by difficulty with initiating and maintaining respiration, the depression of the muscle tone and reflexes, the subnormal level of consciousness and often seizures (Nelson \& Leviton, 1991). HIE is the term used to describe the resultant condition due to a deficit in the oxygen supply to the brain. The neonatal brain can have a lack of oxygen through two major pathogenetic mechanisms, hypoxemia, which is a diminished amount of oxygen in the blood supply, and ischemia, which is a diminished amount of blood perfusing the brain. During the perinatal period hypoxemia or ischemia or both occur as a result of asphyxia, an impairement in the exchange of respiratory gases, oxygen and carbon dioxide. The duration and severity of HIE depend of the severity of asphyxia. Neonatal asphyxial encephalopathy occurs in 1-2 per 1000 births in the high income countries (Marlow \& Budge, 2005; Pierrat et al., 2005; Barkovich, 2005), in low-income countries, the incidence is much higher (Ellis et al., 2000; Black et al., 2010).

Despite major advances in obstetric and neonatal care, and the introduction of therapeutic hypothermia, HIE is still a devastating occurrence that results in death or disability in $47 \%$ of infants with moderate or severe HIE (Edwards et al., 2010). As survivours of severe HIE have profound long-term neurologic disability like cerebral palsy, mental retardation and epilepsy (Finer et al., 1992; Perlman, 2006) and a large majority of infants with moderate HIE have cognitive problems (Lindström et al., 2008), sequelae of hypoxic-ischaemic brain injury require significant resources (Lindström et al., 2008).

The principal mechanism of pathogenesis underlying most of the neuropathology attributed to intrapartum hypoxia-ischemia is impaired cerebral blood flow, which occurs most likely because of interruption in placental blood flow and gas exchange (Perlman, 2006).

However, the development of brain injury after hypoxia-ischemia is an evolving process which is initiated during the hypoxic-ischemic insult but extend into the reperfusion period during recovery (Fellman \& Raivio, 1997; Perlman, 2006). The brain damage, following hypoxic-ischemic insults, develops after a delay, being preceeded by the symptom-free interval or even the temporary improvement of the clinical picture immediately after insult (van Bel et al., 1998). The time before the full development of brain damage represents a 
window of opportunity for therapeutic interventions (Robertson \& Edwards, 1998). It is therefore essential to assess the severity of asphyxia in the newborn infant as soon as possible after birth or during the first hours after asphyxia to provide adequate care and treatment before the final damage develops. As the duration and severity of asphyxia may be difficult to estimate and the compensatory capacity of the foetus and newborn is unknown, it may be difficult to predict the severity of organ damage immediately after birth based on the clinical findings only.

The treatment of HIE consists largely of supportive care, which does little to reduce the ongoing injury that develops in the hours immediately following the hypoxic-ischemic insult. Providing standard intensive care support, correcting metabolic acidosis, close monitoring of the fluid status, and seizure control are the main elements of treatment in patients with HIE (Stola \& Perlman, 2008). In recent years, randomised trials of hypothermia (reducing the core temperature by $3.5{ }^{\circ} \mathrm{C}$ for $72 \mathrm{~h}$ ) have convincingly shown a neuroprotective effect (Edwards et al., 2010) and increase intact survival after birth asphyxia (Hagmann et al., 2011). Cooling is recommended after severe birth asphyxia by both the UK NICE (http://www.nice.org.uk/) and the International Consensus of Cardiopulmonary Resuscitation (ILCOR) guidelines (2010) (Perlman et al., 2010). Over the last two decades, promising new neuroprotective strategies designed to limit the extent of brain injury caused by hypoxia-ischemia have been under investigation (Robertson \& Edwards, 1998; Shadid et al., 1998; Thoresen \& Whitelaw, 2000; van Bel et al., 1998). Many of these promising treatment strategies, including hypothermia and the administration of antagonist against excitatory amino acids or free radicals, have a limited window of effectiveness (in some cases as little as 6 hours), making early detection of injury critically important (Shadid et al., 1998; Thoresen \& Whitelaw, 2000; van Bel et al., 1998). Neuroimaging with ultrasonography (US), computed tomography (CT), and magnetic resonance imaging (MRI) have become increasingly valuable in the pre-treatment work-up of patients with HIE as they can provide information on the severity and extent of injury (Huang \& Castillo, 2008).

US is a simple radiological method easily applicable in critically ill children (Ilves et al., 2009a, 2009b). Cranial US is a useful tool to early detect developmental abnormalities and exclude other causes of encephalopathy than hypoxia-ischemia (Epelman et al., 2010; Lowe \& Bailey, 2011a, 2009b). Doppler US is a non-invasive method, which allows repeated and safe assessment of hemodynamics in neonatal units (Ilves et al., 2008, 2009a, 2009b). Several studies have demonstrated that the pulsed Doppler technique can be used to examine the pattern of blood flow velocity (BFV) in the major vessels of the brain (Ehehalt et al., 2005) as well as visceral organs (Weir et al., 1995) in neonates with good reproducibility. The BFV findings have clinical relevance in interpreting the cardiovascular adaptation of distressed newborn infants with a decompensation of circulatory responses and the possible development of multiorgan failure including brain damage (Ilves et al., 2009b).

Although MRI is the reference standard for infant brain imaging (Barkovich, 1992, 2005; Epelman et al., 2010; Martin et al., 1995; Rutherford et al., 1994, 1996), it is expensive, often requires sedation and transportation of the neonate, and may not be possible to perform on critically ill patients (Lowe \& Bailey, 2011a). With the improvement in sonographic equipment, the visualization of a variety of pathology and anatomic variants has become more feasible. Recent studies, using modern ultrasound equipment, have shown that cranial US is diagnostically accurate compared with MRI and useful to determine initial clinical 
management (Epelman et al., 2010; Lowe \& Bailey, 2011a). In experienced hands, US is an excellent tool to detect the most frequently occurring brain abnormalities in the preterm and full-term neonates, to study the evolution of lesions, and to follow brain maturation. However, US also have limitations and MRI is needed in most neonates with (suspected) parenchymal brain injury and/or neurological symptoms (van Wezel Meijler et al., 2010).

\section{Principles of sonographic evaluation and Doppler investigations}

\subsection{Cranial ultrasound technique in HIE}

Imaging findings in HIE are highly variable and depend on a number of factors, including brain maturity, severity and the duration of insult, and the type and timing of imaging studies. Early imaging findings can be subtle and are often overlooked. Therefore, it is essential to be familiar with the different patterns of injury that may be observed and to pay attention to the areas that are most likely to be injured after asphyxia when interpreting studies performed in asphyxiated infants with suspected HIE (Epelman et al., 2010; Huang \& Castillo, 2008). As recent US studies show modern cranial US is required to detect these early subtle findings (Epelman et al., 2010).

Cranial US begins with basic grey-scale imaging using the linear-array transducer via the anterior fontanel in the coronal and sagittal planes (Epelman et al., 2010; Lowe \& Bailey, 2011a). Typically, six to eight coronal images are obtained beginning at the frontal lobes just anterior to the frontal horns and extending to the occipital lobes posterior to the lateral ventricle trigons. The transducer is then rotated 90 degrees, and approximately five images are obtained including a midline sagittal view of the corpus callosum and cerebellar vermis in addition to the bilateral parasagittal image beginning in the midline and progressing laterally through the peripheral cortex. The structure and echogenicity of the brain is evaluated to establish normality through the anterior fontanel (Lowe \& Bailey 2011a). The size and the configuration of the frontal horns of the ventricles, the interhaemispheric fissure and the subarachnoidal space, the visualization of the normal structures (including sulci), the existence of haemorrhages and parenchymal echodensities, the presence, location and size of cystic degeneration will be examined (Ilves et al. 2009a; Rutherford et al., 1994). The ventricular size is measured as the greatest distance perpendicular to the ventricular axis (the greatest axis of the lateral ventricle on the coronal scan), and the maximal interhemispheric distance and the maximal width of the subarachnoideal space on the coronal scan are also measured (Liao et al., 1986).

To complete the modern head US, screening images via other supplemental fontanels and high-resolution linear images are needed (Lowe \& Bailey, 2011a). An attempt must be made to visualize as much as possible of the central and peripheral structures of the brain using multiple acoustic windows, such as the anterior and the posterior fontanels, as well as views through the temporal, mastoid and occipital areas. The angle of the transducer must be varied in an attempt to evaluate the periphery of the brain with particular attention to the subcortical white matter and the grey-white matter differentiation in as much of the cerebral hemispheres as possible (Epelman et al., 2010). The visualization of the cerebellar hemispheres is optimised by obtaining images through the right and left mastoid fontanels, the technique of which has been shown to improve the detection of posterior fossa haemorrhages. Finally, the completion of the modern cranial US requires switching from the 
curved - to a linear -array transducer, which allows high-resolution imaging of the brain with the detailed interrogation of the subarachnoid space and superficial cortex as well as deeper brain structures. Linear images can be adjunctively obtained via any fontanel (Epelman et al., 2010).

North \& Lowe describe some basic cranial US principles, which are helpful in interpretation of the findings, in 2009 (North and Lowe, 2009). First, the grey matter tends to be hypoechoic and the white matter tends to be hyperechoic. When this pattern is reversed, abnormality is indicated. Second, the normal brain is always symmetric, but symmetric findings are not always normal. This principle can avoid overlooking symmetric abnormalities such as bilateral hyperechoic thalami which can develop after thalamic ischemia or infarct during HIE (Huang \& Castillo, 2008; North \& Lowe, 2009). A third principle involves the visualization of all the layers of the normal cortex: the superficial pia mater should be seen as a thin well-defined hyperechoic layer overlying the hypoechoic cortical grey matter, which in turn overlies the hyperechoic white matter (Lowe \& Bailey, 2011a; North \& Lowe, 2009). Failure to distinctly visualize all these normal layers is helpful to identify the areas of abnormality, such as focal haemorrhage or infarct. Fourth, the periventricular white matter is normally homogeneous in echogenicity as is equal to or less echogenic than the adjacent chorioid plexus (Lowe \& Bailey, 2011a; North \& Lowe, 2009). Asymmetric or heterogenic increase echogencity of the periventricular white matter suggests an abnormality, as can occur with developing periventricular leucomalacia. The enlargement of liquor spaces and multicystic encephalomalacy can develop as early as after the first week of life in infants with severe HIE (Ilves et al., 2009a).

\subsection{Cerebral Doppler ultrasound technique}

After viewing cranial structures with the grey-scale US colour Doppler is switched on to screen vascular structures (North \& Lowe, 2009). Colour, spectral, and power Doppler imaging can be performed in the coronal and transverse plane via the anterior or the temporal fontanels, respectively. Power Doppler is used to screen for regions of hyper- or hypovascularity (Epelman et al., 2010; Lowe \& Bailey, 2011). Colour Doppler abnormalities include changes in the RI on spectral tracings and abnormalities of flow velocity in the venous sinuses and arteries. The choice which fontanel is used is based on convenience as well as which vessels the operator wishes to visualize. Colour Doppler images via the posterior fontanel or foramen magnum can also be used to screen for patency of the transverse sinuses (Epelman et al., 2010).

At first, the arterial system is assessed for patency and resistance to flow by obtaining a colour Doppler image of the circle of Willis (Lowe \& Bailey, 2011a). The colour images via the anterior or temporal fontanel are used to localize the middle cerebral artery (MCA), the anterior cerebral artery (ACA), the internal cerebral artery (ICA) or the basilar artery (BA) and to obtain special tracing with velocity curves and the resistance index measurements (Ilves et al., 2008). After the arterial system, the venous system is evaluated with grey-scale US and Doppler techniques to document the patency of the sagittal sinus and the vein of Galen in the sagittal plane (Lowe \& Bailey, 2011; Epelman et al., 2010).

During colour Doppler studies systolic, diastolic and mean velocities and the resistance index are evaluated using spectral Doppler tracing in the major peripheral vessels: ACA, 
MCA, ICA and BA. Care must be taken to visualize as much as possible of each of these arteries to accurately determine the insonation angle. The angle between the estimated direction of blood flow and the Doppler beam must be as low as possible to accurately measure the velocity, suggested less than 20 or less degrees, therefore the evaluation of MCA is suggested via the temporal approach and BA, ACA and ICA via the anterior fontanel. Guided by the velocity signal, displayed on an oscilloscope and an audio signal, the highest possible velocities must be used in the range-gated mode. After a stable velocity recording over 20-30 consecutive beats are received, and at least the 3-5 consecutive waveforms with the highest amplitude recorded can be analysed for: (1) the peak systolic flow velocity (Vs); (2) the end diastolic peak flow velocity (Vd) and (3) the time-mean of the maximum velocity envelope curve (TAMV) and the time averaged mean velocity (TAV), i.e. the integral of the mean frequencies over at least 3-5 complete pulses. The resistance index (RI) is calculated according to the formula $\mathrm{RI}=(\mathrm{Vs}-\mathrm{Vd}) / \mathrm{Vs}$.

For the measurement of BFV in cerebral arteries, the ACA is visualised in the sagittal plane through the anterior fontanel, and the signals are recorded from the point midway between the inferior-most border of the corpus callosum and the vessel's origin from the circle of Willis (Evans et al., 1988; Hayashi et al., 1992; Ilves et al., 2008). The BA is visualized in the sagittal plane just before the pons. The ICA is found in the coronal plane in the base of the skull just below the corner of the sella in the pars petrosa, where the vessel is oriented towards the scanner (Hayashi et al., 1992). The MCA is easy to visualize through the temporal bone in the region above the zygomatic arch in the fold of the temporal lobe from the straight mid-portion of the artery (Evans et al., 1988; Ilves et al., 2008).

\subsection{Visceral Doppler ultrasound technique}

The majority of term infants after severe birth asphyxia develop disturbancies in other organs than the brain (Martin-Ancelet al., 1995, Wayenberg et al., 1998; Shah et al., 2004). The redistribution of the blood to maintain the perfusion of the brain during asphyxia causes long-lasting changes in the blood flow in visceral organs, first of all in kidneys and intestine (Ilves et al., 2009). To evaluate the visceral blood flow changes and possible development of multiorgan failure in HIE patients, the abdominal organs must also be investigated. Kidneys can be scanned from the dorsolateral view, and the size, structure and echogenicity of the kidneys are evaluated to establish normality. The BFV in the renal artery (RA) can be visualized by placing the transducer in the right dorsolateral area of the flank below the costal arch and the sampling volume placed in the proximal portion of the vessel with a simultaneous viewing of the abdominal aorta (Adriani et al., 2001; Agata et al., 1994; Ilves et al., 2008). The origin of the coeliac artery (CA) and the superior mesenteric artery (SMA) is localized from the aorta in a longitudinal plane and the sampling volume is placed in the proximal SMA and CA (Agata et al., 1994; Ilves et al., 2008; Weir et al., 1995).

\subsection{Neonatal US technique}

Neonates can be usually examined without sedation while asleep or in the awake-quiet state with eyes closed and performing no gross body movements, which can disturb the evaluation of the velocity curves. Whenever restlessness occurs, the measurements of the blood flow velocity can be taken only after the infants are properly soothed and quiet. Low cyclical variations in the cerebral BFV, dependent on the sleep state, have been observed in 
term neonates with significantly greater amplitude during quiet sleep (often used in older ages as non rapid eye movement sleep-NREM sleep) compared to active sleep (REM sleep). However, no difference in the median cerebral BFV was observed in different sleep states (Ferrarri, et al., 1994). The BFV measurements of visceral organs must be performed at least 60-90 min after feeding as feeding increases the BFV to CA and SMA (Coombs et al., 1992; Martinussen et al., 1994;).

The study must be performed as rapidly as possible to minimize adverse influences on the infant; however, experienced investigators usually perform the whole study less than 10-15 $\mathrm{min}$. The duration of the ultrasound exposure of one artery is suggested to be not more than $60 \mathrm{sec}$ and the output intensity not to exceed the suggested safety limit of $100 \mathrm{~m} \mathrm{~W} / \mathrm{cm}^{2}$.

The BFV and the RI are influenced by many factors, which must be taken into account during evaluation. The major regulatory mechanism for the cerebral blood flow is autoregulation (Pryds et al, 1990, 1991), but also PaCO2, the oxygen and blood glucose delivery and the total haemoglobin concentration level (Volpe, 2005).

The intracranial and visceral hemodynamics change dramatically after birth in healthy infants. A severe increase in the BFV and the decrease in cerebrovascular resistance take place during the first week of life with largest changes occurring within the first 24 hour as a part of neonatal adaptation after birth (Agata et al., 1994; Hayashi et al., 1992; Ilves et al., 2008; Kempley et al., 1996; Martinussen et al., 1994; Yildirim et al., 2005). Ductal close and progressive increase in the blood pressure may explain the observed increase in BFV and decrease in the RI in healthy infants (Agata et al., 1994; Hayashi et al., 1992; Ilves et al., 2008; Martinussen et al., 1994; Yildirim et al., 2005). Autoregulation of the cerebral blood flow is operative in a normal full term infant (Ilves et al., 2008), however a pressure-passive state of the cerebral circulation is observed in seriously asphyxiated full term infants Pryds et al., 1990, Ilves et al., 2008)

The resistance index is also influenced by the flow velocity, the blood volume, the presence of congenital cardiac anomalies and the peripheral vascular resistance. The RI becomes higher if the diastolic flow decreases. If the intracranial pressure exceeds systemic pressure impending diastolic cerebral blood flow, RI increases as it can happen during cerebral swelling (North \& Lowe, 2009). However, it is very important to keep in mind that the RI becomes unreliable in some cardiac anomalies with left to right shunts which can decrease the RI particularly in the case of persistent ductus arteriosus alone or combined with other cardiac anomalies like truncus arteriosus communis, aortopulmonary shunts, aortic valve insufficiency and also in some extra cardiac shunts like cerebral arteriovenous fistula or the arteriovenous malformation of the great vein of Galen (Lipman et al., 1982, Deeg 1989). The RI becomes lower in the infants with severe birth asphyxia with severe HIE (Ilves et al., 2009a, 2009b), but also in the infants with other causes of increase in diastolic flow as it happens in the infants with cerebral vascular dilatation resulting from acute hypoxia or ischemia of any cause (Lowe \& Bailey, 2011a).

\subsection{Sensitivity of neonatal US}

Modern US equipment with the increased image quality and resolution can effectively depict changes in the brain parenchyma in HIE patients (Epelman et al., 2010; Ilves et al., 2009a), in contrast to the experience with ultrasound maschines in pediatrics in the early 
nineties (Barkovich, 1992; Stark \& Seibert, 1994). If compared to MRI, the sensitivity for modern US was 100\% (CI: 94.1-100), specificity 33.3\% (CI: 7.5-70), positive predictive value

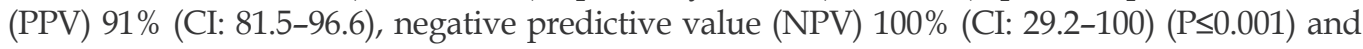
accuracy $95.7 \%$ (Epelman et al., 2010). Because US is operator-dependent, the importance of the aggressive US technique cannot be underestimated in the success of the US findings. The depiction of the lesions by US varies from subtle to diffuse changes in the echogenicity of the brain parenchyma. The subtle changes are often best appreciated during real-time imaging and it is therefore essential for the operator to be fully familiar with subtle changes in echogenicity and the areas where they can be visualized. Such aggressive technical approach showed that US has a high sensitivity and accuracy for documenting parenchymal abnormalities and enabled us to show at least one US abnormality in all 61 neonates with encephalopathy who had an abnormal MRI. There were no false negative US examinations (NPV $=100 \%$ ), which might be interpreted to mean that a negative US should obviate the need for an MRI (Epelman et al., 2010). However, in many cases MRI revealed more extensive and florid injury, suggesting that encephalopathic neonates indeed benefit from MRI. Although MRI is a gold standard, many of these children are too sick to be transported to the MRI suite or to tolerate the examination and in these cases a thorough US examination is invaluable.

\section{Clinical description of hypoxic-ischemic encephalopathy}

\subsection{The stages of hypoxic-ischaemic encephalopathy}

The children born at term with neonatal encephalopathy after birth asphyxia represent a heterogeneous group with respect to etiological factors, clinical symptoms, and outcome (Lindström et al., 2008). The causes of HIE can be separated into antepartum, intrapartum, and postpartum factors. In most cases, HIE in term neonates is associated with antepartum risk factors alone (including maternal hypotension, infertility treatment, multiple gestation, prenatal infection, and thyroid disease) or antepartum factors in combination with intrapartum. Intrapartum factors alone (including forceps delivery, breech extraction, umbilical cord prolapse, abruptio placenta, tight nuchal cord, and maternal fever) are responsible for a small minority of the cases of HIE and only $10 \%$ of the cases of HIE in term neonates are associated with postnatal complications such as severe respiratory distress, sepsis, or shock (Ferriero, 2004).

Traditionally based on the severity of asphyxia and HIE, the children are described to have a mild, moderate or severe stage of HIE. Clinical signs and symptoms of neonatal HIE can be non-specific at birth and usually evolve over a period of days (Ferriero, 2004), but data suggest that infants at the highest risk for having suffered severe HIE can be identified with the reasonable reliability based on clinical findings. These findings include the evidence of intrapartum distress (foetal heart rate abnormality), severe foetal academia, severe functional depression (indicated by a low 5-minute Apgar score), the need for resuscitation in the delivery room, an abnormal early neurologic examination, and an abnormal electroencephalogram (Shalak \& Perlman, 2004). In the first hours following a severe insult, neonates may demonstrate depressed consciousness, periodic breathing with apnoea, or bradycardia. Hypotonia may be present, particularly if there has been an injury to the cortical regions, and seizures may occur in the cases of severe asphyxia. Sarnat and Sarnat (1976) introduced an assessment of the severity of asphyxia in term infants on the basis of the clinical symptoms developing during the first days after birth (Sarnat \& Sarnat, 1976). 


\begin{tabular}{|c|c|c|c|}
\hline & $\begin{array}{l}\text { Mild HIE } \\
\text { Stage I }\end{array}$ & $\begin{array}{l}\text { Moderate HIE } \\
\text { Stage II }\end{array}$ & $\begin{array}{l}\text { Severe HIE } \\
\text { Stage III }\end{array}$ \\
\hline $\begin{array}{l}\text { 1. Level of } \\
\text { consciousness }\end{array}$ & Hyper alert & $\begin{array}{l}\text { Lethargy or } \\
\text { obtunded (difficult } \\
\text { to rouse) }\end{array}$ & $\begin{array}{l}\text { Stupor or coma } \\
\text { (cannot be aroused) }\end{array}$ \\
\hline \multicolumn{4}{|l|}{$\begin{array}{l}\text { 2. Neuromuscular } \\
\text { control }\end{array}$} \\
\hline Muscle tone & Normal & Mild hypotonic & Flaccid \\
\hline Posture & $\begin{array}{l}\text { Mild distal } \\
\text { flexion }\end{array}$ & $\begin{array}{l}\text { Strong distal } \\
\text { flexion }\end{array}$ & $\begin{array}{l}\text { Intermittent } \\
\text { decerebration } \\
\text { (extension) }\end{array}$ \\
\hline \multicolumn{4}{|l|}{$\begin{array}{l}\text { 3. Complex (primitive } \\
\text { reflexes) }\end{array}$} \\
\hline Suck & Normal/weak & Weak or absent & Absent \\
\hline Moro (startle) & $\begin{array}{l}\text { Strong, low } \\
\text { threshold }\end{array}$ & $\begin{array}{l}\text { Weak, incomplete, } \\
\text { high threshold }\end{array}$ & Absent \\
\hline Tonic neck & slight & strong & Absent \\
\hline \multicolumn{4}{|l|}{ 4. Autonomic Function } \\
\hline Pupils & mydrias & miosis & $\begin{array}{l}\text { Variable, often } \\
\text { unequal, poor light } \\
\text { reflex, fixed, dilated }\end{array}$ \\
\hline Heart rate & Tachycardia & Bradycardia & Variable \\
\hline 5. Seizures & None & $\begin{array}{l}\text { Common (focal or } \\
\text { multifocal }\end{array}$ & $\begin{array}{l}\text { Frequent/difficult to } \\
\text { control }\end{array}$ \\
\hline Electroencephalogram & Normal & $\begin{array}{l}\text { Early: low voltage } \\
\text { continuous delta } \\
\text { and theta } \\
\text { Later: periodic } \\
\text { pattern (awake). } \\
\text { Seizures: focal } \\
\text { spike and wave }\end{array}$ & $\begin{array}{l}\text { Early periodic } \\
\text { pattern with } \\
\text { isopotential phases: } \\
\text { Later: totally } \\
\text { isopotential }\end{array}$ \\
\hline Duration & Less than $24 \mathrm{~h}$ & 2-14 days & Hours to weeks \\
\hline
\end{tabular}

Table 1. Clinical stages of hypoxic-ischemic encephalopathy in the full-term newborn infant modified from Sarnat and Sarnat (Sarnat \& Sarnat, 1976)

\subsection{The pathophysiology of HIE}

The perinatal brain can be deprived of oxygen supply by two major pathogenetic mechanisms: hypoxemia and ischemia. Hypoxemia and ischemia can occur because of an event either before and/or during delivery, such as a nuchal cord, placenta previa, abruptio placenta, prolapsed or compressed cord, ruptured uterus, and/or obstetric accident. These conditions can lead to a reduced oxygen and/or blood flow to the infant. Not all the cases of the hypoxic-ischemic insult before and/or during labour will result in HIE. On the other hand in severe cases following the termination of the insult, a cascade of deleterious events results in the development of HIE and in cell death. 
The principal pathogenetic mechanism, underlying most of the neuropathology, attributed to intrapartum hypoxia-ischemia, is the impaired cerebral blood flow, which is most likely to occur because of the interruption in the placental blood flow and gas exchange; it is often referred to as "asphyxia" or severe foetal acidemia. The latter is defined as a foetal umbilical arterial pH level of below 7.00 (Perlman, 2006). With prolonged hypoxemia, cardiac hypoxia occurs, leading to the diminished cardiac output and, ultimately, to brain ischemia. Thus, the brain injury resulting from asphyxia is the consequence of ischemia superimposed on hypoxia. In fact, acute hypoxemia without superimposed ischemia is less likely to cause injury, unless the hypoxic state is prolonged (Biagas, 1999; Huang \& Castillo, 2008).

Severe and prolonged hypoxia and ischemia of any organ will result in cell death and tissue damage, the brain is especially sensitive to a decrease in the cellular oxygen supply (Fellman \& Raivio, 1997). The more severe the hypoxia-ischemic insult, the greater the "primary neuronal death" will be during asphyxia (Fellman \& Raivio, 1997).

After resuscitation, which may occur in utero or postnatally in the delivery room, cerebral oxygenation and perfusion is restored (Perlman, 2006). The restoration of blood flow and oxygen delivery after asphyxia is necessary for organ survival, but is critical, because damage may progress during this "reperfusion period". Therefore, in survivors brain injury is an evolving process that is initiated during the insult and extends long into a recovery period (Fellman \& Raivio, 1997; Perlman, 2006). If the initial energy failure during asphyxia is sufficiently prolonged and severe, the reduction in the cerebral blood flow and oxygen delivery is capable of triggering a cascade of deleterious biochemical events at the cellular level leading to secondary delayed cell death without continuing energy failure (Fellman \& Raivio, 1997; Perlman, 2006; Shadid et al., 1998). However, the process of energy failure recurs from 6 to 48 hours later in a second phase of injury (Perlman, 2006), characterised by a progressive decrease in high energy phosphate levels, in spite of the normal substrate supply, the normal blood pressure and the acid base status as shown in human infants (Wyatt et al., 1989) and newborn piglets (Lorek et al., 1994). In the case of the development of the secondary energy failure, survival without permanent brain damage is very unlikely. In a human infant, the severity of the second energy failure is correlated with adverse neurodevelopmental outcomes at 1 and 4 years (Roth et al., 1997). So the onset of ischemic cell changes during reperfusion is delayed and is called the delayed or "secondary cell death" phenomenon (van Bel et al., 1998).

Hypoxic ischemic insults may lead to neuronal cell death, to necrosis or apoptosis, or both, depending on the severity of the initial insult, the local environment of the affected region of the brain, and the maturational state of the brain. The intensity of the initial insult may determine the mode of death, with severe insult resulting in necrosis, whereas milder insults result in apoptosis (Bonfoco et al., 1995). Whether a particular cell undergoes necrosis or apoptosis may depend upon many factors: the severity of the cellular insult, the cellular energy reserves, and the events associated with delayed energy failure, the ability of the mitochondria to recover function upon resuscitation or trophic support from neighbouring cells (Edwards et al., 1997). Necrosis is a passive process and is characterized by cell swelling, disrupted cytoplasmic organelles, membrane disintegration, cell rupture, release of intracellular contents, and as a consequence, inflammation and phagocytosis. Apoptosis 
is an active process distinguished from necrosis by the presence of cell shrinkage, nuclear pyknosis, and relative preservation of the cellular membrane, genomic fragmentation and death without inflammation (Edwards et al., 1996).

The goal of the management of the newborn infants who have sustained a hypoxic-ischemic insult is to early identify the infant at the highest risk for evolving injury, facilitate adequate perfusion and nutrients to the brain and to provide specific interventions during the short time period when interventions might be still efficacious in reduction of developing brain damage (Perlman, 2006).

\section{The pathogenesis of multiorgan failure in perinatal asphyxia}

The interruption of the placental blood flow during labour and the decompensation of the circulatory responses lead to systemic hypotension and ischemia in different organs. The key to the protection of the central nervous system during the hypoxic-ischaemic insult is the adaptation of the foetal cardiovascular system, which by increasing the cerebral blood flow maintains cerebral oxygen metabolism (Koehler et al., 1985). The compensatory cardiovascular response of the foetus to hypoxemia is an increase of the arterial pressure caused by the increased systemic vascular resistance and the initial increase of cardiac output (Koehler et al., 1985). The redistribution of cardiac output - with an increased flow to the brain, heart, and adrenal glands at the expense of the flow to the kidneys, gut and skin is a major protective mechanism to maintain the perfusion of vital organs during asphyxia and can lead to a visceral organ as well as at last cerebral injury (Koehler et al., 1985; Perlman, 2006; Stola \& Perlman, 2008).

This redistribution of blood can lead to ischemic damage in different organs with multiple organ failure developing in 50-60\% of severely asphyxiated infants (Wayenberg, et al., 1998). Early BFV changes have been documented in critically ill infants following birth asphyxia in the cerebral (Bennhagen et al., 1998; Ilves et al., 2004, 2009a, 2009b), renal or superior mesenteric arteries and the celiac artery (Koc et al., 1998; Luciano et al., 1998).

The central nervous system is disturbed in $70 \%$ of infants after severe birth asphyxia (Martin-Ancel et al., 1995) and the severity of HIE predicts the risk of death and long-term neurodisability (Ilves et al., 2004, 2009a; Lindström et al., 2008; Sarnat \& Sarnat, 1976). However, asphyxia is likely to cause disturbances in a number of organ systems other than the brain. Renal involvement occurred in $40-50 \%$, pulmonary involvement in $25 \%$, cardiac involvement in 25-30\% and gastrointestinal involvement in 30\% of the cases of asphyxiated term infants (Martin-Ancel et al., 1995; Wayenberg et al., 1998). Others show a high proportion of infants with renal, pulmonary, cardiac and liver dysfunctions both in with a good neurodevelopmental outcome (58-88\% of infants) and with an adverse outcome (64$86 \%$ of infants) (Shah et al., 2004).

As the duration and severity of asphyxia may be difficult to estimate and the compensatory capacity of the foetus and newborn is unknown, it is difficult to predict the severity of organ damage immediately after birth. The BFV findings have a clinical relevance in interpreting the cardiovascular adaptation of distressed newborn infants with a decompensation of circulatory responses and the possible development of organ failure (Ilves et al 2009b). 


\section{Acute changes in sonography}

\subsection{Acute changes in grey-scale sonography}

\subsubsection{Factors influencing US findings in HIE patients}

Factors such as brain maturity, the duration and severity of insult, and the type and timing of imaging studies all influence radiological findings in HIE.

The sites in the brain that tend to be most vulnerable to hypoxic injury will be determined largely by the maturity of the brain (Huang \& Castillo, 2008). Severe hypoxia-ischemia in both preterm and term neonates preferentially damages the deep grey matter, with the perirolandic involvement more frequently observed in the term neonates. Less profound insults result in intraventricular haemorrhages and periventricular white matter injury in preterm neonates and parasagittal watershed territory infarcts in term neonates (Huang \& Castillo, 2008).

The duration of insult also seems to be a key determinant of the pattern of injury in HIE, since the insults of short duration usually do not result in brain injury. The episodes of severe hypoxia-ischemia result in a different injury pattern compared with less severe insults (Huang \& Castillo, 2008).

It is well known that global hypoxic-ischemic insults do not affect all brain structures uniformly and certain tissues in the brain are more likely to be injured and are injured earlier than others, a concept known as selective vulnerability. Evidence suggests that the observed patterns of injury reflect the dysfunction of selected excitatory neuronal circuits, which causes a complex cascade of deleterious biochemical events and, ultimately, selective neuronal death (Johnston et al., 2001). In infants with HIE the following areas a most susceptible to hypoxic-ischaemic injury: (a) the areas of the brain with the highest concentrations of glutamate or other excitatory amino acid receptors (primarily located in the grey matter) are more susceptible to the excitotoxic injury following the hypoxicischemic insult; (b) the areas of the brain with the greatest energy demands become energy depleted most rapidly during hypoxia-ischemia, and are therefore injured more easily and earlier; and (c) because of delayed cell death from apoptosis the injury may be expressed in full extent days and even months after the initial insult has occurred (Ilves et al., 2009; Huang \& Castillo, 2008).

Selective neuronal necrosis is the most common variety observed in neonatal HIE with necrosis of the neurons in a characteristic widespread distribution. The neurons of the cerebral cortex are the most vulnerable, most often hippocampus. However, the involvement of diencephalon, basal ganglia (neurons of the caudate, putamen and globus pallidus) and the thalamic injury and even brainstem occur. The mechanism of the neuronal injury is related to ischemia rather than hypoxia alone (Volpe JJ 2008). Regional vascular factors play a certain role because of neuronal injury is more marked in vascular borderzones during ischemia, in the deths of sulci and in parasagittal cerebral cortex (Volpe JJ 2008).

Less severe insults of partial asphyxia result in a different pattern of injury. The experiments performed in animal models have demonstrated that the episodes of prolonged foetal hypoxia result in shunting of blood to vital brain structures, such as the brainstem, thalami, basal ganglia, hippocampus, and cerebellum, at the expense of less metabolically active structures as the cerebral cortex and the white matter (Ashwal et al., 1980). Therefore, the 
brainstem, cerebellum, and the deep grey matter structures are generally spared from injury in mild to moderate hypoxic-ischemic insults, because of autoregulatory mechanisms are able to maintain perfusion to these vital areas of the brain (Barkovich, 2005); however, more prolonged insults result in injury to the intervascular watershed zones, which are relatively hypoperfused as a result of this shunting during a pressure passive state of cerebral circulation (Barkovich, 2005). This injury is visible as a symmetric damage but may be more prominent in one hemisphere than the other. The posterior aspects of the cerebral hemispheres, especially the parietal-occipital regions, are more affected than the anterior (Volpe JJ 2009).

\subsubsection{Acute US findings}

The US abnormalities in HIE patients can be found in the cortical grey matter, in the areas of the subcortical or periventricular white matter, or in deep grey-matter structures, including basal ganglia, thalami and the brainstem (Eken et al., 1995; Epelman et al., 2010; Ilves et al., 2009a). US changes in HIE can be classified as peripheral and central.

\subsubsection{Peripheral brain abnormalities}

include changes in the grey-white matter differentiation (increased, absent or mixed) and changes in the echogenicity of the cortex and subcortical white matter (Figure 2b). Early US findings include a global increase in cerebral echogenicity and the obliteration of the cerebrospinal fluid (CSF)-containing spaces, suggesting diffuse cerebral oedema (Figure 2a). However, the small size of liquor spaces alone is not specific for brain oedema and slitlike ventricles can be seen in healthy newborns during the first 3 days of life (Ilves et al., 2009a). The size of the ventricles, the interhemispheric fissure and the subarachnoideal space is not different between controls and asphyxiated infants with different stages of HIE or with different outcome during the first 3 days after birth (Ilves et al., 2009a).

An increase of parenhymal echogenicity with partial or total obliteration of normally visible structures is correlated with severity of HIE: Although the phenomenon of increased echogenicity (the so- called "bright brain") is fully developed by the second-third day of life, the moderate to severe increase of parenchymal echogenicity as early as at the age of $12 \pm 2 \mathrm{~h}$ has a high sensitivity (88\%) for death or severe disability and can be an early predictor of permanent brain damage in asphyxiated infants (Ilves et al., 2009a). By the second-third day of life, the predictive value of the "bright brain" phenomenon can be as high as 90-100\% (Babcock \& Ball, 1983). A strong correlation exists between the duration of the increase of parenchymal echogenicities and outcome at the age of 18 months. In infants with moderate HIE echogenicity gradually normalizes; long-lasting increase in parenchymal echogenicity (5-7 days and more) is a predictor of poor outcome (Babcock and Ball, 1983; Ilves et al., 2009a).

The power Doppler evaluation of the brain parenchyma is also valuable to determine the presence of hyperaemia or hypovascularity (Figure 2c). In the infants with hyperaemia of the brain parenchyma the prominence of the lenticulostriate vasculature and/or prominent cortical vessels can be observed. However, hyperaemia was a subjective evaluation and was noted as present in 19 of the 53 neonates with encephalopathy (Epelman et al., 2010). The regions of hypovascularity can be seen in the areas of vascular occlusion or infarction (Lowe \& Bailey, 2011a). Cranial US, as well as CT, is of limited use in the detection of superficial cortical and subcortical watershed zone injuries, given their location relative to the calvaria. 
Therefore, MRI imaging is the modality of choice in term infants who experience partial asphyxial events which is more effective to identify these lesions (Figure 2g). Diffusionweighted images are the earliest to change, usually within the first $24 \mathrm{~h}$ (Barkovich, 2005). However, with the good technique described earlier, modern equioment and excellent experience in ultrasonongraphy are essential to detect parencymal echogenisities.

\subsubsection{Central brain abnormalities}

in infants with severe global asphyxia include changes in echogenicity of the deep grey matter (basal ganglia and thalami), brainstem and the periventricular white matter (Epelman et al., 2010) (Figure 2e). Severe asphyxial events in term neonates result in a primarily central pattern of injury involving the deep grey matter (putamina, ventrolateral thalami, hippocampus, dorsal brainstem, and lateral geniculate nuclei) and occasionally the perirolandic cortex. These areas of the brain are actively myelinating (an energy-intensive process) or contain the highest concentrations of NMDA receptors at term (Barkovich, 1992) and are, therefore, the most susceptible to neonatal HIE. The remainder of the cerebral cortex is generally spared or shows mild insults, since it is generally less metabolically active in the immediate perinatal period; however, with more prolonged insults, the remaining cortex will become involved, a finding that generally corresponds to a worse neurologic outcome (Barkovich, 2005). The presence of thalamic echogenicity generally suggests a more severe injury and correlates with a poorer outcome (Connolly et al., 1994; Eken et al., 1994). Hyperechogenicity in the thalamic/basal ganglia region (so-called "bright thalamus") takes time to develop, and a correct US diagnosis will not always be possible during the first $24 \mathrm{~h}$ of age unless there is an antenatal onset of the lesion (Eken et al., 1994). Hypovascularity and/or hyperaemia in basal ganglia can be followed in the infants with severe HIE by the power Doppler. Increased echogenicity in the basal ganglia, thalami, and brainstem is prolonged and is even readily apparent after 7 days after severe birth asphyxia (Babcock \& Ball, 1983; Hertzberg et al., 1987).

Less severe insults of partial asphyxia result in a different pattern of injury. The brainstem, cerebellum, and the deep grey matter structures are generally spared from injury in mild to moderate hypoxic-ischemic insults, since autoregulatory mechanisms are able to maintain perfusion to these areas of the brain (Barkovich, 2005); however, more prolonged insults result in the injury to the intervascular boundary (watershed) zones. If the normal pattern of the hypoechoic grey matter and the hyperechoic white matter is reversed, abnormality is indicated (Huang \& Castillo, 2008; North \& Lowe, 2009). Normal layers of cortex: the superficial pia mater should be seen as a thin well-defined hyperechoic layer overlying the hypoechoic cortical grey matter, which in turn overlies the hyperechoic white matter, will be difficult to distinct. Failure to distinctly visualize these normal layers is helpful to identify the areas of abnormality, such as focal haemorrhage or infarct. However, again MRI imaging is more effective to identify these lesions (Barkovich, 2005). The periventricular white matter hyperechogenicities, uni-or bilateral can be followed as more echogenic than the adjacent chorioid plexus (Lowe \& Bailey, 2011a; North \& Lowe, 2009).

\subsection{Acute changes in the Doppler sonography}

The use of cerebral arterial Doppler US during US evaluation performed in the first few days of life may improve sensitivity and specificity for brain injury, since the presence of 
diminished resistive indexes in the cerebral arteries has been associated with a poor clinical outcome, even in the absence of other US abnormalities (Stark, et al., 1994). Changes in BFV and metabolism after hypoxic-ischaemic insult are the potential mechanisms of subsequent damage during reperfusion (Fellman \& Raivio, 1997; Rosenberg, 1986).

\subsubsection{Initial cerebral hyperaemia}

In animal models, resuscitation after mild to moderate ischemia is accompanied by a period of initial hyperaemia (Rosenberg, 1986). Evidence exists that the initial hyperaemia is a prerequisite for functional recovery, at least following long periods of ischemia (Kangström et al., 1983). A severe hypoxic-ischaemic insult is followed by a failure to reperfuse ischaemic regions even after the restoration of the oxygen supply although the blood pressure is within the autoregulatory range. Failure to reperfuse ischaemic regions after the restoration of circulation is called the "no-reflow" phenomenon and is one of the potential mechanisms of extended injury during the period after ischemia. It is possible that not all structures of the brain are affected by the "no-reflow" phenomenon. In some structures, there may be the evidence of "reactive hyperaemia", whereas others may show perfusion defects (Kangström et al., 1983). Typically, these defects affect striatum, thalamus, and hippocampus, as well as frontal and parietal cortices after complete global ischemia. The duration of ischemia influence the condition of flow restoration in "no-reflow" areas, the longer the period of ischemia, the more structures were affected by the phenomenon in rats (Kangström et al., 1983).

\subsubsection{Delayed cerebral hypoperfusion}

Initial cerebral hyperaemia after the hypoxic-ischemic insult is followed by delayed cerebral hypoperfusion. A steady decrease of BFV 12 hours after asphyxia has been described in infants (Ilves et al., 2004; Shadid et al., 1998; van Bel et al., 1998) and in animal models (Kangström et al., 1983). It has been speculated that the delayed hypoperfusion may add a secondary ischaemic insult to the tissue, especially if the reduction in BFV is not matched to the metabolic rate (Kangström et al., 1983). The severity of posthypoxic hypoperfusion increases with the increasing duration of a preceding cerebral ischemia (Kangström et al., 1983). Among infants with severe birth asphyxia, babies with the severest stage of HIE demonstrated the greatest decrease in BFV (van Bel et al., 1998). We have shown that some asphyxiated infants mostly with moderate HIE have extremely low cerebral BFV velocities (below 3SD) with a high RI during the first day of life which is associated with poor prognosis (Ilves et al., 2004, 2009a, 2009b)(Figure 2d). This delayed postasphyxial cerebral hypoperfusion could be related to an excess of vasoconstrictor molecules (Fellman \& Raivio, 1997). Low cerebral blood volume during the first 28 days of life was described in a small group of infants probably with mild to moderate HIE, developing cerebral paralysis later (Fukuda et al., 2008).

\subsubsection{Delayed cerebral hyperperfusion}

Several authors have reported high BFV and significantly lowered RI in infants with severe HIE at the age of 24-72 h (Levene et al., 1989; Bennhagen et al., 1998; Ilves et al., 2009 a, 2009b) (Figure 2f). BFV has been recorded higher in infants with permanent brain damage 
during the first days of life (Pryds, 1991; Ilves et al., 2009 a, 2009b). Severe increase in cerebral BFV and the decrease in RI take place in asphyxiated infants with severe HIE and a poor prognosis by the second half of the first day, being most severe during the first 3 days (Bennhagen et al., 1998; Ilves et al., 2009a; Meek et al., 1999; Pryds et al., 1990). The maximum BFV values are registered at the age of 36-71 $\mathrm{h}$ (Ilves et al., 2009a) decreasing gradually by the age of 1 week (Figure 1). However, in very severe cases with a poor prognosis high BFV is seen already at the age of $12 \mathrm{~h}$ or ealier suggesting a very short therapeutic window in severely asphyxiated infants (Ilves et al., 2009a). Animal models have also shown that initial posthypoxic hypoperfusion is followed by delayed vasodilatation, increased BFV and cerebral energy failure 24-72 $\mathrm{h}$ after the hypoxic-ischemic insult (Lorek et al., 1994). In this situation, dissociation exists between the cerebral metabolic rate and the over-abundant BFV relative to the metabolic needs of the brain tissue, the socalled luxury perfusion (Levene et al., 1989). Markedly high BFV despite isoelelctric EEG activity is found in these infants (Pryds, 1991). The BFV was higher, but the cross-brain oxygen extraction was lower in asphyxiated infants who died, compared to the neonates who survived with a normal neurologic outcome (Frewen et al., 1991). Delayed hyperaemia often occurs as a focal phenomenon, but may also be generalised (Levene et al., 1989).

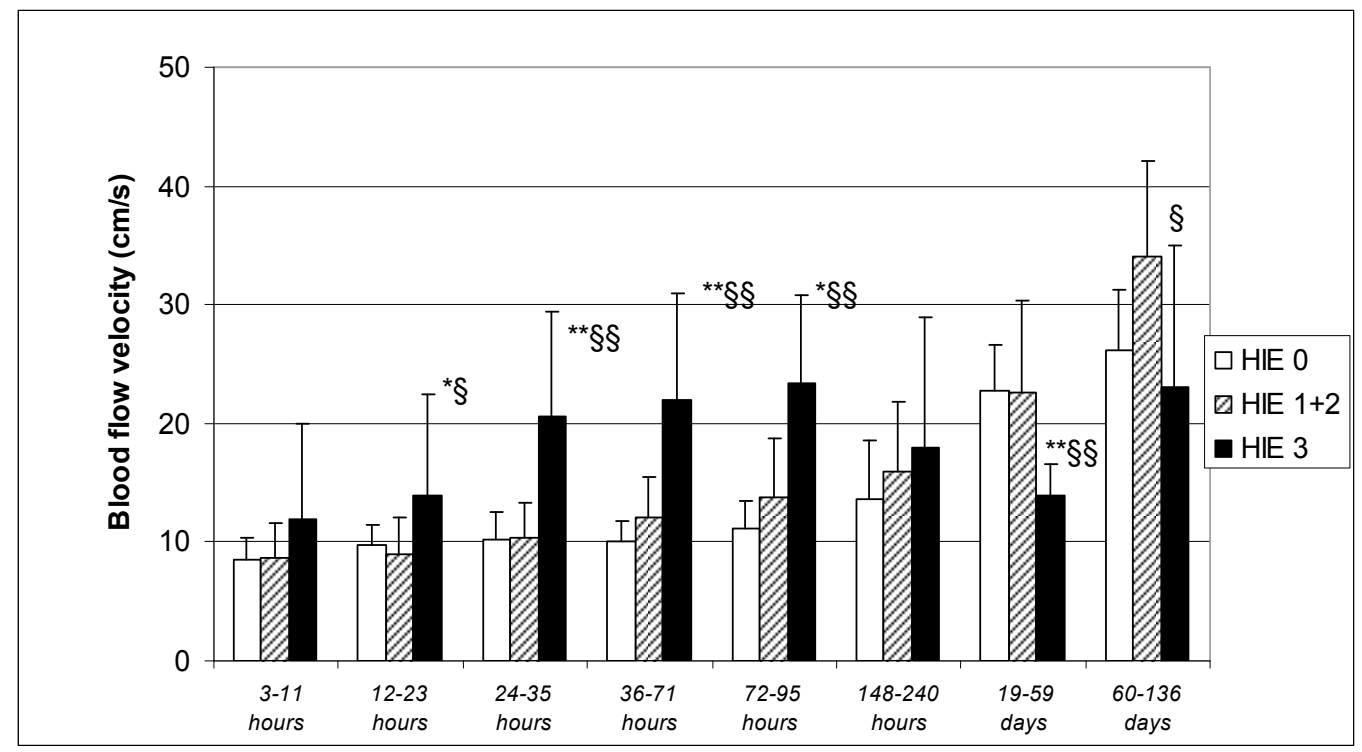

Fig. 1. Mean cerebral blood velocity (TAV) in a anterior cerebral artery infants with HIE with HIE 0 ( $n=115$ infants); HIE I+II ( $n=58$ infants); HIE III ( $n=25$ infants); * $p<0.05$ HIE III vs. HIE I+II; **p < 0.001 HIE III vs. HIE I+II; $\$ p<0.05$ HIE III versus HIE $0 ; \S \S p<0.001$ HIE III versus HIE

In 1986, Archer et al. (Archer, et al., 1986) introduced the cerebral vascular resistance index (RI) for predicting clinical outcome from $24 \mathrm{~h}$ of age in the infants with moderate or severe HIE (Figure 2f). In severe HIE patients the RI below 0.55 had a positive predictive value (PPV) for death or disability of $75 \%$ and a negative predictive value (NPV) of $100 \%$. Bennhagen and co-workers have found the RI increased in infants with HIE during the first 
day of life developing low RI by the second day of life (Bennhagen et al., 1998). In some infants with poor prognosis, the RI is already significantly lower compared to the control group as early as at the age of 2-11.9 h (Ilves et al., 2009a). Although the low RI soon after birth had a poor outcome, the high RI was found in most infants who subsequently had a poor outcome (Eken et al., 1995). RI was independent of the angle between the ultrasound beam and the blood flow in the artery being examined and could be obtained from any intracerebral arter giving a good-quality signal.

The reason for this cerebral hyperaemia and the low RI during the second-third day after asphyxia may be a severe vasoparalysis, a form of irreversible cerebral vascular injury (Levene et al., 1989; Pryds et al., 1990; Meek et al., 1999). In infants with birth asphyxia hyperperfusion is thought to be a sign of permanent brain damage with a poor neurodevelopemental prognosis (Bennhagen et al., 1998; Ilves et al., 2009a; Meek et al., 1999; Pryds et al., 1990). The severity of vasoparalysis and cerebral hyperaemia is thought to correlate with the degree of brain injury and presumably the severity of asphyxia insult (Levene et al., 1989; Pryds et al., 1990). The infants with the poorest neurologic outcome (isoelectric EEG and death) had the highest values for BFV and no cerebrovascular autoregulation or $\mathrm{CO} 2$ reactivity; the infants with the burst suppression EEG and moderate to severe brain injury has slightly elevated values of BFV and impaired autoregulation, but a sustained reactivity to Pa CO2 (Pryds et al., 1990). The infants without an evidence of brain injury had normal values for $\mathrm{BFV}$, intact autoregulation and reactivity to $\mathrm{PaCO} 2$ (Pryds et al., 1990). The mechanism of such luxury cerebral perfusion following ischaemic brain injury is still unclear. The neuronal disruption causing release of vasoactive substances such as adenosine and lactate, or of excitatory amino acids (Pryds, 1991), irreversible cerebral vascular injury with a complete loss of tone in resistance vessels may play a role in the pathogenesis of delayed hyperaemia (Fellman \& Raivio, 1997; Levene et al., 1989; Pryds et al., 1990).

Severe increase in the cerebral blood flow velocity and the decrease in RI have been described in infants with severe HIE/ poor outcome (Eken et al., 1995; Ilves et al., 2004, 2009a; Levene et al., 1989; Pryds et al., 1990; Stark et al., 1994). However, recent studies show that even in the infants with mild / moderate HIE the mean cerebral BFV was higher than in the control group in all the cerebral arteries during the period 36-120 h (Ilves et al., 2009a). Moderately but statistically significantly increased mean cerebral BFV in the infants with mild-moderate HIE from $36 \mathrm{~h}$ lasting as late as 7-10 days after asphyxia can indicate the ongoing subacute process of cell damage in these infants. Although only a small number (2 of 58 infants) with moderate HIE developed severe disability (Ilves et al., 2009a) at the age of 18 months, recent data in the long-term follow-up study of the infants with moderate HIE show a high rate of cognitive dysfunctions in these children (Lindström et al., 2008).

All the previously published literature on RI and BFV and the prediction of outcome has been obtained on infants at normal temperature. It is possible that hypothermia will affect the cardiovascular system in the ways that might change RI (Elstad et al., 2011; Thoresen \& Whitelaw, 2000). Elstad with cowoorker have described: “Among the cooled infants, the PPV of any RI below 0.55 for poor outcome was only $60 \%$. This is lower than the PPV in any previous studies in infants with HIE treated at normothermia (median value $84 \%$ ). The initial data show that RI $>0.55$ is a good negative predictor, but RI below 0.55 is not as good 
positive predictor of poor outcome as reported earlier in normothermic infants. These findings mean that infants, who would have been predicted to have poorer outcome at normothermia, have better outcome treated when cooled. A low RI is significantly less predictive of poor outcome during hypothermia than normothermia. The lower PPV of RI may be because hypothermia affects cerebral vasculature or protects the brain solely at a molecular and cellular level. Infants, who would have been predicted from their RI to have poorer outcome at normothermia, have better outcome when cooled" (Elstad et al., 2011). RI should never be used for prediction alone; it is only one piece of information to be put into the clinical puzzle. However, as ultrasound Doppler is safe, cheap and readily available, RI measurement is recommended on day 2 (24-48 h after birth) and three (48-72 h) for infants with HIE treated with hypothermia as RI is lowest at 48 h" (Elstad et al., 2011).

The animal model using contrast-enhanced ultrasound (CEUS) and MRI shows that both can detect an early temporal evolution of cerebral perfusion in perinatal hypoxia and resuscitation, reversible after 7 hours (de Lange et al., 2011). Hyperoxic resuscitation caused early decreased cerebral perfusion, not present in the normoxic group. These findings show that the combined use of CEUS can provide important diagnostic information and give new insights into perinatal vascular hypoxia mechanisms (de Lange et al., 2011).

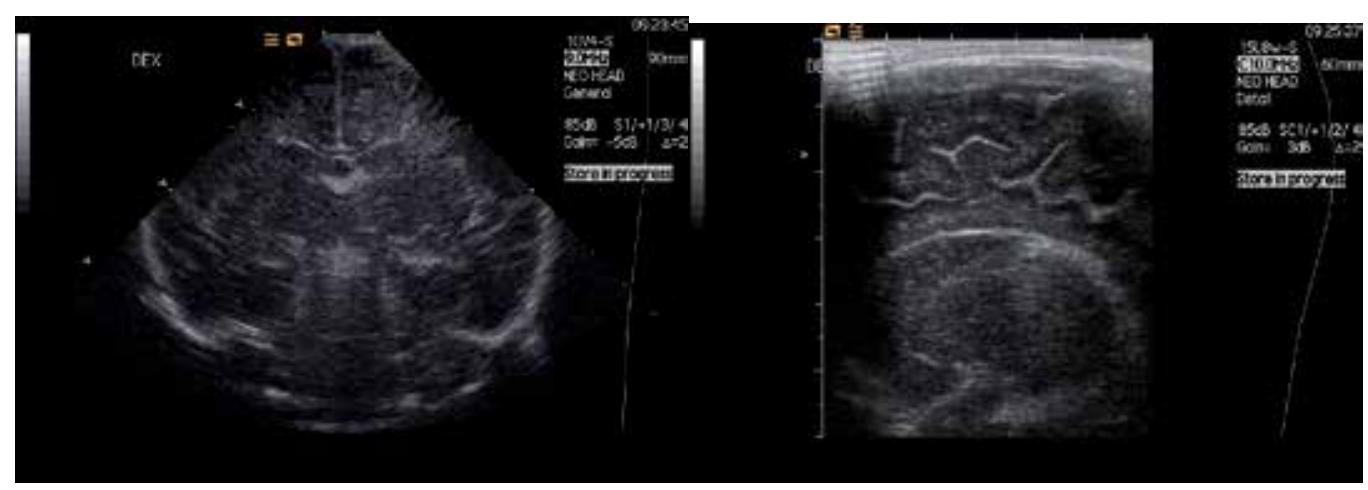

a

b

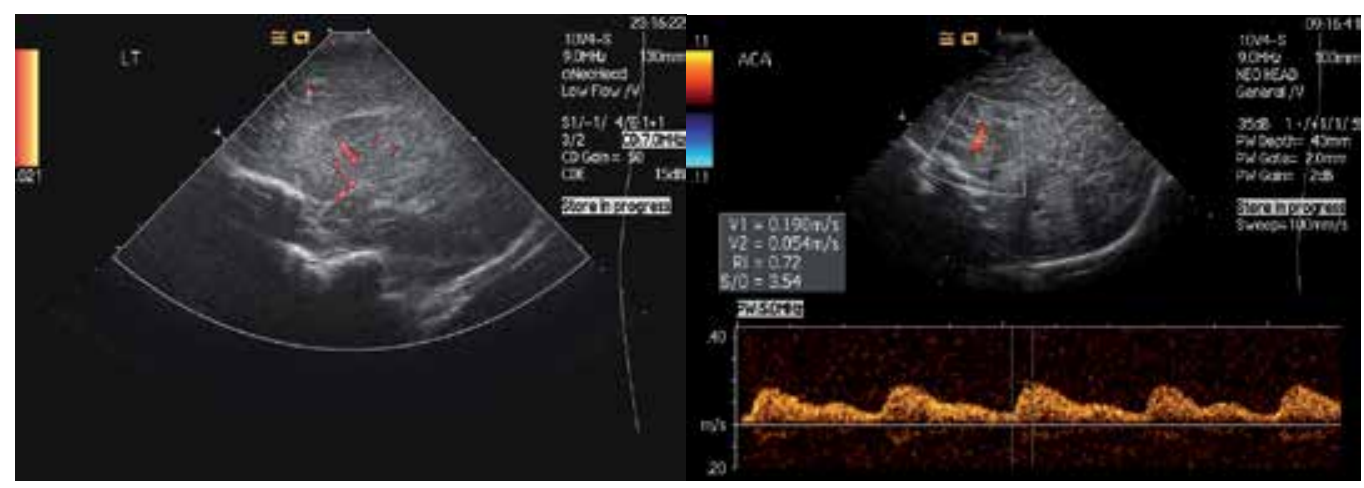

C

d 


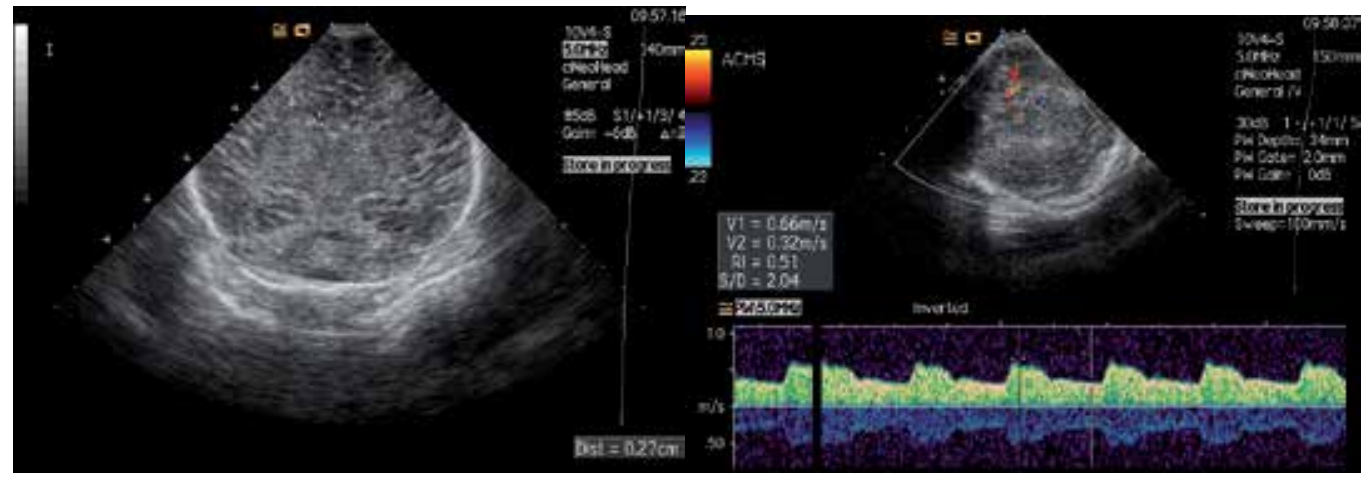

e

$\mathrm{f}$

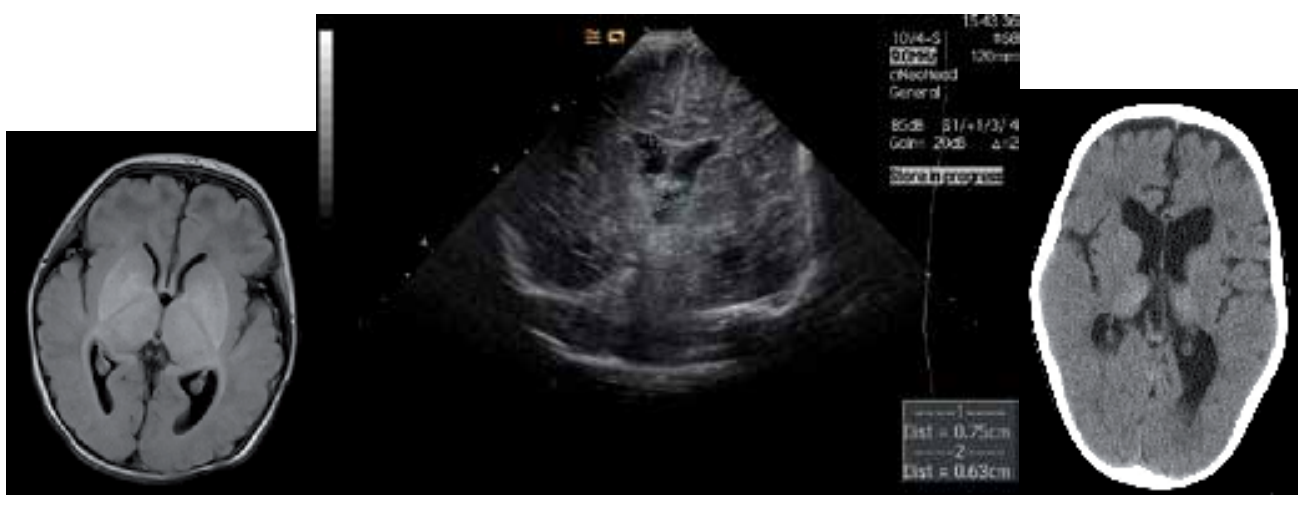

g

h

i

a) Coronar US image shows ill-defined structures including basal ganglia, and slitlike ventricles at the age of $12 \mathrm{~h}$

b) Magnified sagittal view using linear transducer shows the accentuation of the grey -white matter differentiation with prominent sulci at the age of $12 \mathrm{~h}$

c) Left parasagittal view shows hyperaemia in the region of basal ganglia with power Doppler at the age of $12 \mathrm{~h}$

d) Colour Doppler evaluation shows a low cerebral blood flow velocity with normal resistance in the right anterior cerebral artery at the age of $12 \mathrm{~h}$

e) Coronar US image shows still ill-defined structures with general increase in echogenicty including basal ganglia, and still slitlike ventricles at the age of 3 days

f) Colour Doppler evaluation shows a high cerebral blood flow velocity with low resistance in the left medial cerebral artery at the age of 3 days

g) MRI FLAIR image with pathological signal intensity bilaterally in basal ganglia at the age of 8 days

h) Coronar US with moderately enlarged side ventricles and ill-defined basal ganglia at the age of 1 month

i) CT image with atrophy and increased attenuation in basal ganglia at the age of 3 years

Fig. 2. $12 \mathrm{~h}$ old girl born at 39 weeks of gestation with a history of perinatal asphyxia after total abruption placenta and the respective Apgar scores 1-2-3 at the age of 1-5-10 minutes with severe HIE who survived with cerebral paralysis, tetraplegia, seizures and bulbar paralysis 


\section{Long-term changes in sonography}

\subsection{Long-term changes in gray-scale sonography}

Late findings of US include the resolution of the initial findings and the progressive prominence of the ventricles and the extraaxial CSF containing spaces, likely due to atrophy and the development of multicyctic encephalopathy.

By the age of the 7 days the size of the ventricles, the interhemispheric fissure and the subarachnoideal space were significantly increased in the infants with a severe HIE/poor prognosis compared to the infants with a mild to moderate HIE or the infants with a normal outcome/mild impairments respectively (Ilves et al., 2009a). By the age of 5 months the progressive enlargement of the size of the ventricles, the interhemispheric fissure and the subarachnoideal space occurred in the infants with a severe HIE/poor prognosis (Ilves et al. 2009a) (Figure 2h). The size of the ventricles and the interhemispheric fissure at the age of 21-159 days was significantly correlated with the severity of HIE and the psychomotor development at the age 18 months.

Multicystic encephalopathy developed in 18 of 25 infants with severe HIE, all had a poor outcome: 10 infants died and eight infants were with severe disability at the age of 18 months. Parenchymal cysts mostly in the frontoparietal region were first seen as early as at the age of 7 days in five infants, all died during the first month. In 12 infants with severe HIE cysts could be visualized between the age of 14-21 days and only in 1 of 18 infants the cysts were first seen after the age of 21 days (Ilves et al., 2009a). Small cysts are no longer visible by the age of 3 months, therefore it seems reasonable to perform US in the infants with a moderate or severe stage of HIE at the age of 1 month in order to detect all the infants with cystic degeneration (Ilves et al., 2009a).

The intensity of the initial insult may determine the mode of cell death resulting in necrosis in severe injury whereas milder insults result in apoptosis (Bonfoco et al., 1995). It is likely that the enlargement of the side ventricles and the appearance of the parenchymal cysts visible already by the age of 7 days in the infants who died can be the signs of rapid cell death and probably represent cell necrosis, which accounts for the significant proportion of the cell loss in asphyxiated infants (Bonfoco et al., 1995).

CT and MRI studies indicate the temporal profile of the signs of the brain swelling during the first week of life being most severe 36-72 hours after asphyxia (Rutherford et al., 1995; Yoxall \& Weindling, 1998) (Figure 2h). The infants with a severe HIE/poor prognosis and the progressive enlargement of the side ventricles and the subarachnoidal space with the development of parenchymal cysts during the first months of life have also suboptimal head growth during the first months of life (Ilves et al., 2009a). This enlargement of side ventricles progresses during several months (Ilves et al., 2009a). Decrease in the periventricular white matter has been described earlier in MRI studies in the infants older than 3 weeks (Rutherford et al., 1995). Serial head circumference investigations also show that the appearance of microcephaly may be delayed for more than 12 months (Cordes et al., 1994). The findings suggest that cell death can be long-lasting with on-going progressive cell loss days and may be weeks and months after asphyxia. Apoptosis that is aslower process can play a role in the long-lasting brain damage in the infants with severe asphyxia. This can indicate that neuroprotection probably must be lasting more than only first days after 
asphyxia. Therapeutic hypothermia for moderate to severe HIE's during the first days after asphyxia is currently the only clinically proven treatment for infants with neonatal asphyxia (Hoehn et al., 2008). The potential strategies to prevent or reduce reperfusion injury in asphyxiated infants probably need to include the interventions that target both processes: cell necrosis during the first days after asphyxia and apoptosis on going probably during the longer period after asphyxia.

\subsection{Long-term changes in Doppler sonography}

In normal infants and the infants with a mild/moderate HIE or good prognosis, steady increase in the mean cerebral BFV and the decrease in RI occurred in all the cerebral arteries during the first months of life (Ilves et al., 2008, 2009a; Kojo et al., 1996; Mercuri et al., 2000), probably reflecting the increasing cerebral blood flow during the first year of life. The infants in the control group and the infants with a mild/moderate HIE or good prognosis also showed steady head growth with no significant differences in the head circumference or the ventricle size between these groups during the first months of life. The steady increase in cerebral BFV in healthy infants appears to reflect the rising metabolic demand of the growing brain during the neonatal period (Kelner et al., 2003). The increase in blood flow has been shown during the first year of life, the cause of which can be rapid myelination and the explosive increase of the synapses with decrease in nerve cell density (Takahashi et al., 1999). The increasing demand for metabolic fuels during the first months after birth is found in the studies investigating cerebral glucose consumption using positron emission tomography (PET) and measuring cerebral oxygen consumption with NIRS (Chugani et al., 1986; Kinnala et al., 1996; Yoxall et al., 1998). This extreme increase in the mean BFV in infants with a severe HIES/poor outcome is temporary and the cerebral BFV gradually decreases after the first week of life (Ilves et al., 2009a). The findings are consistent with the studies of the cerebral glucose metabolism measured by PET in term infants with hypoxic-ischemic encephalopathy, showing hypermetabolism during the acute period 2-5 days after birth asphyxia (Blennow et al., 1995) and lower metabolism during the subacute period (at the age of 4-24 days) after asphyxia (Thorngren-Jerneck et al., 2001).

Such steady increase of the mean BFV is not found in infants with a severe HIE/poor prognosis during the first months after asphyxia (Figure 1). After the extremely high cerebral BFV during the first days of life significantly lower mean cerebral BFV develops during the first months after asphyxia in severe HIE infants. At the same age the enlargement of lateral ventricles, multicystic encephalopathy and the suboptimal head circumference develops in infants with a severe HIE / poor neurodevelopmental outcome suggesting decreased brain volume due to a massive loss of the brain tissue in infants with a severe HIE/ poor prognosis by this time (Ilves et al., 2009a).

It is not known whether this decrease in the mean BFV in infants with a severe HIE/poor prognosis is caused only by the low brain volume or whether these infants have lower metabolism or because of both problems. Severe HIE infants had a low head circumference with wide liquor spaces and hence the low brain volume and therefore the required cerebral BFV to fulfil energy requirements for the damaged decreased cerebral parenchyma can be lower. Such decreased brain volume probably no longer needs a normal blood flow. It is also possible that the requirement for energetic fuel is low because of the low metabolism in 
the damaged cells in the cases of severe brain damage. The only published case report, following longitudinal changes in cerebral metabolism in an infant with HIE and subsequent cerebral palsy, has demonstrated transient hypermetabolism in the neonatal period followed by severe hypometabolism later during childhood (Batista et al., 2007). More longitudinal data about cerebral blood flow and metabolism is needed to understand the causes of long term low cerebral blood flow velocities in infants with a severe HIE/poor prognosis months after birth asphyxia.

\subsection{US and Doppler findings in visceral organs}

Although the central nervous system is disturbed in $70 \%$ of infants after severe birth asphyxia (Martin-Ancel et al., 1995), up to $80-90 \%$ of infants develop disturbances in other organs (Martin-Ancel et al., 1995; Shah et al., 2004; Wayenberg et al., 1998). The interruption of the placental blood flow during labour and the decompensation of the circulatory responses lead to systemic hypotension and ischemia in different organs. Redistribution of the blood during asphyxia is the major protective mechanism in order to maintain the perfusion of vital organs such as the brain, heart and adrenal glands, but it causes ischemic damage to the visceral organs like kidneys and gut (Perlman, 2006; Stola \& Perlman, 2008). Early BFV changes have been documented in critically ill infants following birth asphyxia in the cerebral (Bennhagen et al., 1998; Ilves, et al., 2009b), renal (RA) (Ilves et al., 2009b; Luciano et al., 1998), superior mesenteric arteries (SMA) (Ilves et al., 2009b; Koc et al., 1998) or in CA (Ilves et al., 2009b).

Different patterns of post-injury BFV changes exist in the visceral arteries compared to the cerebral arteries in asphyxiated infants with HIE. The mean BFV in the SMA and CA (but not in the RA) increased in the infants with mild-moderate HIE during the first $12 \mathrm{~h}$ after asphyxia (Ilves et al., 2009a, 2009b). The reperfusion of ischemic intestine and gastric mucosa is characterised by an initial hyperaemia with ensuing mucosal repair, as shown in animal models (Meleagros et al., 1994). The increase in BFV, seen in the SMA and CA but not in the RA during the first hours after asphyxia, can be the compensatory hyperaemia in the gastrointestinal tract, stomach and liver in the infants with mildmoderate HIE. Such differences in the compensatory capabilities in the RA, SMA and CA can explain the differences in the frequency of severe organ failure found in asphyxiated infants. Renal failure is found to develop more often in $40-57 \%$ of asphyxiated infants compared to a lower rate $(30 \%)$ of gastrointestinal complications in asphyxiated term infants; liver failure is a rare complication in the infants with severe HIE (Martin-Ancel et al., 1995; Perlman et al., 1989).

The lack of hyperaemia in the SMA and CA and a decrease in BFV in the RA already during the first hours after asphyxia in severe HIE infants, despite vasoactive treatment and normal BP shows decreased compensatory capabilities in the infants with severe HIE to the restore blood flow in the visceral arteries. Extremely low BFV in all the visceral arteries was found during the first day in infants with a very long resuscitation period and the fatal outcome. These infants had normal blood pressure but a very high BFV in the cerebral arteries and a very low BFV in the visceral arteries already during the first day of life with the development of severe HIE, severe renal, gastrointestinal and hepatic failure as well as cardiac failure. Animal models have indicated that reperfusion is the main mechanism of injury after intestinal or renal ischemia; however, the post-ischemic 
reperfusion injury is related primarily to the magnitude and time of the total ischemia (Rangan \& Bulkley, 1993).

In infants with severe HIE or poor outcome, a progressive decrease in the mean BFV was found in the RA and SMA, being the lowest during the second day of life (Akinbi et al., 1994; Ilves et al., 2009b; Luciano et al., 1998). A decrease in the renal blood flow during the first day of life carries a high risk of the development of acute renal failure (Akinbi et al., 1994; (Ilves et al., 2009b; Luciano et al., 1998). A number of animal studies have provided evidence that oxygen metabolites, different vasoconstrictive molecules and intravascular clotting may play a role in the microcirculation derangement in the delayed progressive postischemic reperfusion injury (Fellman \& Raivio, 1997; Perlman, 2006). In normal infants, vasodilation secondary to the copious production of endothelium-derived nitric oxide (NO) is favoured, but endothelial cell injury could shift this balance in favour of endothelin (ET)1-mediated vasoconstriction and would limit NO production, leading to intestinal ischemia and the tissue injury as from humans with NEC (Nankervis et al., 2008). A hypoxic/ischemic insult with the resulting activation of the coagulation cascade also plays a role in renal hypoperfusion (Andreoli, 2004).

During the first day of life, pressure-passive BFV in the RA and SMA but not in the CA has been described earlier in healthy infants in the presence of open ductus arteriosus (Ilves et al., 2008). This may reflect the relatively good autoregulation of the blood flow in the CA in healthy and asphyxiated newborn infants with vasoactive treatment having a good effect; however, extra factors can redistribute the blood flow, sparing the blood flow to the CA and decreasing the blood to the SMA and RA.

Our findings indicate that differences exist in postasphyxic changes in BFV in the cerebral and visceral organs in different severities of HIE. Increased BFV develops in the SMA and $\mathrm{CA}$ in the infants with mild to moderate HIE early postinjury. A severe increase in BFV in the cerebral arteries, but a decrease in the renal and superior mesenteric arteries develops by the second day of life in a severe HIE infant, indicating that a therapeutic window exists for visceral damage. As in healthy infants, a relatively spared blood flow in the CA is found in asphyxiated newborn infants. No data exists how the BFV in visceral organs change during postasphyxial hypothermia.

\section{Outcome}

Outcomes of babies with HIE vary according to the severity of the injury. The children with mild HIE can be expected to recover and the children with the severe form either develop severe disability or die. Between $15 \%$ and $20 \%$ of infants suffering HIE die during the neonatal period, and an additional 25\% develop permanent neurologic deficits (Vannucci \& Perlman, 1997). The infants who survive a severe hypoxic insult with severe HIE typically go on to develop quadriparesis, choreoathetosis, severe seizure disorders, or mental retardation. The patients with moderate injuries invariably can develop spastic diplegia or quadriplegia (often falling under the umbrella term cerebral palsy) (Barkovich, 2005).

The children with moderate encephalopathy have a less certain prognosis (Ilves et al., 2009a), however the majority of infants with moderate HIE have major and/or cognitive disability in a long-term follow-up if followed during adolescence (Lindström et al., 2008) 


\section{Conclusions}

Sonography is an excellent tool in screening of asphyxiated infants with HIE and evaluating the prognosis of asphyxiated infants. Advances in the US equipment now permit the visualization of structures not previously shown. Despite some limitations, the US technique effectively depicts parenchymal abnormalities of the neonatal brain, showing good correlation with MRI findings. The parenchymal abnormalities depicted on US are not as florid or clearly visualized as on MRI and US might not depict as many focal lesions as MRI, especially those placed cortically. Therefore, US might severely underestimate the degree of injury, and the findings might indeed represent the tip of the iceberg. Despite this, US is a more effective modality in this group of patients than it has been suggested in the literature and remains an excellent screening modality for use in neonates too critically ill to be transported to MRI. MRI should ideally evaluate every infant with neonatal encephalopathy as early as possible, as the window required to demonstrate injury in DWI is small. However, high-quality US is very sensitive in detecting injuries and is often the only study available during the initial evaluation of these critcally ill patients or in those under hypothermic treatment. US provides an option for the interval follow-up assessment of these infants, even if the follow-up MRI is planned (Epelman et al., 2010). Differences exist in the blood flow velocity in the brain and in visceral organs in the infants with a different severity of HIE with a high cerebral blood flow and a low RI developing in the infants with a poor prognosis. In the infants with HIE treated with therapeutic hypothermia, the low resistance index is probably not as good a predictor of a poor outcome as in normothermia and further studies are needed in this area.

\section{References}

Adriani, G., Persico, A., Tursini, S., Ballone, E., D Cirotti \& Chiesa, PL. The renal-resistive index from the last 3 months of pregnancy to 6 months old. BJU International 2001; 87: 562.

Agata, Y., Hiraishi, S., Misawa, H., Hirota, H., Nowatari, M., Hiura, K., Fujino, M., Oguchi, K. \& Horiguchi, Y. Regional blood flow distribution and left ventricular output during early neonatal life: A quantitative ultrasonographic assessment. Pediatr Res 1994; 36: 805-810.

Akinbi, H., Abbasi, S., Hilpert, PL. \& Bhutni, VK. Gastrointestinal and renal blood flow velocity profile in neonates with birth asphyxia. J Pediatr 1994; 125: 625-627.

Andreoli, SP. Acute renal failure in the newborn. Semin Perinatol 2004; 28: 112-123.

Archer, LN., Levene, MI. \& Evans, DH. Cerebral artery Doppler ultrasonography for prediction of outcome after perinatal asphyxia. Lancet 1986; 2: 1116-1118.

Ashwal, S., Majcher, JS., Vain, N., \& Longo, LD. Patterns of fetal lamb regional cerebral blood flow during and after prolonged hypoxia. Pediatr Res 1980;14: 1104-1110.

Babcock, DS. \& Ball, W. Jr. Postasphyxial encephalopathy in full-term infants: US diagnosis. Radiology 1983; 148: 417-423.

Barkovich, AJ. 2005. Brain and spine injuries in infancy and childhood. In: Barkovich AJ, ed. Pediatric neuroimaging: 190-290, 4th ed, Lippincott Williams \& Wilkins, ISBN 978-14160-4918-0, Philadelphia. 
Barkovich, AJ. MR and CT evaluation of profound neonatal and infantile asphyxia. Am J Neuroradiol 1992; 13: 959-972.

Batista, CE., Chugani, HT., Juhasz, C., Behen, ME. \& Shankaran, S. Transient hypermetabolism of the basal ganglia following perinatal hypoxia. Pediatr Neurol 2007; 36: 330-333.

Bennhagen, RG., Weintraub, RG., Lundström, NR. \& Svenningsen, NW. Hypoxic-ischaemic encephalopathy is associated with regional changes in cerebral bblood flow velocity and alterations in cardiovascular function. Biol Neonate 1998;75:275286.

Biagas, K. Hypoxic-ischemic brain injury: advancements in understanding of mechanisms and potential avenues for therapy. Curr Opin Pediatr 1999; 11: 223-228.

Black, RE., Cousens, S., Johnson, HL., Lawn, JE., Rudan, I., Bassani, DG., Jha, P. et al. Global, regional, and national causes of child mortality in 2008: a systematic analysis. Lancet 2010; 375: 1969-1987 .

Blennow, M., Ingvar, M., Lagercranz, H., Stone-Elander, S., Eriksson, L., Forssberg, H., Ericson, K. \& Flodmark, O. Early 18FFDG positron emissionn tomography in infants with hypoxic/ischemic encephalopathy shows hypermetabolism during the psotasphyctic period. Acta Paediatr 1995; 84: 1289-95.

Bonfoco, E., Kraine, D., Ankarcroma, M., Nicotera, P. \& Lipton, SA. Apoptosis and necrosis: two distinct events, induced respectively by mild and intense insults with Nmethyl-Daspartate or nitri oxide/superoxide in cortical cell cultures. Proc Natl Acad Sci 1995; 92: 7162-7166.

Chugani, HT. \& Phelps, ME. Maturational changes in cerebral function in infants determined by 18FDG positron emission tomography. Science 1986; 231: 840-843.

Connolly, B., Kelehan, P., O’Brien, N., Gorman, W., Murphy, JF., King, M. \& Donoghue, V. The echogenic thalamus in hypoxic ischaemic encephalopathy. Pediatr Radiol 1994; 24: $268-271$.

Coombs, RC., Morgan, ME., Durbin, GM., Booth, IW. \& McNeish, AS. Doppler assessment of human neonatal gut blood flow velocities: postnatal adaptation and response to feeds. J Pediatr Gastroenterol Nutr 1992; 15: 6-12.

Cordes, I., Roland, EH., Lupton, BA. \& Hill, A. Early prediction of the development of microcephaly after hypoxic-ischemic encephalopathy in the full-term newborn. Pediatrics 1994; 93: 703-707.

Deeg K-H Zerebrale Dopplersonographie im Kindersalter. Springer Verlag Berlin Heidelberg 1989

de Lange, C., Braband, K., Emblem, KE., Bjornerud, A., Løberg, EM., Saugstad, OD. \& Munkeby, BH. Cerebral Perfusion in Perinatal Hypoxia and Resuscitation Assessed by Transcranial Contrast-Enhanced Ultrasound and 3 T MRI in Newborn Pigs. Invest Radiol 2011; Jul 1.

Edwards, AD. \& Mehmet, H. Apoptosis in perinatal Hypoxic-ischaemic cerebral damage. Neuropathol Appl Neurobiol 1996; 22: 494-498. 
Edwards, AD., Yue, X., Cox, P., Hope, PL., Azzopardi, DV., Squier, MV. \& Mehmet, H. Apoptosis in the brains of infants suffering intrauterine cerebral injury. Pediatr Res 1997; 42: 684-689.

Edwards, AD., Brocklehurst, P., Gunn, AJ., Halliday, H., Juszczak, E., Levene, M., Strohm, B. et al. Neurological outcomes at 18 months of age after moderate hypothermia for perinatal hypoxic-ischaemic encephalopathy: synthesis and meta-analysis of trial data. BMJ 2010; 340: c363.

Ehehalt, S., Kehrer, M., Goelz, R., Poets, C. \& Schöning, M. Cerebral blood flow volume measurements with ultrasound: Intraobserver reproducibility in preterm and term neonates. Ultrasound Med Biol 2005; 31: 191-196.

Eken, P., Toet, MC. Groenendaal, F. \& de Vries, LS. Predictive value of early neuroimaging, pulsed Doppler and neurophysiology in full term infants with hypoxic-ischaemic encephalopathy." Arch Dis Child 1995; 73: F75-80.

Ellis, M., Manandhar, DS., Wyatt, J., Bolam, AJ. \& Costello, AM. Stillbirths and neonatal encephalopathy in Kathmandu, Nepal: an estimate of the contribution of birth asphyxia to perinatal mortality in a low-income urban population. Paediatr Perinat Epidemiol 2000; 14: 39-52.

Elstad, M., Whitelaw, A. \& Thoresen, M. Cerebral resistance index is less predictive in hypothermic encephalopathic newborns. Acta Paediatr 2011; 21.

Epelman, M., Daneman, A., Kellenberger, CJ., Aziz, A., Konen, O., Moineddin, R., White, H. \& Blaser, S. Neonatal encephalopathy: a prospective comparison of head US and MRI. Pediatr Radiol 2010; 40: 1640-1650.

Evans, DH., Levene, MI., Shortland, DB. \& Archer, LNJ. Resistance index, blood flow velocity, and resistance-area product in the cerebral arteries of very low birth weight infants during the the first week of life. Ultrasound in Med $\mathcal{E}$ Bio 1988; 14: 103-110.

Fellman, V., \& Raivio, KO. Reperfusion injury as the mechanism of brain damage after perinatal asphyxia. Pediatr Res 1997; 41: 599-606.

Ferrarri, F., Kelsall, AW., Rennie, JM. \& Evans, DH. The relationship between cerebral blood flow velocity fluctuations and sleep state in normal newborns. Pediatr Res 1994; 35: 50-54.

Ferriero, DM. Neonatal brain injury. N Engl J Med 2004; 351: 1985-1995.

Finer, NN., Robertson, CM., Richards, RT., Pinnell, LE. \& KL Peters. Hypoxic-ichemic encephalopathy in term neonates: perinatal factors and outcome. J Pediatr 1992; 98: 112-117.

Frewen, TC., Kissoon, N., Kronick, J. Fox, M., Lee, R., Bradwin, N. \& Chance, G. Cerebral blood flow, cross-brain oxygen extraction, and fontanelle pressure after hypoxicisćhemic injury in newborn infants. J Pediatr 1991; 118, 265-271.

Fukuda, S., Mizuno, K., Kawai, S., Kakita, H., Goto, T., Hussein, MH., Daoud, GA. et al. Reduction in cerebral blood flow volume in infants complicated with hypoxicischemic encephalopathy resulting in cerebral palsy. Brain Dev 2008; 30: 246-253.

Hagmann, CF., Brotschi, B., Bernet, V., Latal, B., Berger, TM. \& Robertson, NJ. Hypothermia for perinatal asphyxial encephalopathy. Swiss Med Wkly 2011; 16, nr 141:w13145. doi: 10.4414/smw.2011.13145. 
Hayashi, T., Ichiyama, T., Uchida, M., Tashiro, N. \& Tanaka, H. Evaluation by colour Doppler and pulsed Doppler sonography of blood flow velocities in intracranial arteries during the early neonatal period. Eur J Pediatr 1992; 151: 461-465.

Hertzberg, BS., Pasto, ME., Needleman, L., Kurtz, AB. \& Rifkin, MD. Postasphyxial encephalopathy in term infants: sonographic demonstration of increased echogenicity of the thalamus and basal ganglia. J Ultrasound Med 1987; 6: 197202.

Hoehn, T., Hansmann, G., Bührer, C., Simbruner, G., Gunn, AJ., Yager, J., Levene, M., et al. Therapeutic hypothermia in neonates. Review of current clinical data, ILCOR recommendations and suggestions for implementation in neonatal intensive care units. Resuscitation 2008; 78: 7-12.

Huang, BY. \& Castillo, M. Hypoxic-ischemic brain injury: imaging findings from birth to adulthood. Radiographics 2008; 28: 417-439.

Ilves, P., Lintrop, M., Talvik, I., Muug, K., Maipuu, L. \& Metsvaht, T. Low cerebral blood flow velocity and head circumference in infants with severe hypoxic ischemic encephalopathy and poor outcome. Acta Paediatr 2009; 98: 459-465.

Ilves, P., Lintrop, M., Talvik, I., Muug, K. \& Maipuu, L. Changes in cerebral and visceral blood flow velocities in asphyxiated term infants with hypoxic ischemic encephalopathy. J Ultrasound Med 2009; 28: 1471-1480.

Ilves, P., Lintrop, M., Talvik, I., Muug, K., Asser, K. \& Veinla, M. Developemntal changes in cerebral and visceral blood flow velocity in healthy neonates and infants. J Ultrasound Med 2008; 27: 199-207.

Ilves, P., Lintrop, M., Metsvaht, T., Vaher, U. \& Talvik, T. Cerebral blood flow velocities in predicting outcome of asphyxiated newborn infants. Acta Paediatr 2004; 93: 523528.

Johnston, MV., Trescher, WH., Ishida, A. \& Nakajima, W. Neurobiology of hypoxic-ischemic injury in the developing brain. Pediatr Res 2001; 49: 735-741.

Kangström, E., Smith, M-L. \& Siesjö, BK. Local cerebral blood flow in the recovery period following incomplete cerebral ischemia in the rat. J Cerebr Blood Flow Metab 1983; 3: 170-182.

Kelner, M., Krägeloh-Mann, I., Goelz, R. \& Schöning, M. The development of cerebral perfusion in healthy preterm and term newborns. Neuropediatrics 2003; 34: 281286.

Kempley, ST., Vyas, S., Bower, S., Nicolaides, KH. \& Gamsu, H. Cerebral and renal artery blood flow velocity before and after birth. Early Human Dev 1996; 46: 165-174.

Kinnala, A., Suhonen-Polvi, H., Äärimaa, H., Kero, T., Korvenranta, H., Ruotsalainen, U., et al. Cerebral metabolic rate for glucose during the first six months of life: an FDG positron emission tomography study. Arch Dis Child 1996; 74: F153-157.

Koc, E., Arsan, S., Ozcan, H., Zenciroglu, A., Erdem, I. \& Ertogan, F. The effect of asphyxia on gut blood flow in term neonates. Indian J Pediatr 1998; 65: 297-302.

Koehler, RC., Traystman, RJ. \& Jones, J. Regional blood flow and O2 transport during hypoxic and CO hypoxia in neonatal and adult sheep. Am J Physiol 1985; 248: H 118-124. 
Kojo, M., Ogawa, T. \& Yamada, K. Normal developmental changes in carotid arterial blood flow measured by Doppler flowmetry in children. Pediatr Neurol 1996; 14: 313-316.

Levene, MI., Fenton, AC., Evans, DH., Archer, LNJ., Shortland, DB. \& Gibson, NA. Severe birth asphyxia and abnormal cerebral blood flow velocty. Dev Med Child Neurol 1989; 31: 427-434.

Liao, MF., Chaou, WT., Tsao LY., Nishida, H. \& Sakanoue, M. Ultrasound measurement of the ventricular size in newborn infants. Brain Dev 1986; 8: 262-268.

Lindström, K., Hallberg, B., Blennow, M., Wolff, K., Fernell, E. \& Westgren, M. Moderate neonatal encepehalopathy: pre- and perinatal risk factors and long-term outcome. Acta Obstet Gynecol Scand 2008; 87: 503-509.

Lipman, B., Serwer, GA. \& Brazy, JE. Abnormal cerebral hemodynamics in preterm infants with patent ductus arteriosus. Pediatrics 1982; 69: 778-781.

Lorek, A., Takei, Y., Cady, E., Wyatt, J., Penrice, J., Edwards, A., et al. Delayed ("secondary") cerebral energy failure after acute hypoxia -ischemia in the newborn piglet: continous 48-hour studeis by phosphorus magnetic resonance spectroscopy. Pediatr Res 1994; 36: 699-706.

Lowe, LH., \& Bailey, Z. Sate-of-the-art cranial sonongraphy: Part I, modern techniques and image interpretation. AJR Am J Roentgenol 2011; 196: 1028-1033.

Lowe, LH.,\& Bailey, Z. State-of-art cranial sonongraphy: Part 2, pitfalls and variants. Am J Roentgenol 2011; 196:1034-1039.

Luciano, R., Gallini, F., Romagnoli, C., Papacci, P. \& Tortorolo, G. Doppler evaluation of renal blood flow velocity as a predictive index of acute renal failure in perinatal asphyxia. Eur J Pediatr 1998; 157: 656-660.

Marlow, N. \& Budge, H. Prevalence, causes, and outcome at 2 years of age of newborn encephalopathy. Arch Dis Child Fetal Neonatal Ed 2005; 90: F 193-194.

Martin, E., \& Barkovich, AJ. Magnetic resonance imaging in perinatal asphyxia. Arch Dis Child 1995; 72: F 62-70.

Martin-Ancel, A., Garcia-Alix, A., Gayá, F., Carbañas, F., Burgueros, M. \& Quero, J. Multiple organ involvement in perinatal asphyxia. J Pediatr 1995; 127: 786-793.

Martinussen, M., Brubakk, AM., Linker, DT., Vik, T. \& Yao, AC. Mesenteric blood flow velocity and it relation to circulatory adaptation during the first week of life in healthy term infants. Pediatr Res 1994; 36: 334-339.

Meek, JH., Elwell, CE., McCormick, DC., Edwards, AD., Townsend, JP., Stewart, A., et al. Abnormal cerebral haemodynamics in perinatally asphyxiated neonates related to outcome. Arch Dis Child Fetal Neonatal Ed 1999; 81: F 110-115.

Meleagros, L., Ghatei, MA. \& Bloom, SR. Release of vasodilator, but not vasoconstrictor, neuropeptides and of enteroglucagon by intestinal ischaemia/reperfusion in the rat. Gut 1994; 35: 1701-1706.

Mercuri, E., Ricci, D., Cowan, FM., Lessing, D., Frisone, MF., Haataja, L., Counsell, SJ., et al. Head growth in infans with hypoxic-ischemic encephalopathy: correlation with neonatal magnetic resonance imaging. Pediatrics 2000; 106: 235-243.

Nankervis, CA., Giannone, PJ. \& Reber, KM. The neonatal intestinal vasculature: contributing factors to necrotizing enterocolitis. Semin Perinatol 2008; 32: 83-91. 
Nelson, KB. \& Leviton, A. How much of neonatal encephalopathy is due to birth asphyxia? Am J Dis Child 1991; 145: 1325-1331.

North, K., \& Lowe, L. Modern head ultrasound: normal anatomy, variants, and pitfalls that may simulate disease. Ultrasound Clin 2009; 4: 497-512.

Perlman, JM., Wyllie, J., Kattwinkel, Atkins, DL., Chameides, L., Goldsmith, JP., Guinsburg, R., et al. Neonatal resuscitation: 2010 International Consensus on Cardiopulmonary Resuscitation and Emergency Cardiovascular Care Science with Treatment Recommendations. Circulation 2010; 122: S 516-538.

Perlman, JM. Summary proceedings from the neurology group on hypoxic-ishemic encephalopathy. Pediatrics 2006; 117: S 28-33.

Perlman, JM., Tack, ED., Martin, T., Shackelford, G. \& Anon, E. Acute systemic organ injury in term infants after asphyxia. Am J Dis Child 1989; 143: 617-620.

Persico, A., Tursini, S., Ballone, E., Cirotti, D. \& Chiesa, PL. The renal-resistive index from the last 3 months of pregnancy to 6 months old. BJU International 2001; 87: 562.

Pierrat, V., Haouari, N., Liska, A., Thomas, D., Subtil, D. \& Truffert, P. Prevalence, causes, and outcome at 2 years of age of newborn encephalopathy: population based study. Arch Dis Child Fetal Neonatal Ed 2005; 90: F 257-261.

Pryds, O. Control of cerebral circulation in the high-risk neonate. Ann Neurol 1991; 30: 321329.

Pryds, O., Greisen, G., Lou, H. \& Friis-Hansen, B. Vasoparalysis accociated with brain damage in asphyxiated term infants. J Pediatr 1990; 117: 119-25.

Rangan, U. \& Bulkley, GB. Prospects for treatment of free radical-mediated tissue injury. $\mathrm{Br}$ Med Bull 1993; 49: 700-718.

Robertson, NJ. \& Edwards, AD. Recent advances in developing neuroprotective strategies for perinatal asphyxia. Current Opinion in Pediatrics 1998; 10: 575-580.

Rosenberg, AA. Cerebral blood flow and $\mathrm{O} 2$ metabolism after asphyxia in neonatal lambs. Pediatr Res 1986; 20: 778-782.

Roth, SC., Baudin, J., Cady, E., Johal, K., Townsend, JP., Wyatt, JS., Reynolds, EO. \& Stewart, AL. Relation of deranged neonatal cerebral oxidative metabolism with neurodevelopmental outcome and head circumference at 4 years. Dev Med Child Neurol 1997; 39: 718-725.

Rutherford, M., Pennock, J., Schwieso, J., Cowan, F. \& Dubowitz, L. Hypoxic-ischaemic encephalopathy: early and late magnetic resonance imaging findings in relation to outcome. Arch Dis Child 1996; 75: F145-151.

Rutherford, MA., Pennock, JM., Schwieso, JE., Cowan, FM. \& Dubowitz, LM. Hypoxicischemic encephalopathy: early magnetic resonance imaging findings and their evolution. Neuropediatrics 1995; 26: 183-191.

Rutherford, MA., Pennock, JM. \& Dubowitz, LM. Cranial ultrasound and magnetic resonance imaging in imaging in hypoxic-ischaemic encephalopoathy: a comparison with outcome. Dev Med Child Neurol 1994; 36: 813-825.

Sarnat, HB. \& Sarnat, MS. Neonatal encephalopathy following fetal distress. Arch Neurol 1976; 33: 698-705. 
Shadid, M., Moison, R., Steendijik, P., Hiltermann, L., Berger, HM. J\& van Bel, F. The effect of antioxidative combination therapy on post hypoxic-ischemic perfusion metabolism and electrical activity of the newbron brain. Pediatr Res 1998; 44: 11924.

Shah, P., Riphagen, S., Beyene, J.\& Perlman, M. Multiorgan dysfunction in infants with post-asphyxial hypoxic-ischaemic encephalopathy. Arch Dis Child 2004; 89: F 152155.

Shalak, L. \& Perlman, JM. Hypoxic-ischemic brain injury in the term infant: current concepts. Early Hum Dev 2004; 80: 125-141.

Stark, JE. \& Seibert, JJ. Cerebral artery Doppler ultrasonography for prediction of outcome after perinatal asphyxia. J Ultrasound Med 1994; 13: 595-600.

Stola, A. \& Perlman, J. Post-resuscitation strategies to avoid ongoing injury following intrapartum hypoxia-ischemia. Semin Fetal Neonatal Med 2008; 13: 424-431.

Takahashi, T., Shirane, R. Sato, S. \& Yoshimoto, T. Developmental changes of cerebral blood flow and oxygen metabolism in children. AM J Neuroradiol 1999; 20: 917-922.

Thoresen, M. \& Whitelaw, A. Cardiovascular changes during mild therapeutic hypothermia and rewarming in infants with hypoxic-ischemic encephalopathy. Pediatrics 2000; 106: 92-99.

Thorngren-Jerneck, K., Ohlsson, T., Sandell, A., Erlandsson, K., Strand, SE., Ryding, E., Svenningsen, NW. Cerebral glucose metabolism measured by positron emission tomography in term infants with hypoxic-ischemic encephalopathy. Pediatr Res 2001; 49: 495-501.

van Bel, F., Shadid, M., Moison, RM., Dorrepaal, CA., Fontijn, J., Monteiro, L., et al. Effect of allopurinol on postasphyxial free radical formation, cerebral hemodynamics, and electrical brain activity. Pediatrics 1998; 101: 185-193.

van Wezel Meijler, G., Steggerda, SJ. \& Leijser, LM. Cranial ultrasonongraphy in neonates: role and limitations. Semin Perinatol 2010; 34: 28-38.

Vannucci, RC. \& Perlman, JM. Interventions for perinatal hypoxic-ischemic encephalopathy. Pediatrics 1997; 100: 1004-1014.

Volpe JJ. Neurology of the newborn. WB Saunders Company. 5th ed 2008.

Wayenberg, JL., Vermeylen, D. \& Damis, E. Definition of asphyxia neonatorum and incidence of neurologic and systemic complications in full-term newborn. Arch Pediatr 1998; 5: 1065-1071.

Weir, FJ., Fong, K., Ryan, ML., Myhr, T. \& Ohlsson, A. Superior mesenteric artery and renal artery blood flow velocity measurements in neonates: technique and interobserver reliability. Pediatr Radiol 1995; 25; 145-148.

Weissner, C., Vogel, P., Neumann-Haefelin, T. \& Hossmann, K-A. Molecular correlates of delayed neuronal death following transient forebarin ischemia in the rat. Acta Neurochir 1996; 66: 1-7.

Wyatt, JS., Edwards, AD., Azzopardi, D. \& Reynolds, EO. Magnetic resonance and near infrared spectroscopy for investigation of perinatal hypoxic-ishaemic brain injury. Arch Dis Child 1989; 64: 953-963.

Yildirim, H., Gungor, S., Cihangiroglu, MM. \& Aygun, AD. Doppler studies in normal kidney of preterm and term neonates: changes in relation to gestational age and birth weight. J Ultrasound Med 2005; 24: 623-627. 
Yoxall, CW. \& Weindling, AM. Measurement of cerebral oxygen consumption in the human neonate using near infrared spectroscopy: cerebral oxygen consumption increases with advancing gestational age. Pediatr Res 1998; 44: 283-290. 


\title{
Neoplasm Related Encephalopathies
}

\author{
Lore Lapeire ${ }^{1}$, Anne Sieben ${ }^{2}$, Patrick Santens ${ }^{2}$ and Simon Van Belle ${ }^{1}$ \\ ${ }^{1}$ Department of Medical Oncology, \\ ${ }^{2}$ Department of Neurology, \\ Ghent University Hospital \\ Belgium
}

\section{Introduction}

Encephalopathy is a common clinical syndrome in cancer patients. The underlying causes of cancer related encephalopathies are various and mostly non-specific, such as metabolic disturbances as seen in critically ill patients. Electrolyte disturbances, hypoxia, hyperammonemia, thiamine deficiency and toxic reactions are all possible causes of encephalopathy in cancer patients. Structural lesions such as brain metastasis or venous thrombosis can evoke intracranial hypertension, also inducing a clinical picture resembling encephalopathy.

However, next to these non-specific causes of cancer related encephalopathy, there are also uncommon but highly specific encephalopathic syndromes related to malignancies and their treatments. In this chapter, we discuss encephalopathy associated with these uncommon clinical syndromes.

Paraneoplastic neurological syndromes (PNS) are clinical syndromes caused by nonmetastatic manifestations of neoplasm outside the nervous system. PNS can be immunemediated, and in those cases an association with circulating anti-neuronal antibodies and cytotoxic T-cells, in combination with specific tumours, can be made.

In non-immune mediated PNS, encephalopathy can be induced by tumour products such as hypertensive encephalopathy in patients with aldosterone-producing adenoma. Encephalopathy can also be the result of a depletion of substrate by direct competition between the central nervous tissue and the tumour, e.g. carcinoid encephalopathy.

In addition to these paraneoplastic neurological syndromes, we will present two typical cancer treatment related neurological conditions causing encephalopathy: PRES (posterior reversible encephalopathy syndrome) and PML (progressive multifocal leukoencephalopathy).

Pathophysiological mechanisms will be discussed, as well as associated clinical symptoms, prognosis and possible treatments.

\section{Paraneoplastic neurological syndromes}

PNS are unusual presentations of cancer, occurring in 1 per 10000 patients with malignancies. Clinically well defined immune-mediated PNS account for $10 \%$ of all non- 
metastatic neurological complications with cancer. However, the percentage of patients with malignancies presenting with peripheral neuropathy or muscle weakness is much higher.

About 3-5\% of patients with small-cell lung cancer (SCLC), 15-20\% of patients with thymoma and about $10 \%$ of patients with plasma-cell dyscrasias develop PNS. In patients with cancer of the breast or ovary, prevalence is well below 1\% (Darnell \& Posner, 2003).

In $2 / 3$ of patients, PNS will precede the diagnosis of malignancy. Identification of the PNS may allow tumour diagnosis when the tumour is small, localised and more responsive to treatment. Therefore, it is very important to recognize PNS with their various clinical presentations and subsequently start screening for malignancy when PNS is suspected.

Clinical presentations of PNS are various and include peripheral symptoms as well as central signs (Table 1). Peripheral symptomatology includes neuropathy, myasthenia and muscle weakness, whereas central signs are heterogeneous and include ataxia, visual disturbances, epilepsy, dysphagia and changes in mental state.

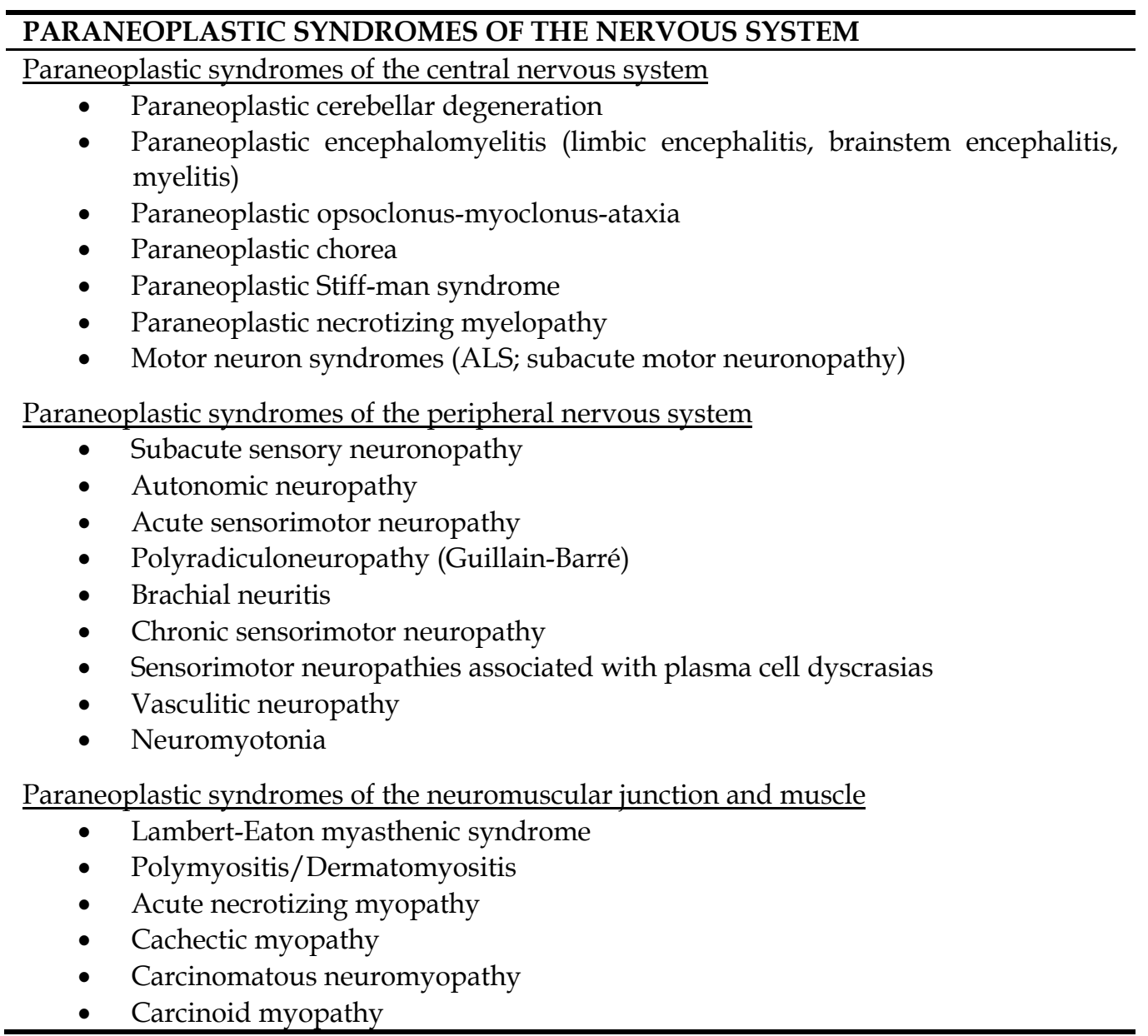

Table 1. Immune Mediated Paraneoplastic Syndromes of the Nervous System (Dalmau et al., 2007) 
The more common PNS are syndromes of the peripheral nervous system and neuromuscular junction: Lambert-Eaton myasthenic syndrome (LEMS) which affects 3\% of patients with SCLC and myasthenia gravis, occurring in 15\% of patients with thymoma.

Paraneoplastic encephalomyelitis is the PNS of the central nervous system most frequently seen.

Tumours which are commonly involved in PNS of the central nervous system express neuroendocrine proteins (e.g. small-cell lung cancer (SCLC)), affect organs with immunoregulatory proteins (e.g. thymoma) or contain mature or immature neuronal tissue (e.g. teratoma).

Tumours originating from tissue producing immunoglobulins (e.g. lymphomas) are more commonly involved in PNS of the peripheral nervous system (Dalmau \& Rosenfield, 2008).

As mentioned above, circulating antibodies against the expressed tumoural proteins can be associated with PNS. Different antibodies can be associated with the same PNS and inversely, the same antibody can be associated with different clinical syndromes (Voltz, 2002). Moreover, several PNS may co-occur in the same patient, particularly if the underlying tumour is SCLC.

The detection of antibodies in serum or cerebrospinal fluid confirms the clinical diagnosis of a paraneoplastic syndrome and allows early identification of an underlying tumour. However, the failure of finding antibodies does not exclude a diagnosis of PNS and inversely, antibodies have been detected in patients without PNS, e.g. anti-Hu antibodies (in low titres) in patients with SCLC can be present without any neurological symptoms (Nath \& Grant, 1997).

Next to immune mediated PNS, there are also non-immunologic mechanisms that can be involved in PNS. Cytokines and hormones produced by tumours can induce an encephalopathy. Another yet uncommon cause of encephalopathy involves the competition between the tumour and nervous tissue for a specific substrate, e.g. carcinoid tumours and tryptophan. This very rare and particular picture will be described further in this chapter.

\subsection{Pathogenetic mechanism of immune-mediated PNS}

The identification in 1985 of anti-neuronal antibodies in the serum of patients with nonmetastatic neurological complications of cancer, led to the hypothesis that paraneoplastic neurological syndromes are immune-mediated (Voltz, 2002). Antibodies against neuronal antigens have since then been extensively described.

The central and peripheral nervous system are considered to be low immunogenetic systems, since the presence of the blood/brain barrier means that antigens which are localised in the cerebrospinal fluid do not normally induce an immune response. Aberrant expression of neuronal antigens by a tumour outside this barrier, can lead to an immunological response which breaches the blood/brain barrier, as such inducing a possible influence on the central and peripheral nervous system.

Before a disorder can be considered to be antibody mediated, several criteria have to be fulfilled (Koch's postulates, as cited in Sutton \& Winer, 2002):

1. The reactivity of the antibody with the antigen results in antigen loss and/or neuronal loss, inducing the clinical phenotype. 
2. A passive transfer of the immunoglobulins of an affected patient to an animal should induce the clinical phenotype.

3. Immunotherapy that leads to a reduction of antibody titres should be associated with clinical improvement or at least stabilisation of the symptomatology.

4. Immunization of an animal with the purified antigen leads to the development of an antibody response, resulting in the clinical phenotype.

In the group of onconeural antibodies, only the antibodies reacting against VGCC (voltagegated calcium channel) and mGluR1 (metabotropic glutamate receptor) have met the above mentioned criteria and can be considered to be pathogenic antibodies, e.g. anti-VGCC antibodies inducing the Lambert-Eaton Myasthenic Syndrome. However, the anti-VGCC antibodies found in paraneoplastic cerebellar degeneration are not found to be pathogenic, whereas anti-GluR1 antibodies are the cause of the cerebellar degeneration in patients with Hodgkin's disease (Sutton \& Winer, 2002).

Anti-VGCC antibodies and anti-GluR1 antibodies are antibodies reactive to antigens on the cell surface of neurones. Up till now, none of the antibodies acting against an intracellular neuronal antigen are found to be pathogenic.

In post-mortem findings of patients with PNS and antibodies against intracellular targets, the finding of infiltration of T-cells in the affected central nervous tissue has opened the possible role of a cytotoxic T-cell mediated immune response.

This pathway includes T-cell immune responses that probably act against the target antigens of the circulating antibodies. E.g. Hu antigen-specific T-cells have been identified in blood and cerebrospinal fluid of patients with circulating anti-Hu antibodies. Support for this additional T-cell mediated mechanism includes the difficulties in treating these disorders with strategies directed at the humoral immune response (Dalmau \& Rosenfield, 2008).

This pathogenesis has been confirmed in the development of paraneoplastic encephalomyelitis in patients with SCLC and a positive anti-Hu antibody serology.

It remains to be investigated whether cytotoxic T-lymphocytes against the Yo-antigen are responsible for the paraneoplastic cerebellar degeneration (Sutton \& Winer, 2002).

Thus, antibodies seem to be in most cases a marginal phenomenon opposed to a causing factor. The importance of detecting the antibody remains however essential, as screening for malignancies can be specified and the prognosis of the PNS can be formulated.

\subsection{Antineuronal antibodies}

Antineuronal antibodies belong to a group of heterogeneous antibodies, which tend to present a diversity of clinical symptoms.

Two groups can be separated: antineuronal antibodies formed in the presence of a malignancy, i.e. paraneoplastic or onconeural antibodies (table 2), while the other group is often but not always associated with neoplasm (table 3).

The majority of antibodies can be detected using indirect immunofluorescence. A confirmation test using Western blot against recombinant antigen is an additional value (Sutton \& Winer, 2002). 


\begin{tabular}{|c|c|c|}
\hline Antibody & Paraneoplastic neurological syndrome & Associated tumours \\
\hline Anti-Yo (APCA1) & $\begin{array}{l}\text { Paraneoplastic cerebellar degeneration } \\
\text { (PCD) }\end{array}$ & $\begin{array}{l}\text { Ovarian cancer, breast } \\
\text { cancer }\end{array}$ \\
\hline $\begin{array}{l}\text { Anti-Hu } \\
\text { (ANNA1) }\end{array}$ & $\begin{array}{l}\text { Subacute sensory neuronopathy } \\
\text { Encephalomyelitis }\end{array}$ & $\begin{array}{l}\text { Small-cell lung cancer } \\
\text { (SCLC) }\end{array}$ \\
\hline & $\begin{array}{l}\text { Autonomic dysfunction } \\
\text { PCD } \\
\text { Limbic encephalitis }\end{array}$ & \\
\hline Anti-CV2 & Chorea & SCLC, thymoma, germ- \\
\hline (CRMP5) & $\begin{array}{l}\text { Sensory neuronopathy } \\
\text { Sensorimotor neuropathy } \\
\text { Encephalomyelitis } \\
\text { PCD } \\
\text { Limbic encephalitis }\end{array}$ & cell tumours of testis \\
\hline Anti-Ri (ANNA2) & $\begin{array}{l}\text { Brainstem encephalitis } \\
\text { Opsoclonus-Myoclonus-Ataxia syndrome } \\
\text { PCD }\end{array}$ & $\begin{array}{l}\text { Breast cancer, ovarian } \\
\text { cancer, SCLC }\end{array}$ \\
\hline Anti-Ma (1\&2) & $\begin{array}{l}\text { Limbic/mesodiencephalic/ hypothalamic } \\
\text { encephalitis }\end{array}$ & $\begin{array}{l}\text { Breast cancer, germ-cell } \\
\text { tumours of testis }\end{array}$ \\
\hline Anti- & Stiff-person syndrome & Breast cancer \\
\hline amphiphysin & Encephalomyelitis & SCLC \\
\hline Anti-Tr (PCA-Tr) & PCD & Hodgkin's disease \\
\hline Anti-ANNA3 & Various PNS of the CNS & SCLC \\
\hline Anti-PCA2 & Various PNS of the CNS & SCLC \\
\hline Anti-Zic & PCD & SCLC \\
\hline Anti-mGluR1 & PCD & Hodgkin's lymphoma \\
\hline \multicolumn{3}{|c|}{$\begin{array}{l}\text { Table 2. Well characterized onconeural antibodies (APCA: anti-Purkinje cell antibody, } \\
\text { ANNA: anti-neuronal nuclear antibody, CRMP: collapsin response mediator proteins, } \\
\text { mGluR: metabotropic glutamate receptor) (Dalmau et al., 2007) }\end{array}$} \\
\hline Antibody & $\begin{array}{l}\text { Paraneoplastic neurological } \\
\text { syndrome }\end{array}$ & Associated tumours \\
\hline Anti-NMDAR & Encephalitis & Ovarian teratoma \\
\hline Anti-VGKC & $\begin{array}{l}\text { Limbic encephalitis } \\
\text { neuromyotonia } \\
\text { other }\end{array}$ & Thymoma, SCLC, other \\
\hline Anti-VGCC & $\begin{array}{l}\text { LEMS } \\
\text { PCD }\end{array}$ & SLCL \\
\hline Anti-AchR & Myasthenia Gravis & Thymoma \\
\hline Anti-nAchR & Subacute pandysautonomia & SCLC, other \\
\hline Anti-GAD & $\begin{array}{l}\text { Stiff-person syndrome } \\
\text { cerebellar ataxia } \\
\text { limbic encephalitis } \\
\text { other }\end{array}$ & Thymoma, other \\
\hline
\end{tabular}

Table 3. Antibodies that occur with and without malignancy (NMDA: N-methyl-D-Asparginic acid receptor, VGKC: voltage-gated potassium channel, VGCC: voltage-gated calcium channel, AchR: acetylcholine receptor, nAchR: neuronal acetylcholine receptor, GAD: glutaminic acid decarboxylase, LEMS: Lambert-Eaton myastenic syndrome) (Dalmau et al., 2007) 


\subsubsection{Anti-Yo antibody (APCA-1 = anti-Purkinje cell antibody type 1)}

Clinical associations

Anti-Yo antibodies are mostly clinically accompanied by a paraneoplastic cerebellar degeneration $(>50 \%)$.

Tumour associations

Most patients are women and associated tumours are almost exclusively gynaecologic (47\% ovarian, $13 \%$ endometrial and tubal carcinoma) or breast cancers $(25 \%)$. Some patients with SCLC, various adenocarcinomas, transitional cell carcinoma of the bladder or lymphoma have been described (Gozzard \& Maddison, 2010).

\section{Antigen}

The Yo-antigens belong to a family of DNA binding proteins. The expression of the antigens is limited to the cytoplasm of Purkinje cells. Anti-Yo antibodies recognize 2 antigens: a cytoplasmatic protein called CDR62 (cerebellar degeneration-related 62kDa protein) and a minor cytoplasmatic protein of $34 \mathrm{kDa}$, named CDR34.

Three genes have been cloned (cdr 1-3). Cdr1 encodes the $34 \mathrm{kDa}$ protein while Cdr2 encodes the CDR62 protein that specifically binds to c-Myc thereby downregulating its activity. Disruption of this interaction by anti-Yo antibodies may increase c-Myc activity, leading to apoptosis of Purkinje cells (Dalmau \& Rosenfield, 2008; Nath \& Grant, 1997; Voltz, 2002).

However, different studies have demonstrated T-lymphocytes in the leptomeninges, brain stem and medulla, without lymphocytic infiltration or signs of inflammation in the cerebellar cortex in patients with a later stage of the cerebellar degeneration (Sutton \& Winer, 2002). It is proposed that the loss of the Purkinje cells represent a final "burn out" stage of a cell-mediated immune process (Voltz, 2002). This hypothesis is supported by the presence of T-lymphocytes in the cerebellar cortex of a patient, who died 4 months after onset of the cerebellar symptomatology (Sutton \& Winer, 2002).

\subsubsection{Anti-Hu antibody (ANNA-1 = anti- neuronal nuclear antibody type 1)}

Clinical associations

As anti-Hu antibodies react with antigens localized in the nucleus of every neuronal cell, anti-Hu antibodies can cause a variety of PNS of the central and peripheral nerve tissue (table 2): paraneoplastic limbic encephalitis, paraneoplastic cerebellar degeneration, paraneoplastic encephalomyelitis and subacute sensory neuronopathy.

Identification of anti-Hu antibodies at a concentration greater than 1/500 in cerebrospinal fluid is highly sensitive for a PNS of the central nervous system, typically limbic encephalitis or paraneoplastic encephalomyelitis.

Tumour associations

$80 \%-93 \%$ of patients with anti-Hu antibodies will have SCLC (Gozzard \& Maddison, 2010); Nath \& Grant, 1997). Extrathoracic tumours in association with anti-Hu antibodies include neuroblastoma (50\%) and prostate cancer (Titulaer et al, 2011). 
Antigen

Anti-Hu antibody reacts with a neuronal nuclear antigen that belongs to a family of RNA binding proteins.

There are 4 antigens which are recognized by anti-Hu antibodies: $\mathrm{HuD}, \mathrm{HuC}, \mathrm{HuR}$ and Hel$\mathrm{N} 1 . \mathrm{HuD}, \mathrm{HuC}$ and Hel-N1 are expressed in terminally differentiated neurons, whereas HuR is expressed in all proliferating cells (Sutton \& Winer, 2002). HuD, HuC and Hel-N1 are expressed by all SCLC's.

Immunologic associations

Patients with paraneoplastic syndromes and anti-Hu antibodies may develop concurrent antibodies to other onconeural antigens. They include anti-CV2 (CRMP5), antiamphiphysin, anti-Ri, anti-VGCC and anti-Zic4 antibodies.

\subsubsection{Anti-Ri antibody (ANNA-2 = anti-neuronal nuclear antibody type 2)}

Immunocytochemistry of serum or CSF shows an anti-neuronal nuclear antibody which is identical to the ANNA-1 antibody on immunocytochemical criteria. With western blotting, a different banding pattern can be seen, distinguishing this antibody from ANNA-1.

Clinical associations

This antibody has mostly been described in patients with opsoclonus-myoclonus-ataxia with SCLC as underlying tumour. Associated PNS can be paraneoplastic cerebellar degeneration (Gozzard \& Maddison, 2010).

Tumour associations

Next to SLCL, other underlying neoplasms can be malignancies of breast, ovary and bladder (Darnell \& Posner, 2003; Gozzard \& Maddison, 2010).

Antigen

The antigen recognized by anti-Ri antibodies is a protein belonging to a family of RNAbinding proteins, mainly expressed in the nervous system. There are 2 Ri-antigens: Nova-1 and Nova-2, widely expressed within the central nervous system.

Recent pathological and functional MRI findings suggest that the disinhibition of the fastigial nucleus of the cerebellum is involved, causing the clinical picture. However, it remains unclear why this structure is most susceptible to the toxicity of the anti-Ri antibody, whilst the inflammatory process is seen in the entire central nervous system (Dalmau \& Rosenfield, 2008; Sutton \& Winer, 2002).

\subsubsection{Anti-CV2 antibodies (=Anti-CRMP5 antibodies)}

Clinical associations

Patients can manifest with different clinical syndromes. Sensorimotor neuropathy and paraneoplastic cerebellar degeneration are most commonly found. Less frequent are limbic encephalitis, encephalomyelitis and LEMS. 
Tumour associations

The tumour most commonly found in patients with anti-CV2 antibodies, is SCLC (60\%$77 \%)$. Further, thymoma (7\%) and neoplasm of the uterus need to be excluded (Voltz, 2002).

Antigen

The target of anti-CV2 antibodies is a family of $\sim 66 \mathrm{kDa}$ proteins that are mainly expressed in the nervous system called CRMP for Collapsin Response Mediator Proteins. In the adult brain, the expression of CRMPs is dramatically down-regulated. However, they remain expressed in structures that have been shown to retain capacity of differentiation and also in a subpopulation of oligodendrocytes. In the peripheral nervous system CRMPs are expressed in a subset of sensory neurons and Schwann cells.

Immunologic associations

Patients with paraneoplastic syndromes and anti-CV2 antibodies may develop concurrent antibodies to other onconeural antigens. They include anti-Hu, anti-amphiphysin, anti-Ri, and anti-Zic4 antibodies.

\subsubsection{Anti-Ma antibodies}

The Ma proteins (Ma1, Ma2 and Ma3) belong to a family of brain-testis-cancer proteins. Ma1 and $\mathrm{Ma} 2$ antigens are expressed in the testis and in neurons. The highest level of expression of $\mathrm{Ma}$ antigens is seen in the neurons of the limbic structures, tegmental nuclei and cerebellar dentate nucleus. Aberrant expression of $\mathrm{Ma}$ antigens are seen in other malignancies, such as breast cancer (Sutton \& Winer, 2002; Voltz, 2002).

Different neurological syndromes can be associated. Logically, mostly paraneoplastic limbic encephalitis and paraneoplastic encephalomyelitis are seen.

\subsubsection{Anti-amphiphysin antibodies}

The antigen is a protein located in synaptic vesicles and may have a role in vesicle endocytosis. This antibody is found in patients with Stiff-person syndrome, encephalomyelitis and subacute sensory neuronopathy. The underlying tumour can be breast cancer, as well as SLCL (Titulaer et al, 2011; Voltz, 2002).

\subsubsection{Anti-Tr antibodies}

This antibody has similarities with the anti-Yo antibody but can be differentiated by a typical immunohistochemical staining pattern of the molecular layer of the cerebellar cortex, in combination with staining of the Purkinje cell layer. Clinical presentation is a cerebellar degeneration. The associated tumour is Hodgkin lymphoma (Sutton \& Winer, 2002; Voltz, 2002).

\subsubsection{Antibodies that occur with and without malignancy}

Anti-VGCC antibodies (anti-voltage gated calcium antibodies)

This antibody is mostly associated with LEMS. In 50\% of patients with LEMS and antiVGCC antibodies, SCLC is found. Rarely, patients present with a paraneoplastic cerebellar degeneration. 
Anti-NMDAR antibodies (anti-N-Methyl-D-Asparginic acid receptor antibodies)

These antibodies are found in young women with a teratoma of the ovary. Clinically, patients present with a limbic encephalitis in combination with psychiatric symptoms and autonomic dysfunction. The neurological symptomatology can reverse after treatment of the tumour, as well as after immunotherapy.

Anti-Zic antibodies

This antibody has a low frequency. A variety of neurological symptoms can occur, with cerebellar ataxia due to paraneoplastic cerebellar degeneration most frequently seen. Underlying tumour is SCLC.

Anti-mGluR1 receptor antibodies

This metabotropic glutamate receptor antibody reacts with the mGluR1 receptor on the surface of Purkinje cells. There is an association with Hodgkin's lymphoma. Clinical presentation is a cerebellar syndrome (Darnell \& Posner, 2003).

Anti-VGKC antibodies (anti-voltage gated potassium channel antibodies)

Patients with a positive serology for this antibody present typically with neuromyotonia. A PNS of the central nervous system occasionally seen is limbic encephalitis, commonly evolving to a less focalized encephalitis. Underlying tumours are SCLC and thymoma (Titulaer et al, 2011).

Anti-GAD antibodies (anti-glutaminic acid decarboxylase antibodies)

This antibody has a well known association with Stiff-Person Syndrome. Recently patients with paraneoplastic cerebellar degeneration or limbic encephalitis were described. This antibody is frequently found in patients with insulin-dependent diabetes mellitus. When a paraneoplastic mechanism is suspected, patients should be screened for a thymoma.

\subsection{Immune-mediated paraneoplastic syndromes of the central nervous system}

As 2/3 of PNS precede the diagnosis of malignancy, it is important to recognize the clinical picture and to start an appropriate screening for neoplasm.

Several features are shared by most PNS of the CNS. The clinical presentation is a fast accumulation of symptoms of central nervous system involvement, evolving over weeks and severely disabling the patient.

MRI findings are non-specific and can show normal findings, relative atrophy of specific regions (e.g. cerebellum) or regional Gadolinium-enhancing lesions. In cerebrospinal fluid, mostly signs of inflammation or immune-mediated mechanisms are withheld: moderate lymphocytic pleocytosis (30-40 white blood cells $/ \mathrm{mm}^{3}$ ), increased protein concentration (50-100 mg/dl), high IgG index and CSF-specific oligoclonal bands (Darnell \& Posner, 2003).

Because of the low prevalence of PNS, the PNS Euronetwork published diagnostic criteria for PNS (table 4) (PNS Euronetwork, 2002; Titulaer et al, 2011). 
Diagnostic Criteria for Paraneoplastic Neurological Syndromes

Definite PNS

1. A classical syndrome and cancer that develops within five years of the diagnosis of the neurological disorder.

2. A non-classical syndrome that resolves or significantly improves after cancer treatment without concomitant immunotherapy, provided that the syndrome is not susceptible to spontaneous remission.

3. A non-classical syndrome with onconeural antibodies (well characterised or not) and cancer that develops within five years of the diagnosis of the neurological disorder.

4. A neurological syndrome (classical or not) with well characterised onconeural antibodies (anti-Hu, Yo, CV2, Ri, Ma2, or amphiphysin), and no cancer.

Possible PNS

1. A classical syndrome, no onconeural antibodies, no cancer but at high risk to have an underlying tumour.

2. A neurological syndrome (classical or not) with partially characterised onconeural antibodies and no cancer.

3. A non-classical syndrome, no onconeural antibodies, and cancer present within two years of diagnosis.

Table 4. Diagnostic Criteria for Paraneoplastic Neurological Syndromes (Graus et al, 2004)

\subsubsection{Paraneoplastic cerebellar degeneration}

Although one of the best known PNS, paraneoplastic cerebellar degeneration is rare, occurring in $0,2 \%$ of patients with cancer.

The clinical presentation is typical. The progression of a paraneoplastic cerebellar degeneration is fast, leaving the patient severely disabled after a small number of weeks.

Patients start complaining of vertigo, dizziness and nausea, followed by ataxia which makes it difficult to walk and to perform activities in daily living. As disease progresses, nystagmus develops causingblurry vision, and dysarthria and dysphagia may occur.

About $20 \%$ of patients present with mild cognitive deficits (Gozzard \& Maddison, 2010).

Initially MRI is normal. However, subsequent MRI scans during the course of the disease, show a progressive cerebellar atrophy. Initial fluoro-deoxy-glucose PET can show cerebellar hypermetabolism. The findings in cerebrospinal fluid are compatible with an immune mediated or inflammatory process (Gozzard \& Maddison, 2010).

Differential diagnosis includes viral cerebellitis, toxic cerebellitis, alcohol-related vitamin deficiency induced cerebellitis and Creutzfeldt-Jakob disease (CJD) (Dalmau et al, 2007).

In the differential diagnosis with CJD, it is important to consider that $12 \%$ of patients with PNS of the CNS have 14-3-3 protein in the CSF whereas high titres of this protein in cerebrospinal fluid are typically found in CJD.

In patients with paraneoplastic cerebellar degeneration, a range of antineuronal antibodies can be detected in serum and in cerebrospinal fluid. Some are specifically related to 
cerebellar involvement, whereas others are non-specific and might reflect a tumour-induced immune response. Dependent on the associated antibody, the clinical course, prognosis and survival may differ (Voltz, 2002).

Most frequently, anti-Yo antibodies are seen, with ovarian cancer or breast cancer as underlying pathology. This PNS occurs consequently more in middle aged women. Median survival of anti-Yo positive patients with breast cancer is 100 months, whereas anti-Yo positive patients with ovarian malignancies survive 22 months.

When small-cell lung cancer is found, anti-Hu antibodies are present in $23-40 \%$ of patients with paraneoplastic cerebellar degeneration. Median survival is 9 to 12 months (Voltz, 2002).

$41 \%$ of patients will develop anti-VGCC (voltage-gated calcium-channel) antibodies. These antibodies are also present in Lambert-Eaton myasthenic syndrome.

Anti-Tr antibody, associated with Hodgkin's lymphoma, is another highly specific antibody. The patients are usually young men and the diagnosis of PNS often follows the diagnosis of lymphoma (Nath \& Grant, 1997).

Rarely other antibodies are found, such as antibodies against collapsing-response mediator protein 5 (CRMP or CV2), amphiphysin or antineuronal nuclear antibody 3 (ANNA3).

The neuropathological hallmark of the paraneoplastic cerebellar degeneration is extensive loss of Purkinje cells in association with atrophy of granular and molecular layers. Microglial proliferation is seen in combination with astrocytosis. More specific, in the beginning of the disease T-cell infiltration in leptomeninges and in cerebellar cortex can be found. As the disease progresses, lymphocytic infiltration diminishes and gliosis develops (Dalmau et al, 2007; Nath \& Grant, 1997; Sutton \& Winer, 2002).

\subsubsection{Paraneoplastic encephalomyelitis}

Paraneoplastic encephalomyelitis (PEM) has a strong association with the anti-Hu antibody (Titulaer et al, 2011). In 75\% of patients with a positive serology, an underlying SCLC is found. Other antibodies less frequently associated with PEM are anti-CV2 (CRMP5) and anti-amphiphysin.

This PNS includes a variety of neurological syndromes, the clinical picture being the result of the variable anatomic involvement. The regions which are frequently involved are the hippocampus, lower brain stem, spinal cord and dorsal root ganglia (Gozzard \& Maddison, 2010). The diversity of neurological dysfunction reflects the fact that anti-Hu antigens, the target of the autoimmune dysfunction, are expressed in all central and peripheral neurones (Sutton \& Winer, 2002).

Most frequently, patients present with symptoms suggestive of subacute sensory neuronopathy, followed by limbic encephalitis, brain stem encephalitis and myelitis.

Patients present often with a symptomatology of one of the above mentioned regions, which eventually, as disease progresses, evolves into a multifocal neurological syndrome (PNS Euronetwork, 2002).

As limbic encephalitis is part of this clinical picture often accompanied with an encephalopathic syndrome, this will be discussed further. 
CSF studies may show pleocytosis and elevated protein levels. MRI brain scans are mostly normal. However, hyperintense T2-weighted lesions in brainstem, thalamus and hippocampus can be seen. Lesions are rarely contrast enhancing.

Extensive neuronal loss is seen in the affected brain regions: hippocampal sclerosis, gliosis in brainstem, spinal cord and dorsal root ganglia.

\subsubsection{Paraneoplastic limbic encephalitis}

This clinical syndrome is seen in $0.3 \%$ of cancer patients (Nath \& Grant, 1997). Patients tend to be of middle age with no sex predilection. Patients develop mood and sleep disturbances, seizures, hallucinations and short term memory loss. This cognitive decline can eventually lead towards dementia (Voltz, 2002).

Electro-encephalographic findings often include epileptic foci, mostly in one or both temporal lobes, next to generalized slow wave activity. In 70 to $80 \%$ of patients, hyperintense foci in the medial part of one or both temporal lobes are seen on MRI fluidattenuated inversion recovery (FLAIR) and T2-weighted sequences. Gadolinium contrast enhanced lesions are rare. Fluoro-deoxy-glucose PET often shows hypermetabolism of the medial parts of the temporal lobes and often precedes MRI findings. In cerebrospinal fluid, findings suggest inflammation (Gozzard \& Maddison, 2010).

Differential diagnoses of limbic encephalitis include viral encephalitis, prion diseases and auto-immune non-neoplastic encephalopathies (Voltz, 2002).

Neuropathological findings can be summarized as an inflammation of the structures of the limbic system. Perivascular lymphocytic infiltration is seen, in combination with microglial proliferation and reactive gliosis. Immunological staining with anti-Hu shows strong staining of neuronal nuclei in central and peripheral nervous tissue with sparing of nucleoli. Cytoplasm is weakly stained. There is no staining of systemic tissue (Nath \& Grant, 1997).

Tumours most frequently involved are SCLC, testicular germ-cell malignancies, thymoma, teratoma or Hodgkin's lymphoma. Dependent on the antibody which is found in serum and cerebrospinal fluid, subtyping of limbic encephalitis is recommended (Dalmau \& Rosenfield, 2008).

Anti-NMDA receptor antibodies and anti-VGKC antibodies act against proteins that are exposed on the cell surface from e.g. hippocampus. Patients with a positive serology for those antibodies, respond well to immunotherapy, whereas patients who are positive for antibodies acting against antigens localised on the cytoplasmatic side of the cell (e.g. amphiphysin) or localised in the cytoplasm or nucleus (e.g. GAD, $\mathrm{Hu}$ ), respond less or not to immunotherapy.

Limbic encephalitis with antibodies to intracellular antigens

In about 1 of 2 patients with anti-Hu antibodies SCLC and limbic encephalitis, the clinical picture starts with the symptomatology of limbic encephalitis but evolves eventually into a more widespread encephalitis, often in combination with myelitis.

Patients can present with anti-CRMP5 (anti-CV2) antibodies. In those cases, the clinical picture can be dominated by optic neuritis and myelitis, whereas symptoms suggestive of 
encephalitis are less explicit. SCLC and thymoma are tumours most commonly involved. The combination of optic neuritis and myelitis resembles a diagnosis of Devic's syndrome (neuromyelitis optica).

Anti-Ma2 antibodies are associated with limbic encephalitis in combination with involvement of the hypothalamus and brainstem. Clinical presentation is variable and can start with hypersomnolence, cataplexy, narcolepsy, REM sleep disturbances, hormonal deficits or hyperphagia. Involvement of the mesencephalon causes vertical gaze palsy, orofacial and jaw dystonia. Differential diagnosis has to be made with Whipple's disease.

Anti-Ma2 antibodies in males younger than 50 years should trigger a screening program for testicular germ-cell tumours. In older patients, SCLC or breast tumours have to be excluded.

Many patients with germ-cell tumours of the testes and anti-Ma2-associated encephalitis benefit from orchiectomy and immunotherapy. In 35\% of patients, neurological signs improve.

Limbic encephalitis with antibodies to neuronal cell-surface antigens

The two main clinical syndromes associated with these antibodies are typical limbic encephalitis and encephalitis with psychiatric symptoms, hallucinations and peripheral nerve hyperexcitability, amongst other signs of autonomic dysfunction.

Antibodies act against the voltage-gated potassium channels.

About $30 \%$ of patients with anti-VGKC antibodies have tumours, of which SCLC and thymoma are most prevalent.

The CSF of those patients shows less inflammation, less pleiocytosis and lower protein concentration compared to other PNS.

$80 \%$ of patients respond to immunotherapy.

Patients with anti-NMDA-receptor antibodies are usually young and female. Symptomatology starts with headaches, memory deficits, epileptic seizures and psychiatric behavioural symptoms. As the disease progresses, autonomic disturbances are seen, leading to supported ventilation or pace-making.

About $65 \%$ of the patients have a malignancy. Underlying tumour is mostly a (cystic) ovarian teratoma. Immunotherapy in combination with treatment of the tumour results mostly in neurological improvement.

\subsubsection{Paraneoplastic opsoclonus-myoclonus-ataxia syndrome}

Opsoclonus is the abnormal movement of the eyes consisting of chaotic, involuntary conjugated saccades with horizontal, vertical and torsional components and without intersaccadic interval. Myoclonus is most often present as myoclonic jerks in limbs and trunk. Occasionally, encephalopathy or cerebellar ataxia is present.

Clinical symptoms often have an acute onset and may fluctuate in the development of the PNS. Rarely, it resolves spontaneously.

Opsoclonus-myoclonus-ataxia syndrome can occur in combination with infections, metabolic disturbances, toxic factors as well as in combination with neoplasm. 
MRI is usually normal. CSF may show mild inflammatory changes, mostly B-lymphocyte pleiocytosis and oligoclonal bands (Gozzard \& Maddison, 2010). EEG may be normal or demonstrate generalized slow activity, without epileptic discharges.

Paraneoplastic opsoclonus-myoclonus-ataxia syndrome is seen in 3 patients groups.

In children, this clinical syndrome is seen in combination with neuroblastoma in $50 \%$ of cases. There is no corresponding antibody identified.

In adult female patients, the tumour most commonly found is cancer of ovary and breast. The associated antibody is anti-Ri.

In adult patients who are anti-Ri antibody negative, the underling tumour is SCLC. A positive serology for anti-Hu and an association with anti-Ma2 has been described in patients with SCLC and opsoclonus-myoclonus-ataxia syndrome (Voltz, 2002).

Recent immunological findings however have demonstrated the presence of antibodies against postsynaptic or cell-surface antigens. Those antibodies, present in the serum of children with neuroblastoma, react with the cell surfaces of cerebellar granular neurons and neuroblastoma cells (Dalmau et al, 2007).

Neuropathology shows a mild perivascular and interstitial lymphocytic infiltration, in combination with a relative loss of Purkinje cells and loss of neurons in the dentate nucleus.

Immunhistochemistry pattern of anti-Ri include strong staining of only central nervous system neuronal tissue, sparing of nucleoli, weak cytoplasmatic staining and no staining of systemic tissues (Nath \& Grant, 1997; PNS Euronetwork, 2002).

\subsection{Treatment of PNS}

There is no standard of care for PNS of the central nervous system. Moreover, in the vast majority of cases, PNS are refractory to any form of treatment.

Clinical experience suggests that treatment and removal of the causing factor, i.e. the tumour is necessary to stabilise or improve symptomatology. Adjuvant corticosteroids, intravenous immunoglobulin therapy, plasma exchange, cyclophosphamide and tacrolimus have not substantially improved the neurological outcome.(Dalmau \& Rosenfield, 2008).

In children with paraneoplastic opsoclonus-myoclonus syndrome, the clinical picture often responds, however only partially, to treatments such as corticosteroids, intravenous immunoglobulin, plasma exchange, cyclophosphamide or rituximab. Symptom relapses can occur during transient illnesses (e.g. viral infections). In adults, paraneoplastic opsoclonusmyoclonus syndrome is less responsive to immunotherapy. Stabilisation or improvement is only seen in patients with cured or controlled malignancies. Rarely, spontaneous remission of neurological symptoms can occur in patients with opsoclonus-myoclonus-ataxia syndrome. (Nath \& Grant, 1997).

\subsection{Non-immune mediated paraneoplastic encephalopathies}

As previously mentioned, non-immune mediated encephalopathies can be provoked by a whole spectrum of etiological factors such as metabolic disorders, electrolyte disturbances 
and excessive production of certain hormones or proteins, elicited by cancer or not. Some specific clinical entities have been described. Neuro-endocrine tumours can synthesize biologically active substances, such as hormones, growth factors or cytokines, which can induce non-immune mediated encephalopathy. For instance, pheochromocytoma, paraganglioma or tumours of the adrenal gland can cause hypertensive encephalopathy through production of vaso-active peptides (Kaltsas et al., 2010).

A peculiar form of non-immune mediated encephalopathy has been described in carcinoid tumours where competition arises between the tumour and the nervous tissue for a specific substance called tryptophan. Active production of serotonin by the carcinoid tumour causes depletion of tryptophan leading to neuronal dysfunction. Associated neurological disorders include carcinoid myopathy and carcinoid encephalopathy. Other mechanisms of carcinoid encephalopathy have also been proposed such as excessive production of serotonin in the brain by CNS metastasis, fulminant hepatic metastasis and aberrant secretion of ACTH of CRH by carcinoid tumours (Lapeire et al., 2010). Treatment of carcinoid encephalopathy is mainly focused on controlling the tumour although more 'targeted' treatment such as oral tryptophan substitution or serotonin receptor antagonism have proved their use in case reports.

\section{Cancer treatment related encephalopathies}

As the treatment modalities of cancer evolve, the side effects subsequently follow. Already decades ago, cytotoxic agents were linked with the development of encephalopathies and with the more recently developed targeted therapies the number of case reports regarding cancer treatment related encephalopathies is rising.

Cancer treatment related encephalopathies can be divided into two groups. On one hand we have toxic encephalopathies, of which posterior reversible encephalopathy syndrome or PRES is an uncommon yet characteristic cliniconeuroradiological syndrome. The second group comprises encephalopathies induced by opportunistic infections with progressive multifocal leukoencephalopathy or PML being most reported and investigated.

\subsection{Posterior reversible encephalopathy syndrome (PRES)}

\subsubsection{Definition of PRES}

In 1996, Hinchey et al. were the first to describe a syndrome characterized by similar cliniconeuroradiological findings in a group of patients with different clinical settings (Hinchey et al., 1996). They suggested the term 'reversible posterior leukoencephalopathy syndrome' (RPLS) given the reversibility of the abnormalities and the typical localization of white-matter lesions in the posterior occipital-parietal regions of the brain.

Afterwards, many critical remarks were postulated on the term RPLS. First of all, some case reports described the development of intracranial hemorrhage as complication with subsequent permanent sequellae or even fatal outcome, suggesting that not all RPLS are 'reversible' (Schwartz, 1996). Secondly, although in most patients the posterior regions were involved, other brain regions were also often affected. Thirdly, the term leukoencephalopathy is too restrictive since the white-matter lesions were frequently accompanied with cortical involvement. 
Since then, many other therms were proposed like 'hypertension encephalopathy' (HTE), 'occipito-parietal encephalopathy' (Pavlakis et al., 1997) or 'potentially reversible encephalopathy syndrome' (Narbone et al., 2006). Casey et al. first used the term 'posterior reversible encephalopathy syndrome' or PRES (Casey et al., 2000). Although 'posterior' and 'reversible' are still present, the term PRES became generally accepted and systematically used. A possible explanation for this acceptance is that in time, it became apparent that with a prompt diagnosis and treatment, PRES is reversible in almost every patient. In addition, using the term 'posterior' does not exclude other regions to be involved but merely depicts the fact that occipito-parietal abnormalities are the most common feature on neuro-imaging.

\subsubsection{Clinical presentation}

PRES normally emerges gradually over a few days but can also have an acute, sudden onset. Mild to severe hypertension is a common feature in 70 to $80 \%$ of patients with PRES. Other typical clinical symptoms include headache, nausea/vomiting, seizures and visual disturbances (table 5). Seizures may start focally and evolve into generalized attacks or even status epilepticus. Quite often, seizures are the first presenting symptom of PRES. Many forms of visual changes have been reported ranging from a blurred vision to complete cortical blindness.

Next to the typical symptoms, many patients with PRES suffer from an altered mental status with confusion and diminished concentrating ability. Lethargy is common, sometimes worsening into stupor or even coma. Other neurological aberrations, like dysarthria and ataxia, have also been associated with PRES.

\begin{tabular}{|l|}
\hline Clinical symptoms of PRES \\
\hline Typical: \\
Hypertension \\
Headache \\
Nausea/vomiting \\
Seizures \\
Focal \\
Tonic-clonic/generalized \\
Status epilepticus \\
Visual disturbances \\
$\quad$ Blurred vision \\
$\quad$ Hemianopsy \\
$\quad$ Visual neglect \\
Cortical blindness \\
\\
Other: \\
Confusion \\
Attention deficit \\
Lethargy \\
Stupor \\
Coma \\
Ataxia \\
Dysarthria
\end{tabular}

Table 5. Clinical symptoms of PRES 


\subsubsection{Etiology}

The name PRES was given to common cliniconeuroradiological findings in patients with very divergent underlying pathologies as summarized in table 6. All these clinical conditions induce a form of 'systemic toxicity', making the patient vulnerable for developing PRES.

With regard to cancer treatment, PRES was mostly seen after the administration of highdose (combination) chemotherapy for hematologic malignancies. However, PRES has also been reported in patients treated with a single chemotherapeutic agent like cisplatin, gemcitabine or cytarabine. Moreover, the newer 'targeted therapies' like the monoclonal antibody bevacizumab and the tyrosine kinase inhibitors sorafenib and vandetanib have recently been linked with the development of PRES (Broniscer et al., 2010; Glusker et al., 2006; Govindarajan et al., 2006).

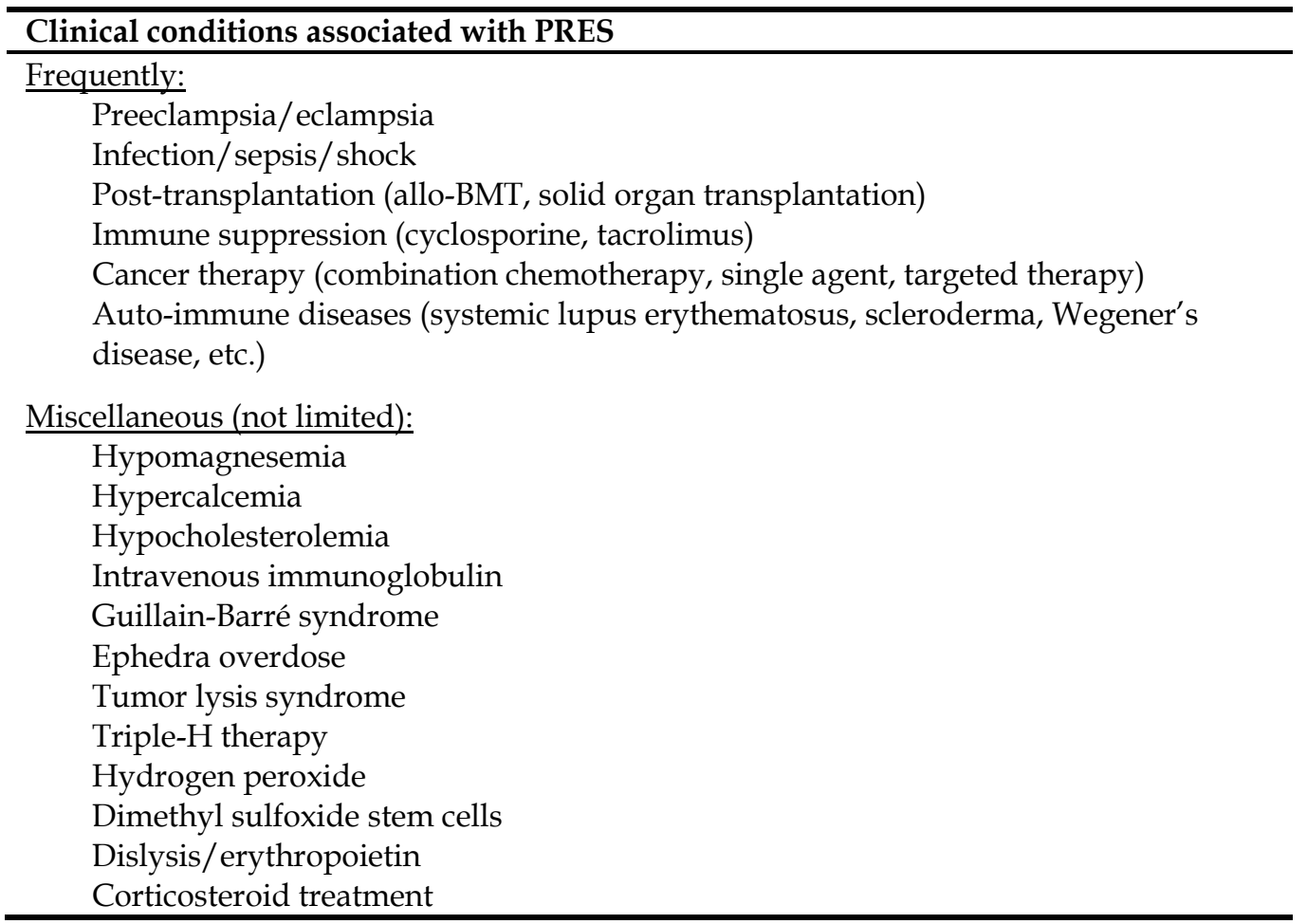

Table 6. Clinical conditions associated with PRES

\subsubsection{Pathophysiology}

The pathophysiological mechanism behind PRES still remains a matter of debate.

When PRES was initially described, the hypodense parietal-occipital regions on CT imaging prompted investigators to suggest that ischemia was the causative factor of the observed abnormalities. The hypothesis was that the 'systemic disease' of the patient elevates the blood pressure which is counteracted by an auto-regulatory vasoconstriction in the brain, leading to reduced blood flow, ischemia and subsequent vasogenic edema. 
As more cases were described and more research was performed, a new hypothesis was proposed based upon the hypertension/hyperperfusion theory. This hypothesis stated that PRES was the result of acute and severe hypertension beyond the autoregulatory limits, leading to a breakthrough of the blood-brain barrier, hyperperfusion, capillary bed injury, fluid leakage and vasogenic edema (Bartynski, 2008b, Staykov and Schwab, 2011). Different animal studies supporting the hypertension/hyperperfusion theory and the success of immediate anti-hypertensive treatment in clinical setting strengthened the belief in this more recent hypothesis.

However, several remarks have been posted questioning the reliability of this mechanism. For instance, hypertension is not or only mildly present in about $25 \%$ of patients with PRES, certainly not reaching the upper limits of autoregulation (Bartynski, 2008b, Staykov and Schwab, 2011). Secondly, solid evidence of hyperperfusion is scarce. Thirdly, the results from animal studies can not merely be extrapolated to humans. In patients with PRES, hypertension is induced by a 'systemic process/disease' while in animal studies hypertension is induced in healthy animals (Bartynski, 2008b and Staykov, 2011). At last, more advanced imaging techniques like cerebral angiography and MR angiography showed unexpectedly an inverse relation between the severity of hypertension and the extent of brain edema (Bartynski and Boardman, 2008).

In addition, as our knowledge of PRES expands, new insights in the pathophysiological mechanisms arise, pointing towards the older vasoconstriction-hypoperfusion theory. For example, all the well-known underlying pathologies (table 6) show activation of the cellular immune system, production of inflammatory cytokines and endothelial activation and injury, leading to a vasculopathy with vasoconstriction, hypoperfusion and subsequent ischemia as possible outcome (Bartynski, 2008b). It is clear that more investigations in this area are needed to elucidate this complex subject.

\subsubsection{Diagnosis}

The diagnosis of PRES is mainly based upon the combination of typical clinical symptoms and specific abnormalities on brain imaging.

A laboratory work-out can give an indirect hint towards PRES by detecting thrombocytopenia, schistocytes and an increased serum lactate dehydrogenase (LDH) caused by endothelial injury which almost always accompanies PRES (Bartynski, 2008a; Staykov and Schwab, 2011).

Affected brain regions can be recognized by a hypodense signal on CT-scan but an MRI of the brain with fluid-attenuated inversion recovery (FLAIR) is the gold standard for the diagnosis of PRES because of its higher accuracy in detecting all affected areas (Casey et al., 2000). A pattern of symmetrical edema of the parietal-occipital regions is most frequently seen on neuro-imaging (Picture 1), but involvement of the frontal lobes, basal ganglia, brain stem and cerebellum have also been reported (Bartynski, 2008a; Hinchey et al., 1996; Staykov and Schwab, 2011).

Focal aberrations can be present along with the symmetrical edema but in absence of the latter, the diagnosis can be quite difficult. Three major patterns of PRES were proposed by Bartynski, namely a holohemispheric watershed pattern, a superior frontal sulcus pattern 
and a dominant parietal-occipital pattern. These three typical patterns were seen in 99 of 136 patients with PRES. The other patients showed a partial or asymmetric variation of one of these major patterns (Bartynski and Boardman, 2007). Awareness of these different patterns is of great importance for a quick diagnosis and treatment, subsequently leading towards a better outcome.
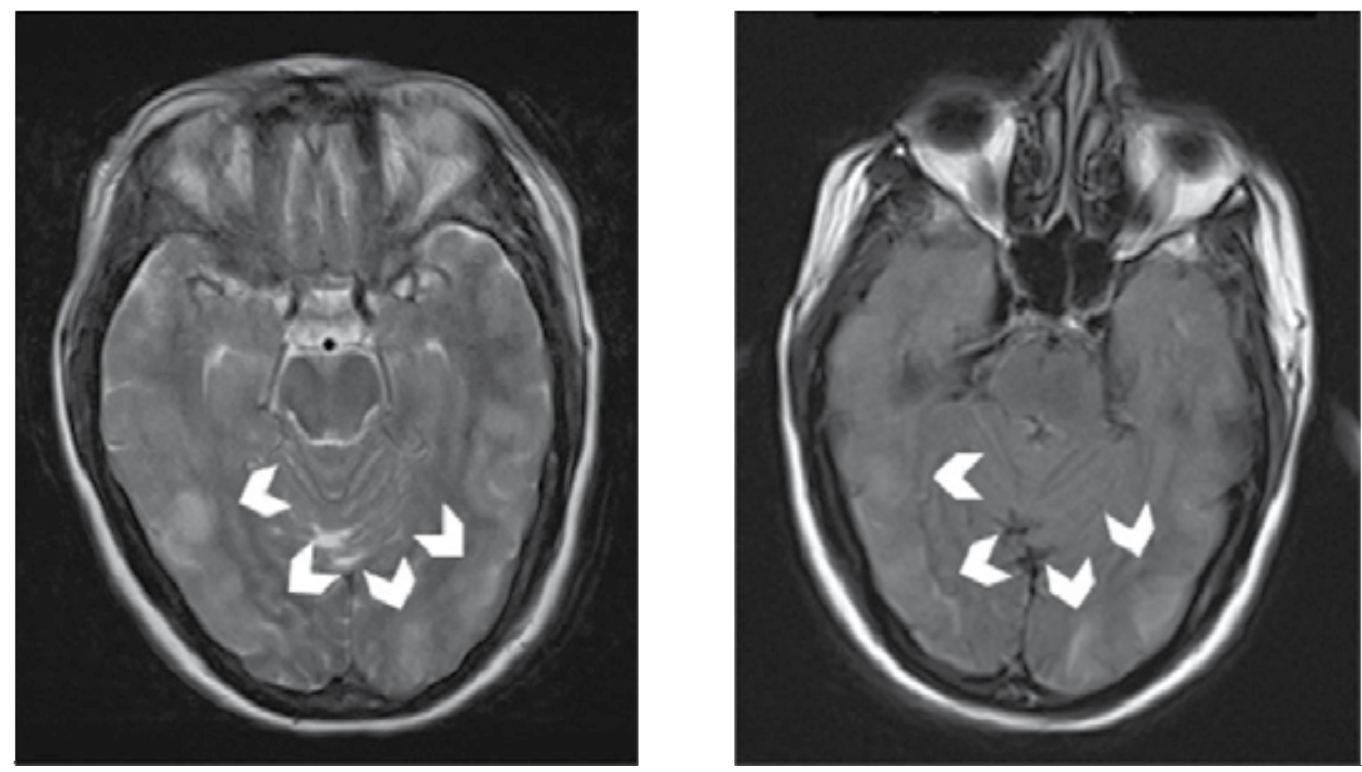

Picture 1. An example of NMR imaging in PRES: hyperintense signal (white arrowheads) on T2-weighted (left) and FLAIR (right) images of symmetrical affected parieto-occipital regions.

MR diffusion weighted imaging (DWI) with calculation of the apparent diffusion coefficient (ADC) may have an additional value in distinguishing a vasogenic edema (in PRES) from a cytotoxic edema (infarction), especially given the differences in treatment strategies (Doelken, 2007).

Cerebral angiography (CA) and MR angiography (MRA) commonly show signs of diffuse or focal vasoconstriction (with sometimes a typical string-of-beads appearance) and vasculopathy (Bartynski and Boardman, 2008). MR perfusion mostly reveals a reduced blood flow in the affected areas (Bartynski, 2008a; Brubaker et al., 2005).

MR spectroscopy describes reduced $\mathrm{N}$-acetylaspartate:choline and $\mathrm{N}$-acetylaspartate: creatinine ratios suggesting neuronal, axonal or synaptic dysfunction (Bartynski, 2008a; Pavlakis, 1997; Staykov and Schwab, 2011). The presence of lactate in PRES accompanied by vasoconstriction is suggestive for ischemia (Bartynski, 2008a; Staykov and Schwab, 2011). It is clear that information and results obtained from the more recent and advanced imaging techniques like CA, MRA, MR perfusion and others are more in favor of the old vasoconstriction/hypoperfusion theory. 


\subsubsection{Treatment}

Patients with PRES should be treated in a (neuro)critical care unit with close hemodynamic monitoring and the opportunity for intubation and ventilation in case of severe decrease in mental state. Cornerstone in the treatment of PRES is removing the causative pathology or drug. Specifically in cancer, the responsible chemotherapeutic agent(s) or targeted therapy should be reduced or even completely stopped when possible. About $75 \%$ of patients will present with a mild to severe elevated blood pressure which needs to be corrected with antihypertensive drugs. Nitroglycerine however should be avoided as it has been reported to aggravate the cerebral edema in PRES (Finsterer, 2003, as cited in Staykov and Schwab, 2011). In case of seizures or status epilepticus, benzodiazepines are given in the acute phase followed by long-term anti-convulsive therapy. Controversy remains about the duration of the anti-convulsive therapy. In some cases the treatment was successfully stopped after a few days, others continued the drugs for a couple of months. Seizures in pregnant women with eclampsia can be treated with magnesium sulfate.

\subsubsection{Prognosis}

As can be derived from the ' $R$ ' in the term PRES, this syndrome is usually completely reversible in a few days to weeks. A prompt diagnosis and correct treatment are therefore imperative. Sequellae however can arise, even with adequate treatment. Ischemia, parenchymal hemorrhage and epilepsy are possible complications of PRES with long-term consequences and a detrimental effect on quality of life (Staykov and Schwab, 2011).

Reoccurence of PRES has been reported anecdotally (Sweany et al., 2007). In cancer patients the question remains if the causative drug can safely be reintroduced after recovery from PRES. Many case reports describe a successful completion of the planned treatment scheme suggesting that the cancer treatment is an evoking but not sole causative factor in cancer treatment related PRES.

\subsection{Progressive multifocal leukoencephalopathy (PML)}

\subsubsection{Introduction}

Progressive multifocal leukoencephalopathy is a demyelinating disease of the white matter of the human brain. In 1958, Astrom et al. were the first to utter the term progressive multifocal leukoencephalopathy after their experience of similar clinical, neurological and pathological findings in two patients with chronic lymphocytic leukemia and one patient with Hodgkin's lymphoma (Astrom et al., 1958). At that time, the underlying cause of the disease was unknown. A few years later, electron microscopy of the brain lesions associated with PML revealed intranuclear particles in the oligodendrocytes which were later identified as virions derived from a papovavirus.

Eventually, more than ten years after the first description of PML, Padgett et al. were able to culture the virus out of a brain lesion in human fetal glial cells (Padgett et al., 1971). They named it the JC virus (JCV) according to the initials of the donor patient and it was later characterized as a human polyomavirus.

More recently, next to PML, other neurological disorders have been associated with JCV, namely JC virus granule cell neuronopathy, JC virus encephalopathy and JC virus 
meningitis (Tan and Koralnik, 2010). JCV is even thought to play a role in different types of brain tumors, gastro-intestinal cancers and lung cancer, although its contribution to the development of these cancers remains unraveled.

\subsubsection{Clinical manifestation}

The brain lesions associated with PML are asymmetric and variable in size, subsequently leading to a variety of possible clinical symptoms. In contrast with PRES, PML is characterized by a subacute onset of symptoms, gradually progressing over weeks to months.

Signs of subcortical dementia with cognitive impairment are a common feature and often the first symptoms indicating the development of PML (Major, 2010). Motor dysfunctions often accompany the cognitive problems and can range from walking difficulties and deranged coordination to ataxia and hemiparesis (Major, 2010; Brew et al., 2010). Visual changes are also frequently reported with hemianopsia being the most prevalent one. Seizures have been described in about twenty percent of PML cases and can be expected if the lesions are in close contact with the cortex (Brew et al., 2010).

Other possible symptoms are headaches, dysartria, aphasia and abulia (Lima et al., 2010).

As the disease progresses, the patient gradually develops global impairment eventually leading to death if treatment fails.

\subsubsection{Etiology}

Infection with the JC virus is imperative for the possible development of PML but the JC virus on its own is not enough to cause this neurological disorder. The presence of a predisposing immunocompromising condition is also an absolute necessity.

Until the 1980's, PML was a rare neuropathological disease mostly associated with hematological malignancies and systemic inflammatory disorders (Brew et al., 2010). With the outbreak of the AIDS pandemia in the mid-1980's, the incidence of PML rose spectacularly to a 50-fold increase. AIDS became the most important predisposing factor for developing PML, being present in about 3\% of HIV-1 positive individuals (Major, 2010).

More recently, new case reports of PML arose from a rather unexpected corner, namely the treatment of patients with immunomodulatory drugs, with the monoclonal antibodies natalizumab, efalizumab and rituximab as best described entities. The first cases of PML were seen in 2005 in different phase III trials where patients received natalizumab for the treatment of multiple sclerosis (MS) or Chron's disease (Kleinschmidt-DeMasters and Tyler, 2005; Langer-Gould et al., 2005 and Van Assche et al., 2005 as cited in Berger et al., 2009 and Weissert, 2011). Diagnoses of PML in patients treated with efalizumab for psoriasis or with rituximab for lymphoproliferative disorders followed but to a lesser extent than natalizumab.

Each monoclonal antibody has its specific immunomodulatory characteristics (Berger et al., 2009) possibly providing the reason why some monoclonal antibodies are more frequently associated with PML than others. In addition, the disorder requiring immunomodulatory therapy can also be a predisposing condition for developing PML making it difficult to 
assess the real influence of the therapy on the incidence of PML. For example, rituximab is used for the treatment of lymphoproliferative disorders and both are linked with PML.

Next to monoclonal antibodies, several anti-neoplastic and immunosuppressive therapies like methotrexate, cyclophosphamide, carboplatinum/gemcitabine, fludarabine and mycophenolate mofetil have been reported as causative agents for PML (Brew et al., 2010; Palmieri et al., 2010).

\subsubsection{Pathophysiology}

The pathophysiological mechanism behind PML is complicated and until now not fully elucidated. However, to some degree there is a consensus about the major steps that need to be fulfilled for the development of PML (table 7). These steps include: primary infection with JCV, period of latent infection, reactivation of JCV and eventually invasion into the brain (Brew et al., 2010; Major, 2010; White and Khalili, 2011).

Primary infection:

A primary infection with the JC virus is indispensable for the possible development of PML. Primary infection occurs mainly in the childhood but continues into middle age. JCV is a hemagglutinating virus and hemagglutination inhibition assays were able to detect JCV antibodies in 50-90\% of adults (Taguchi et al., 1982 and Walker and Padgett, 1983 as cited in White and Khalili, 2011). The route of transmission is believed to be inhalation of the virus with infection of the tonsils or ingestion of contaminated water or food and uptake of the virus by the gastro-intestinal tract. A primary infection usually occurs without symptoms while some organs stay behind with latent infection.

Period of latency:

Based upon detection of JCV in urine and the presence of JCV DNA in tissue of PML and non-PML patients, at least 3 organs are believed to harbor latent infection: the kidney, the bone marrow and lymphoid tissues like the tonsils, spleen, B-lymphocytes and CD 34+ hematopoetic cells (Major, 2010; White and Khalili, 2011).

Molecular and genetic characterization of JCV found in these tissues and cells led to the discovery of two different types, namely archetype JCV and prototype JCV. The difference between these two types is the appearance of rearrangements in the non-coding control region (NCCR) of the virus leading to a neurotropic prototype JCV (Berger et al., 2009).

Based upon these findings, following hypothesis was put forward: archetypal JCV is responsible for the primary infection, possesses a normal NCCR and is not able to infect human glial cells. During latency, rearrangements in the NCCR occur leading to the more aggressive, neurotropic prototype JCV. This hypothesis was supported by the intriguing observation of possible latency in B-cells, possessing all the necessary machinery for gene rearrangement (Berger et al, 2009). Moreover, the fact that PML remains a rare entity despite the high prevalence of JCV-infection points towards the necessity of this genetic alteration.

Of note, controversy remains regarding the brain as possible site of latency. Although prototype JCV-DNA has been found in normal non-PML brain tissue (White and Khalili, 2011), some investigators question the possibility of the brain as a functional latency site 
given the rarity of PML and the unlikeliness of establishing a long-term latency without inflammatory consequences during viral clearance (Major, 2010).

Reactivation of JCV:

The most popular theory is reactivation of JCV in peripheral blood mononuclear cells causing hematogenous dissemination of the virus across the body with B-lymphocytes having the ability to cross the blood-brain barrier (Berger et al., 2009; Brew et al, 2010; White and Khalili, 2011).

An alternative model was proposed by White and Khalili where extracellular cytokines cause a reactivation of latent JCV in the brain (White and Khalili, 2011). In both models, reactivation and active replication of JCV is only possible in light of a reduction in immunosurveillance (as described in partim etiology).

Invasion of the brain:

Regardless of the 2 different models, eventually, activated neurotropic JC virus is present in the brain and has the capability of infecting oligodendrocytes and astrocytes, leading to cell lysis and further spreading of the virus to adjacent cells. Lysis of oligodendrocytes causes demyelination, a process responsible for the typical clinical and neuroradiological features of PML.

\section{Proposed stages of PML development}

Primary infection

Initial JCV infection, mainly in childhood or adulthood, through inhalation (tonsils) or ingestion of contaminated food or water (gastro-intestinal system)

\section{Latent infection}

Establishment of latent infection in kidneys, bone marrow and lymphoid tissues (brain as functional site of latent infection remains controversial)

Rearrangement in the non-coding control region of JCV (within B-cells) leading to a neurotropic JCV strain

\section{Reactivation}

Failed immunosurveillance in the periphery

Reactivation of JCV in PBMCs with hematogenous dissemination through the whole body and crossing the blood-brain barrier via B-cells

\section{Brain infection}

Entry of neurotropic JCV into the brain (via B-cells)

Establishment of productive infection of oligodendrocytes

Failed immunosurveillance in the brain

Table 7. Proposed stages of PML development

\subsubsection{Diagnosis}

The diagnosis of PML is based on typical clinical manifestations, specific neuroradiological findings and detection of JCV-DNA in the cerebrospinal fluid (CSF). 
A brain CT scan shows low-density lesions in the subcortical white matter with no mass effect and no contrast enhancement. MRI of the brain has proven its superiority to CT scanning in documenting the number and extent of the lesions (Post MJ et al., 1986 as cited in Major et al., 1992) and in detecting the lesions in the initial stages of the disease (Shah et al., 2010).

PML mainly presents itself on MRI as single or asymmetric multifocal white matter lesions, enlarging and becoming confluent as the disease progresses. The lesions are typically hypointense on T1- and hyperintense on T2-weighted and FLAIR images compared to the normal white matter (Picture 2) (Shah et al., 2010). There usually is no mass effect, no edema and no gadolinium enhancement. However, gadolinium enhancement is frequently seen in natalizumab treated patients as documented in $43 \%$ of cases (Calabrese et al., 2007 and Clifford et al., 2010 as cited in Brew et al., 2010). Involvement of the parieto-occipital white matter, corpus callosum and posterior fossa is commonly seen while lesions restricted to the cerebellum, brainstem or pyramidal tract are also possible. Involvement of the spinal cord is rare, certainly in the beginning of PML, and when present alternative causes should be considered first. Although PML is known for its white matter lesions, involvement of the grey matter has been detected in up to $50 \%$ of patients with PML (Mark AS and Atlas SW, 1989 as cited in Shah et al., 2010).
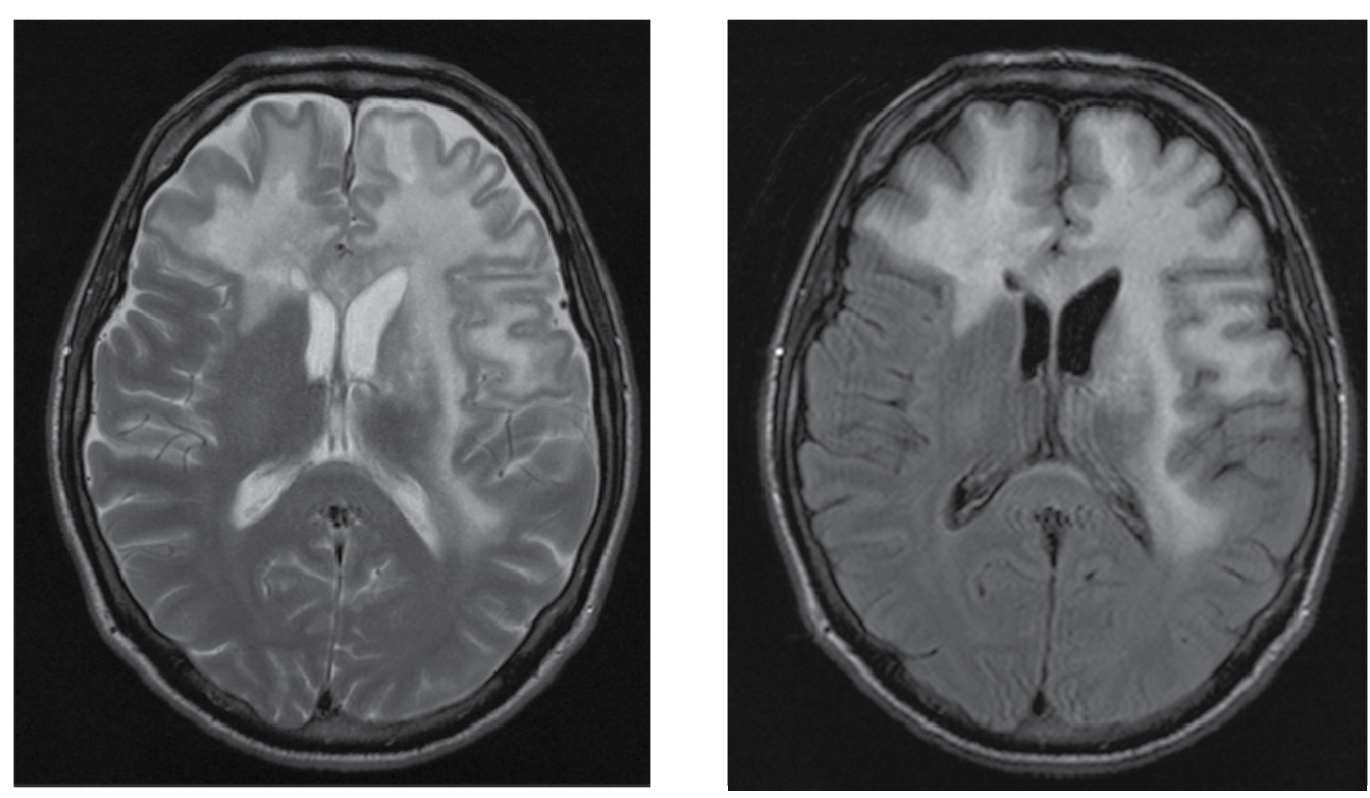

Picture 2. An example of NMR imaging in PML: hyperintens signal on T2-weighted (left) and FLAIR (right) images indicating asymmetric white matter lesions.

DWI MRI with ADC mapping provides additional information for characterizing the detected aberrations on MRI. A high signal on DWI with a low ADC value indicates regions with active infection as seen in new lesions and at the border of larger lesions while the center of these larger lesions usually have elevated ADC values (Brew et al., 2010 and Shah et al., 2010). 
MR spectroscopy in PML may reveal a diminished $\mathrm{N}$-acetylaspartate linked with neuronal loss and dysfunction, increased choline indicating demyelination, high lactate and lipid levels correlated with necrosis, and a temporary increase of myoinositol in evolving lesions (Brew et al., 2010 and Shah et al., 2010). None of these findings, however, are specific for PML and therefore can not be used for definitive diagnostic purposes.

Detection of JCV-DNA in CSF by PCR amplification is imperative for diagnosing PML. However, negative results do not simply exclude PML as JCV-DNA can still be undetectable in the beginning of the disease course. Moreover, the quality of JCV-DNA testing can variate substantially between different testing facilities. In case of a negative result and strong clinical and imaging evidence, a repeat lumbar puncture is recommended. If JCV-DNA remains undetected in the CSF, a stereotatic brain biopsy can be helpful. Although this is a highly invasive technique, it may often provide the necessary information for a correct diagnosis.

Histopathological features of PML are areas of demyelination. These lesions contain a moderate number of foamy astrocytes and only scant perivascular lymphocytes. Lymphocytic infiltration may be more common in HIV-positive PML and may be associated with a slightly better prognosis. A striking feature, particularly in older lesions, is the presence of very large astrocytes with bizarre, pleiomorphic nuclei. Viral inclusions are seen in the periphery of the foci of demyelination and are localized in the enlarged nuclei of oligodendrocytes (Brew et al., 2010; Ellison et al., 2004). JCV-DNA can be detected by in situ hybridization. Immunohistochemistry is often used to confirm diagnosis by revealing the presence of JCV antigen (Picture 3) (Brew et al., 2010; Major et al., 1992).
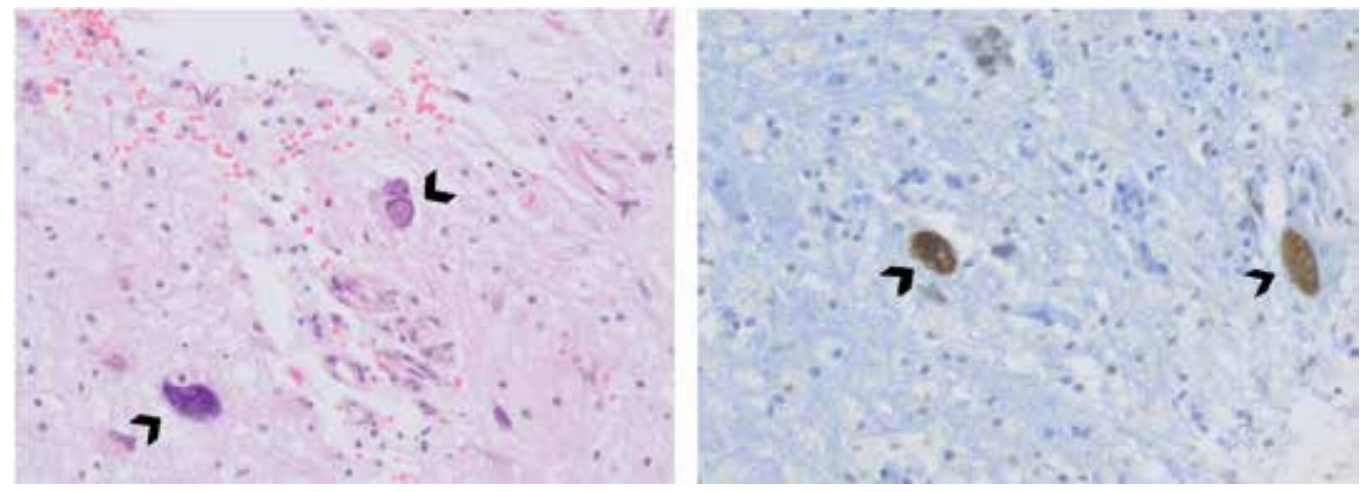

Picture 3. Hematoxylin-eosin stain (left) in a case of PML (magnification 200x) reveals foci of demyelination with macrophages and scattered glial cells showing hyperchromatic and atypical nuclei (arrows), sometimes with ground glass appearance. The diagnosis of PML is confirmed by immunostain for JC virus (right) in the affected nuclei (arrows).

\subsubsection{Treatment}

Although different agents with antiviral activity have been tried, there is no adequate treatment for PML as we speak.

Cytarabine was able to inhibit JCV replication in vitro but failed to show improvement of survival in randomized controlled trials (Hall et al., 1998 as cited in Brew et al., 2010). 
Cidofovir, an antiviral agent against cytomegalovirus infection, was tested in different trials with HIV-positive PML patients and showed contradictory results in terms of survival or PML-related residual disability (Tan and Koralnik, 2011).

The discovery that JCV binds the 5- $\mathrm{HT}_{2 \mathrm{~A}}$ receptor before infecting the cell led to the use of mirtazapine (a serotonin receptor blocker) as possible treatment for PML. However, the positive results from case reports have not been consolidated by large studies (Brew et al, 2010; Tan and Koralnik, 2011).

More recently, promising in vitro data were reported using the anti-malaria drug mefloquine. This led to the set up of a momentarily ongoing large clinical trial evaluating the use of mefloquine in the treatment of PML (clinicaltrials.gov, 2009).

With the lack of an effective antiviral agent the treatment of PML is mainly focused on improvement of the impaired immune status of the host. For cancer patients, this usually means reducing or even stopping the immunosuppressive agents allowing the immune system to recover with, hopefully, subsequent containment of the JCV infection.

In case of immunomodulatory monoclonal antibody associated PML, some specific characteristics should be considered. For example, natalizumab has a half-life of 11 days in the blood and even after immediate cessation of the drug, it still has biological activity for 3 months during which PML can further progress (Tan and Koralnik, 2011). Plasma exchange for quick removal of serum natalizumab has been tried but exposes the patient to a high risk of developing immune reconstitution inflammatory syndrome (IRIS) with possible fatal outcome (described in partim prognosis). Rituximab on the other hand, induces a long-term decrease in B-cells and has already cleared the circulation long before PML occurs. Thus, immediate cessation of the drug or plasma exchange are of no use in this situation.

Better understanding of the specific role of the different monoclonal antibodies in JCV reactivation and more knowledge regarding the development of IRIS during immune reconstitution will be necessary for a better management of immunomodulatory monoclonal antibody associated PML (Tan and Koralnik, 2011).

\subsubsection{Prognosis}

PML is a deleterious disease with mortality rates up to $90 \%$ within a few months when left untreated. However, if the immune response can be restored, survival significantly improves. A clinical neurologic recovery, contrast enhancement of PML lesions on neuroimaging, high CD4+ T-lymphocyte count at disease onset and the appearance of JCVspecific CD8+ cytotoxic T-lymphocytes in the blood within three months of the diagnosis are considered positive prognostic factors (Berger et al., 1998; Marzocchetti et al., 2009). A high JCV-DNA load in CSF is considered a determinant of poor survival.

The outcome in long-term survivors of PML can range from complete recovery to sustained severe neurologic disabilities requiring institutionalization (Lima et al., 2010). Patients with small PML-lesions and a short symptom-to-diagnosis time appear to have a better outcome while involvement of the cerebellum or brainstem may indicate a worse prognosis (Brew et al., 2010; Lima et al., 2010; Vermersch et al., 2011).

Although reconstitution of the immune system is a key factor for a better survival in PML, it can be accompanied by an acute inflammatory reaction called immune reconstitution 
inflammatory syndrome or IRIS, marked by a transient worsening of the neurological symptoms and contrast enhancement of the PML lesions on brain imaging. In severe cases of IRIS, edema with mass effect and brain herniation may occur with possible fatal outcome.

This might give IRIS a negative accent while in fact, development of IRIS in PML does not negatively influence PML survival and may even be potentially linked with prolonged survival and possible cure given the fact that IRIS actually reflects a (too) fast recovery of the immune system (Tan and Koralnik, 2011; Weissert, 2011).

Successful treatment of IRIS has been reported with high dose steroids to suppress the inflammation, although this remains controversial since these are also immunosuppressants with possible detrimental effects on the underlying immunocompromising diseases responsible for inducing PML in the first place (Tan and Koralnik, 2011).

\section{Conclusion}

Encephalopathy is a clinical syndrome, seen in many patients with underlying systemic diseases. In this chapter, we focused on uncommon clinical pictures presenting as an encephalopathy and discussed the causing pathophysiology.

As mentioned above, paraneoplastic neurological syndromes precede a diagnosis of malignancy in $60 \%$ of all cases, emphasizing that recognition of this syndrome may lead to early detection of an associated malignancy, with subsequent advantages in therapy response and prognosis.

Initially an auto-immune mediated pathophysiological mechanism was assumed, but the mechanisms of most paraneoplastic neurological syndromes could not be elucidated up till now. We hope that further research in this intriguing immune-neuro-oncological field will help understand the mechanisms of cancer and PNS and that manipulating the immune system can lead to tumour treatment and regression.

Although PRES and PML are rare diseases, recognizing these clinical entities can be of vital importance. Their prompt diagnosis and correct management not only positively influences the clinical outcome and survival but also reduces a possible decrease in the patients' quality of life which is already greatly impaired due to the seriousness of their underlying diseases. For both PRES and PML, predictive markers and a better understanding of the pathophysiological mechanisms could be useful in terms of prevention and more adequate treatment. Further research comprising these topics is ongoing.

With the fast changing world of cancer therapies, especially the targeted therapies, more reports of PRES and PML are expected but also new encephalopathies can arise. It is our responsibility to closely monitor patients during and after cancer treatment and to be vigilant for unexpected adverse events.

\section{Acknowledgements}

The authors would like to thank Dr. Caroline Van Den Broecke for kindly providing the anatomopathological images of PML. 


\section{References}

Astrom, K.E.; Mancall, E. \& Richardson, E.P. (1958). Progressive multifocal leukoencephalopathy; a hitherto unrecognized complication of chronic lymphatic leukemia and Hodgkin's disease. Brain,Vol. 81, No. 1, (March 1958), pp. 93-111

Bartynski, W.S. \& Boardman, J.F. (2007). Distinct Imaging Patterns and Lesion Distribution in Posterior Reversible Encephalopathy Syndrome. American Journal of Neuroradiology, Vol. 28, No. 7, (August 2007), pp. 1320-7

Bartynski, W.S. \& Boardman, J.F. (2008). Catheter Angiography, MR Angiography, and MR Perfusion in Posterior Reversible Encephalopathy Syndrome. American Journal of Neuroradiology, Vol. 29, No. 3, (March 2008), pp. 447-55

Bartynski, W.S. (2008a). Posterior Reversible Encephalopathy Syndrome, Part 1: Fundamental Imaging and Clinical features. American Journal of Neuroradiology, Vol. 29, No. 6, (June-July 2008), pp. 1036-42

Bartynski, W.S. (2008b). Posterior Reversible Encephalopathy Syndrome, Part 2: Controversies surrounding Pathophysiology of Vasogenic Edema. American Journal of Neuroradiology, Vol. 29, No. 6, (June-July 2008), pp. 1043-49

Berger, J.R.; Levy, R.M.; Flomenhoft, D. \& Dobbs, M. (1998). Predictive factors for prolonged survival in acquired immunodeficiency syndrome-associated progressive multifocal leukoencephalopathy. Annals of Neurology, Vol. 44, No. 3, (September 1998), pp. 341-9

Berger, J.R.; Houff, S.A. \& Major, E.O. (2009). Monoclonal antibodies and progressive multifocal leukoencephalopathy. MAbs, Vol. 1, No. 6, (November-December 2009), pp. 583-9

Brew, B.J.; Davies, N.W.; Cinque, P.; Clifford, D.B. \& Nath, A. (2010). Progressive multifocal leukoencephalopathy and other forms of JC virus disease. Nature Reviews Neurology, Vol. 6, No. 12, (December 2010), pp. 667-79

Broniscer, A.; Baker, J.N.; Tagen, M.; Onar-Thomas, A.; Gilbertson, R.J.; Davidoff, A.M.; Pai Panandiker, A.S.; Leung, W.; Chin, T.K.; Stewart, C.F.; Kocak, M.; Rowland, C.; Merchant, T.E.; Kaste, S.C. \& Gajjar, A. (2010). Phase I study of vandetanib during and after radiotherapy in children with diffuse intrinsic pontine glioma. Journal of Clinical Oncology, Vol. 28, No. 31, (November 2010), pp. 4762-8

Brubaker, L.M.; Keith Smith, J.; Lee, Y.Z.; Lin, W. \& Castillo M. (2005). Hemodynamic and Permeability Changes in Posterior Reversible Encephalopathy Syndrome Measured by Dynamic Susceptibility Perfusion-Weighted MR Imaging. American Journal of Neuroradiology, Vol. 26, No. 4, (April 2005), pp. 825-30

Casey, S.O.; Sampaio, R.C.; Michel, E. \& Truwit, C.L. (2000). Posterior Reversible Encephalopathy Syndrome. American Journal of Neuroradiology, Vol. 21, No. 7, (August 2000), pp. 1199-206

Clinicaltrials.gov [online]. (2009). Study to explore the effect of mefloquine in subjects with progressive multifocal leukoencephalopathy. http://clinicaltrials.gov/ct2/show/NCT00746941

Dalmau, J.; Gilberto Gonzalez, R. \& Lerwill, M.F. (2007). Case 4-2007: A 56-Year-Old Woman with Rapidly Progressive Vertigo and Ataxia. New England Journal of Medicine, Vol. 356, No.6, (February 2007), pp. 612-620

Dalmau, J. \& Rosenfield, M.R. (2008). Paraneoplastic syndromes of the CNS. Lancet Neurology,Vol.7, (2008), pp. 327-340 
Darnell, R.B. \& Posner, J.B. (2003). Paraneoplastic syndromes involving the nervous system. New England Journal of Medicine, Vol. 349, No. 16, (October 2003), pp. 1543-54

Doelken, M.; Lanz, S.; Rennert, J.; Alibek, S.; Richter, G. \& Doerfler, A. (2007). Differentiation of cytotoxic and vasogenic edema in a patient with reversible posterior leukoencephalopathy syndrome using diffusion-weighted MRI. Diagnostic and Interventional Radiology, Vol. 13, No. 3, (September 2007), pp. 125-8

Ellison, D.; Love, S.; Chimelli, L.; Harding, B.N.; Lowe, J. \& Vinters, H.V. (2004). Chapter 13: Chronic and subacute viral infections of the CNS, In: A reference text of CNS pathology, Houston, M.J., pp. 298-300, Elsevier Limited, ISBN 0723432392

Glusker, P.; Recht, L. \& Lane, B. (2006). Reversible posterior leukoencephalopathy syndrome and bevacizumab. New England Journal of Medicine, Vol. 354, No. 9, (March 2006), pp. 980-2

Govindarajan, R.; Adusumilli, J.; Baxter, D.L.; El-Khoueiry, A. \& Harik, S.I. (2006). Reversible posterior leukoencephalopathy syndrome induced by RAF kinase inhibitor BAY 43-9006. Journal of Clinical Oncology, Vol. 24, No. 28, (October 2006), pp. e48

Gozzard, P. \& Maddison, P. (2010). Which antibody and which cancer in which paraneoplastic syndromes? Practical Neurology, Vol. 10, (2010), pp. 260-270

Graus, F.; Delattre, J.Y.; Antoine, J.C.; Dalmau, J.; Giometto, B.; Grisold, W.; Honnorat, J.; Sillevis Smitt, P.; Vedeler, Ch.; Verschuuren, J.J.G.M.; Vincent, A. \& Voltz, R. for the Paraneoplastic Neurological Syndrome Euronetwork. (2004). Recommended diagnostic criteria for paraneoplastic neurological syndrome. Journal of Neurology, Neurosurgery and Psychiatry,Vol.75, (2004), pp. 1135-1140

Hinchey, J.; Chaves, C.; Appignani, B.; Breen, J.; Pao, L.; Wang, A.; Pessin, M.S.; Lamy, C.; Mas, J. \& Caplan, L.R. (1996). A Reversible Posterior Leukoencephalopathy Syndrome. New England Journal of Medicine, Vol. 334, No. 8, (february 1996), pp. 494-500

Kaltsas, G.; Androulakis, I.I.; de Herder, W.W. \& Grossman, A.B. (2010). Paraneoplastic syndromes secondary to neuroendocrine tumours. Endocrine-Related Cancer, Vol. 17, (2010), pp. R173-R193

Lapeire, L.D.; Tansens, A., Lemmens, G.M. \& Van Belle, S. (2010). Carcinoid encephalopathy: A single entity or a spectrum of different disorders? Acta Oncologica, Vol. 49, No. 2, (February 2010), pp. 268-70

Lima, M.A.; Bernal-Cano, F.; Clifford, D.B.; Gandhi, R.T. \& Koralnik, I.J. (2010). Clinical outcome of long-term survivors of progressive multifocal leukoencephalopathy. Journal of Neurology, Neurosurgery and Psychiatry, Vol. 81, No. 11, (November 2010), pp. $1288-91$

Major, E.O.; Amemiya, K.; Tornatore, C.S.; Houff, S.A. \& Berger, J.R. (1992). Pathogenesis and molecular biology of progressive multifocal leukoencephalopathy, the JC virus-induced demyelinating disease of the human brain. Clinical Microbiology Reviews, Vol. 5, No. 1, (January 1992), pp. 49-73

Major, E.O. (2010). Progressive multifocal leukoencephalopathy in patients on immunomodulatory therapies. Annual Review of Medicine, Vol. 61, pp. 35-47

Narbone, M.C.; Musolino, R.; Granata, F.; Mazzù, I.; Abbate, M. \& Ferlazzo, E. (2006). PRES : posterior or potentially reversible encephalopathy syndrome? Neurological Science, Vol. 27, No. 3, (July 2006), pp. 187-9 
Nath, U. \& Grant, R. (1997). Neurological Paraneoplastic Syndromes. Journal of Clinical Pathology,Vol. 50, (1997), pp. 975-980

Padgett, B.L.; Walker, D.L.; ZuRhein, G.M.; Eckroade, R.J. \& Dessel, B.H. (1971). Cultivation of papova-like virus from human brain with progressive multifocal leucoencephalopathy. Lancet, Vol. 1, No. 7712, (June 1971), pp. 1257-60

Palmieri, A.; Valentinis, L.; Bazzano, S.; Baldi, A.; Orlando, F.; Tenaglia, S. \& D'Anna, S. (2011). Progressive multifocal leukoencephalopathy following chemotherapy for lung cancer. Neurological Science, Vol. 32, No. 4, (August 2011), pp. 683-5

Pavlakis, S.G.; Frank Y.; Kalina, P.; Chandra M. \& Lu D.(1997). Occipital-Parietal Encephalopathy: A New Name for an Old Syndrome. Pediatric Neurology, Vol. 16, No. 2, (February 1997), pp. 145-8,

PNS Euronetwork. (2002). http://www.PNSeuronet.org

Schwartz, R.B. (1996). A Reversible Posterior Leukoencephalopathy syndrome. New England Journal of Medicine, Vol. 334, No. 26, (June 1996), pp. 1743

Shah, R.; Bag, A.K.; Chapman, P.R. \& Curé, J.K. (2010). Imaging manifestations of progressive multifocal leukoencephalopathy. Clinical Radiology, Vol. 65, No. 6, (June 2010), pp. 431-9

Staykov, D. and Schwab S. (2011). Posterior Reversible Encephalopathy Syndrome. Journal of Intensive Care Medicine, (February 2011), epub ahead of print

Sutton, I., \& Winer, B. (2002). The immunopathogenesis of paraneoplastic neurological syndromes. Clinical Science, Vol.102, (2002), pp.475-486

Sweany, J.M.; Bartynski, W.S. \& Boardman, J.F. (2007). “Recurrent" Posterior Reversible Encephalopathy Syndrome: Report of 3 Cases - PRES Can Strike Twice! Journal of Computer Assisted Tomography, Vol. 31, No. 1, (January-Februari 2007), pp. 148-56

Tan, C.S. \& Koralnik, I.J. (2010). Progressive multifocal leukoencephalopathy and other disorders caused by JC virus: clinical features and pathogenesis. Lancet Neurology, Vol. 9, No. 4, (April 2010), pp. 425-37

Titulaer, M.J.; Soffietti, R.; Dalmau, J.; Gilhus, N.E.; Giometto, B.; Graus, F.; Grisold, W.; Honnorat, J.; Sillevis Smitt, P.A.E.; Tanasescu, R.; Vedeler, C.A.; Voltz, R. and Verschuuren, J.J.G.M. (2011). Screening for tumours in paraneoplastic syndromes: report of an EFNS Task Force. European Journal of Neurology, Vol. 18, No. 1, (January 2011), 19-e3

Vermersch, P.; Kappos, L.; Gold, R.; Foley, J.F.; Olsson, T.; Cadavid, D.; Bozic, C. \& Richman, S. (2011). Clinical outcomes of natalizumab-associated progressive multifocal leukoencephalopathy. Neurology, Vol. 76, No. 20, (May 2011), pp. 1697-704

Voltz, R. (2002). Paraneoplastic neurological syndromes: an update on diagnosis, pathogenesis, and therapy. The Lancet Neurology,Vol.1, (September 2002), pp 294305

Weissert, R. (2011). Progressive multifocal leukoencephalopathy. Journal of Neuroimmunology, Vol. 231, No. 1-2, (February 2011), pp. 73-7

White, M.K. \& Khalili, K. Pathogenesis of progressive multifocal leukoencephalopathy-revisited. Journal of Infectious Diseases, Vol. 203, No. 5, (March 2011), pp. 578-86 


\title{
Hepatic Encephalopathy
}

\author{
Jeffrey E. Juneau and Brendan M. McGuire \\ University of Alabama at Birmingham, Department of Medicine, \\ Division of Gastroenterology and Hepatology \\ Birmingham, $A L$ \\ USA
}

\section{Introduction}

Hepatic encephalopathy (HE) is a neuropsychiatric condition affecting patients with cirrhosis, acute liver failure and portal-systemic bypass ${ }^{1,2}$, 3. The neuropsychiatric abnormalities include changes in levels of consciousness, behavior, intellect, and neurologic exam. The changes in levels of consciousness can range from altered sleep habits to coma. $\mathrm{HE}$ is a frequent complication in patients with cirrhosis and is broadly classified as minimal HE and overt HE. Minimal HE is defined as subclinical cognitive dysfunction, only detected by neuropsychiatric testing, and is present in up to $80 \%$ of patients with cirrhosis ${ }^{4}$. Overt HE is defined in patients with active HE or a previous episode of $\mathrm{HE}$ and is present in $30-45 \%$ of patients with cirrhosis ${ }^{5}$.

HE results from the overproduction or impaired metabolism of one or more neurotoxin. The general consensus is that ammonia with inflammation in the serum is believed to be the underlying trigger to developing HE. Normally, ammonia enters the portal system from the gut. When the liver is unable to clear ammonia, secondary to a limited number of viable hepatocytes or portal-systemic shunting, ammonia can enter the systemic circulation and enter brain tissue. Elevated ammonia with inflammation can impair normal neurotransmission and result in a variety of neuropsychiatric abnormalities ${ }^{6}$. The inflammatory component is usually related to bleeding, dehydration or sepsis ${ }^{4}$.

\subsection{Classification}

The most common classification used for research purposes is the West Haven Criteria (also called Conn Score) (table I-1) ${ }^{8}$. The West Haven Criteria is graded with a range of $0-4$. The far ends of the spectrum, grades 0 and 4, have fairly obvious clinical manifestations with no abnormalities and comatose, respectively 8,9 . However, the major criticism of the West Haven Criteria is deciphering the middle categories, 1-3, which can be difficult to reproduce due to the subjectivity involved with the assessment.

The World Congress of Gastroenterology developed a classification system for HE in 1998 based on underlying hepatic abnormalities. The three types are $\mathbf{A}, \mathbf{B}$, and $\mathbf{C}$ and are associated with Acute liver failure, portal systemic Bypass without intrinsic liver disease, and Cirrhosis, respectively 2,3,10. Type $C$ can be subcategorized into persistent, episodic, and 
minimal HE depending on symptoms and presentation. Persistent HE is subdivided into mild, severe, and treatment-dependent. Persistent HE presents with cognitive defects that negatively affect social and occupational functioning. The differences between mild and severe persistent HE depend on the West Haven Criteria. Mild persistent HE is present when a patient has met the persistent HE definition as well as having West Haven grade one, whereas severe persistent HE is present with grade two, three, or four of the West Haven Criteria. Treatment-dependent persistent HE implies that overt symptoms develop when treatment stops ${ }^{10}$. Episodic HE is subdivided into recurrent, precipitated, and spontaneous. Episodic is essentially viewed as delirium secondary to a general medical condition, with delirium defined as a disturbance of consciousness that is accompanied by a change in cognition that cannot be accounted for by a pre-existing or evolving dementia. Recurrent episodic HE is defined as having had two or more episodes in one year. Precipitated episodic HE is caused by an identifiable precipitant which led to the HE episode. These precipitants are listed in Table P-1. Spontaneous episodic HE is diagnosed only if no precipitant can be identified and all other causes of mental status alteration have been ruled out. Minimal HE is diagnosed by specialized tests due to the subtlety of its presentation. The most commonly used clinical categorization of HE is minimal and overt HE. Overt HE includes both persistent and episodic HE. But neither the World Congress of Gastroenterology nor the minimal and overt HE Classification systems are scientific or precise enough to offer a clear idea of the patient's clinical condition.

Due to the inconsistencies between HE severity scores, Bajaj and Cordoba created the Spectrum of Neurocognitive Impairment in Cirrhosis (SONIC) to eliminate the phrasing of minimal, overt and normal, as well as the scoring system of West Haven Criteria7. In utilizing a spectrum, the physician can follow the patient's mental status on a day-to-day basis with standardization in place to allow all providers to understand the clinical course of the patient. Although this spectrum was created to eliminate other severity classifications, the use of both West Haven Criteria and minimal/overt HE descriptions is widely practiced. Other scoring systems have been used in small studies such as the HE Scoring Algorithm $(\mathrm{HESA})^{11}$ and clinical hepatic encephalopathy staging scale (CHESS) ${ }^{162}$ but they have not been tested in large, multicenter trials ${ }^{7}$.

\begin{tabular}{|l|l|l|l|}
\hline Stage & Consciousness & Intellect/behavior & Neurological findings \\
\hline 0 & Normal & Normal & Normal exam \\
\hline 1 & $\begin{array}{l}\text { Mild lack of } \\
\text { awareness }\end{array}$ & $\begin{array}{l}\text { Shortened attention span, } \\
\text { impaired addition/subtraction }\end{array}$ & Mild asterixis or tremor \\
\hline 2 & Lethargic & $\begin{array}{l}\text { Disoriented; inappropriate } \\
\text { behavior }\end{array}$ & $\begin{array}{l}\text { Obvious asterixis; slurred } \\
\text { speech }\end{array}$ \\
\hline 3 & Somnolent & $\begin{array}{l}\text { Gross disorientation; bizarre } \\
\text { behavior }\end{array}$ & $\begin{array}{l}\text { Muscular rigidity, clonus, } \\
\text { hyperreflexia }\end{array}$ \\
\hline 4 & Coma & Coma & Decerebrate posturing \\
\hline
\end{tabular}

Used with permission from Alimentary Pharmacology and Therapies "Modern management of Hepatic Encephalopathy" 2010.

Table I-1. West Haven Criteria for Hepatic Encephalopathy 


\section{Pathophysiology}

Due to the various etiologies of the liver dysfunction, the etiology of HE is not completely understood. Nevertheless, several mechanisms are known to be associated with the development of HE and elevated serum ammonia seems to be the spark along with other precipitating factors. Precipitating factors can be found in $80 \%$ of cases with overt $\mathrm{HE}^{12}$. These precipitants work by increasing the underlying inflammatory milieu, or altering the ammonia threshold, or a combination of the above. The precipitants are listed in the table below (Table P-1).

\begin{tabular}{|c|c|c|c|c|}
\hline Increased & \multicolumn{2}{|c|}{ Decreased toxin clearance } & \multirow{2}{*}{$\begin{array}{l}\begin{array}{l}\text { Altered } \\
\text { neurotransmission }\end{array} \\
\text { Benzodiazepines }\end{array}$} & \multirow{2}{*}{$\begin{array}{l}\text { Hepatocellular } \\
\text { damage }\end{array}$} \\
\hline $\begin{array}{l}\text { Excess dietary } \\
\text { protein }\end{array}$ & \multirow[t]{4}{*}{ Dehydration } & $\begin{array}{l}\text { Abdominal } \\
\text { paracentesis }\end{array}$ & & \\
\hline Constipation & & $\begin{array}{l}\text { Fluid } \\
\text { restriction }\end{array}$ & Psychoactive drugs & $\begin{array}{l}\text { Development of } \\
\text { hepatocellular } \\
\text { carcinoma }\end{array}$ \\
\hline GI bleed & & Diuresis & & \\
\hline $\begin{array}{l}\text { Blood } \\
\text { transfusion }\end{array}$ & & $\begin{array}{l}\text { Diarrhea } \\
\text { secondary to } \\
\text { laxatives }\end{array}$ & & \\
\hline \multirow[t]{2}{*}{ Azotemia } & \multirow[t]{2}{*}{ Hypotension } & Bleeding & & \\
\hline & & Vasodilation & & \\
\hline Infection & \multicolumn{2}{|l|}{ Anemia } & & \\
\hline Hypokalemia & \multicolumn{2}{|c|}{ Portal-systemic shunts } & & \\
\hline
\end{tabular}

Table P-1 Common precipitants of hepatic encephalopathy and the underlying mechanisms

\subsection{Ammonia}

The pathophysiology is based largely on the five organs involved in the ammonia metabolism - gut, muscles, kidneys, liver, and brain ${ }^{13}$. The gut, kidneys and muscles are involved in the production of ammonia into the blood system. In the gut, ammonia is mainly produced by urease producing gut flora, which cleaves protein enzymatically to ammonia (NH3), and then absorbed into the portal-system through the colon ${ }^{14}$. In addition, enterocytes contain glutaminase, which converts glutamine to glutamate and ammonia ${ }^{15}$. The kidneys also express glutaminase and are involved in ammonia production during acidbase balance and dehydration ${ }^{16}$. With intense exercise or seizure, ammonia is produced in the skeletal muscles ${ }^{14}$.

Normally, ammonia is metabolized to urea in the liver for renal clearance. Eighty to ninety percent of ammonia enters the urea cycle via the liver and is ultimately cleared via the kidneys. Ammonia clearance also occurs within the kidneys, skeletal muscles, and brain. Ten to twenty percent of ammonia is metabolized by the skeletal muscles and brain via glutamine synthetase. Glutamine synthetase is the enzyme responsible for the condensation of glutamate and ammonia to form glutamine. In patients with cirrhosis, muscle wasting is 
common, thus limiting the ability of skeletal muscles to clear ammonia. The kidney expresses glutamine synthetase and is capable of increasing ammonia metabolism and excretion ${ }^{17}$. The shunting of blood (physiologic or iatrogenic) away from the liver decreases the ammonia metabolism in the liver, and increases systemic circulatory ammonia levels 16,17 .

In the brain, astrocytes are the only brain cells that can metabolize ammonia into glutamine via glutamine synthetase ${ }^{18}$. Astrocytes have several functions including neurodevelopment, maintaining appropriate ion concentrations in extracellular space, and maintaining the blood brain barrier and synaptic transmission. They also have the power to regenerate, which allows them to aid in repairing neuronal tissue ${ }^{19}$. When ammonia levels are elevated in the brain, glutamine levels are elevated which leads to an influx of water and subsequent edema. With chronic exposure to ammonia as well as manganese, astrocytes can undergo a morphologic change to Alzheimer type 2 astrocytosis characterized by cell swelling, large nuclei, prominent nucleoli, marginated chromatin, and the inability of cells to function 20,21 , 22. The swelling of the astrocytes can cause cerebral edema and intracranial hypertension, which leads to global effects on the brain including agitation to seizures ${ }^{14,23-25}$. This cerebral edema has been demonstrated on MRI by reduced magnetization transfer ratios in patients with overt HE ${ }^{26}$. The dysfunction of the astrocytes can lead to permanent damage of the brain. The permanent neurologic damage was seen in patients who received a liver transplant after recurrent episodes of overt HE. The changes included decreased brain volume seen on MRI, loss of the N-acetylaspartate/Creatine-phosphocreatine neuronal marker, and gross motor dysfunction ${ }^{27}$. When acute elevation of arterial ammonia to levels greater than $200 \mathrm{Umol} / \mathrm{L}$ occurs, cerebral autoregulation is lost and cerebral edema and intracranial hypertension may result in herniation 24,25 .

\subsection{Inflammation}

High levels of ammonia do not cause the symptoms of HE alone; a spark has to be present inflammation. Inflammatory markers are elevated in patients with $\mathrm{HE}$ and they can affect the liver and neurologic functions. Inflammatory markers were present in a number of studies, and they are relevant in the presence of all types of HE. Inflammatory markers including C-reactive protein, white blood cell levels, and interleukin-6 are higher in patients with minimal $\mathrm{HE}$ than those without minimal $\mathrm{HE}^{28}$. In overt $\mathrm{HE}$, cytokines are released by the astrocytes in response to ammonia-related injury. Tissue necrosis factor (TNF), which arises during inflammation, stimulates more cytokine production as well as altering the blood brain barrier (BBB). The permeability of the BBB to ammonia increases with increasing severity of liver disease ${ }^{29}$. Over-active neutrophils have been associated with HE and exhibit excessive degranulation and release of reactive oxygen species (ROS). The cytokines increase permeability of ammonia to the BBB. Ammonia swells the astrocytes with osmotic stress, and oxidative and nitrosative stress may cause apoptosis ${ }^{30}$. Thus, ammonia and the inflammation alter the environment of the astrocytes and BBB in a synergistic fashion to create a spectrum of neurologic changes ${ }^{31}$.

\subsection{Amino acids}

An elevation in aromatic amino acids to branched chain amino acids (BCAA) ratio is present in a large portion of patients with cirrhosis and HE. Aromatic amino acids (AAA) are elevated relative to baseline in patients with HE. BCAA are utilized more in the presence of 
hyperammonemia to help in detoxification of ammonia by skeletal muscles. Chronic liver disease and portal-systemic shunts are characterized by reduced levels of BCAA ${ }^{32}$. Aromatic amino acids are associated with an altered BBB, due to the transportation of glutamine out of the astrocytes which brings AAA into the cells. The imbalance may inhibit normal neurotransmitters such as dopamine, serotonin, and norepinephrine 33,34 or hinder their synthesis ${ }^{35}$. This change in concentration has led to treatment options involving BCAA. However, clinical trials of specialized BCAA formulas have yielded mixed results ${ }^{36}$.

\subsection{Neurologic alterations}

Because of the uncertainty surrounding all of the events in HE, a great deal of substances have been observed to be elevated or reduced with HE episodes. GABA, translocator protein $18-\mathrm{kDa}$ (TSPO) receptors, endogenous benzodiazepine, glutamate receptors, serotonin, catecholamines, melatonin, and histamine, which all have a role in cognitive function, have been altered during HE.

The GABA-benzodiazepine neurotransmitter system has been implicated in HE pathogenesis on a number of levels. The GABA receptors were once thought to be a major factor in the development of HE; however the receptors have not been shown to be more numerous or active in patients with HE. In the clinical setting, patients with liver failure are more sensitive to the sedative effects of benzodiazepines than those without liver failure. In rat models, ROS and reactive nitrogen species (RNS) are enhanced in the presence of benzodiazepines ${ }^{30,37}$. Antagonists to GABA-benzodiazepine receptor complex administered to rats with mild to severe hepatic encephalopathy produced a transient improvement in clinical status ${ }^{38}$. Neurosteroids, which are metabolites synthesized by cholesterol or steroid precursors, have also been associated with the GABA-A receptors. Neurosteroids are positive allosteric modulators of GABA-A receptor, thus increasing the GABAergic tone. The increased GABAergic tone has been associated with the reduced oxygen requirements and subsequent reduced blood flow and metabolism in the brain ${ }^{39}$. Endogenous benzodiazepines, which have also been involved with the activation GABA-Aergic neurotransmission, have been identified with contributing to HE. The concentrations of these endogenous benzodiazepines have been directly correlated with the severity of $\mathrm{HE}$ in animal models, but the role in humans has not be completely elucidated ${ }^{40}$.

In patients with $\mathrm{HE}$, translocator protein 18-kDa (TSPO) (formerly known as peripheral-type benzodiazepine receptor (PTBR)) is upregulated in microglial cells that are activated by inflammation ${ }^{41}$. This receptor is responsible for increasing the neurosteroid concentrations that stimulates the GABAergic tone as described above. The TSPO is activated by increases in ammonia and manganese 40,42 . Ammonia's presence as an activator of TSPO seems appropriate. Manganese has a role as well. Serum manganese levels are not elevated in HE, but deposits in the basal ganglia have been seen on MRI. Manganese toxicity has similar clinical manifestations as HE, with the addition of Parkinsonian symptoms, which may or may not be present in $\mathrm{HE}^{21,22}$.

Glutamate receptors have been implicated mainly through the effects of the glutamate, ROS and RNS. As part of the "Trojan Horse Hypothesis," ammonia is thought to induce oxidation when produced from glutamine within the mitochondria of astrocytes. The RNA oxidation induces N-methyl D-aspartic acid (NMDA) activation ${ }^{43}$. NMDA receptors are 
used in the brain function as a means to compartmentalize glutamine and glutamate. Under normal conditions, astrocytes support adjacent neurons with ATP, glutamine, and cholesterol. The neuron metabolizes glutamine to glutamate, and the NMDA receptors take in the glutamate to be recycled back to glutamine. With excess ammonia being metabolized to glutamine, the compartmentalization of glutamine and glutamate is altered. Glutamate is now in excess, and has been found in the hippocampus and cerebral cortex in patients with acute liver failure. Glutamate is an excitatory neurotransmitter, which could lead to agitation and possible seizure. In patients chronically exposed to ammonia, these receptors are down-regulated, which results in less neuroexcitation from glutamate $14,23-25,44$. Meanwhile, glutamine's concentration increases within the astrocytes and ultimately leads to further release of ROS and RNS and swelling.

Neurochemical alterations have consistently been present in various models of HE. In a study by Yurdaydin, the extent of the neurochemical involvement was dependent on the severity of the HE. Nevertheless, the study proposed an increase in serotonic and noradrenergic neurotransmission in patients with $\mathrm{HE}^{40}$.

Serotonin is a neurotransmitter involved in the CNS, and it is also a hormone which interacts with various other organ systems. Serotonin metabolites are increased in patients with HE. In addition, the number of serotonin receptors is altered and catabolizing enzymes are elevated ${ }^{40}$. However, the amount of biologically active serotonin has not been found to be elevated. In some studies, it has been reduced in the synaptic clefts ${ }^{45}$. Despite the alterations in the serotonin cycle, the role of serotonin in $\mathrm{HE}$ is not clear.

Catecholamines, particularly altered concentrations of norepinephrine (NE), have been implicated in contributing to HE. Catecholamine concentrations are closely linked to the AAA:BCAA ratio, thus suggesting a role in amino acid production. BCAA are thought to either competitively inhibit catecholamines or hinder their synthesis. NE is typically reduced in the brain of rats in acute or chronic liver failure. This reduction in NE would result in general slowing of cognitive processes most notably seen in minimal HE or overt HE types B and C. The possible cause of reduced levels of NE may also be directly related to hyperammonemia. Elevated levels of ammonia could potentially cause an increase in the noradrenergic transmission ${ }^{46}$.

Melatonin and histamine are both involved in the circadian rhythm, which dictates the sleepwake cycle. Histamine levels are elevated in the frontal cortex and central histamine concentrations have been linked to the control of arousal in the circadian rhythm ${ }^{47}$. Melatonin levels are altered in patients with liver disease. These changes yield the symptoms of altered sleep patterns and insomnia usually reported early in the disease state ${ }^{48}$.

\subsection{Precipitating factors}

HE is precipitated by an event that impacts the patient's functioning in the vast majority of cases. This event may be an increase in nitrogen, decreased ability to clear toxins, altered neurotransmission, or further hepatocellular damage ${ }^{49}$. The most common precipitating factors are sepsis, dehydration (secondary to treatment), and variceal bleeding ${ }^{12}$. Treating the precipitating factor is a pillar in treatment of HE. In the event that a precipitating factor cannot be identified, the diagnosis of spontaneous HE should be considered. A table of precipitating factors is listed in P-1. 
A large nitrogen load can precipitate an episode of HE. Excess dietary protein, constipation, and hypokalemia are all intake/output related, and can be treated with relative ease. Hypokalemia's effects stem from altering the BBB, and allowing more ammonia to cross. GI bleeding can also precipitate an episode of HE. GI bleeding leads to an excess of ammonia in the blood by digesting and absorbing proteins from the breakdown of red blood cells in the intestines. In addition, GI bleeding leads to the production of ammonia from the kidney resulting in hyperammonemia as well as potential hypovolemia ${ }^{17,50}$. Blood transfusions can be a less obvious cause, but must be considered as a potential precipitating event in HE, especially after an acute GI bleed.

Infection is a common contributor to HE. Infection or sepsis is usually apparent with a combination of increased temperature, white blood cell count, heart rate and respiratory rate. Cirrhotics are also viewed as functionally immunocompromised, therefore, their risk of infection is increased ${ }^{31}$. Because infection is such a common cause of HE, it is standard of care to evaluate all patients with HE with an infection workup including blood and urine cultures, chest $x$-ray, skin exam and diagnostic paracentesis ${ }^{4}$.

Decreased toxin clearance is generally associated with hypovolemia (dehydration, hypotension, or anemia) and portal-systemic shunting (either iatrogenic or spontaneous). Shunting of blood away from the liver bypasses hepatic cells and minimizes the clearance of toxins ${ }^{51}$. Dehydration and anemia, which are usually associated with hypotension, can lead to ineffective clearance of toxins by the kidneys. On occasion, hypotension can be due to systemic vasodilation which has the same result of ineffective toxin clearance. The lack of clearance in all of these processes leads to increased levels of toxins, most notably ammonia.

As noted in the astrocyte physiology, neurotransmission is vital to neurologic functioning. Altered neurotransmission is known to be a precipitating factor in HE. Patients placed on benzodiazepines or any psychoactive drug may react drastically for separate, yet related reasons. Endogenous benzodiazepines are elevated during $\mathrm{HE}$, and increasing the concentration of these would severely affect the patient ${ }^{40}$. With the hepatocytes damaged from the liver disease, blood shunted away from the liver, and various transporter proteins downregulated as a result of the liver disease, drugs affecting the cognitive function of a patient will have a more pronounced effect including stimulants (caffeine, amphemtamines, methylphenidate) and depressants (sedatives, narcotics) ${ }^{52}$.

Although hepatocellular damage is already present, in certain situations it can be worsened. Continued abuse of alcohol may cause worsening hepatic function, which can lead to a spontaneous shunt and a buildup of toxins in the blood and brain. Also, the development of hepatocellular carcinoma in the setting of cirrhosis may worsen the patient's status ${ }^{53}$.

\section{Diagnosis}

The diagnosis of overt HE is generally made by the presence of clinical signs and symptoms, elevated ammonia levels, and the absence of other possible causes of altered mental status. The signs and symptoms seen early are alterations in sleeping patterns, which can progress to somnolence and on to obtundation ${ }^{48}$. Some patients may become more agitated usually occurring in Type A HE54-56. Types B and C HE presentations are more associated with neuroinhibitory symptoms ${ }^{57-59}$. Nevertheless, the approach to anyone suspected of having 
HE is to exclude other possible causes of encephalopathy, identify precipitating factor, and empiric treatment for HE.

Clinical features of overt HE include those associated with consciousness, intellect and behavior, and gross neurological findings. In regards to consciousness, the patient can range from no impairment or mild lack of awareness to lethargy, somnolence or coma. Intellect and behavior changes include shortened attention span, disorientation, and inappropriate or bizarre behavior ${ }^{60}$. Neurological findings on exam include impaired psychomotor testing, varying extent of asterixis, slurred speech, rigidity and hyperreflexia, and transient posturing 61 . These signs and symptoms are all recognized by the West Haven Criteria and are graded by the severity of HE (Table I-1).

A detailed physical exam must be performed mainly to rule out other causes of altered mental status and to help elucidate possible precipitating factors. The general appearance of jaundice signifies underlying liver dysfunction, and often clues the physician as to the etiology of the altered mental status. Other evidence of liver failure, such as ascites, palmar erythema, edema, spider telangiectasias, muscle wasting and fetor hepaticus, may also be present in patients with overt $\mathrm{HE}^{62}$. Monitoring the patient's vital signs may also help with determining the precipitating factor(s) that induced the overt HE. A digital rectal exam should also be performed to assess for constipation and fecal occult blood as precipitating factors for the HE.

The neurologic exam is critical to assess the patient's altered mental status as disorientation and that other etiologies of the encephalopathy can be excluded. The alertness and orientation are aspects of the neurologic exam that usually separates the various grades of HE using the West Haven Criteria, and it is a critical symptom that may determine the need for hospitalization (grades 3 and 4). Mental status evaluations should be assessed to exclude other causes such as Alzheimers and neurovascular disease as well as to grade the encephalopathy according to the West Haven Criteria ${ }^{63}$. A detailed neurologic exam must be performed to rule out a neurologic cause contributing to the change in mental status ${ }^{64}$. Overt HE typically causes a global process, and any focal findings in the neurologic exam must be further pursued. Focal findings such as hemiplegia is an extremely rare presentation for overt HE. Focal neurologic signs are not prognostic and are usually reversible when associated with $\mathrm{HE}^{65}$. Asterixis is a common physical sign associated with overt HE. The presence and severity of asterixis can help grade the HE when using the West Haven Criteria. The assessment of asterixis requires an outstretched arm with extension of the wrist and is positive if a flapping tremor is present ${ }^{66}$. Alternatively, asterixis can be evaluated having the patient grip the evaluator's fingers in steady fashion and is present if the patient's grip tension oscillates. Parkinsonian-like symptoms may be present in the form of rigidity and tremors 4 .

Lab values play a vital role in the diagnosis of $\mathrm{HE}$ with the close monitoring of the ammonia levels. The ammonia level is typically elevated to quantities well above the upper normal limits during an episode of HE. The numerical value of the ammonia levels generally correlates to the extent of mental status change. However, there is a delay in the resolution of symptoms after ammonia levels return to normal, and routine ammonia measurements after diagnosis is not recommended 63,67 . In the event that ammonia is elevated and no underlying liver disease was present, labs should be collected to rule out inborn errors in 
metabolism (IEM) of urea. The IEM that present in adulthood include ornithine transcarbamylase, carbamyl synthetase, and arginosuccinate lyase deficiencies with each having measurable enzyme lab values ${ }^{13}$.

Other labs should be checked to determine the precipitating factor, which may have induced the overt HE. Discovering this factor is vital to the treatment of HE and the ultimate resolution of symptoms. For this reason the serum electrolytes must be checked due to the precipitating factors of hypernatremia, hypokalemia, hyperglycemia, and hypoglycemia 49 . The white and red blood cell counts should also be checked to evaluate for possibility of infection or GI bleed, respectively.

Obtaining pharmacologic levels of potentially toxic medications should be obtained in patients with liver disease receiving multiple drugs and having altered first pass liver metabolism. In addition, a urine drug screen may provide answers to patients with altered mental status.

Cultures should be drawn from the blood, urine, and ascitic fluid to evaluate for infection as precipitating factor of $\mathrm{HE}^{4}$. A chest x-ray may be necessary to rule out pneumonia. A lumbar puncture may be necessary to rule out meningitis. Cultures usually take 24 hours for preliminary results and therefore antibiotics should be started empirically if clinical suspicion is high for infection. Although infection may induce overt HE, sepsis may present with altered mental status with an ammonia level within the normal range ${ }^{63}$. White blood cell count, respiratory rate, heart rate, and temperature should be closely monitored due to differing treatments between overt HE and sepsis.

Radiologic imaging has a minimal role in diagnosing HE. When an unknown patient presents with altered mental status or general somnolence, it is appropriate to rule out an acute intracranial process with a non-contrast CT of the head. Because many patients with liver disease also have coagulopathies and are subject to falls, the head CT is clearly warranted. Imaging must be used in these instances when ruling out an intracranial process that could either be the etiology of the change in mental status or exacerbating $\mathrm{HE}^{68}$. However, in the event of diagnosing HE, radiologic images have not been shown to be necessary. Brain MRI's are increasingly being used to identify low-grade cerebral edema in $\mathrm{HE}$, but this is often used for research purposes. As discussed in the sections above, several imaging modalities have identified abnormalities used in deciphering the pathophysiology of HE. For instance, magnetic transfer ratio was reduced in patients with HE, but reverses with liver transplantation. This finding suggests that the metabolic effects of HE maybe reversible ${ }^{69}$. T1-weighted imaging showed manganese in the basal ganglia suggesting a role of manganese in the pathophysiology of HE ${ }^{70-72}$. Proton spectroscopy (MRS) is mentioned as the evidence of glutamate involvement within the astrocytes during HE59.

Although radiologic imaging has a minimal role in diagnosis and treatment, several modalities may be seen in the future for staging liver disease and the severity of HE. MRS changes directly correlate with severity of HE. This correlation could be used in the staging of liver disease as an illustrative way of monitoring the toll the disease is having on the brain. Although its variability and costs prevent it from being used routinely today, future management of chronic liver disease may include MRS73. T1 mapping with partial inversion recovery (TAPIR) is another method that correlates changes with HE severity. This method maps the brain through a series of T1 MRI scans. Unlike MRS, TAPIR offers measurements in short, clinically relevant acquisition times $^{74}$. 
Electroencephalography (EEG) is a tool used primarily in the research setting for diagnosis of HE. HE is associated with a decrease in mean frequency of electrical activity in the brain in the spectral EEG. Triphasic waves are noted in advanced HE75. However, EEG readings have demonstrated subjectivity, and the test has a low sensitivity. Furthermore, the equipment is expensive and the test has not been validated 76,77 .

\subsection{Specialized tests}

Specialized tests have been developed to quantify the degree of HE and also to monitor recovery or progression. Minimal HE is not diagnosed clinically, but it is diagnosed with neuropsychometric (NP) tests. These tests are useful tools in assessing progression of minimal $\mathrm{HE}$ as well as the initial diagnosis by identifying impairments in visuospatial functioning, attention, processing speed and response inhibition ${ }^{4}, 78$. Minimal HE does not have a gold standard for diagnosis or assessment through neuropsychometric testing. Two tests, Psychometric HE Score (PHES) and Repeated Battery for the Assessment of Neuropsychological status (RBANS), are recognized by prominent organizations worldwide, although no single test has gained significant popularity or validation in the US62.

Psychometic HE Score (PHES) (also referred to as the PSE Syndrome Test) consists of a battery of five tests; Number connection tests (NCT)-A, NCT-B, line tracing test, serial dotting tests and digit symbol test. The diagnosis of minimal HE is made if any two of the $\mathrm{NP}$ tests are impaired beyond two standard deviations from normal ${ }^{64}$. The whole test requires an educated instructor, paper, and pencil. The PHES typically requires 10-20 minutes to complete ${ }^{75}$. The PHES was recognized by the World Congress of Gastroenterology in 1998 and is validated in Germany, Italy, and Spain. The Working Group of Hepatic Encephalopathy has recommended the PHES, but validation of this test has yet to be completed in the United States (US) ${ }^{79-81}$.

The Repeated Battery for the Assessment of Neuropsychological status (RBANS) is a test similar to PHES with the addition of verbal, visual, and working memory domains. The test requires 20-25 minutes to complete, and is administered by paper and pencil. RBANS was created to gauge Alzheimer's Disease, schizophrenia, traumatic brain injury, and in some patients with cirrhosis awaiting liver transplantation. The International Society for the Study of HE and Nitrogen Metabolism (ISHEN) has recommended this test for diagnosis of minimal HE along with PHES. A modified version of RBANS is used in the US for overt HE, and has been proven to be effective in screening patients for minimal HE ${ }^{82-84}$. The RBANS recommended by ISHEN is currently being validated in the US in a multicenter trial 85,86 .

Performing the PHES or the RBANS require supervision by a neurologist, specialized equipment, trained personnel, and time. These tests rely on motor activity, which are often limited in patients with HE by the West Haven Criteria grades 2 to 4 . Thus additional testing of HE has been used. The Critical Flicker Frequency (CFF) is a test of functional efficacy of the cortex, and it is a highly objective and sensitive measure of minimal HE. This test consists of a patient indicating the minimum frequency at which a flickering light is still perceived as flickering and not fused. In Spain and India, it has been effective in differentiating minimal $\mathrm{HE}$ or early stage $\mathrm{HE}$ from no $\mathrm{HE}^{87}$, 88. It positively correlates with NP tests, and it is not influenced by gender, education level, occupation, and only slightly by age. It was validated for assessment of patients with $\mathrm{HE}$ in 2002 and for patients with HE who were undergoing TIPS placement in 200966, 89. For patients with reduced motor skill 
function undergoing HE assessment, pressing a button instead of performing a detailed pencil and paper test is more appropriate. Reaction times are directly measured and should increase the accuracy of assessing HE ${ }^{85}$.

Computerized psychometric tests are gaining popularity and could revolutionize the assessment of patients with $\mathrm{HE}^{78}$. The inhibitory control test is the most popular in the US. Its focus is primarily on response inhibition and attention. It has shown close correlations with other NP tests, but investigations have been limited to only Wisconsin and Virginia, USA. The inhibitory control test offers promise in that training of the technician is minimal, and the cost of the test may be cheaper than the NP tests. Furthermore, the inhibitory control test can also predict overt $\mathrm{HE}$, and it is associated with driver simulator performance and traffic accidents in the US87, 90,91. Cognitive Drug Research Ltd computerized assessment system was designed specifically for cirrhosis and minimal HE, and it focuses on power of attention, continuity of attention, quality of episodic memory, quality of continuous memory and speed of memory. This test is popular in the UK and has shown good correlation with PHES92.

\section{Treatment}

Treatment of overt HE is based on a multifaceted approach of reducing the ammonia levels, treating the precipitating factor, and evaluating the patient for liver transplantation. After the overt HE resolves, the focus of therapy shifts to preventing recurrent $\mathrm{HE}$, improving daily functioning and quality of life, and continuing evaluation for liver transplantation. Therapies can be viewed as short-term treatments, long-term treatments, and sometimes they can be used in both circumstances.

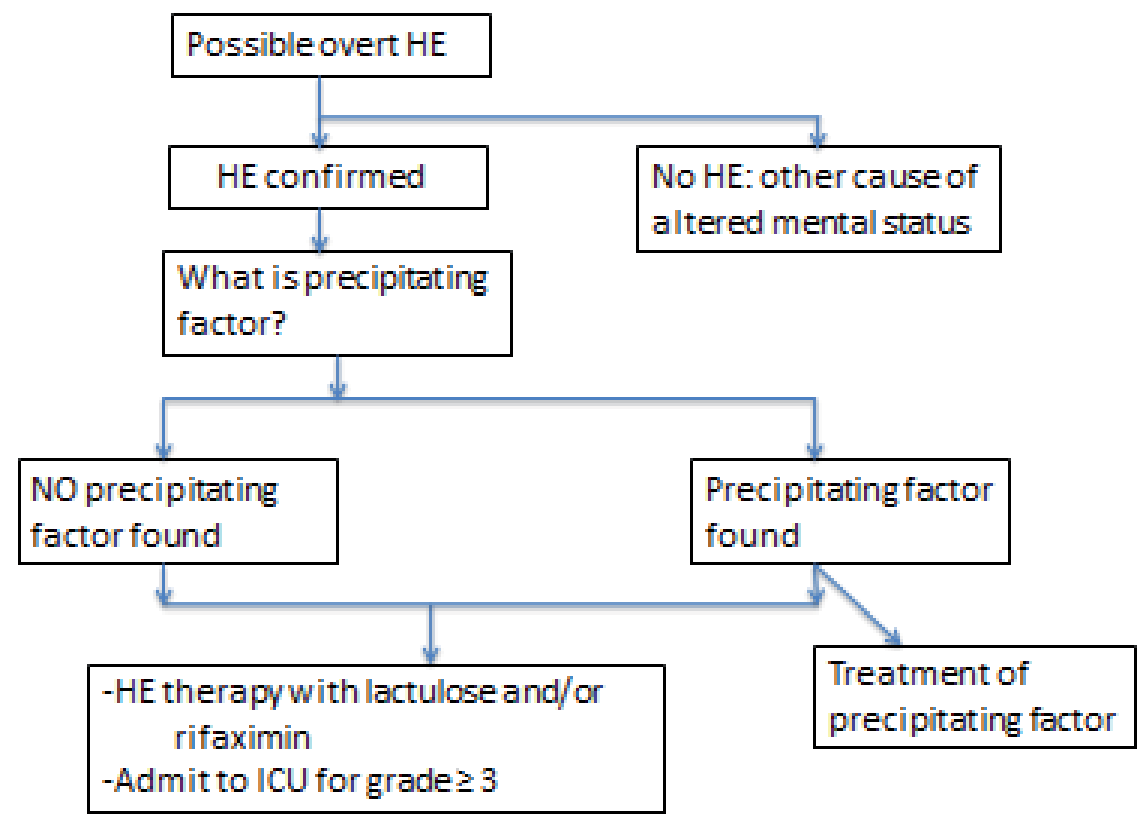

Adaptedfrom Bajaj 


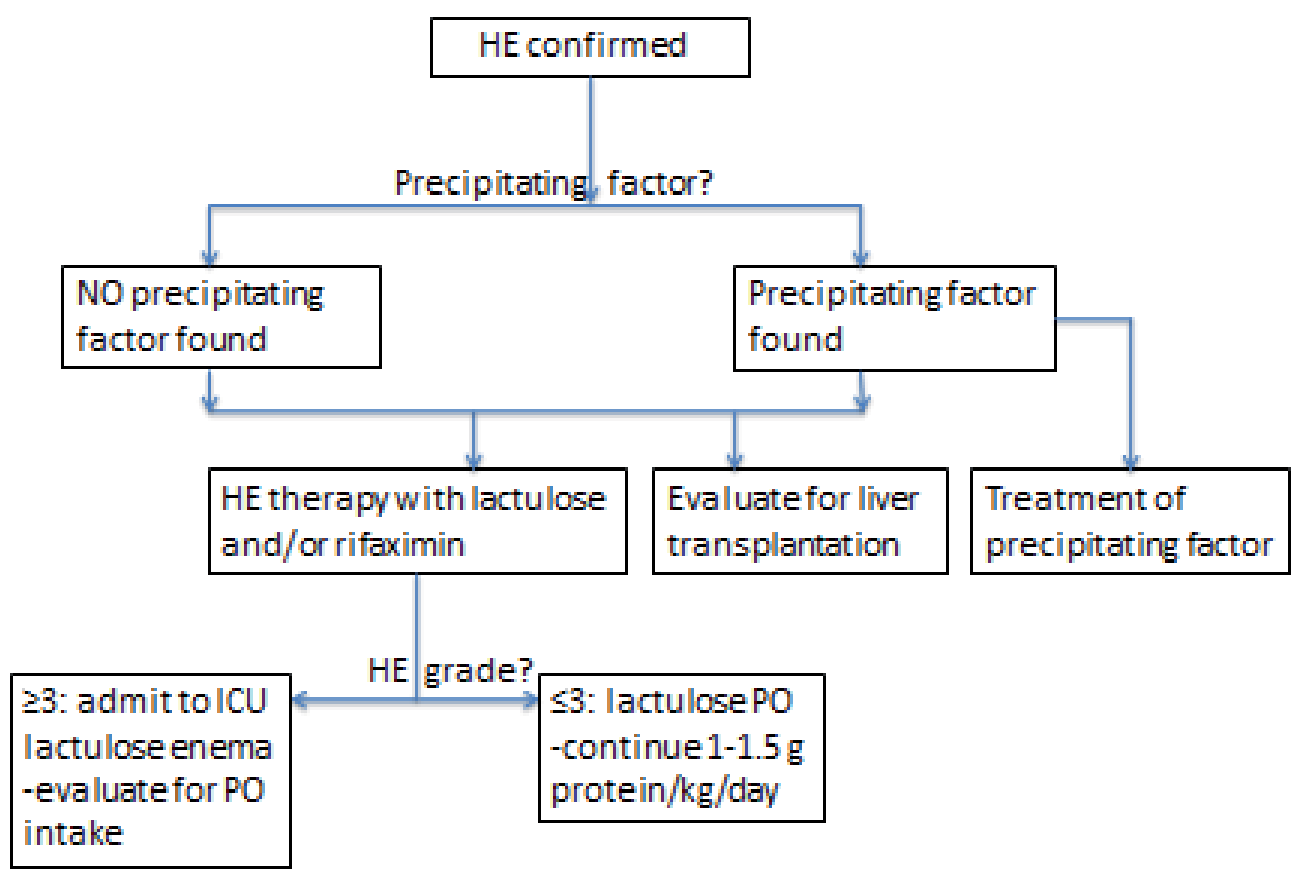

Diagram T-1. Treatment of Hepatic Encephalopathy

Patient safety is another important aspect of therapy. Patients with Type A HE are typically more agitated, and they pose greater risk to hurt themselves, family, and staff. For this reason and because of the possible effects of sedatives, judicious use of restraints is recommended. Haloperidol is a safer sedative than benzodiazepines most likely due to the endogenous benzodiazepines or increased benzodiazepine receptors produced in HE and liver disease. However, there is little evidence to support haloperidol's use in sedation of patients with $\mathrm{HE}^{93}$.

For patients with minimal HE, treatment is focused on family and patient discussions of potential complications that can develop as patients transition to overt HE and to discuss limitations in activities of daily living. In particular, several studies have shown the effect that minimal HE has on the patient's driving performance. One study showed patients with cirrhosis and minimal HE were involved in $17 \%$ of crashes compared to cirrhosis without minimal HE $0 \%{ }^{91}$. It should be noted that patients often overestimate their fitness to drive ${ }^{94}$. Patients and their family members must be aware that limiting or revoking the patient's drivers' license may be necessary.

Once overt HE has been diagnosed, the clinician must search for precipitating factor(s). Once the precipitating factor(s) has been identified, treatment must be directed to resolve the offending agent. It is also important to identify the precipitating factor to prevent recurrence. While treating the precipitating factor will resolve the primary mechanism responsible for the overt $\mathrm{HE}$, reducing the ammonia will help alleviate the cognitive dysfunction. 


\subsection{Ammonia treatments}

Lactulose is a synthetic non-absorbable dissacharide which is used as a cathartic to help clear ammonia through bowel movements. Lactulose also lowers the $\mathrm{pH}$ in the colon by being catabolized to lactic and acetic acid, which reduces ammonia levels in two additional ways. A lower $\mathrm{pH}$ will facilitate the conversion of ammonia $\left(\mathrm{NH}_{3}\right)$ to ammonium $\left(\mathrm{NH}_{4}{ }^{+}\right)$, which is poorly absorbed across the colon ${ }^{95}$. A lower $\mathrm{pH}$ also makes the environment more unfit for urease-positive bacteria, which decreases production of ammonia ${ }^{96-98}$. Lactulose has been shown in numerous studies to be effective, and when used appropriately it is as effective as any new medications. Dosage and administration depends on grade of HE and goal of therapy. For overt HE (grades 1-2), recommendations is to start lactulose orally with $20 \mathrm{~g} / 30 \mathrm{ml}$ every 1-2 hours until patient has 2-4 soft bowel movements. The dosage should always be titrated so that the patient has $2-4$ semisoft bowel movements each day ${ }^{2,61}$. If the HE grade is $\geq 3$, lactulose should be administered via enema due to risk of aspiration. The starting enema dosage is $200 \mathrm{gm} / 300 \mathrm{ml}$ with $700 \mathrm{ml}$ water or saline every 4 hours titrated. Although lactulose has been proven to be more effective than placebo in treating HE, the use of lactulose has not been proven to reduce mortality ${ }^{99}$.

Lactulose can also be used in the long-term treatment of patients with chronic liver diseases to prevent recurrence of $\mathrm{HE}$ and in the treatment of minimal HE ${ }^{100}$. With proper adherence, lactulose's effectiveness has been proven in multiple studies for long-term prevention of recurrent HE. Being inexpensive and effective makes lactulose a $1^{\text {st }}$ line agent for both shortand long-term therapy 61,101 .

Diarrhea, abdominal pains, and fatigue are the most common side effects associated with lactulose. The side effects can be severe enough that patients discontinue using the medication. This discontinuation has been well chronicled in various trials ${ }^{102}$. The side effects of diarrhea can be severe enough that the overuse of lactulose itself could lead to hypovolemia or an electrolyte imbalance that induces a new episode of overt HE ${ }^{103}$. In a lactulose adherence trial conducted, nearly half of the patients with recurrent HE were nonadherent or had $\geq 5$ bowel movements which contributed to the development of overt HE 102. Adherence has been reported as low as $31 \%$ in patients prescribed lactulose $\mathrm{l}^{104}$. Adherence is low due to a lesser quality of life secondary to the side effects of lactulose. Patients on lactulose should be closely monitored, as adherence is affected by presence of adverse events, lack of insight into disease, inability to self-titrate, consequences of nonadherence not fully explained, and absence of close follow-up ${ }^{102}$.

Lacitol has a similar mechanism to Lactulose, but it is unavailable in the US. It is frequently used in England with success similar to lactulose and better adherence. The better adherence is due to its superior palatability and less gastrointestinal side effects that are associated with lactulose ${ }^{103}$. Lacitol was also found to have a faster clinical response than lactulose ${ }^{104}$.

The US Food and Drug Administration (FDA) approved rifaximin in 2010 for the treatment of $\mathrm{HE}$ in liver disease. Over $90 \%$ of the patients in the registration trial were also receiving lactulose. While lactulose's effects are limited by the adverse side effects, rifaximin has minimal side effects. Rifaximin is an antibiotic taken orally with minimal systemic absorption ${ }^{107}$. Its antibiotic properties are thus limited to the gastrointestinal tract, where antibiotic coverage includes gram-positive and gram-negative bacteria, although it has 
minimal effects on gut normal flora ${ }^{108}$. A review comparing rifaximin to lactulose concluded that rifaximin is equally effective and in some studies superior to nonabsorbable disaccharides and other antimicrobials in relieving the signs and symptoms observed in cirrhotic patients with mild-to-moderately severe $\mathrm{HE}^{109}$. However, due to the lack of side effects associated with rifaximin, patient adherence and satisfaction are significantly greater in rifaximin 110,111, 112, 113. When factoring in patient adherence to the comparison of lactulose and rifaximin, rifaximin is superior.

Patient's adherence to rifaximin make it a more practical option for long-term therapy than lactulose, however, the cost of rifaximin is substantially higher (approximately 40 dollars a day) than that of lactulose (approximately 40-100 dollars a month) ${ }^{114}$. Thus, rifaximin and lactulose compare beyond the medical and physical aspects, they also must be compared in the psychosocial realm. A single center retrospective chart review comparing rifaximin to lactulose found that patients on rifaximin therapy were less likely to be hospitalized for recurrent HE, HE severity was reduced, and hospital stay was shorter than patients on lactulose ${ }^{110}$. These results suggest that rifaximin may be more cost effective in the long run, due to less expense associated with hospitalizations. However, multi-center prospective studies are lacking to support this claim. Two cost effective analyses reviewing different strategies in HE management, found that initial therapy of lactulose monotherapy with cross over to rifaximin was the best treatment. In other words, if the patient was intolerant to lactulose or responded poorly, then rifaximin was administered 107,115 .

Rifaximin administration has been the subject of debate. In Italy, pulse therapies with rifaximin achieved therapeutic effect without developing resistance or clostridium difficile infection ${ }^{116}$. Currently in the US, continuous therapy of $550 \mathrm{mg}$ bid for HE is most widely accepted. Although rifaximin's effects were suffice to warrant US FDA approval, studies must continue to investigate maximizing the drug's benefits.

Neomycin is another antibiotic that has been approved in the treatment of overt HE. Trials with neomycin have not yielded the same results as rifaximin or lactulose. In fact, in a randomized study, neomycin was shown to be no better than placebo in 39 patients with HE117. In addition, the potential side effects of ototoxicity and nephrotoxicity that can occur with systemic absorption has limited its use clinically for treating HE118, 103 .

Metronidazole has been used for the treatment of HE, but has not been approved by the US FDA. Small single center studies suggest it may have some benefit in treating HE ${ }^{119}$. However, the major long-term side effect of neurotoxicity, limits its routine use for the management of HE103.

Ornithine-aspartate is a substrate that is used in the urea cycle to convert ammonia to urea and glutamine in both hepatic and muscular metabolism. It has been shown in several controlled trials to lower ammonia concentrations and provide improvements of mental status and cognitive function in patients with mild to moderate HE120 - 122. In a doubleblinded randomized placebo-controlled trial treating patients with acute liver failure, ornithine-aspartate did not lower serum ammonia levels or improved survival ${ }^{123}$. Fifty percent of patients report side effects of mild gastrointestinal disturbance, but no major side effects have been reported 121 - 123. Most trials administered ornithine-aspartate 18-30 g IV daily for 1-2 weeks. Additional studies are required before this agent can be recommended in the treatment of HE. 
Sodium benzoate/sodium phenylacetate has been shown to enhance tissue metabolism of ammonia. Benzoate interacts with glycine to form hippurate, which is renally excreted. Phenylacetate conjugates with glutamine to form phenylacetylglutamine, which is renally excreted as well. Both hippurate and phenylacetylglutamine contain nitrogen from ammonia. In a prospective, randomized, double-blind study on 74 patients with overt HE, an oral dose of 5 grams of sodium benzoate twice daily and lactulose were similar in symptomatic improvement as well as adverse effects ${ }^{124}$. Adverse effects included unpleasant taste, nausea, and salt overload. A combination of sodium benzoate and sodium phenylacetate intravenously is US FDA approved for the treatment of hyperammonemia in urea cycle defects, however, it has not been rigorously studied for HE treatment ${ }^{125,126 .}$

Acarbose is an inhibitor of a-glycosidase, which inhibits the conversion of carbohydrates into monosaccharides for diabetic patients. It also facilitates the reduction of proteolytic bacteria flora that produce ammonia. In a single center randomized control trial comparing acarbose at dosage of 150 to $300 \mathrm{mg} /$ day versus placebo in diabetic patients noted serum ammonia levels were significantly reduced and symptomatic improvement of HE were seen $^{127}$. This drug is not routinely used for the management of HE due to the product information sheet stating acarbose is contraindicated in patients with cirrhosis.

Dietary protein restrictions were once thought to be necessary in the treatment of HE to help resolve hyperammonemia. After many studies were undertaken to examine this belief, it is now recommended that dietary protein should remain constant during an overt HE episode. The prevalence of malnutrition in cirrhotic patients is estimated to be $65-100 \% 128,129$. Muscle wasting is also common in patients with cirrhosis, thus severely limiting ammonia removal from skeletal muscles. Protein intake, therefore, should remain constant during any episode of HE. The suggested protein requirement of 1-1.5 gram/ $\mathrm{kg}$ per day for patients with liver cirrhosis should be continued during an episode of HE130.

Probiotics are thought to work by introducing bacteria that do not produce urease. Probiotics have been associated with reduced serum ammonia concentrations ${ }^{116}$. Single center studies have demonstrated that probiotics improved cognitive function in patients with minimal HE131. A meta-analysis of prebiotics, probiotics, and synbiotics in minimal HE showed clinical improvement of minimal HE, but not as great as lactulose ${ }^{132}$. Probiotics and symbiotics were associated with fewer side effects compared to lactulose. While treatment options for HE with prebiotics, probiotics, and symbiotics are still being investigated, many clinicians are reluctant to introduce live bacteria to functionally immunosuppressed patients.

\subsection{Neurotransmission}

BCAA are decreased and AAA are increased in patients with cirrhosis and HE. As mentioned in the pathophysiology section, this imbalance inhibits production of catecholamines. Restoring balance would seem like a logical treatment, but trials have shown inconsistent results. Perhaps the greatest benefit of BCAA therapy is in the ability to increase protein intake without compromising cerebral function ${ }^{133}$. It has been associated with increased serum albumin levels as well as improved cellular metabolism in cirrhotic patients ${ }^{134}$. 
Timing of BCAA therapy may be a crucial factor in its efficacy. In a small study of 12 patients with cirrhosis, nocturnal administration was associated with increased serum albumin levels at three months while daytime administration was not. It is speculated that these amino acids are used as calories during the day and protein synthesis when infused at night ${ }^{135}$.

Two meta-analyses have produced conflicting results. A meta-analysis of 5 clinical trials suggested that long-term supplementation of oral BCAA was associated with significant improvement in mental recovery from $\mathrm{HE}$ and improvement from chronic HE ${ }^{136}$. However, a recent meta-analysis of 11 trials found no convincing evidence that BCAA supplementation is beneficial 137 . Thus, if protein replacement is clinically indicated, BCAA supplementation may help with the patient recovery through the increase in protein synthesis.

Flumazenil is the short-acting benzodiazepine receptor antagonist, and has been studied for treating patients with HE. Most studies have reported limited success with flumazenil in short-term improvement in mental status. In a systematic review, flumazenil was associated with a significant symptomatic improvement in patients with HE when compared to placebo138. However, clinical improvements are short-term since flumazenil is given intravenously and has a half-life of less than an hour ${ }^{138}$. In addition, caution is advised in patients with hepatic impairment and side effects are significant (seizure, nausea and agitation) limiting its use clinically ${ }^{49}$.

Because the pathogenesis of HE may involve dopaminergic neurotransmission, bromocriptine has been studied as a possible treatment option. Bromocriptine stimulates dopamine receptors and is approved for Parkinson's disease treatment. In a small randomized study, bromocriptine was no more effective than placebo in resolution of HE. However, when bromocriptine was added to more conventional therapies, improvement of extrapyramidal symptoms was reported ${ }^{139}$. Bromocriptine at $30 \mathrm{mg}$ orally twice daily can be considered in patients who are refractory to other therapies with extrapyramidal symptoms ${ }^{64}$.

Zinc deficiency is common among patients with cirrhosis and HE. Zinc is a cofactor of urea cycle enzymes $^{140}$. Zinc supplementation is thought to decrease serum ammonia and GABA and norepinephrine in the brain, but studies have shown conflicting results. In a blinded study of short-term zinc supplementation in patients with $\mathrm{HE}$, no clinically significant benefit was associated with the zinc supplementation ${ }^{141}$. However, in a study evaluating supplementation for 3 months resulted in an improvement of HE142. Zinc toxicity is a concern as well as the adverse effects including nausea, vomiting, loss of appetite, abdominal cramps, diarrhea, and headaches ${ }^{49}$.

\subsection{Surgery}

Shunting of blood away from the liver through a portal-systemic shunt predisposes the patient to recurrent and persistent HE in patients with cirrhosis, surgical shunts, or TIPS placements. Shunting blood away from the liver, limits metabolism of ammonia by the liver. A majority of cirrhotic patients with HE is secondary to portal-systemic shunts and not hepatocellular dysfunction ${ }^{143}$. Obliteration of large spontaneous portal-systemic anastomoses, surgical shunts, or TIPS can be a therapeutic option among persistent HE 
patients ${ }^{49}$. As with any surgical procedure in a patient with cirrhosis, the risk of bleeding and hepatic decompensation must be considered when weighing this treatment option.

The ultimate surgical treatment of HE and liver disease is liver transplantation. Liver transplant recipients rarely present with additional HE episodes. HE episodes before liver transplantation has been associated with increased morbidity and worse patient functioning after transplantation ${ }^{69}$. For this reason, a patient experiencing his first overt HE should be referred to a liver transplant center ${ }^{1}$. The model for end-stage liver disease (MELD) is the scoring system used to list patients with cirrhosis in the US. The clinical data for calculating the MELD score includes objective data (serum creatinine, serum total bilirubin, and international normalized ratio of prothrombin time), but does not include any subjective data, such as episodes of HE or ascites. It has been shown that overt HE worsens prognosis over and above the MELD score and results in poorer survival rate with higher grades of HE for patients admitted to a hospital ${ }^{144}$. This further emphasizes the necessity of beginning the liver transplant workup following the first episode of overt HE.

\subsection{Dialysis}

Peritoneal dialysis, hemodialysis, continuous venovenous hemofiltration and continuous arteriovenous hemodiafiltration have the ability to remove ammonia directly146-151. These therapies have proven to be effective in children and adults with urea cycle defects. They could serve as a bridge for adults with fulminant hepatic failure while they are awaiting liver transplantation.

\subsection{Future/experimental therapies}

Hypothermia is a popular treatment modality for patients who are comatose after a cardiac arrest. In patients with $\mathrm{HE}$, the benefits of hypothermia are associated with the reduction of free radical production, astrocyte swelling, and inflammation ${ }^{152}$. This improves cerebral blood flow and autoregulation, which reduces potential of intracranial hypertension. It also slows the ammonia production in both the gut from urease-positive bacteria and in the kidney. Hypothermia has also been shown to slow protein catabolism and prevents the neutrophil dysfunction 153,154 . Based on all of these mechanisms for clinical improvement, hypothermia could be a valuable tool in liver failure and grade 3-4 HE, but large randomized trials are needed before routine clinical use ${ }^{31}$.

Indomethacin is a nonsteroidal anti-inflammatory drug (NSAID) used in rheumatic disease and other inflammatory processes. The role of indomethacin in $\mathrm{HE}$ is to reduce inflammation and reduce cerebral blood flow, thus decreasing intracranial hypertension 24,99 , 155. There is excitement surrounding TLR-2, TLR-4, and TLR-9 inhibitors which would down-regulate neutrophil activation and therefore inflammation ${ }^{31}$. The side effect of indomethacin and other NSAIDs causing renal failure in cirrhotic patients may limit its use.

Levocarnitine is a metabolite of lysine degradation and serves as a carrier for short-chain fatty acids across mitochondrial membrane. It has been shown to stimulate the urea cycle and a delayed onset of HE in hyperammonemic mice, which is thought to be protective against ammonia neurotoxicity ${ }^{156}$. In the two studies conducted on patients with HE, 
levocarnitine reduced ammonia levels and improved mental status in one, while it provided no significant benefit in the other ${ }^{157,158}$. Future studies are needed before clinical use.

The glutamatergic neurotransmitter system has been implicated in the pathogenesis of HE in several models. Memantine is an NMDA receptor antagonist, which is hypothesized to help reduce the overactivity of NMDA receptors. It is currently approved for the treatment of Alzheimer's Disease. In rats with hyperammonemia and neurologic symptoms, memantine administration improved HE grade and neurologic symptoms ${ }^{159}$. The human studies investigating memantine are pending.

The Molecular Adsorbent Recirculating System (MARS) is an albumin dialysis system that allows the removal of protein-bound and water-soluble toxins. A US multicenter randomized clinical trial in 70 patients with cirrhosis and grade III and IV HE showed significant benefit of MARS versus standard therapy with regard to time to improvement of HE160. However, there is no extracorporeal albumin dialysis approved by the US FDA for the treatment of HE.

Spherical Carbon Adsorbent (AST-120) when given orally acts as a sink for neurotoxins and hepatotoxins in the gut. AST-120 has been study in rats with cirrhosis and have shown decreased arterial ammonia levels, normalization of brain water content and normalization of locomotor activity ${ }^{161}$. Clinical trials are being conducted in humans.

\section{Summary}

The development of HE is a severely debilitating event for patients. Overt HE is a socially isolating event for patients, preventing them from working, driving a car and performing activities of daily living. Elevated serum ammonia with inflammation in the serum has been implicated as the underlying trigger to developing HE. The diagnosis should be considered in any patient with underlying liver disease who present with mental status changes and asterixis. After a thorough workup to rule out any acute neurologic cause of the patient's condition, a major effort must be made to find the precipitating factor which induced the current overt HE. Knowing the precipitating factor will allow both aspects of the HE to be treated - the cause and the effect. Treatments should start by eliminating the precipitating factor, which will ultimately improve the patient's condition. Standard treatments are focused on the prevention of additional ammonia from entering the blood system. Lactulose is a cheap and effective medication that has been studied extensively and it has been proven to be as effective as any medication on the market. Rifaximin is another effective medication, with minimal side effects. However, these agents do not resolve HE completely, but only minimize the number of severe episodes and partially improve mental function in the persistent cases of overt HE. Other potential treatments have shown some clinical benefits in the treatment of $\mathrm{HE}$ and can be considered as third line agents (zinc and sodium benzoate).

Several therapies are currently being studied, and they offer hope to those who currently suffering from recurrent HE. As these therapies become available, the treatment and prevention of HE will become easier to both patients and physicians. Until then, liver transplantation offers resolution of liver failure and HE, improves patient survival, improves quality of life, and decreases healthcare costs. 


\begin{tabular}{|c|c|c|c|}
\hline Line & Agent & Dosage & Side effects \\
\hline \multirow[t]{3}{*}{ First } & \multirow[t]{3}{*}{ lactulose } & $\begin{array}{l}\text { Acute -- enemas: } 300 \mathrm{ml} \text { in } 1000 \mathrm{ml} \\
\text { every } 2 \text { hour until clinical } \\
\text { improvement. }\end{array}$ & \multirow{3}{*}{$\begin{array}{l}\text { Abdominal } \\
\text { cramps, } \\
\text { diarrhea, } \\
\text { dehydration }\end{array}$} \\
\hline & & $\begin{array}{l}\text { Acute -- oral: } 45 \mathrm{ml} \text { each hour until } \\
\text { bowel movement and clinical } \\
\text { improvement }\end{array}$ & \\
\hline & & $\begin{array}{l}\text { Chronic -- oral: } 15-45 \mathrm{ml} \text { tid or bid } \\
\text { continuous until } 2 \text { to } 3 \text { bowel } \\
\text { movements per day. }\end{array}$ & \\
\hline Second & Rifaximin & Chronic -- oral: $400-550 \mathrm{mg}$ po bid & Flatulence \\
\hline \multirow[t]{2}{*}{ Second } & \multirow[t]{2}{*}{ Neomycin } & $\begin{array}{l}\text { Acute -- oral: } 1 \mathrm{~g} \text { every } 6 \text { hours for } \\
\text { up to } 6 \text { days }\end{array}$ & \multirow[t]{2}{*}{$\begin{array}{l}\text { Ototoxicity, } \\
\text { nephrotoxicity }\end{array}$} \\
\hline & & Chronic -- oral: $1-2 \mathrm{~g} /$ day & \\
\hline Second & metronidazole & Chronic -- oral: $250 \mathrm{mg}$ bid & Neurotoxicity \\
\hline Third & $\begin{array}{l}\text { Sodium } \\
\text { benzoate/sodium } \\
\text { phenylacetate }\end{array}$ & $\begin{array}{l}\text { Chronic -- oral: titrate up to clinical } \\
\text { improvement or a maximum dose } \\
\text { of } 5 \mathrm{~g} \text { bid }\end{array}$ & Emesis \\
\hline Third & $\begin{array}{l}\text { Zinc acetate, Zinc } \\
\text { gluconate, zinc } \\
\text { sulfate }\end{array}$ & $\begin{array}{l}\text { Chronic -- oral: } 11 \mathrm{mg} \text { in adult } \\
\text { males and } 8 \mathrm{mg} \text { in adult females } \\
\text { every day }\end{array}$ & Nausea \\
\hline Third & bromocriptine & Chronic -- oral: $30 \mathrm{mg}$ bid & $\begin{array}{l}\text { Nausea, } \\
\text { hypotension, } \\
\text { headache, } \\
\text { dizziness }\end{array}$ \\
\hline Fourth & Surgical & $\begin{array}{l}\text { Chronic -- surgical obliteration of } \\
\text { large spontaneous portal-systemic } \\
\text { anatomoses }\end{array}$ & $\begin{array}{l}\text { Bleeding, } \\
\text { infection }\end{array}$ \\
\hline
\end{tabular}

Table S-1. Treatment Summary

\section{References}

[1] Ferenci P. Hepatic encephalopathy. In: Haubrich WS, Schaffner F, Berk JE, eds. Bockus Gastroenterology. 5th ed. Philadelphia, Pa: WB Saunders; 1995:1998-2003.

[2] Blei A, Cordoba J. Practice Parameters Committee of the American College of Gastroenterology: Hepatic encephalopathy. Am J Gastroenterol. 2001; 96: 1968-1976.

[3] Mendler M, Donovan J, Blei A. Central nervous system and pulmonary complications of end-stage liver diseases. In Textbook of Gastroenterology, $4^{\text {th }}$ Ed., Vol 2. Edited by Yamada J, Alpers D, Kaplowitz N, et al. Philadelphia: Lippincott Williams \& Wilkins;2003: 2445-2467

[4] Bajaj JS. Review article: the modern management of hepatic encephalopathy. Aliment Pharmacol Ther 2010; 31: 537-547.

[5] Poordad FF. Review article: the burden of hepatic encephalopathy. Aliment Pharmacol Ther 2007; 25(Suppl 1): 3-9. 
[6] Chatauret N, Butterworth RF. Effects of liver failure on inter-organ trafficking of ammonia: Implications for the treatment of hepatic encephalopathy. J Gastroenterol Hepatol. 2004;19: S219-23.

[7] Bajaj JS, Cordoba J, Mullen KD, Amodio, P, Shawcross DL, Butterworth RF, Morgan MY. Review article: the design of clinical trials in hepatic encephalopathy - an International Society for Hepatic Encephalopathy and Nitrogen Metabolism (ISHEN) consensus statement. Aliment Pharmacol Ther 2011; 33: 739-47.

[8] Conn HO, Leevy CM, Vlahcevic ZR, et al. Comparison of lactulose and neomycin in the treatment of chronic portal-systemic encephalopathy. A double blind controlled trial. Gastroenterology 1977; 72: 573-83.

[9] Parsons-Smith BG, Summerskill WHJ, Dawson AM, Sherlock S. The electroencephalograph in liver disease. Lancet 1957; 2: 867-71.

[10] Ferenci P, Lockwood A, Mullen K, et al. Hepatic encephalopathy-definition, nomenclature, diagnosis, and quantification: final report of the working party at the11th World Congresses of Gastroenterology, Vienna, 1998. Hepatology 2002; 35:716-21.

[11] Hassanein, TI, Hilsabeck RC, Perry W. Introduction to the Hepatic Encephalopathy Scoring Algorithm (HESA). Dig. Dis. Sci. 2008; 53: 529-38.

[12] Fessel JN, Conn, HO. An analysis of the causes and prevention of hepatic coma. Gastroenterology. 1972; 62:191.

[13] Clay, AS, Hainline BE. Hyperammonemia in the ICU. Chest 2007; 132:1368-78.

[14] Bachmann, C. Mechanisms of hyperammonemia. Clin Chem Lab Med. 2002; 40,653-662.

[15] Vince, A, Dawson, AM, Park, N, et al. Ammonia production by intestinal bacteria. Gut 1973;14,171-177.

[16] Karim, Z, Szutkowska, M, Vernimmen, C, et al Renal handling of NH3/NH4+: recent concepts. Nephron Physiol 2005;101,77-81.

[17] Olde Damink, SW, Dejong, CH, Deutz, NE, et al Kidney plays a major role in ammonia homeostasis after portasystemic shunting in patients with cirrhosis. Am J Physiol Gastrointest Liver Physiol 2006;291,G189.

[18] Cooper AJ, Plum F. Biochemistry and physiology of brain ammonia. Physiolo. Rev. 1987; 67, 440-519.

[19] Waxman SG, "Chapter 2. Development and Cellular Constituents of the Nervous System" (Chapter). Waxman SG: Clinical Neuroanatomy, 26e

[20] Norenberg M. Hepatic encephalopathy: studies with astrocyte cultures. In: Norenberg M, Hertz L, Schousboe A, eds. The Biochemical Pathology of Astrocytes. New York: Alan R. Liss; 1988:451-464.

[21] Aggarwal A et al. Reversible Parkinsonism and T1W pallidal hyperintensities in acute liver failure. Mov Disord. 2006; 21: 1986-1990

[22] Krieger D et al. Manganese and chronic hepatic encephalopathy. Lancet 1995; 346: 270 74.

[23] Butterworth, RF Effects of hyperammonaemia on brain function. J Inherit Metab Dis 1998; 21:6-20.

[24] Vaquero, J, Chung, C, Cahill, ME, et al Pathogenesis of hepatic encephalopathy in acute liver failure. Semin Liver Dis 2003; 23:259-269.

[25] Shawcross, D, Jalan, R. The pathophysiologic basis of hepatic encephalopathy: central role for ammonia and inflammation. Cell Mol Life Sci 2005; 62:2295-2304. 
[26] Cordoba J, Alonso J, Rovira A, Jacas C, Sanpedro F, Castells L, et al. The development of low-grade cerebral oedema in cirrhosis is supported by the evolution of $1 \mathrm{H}$ magnetic resonance abnormalities after liver transplantation. J Hepatol 2001; 35:598604.

[27] Garcia-Martinez R, Rovira A et al. Hepatic encephalopathy is associated with posttransplant cognitive function and brain volume. Liver Transpl 2011; 17:38-46.

[28] Shawcross DL, Davies NA, Williams R, Jalan R. Systemic inflammatory response exacerbates the neuropsychological effects of induced hyperammonemia in cirrhosis. J Hepatol 2004; 40:247-254.

[29] Lockwood A, Yap E, Wong W. Cerebral ammonia metabolism in patients with severe liver disease and minimal hepatic encephalopathy. J Cereb Blood Flow Metab 1991; 11:337-341.

[30] Murthy CR, Rama Rao KV, Bai G, Norenberg MD. Ammonia-induced production of free radicals in primary cultures of rat astrocytes. J Neurosci. Res 2001; 66:282-298.

[31] Shawcross DL, Shabbir SS, Taylor, NJ, Hughes RD. Ammonia and the neutrophil in the pathogenesis of hepatic encephalopathy in cirrhosis. Hepatology 2010; 51:1062-1069.

[32] Soeters PB, Fischer JE. Insulin, glucagon, aminoacid imbalance, and hepatic encephalopathy. Lancet 1976; 2(7991):880-2.

[33] Ferenci P, Wewalka F. Plasma amino acids in hepatic encephalopathy. J Neural Transm Suppl. 1978;14(suppl): 87-94.

[34] Morgan MY, Milsom JP, Sherlock S. Plasma ratio of valine, leucine and isoleucine to phenylalanine and tyrosine in liver disease. Gut. 1978;19: 1068-1073.

[35] James, JH, Ziparo V, Jeppsson B, Fischer JE. “Hyperammonaemia, plasma aminoacid imbalance, and blood-brain aminoacid transport: a unified theory of portalsystemic encephalopathy." Lancet 1979; 2(8146):772.

[36] Naylor CD, O'Rourkee K, Detsky AS, Baker JP. Parenteral nutrition with branchedchain amino acids in hepatic encephalopathy a meta- analysis. Gastroenterology. 1989; 97: 1033.

[37] Schliess F, Gorg B, Haussinger D. Pathogenetic interplay between osmotic and oxidative stress: the hepatic encephalopathy paradigm. Biol. Chem. 2006; 387:13631370.

[38] Bassett ML, Mullen KD, Skolnick P, Jones EA. Amelioration of hepatic encephalopathy by pharmacologic antagonism of the GABAA-benzodiazepine receptor complex in a rabbit model of fulminant hepatic failure. Gastroenterology. 1987; 93(5):1069.

[39] Ahboucha S, Butterworth RF. Pathophysiology of hepatic encephalopathy: a new look at GABA from the molecular standpoint. Metab Brain Dis 2004; 19:331-343.

[40] Yurdaydin C, Gu ZQ, Nowak G, Fromm C, Holt AG, Basile AS. Benzodiazepine receptor ligands are elevated in an animal model of hepatic encephalopathy: relationship between brain concentration and severity of encephalopathy. J Pharmacol Exp Ther. 1993;265(2):565.

[41] Cagnin A, Taylor-Robinson SD, Forton DM, Banati RB. In vivo imaging of cerebral "peripheral benzodiazepine binding sites" in patients with hepatic encephalopathy. Neurochem. Int. 2008; 52:575-587.

[42] Ahboucha S, Butterworth RF. The neurosteroid system: an emerging therapeutic target for hepatic encephalopathy. Metab Brain Dis 2007; 22:291-308. 
[43] Reinehr R, Becker S, Qvartskhava N, Bidmon HJ, Selbach O, Haas HL, Schliess F. Hypoosmotic swelling and ammonia increase oxidative stress by NADPH oxidase in cultured astrocytes and vital brain slices. Glia. 2007;55(7):758.

[44] Butterworth, RF Glutamate transporter and receptor function in disorders of ammonia metabolism. Ment Retard Dev Disabil Res Rev 2001;7,276-279

[45] Mousseau DD, Butterworth RF. "The [3H]tryptamine receptor in human brain: kinetics, distribution, and pharmacologic profile." J Neurochem. 1994;63(3):1052.

[46] Yurdaydin C, Hörtnagl H, Steindl P, Zimmermann C, Pifl C, Singer EA, Roth E, Ferenci $\mathrm{P}$. Increased serotoninergic and noradrenergic activity in hepatic encephalopathy in rats with thioacetamide-induced acute liver failure. Hepatology. 1990;12(4 Pt 1):695.

[47] Lozeva V, Tuomisto L, Sola D, Plumed C, Hippeläinen M, Butterworth R. Increased density of brain histamine $\mathrm{H}(1)$ receptors in rats with portacaval anastomosis and in cirrhotic patients with chronic hepatic encephalopathy. Hepatology. 2001;33(6):1370.

[48] Córdoba J, Cabrera J, Lataif L, Penev P, Zee P, Blei AT. High prevalence of sleep disturbance in cirrhosis. Hepatology. 1998;27(2):339.

[49] Al Sibae MR, McGuire BM. Current trends in the treatment of hepatic encephalopathy. Ther Clin Risk Manag 2009; 5: 617-626.

[50] Olde Damink, SW, Dejong, $\mathrm{CH}$, Deutz, NE, et al. Upper gastrointestinal bleeding: an ammoniagenic and catabolic event due to the total absence of isoleucine in the haemoglobin molecule. Med Hypotheses 1999; 52, 515-519.

[51] Chatauret N, Butterworth RF. Effects of liver failure on inter-organ trafficking of ammonia: Implications for the treatment of hepatic encephalopathy. J Gastroenterol Hepatol. 2004;19: S219-223.

[52] Rodighiero V. "Effects of liver disease on pharmacokinetics. An update." Clin Pharmacokinet 2000; 38(6):491.

[53] Kumar V, Fausto N, Abbas A (editors) (2003). Robbins \& Cotran Pathologic Basis of Disease (7th ed.). Saunders. pp. 914-7

[54] Rose C, Kresse W, \& Kettenmann H. Acute insult of ammonia leads to calciumdependent glutamate release from cultured astrocytes, an effect of $\mathrm{pH}$. J. Biol. Chem. 2005; 280:20937-20944.

[55] Rose C. Effect of ammonia on astrocytic glutamate uptake/release mechanisms. J. Neurochem. 2006; 97(suppl 1):11-15.

[56] Hertz L, Murthy CR, Lai JC, Fitzpatrick SM, \& Cooper AJ. Some metabolic effects of ammonia on astrocytes and neurons in primary cultures. Neurochem. Pathol. 1987; 6:97-129.

[57] Cordoba J, Sanpedro F, Alonso J, \& Rovira A. ${ }^{1} \mathrm{H}$ Magnetic resonance in the study of hepatic encephalopathy in humans. Metab. Brain Dis. 2002; 17:415-416.

[58] Haussinger D. Low grade cerebral edema and the pathogenesis of hepatic encephalopathy in cirrhosis. Hepatology 2006; 43:1187-1190.

[59] Rovira A, Alnoso J \& Cordoba J. MR imaging findings in hepatic encephalopathy. AJNR Am. J. Neuroradiol. 2008; 29:1612-1621.

[60] Mullen K Hepatic encephalopathy. Hepatology: A Textbook of Liver Disease, 5rd Ed., Vol. 1. Edited by Zakim D, Boyer TD ,. Philadelphia, Pa: WB Saunders;2006, 311-331. 
[61] Mullen KD, Amodio P, Morgan MY. Therapeutic studies in hepatic encephalopathy. Metab. Brain Dis. 2007; 22:407-23.

[62] Ferenci P. Clinical Manifestations of Hepatic Encephalopathy. (online) http://www.uptodate.com 2010.

[63] Sotil EU, Gottstein J, Ayala E, Randolph C, Blei AT. Impact of preoperative overt hepatic encephalopathy on neurocognitive function after liver transplantation, Liver Transpl 2009; 15: 184-92.

[64] Ferenci P, Lockwood A, Mullen K, Tarter R, Weissenborn K, Blei AT. Hepatic encephalopathy-definition, nomenclature, diagnosis, and quantification: final report of the working party at the $11^{\text {th }}$ World Congress of Gastroenterology, Vienna, 1998. Hepatology 2002; 35: 716-21.

[65] Cadranel JF, Lebiez E, Di Martino V, Bernard B, El Koury S, Tourbah A, Pidoux B, Valla D, Opolon P. Focal neurological signs in hepatic encephalopathy in cirrhotic patients: an underestimated entity? Am J Gastroenterol. 2001;96(2):515.

[66] Kircheis G, Wettstein M, Timmermann L, Schnitzler A, Haussinger D. Critical flicker frequency for quantification of low-grade hepatic encephalopathy. Hepatology 2002: 35: 357-66.

[67] Bass NM. Review article: the current pharmacological therapies for hepatic encephalopathy. Aliment Pharmacol Ther 2007; 25(Suppl 1): 23-31.

[68] Tsuji H. et al. A case of liver cirrhosis associated with chronic subdural hematoma and hepatic encephalopathy (Japanese). Fukucka Igaku Zasshi 1991; 82: 528-532.

[69] Garcia-Martinez R, Rovira A, Alonso J, Jacas C, Simon-Talero M, Chavarria L, Vargas V, Cordoba J. Hepatic Encephalopathy is Associate with Posttransplant Cognitive Function and Brain Volume. Liver Transpl 2010; 17: 38-46.

[70] Mullen KD \& Jones EA. Natural benzodiazepines and hepatic encephalopathy. Semin. Liver Dis. 1996; 16: 255-264.

[71] Mullen KD, Cole M \& Foley JM. Neurological deficits in "awake" cirrhotic patients on hepatic encephalopathy treatment: missed metabolic or metal disorder? Gastroenterology 1996; 111: 256-257.

[72] Rose C. et al. Manganese deposition in basal ganglia structures results form both portal-systemic shunting and liver dysfunction. Gastroenterology 1999; 117: 640-644.

[73] Haseler, LJ, Sibbitt, WL, Mojtahedzadeh, HN, et al. Proton MR spectroscopic measurement of neurometabolites in hepatic encephalopathy during oral lactulose therapy. AJNR Am J Neuroradiol 1998; 19:1681.

[74] Shah NJ, Neeb H, Zaitsev M, Steinhoff S, Kircheis G, Amunts K, Häussinger D, Zilles K. "Quantitative T1 mapping of hepatic encephalopathy using magnetic resonance imaging." Hepatology 2003;38(5):1219.

[75] Amodio P, Campagna F, Olianas S, et al. Detection of minimal hepatic encephalopathy: normalization and optimization of the Psychometric Hepatic Encephalopathy Score. A neuropsychological and quantified EEG study. J Hepatol 2008; 49: 346-53.

[76] Montagnese S, Jackson C, Morgan MY. Spatio-temporal decomposition of the electroencephalogram in patients with cirrhosis. J Hepatol 2007; 46: 447-58.

[77] Amodio P, Montagnese S, Gatta A \& Morgan MY. Characteristics of minimal hepatic encephalopathy. Metab Brain Dis 2004; 19: 253-267.

[78] Prakesh R, \& Mullen KD. Mechanisms, diagnosis and management of hepatic encephalopathy. Nat rev Gastroenterol Hepatol 2010; 7: 515-525. 
[79] Montagnese S, Amodio P, \& Morgan MY. Methods for diagnosing hepatic encephalopathy in patients with cirrhosis: a multidimensional approach. Metab Brain Dis 2004; 19: 281-312.

[80] Weissenborn K, et al. Neuropsychological characterization of hepatic encephalopathy. J Hepatol 2001; 29: 768-773.

[81] RED Hepatic Encephalopathy (Spanish). Spanish normative data for hepatic encephalopathy (online), http://www.redeh.org/ 2010.

[82] Randolph C et al. Neuropsychological assessment of hepatic encephalopathy: ISHEN practice guidelines. Liver Int 2009; 29: 629-635.

[83] Meyer T, Eshelman A, \&Abouljoud M. Neurpsychological changes in a large sample of liver transplant candidates. Transplant Proc 2006; 38: 3559-3560.

[84] Sorrell JH, Zolnikov BJ, Sharma A, \& Jinnai I. Cognitive imparment in people diagnosed with end-stage liver disease evaluated for liver transplantation. Psychiatry Clin Neurosci 2006; 60: 174-181.

[85] Iduru S, Mullen KD. The demise of the pencil? New computer-assisted tests for minimal hepatic encephalopathy. Gastroenterology 2008; 135: 1455-1456.

[86] Randolph C. Randolph, C. (1998). Repeatable Battery for the Assessment of Neuropsychological Status (RBANS). San Antonio: Harcourt, TX: The Psychological Corporation.

[87] Bajaj JS, Hafeezullah M, Franco J, et al. Inhibitory control test for the diagnosis of minimal hepatic encephalopathy. Gastroenterology 2008; 135: 1591-1600e1.

[88] Sharma P, Sharma BC, Puri V, Sarin SK. Critical flicker frequency: diagnostic tool for minimal hepatic encephalopathy. J Hepatol 2007; 47: 67-73.

[89] Kircheis G, Wettstein M, Timmerman L, Schnitzler A, \& Haussinger D. Diagnostic and prognostic values of critical flicker frequency determination as new diagnostic tool for objective HE evaluation in patients undergoing TIPS implantation. Eur J Gastroenterol Hepatol 2009; 1383-1394.

[90] Bajaj JS, et al. Inhibitory control test is a simple method to diagnose minimal hepatic encephalopathy and predict development of overt hepatic encephalopathy. Am J Gastroenterol 2007; 102: 754-760.

[91] Bajaj JS, Hafeezullah M, Zadvornova Y, et al. The effect of fatigue on driving skills in patients with hepatic encephalopathy. Am J Gastroenterol 2009; 104: 898-905.

[92] Mardini H, Saxby BK, \& Rcord CO. Computerized psychometric testing in minimal encephalopathy and modulation by nitrogen challenge and liver transplant. Gastroenterology 2008; 135: 1582-1590.

[93] Prabhakar S, Bhatia R. "Management of agitation and convulsions in hepatic encephalopathy." Indian J Gastroenterol. 2003;22 Suppl 2:S54.

[94] Kircheis G, Knoche A, Hilger N, Manhart F, Schnitzler A, Schulze H, Haussinger D. Hepatic encephalopathy and fitness to drive. Gastroenterology 2009; 137(5): 17061715.

[95] Eroglu Y, Byrne W. Hepatic encephalopathy. Emerg Med Clin N Am 2009; 27: 401-414

[96] Morgan MY, Blei A, Grungreiff K, et al. The treatment of hepatic encephalopathy. Metab Brain Dis 2007; 22: 389-405.

[97] Cordoba J, Blei AT. Treatment of hepatic encephalopathy. Am J Gastroenterol 1997; 92(9): 1429-39. 
[98] Clausen MR, Mortensen PB. Lactulose, disaccharides and colonic flora: clinical consequences. Drugs 1997; 53(6): 930-942.

[99] Jalan, R Intracranial hypertension in acute liver failure: pathophysiological basis of rational management. Semin Liver Dis 2003;23,271-282.

[100] Sharma BC, Sharma P, Agrawal A, Sarin SK. Secondary prophylaxis of hepatic encephalopathy: an open label randomized controlled trial of lactulose versus placebo. Gastroenterology 2009; 137: 885-91.

[101] Als-Nielsen B, Gluud LL, Gluud C. Non-absorbable disaccharides for hepatic encephalopathy: systematic review of randomized trials. BMJ 2004; 328: 1046.

[102] Bajaj JS, Sanyal AJ, Bell D, Gilles H, \& Heuman DM. Predictors of the recurrence of hepatic encephalopathy in lactulose-treated patients. Aliment Pharmacol Ther 2010; 31: 1012-1017.

[103] Phongsamran PV, Kim JW, Abbott JC, \& Rosenblatt A. Pharmacotherapy for Hepatic Encephalopathy. Drugs 2010; 70(9): 1131-1148.

[104] Leevy CB, Phillips JA. Hospitalizations during the use of rifaximin versus lactulose for the treatment of hepatic encephalopathy. Dig Dis Sci 2007; 52: 737-741.

[105] Heredia D, Teres J, Orteu N, et al. Lactitol vs. lactulose in the treatment of chronic recurrent portal-systemic encephalopathy. J Hepatol 1988; 7: 106-10.

[106] Morgan MY, Hawley KE. Lactitol vs lactulose in the treatment of acute hepatic encephalopathy in cirrhotic patients: a double-blind, randomized trial. Hepatology 1987; 7 (6): 1278-84.

[107] Huang DB, DuPont HI. Rifaximin: a novel antimicrobial for enteric infections. J Infect 2005; 50: 97-106.

[108] Brigidi P, Swennen E, Rizzello F, et al. Effects of rifaximin administration on the intestinal microbiota in patients with ulcerative colitis. J Chemother 2002; 14: 290295.

[109] Lawrence KR, Klee JA. Rifaximin for the treatment of hepatic encephalopathy. Pharmacotherapy. 2008 Aug;28(8):1019-32.

[110] Leevy CB, Phillips JA. Hospitalizations during the use of rifaximin versus lactulose for the treatment of hepatic encephalopathy. Dig Dis Sci 2007; 52: 737-41.

[111] Jiang Q, Jiang $\mathrm{XH}$, Zheng $\mathrm{MH}$, Jiang LM, Chen YP, Wang L. Rifaximin versus nonabsorbable disaccharides in the management of hepatic encephalopathy: a meta-analysis. Eur J Gastroenterol Hepatol 2008; 20: 1064-70.

[112] Bass NM. The current pharmacological therapies for hepatic encephalopathy. Aliment Pharmacol Ther 2007; 25: Suppl 1: 23-31.

[113] Maclayton DO, Eaton-Maxwell A. Rifaximin for treatment of hepatic encephalopathy. Ann Pharmacother 2009; 43: 77-84.

[114] PRICING - (data from drugstore.com)

[115] Sama Ca, Morselli-Labate AM, Pianta P, et al. Clinical effects of rifaximin in patients with hepatic encephalopathy intolerant or nonresponsive to previous lactulose treatment: an open-label, pilot study. Clin Ther Res 2004; 65 (5): 413-420.

[116] Longuercio C, Federico A, De Girolamo V, Ferrieri A, Del Vecchio, Blanco C. Cyclic treatment of chornic hepatic encephalopathy with rifaximin. Results of a doubleblind clinical study. Minerva Gastroenterol Dietol 2003; 49: 53-62. 
[117] Strauss E, Tramote R, Silva EP, Caly WR. Double-blind randomized clinical trial comparing neomycin and placebo in the treatment of exogenous hepatic encephalopathy. Hepatogastroenterology. 1992;39: 542.

[118] Greenberg LH, Momary H. Audiotoxicity and nephrotoxicity due to orally administered neomycin. JAMA 1965; 194: 237-238.

[119] Tarao K, Ikeda T, Hayashi K, et al. Successful use of vancomycin hydrochloride in the treatment of lactulose resistant chronic hepatic encephalopathy. Gut 1990; 31: 702706.

[120] Staedt U, Leweling H, Gladisch R, et al. Effects of ornithine aspartate on plasma ammonia and plasma amino acids in patients with cirrhosis: a double-blind, randomized study using a four-fold crossover design. J Hepatol 1993; 19: 424-430.

[121] Kircheis G, Nilius R, Held C, et al. Therapeutic efficacy of L-ornithine-L-aspartate infusions in patients with cirrhosis and hepatic encephalopathy: results of a placebo-controlled, double-blind study. Hepatol 1998; 25: 1351-1360.

[122] Stauch S, Kircheis G, Adler G, et al. Oral L-ornithine L-aspartate therapy of chronic hepatic encephalopathy: results of a placebo-controlled double-blind study. J Hepatol 1998; 28: 856-64.

[123] Acharya SK, Bhatia V, Sreenivas V, et al. Efficacy of L-ornithine L-aspartate in acute liver failure: a double-blind, randomized, placebo-controlled study. Gastroenterol 2009; 136: 2159-2168.

[124] Sushma S, Dasarathy S, Tandon RK, et al. Sodium benzoate in the treatment of acute hepatic encephalopathy: a double-blind randomized trial. Hepatology 1992; 16: 138144.

[125] Mendenhall CL, Rouster S, Marshall L, et al.: A new therapy for portal systemic encephalopathy. Am J Gastroenterol 1986, 81:540-543.

[126] Batshaw ML, MacArthur RB, Tuchman M. Alternative pathway therapy for urea cycle disorders: twenty years later. J Pediatr. Jan 2001;138(1 Suppl):S46-54; discussion S545.

[127] Gentile S, Guarino G, Romano M, et al. A randomized controlled trial of acarbose in hepatic encephalopathy. Clin Gastroenterol Hepatol 2005; 3: 184-91.

[128] Mendenhall C, Roselle GA, Gartside P, and Moritz T. Relationship of protein calorie malnutrition to alcoholic liver disease: a reexamination of data from two Veteran Administration Cooperative Studies. Alcoholism: Clinical and Experimental Research 1995; 19 (3): 602-609.

[129] Campillo B, Richardet JP, Scherman E, \& Bories PN. Evaluation of nutritional practice in hospitalized cirrhotic patients: results of a prospective study. Nutrition 2003; 19 (6): 515-564.

[130] Plauth M, Cabre E, Riggio O, et al. ESPEN Guidelines on Enteral Nutrition: liver disease. Clinical Nutrition 2006; 25 (2): 285-294.

[131] Liu Q, Duan ZP, Ha da K, et al. Synbiotic modulation of gut flora: Effect on minimal hepatic encephalopathy in patients with cirrhosis. Hepatology. 2004;39: 1441.

[132] Shukla S, Shukla A, Mehboob S, \& Guha S. Meta-analysis: the effects of gut flora modulation using probiotics, probiotics and synbiotics on minimal hepatic encephalopathy. Aliment Pharmacol Ther 2011; 33: 662-671. 
[133] Marchesini G, Dioguardi FS, Bianchi GP, et al. Long-term branched chain amino acid treatment in chronic hepatic encephalopathy. J Hepatol 1991; 11: 92-101.

[134] Nakaya Y, Okita K, Suzuki K, et al. BCAA-enriched snack improves nutritional state of cirrhosis. Nutrition 2007; 23 (2): 113-120.

[135] Fukushima H, Miwa Y, Ida E, et al. Nocturnal branched-chain amino acid administration improves protein metabolism in patients with liver cirrhosis: comparison with daytime administration. Journal of Parenteral and Enteral Nutrition 2003; 27 (5): 315-322.

[136] Naylor CD, O'Rouke K, Detsky As, et al. Parenteral nutrition with branched-chain amino acids in hepatic encephalopathy: a meta-analysis. Gastroenterology 1989; 97: 1033-42.

[137] Als-Nielsen B, Koretz RL, Kjaergard LL, et al. Branched-chain amino acids for hepatic encphlaopathy. Cochrane Database Syst Rev 2003; (2): CD001939.

[138] Als-Nielsen B, Kjaergard LL, Gluud C. Benzodiazepine receptor antagonists for acute and chronic hepatic encephalopathy. Cochrane Database Syst Rev 2004; (2): CD 002798

[139] Uribe M, Farca A, Marquez MA, Garcia-Ramos G, Guevara L. Treatment of chronic portal systemic encephalopathy with bromocriptine: A double-blind controlled trial. Gastroenterology. 1979;76: 1347-1351.

[140] Loomba V, Pawar G, Khar KL, et al. Serum zinc levels in hepatic encephalopathy. Indian Gastroenterol 1995; 14: 51-53.

[141] Riggio O, Ariosto F, Merli M, et al. Short-term oral zinc supplementation does not improve chronic hepatic encephalopathy: results of a double-blind crossover trial. Dig Dis Sci 1991; 36: 1204-1208.

[142] Marchesini G, Fabbri A, Bianchi G, et al. Zinc supplementation and amino acidnitrogen metabolism in patients with advanced cirrhosis. Hepatology 1996; 23: 10841092.

[143] Riggio O, Efrati C, Catalano C, et al. High prevalence of spontaneous portal-systemic shunts in persistent hepatic encephalopathy: a case-control study. Hepatology 2005; 42: 1158-1165.

[144] Stewart CA, Malinchoc M, Kim WR, Kamath PS. Hepatic encephalopathy as a predictor of survival in patients with end-stage liver disease. Liver Transpl 2007; 13: 1366-71.

[145] Chang MY, Fang JT, Chen YC, et al. Continuous venovenous haemofiltration in hyperammonaemic coma of an adult with non-diagnosed partial ornithine transcarbamylase deficiency. Nephrol Dial Transplant 1999; 14, 1282-1284.

[146] Donn, SM, Swartz, RD, Thoene, JG. Comparison of exchange transfusion, peritoneal dialysis, and hemodialysis for the treatment of hyperammonemia in an anuric newborn infant. J Pediatr 1979; 95, 67-70.

[147] Treem WR. Inherited and acquired syndromes of hyperammonemia and encephalopathy in children. Semin Liver Dis 1994; 14, 236-258.

[148] Rutledge SL, Havens PL, Haymond MW, et al. Neonatal hemodialysis: effective therapy for the encephalopathy of inborn errors of metabolism. J Pediatr 1990; 116, 125-128.

[149] Falk MC, Knight JF, Roy LP, et al. Continuous venovenous haemofiltration in the acute treatment of inborn errors of metabolism. Pediatr Nephrol 1994; 8, 330-333. 
[150] Mathias RS, Kostiner D, Packman S. Hyperammonemia in urea cycle disorders: role of the nephrologist. Am J Kidney Dis 2001; 37, 1069-1080.

[151] Summar M, Pietsch J, Deshpande J, et al. Effective hemodialysis and hemofiltration driven by an extracorporeal membrane oxygenation pump in infants with hyperammonemia. J Pediatr 1996; 128, 379-382.

[152] Stahl J. Studies of the blood ammonia in liver disease: Its diagnostic, prognostic, and therapeutic significance. Ann Intern Med. Jan 1963; 58: 1-24.

[153] Shawcross D, Davies N, Hodges S, Wright G, Jalan R. Hypothermia abolishes ammonia-induced neutrophil spontaneous oxidative burst. Hepatology 2006; 44 (Suppl 1): 363A.

[154] alan R, Olde Damink SW, Deutz NE, Hayes PC, Lee A. Moderate hypotherma in patients with acute liver failure and uncontrolled intracranial hypertension. Gastroenterology 2004; 127: 1338-1346.

[155] Clemmesen J, Hansen B, Larsen FS. Indomethacin normalizes intracranial pressure in acute liver failure: a twenty-three year old woman treated with indomethacin. Hepatology 1997; 26, 1423-1425.

[156] Therrien G, Rose C, Butterworth J, Butterworth RF. Protective effect of L-carnitine in ammonia-precipitated encephalopathy in the portacaval shunted rat. Hepatology. 1997;25: 551.

[157] Malaguarnera M, Pistone G, Elvira R, et al. Effects of L-carnitine in patients with hepatic encephalopathy. World J Gastroenterol 2005; 11: 7197-7202.

[158] del Olmo JA, Castillo M, Rodrigo JM, et al. Effects of L-carnitine upon ammonia tolerance test in cirrhotic patients. Adv Exp Med Biol 1990; 272: 197-208.

[159] Vogels BA, Maas MA, Daalhuisen J, et al. Memantine: A noncompetitive NMDA receptor antagonist improves hyperammonemia-induced encephalopathy and acute hepatic encephalopathy in rats. Hepatology. 1997; 25: 820.

[160] Hassanein TI, Tofteng F, Brown RS Jr, McGuire B, Lynch P, Mehta R, Larsen FS, et al. Randomized controlled study of extracorporeal albumin dialysis for hepatic encephalopathy in advanced cirrhosis. Hepatology 2007;46:1853-62.

[161] Bosoi CR, Rarent-Robitaille C, Anderson K, Tremblay M, Rose FR. AST-120 (spherical carbon adsorbent) lowers ammonia levels and attenuates brain edema in bile ductligated rats. Hepatology 2011;53:1995-2002.

[162] Ortiz M, Cordoba J, Doval E, et al. Development of a clinical hepatic encephalopathy staging scale. Aliment Pharmacol Ther 2007; 26:859-67. 


\title{
Hypoxic Encephalopathy
}

\author{
Mireia Moragas Garrido and Jordi Gascón Bayarri \\ Hospital Universitari de Bellvitge \\ Spain
}

\section{Introduction}

Encephalopathy is a broad concept that refers to a syndrome of overall brain dysfunction. Various systemic diseases can provoke encephalopathy, and the prominent related neurological signs and symptoms may mask the underlying disorder. Lack of oxygen and of blood flow to the brain are main causes of this condition. Often both mechanisms are related to heart failure and circulation, and of the lungs and respiration. It is difficult to ascertain which predominates. Hence, physicians use the ambiguous term of cardiorespiratory failure.

Hypoxic/anoxic brain injury can result from insufficient cerebral blood flow, reduced oxygen availability, reduced oxygen carriage by blood, or metabolic interference with the use of available oxygen (Commichau, 2006).

Ischemic - Hypoxic encephalopathy is often seen in emergency departments and can have a disastrous prognosis. A large number of medical conditions can lead to this situation, mainly (Ropper \& Brown, 2005):

1. Heart failure followed by respiratory depression secondary to massive blood loss, septic or traumatic shock, and heart disease, such as myocardial infarction or ventricular arrhythmia.

2. Respiratory failure followed by cardiac arrest as a result of low oxygen intake (in tracheal compression or obstruction, drowning, strangulation, aspiration of gastric content, or during general anesthesia if the inspired gas is poor in oxygen), respiratory muscles weakness in neurological diseases (Guillain-Barré syndrome, amyotrophic lateral sclerosis, myasthenia gravis) or central nervous system injury (mainly spinal cord injury).

3. Reduced oxygen carriage by the blood in carbon monoxide poisoning.

4. Histotoxicity in cyanide poisoning.

Physicians should also be aware of the delayed form, a rare condition that is difficult to diagnose. Delayed postanoxic encephalopathy (DPE) should be suspected in patients who have experienced respiratory depression or when a patient presents with subacute onset of cognitive and neuropsychiatric deficits. We describe a case of delayed postanoxic encephalopathy following acute intake of cocaine and heroin.

\section{Epidemiology}

In industrialized countries, out-of-hospital cardiac arrest occurs in 0.04 to $0.13 \%$ of the total population per year (Callans, 2004). Despite advances in prevention and treatment of 
cardiac and respiratory failure, the prognosis after a cardiopulmonary arrest remains poor. This condition represents an important cause of death in developed countries. However, due to its high incidence, even with low survival rates, the prevalence of survivors is surprisingly high (Callans, 2004; Young, 2009). Even when cardiac arrests and resuscitation take place in the hospital, fewer than one in five patients survive to discharge (Pederby, 2003). After a severe hypoxic insult, the brain may have become critically injured and patients may remain comatose, leading ultimately to severe cognitive impairment with a fully dependence for the basic life activities or to a vegetative state (Levy et al., 1985; Longstreth et al., 1983). The economical impact of these patients is substantial since their need for long-term care is unavoidable (Gray et al., 1991).

\section{Pathophysiology}

Brain hypoxia and ischemia due to systemic hypoxemia, reduced cerebral blood flow, or both are the primary physiological processes that lead to hypoxic-ischemic encephalopathy. The brain damage that occurs following hypoxic insult differs depending on the underlying mechanism. Ischemia (low cerebral blood flow) usually results in patchy infarctions in border zones between the major cerebral arteries (Fig. 1), whereas anoxia (reduced oxygen carriage by the blood) provokes neuronal death, mainly in the hippocampus, deep folia of the cerebellum, and cerebral cortex. In extreme cases of hypoxia and ischemia, more generalized damage occurs in the cerebral cortex, deep nuclei, and cerebellum. The deep grey matter of the brainstem is highly resistant to anoxia and tends to survive after relatively severe and prolonged anoxia when extensive cortical damage has been inflicted (Plum \& Posner, 1980).

At the cellular level, neuronal injury in hypoxic-ischemic encephalopathy is a progressive process that starts with necrosis in which the magnitude of the final neuronal damage depends on the duration and severity of the initial insult combined to the effects of reperfusion injury, and apoptosis. The necrotic tissue swells rapidly, mainly because of excessive of both intracellular and intercellular water content. Initially, venous blood darkens owing to an increase in reduced hemoglobin. The tissue becomes pale and arteries and arterioles become narrowed, especially in the pale areas. Eventually, during the reperfusion phase, the sequence is reversed and slight hyperemia might be present. Necrosis not only affects neurons but also oligodendroglial cells in the white matter (Lo et al., 2003).

Furthermore, the injury to both neurons and oligodendroglial cells secondary to necrosis is followed by an inflammatory response, activating endothelial cells to secrete proteases and cytokines and to express cell adhesion molecules that attract additional inflammatory cells (Lo et al., 2003).

At the molecular level, ischemia and anoxia lead to malfunction of the essential processes that sustain the Krebs cycle and electron transport system (Singh et al., 1992). In situations of severe, persistent hypoxia, neurons catabolize themselves to maintain activity, tissue necrosis ensues, and there is an accumulation of catabolic products and excitatory neurotransmitters, such as glutamate. Ultimately, massive intracellular influx of calcium leads to diffuse cell destruction (DW Choi \& Rothman, 1990). 


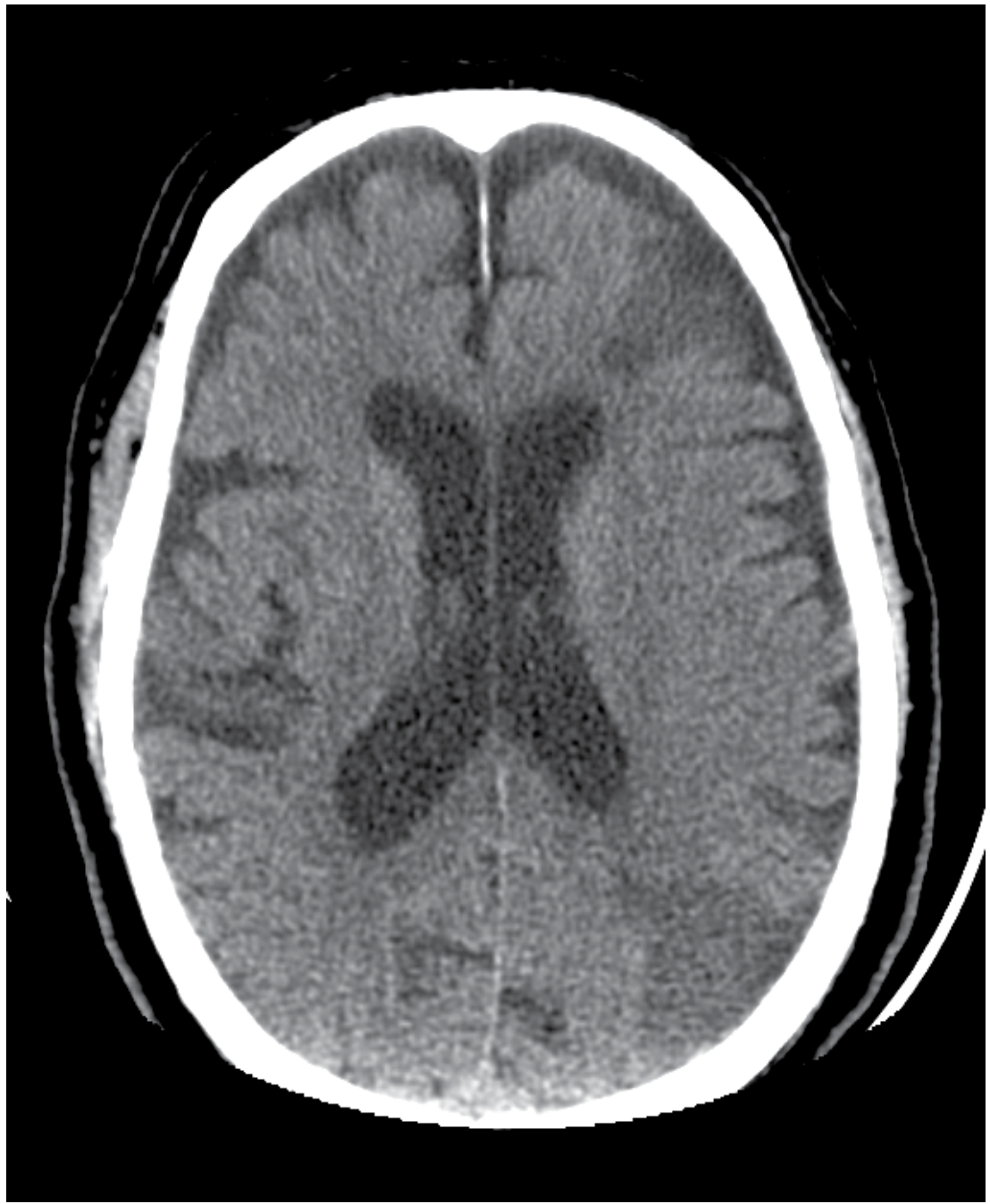

Fig. 1. Cranial CT after cardiac arrest demonstrating watershed infarction between the anterior and middle and between the middle and posterior cerebral arteries.

After transient recovery of cerebral energy metabolism, a secondary phase of apoptotic neuronal death occurs leading to a delayed injury (Lo et al., 2003). Experimental studies have suggested that delayed apoptosis can be triggered during anoxia, causing demyelination and neuronal death some time after the anoxic insult (Sheth \& Bodensteiner, 1998; Kim HY et al., 2002). The physiopathogenic mechanism of delayed lesions remains unclear although two main hypotheses have been proposed: neuronal apoptosis (Sheth \& Bodensteiner, 1998; Kim HY et al., 2002) and demyelinization (Barker et al., 1999). In DPE, the initial anoxia should be severe enough for a threshold to be exceeded triggering apoptosis, which leads to neuronal death. The demyelinization visible in cranial MRI is attributed to oligodendroglial dysfunction and attempts have been made to relate this to an arylsulphatase A deficiency (Gottfried, 1997; Barker et al., 1999). 
Carbon monoxide (CO) is an exogenous toxin which produces a unique anoxia frequently associated with delayed neurologic deterioration (Choi IS, 1983). CO possesses a great affinity for hemoglobin (over 200 times that of oxygen) and drastically displaces the oxygen content of blood, leading to prolonged hypoxia and acidosis. Cardiac toxicity and hypotension generally follow and contribute to brain damage. After $\mathrm{CO}$ poisoning, two hystopathological patterns have been reported in the literature. One pattern comprises degeneration of the cortical laminae and basal ganglia and is supposed to occur short after $\mathrm{CO}$ poisoning and the other one entails different degrees of demyelinization in the centrum semiovale, occurring in close relation to the delayed form of encephalopathy (Custodio \& Bradsford, 2004; Hori et al., 1991).

Cyanide $(\mathrm{CN})$ makes the cells of an organism unable to use oxygen through the inhibition of cytochrome $\mathrm{C}$ oxidase affecting primarily the myocardium and secondarily the brain (Beasley et al., 1998).

\section{Clinical syndromes}

Different clinical syndromes can occur after an anoxia, depending on the severity, the duration and the underlying mechanism of the hypoxic insult. Neurological syndromes occurring after anoxia are summarized in Table 1.

Blood oxygen saturation is used as an objective measurement to predict the severity of cerebral hypoxia: $95-100 \%$ saturation is considered normal, $91-94 \%$ is considered mild, 86 $90 \%$ is considered moderate and anything below $86 \%$ is considered severe (Butterworth \& Roger). It is important, though, that the phsysician bears in mind that if $\mathrm{CO}$ poisoning is suspected, blood oxygen saturation is not reliable and carboxyhemoglobine should be measured.

1) Mild sustained hypoxia

(a) Cognitive impairment

(b) Confusional states

(c) Delirium

2) Brief anoxic-ischemic events

(a) Syncope

(b) Abortive or actual generalized seizure activity

3) Sustained severe hypoxia

(a) Coma with residual neurological deficits

1. Dementia

2. Vegetative state

3. Brain death

(b) Seizure activity

(c) Watershed infarction of cerebrum, cerebellum, spinal cord

(d) Infarction distal to a pre-existing arterial stenosis or occlusion

(e) Postanoxic demyelination

Table 1. Clinical syndromes following anoxia (Caronna \& Finklestein, 1978). 


\subsection{Postanoxic coma, sequelae, and clinical prognosis}

Because of its autoregulatory capability, the brain can respond to a decrease in oxygen supply by increasing the cerebral blood flow. If this response suffices to maintain the minimum oxygen required, the subject will remain asymptomatic. When this increase is insufficient, symptoms of cerebral hypoxia will gradually develop (Butterworth \& Roger). Mild hypoxia results in less severe symptoms, including inattentiveness, difficulties with complex tasks, impaired short-term memory, and motor incoordination. If the oxygen deprivation is prolonged or severe, it can result in loss of consciousness, seizures, deep coma, cessation of brainstem reflexes, and ultimately, brain death (Safar, 1986).

The duration of anoxia necessary to provoke brain damage has not been established (Safar, 1986). There are several potentially important related factors, such as prearrest blood glucose levels, preischemic medication (aspirin and calcium channel blockers), associated hypothermia (Müllner et al., 1998), and age (Kriel et al., 1994). Neurons can tolerate approximately 20 minutes of normothermic ischemic anoxia, but cerebral recovery will be impeded by secondary brain damage after reperfusion. It is generally accepted that more than five minutes of anoxia during circulatory arrest can cause serious brain injury and result in sequelae (Safar, 1986).

After cardiac arrest, postanoxic patients remain in deep coma in which even brainstem function may be absent, and ventilation support becomes necessary. The duration of this state depends on the length of the anoxic insult, but generally, patients regain brainstem function in one to three hours. From the initial flaccidity, patients can develop decerebrate or decorticate posturing before awakening. Awakening is gradual and has different patterns. Patients who awake within 24 hours after cardiac arrest may be agitated and confused for some hours or days until cognitive functioning recovers. Arousal in other patients can take longer, and in the interim they show reflex motor posturing, grasping, eye opening and, finally, arousal. At this time, previously hidden signs secondary to focal or multifocal infarction may appear. Diffuse cortical injury mainly presents as short-term memory loss, inattention, emotional lability, hallucinations, and difficulty in the flow of thoughts, whereas basal ganglia injury manifests as parkinsonian syndrome or chorea. Throughout this process, seizures of any kind can appear (Snyder et al., 1980). Axial myoclonus or asynchronous distal limb myoclonus are common within the first 12 hours after cardiac arrest (Snyder et al., 1980). Generalized tonic-clonic seizures occasionally occur. Partial simple or complex seizures can also develop and be misdiagnosed as a prolongation of the patient's postanoxic stupor or confusional state (Wijdicks, 1995).

The prognosis after anoxic coma is difficult to ascertain, but guidelines have been designed based on a series of 210 patients (Levy et al., 1985) (Table 2).

In a more extensive review of this subject (Wijdicks et al., 2006), the following factors were cited as indicators of a poor prognosis after cardiopulmonary arrest: absent papillary light response or corneal reflexes; absent motor response or extensor response to pain on the third day in observation, and myoclonus status epilepticus. Bilateral absent cortical response on somatosensory evoked potential studies recorded three days after anoxia also indicate a bad prognosis. A burst suppression pattern on electroencephalography (EEG) or generalized epileptiform discharges predicts a poor outcome, but with insufficient prognostic accuracy. Serum neuron-specific enolase above $33 \mu \mathrm{g} / \mathrm{L}$ is also predictive of a poor outcome. 


\begin{tabular}{lll}
\hline $\begin{array}{l}\text { Time since cardiac } \\
\text { arrest }\end{array}$ & $\begin{array}{l}\text { Patients with virtually no chance } \\
\text { of regaining independence }\end{array}$ & $\begin{array}{l}\text { Patients with best chance of } \\
\text { regaining independence }\end{array}$ \\
\hline Initial examination & $\begin{array}{l}\text { No pupillary light reflex in the } \\
\text { absence of other causes }\end{array}$ & $\begin{array}{l}\text { Pupillary light reflexes present; } \\
\text { motor response decorticate or } \\
\text { decerebrate posturing; } \\
\text { spontaneous eye movements } \\
\text { conjugately roving or orienting } \\
1 \text { day }\end{array}$ \\
& $\begin{array}{l}\text { 1-day motor response no better } \\
\text { than decorticate posturing; } \\
\text { spontaneous eye movements } \\
\text { neither orienting nor conjugate; } \\
\text { roving }\end{array}$ & $\begin{array}{l}\text { withdrawal or better; 1-day eye } \\
\text { opening to noise or } \\
\text { spontaneously }\end{array}$ \\
& $\begin{array}{l}\text { 3-day motor response no better } \\
\text { than decorticate posturing }\end{array}$ & $\begin{array}{l}\text { Motor response withdrawal or } \\
\text { better; spontaneous eye } \\
\text { movements normal }\end{array}$ \\
& $\begin{array}{l}\text { No obeying commands; } \\
\text { spontaneous eye movements } \\
\text { neither orienting nor conjugate }\end{array}$ & \\
\hline week & & \\
&
\end{tabular}

Table 2. Guidelines to identify patients with a poor or favorable prognosis after cardiopulmonary arrest (Levy et al., 1985)

\subsection{Persistent vegetative state}

Some patients who achieve arousal are otherwise unaware of environmental stimuli. This tragic situation is known as persistent vegetative state (previously vegetative coma).

Patients in a vegetative state can present brainstem reflexes and sleep-wake cycles, and achieve certain behavior patterns, such as yawning, smiling, crying, sneezing, or blinking to a threat. The motor responses tend to be decorticated or decerebrate posturing - Non consistent, nonreflexive response to stimulation and following moving objects with gaze can be achieved. If this condition is maintained a month after the resuscitation, it is considered a persistent vegetative state. (American Neurological Association Committee on Ethical Affairs, 1993).

The underlying pathologic substrate of this state is diffuse prosencephalon injury with brainstem preservation. The EEG is, therefore, abnormal and shows a complete absence of cortical alpha activity, although arousal changes can be seen (Commichau, 2006). CT scans may initially show diffuse and multifocal cerebral damage and eventually the development of progressive and severe cerebral atrophy. (American Neurological Association Committee on Ethical Affairs, 1993).

\subsection{Cerebral edema}

Some, but not all patients who experience cardiopulmonary arrest have subsequent cerebral edema (Fig. 2). This is not accompanied by papilledema, and herniation is uncommon (Commichau, 2006). Autopsy study has shown that edema is more likely to be found in patients with deep coma and prolonged survival, which indicates that it might be a postnecrotic feature rather than a result of inflammation (Commichau, 2006). Intracranial 
hypertension may be more likely to develop when the hypoxic insult is due to respiratory failure. Some authors consider that this could be related to the presence of hypercapnia and acidosis previous to the cardiopulmonary arrest, but the value of these parameters as prognostic factors is poor (Müllner, 1997; Müllner 1998).

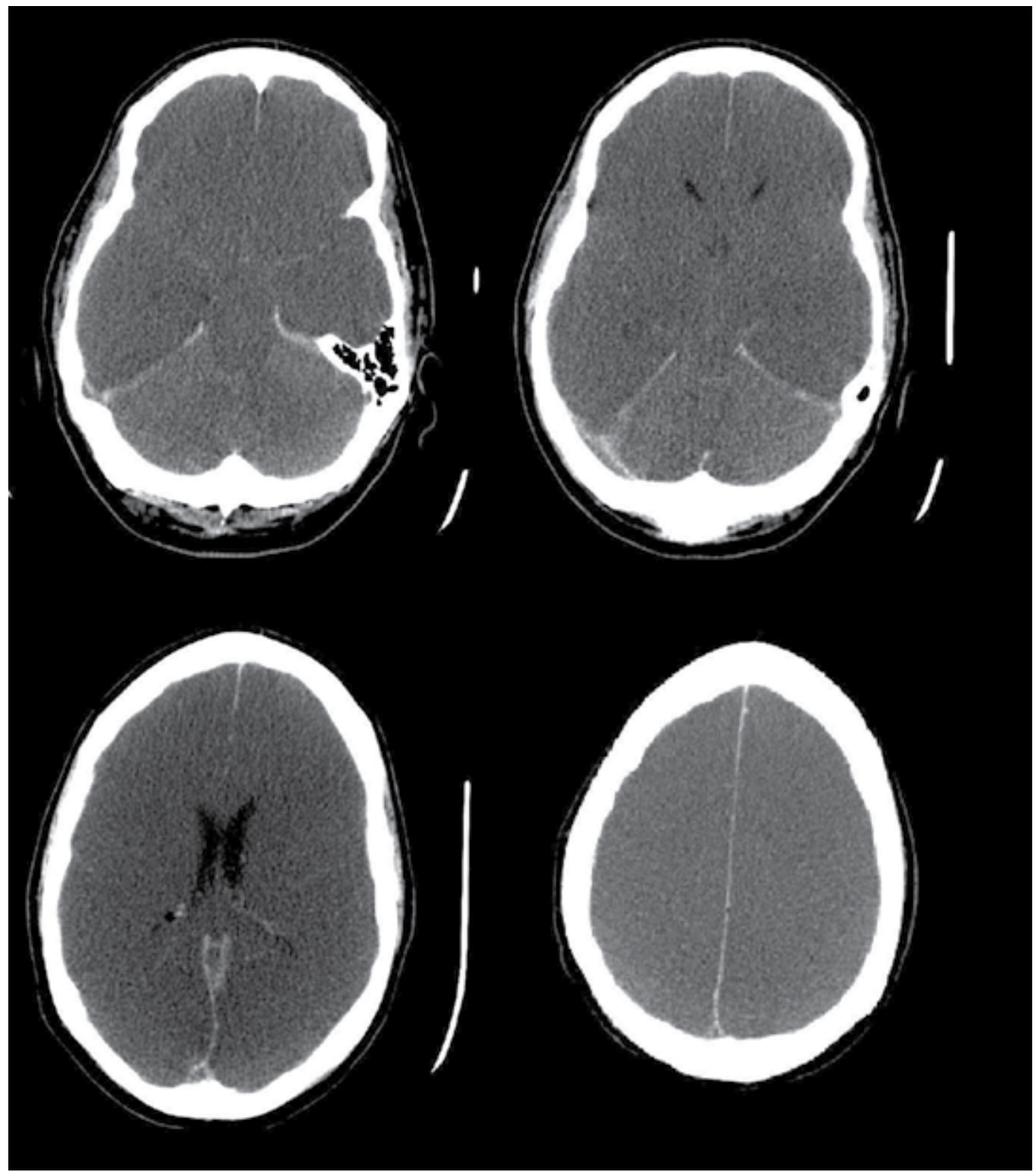

Fig. 2. Cranial CT without contrast infusion after cardiac arrest demonstrating the loss of distinction between gray and white matter throughout the cerebral hemispheres. The patient remained comatose and became vegetative. 
The early presence of high intracranial pressure secondary to cerebral edema after a cardiopulmonary arrest may predict a poor prognosis in relation to the global tissue death (Commichau, 2006). There is no evidence that treatment of this condition with; hyperventilation (ECC, 2005), corticosteroids (Jastremski et al., 1989), osmotic diuretics, barbiturate-induced coma, or ventriculostomy might be effective (Brain Resuscitation Clinical Trial I Study Group, 1986).

\subsection{Brain death}

Definition of death in the adult can be established in two different situations: 1) when there is an irreversible cessation of cardiopulmonary function and 2) when the irreversible cessation function is that of the central nervous system. Diagnosis in the former group is acquired after clinical examination. In the latter group, patients are supported artificially by mechanical ventilation, then this diagnosis becomes a difficult issue.

The ultimate and most severe consequence of anoxia is brain death, in which there is no cerebral or brainstem activity, patients cannot breathe on their own, and only blood pressure and cardiac function are preserved. The diagnosis of brain death is based on persistent absence of all cerebral and brainstem function, including a lack of response to external stimuli, and no brainstem reflexes (eg, pupillary response, and oculocephalic, corneal, and vestibular reflexes). Reevaluation of the patient in six hours is recommended, and a 24-hour observation period is needed in cases of postanoxic damage (Wijdicks, 1995). It is mandatory to rule out other causes of brain suppression in these cases. Therefore, glycemia, toxic agents, and anesthetics must be checked, and hypothermia reversed (Commichau, 2006).

The diagnosis can be supported by EEG study, which shows a complete absence of electric activity (isoelectricity), and other complimentary tests (transcranial Doppler ultrasound or radionuclide brain diffusion studies), which confirm an absence of cerebral blood flow Commichau, 2006).

The legal criteria may vary between countries, but usually, two different doctors are required to demonstrate complete absence of brain activity, and this fact can be supported by two flat EEGs taken 24 hours apart (Wijdicks, 1995).

\subsection{Posthypoxic neurologic sequelae}

After a hypoxic event, patients can develop various sequelae, including a persistent vegetative state, as mentioned above, cognitive impairment with or without extrapyramidal signs, Korsakoff syndrome, parkinsonian syndrome with cognitive impairment (mostly related to $\mathrm{CO}$ poisoning), choreoathetosis, cerebellar ataxia, intention (action) myoclonus, and seizures. When ischemia is prominent, two main syndromes are seen, visual agnosia (Balint syndrome and cortical blindness) and "man in the barrel" syndrome (severe bilateral arm weakness) Commichau, 2006).

\section{Electrodiagnostic and neuroimaging studies}

Electroencephalography is useful for diagnosing seizures and nonconvulsive status, monitoring the response to antiepileptic drugs, and assessing the patent's prognosis. 
However, EEG findings should be interpreted in keeping with the patients' clinical features, and the physician should rule out the presence of drugs, anesthetics, and previous abnormalities. Several abnormal patterns can be seen during and after cardiopulmonary arrest. The absence of detectable EEG activity indicates a poor prognosis. Several authors have attempted to grade EEG activity in postanoxic coma to predict the prognosis. In general, normal or near-normal early activity is assigned a low grade, which represents a favorable prognosis, and conversely, delta activity or a burst-suppression pattern (Fig. 3) are classified as high grades, denoting a poor prognosis (Yamashita et al., 1995). Serial EEG studies can be used to assess the prognosis according to the changes in the patient's grading.

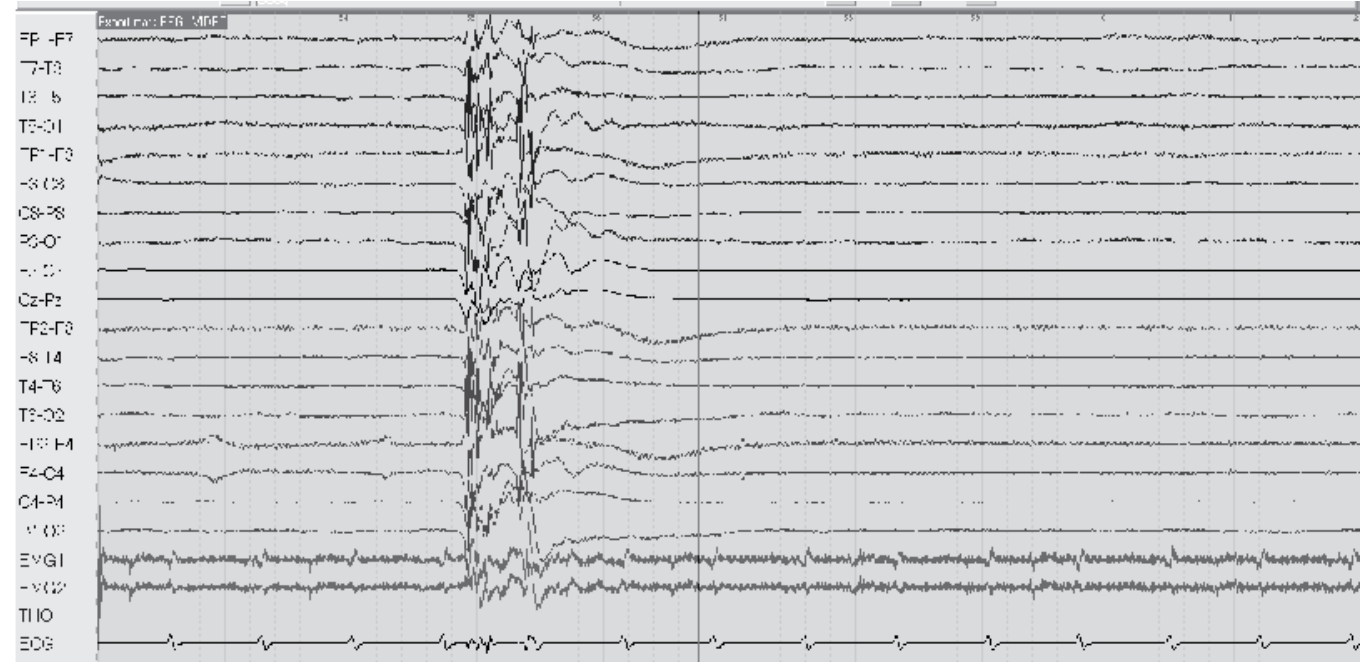

Fig. 3. EEG showing burst-suppression pattern. Courtesy of Dr. M. Veciana.

Evoked potential (EP) studies are also helpful in this regard. Absence of the early cortical components (N20-P27 complex) of somatosensory evoked potentials (SSEPs) (Fig. 4) in a comatose patient three days after cardiopulmonary arrest indicates an ominous prognosis (Wijdicks et al., 2006). To better predict the prognosis in hypoxic/anoxic encephalopathy, clinical examination, EEG, and SSEP results can be combined (Chen et al., 1996).

Cranial CT might not be useful in the first few hours after cardiopulmonary arrest. In a study of patients with nontraumatic out-of-hospital cardiopulmonary arrest, early CT (within 24 hours) was normal in $88 \%$. In the remaining patients, CT showed varying degrees of cerebral edema, usually without herniation. Only one patient showed acute subarachnoid hemorrhage (Cocchi et al., 2010). The most frequent abnormalities found after 72 hours are bilateral hypodensity of the globi pallidi, diffuse leukoencephalopathy, and hypodensities corresponding to ischemia of watershed zones (visual cortex, visual association areas, superior parietal lobules, primary sensory and motor cortices, cerebellar cortex, basal ganglia, and thoracic spinal cord) (Commichau, 2006). MRI can provide higher resolution for brain imaging, but it may be difficult to perform in a patient under ventilatory support.

Lastly, one biomarker found to be of use is serum neuron-specific enolase concentration above $33 \mu \mathrm{g} / \mathrm{L}$, which is predictive of a poor outcome (Wijdicks et al., 2006), although this cut off is currently under revision (Oddo \& Rossetti, 2011). 


\section{SEP R}

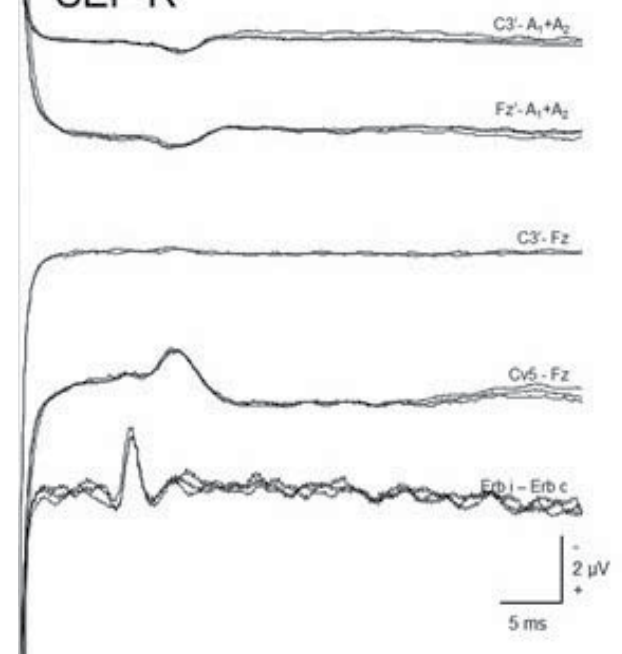

A

\section{SEP R}

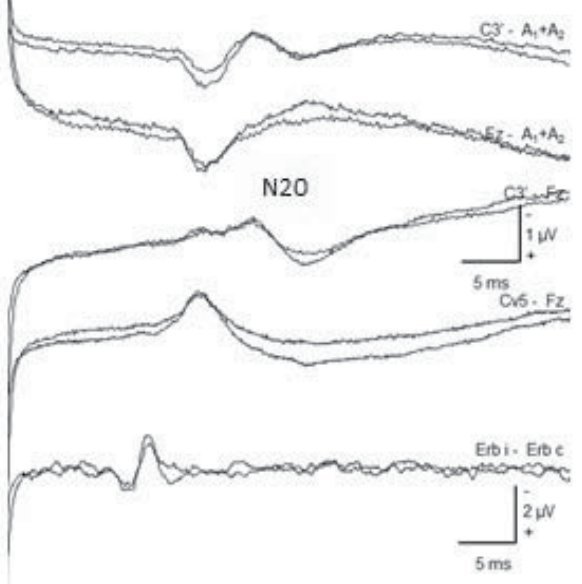

B

Fig. 4. Somatosensory evoked potential (SSEPs) registers. A: Absence of the early cortical components (N20-P27 complex). B: Normal SSEP. Courtesy of Dr. M. Veciana.

\section{Treatment}

Treatment for postanoxic encephalopathy can be divided into strategies focussed on treating the acute or chronic associated complications and approaches directed toward protecting the brain during the recovery process and preventing further hypoxic injury.

It is critical to reestablish cardiac and pulmonary function as soon as possible. Once this is accomplished, there is considerable evidence that hypothermia can be of use to avoid further brain damage (Arrich, 2010; Hypothermia after Cardiac Arrest Study Group, 2002; Bernard et al., 2002). Therefore, following hypoxic/anoxic injury to the brain, patients should undergo conventional cooling to achieve mild therapeutic hypothermia (Arrich, 2010). Other treatments such as administration of barbiturates (Brain Resuscitation Clinical Trial I Study Group, 1986), vasodilator drugs (Geocadin et al., 2008), calcium channel blockers (Brain Resuscitation Clinical Trial II Study Group, 1991), and steroids (Jastremski et al., 1989), although theoretically of use, have not shown substantial benefit. Oxygen could be beneficial within the first hours, but does not seem helpful once the blood becomes oxygenated (Brain Resuscitation Clinical Trial I Study Group, 1986). 
Efforts should be directed to maintain the balance of other factors such as cerebral perfusion, and avoid additional potential injury related to cerebral edema, intracranial hypertension, fever, and hyperglycemia. To maintain cerebral perfusion, a median arterial blood pressure of 80 to $100 \mathrm{~mm} \mathrm{Hg}$ is suggested to be beneficial within the first 24 hours after cardiac arrest (Bell et al., 2005). Monitoring by neurological examination is useful to promptly detect any worsening due to intracranial hypertension and brain herniation. Hypoxia, hypotension, and hypercapnia can worsen brain damage and should be avoided. Hyperventilation can also be harmful. Therefore, it has been suggested that comatose patients should be mechanically ventilated to achieve normocapnia (ECC, 2005). Hypoglycemia and hyperglycemia can both produce brain damage after any brain injury; hence, strict control of glycemia is needed during this period (Losert et al., 2008).

Management of seizures requires special attention, as there is evidence that strict seizure control can improve the prognosis of patients who have experienced cardiopulmonary arrest (Rossetti et al., 2009). Seizures and myoclonus are common following anoxic brain injury (Koenig \& Geocadin, 2005), although prophylactic treatment for these events is not an extended practice. Whenever seizures are suspected, EEG study should be performed, and if positive, antiepileptic drugs should be promptly administered. Axial myoclonus responds poorly to anticonvulsant therapy. Benzodiazepines and sodium valproate may be effective, although more aggressive treatment, such as neuromuscular blockade or deep sedation with midazolam or propofol, is sometimes required (Koenig \& Geocadin, 2005).

On a longer-term basis, complications such as mood disorders (depression, disinhibition, agitation, apathy, and mania) will require proper medical therapy. Cognitive impairment can be managed with cognitive stimulation, and motor disorders with rehabilitation therapy.

\section{Delayed postanoxic encephalopathy}

Lastly, and regarding our case report stated below, we will take a moment to discuss delayed postanoxic encephalopathy. DPE is a rare condition that presents after apparent recovery from acute cerebral anoxia (Custodio \& Basford, 2004; Plum \& Posner, 1992; Choi, 1986). It appears within one to three weeks after anoxia and has an insidious onset characterized by cognitive, neuropsychiatric, motor, and extrapyramidal abnormalities (Custodio \& Basford, 2004; Plum \& Posner, 1992). At onset, the patient shows apathy, confusion, attentional and memory deficits, irritability, and aggressiveness, followed by altered gait, spasticity, and extrapyramidal manifestations, which, in some cases, eventually lead to coma or death (Plum \& Posner, 1992; Choi, 1986). Some patients stabilize and are left with mild or moderate sequelae, whereas others recover completely (Custodio \& Basford, 2004).

DPE has been related to acute CO poisoning (Custodio \& Basford, 2004), connatal and postoperative anoxia, cardiopulmonary arrest of any cause (heart disease, heroin intoxication (Protass, 1976)), hypovolemic shock, hypoglycemia, and strangulation (Custodio \& Basford, 2004; Ginsberg, 1979; Hori et al., 1991). 
The main histopathological patterns seen in this syndrome are demyelination in the centrum semiovale and necrosis of the cortical laminae and basal ganglia, which correlate with cranial MRI features (Custodio \& Basford, 2004; Hori et al., 1991). On neuroimaging, DPE patients show increased signal intensity on T2-weighted and FLAIR sequences, repercussions in diffusion-weighted images and ADC mapping of the centrum semiovale and periventricular white matter, and bilateral signal abnormalities in the globi pallidi, with low signal intensity on T1-weighted and high signal intensity on T2-weighted and FLAIR images ( JH Kim et al., 2003; Inagaki et al., 1997). It is reasonable to assume that the abnormal features in the pallidal nuclei are secondary to acute damage after anoxic injury, whereas the white matter findings correspond to delayed damage.

Outcome in DPE ranges from complete recovery to death, and seems not to be related to the medication administered (Custodio \& Basford, 2004; Kwon et al., 2004). Severity and persistence of DPE sequelae have been correlated to the presence and persistence of low signal intensity in the ADC map on cranial MRI ( JH Kim et al., 2003). The outcome in our patient was favorable and coincided with an improvement in the white matter lesions on FLAIR imaging, despite the presence and persistence of low signal intensity in the ADC map.

\section{Case report}

A 34-year-old man with a history of weekly inhaled cocaine use was brought unconscious to the emergency room. He gradually regained consciousness and became responsive and alert. Neurological examination and cranial computed tomography (CT) findings were normal. Urine tests were positive for heroin and cocaine. Based on this fact and the absence of other causes of respiratory depression, a diagnosis of acute respiratory depression secondary to acute cocaine and heroin intoxication was established.

Two weeks later, the patient presented progressive deterioration of cognitive tasks and was unable to carry out daily life activities. His performance at work was impaired and he showed memory loss, attentional deficit, indifference, irritability, and dysarthria. The neurological examination revealed bilateral asterixis, anterograde memory disorder, inattention, and anosognosia; the Mini-Mental score was 17/30. Cranial CT showed bilateral hypodense areas in the globi pallid (Fig. 5).

Cranial magnetic resonance imaging (MRI) revealed high signal intensity on T2-weighted, FLAIR and isotropic diffusion-weighted images, with low ADC values (Fig. 6), consistent with diffuse supratentorial leukoencephalopathy. Neuropsychological examination disclosed a frontal dysexecutive syndrome with moderate memory deficits.

One month later, the patient and his family reported a slight subjective improvement as indicated by a greater ability to concentrate and retain new information. This was in accordance with a reduction in white matter damage on cranial MRI (Fig. 7) and an improvement in the neuropsychological examination.

One year later, the patient showed further improvement and was almost asymptomatic, with only some persistent irritability and attentional deficit. He refused additional cranial MRI and neuropsychological testing, considering himself cured. 


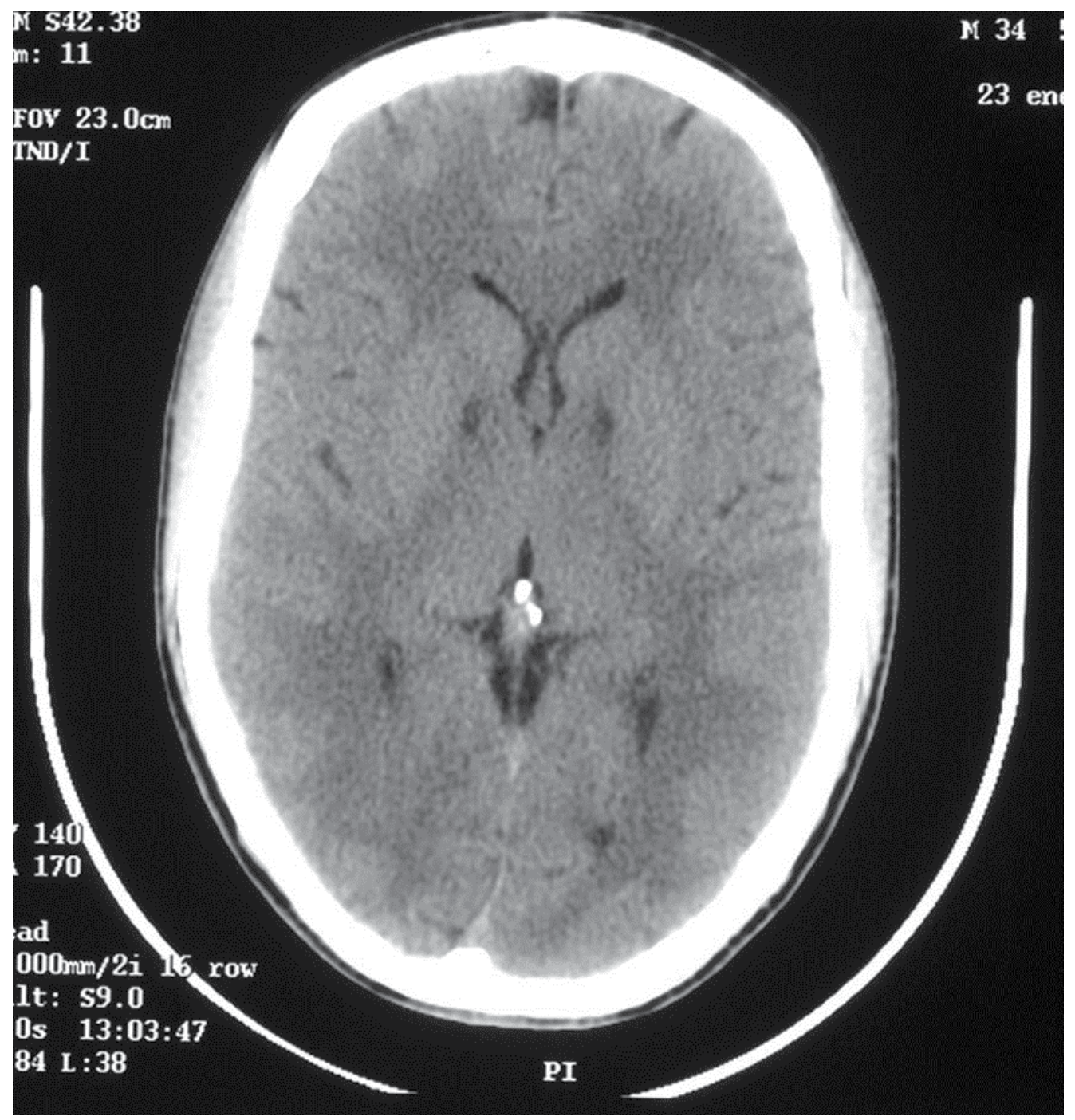

Fig. 5. Cranial CT showing bilateral hypodense areas in the globi pallid 

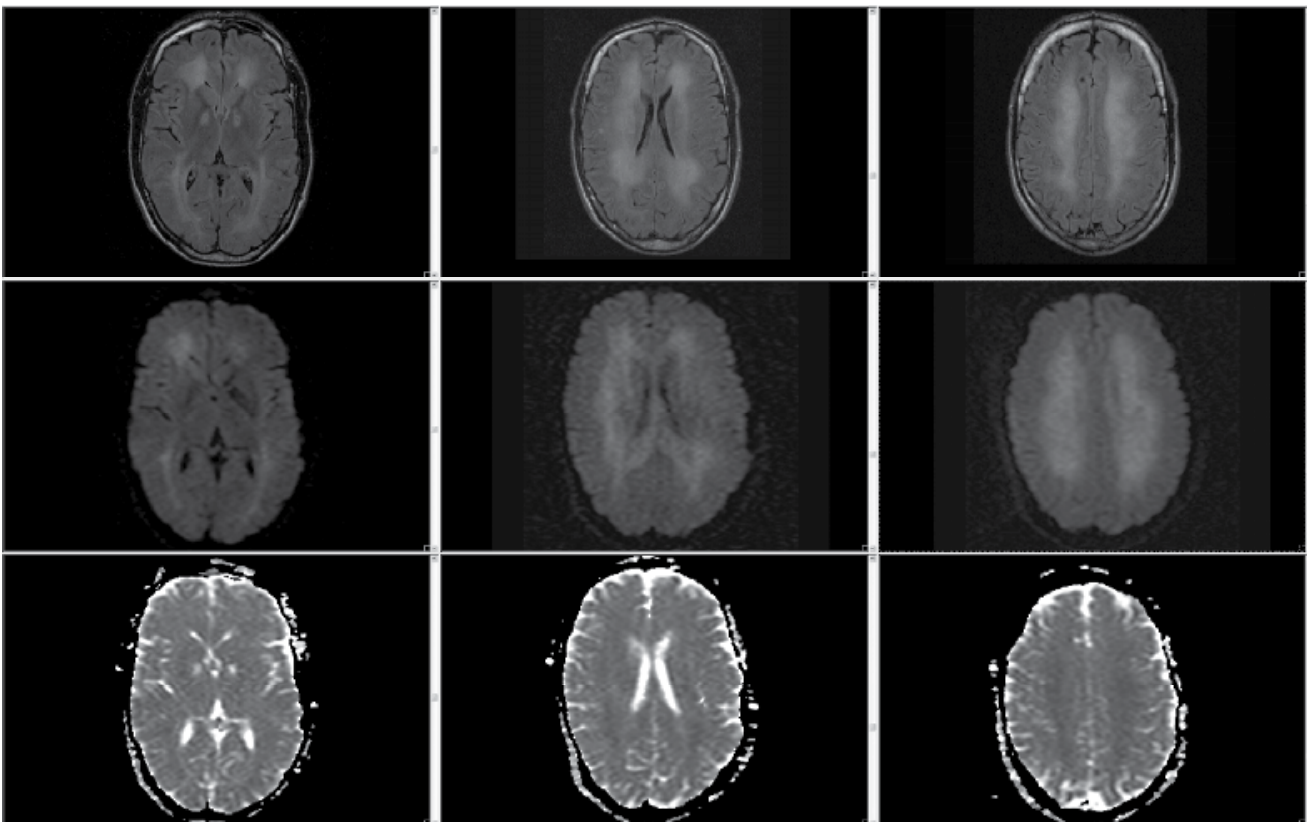

Fig. 6. Initial magnetic resonance imaging findings. FLAIR images (a) show high signal intensity areas in the bilateral periventricular white matter and pallidal nuclei. DWI imaging (b) show similar high signal intensity in the periventricular white matter, with diffuse low signal intensity in the ADC map (c).
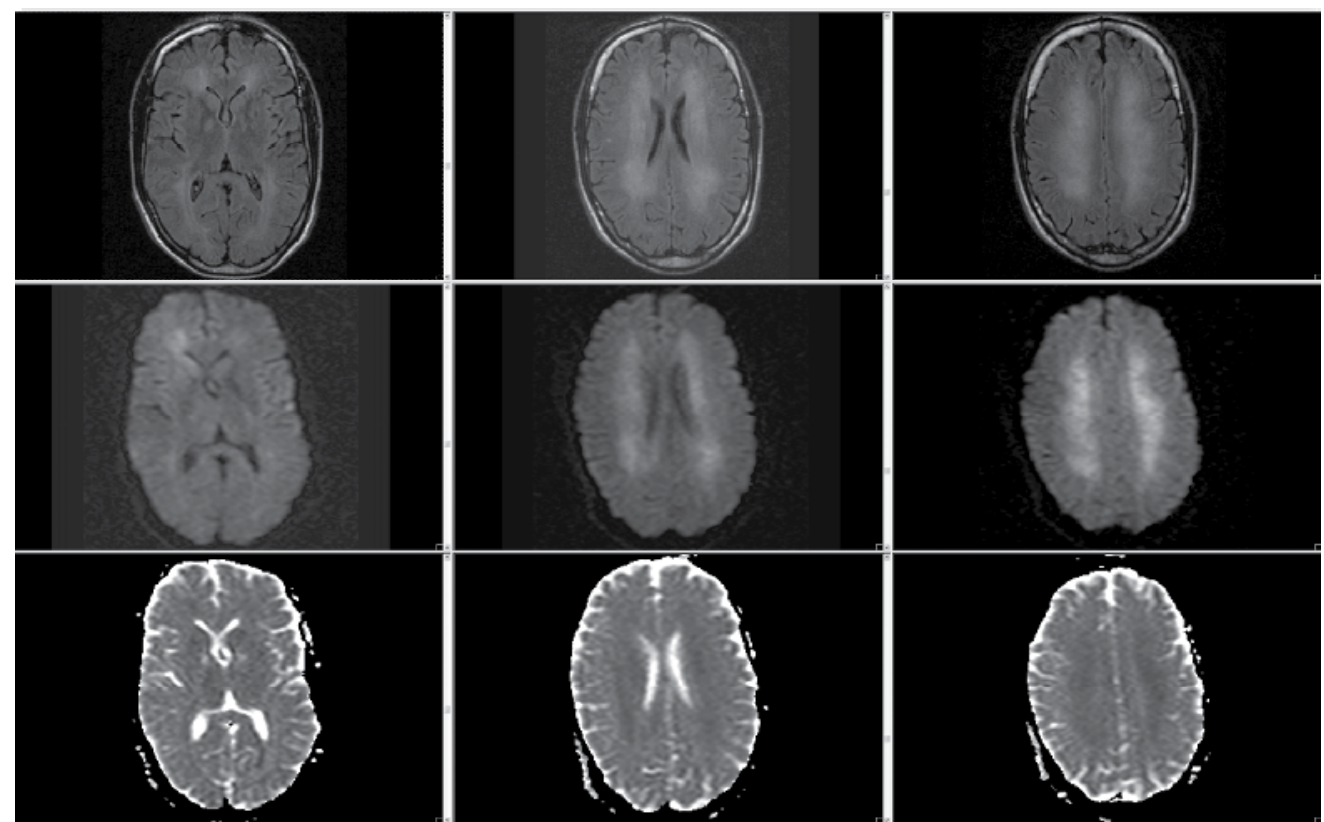

Fig. 7. Follow-up magnetic resonance, one month later, shows a reduction of white matter damage in the bilateral frontal corona radiata and pallidal nuclei (a), and persistence of DWI findings (b) with diffuse low signal intensity in the ADC map (c). 


\section{Summary}

Although huge advances have been made in intensive care medicine and cardiovascular therapy, brain injury remains the main cause of disability in patients who experience cardiopulmonary arrest. Complications are common and their treatment, of crucial importance, although a great amount of medical resources are required. The results of research in various neuroprotective strategies have been discouraging, with the exception of hypothermia, which seems to be a successful and worthwhile measure, as well as therapies focused on seizures. Efforts must be placed on creating new hospital protocols that emphasize the importance of achieving mild hypothermia within the first hours after cardiopulmonary arrest, as well as detecting and promptly treating any kind of seizure.

Regarding delayed postanoxic encephalopathy, we should suspect this syndrome in patients who have suffered respiratory depression or cardiac failure of any cause, or when a patient presents with a subacute onset of cognitive and neuropsychiatric deficits. The clinical evolution of this condition may be favorable, does not seem to depend on any medical treatment, and could be related to an improvement in white matter lesions.

\section{References}

American Neurological Association Committee on Ethical Affairs. Persistent vegetative state. Ann Neurol 1993; 33:386-390.

Arrich J, Holzer M, Herkner H, Müllner M. Cochrane corner: hypothermia for neuroprotection in adults after cardiopulmonary resuscitation. Anesth Analg 2010; 110:129.

Barker RA, Dick DJ, Cochius JI. Delayed postanoxic encephalopathy and pseudodeficiency of arylsulphatase A. J Neurol 1999;246:1103-1104.

Beasley DM, Glass WI. Cyanide poisoning: pathophysiology and treatment recommendations. Occup Med (Lond) 1998; 48:427-431.

Bell DD, Brindley PG, Forrest D, Al Muslim O, Zygun D. Management following resuscitation from cardiac arrest: recommendations from the 2003 Rocky Mountain Critical Care Conference. Can J Anaesth 2005; 52:309-322.

Bernard SA, Gray TW, Buist MD, Jones BM, Silvester W, Gutteridge G, Smith K. Treatment of comatose survivors of out-of-hospital cardiac arrest with induced hypothermia. N Engl J Med 2002; 346:557-563.

Brain Resuscitation Clinical Trial I Study Group. Randomized clinical study of thiopental loading in comatose survivors of cardiac arrest. N Engl J Med 1986; 314:397-403.

Brain Resuscitation Clinical Trial II Study Group. A randomized clinical study of a calciumentry blocker (lidoflazine) in the treatment of comatose survivors of cardiac arrest. N Engl J Med 1991; 324:1225-1231.

Butterworth, Roger F. Hypoxic Encephalopathy. In: Siegel, George J. et al. (eds.) Basic Neurochemistry: Molecular, Cellular and Medical Aspects, 6th edition, Philadelphia: Lippincott Williams \& Wilkins.

Callans DJ. Out-of-hospital cardiac arrest-the solution is shocking. N Engl J Med 2004; 351:632-634.

Caronna JJ, Finklestein S. Neurological syndromes after cardiac arrest. Stroke 1978; 9:517520. 
Chen R, Bolton CF, Young B. Prediction of outcome in patients with anoxic coma: a clinical and electrophysiologic study. Crit Care Med 1996; 24:672-678.

Choi DW, Rothman SM. The role of glutamate neurotoxicity in hypoxic ischemic neuronal death. Annu Rev Neurosci 1990; 13:171.

Choi IS. Delayed neurologic sequelae in carbon monoxide intoxication. Arch Neurol 1983; 40:433-435.

Cocchi MN, Lucas JM, Salciccioli J, Carney E, Herman S, Zimetbaum P, Donnino MW. The role of cranial computed tomography in the immediate post-cardiac arrest period. Intern Emerg Med 2010; 5:533-538.

Commichau C. (2006). Hypoxic-Ischemic encephalopathy, in Neurological therapeutics principles and practice, Noseworthy JH. (528-537), Informa Healthcare, ISBN 139781841845833.

Custodio CM, Basford JR. Delayed postanoxic encephalopathy: a case report and literature review. Arch Phys Med Rehabil 2004; 5:502-505.

Emergency Cardiovascular Care (ECC) Committee. American Heart Association Guidelines for Cardiopulmonary Resuscitation and Emergency Cardiovascular Care. Part 7.5: Postresuscitation Support. Circulation 2005; 112:84-88.

Geocadin RG, Koenig, MA, Jia X, Stevens RD, Peberdy MA. Management of brain injury after resuscitation from cardiac arrest. Neurol Clin, 2008; 26:487-506.

Ginsberg MD. Delayed neurological deterioration followinghypoxia. Adv Neurol 1979; 26:21-44.

Gottfried JA, Mayer SA, Shungu DC, Chang Y, Duyn JH. Delayed posthypoxic demyelination. Association with arylsulfatase A deficiency and lactic acidosis on proton MR spectroscopy. Neurology 1997; 49:1400-1404.

Gray WA, Capone RJ, Most AS. Unsuccessful emergency medical resuscitation - are continued efforts in the emergency department justified? N Engl J Med 1991; 325:1393-1398.

Hori A, Hirose G, Kataoka S, Tsukada K, Furui K, Tonami H. Delayed postanoxic encephalopathy after strangulation: serial neuroradiological and neurochemical studies. Arch Neurol 1991; 48:871-874.

Hypothermia after Cardiac Arrest Study Group. Mild therapeutic hypothermia to improve the neurologic outcome after cardiac arrest. N Engl J Med 2002; 346:549-556.

Inagaki T, Ishino $\mathrm{H}$, Seno $\mathrm{H}$, Umegae $\mathrm{N}$, Aoyama T. A long-term study of serial magnetic resonance images en patients with delayed encephalopathy after acute carbon monoxide poisoning. Psychiatry Clin Neurosci 1997; 51:421-423.

Jastremski M, Sutton-Tyrrell K, Vaagenes P, Abramson N, Heiselman D, Safar P. Glucocorticoid treatment does not improve neurological recovery following cardiac arrest. Brain Resuscitation Clinical Trial I Study Group. JAMA 1989; 262:3427-3430.

Kim HY, Kim BJ, Moon SY, et al. Serial diffusion-weighted MR imaging in delayed postanoxic encephalopathy. A case study. J Neuroradiol 2002;29(3):211-215.

Kim JH, Chang KH, Song IC, Kim KH, Kwon BJ, Kim HC, Kim HY, Han MH. Delayed encephalopathy of acute carbon monoxide intoxication: diffusivity of cerebral white matter lesions. Am J Neuroradiol 2003; 24:1592-1597.

Koenig M, Geocadin R. Global hypoxia-ischemia and critical care seizures. In: Varelas P, editor. Seizures in critical care. Totowa (NJ): Humana Press; 2005. p. 119-38. 
Kriel RL, Krach LE, Luxenberg MG, Jones-Saete C, Sanchez J. Outcome of severe anoxic/ischemic brain injury in children. Pediatr Neurol 1994; 10:207-212.

Kwon OY, Chung SP, Ha R, Yoo IS, Kim SW. Delayed postanoxic encephalopathy after carbon monoxide poisoning. Emerg Med J 2004; 21:250-251.

Levy DE, Caronna JJ, Singer BH, Lapinski RH, Frydman H, Plum F. Predicting outcome from hypoxic-ischemic coma. JAMA 1985; 253:1420-1426.

Lo EH, Dalkara T, Moskowitz MA. Mechanisms, challenges, and opportunities in stroke. Nat Rev Neurosci 2003; 4:399.

Longstreth WT Jr, Diehr P, Inui TS. Prediction of awakening after out-of-hospital cardiac arrest. N Engl J Med 1983; 308:1378-1382.

Losert H, Sterz F, Roine RO, Holzer M, Martens P, Cerchiari E, Tiainen M, Müllner M, Laggner AN, Herkner H, Bischof MG. Strict normoglycaemic blood glucose levels in the therapeutic management of patients within $12 \mathrm{~h}$ after cardiac arrest might not be necessary. Resuscitation 2008; 76:214-220.

Müllner M, Sterz F, Domanovits H, Behringer W, Binder M, Laggner AN. The association between blood lactate concentration on admission, duration of cardiac arrest, and functional neurological recovery in patients resuscitated from ventricular fibrillation. Intensive Care Med 1997; 23:1138-1143.

Müllner M, Sterz F, Behringer W, Schörkhuber W, Holzer M, Laggner AN. The influence of chronic prearrest health conditions on mortality and functional neurological recovery in cardiac arrest survivors. Am J Med 1998; 104:369-373.

Oddo M, Rossetti AO. Predicting neurological outcome after cardiac arrest. Curr Opin Crit Care. 2011 Jun;17(3):254-259

Peberdy MA, Kaye W, Ornato JP. Cardiopulmonary resuscitation of adults in the hospital: a report of 14,720 cardiac arrests from the National Registry of Cardiopulmonary Resuscitation. Resuscitation 2003; 58:297-308.

Plum F, Posner JB, Hain RF. Delayed neurological deterioration after anoxia. Arch Inter Med 1962; 110:18-25.

Plum F, Posner JB: Diagnosis of Stupor and Coma, 3rd ed. Philadelphia, Davis, 1980.

Protass LM. Delayed Postanoxic Encephalopathy after heroin use. Ann Int Med 1971; 74:738739.

Ropper AH, Brown RH. (2005). Adams and Victor's principles of neurology, Mc Graw Hill, ISBN 0-07-146971-0, China.

Rossetti AO, Oddo M, Liaudet L, Kaplan PW. Predictors of awakening from postanoxic status epilepticus after therapeutic hypothermia. Neurology 2009; 72:744-749.

Safar P. Cerebral resuscitation after cardiac arrest: a review. Circulation. 1986; 74:138-153.

Sheth RD, Bodensteiner JB. Delayed postanoxic encephalopathy: possible role for apoptosis. J Child Neurol 1998; 13:347-348.

Singh NC, Kochanek PM, Schiding JK, Melick JA, Nemoto EM. Uncoupled cerebral blood flow and metabolism after severe global ischemia in rats. J Cereb Blood Flow Metab 1992;12:802-808.

Snyder BD, Hauser WA, Loewenson RB, Leppik IE, Ramirez-Lassepas M, Gumnit RJ. Neurologic prognosis after cardiopulmonary arrest: III. Seizure activity. Neurology 1980; 30:1292-1297. 
Wijdicks EF, Hijdra A, Young GB, Bassetti CL, Wiebe S. Practice Parameter: Prediction of outcome in comatose survivors after cardiopulmonary resuscitation (an evidencebased review): report of the Quality Standards Subcommittee of the American Academy of Neurology. Neurology. 2006; 67:203-210.

Wijdicks EF. Determining brain death in adults. Neurology 1995; 45:1003-1011.

Yamashita S, Morinaga T, Ohgo S, Sakamoto T, Kaku N, Sugimoto S, Matsukura S. Prognostic value of electroencephalogram (EEG) in anoxic encephalopathy after cardiopulmonary resuscitation: relationship among anoxic period, EEG grading and outcome. Intern Med 1995; 34:71-76.

Young, GB. Neurologic prognosis after cardiac arrest. New England Journal of Medicine 2009; 361:605-611. 


\title{
Encephalopathy Associated with Psychotropic Drug Therapy
}

\author{
Yuji Odagaki \\ Department of Psychiatry, Faculty of Medicine, Saitama Medical University \\ Department of Psychiatry, Moro Hospital \\ Japan
}

\section{Introduction}

All therapeutic interventions are double-edged swords with benefits and adverse effects, and pharmacotherapy is not an exception. Shortly after the introduction of conventional antipsychotic drugs into clinical practice, relatively rare but serious complications with hyperthermia, muscle rigidity, autonomic instability, and disturbed mental status were recognized to develop in some patients treated with antipsychotics. This type of encephalopathy induced by the use of antipsychotics was referred to as neuroleptic malignant syndrome (NMS), and almost all physicians prescribing antipsychotics are nowadays aware of this adverse phenomenon. Another well-known type of encephalopathy associated with psychotropic drug therapy is serotonin toxicity (ST) or serotonin syndrome (SS), which is characterized by autonomic and neuromuscular symptoms and altered mental status. In contrast with the idiosyncratic nature of NMS, ST is a spectrum pathophysiological state assumed to derive from excess serotonergic neural transmission caused by serotonin-related psychotropic agents. In these two decades, pharmacotherapy with psychotropic drugs for mentally ill patients has been dramatically changed, and classical prototypal antipsychotics and antidepressants have been replaced with atypical antipsychotics and selective serotonin reuptake inhibitors (SSRIs), respectively. These newly developed psychotropic drugs are generally safer and more tolerable than older drugs. However, atypical antipsychotics are not free of the risk of development of NMS, and the explosive prevalence of SSRIs prescribed not only for depression but also for a number of psychiatric diagnoses such as anxiety, eating, impulse-control, and personality disorders may increase the incidence of ST. Therefore, these two pathological states still remain as major adverse effects of psychotropic drugs involving altered functioning of the central nervous system (CNS), to which all clinicians prescribing psychoactive drugs should pay attention. The popularity of SSRIs also increased the case reports of patients suffering from discontinuation syndrome, which sometimes includes CNS symptoms like anxiety and irritability. In this chapter, the author provides a comprehensive overview of the abovementioned adverse effects affecting the CNS function associated with psychotropic pharmacotherapy. In addition, several other pathological conditions potentially causing encephalopathic symptoms in psychiatric patients treated with psychotropic drugs, e.g., hyponatremia, valproate-induced hyperammonemia, transient splenial lesion of the corpus callosum, and so on, are also described. 


\section{Neuroleptic malignant syndrome}

\subsection{Historical background}

In the late 1950s, immediately after the introduction of antipsychotics into the clinical practice, single case reports of encephalopathic reaction to antipsychotic agents with fever, muscle rigidity, and autonomic dysfunction were already described (Kinross-Wright, 1958; Preston, 1959). In 1960s, French psychiatrists took notice of such cases and designated them as NMS (Delay \& Deniker, 1968). However, the syndrome had been thereafter reported only occasionally and little attention had been paid to the concept of NMS, especially in the United States, until Caroff (1980) reviewed 60 cases of NMS reported in the scientific literature to that time. His review article renewed interest of clinicians and researchers all over the world and proliferated the reports on the epidemiology, risk factors, symptomatology, diagnostic criteria, differential diagnoses, pathophysiology, and treatment of the syndrome. At the present time, NMS is one of the most popularly recognized complications of psychotropic pharmacotherapy and described in most standard medical textbooks.

\subsection{Epidemiology}

Along with an increase in awareness of its concept after the Caroff's review (1980), many patients diagnosed as NMS were reported throughout the world and it was recognized that NMS was not so rare than formerly supposed. In the review by Keck et al. (1991) on the epidemiology of NMS, the estimated frequency of NMS was reported to be within a wide range from $0.02 \%$ to $2.4 \%$. The high incidence of NMS reported in earlier studies from the United States is, however, evidently overestimated due to a variety of factors, e.g., retrospective study design, loose diagnostic criteria, adherence to an amorphous "spectrum concept", and clinical practices in vogue (Adityanjee et al., 1999a). Based on the more recent reports (Spivak et al., 2000; Montoya et al., 2003; Shiloh et al., 2003) as well as my own clinical experience as a psychiatrist at the front for almost three decades, the incidence of definite NMS does not appear to exceed 2-3 cases out of one thousand consecutive patients treated with antipsychotics, as long as precautionary measures and monitoring are cautiously and sufficiently employed for the early recognition and prevention of NMS.

\subsection{Clinical features}

The principal clinical manifestations of NMS are characterized by the symptoms related to the following 4 major areas: (1) hyperpyrexia, (2) extrapyramidal symptoms (EPS), (3) altered mental status, and (4) autonomic instability. Hyperthermia is prerequisite to being diagnosed as NMS (Kurlan et al., 1984; Levenson, 1985; Addonizio et al., 1987; Caroff \& Mann., 1988; Rosebush \& Stewart, 1989), with a body temperature exceeding $38^{\circ} \mathrm{C}$ in most cases, and as high as $40-41^{\circ} \mathrm{C}$ in some patients. The EPS are also noted in almost all cases reported as NMS, typically represented as muscle rigidity accompanied by tremors, which is often described as "lead-pipe" or "plastic". Other forms of EPS including focal dystonia, sialorrhea, dysphagia, dysarthria, opisthotonus, oculogyric crisis, chorea and dyskinesia are sometimes observed as accompanying symptoms. Altered mental status includes varied levels of consciousness disturbances ranging from drowsiness to coma, confusion, agitation, delirium, stupor, mutism and so on. Involvement of the autonomic system is manifested by severe tachycardia, labile blood pressure, profuse diaphoresis, dyspnea and incontinence. 
Although laboratory abnormalities of NMS are nonspecific, elevation of serum creatine phosphokinase (CPK) (>99 \% derived from muscle fraction) is noted in almost all cases, which reflects significant muscle damage during development of NMS. In severely affected cases, it often exceeds 10,000 IU/liter with a high risk of development of myoglobinuria and renal failure. Leukocytosis is also seen in almost all patients with NMS. Hepatic enzymes are occasionally elevated but less dramatically than CPK.

Diagnosis of NMS is made based on the above-mentioned clinical symptoms and laboratory findings when these abnormalities develop subsequent to the initiation of or increase in antipsychotics and other medical conditions can be excluded. The typical cases with "fullblown" symptoms appear to be diagnosed with ease if the physician is aware of the concept of NMS. Several diagnostic criteria for NMS have been proposed by different researchers (Levenson, 1985; Addonizio et al., 1986; Pope et al., 1986; Adityanjee et al., 1988; J.H. Friedman et al., 1988; Keck et al., 1989a, 1989b; Caroff et al., 1991; Nierenberg et al., 1991; Caroff \& Mann, 1993; Adityanjee et al., 1999b), and here presented in Table 1 is the research criteria for NMS described in DSM-IV-TR (Frances et al., 2000).

\begin{tabular}{l} 
A. The development of severe muscle rigidity and elevated temperature associated \\
with the use of neuroleptic medication. \\
\hline B. Two (or more) of the following: \\
(1) diaphoresis \\
(2) dysphagia \\
(3) tremor \\
(4) incontinence \\
(5) changes in level of consciousness ranging from confusion to coma \\
(6) mutism \\
(7) tachycardia \\
(8) elevated or labile blood pressure \\
(9) leucocytosis \\
(10) laboratory evidence of muscle injury (e.g., elevated CPK) \\
C. The symptoms in Criteria A and B are not due to another substance (e.g., \\
phencyclidine) or a neurological or other general medical condition (e.g., viral \\
encephalitis). \\
\hline D. The symptoms in Criteria A and B are not better accounted for by a mental disorder \\
(e.g., Mood Disorder With Catatonic Features).
\end{tabular}

Table 1. Research criteria for NMS described in DSM-IV (Frances et al., 2000)

\subsection{Precipitating agents}

Virtually all classes of dopamine $\mathrm{D}_{2}$ receptor antagonists have been associated with NMS. This includes not only neuroleptics in a narrow sense (either typical or atypical antipsychotics) prescribed for the control of psychotic symptoms, but also several classes of drugs with the potential to block $\mathrm{D}_{2}$ receptors in CNS used as antidepressants, antiemetics, anesthetics, and sedatives (e.g., Robinson et al, 1985; L.S. Friedman et al., 1987; Taylor \& Schwartz, 1988; Madakasira, 1989; Spirt et al., 1992; Chan-Tack, 1999). 
Aside from cases with NMS associated with the use of antagonists for dopamine $\mathrm{D}_{2}$ receptors, the clinical features identical or closely resemblant to NMS have been occasionally reported to develop during the course of the treatment with antiparkinsonian drugs, especially after the withdrawal or reduction of them, in patients with Parkinson's disease (Henderson \& Wooten, 1981; Toru et al., 1981; J.H. Friedman et al., 1985; Y. Yamawaki \& Ogawa, 1992; Serrano-Dueňas, 2003; Takubo et al., 2003). In these cases, it may be inappropriate to use the term NMS since the patients were not receiving "neuroleptics" (Addonizio et al., 1987), and some strict researchers refer to such cases as "NMS-like state" (Toru et al., 1981), "malignant dopaminergic syndrome" (Serrano-Dueňas, 2003), or simply as "malignant syndrome" (Takubo et al., 2003). Irrespective of how it is called, the underlying pathophysiology to precipitate the clinical course in these patients is supposed to be dopaminergic hypofunction in CNS, which is identical to that in the patients with NMS induced by the use of antipsychotics. Therefore, it appears acceptable to consider the episode fulfilling the clinical features of NMS in patients with Parkinson's disease as a variant of NMS, even if it is not induced by the use of neuroleptics.

The similar syndromes have also been reported to be caused by numerous other classes of drugs with different mechanisms of action [e.g., lithium, clomipramine, nortryptiline, SSRIs, benzodiazepines] (Kellam, 1987a; Ananth et al., 2004a, but see also Assion et al., 1998), and even in the absence of any pharmacotherapeutic intervention (the so-called lethal catatonia), and thus some researchers have commented that NMS is a misnomer (Singh \& Maguire, 1987; Brennan et al., 1988). They proposed novel names such as "iatrogenic malignant syndrome" (Singh \& Maguire, 1987) or "pyrexial catatonia" (Kellam, 1987b) instead of NMS. Heyland \& Sauvé (1991) suggested a new label "drug-induced central hyperthermic syndrome" for such cases. In my opinion, however, these designations are too vague as a diagnosis in clinical settings, and it should be avoided to broaden the concept of NMS to too much extent.

\subsection{Risk factors}

As shown in Table 2, there had been many potential risk factors for NMS identified or postulated in numerous reviews, case series, and reports published until 1989 (Keck et al., 1989a). Among them, demographic items such as sex, age, and psychiatric diagnosis are not of essential importance as risk factors (Caroff \& Mann, 1993). It should be kept in mind that NMS can develop regardless of sex, age, and psychiatric diagnosis, when antipsychotic agents are administered to a patient. Case-controlled studies (Keck et al., 1989a; Berardi et al., 1998; Viejo et al., 2003) have indicated psychopathological features such as agitation, confusion, disorganized behavior, and catatonia, as well as pharmacological features such as higher neuroleptic dose at greater rates of dose increase and parenteral neuroleptic injections, as risk factors. It has been reported that antecedent existence of EPS including akathisia is also a risk factor of NMS (Berardi et al., 1998). NMS occurs independent of climate and ambient conditions (Caroff \& Mann, 1993), and Viejo et al. (2003) were unable to find a significant difference in environmental temperature at onset of clinical symptoms of NMS between the cases and matched-controls. Considering various cases of NMS reported to develop during heat waves (Shalev et al., 1988; Fitzgerald et al., 1997), however, adverse climate conditions with high temperature and excessive humidity may be important in some cases to trigger the development of NMS. High serum CPK levels during psychotic episodes may also be a risk factor for NMS (Hermesh et al., 2002). 


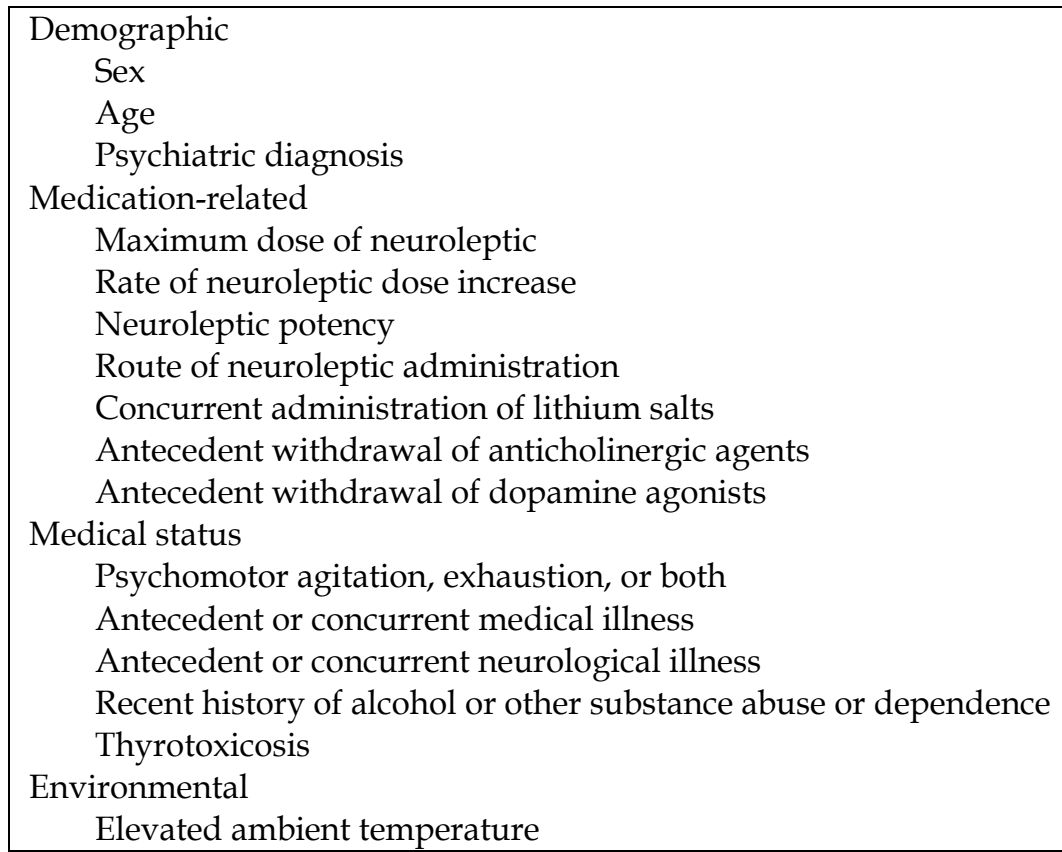

Table 2. Risk factors for NMS identified or postulated until the report by Keck et al. (1989a)

Familial occurrence of NMS has been reported occasionally (Deuschl et al., 1987; Otani et al., 1991; Ziegenbein et al., 2006), suggesting that a predisposition to NMS is genetically governed in some cases. Extreme caution should be then paid to a patient with a family history of NMS, to whom it is necessary to initiate pharmacotherapy with antipsychotics.

\subsection{Clinical course and outcome}

Although the reported duration of exposure to antipsychotics prior to the development of NMS is extremely varied, the majority of cases occur within 1 to 2 weeks after the initiation of an offending drug (Shalev \& Munitz, 1986; Addonizio et al., 1987; Caroff et al., 1988). Though nonspecific, the following signs and symptoms have been reported to precede NMS in some cases: unexpected changes in mental status, particularly obtundation or new-onset catatonia; episodic tachycardia, tachypnea, or hypertension; incontinence; low-grade temperature elevations; dysarthria, dysphagia, diaphoresis, sialorrhea, rigidity, myoclonus, tremor or other EPS unresponsive to antiparkinsonian agents; and unexplained elevation in serum CPK (Caroff et al., 1991; Caroff \& Mann, 1993). Since almost all of these are themselves constituent symptoms of NMS (see above), it may be possible to diagnose a patient with such prodromal or early symptoms as NMS, depending on the diagnostic criteria of NMS adopted. At any rate, it is practically very important to be aware of sequential progression of symptoms of NMS in order to facilitate prompt recognition and interventions to abort the symptoms in its incipient stage. Analysis of the temporal sequence of the four predominant clinical features of NMS has indicated that either altered mental status or muscle rigidity precedes hyperthermia and autonomic dysfunction in the majority of fulminant NMS cases, most typically with the following order: (1) changes in mental status; (2) muscle rigidity; (3) hyperthermia; and (4) autonomic dysfunction (Velamoor et al., 
1994). Woodbery \& Woodbery (1992) proposed five discrete stages toward the progression of NMS, from stage I or drug-induced parkinsonism to stage V (the severest form of fulminant NMS). This spectrum-based concept of NMS (Fig. 1) may be practically useful to comprehend the progression of symptoms, at least in typical cases (Odagaki, 2009).

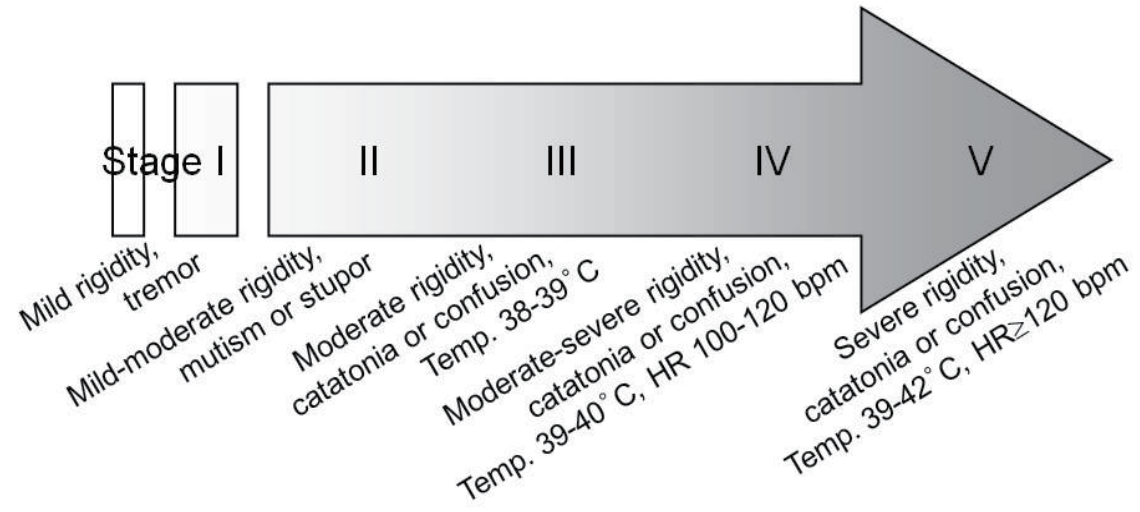

Fig. 1. The typical progression of symptoms of NMS from the mildest stage I (drug-induced parkinsonism) through stage V or the severest form of NMS (Odagaki, 2009). Stages I-V are originally proposed by Woodbury and Woodbury (1992) and adapted by Strawn et al. (2007).

Once antipsychotics are stopped, NMS is self-limited barring complications, with the duration to resolution of NMS up to two weeks in the majority of cases (Shalev \& Munitz, 1986; Addonizio et al., 1987; Caroff et al., 1988). The notion that prompt withdrawal of the offending agent at early stage can prevent progression to definite NMS has been corroborated by the study reported by Shiloh et al. (2003).

The clinical course of NMS can be modified by concurrence of many complications. Although most medical problems are reported as complications (Addonizio et al., 1987), one of the most common and serious complications of NMS is acute renal failure caused by rhabdomyolysis (Levenson, 1985). As it has been reported that renal failure and myoglobinemia are associated with a significantly higher risk of mortality in NMS (Shalev et al., 1989), careful monitoring is necessary as to serum CPK levels, myoglobinemia /myoglobinuria, and renal functions throughout the course of NMS. As impressed with its designation, NMS is potentially lethal (Kellam, 1987a). The mortality rate was, however, reported even in the late 1980s to be apparently declined as compared with the former reports, and the overall rate of mortality for patients without concurrent organic diseases was reported as less than $10 \%$ since 1984 (Shalev et al., 1989). The survey on NMS cases conducted in Japan also showed a clear chronological decline of mortality rate, which reached below $10 \%$ in the latest years of the survey (S. Yamawaki et al., 1990).

Although persistent, long-term clinical sequelae of NMS were historically considered rare, there have been substantial case reports with deficits related to complications or severity of the syndrome, particularly hypoxia or hyperthermia. As neuropsychiatric sequelae of NMS, Adityanjee et al. (2005) raised CNS dysfunctions manifested as cognitive impairment or as 
parkinsonian syndrome, peripheral neurologic impairment such as peripheral neuropathy, and psychological impairment including severe depression.

\subsection{Differential diagnosis}

Differential diagnosis is of prime importance, because NMS constituted with nonspecific symptoms and laboratory abnormalities is a diagnosis of exclusion. The disorders listed in Table 3 (Strawn et al., 2007) should be considered as differential diagnoses.

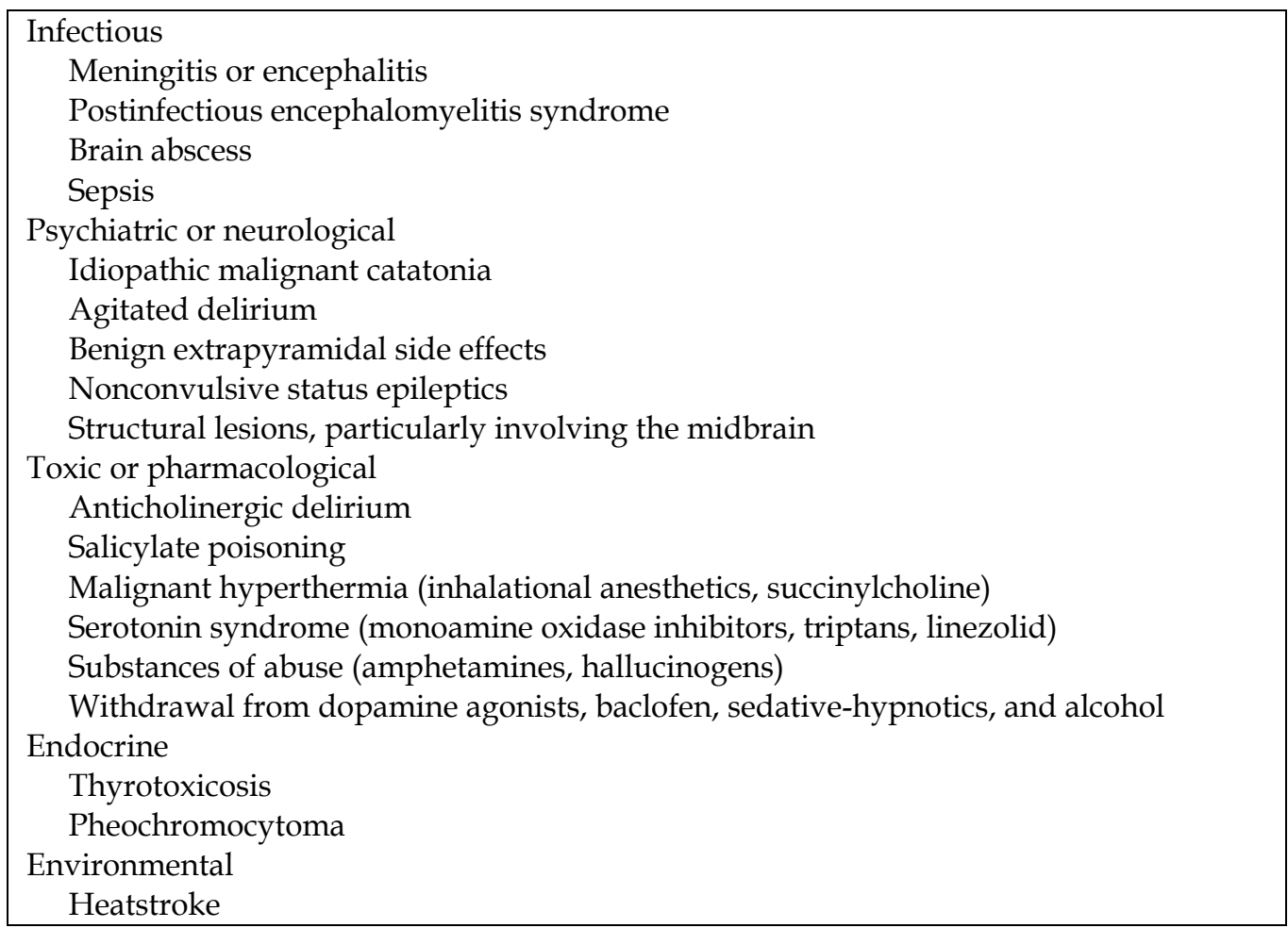

Table 3. Differential diagnosis of NMS (Strawn et al., 2007)

Among these, idiopathic malignant (lethal) catatonia and SS are of special importance from the practical as well as theoretical viewpoints. Idiopathic malignant catatonia, a lifethreatening febrile neuropsychiatric syndrome that had been described well several decades before the advent of antipsychotic drugs, is clinically quite similar to, and often indistinguishable from, NMS (Mann et al., 1986). Some researchers indeed regard NMS as a drug-induced iatrogenic form of malignant catatonia with the same underlying pathophysiology (Mann et al., 1986; White, 1992; Fink, 1996a; Carroll \& Taylor, 1997; Fricchione et al., 2000). On the other hand, Castillo et al. (1989) emphasized the importance of differentiating these two syndromes clinically. In response to the editorial by Fink (1996a), Northhoff (1996) also contributed a correspondence against the notion that NMS and catatonia were variants of the same disorder. The similar debate has been held on the discrimination between NMS and SS (Fink, 1996b; Kontaxakis et al., 2003; Odagaki, 2009; Steele et al., 2011). It is beyond the scope of this article to have a detailed discussion on the 
possibility that NMS belongs to a spectrum of the same disorder that contains catatonia and/or SS. Nevertheless, clinicians should keep an effort to differentiate NMS from other pathological conditions as long as there may be some differences in etiology, pathophysiology, and treatment.

\subsection{NMS associated with atypical antipsychotics}

With widespread use of atypical antipsychotics in recent years, NMS induced by these drugs has attracted much attention of clinicians and researchers (Caroff et al., 2000; Farver, 2003; Ananth et al., 2004b; Trollor et al., 2009). At the same time, great interest has also been taken in the concept of "atypical NMS", which is often presumed to be more associated with atypical antipsychotics than with conventional drugs (Picard et al., 2008). Though still controversial, the review of Trollor et al. (2009) suggests that NMS associated with atypical antipsychotics manifests in a typical manner, with one notable exception of clozapineinduced NMS which appears to be less associated with EPS. It should be borne in mind that even the atypical antipsychotics have the potential to precipitate the patient to NMS, as long as they are dopamine $\mathrm{D}_{2}$ receptor antagonists.

\subsection{Pathophysiology}

Although the precise pathophysiological mechanisms underlying NMS are still unknown, antipsychotic-induced dopamine $\mathrm{D}_{2}$ receptor blockade in the striatum and hypothalamus likely plays a pivotal role, at least at its initial stages, for the development of NMS (Mann et al., 2000). In addition to dopaminergic dysfunction, complex alterations in functioning of different neurotransmitters in various brain regions, e.g., norepinephrine, serotonin, $\gamma$ aminobutyric acid (GABA), and glutamate, are believed to be involved in the expression of multifarious clinical features of NMS (Ananth, 2004a). Through complex neurochemical as well as structural interactions among multiple neural transmission systems, a vicious circle likely develops to end a failure of homeostasis, irreversibly and lethal in some cases, that is involved in maintenance of consciousness and regulation of extrapyramidal motor function, body temperature, and autonomic functions. Especially in the fulminant cases with severe autonomic instability, uncoordinated hyperactivity of the peripheral sympathoadrenal systems, culminating in an end-stage hypermetabolic syndrome, is hypothesized (Feibel \& Schiffer, 1981; Gurrera \& Romero, 1992; Gurrera, 1999).

\subsection{Treatment}

One of the most important, and probably the most effective measures to avoid "malignant" sequence of the syndrome is a removal of the potentially offending drug(s) as prompt as possible. Although the treatment of NMS should be individualized for each patient depending on the clinical features and situations, the mainstay is good supportive medical care against extreme hyperthermia, dehydration, metabolic acidosis, nutritive and electrolyte imbalance, and occurrence of the complications including cardiorespiratory and/or renal failures, aspiration pneumonia, rhabdomyolysis, and coagulapathies. Several specific treatments recommended for management of NMS (Table 4) should be taken into consideration as additional therapeutic options, based on the severity and clinical course (Sakkas et al., 1991a, 1991b; Davis et al., 2000). However, it should be stressed that these 
specific remedies for NMS are derived from empirical reports or theoretical grounds, rather than based on prospective, randomized controlled studies (Susman, 2001). For instance, conflicting results have been shown even about the usefulness of dantrolene and bromocriptine, the two most well established drugs for treatment of NMS (Rosenberg \& Green, 1989; Rosebush et al., 1991; Reulbach et al., 2007).

\begin{tabular}{|cl|}
\hline Treatment with & Comments \\
\hline Anticholinergics & $\begin{array}{l}\text { Still controversial, but may be useful in mild or early cases } \\
\text { to help attenuate EPS (Woodbury and Woodbury, 1992) } \\
\text { May be useful when catatonic symptoms predominate } \\
\text { (Francis et al., 2000) }\end{array}$ \\
$\begin{array}{c}\text { Dopaminergic agonists } \\
\text { Bromocriptine }\end{array}$ & $\begin{array}{l}\text { One of the most well established pharmacologic agents for } \\
\text { NMS (Rosenberg \& Green, 1989; Sakkas et al., 1991a, 1991b) }\end{array}$ \\
Levodopa & $\begin{array}{l}\text { Successful cases reported (Harris et al., 1987; Sakkas et al., } \\
\text { 1991a; Nisijima et al., 1997) }\end{array}$ \\
Amantadine & $\begin{array}{l}\text { Its use associated with a statistically significant decrease in } \\
\text { mortality (Sakkas et al., 1991a, 1991b) }\end{array}$ \\
Dantrolene & $\begin{array}{l}\text { One of the most well established pharmacologic agents for } \\
\text { NMS (Rosenberg \& Green, 1989; Sakkas et al., 1991a, 1991b) }\end{array}$ \\
Electroconvulsive & Should be considered when pharmacotherapy has failed or \\
therapy (ECT) & when idiopathic malignant catatonia cannot be ruled out \\
& (Davis et al., 1991; Trollor \& Sachdev, 1999) \\
\hline
\end{tabular}

Table 4. Specific therapeutic remedies proposed for treatment of NMS

\section{Serotonin toxicity (serotonin syndrome)}

\subsection{Historical background}

The term "serotonin syndrome (SS)" was originally derived from the experimental behavioral model in rodents (Jacobs, 1976; Gerson \& Baldessarini, 1980), and introduced into clinical medicine in 1982 for the first time to describe the toxic reactions to the coadministration of a monoamine oxidase inhibitor (MAOI) clorgyline and a tricyclic antidepressant (TCA) clomipramine in two obsessive-compulsive disorder patients (Insel et al., 1982). In fact, however, many similar case reports date back to 1950s (e.g., Mitchell, 1955), the era of prevailing use of MAOIs such as isoniazid and iproniazid as antidepressants or antituberculous drugs (Gillman, 1998). The first review article on SS, based on the summary of 12 clinical reports between 1982 and 1990 (Sternbach, 1991), was instrumental in popularizing the syndrome to physicians, and awareness of SS as a distinct clinical entity increased the number of reported cases diagnosed as SS in the following two decades.

As pointed out by Isbister \& Buckley (2005), the term SS is often equivocally used and the clinical literature on this subject is sometimes inconsistent and confused. In the present article, the term "serotonin toxicity (ST)" is generally used to refer to the clinical state with any signs or symptoms reasonably attributable to excess serotonin caused by serotonergic agents. 


\subsection{Clinical features and diagnosis}

According to the conventional definition based on relatively severe cases reported in the literature (Sternbach, 1991), SS can be described as a clinical triad of (1) mental-status changes, (2) autonomic hyperactivity, and (3) neuromuscular abnormalities. The diagnostic criteria for SS suggested by Sternbach (1991) are shown in Table 5.

A. Coincident with the addition of or increase in a known serotonergic agent to an established medication regimen, at least three of the following clinical features are present:

1) mental status changes (confusion, hypomania)

2) agitation

3) myoclonus

4) hyperreflexia

5) diaphoresis

6) shivering

7) tremor

8) diarrhea

9) incoordination

10) fever

B. Other etiologies (e.g., infectious, metabolic, substance abuse or withdrawal) have been ruled out.

C. A neuroleptic had not been started or increased in dosage prior to the onset of the signs and symptoms listed above.

Table 5. Suggested diagnostic criteria for SS (Sternbach, 1991)

In practice, the signs and symptoms of ST range from tremor and diarrhea in mild cases to delirium, neuromuscular rigidity, and excessive hyperthermia in life-threatening cases, depending on the extent of serotonin excess. The clinical sequelae of serotonin excess are best thought of as a spectrum of toxicity, rather than a defined clinical entity (syndrome) with clear prognostic importance (Dunkley et al., 2003). Randomski et al. (2000) divided the cases into: (1) mild state of serotonin-related symptoms; (2) serotonin syndrome (full-blown form); and (3) toxic states (Fig. 2).

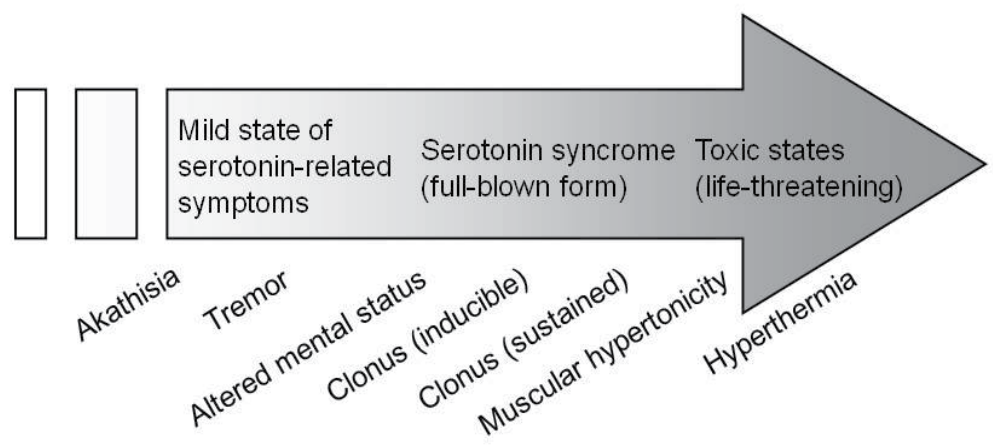

Fig. 2. Spectrum concept of ST (Adapted from Boyer \& Shannon, 2005), with three clinical stages designated by Radomski et al. (2000). 
To assess the severity of ST, the scale developed by Hegerl et al. (1998) may be useful. When adapted to the depressed patients treated with paroxetine, it was positively correlated with paroxetine concentrations and inversely correlated with auditory evoked potential (an indirect measure of serotonergic activity). Their scale consists of the following nine items each rated from 0 to 3: agitation, disorders of orientation, myoclonus, hyperreflexia, tremor, dizziness, hyperthermia, sweating, and diarrhoea.

Dunkley et al. (2003) analyzed the clinical data in 2222 consecutive cases of self-poisoning with serotonergic drugs, and found that several clinical features were associated with the diagnosis of SS. They developed the diagnostic decision rules for ST (Fig. 3), based on the results of their analysis as well as by including muscular hypertonicity and hyperthermia, both characteristic symptoms of the severe, life-threatening SS cases.

The onset of symptoms is usually rapid, with clinical findings often occurring within minutes, and in most cases within 24 hours, after initial use, an overdose, or a change in dosing of the offending drug(s) (Mason et al., 2000). It should be noted that the syndrome is often misdiagnosed initially, especially as exacerbation of psychiatric disorder with anxiety or agitation (MacKay et al., 1999; Attar-Herzerg et al., 2009). Patients with mild manifestations may present with subacute or chronic symptoms, whereas severe cases may progress rapidly to death (Sporer, 1995; Boyer \& Shannon, 2005). The most notorious example of such fatal patients is the 'Libby Zion' case (Asch \& Parker, 1988). Other severe complications of SS include seizures, disseminated intravascular coagulation, respiratory failure, severe hyperthermia, ventricular arrhythmia, and rhabdomyolysis (Sporer, 1995). Milder cases show resolution of the symptoms typically within 24 hours once the offending agents are discontinued (Sternbach, 1991).

In the presence of a serotonergic agent:

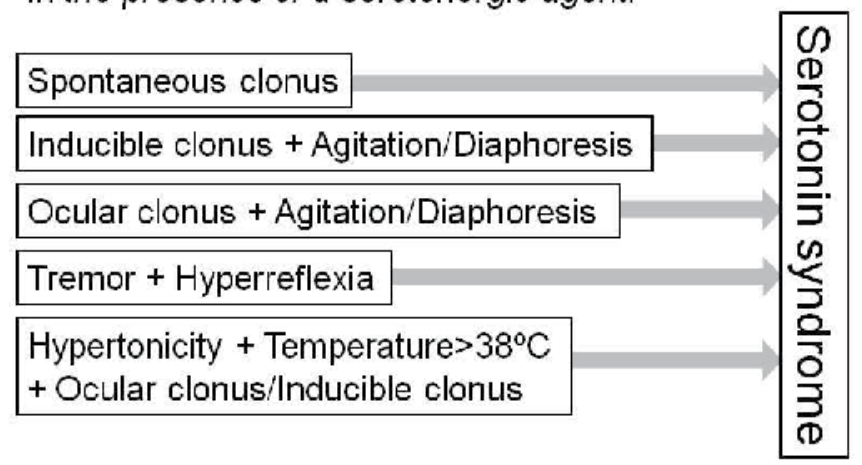

Fig. 3. Diagnostic criteria for SS proposed by Dunkley et al. (2003).

There are no confirmatory or specific laboratory tests available for the diagnosis of SS. Laboratory tests are best used to identify potential complications and assist in overall patient care. Elevated CPK has been reported in 18 \% (Mills, 1997) or 34 \% (Keck \& Arnold, 2000) of the cases, usually associated with muscle rigidity and rhabdomyolysis. Electroencephalograms (EEG) may show general slowing, consistent with diffuse encephalopathy (Mills, 1997). Highly abnormal EEG findings, such as (poly-)spikes and waves (Lejoyeux et al., 1992) and pronounced triphasic waves (Dike, 1997), were also reported in patients with SS. 


\subsection{Precipitating agents}

Virtually all medications that potentiate serononergic neurotransmission in the CNS have been reported in association with the cases of SS (Keck \& Arnold, 2000), either alone or in combination. As shown in Table 6, the most common drug combinations causing the SS are MAOIs and antidepressants (SSRIs or TCAs) (Sporer, 1995). The severity of ST precipitated by the offending drug(s) is predictable well according to the serotonin levels measured in animal experiments (Gillman, 2006). The highest serotonin levels determined by microdialysis studies is achieved by the combination of MAOI and serotonin reuptake inhibitor (SRI) (100-fold as compared with baseline), followed by MAOI plus L-tryptophan (15-fold), MAOI alone (10-fold), moclobemide plus 3,4-methylenedioxymethamphetamine (MDMA) (10-fold), MDMA alone (5-fold), SSRI alone (3-fold), venlafaxine alone (3-fold), moclobemide alone (1.5-fold), TCA alone (1.25-fold), and mirtazapine alone (1.2-fold). Although mirtazapine alone stands at the lowest risk for ST, there has been a case report of severe SS induced by mirtazapine monotherapy (Hernández et al., 2002).

\begin{tabular}{|ll|}
\hline Drug combination & Number of cases \\
\hline MAOI + Pethidine (Meperidine) & 9 \\
MAOI + Dextromethorphan & 4 \\
MAOI + Tryptophan & 9 \\
Reversible MAOI + TCA & 2 \\
Reversible MAOI + SSRI & 5 \\
MAOI-B + SSRI & 6 \\
MAOI-B + TCA & 2 \\
MAOI + SSRI & 24 \\
MAOI + TCA & 16 \\
SSRI + Dextromethorphan & 1 \\
SSRI + Tryptophan & 5 \\
SSRI + Lithium & 1 \\
SSRI + Pentazocine & 1 \\
Buspirone + Trazodone & 1 \\
TCA + Lithium & 1 \\
Bromocriptine + Levodopa & 1 \\
Phenelzine + Trazodone + Dextropropoxyphene & 1 \\
Clomipramine & 1 \\
MDMA & 2 \\
Fentanyl + Sertraline & 1 \\
Sertraline & 1 \\
Ademetionine (S-Adenosyl methionine) + Clomipramine & 1 \\
\hline
\end{tabular}

Table 6. List of the agents reported to cause SS (Sporer, 1995)

\subsection{Differential diagnosis}

The differential diagnosis of SS involves disorders producing cognitive and behavioral, neuromuscular, and autonomic nervous system dysfunction with or without hyperthermia (Keck \& Arnold, 2000), such as anticholinergic poisoning, malignant hyperthermia, and NMS (Boyer \& Shannon, 2005). SS can be confused with NMS, particularly when both 
serotonergic and neuroleptic drugs had been used concomitantly in a patient showing severe life-threatening symptoms and/or complications described before. The following features are raised by Gillman (1999) for differentiation between the two syndromes: NMS, slow onset (days to weeks) and slow progression over 24-72 hours in association with neuroleptics versus SS, both rapid onset and rapid progression (minutes to hours) in association with a combination of serotonergic drugs; NMS, bradykinesia and lead pipe rigidity versus SS, hyperkinesias and clonus; and NMS, an idiosyncratic reaction to therapeutic dosages versus SS, a manifestation of toxicity (usually to a combination of drugs) to which everyone is liable. The differential diagnosis between SS and NMS can be further complicated when atypical antipsychotics are included in the prescription, because some of atypical antipsychotics are $5-\mathrm{HT}_{1 \mathrm{~A}}$ receptor agonist by themselves (Odagaki, 2009).

\subsection{Pathophysiology}

It has been well accepted that excess serotonergic signal transduction in CNS, resulting mostly from increase in serotonin concentrations by several mechanisms, explains most, if not all, of the clinical features of ST or SS. However, it should be noticed that much of our understanding of the pathophysiology of SS derives from preclinical data. As indicated by Isbister \& Buckley (2005), there is no evidence that serotonin behavioral syndrome defined in experimental behavioral pharmacology and ST/SS described in clinical medicine are even similar in terms of phenomenology or underlying mechanisms. The significant confusion in the literature has been created especially as to the pathophysiological roles of 5-HT $\mathrm{HA}_{1 \mathrm{~A}}$ and 5$\mathrm{HT}_{2 \mathrm{~A}}$ receptors implicated in SS. As discussed earlier (Isbister \& Buckley, 2005; Odagaki, 2009), the possible important roles of $5-\mathrm{HT}_{2 \mathrm{~A}}$ receptors in pathogenesis of SS, in particular the severe cases with hyperthermia and/or muscle hypertonicity, should be taken into consideration.

\subsection{Treatment}

Of most importance are prompt recognition of toxicity and discontinuation of offending medications in management of ST. Most cases of ST, even if represented as a full-blown form of SS, are self-limiting, if medications are withdrawn promptly at their early stages and no complications occur. In some patients, however, life-threatening severe toxicity can develop. Severe toxicity is more likely in patients ingesting overdosed serotonergic agent(s) or at least two drugs with different mechanisms of action, with combinations of SSRIs and a MAOI being the most common (Isbister \& Buckley, 2005). The Toxic Exposure Surveillance System in the United States reported over 100 deaths related to ingestion of SSRIs in 2004 (Watson et al., 2005), though it was unclear whether all of these cases could be diagnosed clinically as SS.

Most cases with ST can be managed by means of supportive care with intravenous fluids as well as with symptomatic therapies according to the clinical features and severity, e.g., aggressive external cooling for hyperthermia, benzodiazepines or barbiturates for seizures, and benzodiazepines for muscle rigidity, myoclonus, or agitation. The use of nondepolarizing paralyzing agents under mechanical ventilation is indicated when muscle rigidity remains refractory to benzodiazepines (Mills, 1995; Isbister \& Buckley, 2005). 
In the severe SS cases, more specific therapy may be considered. The most commonly used drugs have been methysergide, cyproheptadine, and chlorpromazine (Mills, 1995; Gillman, 1999). However, the SS cases reported to be successfully treated with these medications are all anecdotal, with randomized controlled trials lacking. Based on the pharmacological properties of these drugs as well as on the experimental results using an animal model of SS (Nisijima et al., 2007), Isbister \& Buckley (2005) suggested that nonselective 5-HT2 antagonists such as ketanserin or selective $5-\mathrm{HT}_{2 \mathrm{~A}}$ antagonists such as atypical antipsychotics may be effective for the treatment of SS. The use of atypical antipsychotics for treating SS is, however, not recommended, at least when NMS is not clearly excluded. There has been a report of the patient who showed SS caused by sertraline intake and consecutively NMS induced by risperidone (J.-M. Kim et al., 2007).

\section{SSRI discontinuation syndrome}

\subsection{Background}

Pharmacotherapy for mood and anxiety disorders has been dramatically changed during the last two decades, especially with an expanding increase in use of SSRIs for the treatment of broad spectrum including depression, varied types of anxiety, eating, obsessivecompulsive, and impulse-control disorders. In parallel with the wide-spread use of SSRIs, reports of SSRI discontinuation syndrome have been increased. As described below, it sometimes involves alterations in mental function, which may be interpreted as a relapse or worsening of the original disorders. This is the reason why the author intends to summarize the syndrome in brief here in this chapter.

Antidepressant discontinuation syndrome (Dilsaver \& Greden, 1984; Dilsaver et al., 1987; Lejoyeux et al., 1996) had been well identified since first reported for imipramine in 1959 (Andersen \& Kristiansen, 1959; Mann \& MacPherson, 1959, as cited in these three reviews). The initial SSRI discontinuation syndrome was described for fluoxetine in 1988 (Cooper, 1988), and subsequent many case reports and review articles indicated it can develop for all SSRIs. Some researchers include discontinuation symptoms related with other antidepressants than SSRIs, such as venlafaxine, nefazodone, trazodone, and mirtazapine, in the same syndrome (Schatzberg et al., 1997a; Tamam \& Ozpoyraz, 2002).

Although the terms discontinuation and withdrawal are sometimes used interchangeably, the latter should be avoided for the patients treated with antidepressants including SSRIs, since these drugs are, unlike opiates or sedative-hypnotics, non-addictive (Schatzberg et al., 1997b; Shelton, 2006).

\subsection{Clinical features and diagnosis}

Schatzberg et al. (1997b) raised the hallmark features of SRI discontinuation syndrome, as shown in Table 7. The clinical symptoms of SSRI discontinuation syndrome resemble those of TCA discontinuation syndrome, which had been classified into the following five main categories (Dilsaver \& Greden, 1984; Dilsaver et al., 1987; Lejoyeux et al., 1996; Haddad, 1997; Taman \& Ozpoyraz, 2002): (1) gastrointestinal and general somatic distress symptoms, e.g., anxiety, agitation, muscle tension, nervousness, flu-like symptoms (fatigue, headache, sweating, myalgia), lethargy, nausea, vomiting, asthenia; (2) sleep disturbances, such as insomnia and excessive and vivid dreams, (3) movement disorders, e.g., akathisia, 
parkinsonism, unsteady gait, abnormal movements of mouth and tongue; (4) behavioral activation, such as panic attacks, delirium, mania or hypomania; and (5) miscellaneous symptoms, such as cardiac arrhythmias. In the case of SSRI discontinuation syndrome, however, cardiac arrhythmias are seldom or never observed (Haddad, 1998), probably due to their minimal or negligible antimuscarinic activity and cardiotoxicity. In addition to, and probably overlapped with, these symptoms commonly seen in TCA discontinuation syndrome, further three symptom clusters are described to follow SSRI termination, i.e., (1) problems with balance (dizziness, ataxia, vertigo); (2) sensory abnormalities (electric shocklike sensations, paresthesia); and (3) aggressive and impulsive behavior (suicide attempts, hoarding during discontinuation) (Haddad, 1998). The proposed diagnostic criteria (Black et al., 2000) are shown in Table 8.

\section{Hall mark features of SRI discontinuation syndrome}

1. Not attributable to other causes

2. Emergent upon abrupt discontinuation, intermittent noncompliance (e.g., missed doses, drug holidays), and less frequently, with dose reduction

3. Generally mild and short-lived

4. Self-limiting but can be distressing

5. Rapidly reversed by the reintroduction of the original medication or the substitution of one that is pharmacologically similar

6. Minimized by slow tapering or by using a drug with an extended half-life

Table 7. Hall mark features of SRI discontinuation syndrome (Schatzberg et al., 1997b)

\begin{tabular}{|ll|}
\hline Criterion & Description \\
\hline A & $\begin{array}{l}\text { Discontinuation of or reduction in dose of an SSRI after a period of use of at } \\
\text { B }\end{array}$ \\
least 1 month \\
Two (or more) of the following, developing within 1 to 7 days of criterion A \\
Dizziness, light-headedness, vertigo or feeling faint \\
Shock-like sensations or paresthesia \\
Anxiety \\
Diarrhea \\
Fatigue \\
Gait instability \\
Headache \\
Insomnia \\
Irritability \\
Nausea and/or emesis \\
Tremor \\
Visual disturbances \\
The symptoms in Criterion B cause clinically significant distress or \\
impairment in social, occupational or important areas of functioning \\
The symptoms are not due to a general medical condition and are not better \\
accounted for by recurrence of symptoms of the mental disorder for which \\
the SSRI was originally prescribed, or by concurrent discontinuation (or \\
reduction in use) of another psychoactive substance
\end{tabular}

Table 8. The proposed diagnostic criteria for SSRI discontinuation syndrome (Black et al., 2000) 
SSRI discontinuation symptoms occur within 1 to 3 days subsequent to cessation of treatment or reduction in dose in the majority of cases (Black et al., 2000). In the case of fluoxetine, however, the symptoms can develop slower (Einbinder, 1995) due to its longer half-life compared with those of other SSRIs (Table 9). In most cases, the syndrome is mild and short-lived, with persisting duration usually not exceeding 1-3 weeks even if untreated (Coupland et al., 1996; Black et al., 2000).

\begin{tabular}{|c|c|c|c|}
\hline \multirow[t]{2}{*}{ SSRI } & \multicolumn{2}{|c|}{ Half-life (Haddad, 1998) } & Half-life \\
\hline & Single dose & Multiple dose & (Schatzberg et al., 1997a) \\
\hline Paroxetine & $10 \mathrm{~h}$ & $21 \mathrm{~h}$ & $21 \mathrm{~h}$ \\
\hline Fluvoxamine & $15 \mathrm{~h}$ & $22 \mathrm{~h}$ & $15 \mathrm{~h}$ \\
\hline Sertraline & $26 \mathrm{~h}$ & $26 \mathrm{~h}$ & $26 \mathrm{~h}(66 \mathrm{~h})^{*}$ \\
\hline Citalopram & $33 \mathrm{~h}$ & $33 \mathrm{~h}$ & \\
\hline Fluoxetine & $1.9 \mathrm{~d}$ & $5.7 \mathrm{~d}(7-15 \mathrm{~d})^{*}$ & $84 \mathrm{~h}(4-16 \mathrm{~d})^{*}$ \\
\hline
\end{tabular}

"Half-life for an active metabolite shown in parentheses. $\mathrm{h}=$ hours, $\mathrm{d}=$ days.

Table 9. Half-lives of SSRIs (Schatzberg et al., 1997a; Haddad, 1998)

The half-life of the SSRI and its active metabolite (Table 9) probably contributes also to the likelihood of developing discontinuation syndrome for each drug. As anticipated from the half-life, the discontinuation events are more frequently and profoundly experienced with SSRIs with shorter half-life, in particular with paroxetine, and least often with fluoxetine that has the longest half-life (Coupland et al., 1996; Price et al., 1996; Rosenbaum et al., 1998).

\subsection{Pathophysiology}

As indicated above, plasma half-life of the SSRI is apparently one of the most important factors for occurrence of discontinuation syndrome, and thus the pathogenic mechanisms underlying SSRI discontinuation syndrome have to be supposed in accordance with this fact. The simplest and most plausible explanation is an abrupt decrease in availability of synaptic serotonin caused by withdrawal or decrease of the SSRI, in the face of downregulated serotonin receptors as a result of synaptic adaptation to long-term SSRI treatment (Schatzberg et al., 1997a).

In addition to this hypothesis, Schatzberg et al. (1997a) also raised the following three other mechanisms that possibly contribute to some clinical features of the syndrome: (1) secondary effects on other neurotransmitters than serotonin; (2) individual genetic or psychological differences; and (3) cholinergic rebound.

\subsection{Clinical implications and treatment}

Rosenbaum \& Zajecka (1997) indicated practical strategies for management of SRI discontinuation syndrome as follows: (1) reassuring patients that the symptoms are likely to be short-lived and mild; (2) for severe and distressing symptoms, the dosage of the drug prescribed immediately before the onset of discontinuation symptoms should be reinstituted and the rate of taper should be slowed; (3) all SRIs, with the exception of fluoxetine, should be gradually tapered; and (4) using or switching to agents with an extended half-life, such as fluoxetine, can help reduce the incidence of SRI discontinuation syndrome. 
The rate of tapering of the drug should depend on its pharmacokinetic and pharmacological profile, current dose, duration of treatment, other drugs concomitantly prescribed, the patient's physical, psychological, and social situations, and so on. Physicians who prescribe SSRIs should be aware of the risk of this syndrome at the termination of treatment, or even during maintenance therapy with a fixed dose. Missing even as few as two doses of a SSRI with a short half-life, which is very often unreported to the physician, might lead to the discontinuation symptoms (Kaplan, 1997). It should be noted some discontinuation symptoms, e.g., anxiety, irritability, fatigue, and insomnia, are sometimes hardly distinguishable from those of a relapse or recurrence of the primary affective or anxiety disorders.

\section{Other encephalopathic symptoms associated with psychotropic drugs}

\subsection{Hyponatremic encephalopathy}

Hyponatremia is among the most common electrolyte abnormalities encountered in clinical practice (Upadhyay et al., 2006, 2009), and it is not rare to experience encephalopathic symptoms such as seizures and altered consciousness due to severe hyponatremia, usually in association with psychogenic polydipsia (Dundas et al., 2007) or self-induced water intoxication (Vieweg et al., 1987; Riggs et al., 1991), in psychiatric patients treated with psychotropic drugs (de Leon et al., 1994).

Clinical severity of hyponatremia is related not only to the absolute level of serum sodium concentration, but also to the rate at which hyponatremia develops. Premonitory or early symptoms include nausea, vomiting, anorexia, disorientation, headache, fatigue, weakness, irritability, lethargy, confusion, and muscle cramps. Acute severe hyponatremia causes cerebral edema that can lead to coma, irreversible neurologic damage, and supratentorial cerebral herniation resulting in respiratory arrest from brain stem compression and death. In contrast, patients with chronic hyponatremia are often asymptomatic, even with serum sodium level as low as $120 \mathrm{mEq} / \mathrm{L}$ (Adroqué, 2005). As hyponatremia progresses, abovementioned premonitory, nonspecific symptoms are followed by neuropsychiatric symptoms such as seizures, hemiplegia, dysarthria, hallucinations, tremor, and coma. Patients may demonstrate an impaired response to verbal and painful stimuli, and exhibit bizarre behavior or experience auditory or visual hallucinations (Fraser \& Arieff, 1997). These psychiatric symptoms may mimic the manifestations of primary psychiatric disorders such as schizophrenia, and thus clinicians should be careful not to be misled into a judgment that the primary disorder is exacerbated. It also should be considered that compulsive water consumption in the late afternoon and evening may impair mental status and further exacerbate psychiatric symptoms (Siegel, 2008).

Although hyponatremia is derived from a variety of etiologies and classified into several types according to plasma and urine osmolarity as well as the volume of the total body water (Reddy \& Mooradian, 2009), hyponatremic encephalopathy in psychiatric patients is usually the so-called polydipsia-hyponatremia syndrome (psychogenic polydipsia) with hypotonic plasma osmolarity and euvolemic status of the total body water. However, compulsive water drinking alone is usually not sufficient to induce marked hyponatremia, because the normal kidney should theoretically be able to excrete water in excess of 20 liters per day and most patients actually ingest less water than that theoretically required (Vieweg 
et al., 1987; Fraser \& Arieff, 1997). As demonstrated by Goldman et al. (1988), the patients with polydipsia and hyponatremia are accompanied with impaired maximal urinary dilution and free-water clearance in response to water loading, suggesting renal abnormally enhanced sensitivity to low concentrations of arginine vasopressin (AVP). They also showed that the patients with a history of polydipsia and hyponatremia demonstrated a higher serum AVP levels as well as greater thirst independent of their serum osmolarity after receiving an infusion of hypertonic saline, suggestive of a downward shift in the threshold for AVP release and defect of osmoregulation of thirst, respectively (Siegel, 2008).

Most psychiatric patients with polydipsia-hyponatremia syndrome are thus associated with maladapted water and electrolyte homeostasis sustained principally by the action of neurohypophyseal antidiuretic hormone AVP. It has been demonstrated that lots of psychotropic drugs including antipsychotics, antidepressants, and anticonvulsants, can cause syndrome of inappropriate antidiuretic hormone secretion (SIADH) (Spigset \& Hedenmalm, 1995; Bhuvaneswar et al., 2009; Reddy \& Mooradian, 2009), and that antipsychotic-induced hyponatremia is most likely a result of SIADH (Meulendijks, 2010). Vieweg et al. (1987) also postulated that the syndrome of self-induced water intoxication and psychosis (SIWIP) was a subcategory of SIADH, with hypoosmolality and hyponatremia induced synergistically by polydipsia and released AVP, both of which were stimulated by psychosis. In the schizophrenic patients with polydipsia-hyponatremia syndrome, plasma AVP levels were shown to rise sharply after the pharmacological induction of psychotic symptoms with methylphenidate (Goldman et al., 1997). This mechanism may underlie the episodic escalation of polydipsia observed in psychotically exacerbated patients [psychosis, intermittent hyponatremia, and polydipsia (PIP) syndrome (Leadbetter et al., 1994)], which sometimes result in severe, life-threatening hyponatremic encephalopathy (Vieweg et al., 1985).

Treatment of hyponatremic symptoms in psychiatric patients depends on their severity as well as the rate of development (Siegel, 2008). Acute severe hyponatremic encephalopathy could be life-threatening, which thus has to be treated as an emergency with hypertonic saline to prevent cerebral edema. On the other hand, chronic hyponatremia associated with psychogenic polydipsia is optimally managed with behavioral treatments including fluid restriction (Dundas et al., 2007) and removal of, if identified, underlying causes. Careful monitoring of diurnal body weight change for the PIP syndrome patients is useful to recognize hyponatremic episodes at an earlier stage and prevent sequelae due to severe hyponatremic encephalopathy (Leadbetter et al., 1994). In any case, osmotic demyelination, such as central pontine myelinolysis, possibly associated with overaggressive correction of serum sodium concentrations (more than $10 \mathrm{mmol} / \mathrm{L}$ in 24 hours, $18 \mathrm{mmol} / \mathrm{L}$ in 48 hours, and $20 \mathrm{mmol} / \mathrm{L}$ in 72 hours), should be avoided (Sterns et al., 2009). Specific pharmacological treatment with democlocycline or AVP receptor antagonists (aquaretics) for chronic hyponatremia in psychiatric patients may become standardized in the near future (Siegel, 2008).

\subsection{Valproate-induced hyperammonemic encephalopathy}

It has been known that valproic acid occasionally induces hyperammonemic encephalopathy in patients with otherwise normal hepatic function (Carr \& Shrewsbury, 2007; Marie-José, 2007). In most cases, encephalopathic symptoms appear in a few days after 
initiation of valproate therapy, with exceptional case reports with onset after longer therapeutic periods for several months or years. There remains controversy as to whether symptoms have any relationship to daily dose or plasma concentration of valproic acid. In a prospective study, the significantly higher prevalence of asymptomatic hyperammonemia was found in the psychiatric patients treated with valproate than in the control, and there was a positive correlation between serum valproic acid concentrations and ammonia levels (Raja \& Azzoni, 2002). Nevertheless, it should be noted that many cases with symptomatic encephalopathic symptoms reported up to date have serum valproic acid concentrations within therapeutic range (Carr \& Shrewsbury, 2007; Marie-José, 2007).

\subsection{Transient splenial lesion of the corpus callosum}

Since several antiepileptic drugs have been widely prescribed as effective mood stabilizers in psychiatric practice (Grunze, 2010), all psychiatrists should be familiar with adverse phenomena associated with them. With widespread use of magnetic resonance imaging (MRI), a characteristic discrete focal lesion limited to the central area of the splenium of the corpus callosum has been recognized to occur in epileptic patients receiving antiepileptic drugs (S.S. Kim et al., 1999; Polster et al., 2001). Although the pathophysiological mechanisms of this lesion are still obscure, antiepileptics appear to be implicated as pathogenetic or triggering factors at least in some cases. Indeed, the same lesion has been reported also in the non-epileptic patients treated with antiepileptic drugs (Maeda et al., 2003; Honda et al., 2006). Some patients developed the lesion on treatment with antiepileptic drugs (Kim et al., 1999; Polster et al., 2001; Maeda et al., 2003), while others subsequent to withdrawal of them (Polster et al., 2001; Gürtler et al., 2005; Honda et al., 2006).

The lesion is usually detected accidentally by MRI, accompanied with no apparent clinical signs and symptoms. It is characterized with an isolated oval-shaped abnormal signal in MRI located in the central area of the splenium, no enhancement on post-contrast MRI, and complete reversibility without specific treatment. In consideration of its benign outcome, unnecessary invasive examination and therapeutic intervention should be avoided (Maeda et al., 2003).

\subsection{Miscellaneous}

Acute onset alterations in CNS function are derived from a variety of etiological factors. When specific pathogenetic processes directly invading the brain (e.g., vascular, infectious, and neoplastic diseases) are ruled out, other diffuse, multifocal, or metabolic causes should be considered (Posner et al., 2007). If treated with psychotropic drugs, the possibility of direct or indirect contribution of these chemicals to the pathophysiological status should be always evaluated with caution. The SIADH associated with psychotropic drugs and valproate-induced hyperammonemia, both of which have been described above, exemplify such implication. In addition, many psychotropic drugs, especially antidepressants and antipsychotics, have been shown to possess ability to reduce seizure threshold and to provoke epileptic seizures (Alldredge, 1999; Pisani et al., 2002). Antipsychotics, in particular atypical antipsychotics such as clozapine and olanzapine, are reported to be associated with an increased risk of obesity, diabetes, and metabolic syndrome (Scheen \& De Hert, 2007; Smith et al., 2008) and in the worse case it can result in fatal hyperglycemic encephalopathy (Koller \& Doraiswamy, 2002; Wehring et al., 2003). Lithium, a widely used mood stabilizer, 
increases the risk of hypothyroidism, which can range from subclinical to life-threatening myxedema coma (Bhuvaneswar et al., 2009; Thomas et al., 2010). A case of Hashimoto's encephalopathy possibly induced by lithium was also reported (Nagamine et al., 2008).

\section{Concluding remarks}

In these two decades, pharmacotherapy with psychotropic drugs for mentally ill patients has been dramatically changed. Classical prototypal antipsychotics, such as chlorpromazine and haloperidol, have been replaced with atypical antipsychotic drugs for the treatment of psychotic patients. As for the pharmacotherapy for mood disorders, total antidepressant prescribing has increased, especially in 1990s with exponential increase in prescriptions of SSRIs (Donoghue, 1998; Middleton, et al., 2001). This remarkable rise in SSRIs prescribing experienced in the United Kingdom may be attributable, in part, to the Defeat Depression Campaign (Paykel et al., 1997), which was undertaken from 1992 to 1996. However, the similar trends in antidepressant prescriptions have also been observed in all other developed countries worldwide. The vigorous marketing promoted by pharmaceutical companies probably contributed to the overwhelming spread of SSRIs in 1990s to a great extent (McHenry, 2005). In addition, SSRIs have been more and more prescribed not only for depression but also for a number of other psychiatric diagnoses such as anxiety, eating, impulse-control, and personality disorders. Many antiepileptic drugs are also prescribed generally for psychiatric patients in anticipation of their mood stabilizing effects.

In general, the newly developed psychotropic drugs are believed to be safer and more tolerable than older drugs. The apparent lowered risk of adverse effects of novel drugs may facilitate the clinician's attitude for prescribing these psychotropic drugs automatically, and sometimes indiscreetly. However, there is no efficacious drug without adverse side effects. All clinicians who have an occasion to prescribe psychotropic drugs, or to see the patients treated with them, should be familiar with adverse symptoms associated with them.

This chapter focuses on the adverse effects of psychotropic drugs involving altered CNS function. Although nonspecific CNS reaction due to overdosed psychotropic drugs has not been included, it should be always taken into consideration when the patients treated with psychotropic drugs exhibit inexplicable CNS symptoms. Lithium-induced toxic encephalopathy has been reported to occur even when serum lithium levels are kept within a therapeutic range (Sheean, 1991). When adverse effects on CNS function are presented apparently as psychiatric symptoms, these should not be misdiagnosed as exacerbation of the primary psychiatric disorder. Such misjudgment may lead to the clinical decision to increase the offending drugs or to add other kinds of psychotropic drugs, which could further complicate matters and delay appropriate intervention. Even in the case of the idiosyncratic adverse effects such as NMS, the most efficacious way to abort the syndrome without serious sequelae should be early recognition of, and prompt appropriate intervention in, the clinical symptoms at the incipient stage.

Pharmacotherapy becomes meaningfully efficacious only when it is considered in the whole therapeutic framework and associated synergistically with other psychosocial therapies. In general, most psychotropic drugs have only limited therapeutic effects, which never cure the psychiatric diseases themselves but relieve the symptoms partially. The efficacy of SSRIs in depressed patients over placebo has been shown to be minimal, if any, by recent meta- 
analyses (Moncrieff \& Kirsch, 2005). While it is true that pharmacotherapy bring enormous benefit to some patients, I have also been aware of many cases at a disadvantage associated with thoughtless or unnecessary use of psychotropic drugs. The prudent and conscientious decision-making for pharmacotherapy in consideration of the quality of life of the individual patient is highly warranted.

\section{Acknowledgment}

This review was envisioned while the author had been on loan to Moro Hospital, and was financially supported by the Grant for Research Work from the Saitama Medical University, Japan. The author is grateful to Professor Ryoichi Toyoshima, the chair of Department of Psychiatry, Faculty of Medicine, Saitama Medical University, and Dr. Taeko Maruki, the director of Moro Hospital, for their support and encouragement.

\section{References}

Adityanjee, Singh, S., Singh, G. \& Ong, S. (1988). Spectrum Concept of Neuroleptic Malignant Syndrome. British Journal of Psychiatry, Vol.153, (July 1988), pp. 107-111, ISSN 0007-1250

Adityanjee, Aderibigbe, Y.A. \& Mathews, T. (1999a). Epidemiology of Neuroleptic Malignant Syndrome. Clinical Neuropharmacology, Vol.22, No.3, (May/June 1999), pp. 151-158, ISSN 0362-5664

Adityanjee, Mathews, T. \& Aderibigbe, Y.A. (1999b). Proposed Research Diagnostic Criteria for Neuroleptic Malignant Syndrome. International Journal of Neuropsychopharmacolgy, Vol.2, No.2, (June 1999), pp. 129-144

Adityanjee, Sajatovic, M. \& Munshi, K.R. (2005). Neuropsychiatric Sequelae of Neuroleptic Malignant Syndrome. Clinical Neuropharmacology, Vol.28, No.4, (July/ August 2005), pp. 197-204, ISSN 0362-5664

Addonizio, G., Susman, V.L. \& Roth, S.D. (1986). Symptoms of Neuroleptic Malignant Syndrome in 82 Consecutive Inpatients. American Journal of Psychiatry, Vol.143, No.12, (December 1986), pp. 1587-1590, ISSN 0002-953X

Addonizio, G., Susman, V.L. \& Roth, S.D. (1987). Neuroleptic Malignant Syndrome: Review and Analysis of 115 Cases. Biological Psychiatry, Vol.22, No.8, (August 1987), pp. 1004-1020, ISSN 0006-3223

Adrogué, H.J. (2005). Consequences of Inadequate Management of Hyponatremia. American Journal of Nephrology, Vol.25, No.3, (May/June 2005), pp. 240-249, ISSN 0250-8095

Alldredge, B.K. (1999). Seizure Risk Associated with Psychotropic Drugs: Clinical and Pharmacokinetic Considerations. Neurology, Vol.53, No.5, Suppl.2, (September 1999), pp. S68-S75, ISSN 0028-3878

Ananth, J., Aduri, K., Parameswaran, S. \& Gunatilake, S. (2004a). Neuroleptic Malignant Syndrome: Risk factors, Pathophysiology, and Treatment. Acta Neuropsychiatrica, Vol.16, No.4, (August 2004), pp. 219-228

Ananth, J., Parameswaran, S., Gunatilake, S., Burgoyne, K. \& Sidhom, T. (2004b). Neuroleptic Malignant Syndrome and Atypical Antipsychotic Drugs. Journal of Clinical Psychiatry, Vol.65, No.4, (April 2004), pp. 464-470, ISSN 0160-6689 
Andersen, H. \& Kristiansen, E.S. (1959). Tofranil-Treatment of Endogenous Depressions. Acta Psychiatrica et Neurologica Scandinavica, Vol.34, No.4, pp. 387-397, ISSN 0001$690 \mathrm{X}$

Asch, D.A. \& Parker, R.M. (1988). The Libby Zion Case. One Step Forward or Two Steps Backward? New England Journal of Medicine, Vol.318, No.12, (March 1988), pp. 771775, ISSN 0028-4793

Assion, H.J., Heinemann, F. \& Laux, G. (1998). Neuroleptic Malignant Syndrome under Treatment with Antidepressants? A Critical Review. European Archives of Psychiatry and Clinical Neuroscience, Vol.248, No.5, (October 1998), pp. 231-239, ISSN 0940-1334

Attar-Herzberg, D., Apel, A., Gang, N., Dvir, D. \& Mayan, H. (2009). The Serotonin Syndrome: Initial Misdiagnosis, Israel Medical Association Journal, Vol.11, No.6, (June 2009), pp. 367-370, ISSN 1565-1088

Berardi, D., Amore, M., Keck, P.E. Jr., Troia, M. \& Dell'Atti, M. (1998). Clinical and Pharmacologic Risk Factors for Neuroleptic Malignant Syndrome: A Case-Control Study. Biological Psychiatry, Vol.44, No.8, (October 1998), pp. 748-754, ISSN 00063223

Bhuvaneswar, C.G., Baldessarini, R.J., Harsh, V.L. \& Alpert, J.E. (2009). Adverse Endocrine and Metabolic Effects of Psychotropic Drugs. CNS Drugs, Vol.23, No.12, (December 2009), pp. 1003-1021, ISSN 1172-7047

Black, K., Shea, C., Dursun, S. \& Kutcher, S. (2000). Selective Serotonin Reuptake Inhibitor Discontinuation Syndrome: Proposed Diagnostic Criteria. Journal of Psychiatry and Neuroscience, Vol.25, No.3, (May 2000), pp. 255-261, ISSN 1180-4882

Boyer, E.W. \& Shannon, M. (2005). The Serotonin Syndrome. New England Journal of Medicine, Vol.352, No.11, (May 2005), pp. 1112-1120, ISSN 0028-4793

Brennan, D., MacManus, M., Howe, J. \& McLoughlin, J. (1988). 'Neuroleptic Malignant Syndrome' without Neuroleptics. British Journal of Psychiatry, Vol.152, No.4, (April 1988), pp. 578-579, ISSN 0007-1250

Caroff, S.N. (1980). The Neuroleptic Malignant Syndrome. Journal of Clinical Psychiatry, Vol.41, No.3, (March 1980), pp. 79-83, ISSN 0160-6689

Caroff, S.N. \& Mann, S.C. (1988). Neuroleptic Malignant Syndrome. Psychopharmacology Bulletin, Vol.24, No.1, pp. 25-29, ISSN 0048-5764

Caroff, S.N. \& Mann, S.C. (1993). Neuroleptic Malignant Syndrome. Medical Clinics of North America, Vol.77, No.1, (January 1993), pp. 185-202, ISSN 0025-7125

Caroff, S.N., Mann, S.C., Lazarus, A., Sullivan, K. \& MacFadden, W. (1991). Neuroleptic Malignant Syndrome: Diagnostic Issues. Psychiatric Annals, Vol.21, No.3, (March 1991), pp. 130-147

Caroff, S.N., Mann, S.C. \& Campbell, E.C. (2000). Atypical Antipsychotics and Neuroleptic Malignant Syndrome. Psychiatric Annals, Vol.30, No.5, (May 2000), pp. 314-321

Caroll, B.T. \& Taylor, R.E. (1997). The Nondichotomy between Lethal Catatonia and Neuroleptic Malignant Syndrome. Journal of Clinical Psychopharmacology, Vol.17, No.3, (June 1997), pp. 235-236, ISSN 0271-0749

Carr, R.B. \& Shrewsbury, K. (2007). Hyperammonemia Due to Valproic Acid in the Psychiatric Setting. American Journal of Psychiatry, Vol.164, No.7, (July 2007), pp. 1020-1027, ISSN 0002-953X 
Castillo, E., Rubin, R.T., Holsboer-Trachsler, E. (1989). Clinical Differentiation between Lethal Catatonia and Neuroleptic Malignant Syndrome. American Journal of Psychiatry, Vol.146, No.3, (March 1989), pp. 324-328, ISSN 0002-953X

Chan-Tack, K.M. (1999) Neuroleptic Malignant Syndrome Due to Promethazine. Southern Medical Journal, Vol.92, No.10, (October 1999), pp. 1017-1018, ISSN 0038-4348

Cooper, G.L. (1988). The Safety of Fluoxetine - An Update. British Journal of Psychiatry, Vol.153, Suppl.3, (September 1988), pp. 77-86, ISSN 0960-5371

Coupland, N., Bell, C.J. \& Potokar, J.P. (1996). Serotonin Reuptake Inhibitor Withdrawal. Journal of Clinical Psychopharmacology, Vol.16, No.5, (October 1996), pp. 356-362, ISSN 0271-0749

Davis, J.M., Janicak, P.G., Sakkas, P., Gilmore, C. \& Wang, Z. (1991). Electroconvulsive Therapy in the Treatment of the Neuroleptic Malignant Syndrome. Convulsive Therapy, Vol.7, No.2, (June 1991), pp. 111-120, ISSN 0749-8055

Davis, J.M., Caroff, S.N. \& Mann, S.C. (2000). Treatment of Neuroleptic Malignant Syndrome. Psychiatric Annals, Vol. 30, No.5, (May 2000), pp. 325-331

Delay, J. \& Deniker, P. (1968). Drug-induced Extrapyramidal Syndromes, In: Handbook of Clinical Neurology, Vol.6, Diseases of the Basal Ganglia, P.J. Vinken \& O.W. Bruyn, (Eds.), pp. 248-266, Elsevier, New York, USA

De Leon, J., Verghese, C., Tracy, J.I., Josiassen, R.C. \& Simpson, G.M. (1994). Polydipsia and Water Intoxication in Psychiatric Patients: A Review of the Epidemiological Literature. Biological Psychiatry, Vol.35, No.6, (March 1994), pp. 408-419, ISSN 00063223

Deuschl, G., Oepen, G., Hermie, L. \& Kindt, H. (1987). Neuroleptic Malignant Syndrome: Observations on Altered Consciousness. Pharmacopsychiatry, Vol.20, No.4, (July 1987), pp. 168-170, ISSN 0176-3679

Dike, G.L. (1997). Triphasic Waves in Serotonin Syndrome. Journal of Neurology, Neurosurgery, and Psychiatry, Vol.62, No.2, (February 1997), pp. 200, ISSN 0022-3050

Dilsaver, S.C. \& Greden, J.F. (1984). Antidepressant Withdrawal Phenomena. Biological Psychiatry, Vol.19, No.2, (February 1984), pp. 237-256, ISSN 0006-3223

Dilsaver, S.C., Greden, J.F. \& Snider, R.M. (1987). Antidepressant Withdrawal Syndromes: Phenomenology and Pathophysiology. International Clinical Psychopharmacology, Vol.2, No.1, (January 1987), pp. 1-19, ISSN 0268-1315

Donoghue, J. (1998) Selective Serotonin Reuptake Inhibitor Use in Primary Care. A 5-Year Naturalistic Study. CNS Drug Investigation, Vol.16, No.6, (December 1998), pp. 453462, ISSN 1173-2563

Dundas, B., Harris, M. \& Narasimban, M. (2007). Psychogenic Polydipsia Review: Etiology, Differential, and Treatment. Current Psychiatry Reports, Vol.9, No.3, (June 2007), pp. 236-241, ISSN 1523-3812

Dunkley, E.J.C., Isbister, G.K., Sibbritt, D., Dawson, A.H. \& Whyte, I.M. (2003). The Hunter Serotonin Toxicity Criteria: Simple and Accurate Diagnostic Decision Rules for Serotonin Toxicity. QJM, Vol.96, No.9, (September 2003), pp. 635-642, ISSN 14602725

Einbinder, E. (1995). Fluoxetine Withdrawal? American Journal of Psychiatry, Vol.152, No.8, (August 1995), pp. 1235, ISSN 0002-953X

Farver, D.K. (2003). Neuroleptic Malignant Syndrome Induced by Atypical Antipsychotics. Expert Opinion on Drug Safety, Vol.2, No.1, (January 2003), pp. 21-35, ISSN 1474-0338 
Feibel, J.H. \& Schiffer, R.B. (1981). Sympthoadrenomedullary Hyperactivity in the Neuroleptic Malignant Syndrome: A Case Report. American Journal of Psychiatry, Vol.138, No.8, (August 1981), pp. 1115-1116, ISSN 0002-953X

Fink, M. (1996a). Neuroleptic Malignant Syndrome and Catatonia: One Entity or Two? Biological Psychiatry, Vol.39, No.1, (January 1996), pp. 1-4, ISSN 0006-3223

Fink, M. (1996b). Toxic Serotonin Syndrome or Neuroleptic Malignant Syndrome? Pharmacopsychiatry, Vol.29, No.4, (July 1996), pp. 159-161, ISSN 0176-3679

Fitzgerald, B., Middleton, J.K. \& Cooper, S.A. (1997). Adverse Effects of Summer amongst People with Learning Disabilities: Neuroleptic Malignant Syndrome. Journal of Intellectual Disability Research, Vol.41, No.3, (June 1997), pp. 273-277, ISSN 0964-2633

Frances, A., Pincus, H.A. \& First, M.B. (2000). Neuroleptic Malignant Syndrome, In: Diagnostic and Statistical Manual of Mental Disorders, Fourth Edition, Text Revision (DSM-IV-TR), American Psychiatric Association (Ed.), pp. 795-798, ISBN 0-89042024-6, Washington, DC

Francis, A., Chandragiri, S., Rizvi, S., Koch, M. \& Petrides, G. (2000). Is Lorazepam a Treatment for Neuroleptic Malignant Syndrome? CNS Spectrum, Vol.5, No.7, (July 2000), pp. 54-57, ISSN 1092-8529

Fraser, C.L. \& Arieff, A.I. (1997). Epidemiology, Pathophysiology, and Management of Hyponatremic Encephalopathy. American Journal of Medicine, Vol.102, No.1, (January 1997), pp. 67-77, ISSN 0002-9343

Fricchione, G., Mann, S.C. \& Caroff, S.N. (2000). Catatonia, Lethal Catatonia, and Neuroleptic Malignant Syndrome. Psychiatric Annals, Vol.30, No.5, (May 2000), pp. 347-355

Friedman, J.H., Feinberg, S.S. \& Friedman, R.G. (1985). A Neuroleptic Malignantlike Syndrome due to Levodopa Therapy Withdrawal. JAMA, Vol.254, No.19, (November 1985), pp. 2792-2795, ISSN 0098-7484

Friedman, J.H., Davis, R. \& Wagner, R.L. (1988). Neuroleptic Malignant Syndrome. The Results of a 6-Month Prospective Study of Incidence in a State Psychiatric Hospital. Clinical Neuropharmacolgy, Vol.11, No.4, (August 1988), pp. 373-377, ISSN 0362-5664

Friedman, L.S., Weinrauch, L.A. \& D’Elia, J.A. (1987). Metoclopramide-Induced Neuroleptic Malignant Syndrome. Archives of Internal Medicine, Vol.147, No.8, (August 1987), pp. 1495-1497, ISSN 0003-9926

Gerson, S.C. \& Baldessarini, R.J. (1980). Motor Effects of Serotonin in the Central Nervous System. Life Sciences, Vol.27, No.16, (October 1980), pp. 1435-1451, ISSN 0024-3205

Gillman, P.K. (1998). Serotonin Syndrome: History and Risk. Fundamental and Clinical Pharmacology, Vol.12, No.5, (September/October 1998), pp. 482-491, ISSN 0767-3981

Gillman, P.K. (1999). The Serotonin Syndrome and Its Treatment. Journal of Psychopharmacology, Vol.13, No.1, (January 1999), pp. 100-109, ISSN 0269-8811

Gillman, P.K. (2006). A Review of Serotonin Toxicity Data: Implications for the Mechanisms of Antidepressant Drug Action. Biological Psychiatry, Vol.59, No.11, (June 2006), pp. 1046-1051, ISSN 0006-3223

Goldman, M.B., Luchins, D.J. \& Robertson, G.L. (1988). Mechanisms of Altered Water Metabolism in Psychotic Patients with Polydipsia and Hyponatremia. New England Journal of Medicine, Vol.318, Vol.7, (February 1998), pp. 397-403, ISSN 0028-4793 
Goldman, M.B., Robertson, G.L., Luchins, D.J., Hedeker, D. \& Pandey, G.N. (1997). Psychotic Exacerbations and Enhanced Vasopressin Secretion in Schizophrenic Patients with Hyponatremia and Polydipsia. Archives of General Psychiatry, Vol.54, No.5, (May 1997), pp. 443-449, ISSN 0003-990X

Grunze, H.C.R. (2010). Anticonvulsants in Bipolar Disorder. Journal of Mental Health, Vol.19, No.2, (April 2010), pp. 127-141, ISSN 0963-8237

Gurrera, R.J. (1999). Sympathoadrenal Hyperactivity and the Etiology of Neuroleptic Malignant Syndrome. American Journal of Psychiatry, Vol.156, No.2, (February 1999), pp. 169-180, ISSN 0002-953X

Gurrera, R.J. \& Romero, J.A. (1992). Sympathoadrenomedullary Activity in the Neuroleptic Malignant Syndrome. Biological Psychiatry, Vol.32, No.4, (August 1992), pp. 334-343, ISSN 0006-3223

Gürtler, S., Ebner, A., Tuxhorn, I., Ollech, I., Pohlmann-Eden, B. \& Woermann, F.G. (2005). Transient Lesion in the Splenium of the Corpus Callosum and Antiepileptic Drug Withdrawal. Neurology, Vol.65, No.7, (October 2005), pp. 1032-1036, ISSN 0028-3878

Haddad, P. (1997). Newer Antidepressants and the Discontinuation Syndrome. Journal of Clinical Psychiatry, Vol.58, Suppl.7, pp. 17-22, ISSN 0160-6689

Haddad, P. (1998). The SSRI Discontinuation Syndrome. Journal of Psychopharmacology, Vol.12, No.3, (May 1998), pp. 305-313, ISSN 0269-8811

Harris, M., Nora, L., Tanner, C.M. (1987). Neuroleptic Malignant Syndrome Responsive to Carbidopa/Levodopa: Support for a Dopaminergic Pathogenesis. Clinical Neuropharmacology, Vol.10, No.2, (April 1987), pp. 186-189, ISSN 0362-5664

Hegerl, U., Bottlender, R., Gallinat, J., Kuss, H.-J., Ackenheil, M. \& Möller, H.-J. (1998). The Serotonin Syndrome Scale: First Results on Validity. European Archives of Psychiatry and Clinical Neuroscience, Vol.248, No.2, (May 1998), pp. 96-103, ISSN 0940-1334

Henderson, V.W. \& Wooten, G.F. (1981). Neuroleptic Malignant Syndrome: A Pathogenetic Role for Dopamine Receptor Blockade? Neurology, Vol.31, No.2, (February 1981), pp. 132-137, ISSN 0028-3878

Hermesh, H., Manor, I., Shiloh, R., Aizenberg, D. Benjamini, Y., Munitz, H. \& Weizman, A. (2002). High Serum Creatinin Kinase Level: Possible Risk Factor for Neuroleptic Malignant Syndrome. Journal of Clinical Psychopharmacology, Vol.22, No.3, (June 2002), pp. 252-256, ISSN 0271-0749

Hernández, J.L., Ramos, F.J., Infante, J., Rebollo, M. \& González-Macías, J. (2002). Severe Serotonin Syndrome Induced by Mirtazapine Monotherapy. Annals of Pharmacotherapy, Vol.36, No.4, (April 2002), pp. 641-643, ISSN 1060-0280

Heyland, D. \& Sauvé, M. (1991). Neuroleptic Malignant Syndrome without the Use of Neuroleptics. Canadian Medical Association Journal, Vol.145, No.7, (October 1991), pp. 817-819, ISSN 0820-3946

Honda, K., Nishimiya, J., Sato, H., Munakata, M., Kamada, M., Iwamura, A., Nemoto, H., Sakamoto, T. \& Yuasa, T. (2006). Transient Splenial Lesion of the Corpus Callosum after Acute Withdrawal of Antiepileptic Drug: A Case Report. Magnetic Resonance in Medical Sciences, Vol.5, No.4, (December 2006), pp. 211-215, ISSN 1347-3182

Insel, T.R., Roy, B.F., Cohen, R.M. \& Murphy, D.L. (1982). Possible Development of the Serotonin Syndrome in Man. American Journal of Psychiatry, Vol.139, No.7, (July 1982), pp. 954-955, ISSN 0002-953X 
Isbister, G.K. \& Buckley, N.A. (2005). The Pathophysiology of Serotonin Toxicity in Animals and Humans. Clinical Neuropharmacology, Vol.28, No.5, (September/October 2005), pp. 205-214, ISSN 0362-5664

Jacobs, B.L. (1976). An Animal Behavior Model for Studying Central Serotonergic Synapses. Life Sciences, Vol.19, No.6, (September 1976), pp. 777-786, ISSN 0024-3205

Kaplan, E.M. (1997). Antidepressant Noncompliance as a Factor in the Discontinuation Syndrome. Journal of Clinical Psychiatry, Vol.58, Suppl.7, pp. 31-36, ISSN 0160-6689

Keck, P.E. Jr., Arnold, L.M. (2000). The Serotonin Syndrome. Psychiatric Annals, Vol.30, No.5, (May 2000), pp. 333-343

Keck, P.E. Jr., Pope, H.G. Jr., Cohen, B.M., McElroy, S.L. \& Nierenberg, A.A. (1989a). Risk Factors for Neuroleptic Malignant Syndrome. A Case-Control Study. Archives of General Psychiatry, Vol.46, No.10, (October 1989), pp. 914-918, ISSN 0003-990X

Keck, P.E. Jr., Sebastianelli, J., Pope, H.G. Jr. \& McElroy, S.L. (1989b). Frequency and Presentation of Neuroleptic Malignant Syndrome in a State Psychiatric Hospital. Journal of Clinical Psychiatry, Vol.50, No.9, (September 1989), pp. 352-355, ISSN 01606689

Keck, P.E. Jr., McElroy, S.L. \& Pope, H.G. Jr. (1991). Epidemiology of Neuroleptic Malignant Syndrome. Psychiatric Annals, Vol.21, No.3, (March 1991), pp. 148-151

Kellam, A.M.P. (1987a). The Neuroleptic Malignant Syndrome, So-called. A Survey of the World Literature. British Journal of Psychiatry, Vol.150, (June 1987), pp. 752-759, ISSN 0007-1250

Kellam, A.M.P. (1987b). Correspondence. British Journal of Psychiatry, Vol.151, No.6, (December 1987), pp. 864-865

Kim, J.-M., Lee, S.-T., Song, E.-C., Jung, K.-H., Sinn, D.-I., Chung, H., Chu, K. \& Kim, M. (2007). Neurotoxic Syndrome Developed after Taking Sertraline and Risperidone. Journal of Clinical Neurology, Vol.3, No.3, (September 2007), pp. 165-167, ISSN 17386586

Kim, S.S., Chang, K.-H., Kim, S.T., Suh, D.C., Cheon, J.-E., Jeong, S.-W., Han, M.H. \& Lee, S.K. (1999). Focal Lesion in the Splenium of the Corpus Callosum in Epileptic Patients: Antiepileptic Drug Toxicity? American Journal of Neuroradiology, Vol.20, No.1, (January 1999), pp. 125-129, ISSN 0195-6108

Kinross-Wright, J.V. (1958). Trifluoperazine and Schizophrenia, In: Trifluoperazine. Clinical and Pharmacological Aspects, H. Brill, (Ed.), pp. 62-70, Lea and Febiger, Philadelphia, USA

Koller, E.A. \& Doraiswamy, P.M. (2002). Olanzapine-Associated Diabetes Mellitus. Pharmacotherapy, Vol.22, No.7, (July 2002), pp. 841-852, ISSN 0277-0008

Kontaxakis, V.P., Havaki-kontaxaki, B.J., Christodoulou N.G., Paplos, K.G. \& Christodoulou, G.N. (2003). Olanzapine-Associated Neuroleptic Malignant Syndrome: Is There an Overlap with the Serotonin Syndrome? Annals of General Hospital Psychiatry, Vol.2, No.1, (October 2003), pp. 10, ISSN 1475-2832, Available from http:/ / www.general-hospital-psychiatry.com/content/2/1/10/

Kurlan, R., Hamill, R. \& Shoulson, I. (1984). Neuroleptic Malignant Syndrome. Clinical Neuropharmacology, Vol.7, No.2, (June 1984), pp.109-120, ISSN 0362-5664

Leadbetter, R.A., Shutty, M.S. Jr., Higgins, P.B. \& Pavalonis, D. (1994). Multidisciplinary Approach to Psychosis, Intermittent Hyponatremia, and Polydipsia. Schizophrenia Bulletin, Vol.20, No.2, pp. 375-385, ISSN 0586-7614 
Lejoyeux, M., Fineyre, F. \& Adès, J. (1992). The Serotonin Syndrome. American Journal of Psychiatry, Vol.149, No.10, (October 1992), pp. 1410-1411, ISSN 0002-953X

Lejoyeux, M., Adès, J., Mourad, I., Solomon, J. \& Dilsaver, S. (1996). Antidepressant Withdrawal Syndrome. Recognition, Prevention and Management. CNS Drugs, Vol.5, No.4, (April 1996), pp. 278-292, ISSN 1172-7047

Levenson, J.L. (1985). Neuroleptic Malignant Syndrome. American Journal of Psychiatry, Vol.142, No.10, (October 1985), pp. 1137-1145, ISSN 0002-953X

MacKay, F.J., Dunn, N.R. \& Mann, R.D. (1999). Antidepressants and the Serotonin Syndrome in General Practice. British Journal of General Practice, Vol.49, No.448, (November 1999), pp. 871-874, ISSN 0960-1643

Madakasira, S. (1989). Amoxapine-Induced Neuroleptic Malignant Syndrome. DICP, Annals of Pharmacotherapy, Vol.23, No.1, (January 1989), pp. 50-55, ISSN 1042-9611

Maeda, M., Shiroyama, T., Tsukahara, H., Shimono, T., Aoki, S. \& Takeda, K. (2003). Transient Splenial Lesion of the Corpus Callosum Associated with Antiepileptic Drugs: Evaluation by Diffusion-Weighted MR Imaging. European Radiology, Vol.13, No.8, (August 2003), pp. 1902-1906, ISSN 0938-7994

Mann, S.C., Caroff, S.N., Bleier, H.R., Welz, W.K.R., Kling, M.A. \& Hayashida, M. (1986). Lethal Catatonia, American Journal of Psychiatry, Vol.143, No.11, (November 1986), pp. 1374-1381, ISSN 0002-953X

Mann, S.C., Caroff, S.N., Fricchione, G. \& Campbell, E.C. (2000). Central Dopamine Hypoactivity and the Pathogenesis of Neuroleptic Malignant Syndrome. Psychiatric Annals, Vol.30, No.5, (May 2000), pp. 363-374

Marie-José, C.C.D. Valproate-Induced Hyperammonaemic Encephalopathy: Review of 14 Cases in the Psychiatric Setting. (2007). International Clinical Pshychopharmacology, Vol.22, No, 6, (November 2007), pp. 330-337, ISSN 0268-1315

Mason, P.J., Morris, V.A. \& Balcezak, T.J. (2000). Serotonin Syndrome: Presentation of 2 Cases and Review of the Literature. Medicine, Vol.79, No.4, (July 2000), pp. 201-209, ISSN 0025-7974

McHenry, L. (2006). Ethical Issues in Psychopharmacology. Journal of Medical Ethics, Vol.32, No.7, (July 2006), pp. 405-410, ISSN 0306-6800

Meulendijks, D., Mannesse, C.K., Jansen, P.A.F., van Marum, R.J. \& Egberts, T.C.G. (2010). Antipsychotic-Induced Hyponatremia. Drug Safety, Vol. 33, No.2, (February 2010), pp. 101-14, ISSN 0114-5916

Middleton, N., Gunnell, D., Whitley, E., Dorling, D. \& Frankel, S. (2001). Secular Trends in Antidepressant Prescribing in the UK, 1975-1998. Journal of Public Health Medicine, Vol.23, No.4, (December 2001), pp. 262-267, ISSN 0957-4832

Mills, K.C. (1995). Serotonin Syndrome. American Family Physician, Vol.52, No.5, (October 1995), pp. 1475-1482, ISSN 0002-838X

Mills, K.C. (1997). Serotonin Syndrome. Critical Care Clinics, Vol.13, No.4, (October 1997), pp. 763-783, ISSN 0749-0704

Mitchell, R.S. (1955). Fatal Toxic Encephalitis Occurring during Iproniazid Therapy in Pulmonary Tuberculosis. Annals of Internal Medicine, Vol.42, No.2, (February 1955), pp. 417-424, ISSN 0003-4819

Moncrieff, J. \& Kirsch, I. (2005). Efficacy of Antidepressants in Adults. British Medical Journal, Vol.331(7509), (July 2005), pp. 155-157, ISSN 0959-535X 
Montoya, A., Ocampo, M. \& Torres-Ruiz, A. (2003). Neuroleptic Malignant Syndrome in Mexico. Canadian Journal of Clinical Pharmacology, Vol.10, No.3, (Autumn 2003), pp. 111-113, ISSN 1198-581X

Nagamine, M. Yoshino, A., Ishii, M., Ogawa, T., Kurauchi, S., Yoshida, T., Shigemura, J., Kodera, T., Tanaka, Y. \& Nomura, S. (2008). Lithium-Induced Hashimoto's Encephalopathy: A Case Report. Bipolar Disorders, Vol.10, No.7, (November 2008), pp. 846-848, ISSN 1398-5647

Nierenberg, D., Disch, M., Manheimer, E., Patterson, J., Ross, J., Silvestri, G. \& Summerhill, E. (1991). Facilitating Prompt Diagnosis and Treatment of the Neuroleptic Malignant Syndrome. Clinical Pharmacology and Therapeutics, Vol.50, No.5, (November 1991), pp. 580-586, ISSN 0009-9236

Nisijima, K., Noguti, M. \& Ishiguro, T. (1997). Intravenous Injection of Levodopa Is More Effective Than Dantrolene as Therapy for Neuroleptic Malignant Syndrome. Biological Psychiatry, Vol.41, No.8, (April 1997), pp. 913-914, ISSN 0006-3223

Nisijima, K., Shioda, K. \& Iwamura, T. (2007). Neuroleptic Malignant Syndrome and Serotonin Syndrome, Progress in Brain Research, Vol.162, pp. 81-104, ISSN 0079-6123

Northhoff, G. (1996). Neuroleptic Malignant Syndrome and Catatonia: One Entity or Two? Biological Psychiatry, Vol.40, No.5, (September 1996), pp. 431-432, ISSN 0006-3223

Odagaki, Y. (2009). Atypical Neuroleptic Malignant Syndrome or Serotonin Toxicity Associated with Atypical Antipsychotics? Current Drug Safety, Vol.4, No.1, 84-93, (January 2009), pp. 84-93, ISSN 1574-8863

Otani, K., Horiuchi, M., Kondo, T., Kaneko, S. \& Fukushima, Y. (1991). Is the Predisposition to Neuroleptic Malignant Syndrome Genetically Transmitted? British Journal of Psychiatry, Vol.158, No.6, (June 1991), pp. 850-853, ISSN 0007-1250

Paykel, E.S., Tylee, A., Wright, A., Priest, R.G., Rix, S. \& Hart, D. (1997). The Defeat Depression Campaign: Psychiatry in the Public Arena. American Journal of Psychiatry, Vol.154, No.6, Festschrift Suppl., (June 1997), pp. 59-65, ISSN 0002-953X

Picard, L.S., Lindsay, S., Strawn, J.R., Kaneria, R.M., Patel, N.C. \& Keck, P.E. Jr. (2008). Atypical Neuroleptic Malignant Syndrome: Diagnostic Controversies and Considerations. Pharmacopsychiatry, Vol.28, No.4, (April 2008), pp. 530-535, ISSN 0277-0008

Pisani, F., Oteri, G., Costa, C., Di Raimondo, G. \& Di Perri, R. (2002). Effects of Psychotropic Drugs on Seizure Threshold. Drug Safety, Vol.25, No.2, pp. 91-110, ISSN 0114-5916

Polster, T., Hoppe, M. \& Ebner, A. (2001). Transient Lesion in the Splenium of the Corpus Callosum: Three Further Cases in Epileptic Patients and a Pathophysiological Hypothesis. Journal of Neurology, Neurosurgery, and Psychiatry, Vol.70, No.4, (April 2001), pp. 459-463, ISSN 0022-3050

Pope, H.G. Jr., Keck, P.E. \& McElroy, S.L. (1986). Frequency and Presentation of Neuroleptic Malignant Syndrome in a Large Psychiatric Hospital. American Journal of Psychiatry, Vol. 143, No.10, (October 1986), pp. 1227-1233, ISSN 0002-953X

Posner, J.B., Saper, C.B., Schiff, N.D. \& Plum, F. (Eds.). (2007). Plum and Posner's Diagnosis of Stupor and Coma, Oxford University Press, ISBN 978-0-19-532131-9, New York, USA

Preston, J. (1959). Central Nervous System Reactions to Small Doses of Tranquilizers. Report of One Death. American Practitioner and Digest of Treatment, Vo10, No.4, (April 1959), pp. 627-630 
Price, J.S., Waller, P.C., Wood, S.M. \& MacKay, A.V.P. (1996). A Comparison of the PostMarketing Safety of Four Selective Serotonin Re-Uptake Inhibitors Including the Investigation of Symptoms Occurring on Withdrawal. British Journal of Clinical Pharmacology, Vol.42, No.6, (December 1996), pp. 757-763, ISSN 0306-5251

Radomski, J.W., Dursun, S.M., Reveley, M.A. \& Kutcher, S.P. (2000). An Exploratory Approach to the Serotonin Syndrome: An Update of Clinical Phenomenology and Revised Diagnostic Criteria. Medical Hypotheses, Vol.55, No.3, (September 2000), pp. 218-224, ISSN 0306-9877

Raja, M. \& Azzoni, A. (2002). Valproate-Induced Hyperammonaemia. Journal of Clinical Psychopharmacology, Vol.22, No.6, (December 2002), pp. 631-633, ISSN 0271-0749

Reddy, P. \& Mooradian, A.D. (2009). Diagnosis and Management of Hyponatremia in Hospitalised Patients. International Journal of Clinical Practice, Vol.63, No.10, (October 2009), pp. 1494-1508, ISSN 1368-5031

Reulbach, U., Dutsch, C., Biermann, T., Sperling, W., Thuerauf, N., Kornhuber, J. \& Bleich, S. Managing an Effective Treatment for Neuroleptic Malignant Syndrome. Critical Care, Vol.11, No.1, (January 2007), pp. R4, ISSN 1364-8535, Available from http://ccforum.com/content/11/1/R4

Riggs, A.T., Dysken, M.W., Kim, S.W. \& Opsahl, J.A. (1991). A Review of Disorders of Water Homeostasis in Psychiatric Patients. Psychosomatics, Vol.32, No.2, (Spring 1991), pp. 133-148, ISSN 0033-3182

Robinson, M.B., Kennett, R.P., Harding, A.E., Legg, N.J. \& Clarke, B. (1985). Neuroleptic Malignant Syndrome Associated with Metoclopramide. Journal of Neurology, Neurosurgery, and Psychiatry, Vol.48, No.12, (December 1985), pp. 1304, ISSN 0022-3050

Rosebush, P. \& Stewart, T. (1989). A Prospective Analysis of 24 Episodes of Neuroleptic Malignant Syndrome. American Journal of Psychiatry, Vol.146, No.6, (June 1989), pp. 717-725, ISSN 0002-953X

Rosebush, P.I., Stewart, T. \& Mazurek, M.F. (1991). The Treatment of Neuroleptic Malignant Syndrome. Are Dantrolene and Bromocriptine Useful Adjuncts to Supportive Care? British Journal of Psychiatry, Vol.159, No.5, (November 1991), pp. 709-712, ISSN 0007-1250

Rosenbaum, J.F. \& Zajecka, J. (1997). Clinical Management of Antidepressant Discontinuation. Journal of Clinical Psychiatry, Vol.58, Suppl.7, pp. 37-40, ISSN 01606689

Rosenbaum, J.F., Fava, M., Hoog, S.L., Ascroft, R.C. \& Krebs, W.B. (1998). Selective Serotonin Reuptake Inhibitor Discontinuation Syndrome: A Randomized Clinical Trial. Biological Psychiatry, Vol.44, No.2, (July 1998), pp. 77-87, ISSN 0006-3223

Rosenberg, M.R. \& Green, M. (1989). Neuroleptic Malignant Syndrome. Review of Response to Therapy. Archives of Internal Medicine, Vol.149, No.9, (September 1989), pp. 19271931, ISSN 0003-9926

Sakkas, P., Davis, J.M., Hua, J. \& Wang, Z. (1991a). Pharmacotherapy of Neuroleptic Malignant Syndrome. Psychiatric Annals, Vol.21, No.3, (March 1991), pp. 157-164

Sakkas, P., Davis, J .M., Janicak, P. G. \& Wang, Z. Y. (1991b) Drug Treatment of the Neuroleptic Malignant Syndrome. Psychopharmacology Bulletin, Vol.27, No.3, pp. 381-384, ISSN 0048-5764 
Schatzberg, A.F., Haddad, C.P., Kaplan, E.M., Lejoyeux, M., Rosenbaum, J.F., Young, A.H. \& Zajecka, J. (1997a). Possible Biological Mechanisms of the Serotonin Reuptake Inhibitor Discontinuation Syndrome. Journal of Clinical Psychiatry, Vol.58, Suppl.7, pp. 23-27, ISSN 0160-6689

Schatzberg, A.F., Haddad, C.P., Kaplan, E.M., Lejoyeux, M., Rosenbaum, J.F., Young, A.H. \& Zajecka, J. (1997b). Serotonin Reuptake Inhibitors Discontinuation Syndrome: A Hypothetical Definition. Journal of Clinical Psychiatry, Vol.58, Suppl.7, pp. 5-10, ISSN 0160-6689

Scheen, A.J. \& De Hert, M.A. (2007). Abnormal Glucose Metabolism in Patients Treated with Antipsychotics. Diabetes and Metabolism, Vol.33, No.3, (June 2007), pp. 169-175, ISSN 1262-3636

Serrano-Dueñas, M. (2003). Neuroleptic Malignant Syndrome-Like, or - Dopaminergic Malignant Syndrome - Due to Levodopa Therapy Withdrawal. Clinical Features in 11 Patients. Parkinsonism and Related Disorders, Vol.9, No.3, (January 2003), pp. 175178, ISSN 1353-8020

Shalev, A. \& Munitz, H. (1986). The Neuroleptic Malignant Syndrome: Agent and Host Interaction. Acta Psychiatrica Scandinavica, Vol.73, No.4, (April 1986), pp. 337-347, ISSN 0001-690X

Shalev, A., Hermesh, H. \& Munitz, H. (1988). The Role of External Heat Load in Triggering the Neuroleptic Malignant Syndrome. American Journal of Psychiatry, Vol.145, No.1, (January 1988), pp. 110-111, ISSN 0002-953X

Shalev, A., Hermesh, H. \& Munitz, H. (1989). Mortality from Neuroleptic Malignant Syndrome. Journal of Clinical Psychiatry, Vol.50, No.1, (January 1989), pp. 18-25, ISSN 0160-6689

Sheean, G.L. (1991). Lithium Neurotoxicity. Clinical and Experimental Neurology, Vol.28, pp. 112-127, ISSN 0196-6383

Shelton, R.C. (2006). The Nature of the Discontinuation Syndrome Associated with Antidepressant Drugs. Journal of Clinical Psychiatry, Vol.67, Suppl.4, pp. 3-7, ISSN 0160-6689

Shiloh, R., Valevski, A., Bodinger, L., Misgav, S., Aizenberg, D., Dorfman-Etrog, P., Weizman, A. \& Munitz, H. (2003). Precautionary Measures Reduce Risk of Definite Neuroleptic Malignant Syndrome in Newly Typical Neuroleptic-Treated Schizophrenia Inpatients. International Clinical Psychopharmacology, Vol.18, No.3, (May 2003), pp. 147-149, ISSN 0268-1315

Siegel, A.J. (2008). Hyponatremia in Psychiatric Patients: Update on Evaluation and Management. Harvard Review of Psychiatry, Vol.16, No.1, pp. 13-24, ISSN 1067-3229

Singh, A.N. \& MaGuire, J. (1987). Neuroleptic Malignant Syndrome (NMS): A Misnomer? British Journal of Psychiatry, Vol.151, No.6, (December 1987), pp. 863-864

Smith, M., Hopkins, D., Peveler, R.C., Holt, R.I.G., Woodward, M. \& Ismail, K. (2008). First$V$. Second-Generation Antipsychotics and Risk for Diabetes in Schizophrenia: Systematic Review and Meta-Analysis. British Journal of Psychiatry, Vol.192, No.6, (June 2008), pp. 406-411, ISSN 0007-1250

Spigset, O. \& Hedenmalm, K. (1995). Hyponatraemia and the Syndrome of Inappropriate Antidiuretic Hormone Secretion (SIADH) Induced by Psychotropic Drug. Drug Safety, Vol.12, No.3, (March 1995), pp. 209-225, ISSN 0114-5916 
Spirt, M.J., Chan, W., Thieberg, M. \& Sachar, D.B. (1992). Neuroleptic Malignant Syndrome Induced by Domperidone. Digestive Diseases and Sciences, Vol.37, No.6, (June 1992), pp. 946-948, ISSN 0163-2116

Spivak, B., Maline, D.I., Kozyrev, V.N., Mester, R., Neduva, S.A., Ravilov, R.S. \& Weizman, A. (2000). Frequency of Neuroleptic Malignant Syndrome in a Large Psychiatric Hospital in Moscow. European Psychiatry, Vol.15, No.5, (August 2000), pp. 330-333, ISSN 0924-9338

Sporer, K.A. (1995). The Serotonin Syndrome. Implicated Drugs, Pathophysiology and Management. Drug Safety, Vol.13, No.2, (August 1995), pp. 94-104, ISSN 0114-5916

Steele, D., Keltner, N.L. \& McGuiness, T.M. (2011). Are Neuroleptic Malignant Syndrome and Serotonin Syndrome the Same Syndrome? Perspectives in Psychiatric Care, Vol.47, No.1, (January 2011), pp. 58-62, ISSN 0031-5990

Sternbach, H. (1991). The Serotonin Syndrome. American Journal of Psychiatry, Vol.148, No.6, (June 1991), pp. 705-713, ISSN 0002-953X

Sterns, R.H., Nigwekar, S.U. \& Hix, J.K. (2009). The Treatment of Hyponatremia. Seminars in Nephrology, Vol.29, No.3, (May 2009), pp. 282-299, ISSN 0270-9295

Strawn, J.R., Keck, P.E. Jr. \& Caroff, S.N. (2007). Neuroleptic Malignant Syndrome. American Journal of Psychiatry, Vol.164, No.6, (June 2007), pp. 870-876, ISSN 0002-953X

Susman, V.L. (2001). Clinical Management of Neuroleptic Malignant Syndrome. Psychiatric Quarterly, Vol.72, No.4, (December 2001), pp. 325-328, ISSN 0033-2720

Takubo, H., Harada, T., Hashimoto, T., Inaba, Y., Kanazawa, I., Kuno, S., Mizuno, Y., Mizuta, E., Murata, M., Nagatsu, T., Nakamura, S., Yanagisawa, N. \& Narabayashi, H. (2003). A Collaborative Study on the Malignant Syndrome in Parkinson's Disease and Related Disorders. Parkinsonism and Related Disorders, Vol.9, Suppl.1, (April 2003), pp. S31-S41, ISSN 1353-8020

Tamam, L. \& Ozpoyraz, N. (2002). Selective Serotonin Reuptake Inhibitor Discontinuation Syndrome: A Review. Advances in Therapy, Vol.19, No.1, (January/February 2002), pp. 17-26, ISSN 0741-238X

Taylor, N.E. \& Schwartz, H.I. (1988). Neuroleptic Malignant Syndrome Following Amoxapine Overdose. Journal of Nervous and Mental Disease, Vol.176, No.4, (April 1988), pp. 249-251, ISSN 0022-3018

Thomas, Z., Bandali, F., McCowen, K. \& Malhortra, A. (2010). Drug-Induced Endocrine Disorders in the Intensive Care Unit. Critical Care Medicine, Vol.38, No.6, Suppl., (June 2010), pp. S219-S230, ISSN 0090-3493

Toru, M., Matsuda, O., Makiguchi, K. \& Sugano, K. (1981). Neuroleptic Malignant Syndrome-Like State Following a Withdrawal of Antiparkinsonian Drugs. Journal of Nervous and Mental Disease, Vol.169, No.5, (May 1981), pp. 324-327, ISSN 0022-3018

Trollor, J.N. \& Sachdev, P.S. Electroconvulsive Treatment of Neuroleptic Malignant Syndrome: A Review and Report of Cases. Australian and New Zealand Journal of Psychiatry, Vol.33, No.5, (October 1999), pp. 650-659, ISSN 0004-8674

Trollor, J.N., Chen, X. \& Sachdev, P.S. (2009). Neuroleptic Malignant Syndrome Associated with Atypical Antipsychotic Drugs. CNS Drugs, Vol.23, No.6, (June 2009), pp. 477492, ISSN 1172-7047

Upadhyay, A., Jaber, B.L. \& Madias, N.E. (2006). Incidence and Prevalence of Hyponatremia. American Journal of Medicine, Vol.119, No.7, Suppl. 1(July 2006), pp. S30-S35, ISSN 0002-9343 
Upadhyay, A., Jaber, B.L. \& Madias, N.E. (2009). Epidemiology of Hyponatremia. Seminars in Nephrology, Vol.29, No.3, (May 2009), pp. 227-238, ISSN 0270-9295

Velamoor, V.R., Norman, R.M., Caroff, S.N., Mann, S.C., Sullivan, K.A. \& Antelo, R.E. (1994). Progression of Symptoms in Neuroleptic Malignant Syndrome. Journal of Nervous and Mental Disease, Vol.182, No.3, (March 1994), pp. 168-173, ISSN 00223018

Viejo, L.F., Morales, V., Puňal, P., Pérez, J.L. \& Sancho, R.A. (2003). Risk Factors in Neuroleptic Malignant Syndrome. A Case-Control Study. Acta Psychiatrica Scandinavica, Vol.107, No.1, (January 2003), pp. 45-49, ISSN 0001-690X

Vieweg, W.V., David, J.J., Rowe, W.T., Wampler, G.J., Burns, W.J. \& Spradlin, W.W. (1985). Death from Self-Induced Water Intoxication among Patients with Schizophrenic Disorders. Journal of Nervous and Mental Disorders, Vol.173, No.3, (March 1985), pp. 161-165, ISSN 0022-3018

Vieweg, W.V., Rowe, W.T., David, J.J., Curnow, R.T. \& Spradlin, W.W. (1986). Self-Induced Water Intoxication and Psychosis (SIWIP): Subcategory of the Syndrome of Inappropriate Antidiuresis (SIAD). Psychiatric Medicine, Vol.4, No.3, pp. 277-290, ISSN 0732-0868

Watson, W.A., Litovitz, T.L., Rodgers, G.C. Jr., Klein-Schwartz, W., Reid, N., Youniss, J., Flanagan, A. \& Wruk, K.M. (2005). 2004 Annual Report of the American Association of Poison Control Centers Toxic Exposure Surveillance System. American Journal of Emergency Medicine, Vol.23, No.5, (September 2005), pp. 589-666, ISSN 0735-6757

Wehring, H.J., Kelly, D.L., Love, R.C. \& Conley, R.R. (2003). Deaths from Diabetic Ketoacidosis after Long-Term Clozapine Treatment. American Journal of Psychiatry, Vol.160, No.12, (December 2003), pp. 2241-2242, ISSN 0002-953X

White, D.A.C. (1992). Catatonia and the Neuroleptic Malignant Syndrome - A Single Entity? British Journal of Psychiatry, Vol.161, No.4, (October 1992), pp. 558-560, ISSN 00071250

Woodbury, M.M. \& Woodbury, M.A. (1992). Neuroleptic-Induced Catatonia as a Stage in the Progression toward Neuroleptic Malignant Syndrome. Journal of the American Academy of Child and Adolescent Psychiatry, Vol.31, No.6, (November 1992), pp. 11611164, ISSN 0890-8567

Yamawaki, S., Yano, E. \& Uchitomi, Y. (1990). Analysis of 497 Cases of Neuroleptic Malignant Syndrome in Japan. Hiroshima Journal of Anesthesia, Vol.26, No.1, (March 1990), pp. 35-44

Yamawaki, Y. \& Ogawa, N. (1992). Successful Treatment of Levodopa-Induced Neuroleptic Malignant Syndrome (NMS) and Disseminated Intravascular Coagulation (DIC) in a Patient with Parkinson's Disease. Internal Medicine, Vol.31, No.11, (November 1992), pp. 1298-1302, ISSN 0918-2918

Ziegenbein, M., Kropp, S., Hillemacher, T. \& Bleich, S. (2006). Genetic Predisposition to Neuroleptic Malignant Syndrome in Siblings. Annals of Pharmacotherapy, Vol.40, No.3, (March 2006), pp. 574-575, ISSN 1060-0280 


\title{
The Use of Microdialysis in the Study of Encephalopathies
}

\author{
Liliana Carmona-Aparicio, Liliana Rivera-Espinosa \\ and Hugo Juárez-Olguín \\ Instituto Nacional de Pediatría \\ México, D.F.
}

\section{Introduction}

Microdialysis is a technique that was devised by Bito et al. (1966) in a study to determine free aminoacids and electrolytes in cerebrospinal fluid and blood plasma in dogs. It consists of recollection of samples in specific places by using semi-permeable membranes, used to evaluate compounds (endogenous neurotransmitters or exogenous such as drugs), in extracellular spaces from different tissues as brain, skin, subcutaneous, tumors, kidney, abdominal and femoral adipose tissue, muscle, lung, middle ear fluid, and other extracellular spaces like blood. Currently, one of the main applications of this methodology in the field of neuroscience is to conduct studies to monitor the release of different substances in brain of conscious and unconscious anesthetized animals. The technique has also been used in surgical procedures as well as in monitoring patients in intensive care unit with traumatic brain injury and subarachnoid hemorrhage. It is employed in neuronal diseases like Epilepsy, Alzheimer, Parkinson, Huntington, Amyotrophic Lateral Sclerosis (ALS) and Encephalopathies.

Encephalopathies are a group of diseases affecting brain which are attributed to a wide range of etiologies with spectra of symptomatology. The main causes of encephalopathy are infections, liver damage, anoxia, hypoxia, ischemia, and kidney failure. This pathology can undergo through altered mental state. The mental alteration may be subtle with slow development lasting for years or it may be profoundly obvious with fast development. The cardinal symptoms of this state consist of inattentiveness, poor judgment, or poor motor coordination, lethargy, dementia, seizures, tremors, muscle twitching and coma. The severity and type of symptoms are related to the level of severity and the cause of the brain disease or damage. The diagnosis of encephalopathy is done by clinical tests during the physical memory and coordination tests, and it is made when the altered mental state is accompanied by another primary diagnosis such as chronic liver disease, kidney failure, anoxia, or others. Thus, the complications derived from encephalopathies arise from a primary health problem or a previous primary diagnosis. The need to avoid complications caused by late diagnosis resides on identification and clarification of the mechanisms underlying the neuronal damage and a search for markers that would give rise to timely diagnosis of the pathology. As a consequence, researchers in neurosciences have devoted their time in resolving this issue through various experimental approaches including 
microdialysis. Currently, microdialysis is not only used to determine the neurochemical abnormalities induced by encephalopathy, but also in the determination of the therapeutic effects of various drugs in the treatment of this pathologic condition. In this chapter we will discuss the utility of microdialysis as a tool for the study of encephalopathy in human and experimental models.

\section{Microdialysis technique}

\subsection{General characteristics}

Microdialysis was developed for continuous sample collection in specific brain areas. This procedure permits the exploration, in real time, of the chemical interchange among cells in extracellular space using samples from the extracellular fluid. This is contrary to other sampling schemes like blood sampling which does not reflect specific changes for the mere fact that molecules are diluted into a relatively high compartment that irrigate vats tissues. It also provides information without the need of dissecting tissue samples to gather information that would be a picture of biochemical events (Robinson \& Justice, 1991).

The group of Bito et al (1966) was the first to introduce "bags of dialysis" within the subcutaneous tissue of the neck and within the parenchyma in the cerebral hemisphere of dogs in order to analyze the content of amino acids in this brain region. These experiments introduced the idea of a "compartment" surrounded by a dialyzing membrane balanced with the extracellular space (Robinson \& Justice, 1991).

Microdialysis is an experimental method consisting of a closed system, in which a thin tube with a dialyzing membrane is inserted into a particular region of the brain or parenchyma of some other tissues like kidney, skin or others where the cannula is fixed. The principle of microdialysis is based on the physicochemical phenomenon of passive diffusion, that occurs when a substance is not homogeneously distributed in an environment accumulating a force that tend to equalize the chemical potential of the same throughout the space, thereby producing a molecular flow from the higher chemical potential of this substance to the lower concentration compartment (Bellander et al., 2004; Di Chiara et al., 1996; Hsiao et al., 1990; Kendrick et al., 1988; Kennth et al., 2002; Juhasz et al., 1989).

It is a spontaneous and irreversible process in which there is an expansion of a substance in solution as a result of movement of its particles, to fill all available volume in which they are found. This movement is constant and random molecules can interchangeably move from one direction to another. However, if one takes into account that the number of particles of a given substance is higher in places of higher concentrations, there is a predominant shift to low concentration sites, giving rise to a net diffusion of the substance from the sites of higher concentration to lower concentration. It is worth mentioning that semi-permeable membranes are characterized by pore size, only found in low weight molecules. This principle is schematized in Figure 1 (Hocht, 2007; Hsiao et al., 1990; Jaitovich, 2003; Juhasz et al., 1989; Orlowska-Majdak, 2004; Verbeeck, 2000; Ungerstedt \& Hallström, 1987).

Microdialysis involves the passage of a vehicle (perfusion) at continuous and defined flow through a membrane, which is implanted in the tissue of interest in case of in vivo experimental models (Figure 2), and in humans, in a solution of known concentration in 
case of in vitro experiments. The choice of vehicle in this type of experimentation must be adequate for the environment where the cannula of microdialysis is fixed. Aqueous solutions are commonly used with concentrations of sodium and potassium ions in low concentrations, without the presence of proteins or at very low concentrations that resemble the extracellular space to study. In some cases, the vehicle may contain proteins to prevent drug sticking to the sides of the cannula (Boschi \& Scherrmann, 2000; Elmquist \& Sawchuk, 1997; Orlowska-Majdak, 2004; Plock \& Kloft, 2005; Verbeeck, 2000).

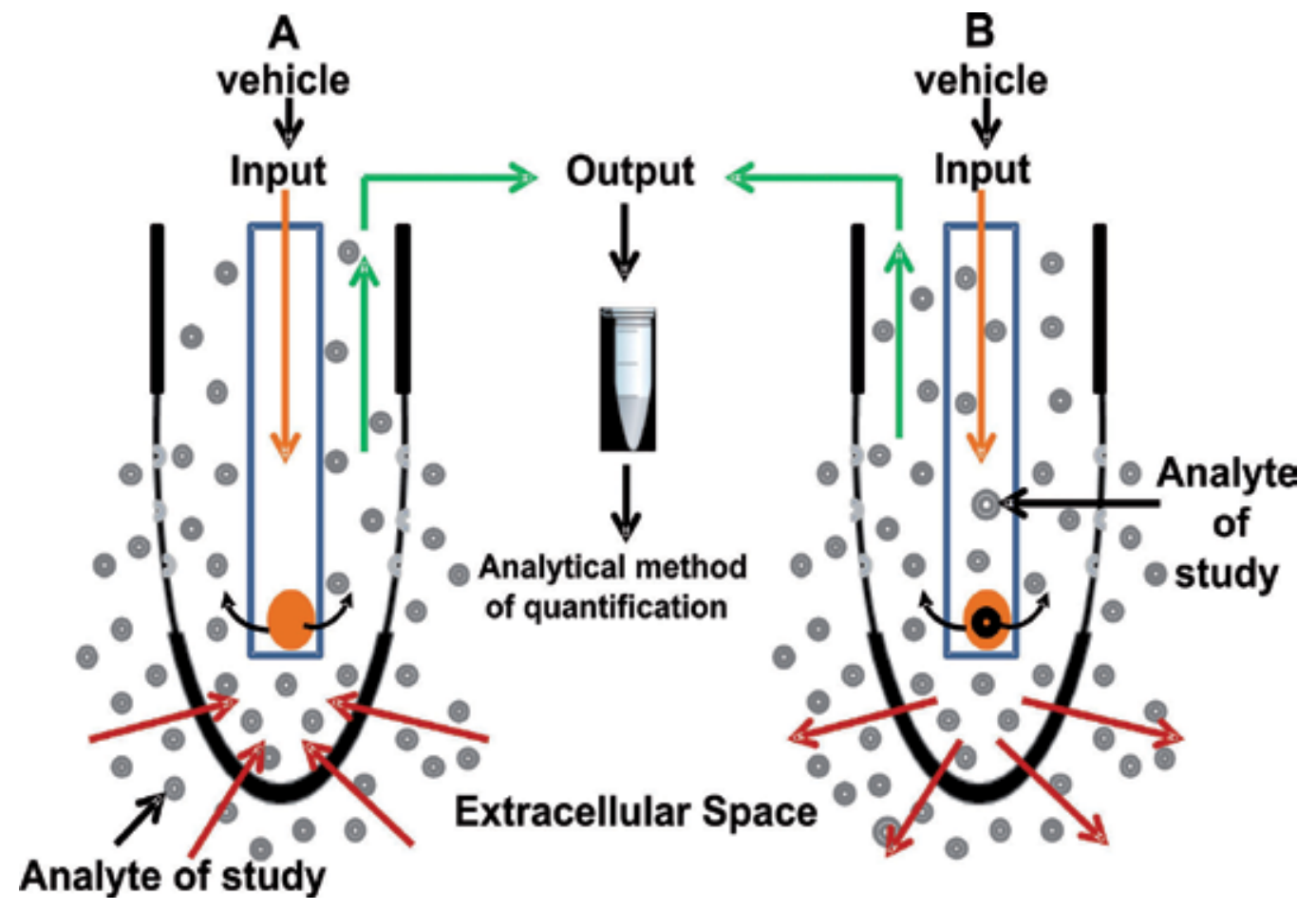

Fig. 1. Directions of molecular migration through dialyzing membrane during microdialysis (A) and retrodialysis (B). The analyte of study in (A) is from extracellular space whereas that in (B) is from external vehicle. Note that the test substance and the case of microdialysis or retrodialysis do not exceed the size of the pores present in the semipermeable membrane. The inlet and outlet terms refer to the vehicle entry and exit respectively. The outlet is always connected to a storage container subsequently quantified by the analytical method with increased sensitivity to the analyte under study.

Vehicles used in microdialysis are of different chemical composition, such as saline, ringer buffers, and artificial cerebrospinal liquid. In the case of retrodialysis, the vehicle is the matrix in which interest analyte is expected to exert a physiological and biochemical effects in the extracellular space of interest. In the process of infusion, the vehicle flow is generated by the movement of analyte that is released by forming a concentration gradient between the two regions separated by a membrane (Figure 3).

The extracellular fluid from the site of membrane location dictates the composition of the infused fluid used in in vivo paradigms. Dissemination occurs through a semipermeable 
membrane. Endogenous compounds such as neurotransmitters, neuropeptides, neurohormones, etc. and exogenous compounds (drugs and metabolites) spread to the interior, while compounds that have been added diffuse out of the vehicle of the retrodialyzer infusion solution (Figure 1).

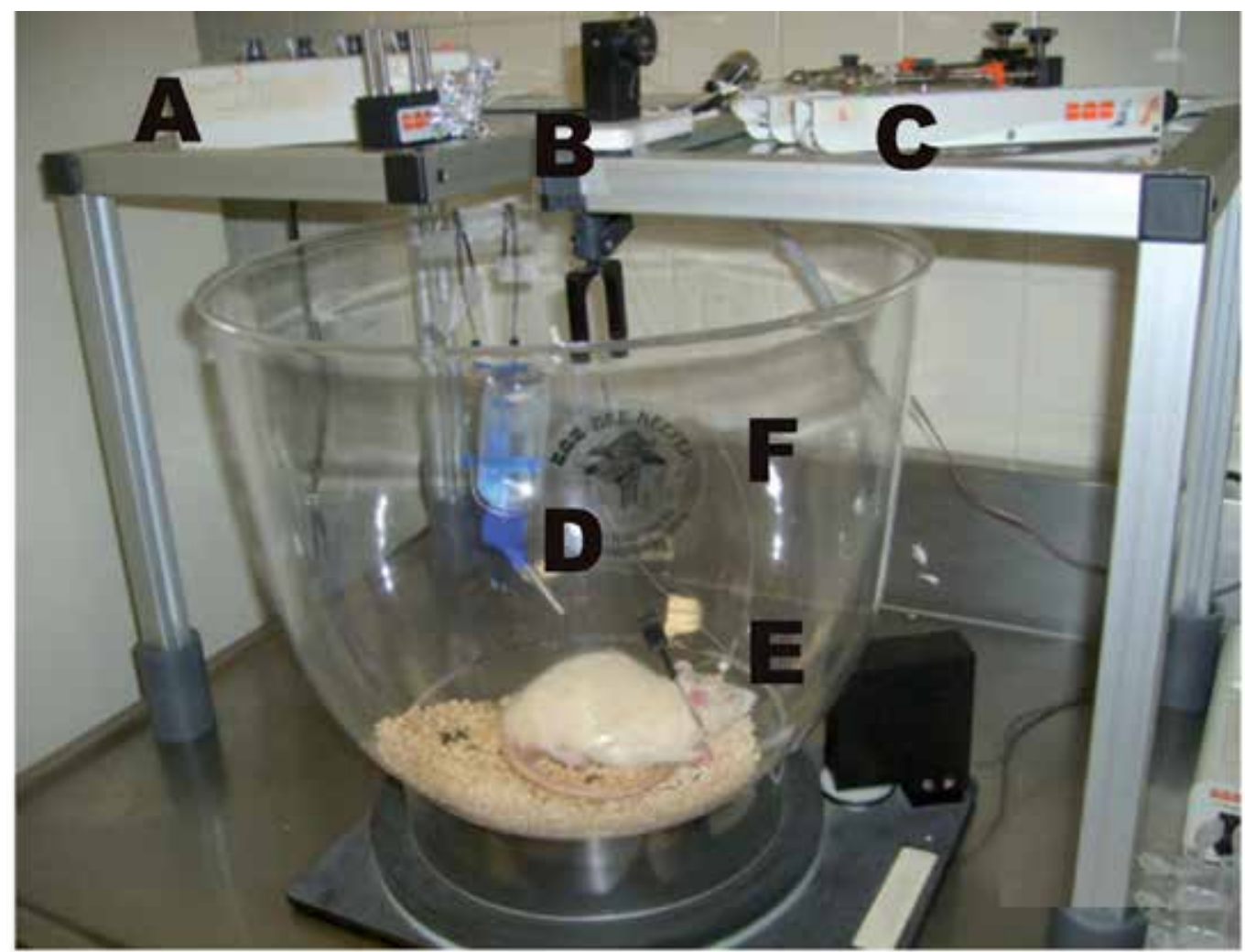

Fig. 2. Representative image of microdialysis system for experimental models in vivo.

A) Velocity controller, B) flow manager, C) booster pump, D) inlet tube, E) clamping collar, and F) Outlet tube.

Several factors affect this diffusion process in the semipermeable membrane of the cannula of microdialysis, and consequently the recovery of the substance of interest. These factors are: a) The flow rate, where the recovery is inversely proportional to the infusion rate and the recovery of the substance of interest is close to one hundred percent when the flow is near zero and it is minimum when it is fast; $b$ ) the membranes composition: There are over 30 different materials for the production of membranes which can be directly derived from natural products, semi-synthetic and fully synthetic; c) the presence of surface charges that reduce the recovery of certain charged molecules, which does not happen with neutral molecules; d) total membrane area (length), where recovery is directly proportional to the total area of the membrane, and e) temperature of the system, where $37^{\circ} \mathrm{C}$ facilitates the diffusion process. 


\subsection{Advantages and disadvantages}

The microdialysis technique has a number of advantages and limitations over other similar techniques such as push-pull cannula. The principal advantages are: a) easy to set up in working routine; b) facilitates the manufacture of probes with homogeneous characteristics; c) can be attached to different systems for chemical analysis; d) systemic or local administration of substances can be easily done; e) stimulation and recording electrical activity can be coupled to microdialyzer system; and f) monitoring abnormal behavior in animals in free movement. The principal disadvantages are: a) it is an invasive technique that generates tissue damage and gliosis, b) it presents low recovery rates of high molecular weight molecules; and d) the final volume presents analytical limitations (Bonate, 1995; Dash et al., 1999; Johnston \& Gupta, 2002; Mendelowitsch, 2001; Orłowska-Majdak, 2004; Parent et al., 2001; Viñas, 2001).

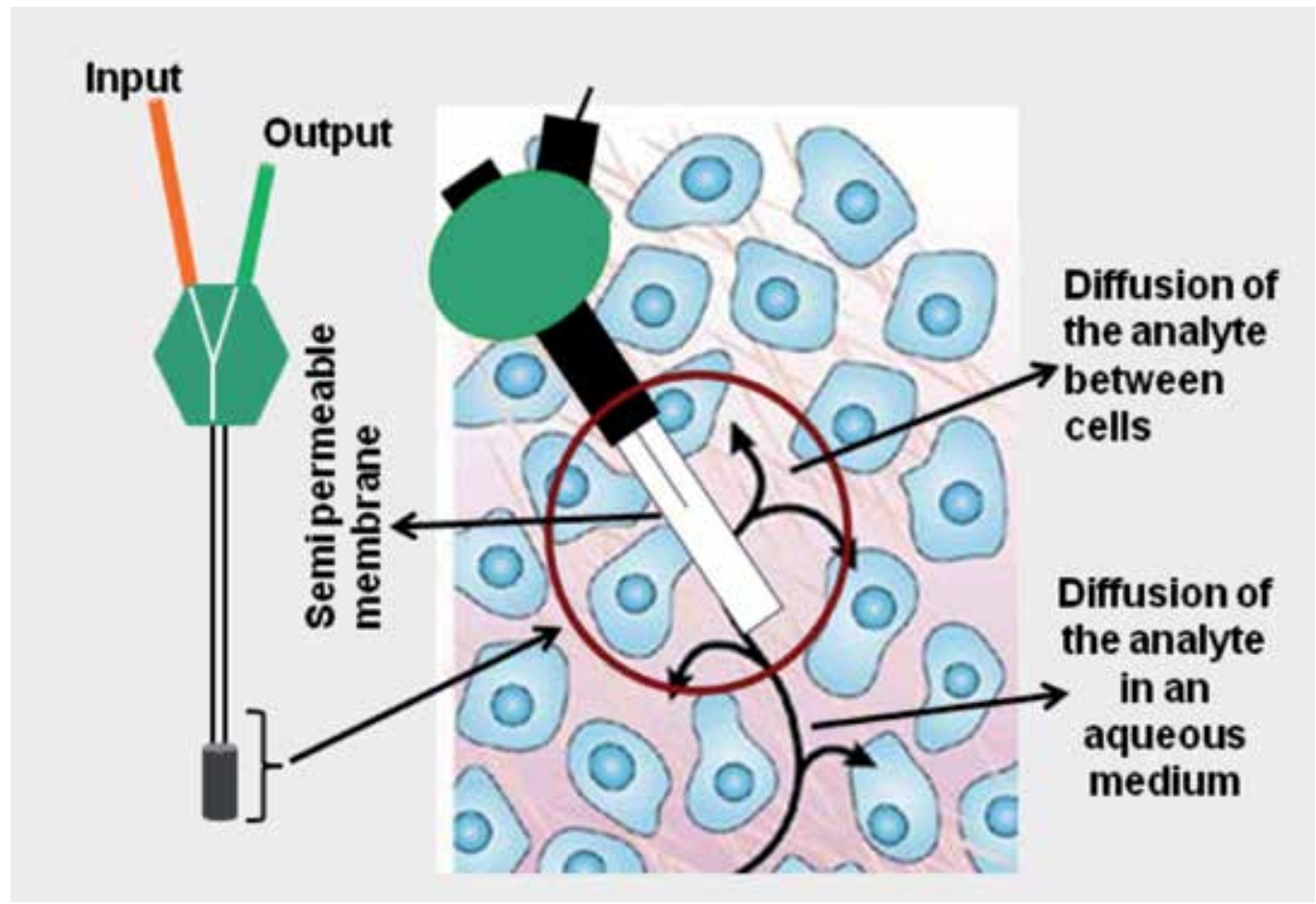

Fig. 3. Schematic representation of the microdialysis cannula place in an in vivo study. The microdialysis cannula membrane highlighted is the active part. The scheme shows the localization of the active part of microdialysis cannula between the extracelullar space of the cells.

One of the principal disadvantages of microdialysis technique is that it does not ensure complete recovery of the substances present in the extracellular space, or externally administered to the space for quantification. This depends on different factors such as the type of membrane, the molecular weight of substances, their permeability through the membrane, and the rate of vehicle flow. The amount of substances crossing the membrane under certain experimental conditions is defined as the recovery percent and it is the result 
of the relationship between the concentrations of the substance measured in the collected sample and their concentration in the outer membrane of microdialysis. This recovery is usually determined in-vitro and expressed as a percentage. The recovery of different probes is calculated for each of the studied substances. The probes are placed in dissolution with known concentration.

The recovery of the substance across the probe is determined by calculating the percentage of the measured amounts in dialysis with respect to the total amount present in the external environment of the probe. For this reason, it is very important to determine the best experimental condition (Boschi \& Schermann, 2000; Dash et al., 1999; Hitzman et al., 2005).

This mathematical relationship is expressed in the formula below:

Relative recovery (RR): $R R=1-(C$ out $/ C$ in $)$

Where " $\mathrm{C}$ in" is the analyte concentration in the perfusate, and " $\mathrm{C}$ out" is the analyte concentration in the dialysate.

\section{General characteristics of encephalopathies}

Encephalopathy is a series of pathological conditions affecting the brain. This includes but not limited to the cerebral cortex intracranial white matter, basal ganglia, hypothalamus, brainstem, and cerebellum (Cash et al., 2010). Encephalopathy is a term that means brain disease, damage, or malfunction and this may present a very broad spectra of symptoms that range from mild, such as memory loss or subtle personality changes, to severe, such as dementia, seizures, coma, or death. In general, encephalopathy is manifested by an altered mental state that is sometimes accompanied by physical manifestations, for example, poor coordination of limb movements (Müller et al., 2008). The term "encephalopathy" is very broad and in most cases, is preceded by various terms that describe the reason, cause, or special conditions of the patient that leads to brain malfunction (Goetz et al., 1999). For example, anoxic encephalopathy means brain damage due to lack of oxygen, and hepatic encephalopathy means brain malfunction due to liver disease. Additionally, some other terms may either describe body conditions or syndromes leading to a specific set of brain malfunctions. Examples of these are metabolic encephalopathy and Wernicke's encephalopathy (Wernicke's syndrome). There are over 150 different terms that modify or precede "encephalopathy" in the medical literature (Randolph et al., 2009).

\subsection{Causes}

The causes of encephalopathy are both numerous and varied. Some examples include: infections (bacteria, viruses, parasites), anoxia (lack of oxygen in the brain), alcohol consumption, liver failure, kidney failure, metabolic diseases, brain tumors, many types of toxic chemicals, alterations in brain pressure, and poor nutrition (Hillbom \& Marttila, 2010). These examples do not cover all the potential causes of encephalopathy but are listed to demonstrate the wide range of causes (Sato \& Moriuchi, 2010). Although numerous causes of encephalopathy are known, the majority of cases are categorized into: infection, liver damage, anoxia, and kidney failure (Chung \& Podolsky, 2005). 


\subsection{Symptoms}

Despite the numerous and varied causes of encephalopathy, at least there is a symptom common to all cases: an altered mental state. The altered mental state may be subtle with slow development during many years (for example, in hepatitis with decreased ability to draw simple designs, termed apraxia). On the other hand, it can be profoundly obvious and develop rapidly (for example, brain anoxia leading to coma or death in a few minutes). Often, symptoms of altered mental status can present as inattentiveness, poor judgment, or poor coordination of movements. Other symptoms that may occur include: lethargy, dementia, seizures, tremors, muscle twitching, and coma. Often, the severity and type of symptoms are related to the severity and the cause of the brain disease or damage. For example, alcohol-induced liver damage (alcoholic cirrhosis) can result in involuntary hand tremors (asterixis), while severe anoxia (lack of oxygen) may result in coma with no movement.

\subsection{Diagnosis}

The diagnosis of encephalopathy is usually by clinical tests done during the physical examination (mental status tests, memory tests, coordination tests) that document an altered mental state. With most cases, findings on clinical tests can either give definitive diagnosis or presumptive diagnosis of encephalopathy (Prakash \& Mullen, 2010). Usually, the diagnosis occurs when the altered mental state is found in conjunction with a primary cause such as chronic liver disease, kidney failure, anoxia, or many other diagnoses. This is contrary to other sampling schemes like blood sampling which does not reflect specific changes for the mere fact that molecules are diluted into a relatively high compartment that irrigate vats tissues. Consequently, physicians may use several different tests at the same time to diagnose both the primary condition (the cause of encephalopathy) and the encephalopathy itself. This approach to diagnosis is common to most physicians, because many doctors view encephalopathy as a secondary complication of a primary underlying health problem.

The most frequently used tests are listed below and with some of the major primary causes, the tests may help in the diagnosis of: a) complete blood count or CBC (infections, loss of blood); b) blood pressure (high or low blood pressure); c) metabolic tests (blood levels of electrolytes, glucose, lactate, ammonia, oxygen, and liver enzyme levels); d) drugs or toxin levels (alcohol, cocaine, amphetamines, and many others); e) blood and body fluid cultures and analysis (infections of many types); f) creatinine (kidney function); g) CT and MRI scans (brain swelling, anatomical abnormalities, infections); h) abnormal blood flow to tissues and abscesses; i) Encephalogram or EEG (brain damage, abnormal brain wave patterns); and j) auto-antibody analysis (dementia caused by antibodies that destroy neurons). This list is not exhaustive, and not all the above tests are needed to reach a diagnosis. Normally, specific tests are usually ordered by the treating physician depending on the symptoms and history of the patient.

There are other Diagnostic Criteria of Encephalopathy. The most relevant are: a) gradual onset (in hours); b) progression in untreated patients; c) gradual decrease in the level of consciousness; d) patients treated with various CNS depressant medications; e) patients with any organ failure, postoperative electrolyte imbalance, endocrine diseases; f) no 
evidence of brain tumor or stroke, but usually no focal hypoglycemia; g) sometimes preceded by focal or generalized seizures; $h$ ) increased spontaneous motor activity (asterixis, myoclonus, rigidity, etc.); i) changes in plasma biochemistry, and hemogram GSA; j) usually normal in imaging studies; k) generalized EEG abnormalities (slowing, triphasic waves); and l) gradual recovery to begin treatment (Randolph et al., 1998).

\subsection{Treatment}

Treatment of encephalopathy varies depending on the primary cause of the symptoms. Consequently, not all cases of encephalopathy are treated in the same way. The best treatments are designed by the treating physician once the patient's primary diagnosis is made. Treatments are highly variable because the causes are so different (Bajaj, 2010). Examples can show how different "encephalopathy treatment" may change depending on the cause: a) short-term anoxia (usually less than two minutes): oxygen therapy; b) longterm anoxia: rehabilitation; c) short- term alcohol toxicity: IV fluids or no therapy; d) longterm alcohol status (cirrhosis or chronic liver failure): oral lactulose, low-protein diet, antibiotics; e) uremic encephalopathy (due to kidney failure): correct the underlying physiological cause, dialysis, kidney transplant; f) diabetic encephalopathy: glucose for hypoglycemia, removal of blood glucose to treat hyperglycemia; g) hypo- or hypertensive encephalopathy: raise (for hypotension) or reduce (for hypertension) blood pressure (Sundaram \& Shaikh, 2009).

The key to treatment of any encephalopathy is to understand the basic cause and thus design a treatment scheme to reduce or eliminate the cause(s). Static encephalopathy (an altered mental state or brain damage that is permanent) is one type of encephalopathy difficult or impossible to treat. The best that can be done in this case if possible is to prevent further damage and implement rehabilitation to allow the individual to live at his or her highest possible functional level (Bismuth et al., 2011).

\subsection{Complications}

Complications of encephalopathy vary from none to profound mental impairments that could lead to death. The complications can be similar in some cases. Also, many investigators consider encephalopathy to be a complication that arises from a primary health problem or primary diagnosis. Complications depend on the primary cause of encephalopathy and can be illustrated by citing a few examples from the wide variety of causes: a) hepatic (liver) encephalopathy (brain swelling with herniation, coma, death); b) metabolic encephalopathy (irritability, lethargy, depression, tremors; occasionally coma or death); c) anoxic encephalopathy (wide range of complications, from none in short-term anoxia to personality changes, severe brain damage and death in long- term anoxic events); d) uremic encephalopathy (lethargy, hallucinations, stupor, muscle twitching, seizures, and death); e) Hashimoto's encephalopathy (confusion, heat intolerance, and dementia); f) Wernicke's encephalopathy (mental confusion, memory loss, and decreased ability for eye movement); g) bovine spongiform encephalopathy or "Mad Cow disease" (ataxia, dementia and myoclonus or muscle twitching without any rhythm or pattern); h) Shigella encephalopathy (headache, stiff neck, delirium, seizures, coma); i) infectious causes of pediatric encephalopathy (irritability, poor feeding, hypotonia or floppy baby syndrome, seizures, death; Nabbout et al., 2011). 
The best way to understand potential complications is to discuss them with the diagnosing doctor who can explain the possible problems associated with the specific cause(s) of the type of encephalopathy (Sima, 2010).

\subsection{Prognosis}

The prognosis of a patient with encephalopathy depends on the initial cause and, in general, the length of time it takes to reverse, stop, or inhibit those causes. Consequently, the prognosis varies from patient to patient and ranges from complete recovery to a poor prognosis that often leads to permanent brain damage or death. One good example of this highly variable prognosis is a patient with hypoglycemic encephalopathy.

When patients with hypoglycemia are given glucose at first signs of encephalopathy (for example, irritability, mild confusion), most of them completely recover from the problem. Delays in correcting hypoglycemia (hours to days) may lead to seizures or coma which may be halted by treatment with complete or partial recovery (minimal permanent brain damage). A long delay or multiple delays in treatment can lead to a poor prognosis with extensive brain damage, coma, or death (Munoz, 2008).

Although the symptoms and time frame vary widely from patient to patient and according to the initial causes of encephalopathy (see above), the prognosis of each case usually follows the pattern described in hypoglycemic cause and depends upon the extent and rapidity with which the underlying cause is treated. The doctor or team of doctors treating the underlying cause of encephalopathy can offer the best information on the individual's prognosis (Häussinger, 2010).

\subsection{Can encephalopathy be prevented?}

Many cases of encephalopathy can be prevented. The key to prevention is to stop or limit the chance of developing any of the multitudes of causes of encephalopathy. If encephalopathy develops, the quicker the underlying cause is treated, the more likely it is to prevent the eruption of severe encephalopathy (Montgomery \& Bajaj, 2011). Examples of prevention and avoidable situations are listed below: a) diabetic encephalopathy: daily check of glucose level and assurance of correct dosage of insulin; b) hepatic encephalopathy: avoidance of alcohol intoxication, drug overdose, and IV injections of illegal drugs; c) anoxic encephalopathy: prevention of food choking, avoidance of risky behavior that could lead to head and neck trauma, and exposure to carbon monoxide; d) hypertensive encephalopathy: monitoring of blood pressure; use of antihypertensive medication as directed and not quitting medications or changing medication without consulting the doctor; e) infectious encephalopathy: eluding physical contact with individuals known to be infected with organisms that may cause encephalopathy such as N. meningitidis or Shigella; f) uremic encephalopathy: do not skip or avoid scheduled dialysis, taking all medications as directed and having frequent assessment of mental status. Methods for prevention of encephalopathy are almost as numerous as the underlying causes. However, some cases of encephalopathy may not be preventable especially those originating from congenital cause or traumatic accident.

Therefore and as mentioned earlier, the key to therapeutic follow-up of any encephalopathy is to understand the underlying mechanism of the pathology and thus propose the best 
treatment scheme. In this sense, microdialysis may contribute to understanding these basic mechanisms of encephalopathy, as well as to the possible application of pharmacological treatments that could restore or improve brain function of the patients, and in this way improve the quality of life of those suffering from this problem.

\section{Microdialysis: Contributions in the study of encephalopathy}

\subsection{Encephalopathy induction mechanisms}

\subsubsection{Hepatic encephalopathy}

Hepatic encephalopathy (HE) is a neuropsychiatric disorder that often occurs as a consequence of acute or chronic liver failure where hyperammonemia plays a major role in the pathogenesis of the disease. Hyperammonemia leads to altered cerebral function and neurological alterations in patients with hepatic encephalopathy. Studies with pigs showed that there is an association between extracellular brain ammonia and intracranial pressure (ICP), given that the concentration of ammonia in the brain, when it gets to about 1,200 microM of arterial accumulated ammonia, increases ICP. This result suggests that ICP could serve as a sensitive marker for HE development (Zwirner et al., 2010). Also, Szerb and Redondo (1993) suggest that ammonia alters the function of astrocytes, facilitating their entry into the brain. This physiological event contributes to the development of HE. This was demonstrated with local administration of fluoroacetate in rats' hippocampal dentate gyrus using microdialysis (Szerb \& Redondo, 1993).

Other studies have permitted the elucidation of the role of glutamatergic system and its receptors in HE. In 1996, Bergqvist et al tried to explain the participation of quinolinic acid (QUIN; a potent N-methyl-D-aspartate, NMDA-receptor agonist) and L-tryptophan (L-TRP) in HE. However, they did not find evidence suggesting their participation on observing that QUIN level and QUIN/L-TRP ratio were significantly low in rats subjected to a portacaval shunt (PCS), and that elevated L-TRP availability increased QUIN levels in a similar degree in both sham and PCS rats (Bergqvist et al., 1996). Whereas in rats with ischemic liver failure induced by portacaval anastomosis followed by a 24 -hr hepatic artery ligation, a significant threefold increase of extracellular glycine (a positive alosteric modulator of NMDA receptor) measured by in vivo cerebral microdialysis was found suggesting the participation of NMDA-mediated excitatory neurotransmission in this pathological condition (Zwingmann et al., 2002). In other studies carried out to determine the role of NMDA receptors, it was shown that blocking these receptors by continuous administration of MK-801 or memantine (NMDA receptor antagonist) induces protection against acute liver failure (ALF) caused by galactosamine in experimental animals. This blockage increased the survival time by two folds. Also, when liver injury is not $100 \%$ lethal $(1.5 \mathrm{~g} / \mathrm{kg}$ galactosamine), blocking NMDA receptors increases the survival rate from 23 to $62 \%$ (Cauli et al., 2008). On the other hand, there is experimental evidence that NMDA activation can be modulated by glutamate-nitric oxide-cyclic guanosine monophosphate (cGMP) pathway in ammonia intoxication in the cerebellum of the rats. However, it was found that this activation is not caused by increased extracellular glutamate (Hermenegildo et al., 2000), but due to the presence of ammonium in high concentration in the brain. This was shown by Suzuki et al in 1992, who studied using microdialysis the brain extracellular fluid (ECF) amino acid metabolism and regional level elevation of ammonia in rats with hyperammonemia, experimentally induced through 
administration of local intracerebral ammonia infusion. They found that total brain ECF amino acid level was increased by the elevation of blood ammonia and that in rats with hyperammonemia, there was an increased permeability of the blood-brain barrier. Also, they observed that only glutamate levels showed a marked elevation suggesting that high ammonia levels may increase the excitability of the brain and this situation may serve as a key to the onset of HE (Suzuki et al., 1992).

In addition, other experimental data showed a significant increase in extracellular hippocampal glutamate concentration in rats thioacetamide-induced liver failure (McArdle et al., 1996). Moreover, other studies suggest that the beneficial effect of hypothermia $\left(35^{\circ} \mathrm{C}\right)$ in rats with hepatic devascularization that induces ALF is mediated via mechanism involving reduced blood-brain transfer of ammonia and/or reduction of extracellular brain glutamate concentrations (Rose et al., 2000). It was also found that extracellular glutamate increased in substantia nigra pars reticulate $(\mathrm{SNr})$ of rats with chronic liver failure due to portacaval shunt (PCS), and that this provoked activation of mGluR1 in SNr which together were responsible for reduced motor activity in these rats (Cauli et al., 2006).

On the other hand, it was demonstrated that the involvement of glutamate-nitric oxidecyclic guanosine monophosphate (cGMP) pathway in brain contributes to cognitive impairment in HE. Rodrigo et al (2007) analyzed glutamate-nitric oxide-cGMP pathway function and nitric oxide synthase (NOS) activation by NMDA in an in vivo microdialysis in cerebral cortex of portacaval shunt (PCS) as well as in control and hyperammonemia rats without liver failure. They demonstrated that basal NOS activity, nitrites and cGMP are increased in cortex of rats with hyperammonemia or liver failure. These results are associated to increased inducible NOS expression. In both animal models and in neurons exposed to ammonia, impairment in NOS activation by NMDA was found while in chronic liver failure, basal NOS activity, nitric oxide and cGMP increased whereas activation of NOS induced by NMDA receptors activation decreased. Their results are evidence that hyperammonemia is responsible for both effects and these alterations could contribute to neurological alterations in HE (Rodrigo et al., 2007). Additionally, there is an evidence indicating that hyperammonemia increases tonic activation of NMDA receptors leading to reduced activity of nNOS and of glutamate--NO--cGMP pathway. This was demonstrated by ElMlili et al (2010), where the blockade of NMDA receptors with MK-801 increases cGMP and NO metabolites in cerebellum in in vivo and in slices from hyperammonemic rats. This was due to the reduction of the phosphorylation and activity of calcium-calmodulinindependent protein kinase II (CaMKII), leading to normalization of nNOS phosphorylation and activity. Also in these experiments, MK-801 increases nNOS in synaptic membranes and reduces it in cytosol (ElMlili et al., 2010).

There is an evidence suggesting that not only glutamatergic neurotransmission is altered. It has been proposed that alterations of excitatory and inhibitory amino acids play a role in the pathogenesis of hepatic encephalopathy in ALF. This was determined in vivo cerebral microdialysis using extracellular concentrations of amino acid samples in the frontal cortex of non-anaesthetized rats at various times during the progression of encephalopathy resulting from ALF (induced by portacaval anastomosis). The amino acids from dialysate were measured by high-performance liquid chromatography (HPLC) with fluorescence detection and the results showed that the deterioration of neurological status was accompanied by two to four-fold increases in extracellular glutamate, glutamine, and 
glycine while concentrations of gamma - aminobutyric acid (GABA) and taurine levels remained unchanged. These results suggest that amino acid could contribute to the pathogenesis of HE induced by ALF (Michalak et al., 1996). However, these results are not convincing because they depend on the method of inducing ALF which can produce different changes in the level of amino acid. This is supported by the studies of Bosman et al (1992), who depicted that following microdialysis determinations in the cerebral cortex of rats with acute hepatic encephalopathy induced by complete liver ischemia, extracellular concentrations of the neuroactive amino acids glutamate, taurine, and glycine increased, whereas extracellular concentrations of aspartate and GABA were unaltered and glutamine decreased (Bosman et al., 1992). Also, in cerebral cortical microdialysates of rats with subclinical hepatic encephalopathy (SHE) induced by two intraperitoneal injections of thioacetamide, it was found that dialysate concentrations of the neuroactive amino acids taurine (Tau), glutamate (Glu) and aspartate (Asp) were 30\% to 50\% higher in SHE rats than in control rats (Albrecht et al., 2000).

Other reports demonstrated that the precursors of monoamines, as well as monoamines together with their metabolites, altered neuronal excitability and contribute to the characteristics of HE. Deshpande et al (2007) showed that in frontal cortex of rats with HE induced by ALF resulting from portacaval anastomosis followed by hepatic artery ligation, the extracellular brain concentrations of aromatic amino acids (AAAs) and of valine and leucine (precursors of monoamine neurotransmitters) elevated by 2 to 4 folds following hepatic devascularization, and these increases were significantly correlated to arterial ammonia concentration (Deshpande et al., 2007; Michalak \& Butterworth, 1997). So, those alterations in monoaminergic system are present in HE. Likewise, the monoaminergic system and the dopaminergic system are affected in HE. Yano et al., (2005) demonstrated that the extracellular concentration of 3,4-dihydroxyphenylacetic acid, a metabolite of dopamine, decreased by $39 \%$ of that in sham-operated animals, although the dopamine level did not change. However, when treated with flumazenil (benzodiacepine antagonist), there was complete abolition of the decrease in the metabolite. Although, in this study the glutamate level in the injured animals decreased to $42 \%$ of that in sham-operated animals, there was no increase in the glutamate levels in animals treated with flumazenil. Also in this study, it was shown that spontaneous motor activity decreased 24 hours after surgery in animals subjected to liver ischemia and that flumazenil treatment improved spontaneous the motor activity 5 minutes after administration, but this effect was not observed after 30 minutes. For this reason, these authors proposed that the restoration of the central dopaminergic function could be a relevant factor in the improvement of HE (Yano et al., 2005).

Other researchers have determined that in hyperammonemic rats there are neurological and cerebral function alterations, as well as alterations on the modulation of motor and neurochemical functions. The locomotion induced by injection of mGluR agonist dihydroxyphenylglycine (DHPG) in the nucleus accumbens increased. Also in control rats, DHPG increased extracellular dopamine $(400 \%)$, but glutamate remained unchanged. Whereas in hyperammonemic rats, DHPG increased extracellular glutamate $(600 \%)$, and when mGluR1 receptor was blocked with 7-hydroxyiminocyclopropan[b]chromen-1acarboxylic acid ethylester (CPCCOEt), this treatment prevented DHPG effects, suggesting that this receptor mediates locomotor and neurochemical effects and showed that modulation of locomotor and neurochemical functions by mGluRs in nucleus accumbens is strongly altered in hyperammonemia (Canales et al., 2003). 
In addition, it was demonstrated that in rats with acute $\mathrm{HE}$ induced by repeated administration of thioacetamide, the impairment of modulation of striatal DA discharge and metabolism by the action of glutamate on NMDA receptors, contribute to motor disturbances in this disease (Borkowska et al., 1999). Cauli et al (2007) demonstrated that the function of the circuits that modulate motor function in rats with liver failure due to portacaval shunt (PCS) is dependent on the kind of neurotransmission. They found this result by analyzing the function of the circuits that modulate motor function in rats with liver failure due to portacaval shunt (PCS) using in vivo brain microdialysis. They inserted cannulae in the NAcc (where injected (S)-3,5-dihydroxyphenylglycine, DHPG) and microdialysis probes in NAcc, ventral pallidum (VP), substantia nigra pars reticulata $(\mathrm{SNr})$, medio-dorsal thalamus (MDT), ventro-medial thalamus (VMT) or prefrontal cortex (PFCx) and analyzed the concentration of extracellular neurotransmitters in these areas. Their results indicate that in control rats, DHPG induces locomotion by activating the normal neuronal circuit: NAcc --> VP --> MDT --> PFCx and in PCS rats this circuit is not activated but DHPG injection in PCS rats activates an alternative circuit: NAcc --> SNr --> VMT --> PFCx that is not activated in control rats. These experiments suggest that dopamine increase induced by DHPG would activate the normal neuronal circuit, while an increase in glutamate would activate the alternative circuit (Cauli et al., 2007).

On the other side, serotonergic system is also affected in HE. This was demonstrated by Bergqvist et al (1996) who showed that frontal neocortical extracellular levels of 5hydroxytryptamine (5-HT) is unaltered while its major metabolite, 5-hydroxyindole-3-acetic acid (5-HIAA), is increased in portacaval shunted rats in an experimental model of chronic hepatic encephalopathy (HE), prior to and after an acute coma-induction by the administration of ammonium acetate $(5.2 \mathrm{mmol} / \mathrm{kg}$, i.p.). These results suggest that an increase in brain ammonia may augment neuronal 5-HT release in HE, which in turn could be a causative of severe stages of HE (Bergqvist et al., 1996).

In addition, in frontal cortex of rats with thioacetamide (TAA)-induced HE, it was found that 5-HIAA and high $\mathrm{K}+-$-evoked 5-HT release increased in HE rats. These data support the idea that serotonergic neurotransmission is altered in this brain area of rats with this pathology (Kaneko et al., 1998).

Like other systems affected in HE, there is an experimental evidence suggesting that noradrenergic system is affected and describes that central noradrenergic mechanisms may contribute to the central nervous system manifestations of HE. Michalak et al (1998) showed that there is an increase of extracellular brain concentrations of noradrenalin (NA), from the extracellular compartment of frontal cortex and thalamus in rats with acute liver failure (Michalak et al., 1998), and this is associated to the loss of NA transporter sites resulting from depletion of central NA stores due to a reserpine-like effect of ammonia which is known to accumulate in brain in ischemic liver failure (Michalak et al., 2010). These experimental data suggested that noradrenergic neurotransmission may play a role in this pathology.

On the other hand, there is an evidence that extracellular glutathione (GSH) is involved in the ammonia toxicity present in rats with HE. This was demonstrated by Hielgier et al (2010) where the administration of ammonium chloride (ammonia) via a microdialysis probe to the rat prefrontal cortex increased GSH. This increase was abrogated by the inhibitor of 
astrocytic energy metabolism of fluoroacetate and inhibitor of glutathione synthesis of buthionine sulfoximine. Their results suggest that in rats with hyperammonemia HA or HE ammonia specifically promote GSH synthesis may improve the availability of precursors for GSH synthesis in neurons and their resistance to ammonia toxicity present in HE (Hilgier et al., 2010).

\subsubsection{Fulminant hepatic encephalopathy}

Fulminant hepatic encephalopathy is one of the encephalopathies with high mortality. To found chemical markers that provide information leading to application of the best therapeutic treatment in these patients is a priority. The microdialysis, being a highly sensitive technique in monitoring cerebral energy metabolism and in early detection of cerebral hypoxia has been used to find these chemical markers. Beuer et al (2004) were the first in reporting a case of monitoring neurochemical markers using intracerebral microdialysis during cardiac resuscitation. They measured the chemical markers of energy metabolism of glucose, lactate, pyruvate, and the marker of cell membrane damage (glycerol) and found that except subcutaneous glucose, all markers showed a sudden and significant increase during resuscitation and a prolonged period afterwards with all values returning to normal after some hours (Bauer et al., 2004). In another study to determine the role of cerebral microdialysis as an adjunct to the management of a 49-year old woman with hepatic encephalopathy secondary to a paracetamol overdose, it was found that the application of the microdialysis technique for the detection of cerebral glucose concentration in the presence of a normal plasma glucose was useful for the continuous on line monitoring of substrate delivery and metabolism, and this may have a relevant role in the therapeutic management of patients with fulminant hepatic failure (Hutchinson et al., 2006).

\subsubsection{Posthypoxic encephalopathy}

Actually, there is only one study that focused on determining the neurochemical alterations in a seemingly posthypoxic encephalopathy. Thoresen et al (1998) studied changes in lactate and pyruvate in gray and white matter in the brain of a newborn pig after a hypoxic insult known to produce seizures and permanent brain damage. They induced hypoxia for fortyfive minutes by reducing the fraction of inspired $\mathrm{O}_{2}$ to the maximum concentration and found that there was no association between onset of electroconvulsive activity and an increase in lactate or lactate/pyruvate $(\mathrm{L} / \mathrm{P})$ ratio which are the biochemical parameters altered in posthypoxic encephalopathy (Thoresen et al., 1998).

\subsubsection{Hypoxic-ischemic encephalopathy}

Pearing et al (1996) studying the effect of hypoxia in-vivo, separately analyzed the hypoxic component of hypoxic-ischemic encephalopathy. They used rats that were prepared such that their arterial oxygen pressure $\left(\mathrm{paO}_{2}\right)$ was maintained at $20 \mathrm{mmHg}$. While maintaining systemic arterial pressures, the brain oxygen concentration and extracellular amino acid concentrations were monitored during 20 minutes. They did not find changes in the extracellular glutamate extracellular levels, and with additional experiments no morphologic injury was detected. Thus, they conclude that hypoxia without ischemia is well tolerated by the brain (Pearigen et al., 1996). 


\subsubsection{Encephalopathy associated with septic shock}

Encephalopathy associated with septic shock as well as neurological disease could be induced by the central nervous formation of reactive oxygen species (ROS) associated with inflammation process. Clement et al (2010) analyzed the effect of peripherally applied lipopolysaccharide (LPS, $100 \mathrm{mug} / \mathrm{kg}$ i.p.) that is used as a model for major depression and results in enhanced inflammatory processes, on the central nervous formation of ROS and interleukin-6 (IL-6) in wild-type mice and in mice lacking NADPH oxidase Nox2 subunit gp91phox using microdialysis paradigm. They found that in the wild-type mice, LPS increased ROS formation in the striatum of wild-type mice and resulted in enhanced IL-6 production. In the mice lacking NADPH oxidase Nox2 subunit gp91phox, LPS did not enhance ROS formation, whereas IL-6 increased. They conclude that gp91phox-containing NADPH oxidase complex was involved in the central nervous ROS formation after peripheral LPS stimulation and these results suggest that ROS determination could be a pharmacological target in patients with this pathological condition (Clement et al., 2010).

\subsubsection{Thiamine deficiency encephalopathy (Wernicke)}

The Wernicke encephalopathy is characterized by thiamine deficiency. This pathology is a neurological disease detected in alcoholics and in patients with compromised nutrition. Todd et al (2001) established the role of glutamate excitotoxicity using in vivo microdialysis in the neuronal cell death due to thiamine deficiency, with the considerations of different factors (blood-brain barrier, tissue reactions to probe implantation) that may affect the probe recoveries (Todd \& Butterworth, 2001). In addition, Todd and Butterworth (1998) evaluated the role of NMDA receptor-mediated glutamate excitotoxicity in the pathogenesis of neuronal loss induced by thiamine deficiency. They also determined extracellular glutamate levels using in vivo cerebral microdialysis in the ventral posterior medial thalamic nucleus. Their result suggests that NMDA receptor-mediated excitotoxicity is not responsible for early neuronal loss in this model of thiamine deficiency encephalopathy (Todd \& Butterworth, 1998).

Other studies were directed to the study of the role of histamine in neuronal degeneration in a rat model of Wernicke's encephalopathy induced by pyrithiamine-induced thiamine deficiency (PTD). They established that the histamine enhancement of glutamate receptor activation suggests that the histamine release could be a participant in glutamate-N-methylD-aspartate (NMDA)-mediated excitotoxic neuronal death in this pathology (Langlais et al., 1994). And, that the release of histamine in rat from nerve terminals and histamine and other vasoactive substances from granulocytes could be responsible for thiamine deficiencyinduced vascular breakdown and perivascular edema in thalamus (McRee et al., 2000).

\subsubsection{Viral encephalopathy}

Espey et al (1998) determined in mice infected with LP-BM5 leukemia retrovirus mixture that develop a progressive immunodeficiency with associated behavioral, histological, and neurochemical alterations consistent with glutamatergic hyperactivation. They clarify the contribution of excitatory amino acids to the neurodegeneration observed in these mice by measuring extracellular glutamate levels in the striated brain area of these animals, and found that infection with an immunodeficiency-inducing retrovirus increases extracellular 
free glutamate levels in this brain region. Based on this, they suggest that these changes contribute to neurodegenerative and cognitive deficits observed in this experimental model of viral encephalopathy (Espey et al., 1998).

\subsubsection{Portal-systemic encephalopathy}

Portal-systemic encephalopathy (PSE) is characterized by psychiatric symptoms progressing through stupor and coma. Actually, there is experimental evidence suggesting that alterations in levels of brain amino acids may play a role in the pathogenesis of PSE. Rao $e t$ al (1995) studying this changes, did found changes in extracellular fluid concentrations of glutamate, aspartate, GABA, tryptophan, leucine, and serine in cerebral frontal cortex of portacaval-shunted rats that were administered ammonium acetate $(3.85 \mathrm{mmol} / \mathrm{kg}$, i.p.) to induce PSE. (Rao et al. 1995).

In addition, PSE is associated with an increased brain tissue of serotonin (5-HT). The involvement of serotoninergic neurotransmission was demonstrated by Bergqvist et al (1996, 1997) who found that potassium chloride (KCI) challenged induced increase of 5-HT release in neocortical region of the rats with a portacaval shunt (PCS) (Bergqvist et al., 1996, 1997).

\subsection{Microdialysis applied in the study of encephalopathy therapeutic treatments}

\subsubsection{Hepatic encephalopathy}

\subsubsection{L-ornithine and L-ornithine-L-aspartate study}

Vogels et al (1997) established that the L-ornithine (ORN) and L-ornithine-L-aspartate (OA) therapeutic treatment had a beneficial effects on the symptomatologies of rats with hyperammonemia-induced encephalopathy by portacaval shunted for the fact that ORN and OA treatments decrease ammonia concentrations in blood by $34 \%$ and $39 \%$, and in brain by $42 \%$ and $22 \%$. Also they found that these substances increased urea production by $39 \%$ and $86 \%$, with a significant smaller increase in brain glutamine and lactate concentrations than in controls. However, the effect of ornithine should be taken with care due to the fact that this substance induces high brain extracellular levels of glutamate and aspartate (excitatory amino acids), suggesting a possible overstimulation of NMDA receptors (Vogels et al., 1997).

\subsubsection{Venlafaxine studies}

Patients with chronic hepatic encephalopathy (HE) may present affective symptoms and antidepressant drug treatment due to HE displays monoaminergic perturbations together with affective symptoms. Venlafaxine (VEN) is an antidepressant, and serotoninnorepinephrine reuptake inhibitor that is used in the treatment of patients with HE. The liver impairment present in HE patients may induce pharmacokinetic alterations of antidepressant drug, which in turn can modify monoaminergic function. Because of this, Wikell et al studied this possible alterations, and determined that in rats with chronic portacaval shunted (PCS; experimental hepatic encephalopathy), VEN (10 mg/kg) administered in a unique doses (subcutaneous) and daily during 14 days (continous delivery by osmotic minipumps) exhibits both pharmacokinetic and pharmacodynamic alterations in these rats (Wikell et al., 1998, 2002). 
When VEN was administered at $5 \mathrm{mg} / \mathrm{kg}$ as a single subcutaneous challenge to portacaval shunted rats, it resulted in elevated levels of VEN in serum, brain parenchyma, and brain dialysate in about 300 minutes after the injection. Therefore, this result suggests that when the dose of VEN administered to experimental HE was reduced 50\%, important pharmacokinetic alterations are presented in these animals (Wikell et al., 2001), in the same way that the studies done with dose of $10 \mathrm{mg} / \mathrm{kg}$ (Wikell et al., 1998, 2002).

\subsubsection{Citalopram studies}

Citalopram (CIT) is an antidepressant drug of the selective serotonin reuptake inhibitor used in patients with HE that display neuropsychiatric symptoms like affective disturbances. The simultaneous pharmacokinetic and pharmacodynamic outcome of the commonly used serotonin-selective thymoleptic drugs in liver-impaired subjects with HE is not totally understood today.

Berqvist et al (1997) studied the effects of neocortical administration of CIT (1.0 microM), and CIT (5 mg/kg subcutaneously) on brain 5-hydroxytryptamine release in portacaval shunted rats with an experimental chronic hepatic encephalopathy. They found that neocortical administration of CIT increased the brain 5-HT output in the same way as in portacaval shunted sham-operated rats. These data do not explain the increased 5-HT turnover and unchanged release in PCS rats by an accelerated brain 5-HT reuptake. For this reason, the administration of CIT ( $5 \mathrm{mg} / \mathrm{kg}$ subcutaneously) resulted in a pronounced decrease of brain 5-HT release in PCS rats than in sham-operated controls. This may be due to a higher susceptibility to indirect 5-HT1A autoreceptor activation in experimental portalsystemic encephalopathy. Their experiments with potassium chloride $(60 \mathrm{mM})$ challenge in the presence of locally CIT ( 1 microM) induced an increase of 5-HT response in PCS rats than in sham-operated rats, confirmed an abnormal increase of 5-HT available for depolarization-induced release in PCS rats. These results suggested that central nervous system 5-HT-active drugs could perhaps pose a potential hazard in patients with liver failure with or without HE (Bergqvist et al., 1997).

In addition, Apelqvist et al (2000) investigated the effects of chronic treatment with CIT (10 $\mathrm{mg} / \mathrm{Kg}$ day) in the frontal neocortex of rats with and without portacaval shunts in 5-HT, 5HIAA, noradrenaline (NA), and dopamine (DA) output. The rats with PCS increased by 2-3 folds the levels of CIT than rats undergoing a sham treatment with CIT in all compartments. This treatment induced in neocortical output differences between PCS rats and control rats within 5-HT and DA systems but not in NA system. Their data suggest pharmacokinetic and pharmacodynamic changes in an equal-dose chronic treatment with CIT in PCS rats, changes that were not observed in sham rats. Interestingly, these authors indicate that although there are pharmacokinetic and pharmacodynamic alterations with CIT-treatment in PCS rats, the beneficial behavioral response remains (Apelqvist et al., 2000).

\subsubsection{Lubeluzole study}

Lubeluzole is a neuroprotectant which is effective in the treatment of experimental stroke in rats, mainly by inhibition of glutamate-activated NO pathway and also by counteracting osmotic stress (mechanism associated to the release of the active amino acid taurine). Zielinska et al (2001) showed that lubeluzole administered intraperitoneally decreases by $25 \%$ the high $(50 \mathrm{mM}) \mathrm{K}+-$ evoked accumulation of Taurine (Tau) in striatal microdialysates 
of healthy rats and by $34 \%$ in rats with thioacetamide-induced hepatic failure, suggesting increased extracellular of Tau in ongoing hepatic encephalopathy. These data indicate that lubeluzole could be effective in ameliorating ionic or osmotic stress in rats with hepatic failure (Zielińska et al., 2001).

\subsubsection{Sildenafil study}

Patients with liver disease with overt or minimal hepatic encephalopathy have impaired intellectual capacity and the underlying molecular mechanism remains unknown. Interestingly, rats with portacaval anastomosis or with hyperammonemia without liver failure also show impaired learning ability and impaired function of the glutamate-nitric oxide-cyclic guanine monophosphate (glutamate-NO-cGMP) pathway in brain. Erceg et al (2005) hypothesized that pharmacological manipulation of this pathway (glutamate-NOcGMP) could restore learning ability. They showed that in vivo brain microdialysis, chronic oral administration of sildenafil (an inhibitor of the phosphodiesterase that degrades cGMP), normalizes the function of the glutamate-NO-cGMP pathway and extracellular cGMP in the brain of rats with portacaval anastomosis or with hyperammonemia. They determined that impairment of learning ability in rats with chronic liver failure or with hyperammonemia are the result of impairment of glutamate-NO-cGMP pathway and that the chronic treatment with sildenafil normalizes the function of the pathway and restores learning ability in rats with portacaval shunts or with hyperammonemia (Erceg et al., 2005).

\subsubsection{Ibuprofen study}

Patients with hepatic encephalopathy show altered motor function, psychomotor slowing, and hypokinesia, which are reproduced in rats with portacaval shunts (PCS, an experimental model of HE). The neurochemical alterations induced by hypokinesia in PCS rats are attributed to the increase of extracellular glutamate in substantia nigra pars reticulate $(\mathrm{SNr})$, but the mechanisms by which liver failure leads to increased extracellular glutamate in $\mathrm{SNr}$ remain unclear. However, it was seen that inflammation acts synergistically with hyperammonemia to induce neurological alterations in hepatic encephalopathy and by this way, the inflammation alterations can contribute to motor alterations in HE. For this reason Cauli et al (2009) assessed if the treatment with an antiinflammatory, ibuprofen, is able to normalize extracellular glutamate in $\mathrm{SNr}$ and/or improve hypokinesia in PCS rats. They found that ibuprofen at 15 or 30 (but not at 5 $\mathrm{mg} / \mathrm{kg} /$ day), completely eliminates hypokinesia and restore normal motor activity. This supports the supposition that inflammation is the principal contributor to the induction of hypokinesia in HE and this data suggests that therapeutic treatment of inflammation in the motor deficits in patients with this pathology could be beneficial (Cauli et al., 2009).

\subsubsection{Hypoxic-ischemic encephalopathy}

\subsubsection{Dichloroacetate study}

Dichloroacetic acid, often abbreviated as DCA, the salts of which has been studied as potential drugs due to their inhibition of pyruvate dehydrogenase kinase enzyme. The common use of DCA is in the treatment of cancer, and actually several studies using adult animal models suggest that DCA may have neuroprotective properties by virtue of its ability to increase rates of metabolism and, therefore, clearance of brain lactic acidosis, 
which may accumulate during cerebral ischemia. Corbett et al (1998) hypothesized that postischemic DCA administration affects lactate and acid clearance in different extents in immature versus mature brain. Their results indicate that postischemic DCA administration helps to resolve cerebral acidosis to a greater degree in immature than in more mature brain, and this suggests that DCA could have cerebroprotective properties for neonatal hypoxicischemic encephalopathy (Corbett et al., 1998).

\subsubsection{Indomethacin study}

Indometacin or indomethacin is a non-steroidal anti-inflammatory drug commonly used to reduce fever, pain, stiffness, and swelling. It works by inhibiting the production of prostaglandins, molecules known to cause these symptoms. To try understanding the role of this drug in the possible protective effect on hypoxic ischemic encephalopathy, Ogasawara et al (1999) examined the effects of indomethacin on extracellular dopamine (DA) in the striatum of immature rats submitted to anoxia using in vivo microdialysis and HPLC to quantify DA. They found that during anoxia the DA level reached $1185+/-400 \%$ of the basal level and the peak levels of DA were only $307+/-63 \%, 153+/-35 \%$ in indomethacin groups. They conclude that the suppression that induces indomethacin is possibly one of the mechanisms that helps to avoid the dopamine alterations on hypoxic ischemic encephalopathy (Ogasawara et al., 1999).

\section{Conclusions}

Microdialysis is a powerful tool widely used in neurosciences. The use in Neurology has been on the increase on the discovery of its importance in understanding the possible mechanisms underlying neuropathies, and its application in the study of new therapeutic substances.

Although, the use in in-vivo experimental studies and in animal models dates back to years, now there is an increase in the tendency to use this technique to study the neuropathology in humans, and this has helped to make the most appropriate decisions for the treatment of patients. It is noteworthy that studies in humans are very limited, since they involve legal and ethical permissions, but however, data from the few studies in human have shown favorable results that have contributed to the implementation of specific treatments.

On the other hand, microdialysis is a technique that allows the measurement of molecules which may be involved in pathophysiological changes of various diseases as encephalopathy, finding molecular targets for early diagnosis and timely treatment of patients with this disease that result in the greater likelihood of remission and minimizing the potential consequences that such conditions could leave, improving the prognosis of these patients.

\section{Acknowledgements}

We thank professor Aristides III Sampieri Hernández for his technical assistance, and also to Dr. Abel Santamaría del Ángel, from the laboratory of excitatory amino acids, National Institute of Neurology and Neurosurgery, Mexico, who kindly provided us with a photography from his microdyalisis equipment to illustrate our work. Finally we thank to Dr Cyrill Ndidi Nwoye a native English speaker for translating this manuscript. 


\section{References}

Albrecht, J., Hilgier, W., Zielińska, M., Januszewski, S., Hesselink, M \& Quack, G. (2000). Extracellular concentrations of taurine, glutamate, and aspartate in the cerebral cortex of rats at the asymptomatic stage of thioacetamide-induced hepatic failure: modulation by ketamine anesthesia. Neurochem Res. Vol.25, No.11, (November 2000), pp 1497-502.

Apelqvist, G., Wikell, C., Carlsson, B., Hjorth, S., Bergqvist, P., Ahlner, J \& Bengtsson, F. (2000). Dynamic and kinetic effects of chronic citalopram treatment in experimental hepatic encephalopathy. Clin Neuropharmacol. Vol.23, No.6, (Nov-Dec 2000), pp 304317.

Bajaj, J. (2010) Review article: the modern management of hepatic encephalopathy. Aliment. Pharmacol. Ther. Vol.31, No.5, (March 2010), pp 537-547.

Bauer, R., Gabl, M., Obwegeser, A., Galiano, K., Barbach, J \& Mohsenipour, I. (2004). Neurochemical monitoring using intracerebral microdialysis during cardiac resuscitation. Intensive Care Med. Vol.30, No.1, (January 2004), pp 159-161.

Bellander, B., Cantais, E., Enblad, P., Hutchinson, P., Nordström, C., Robertson, C., Sahuquillo, J., Smith, M., Stocchetti, N., Ungerstedt, U., Unterberg, A \& Olsen N. (2004). Consensus meeting on microdialysis in neurointensive care. Intensive Care Med. Vol.30, No.12, (December 2004), pp 2166-2169.

Bergqvist, P., Heyes, MP., Apelqvist, G., Butterworth, R \& Bengtsson, F. (1996). Brain extracellular quinolinic acid in chronic experimental hepatic encephalopathy as assessed by in vivo microdialysis: acute effects of L-tryptophan. Neuropsychopharmacology. Vol.15, No.4, (October 1996), pp 382-389.

Bergqvist, P., Hjorth, S., Apelqvist, G \& Bengtsson, F. (1996). Acute effects of L-tryptophan on brain extracellular 5-HT and 5-HIAA levels in chronic experimental portalsystemic encephalopathy. Metab Brain Dis. Vol.11, No.3, (September 1996), pp 269278.

Bergqvist, P., Hjorth, S., Audet, R., Apelqvist, G., Bengtsson, F \& Butterworth, R. (1996). Ammonium acetate challenge in experimental chronic hepatic encephalopathy induces a transient increase of brain 5-HT release in vivo. Eur Neuropsychopharmacol. Vol.6, No.4, (November 1996), pp 317-322.

Bergqvist, P., Hjorth, S., Apelqvist, G \& Bengtsson, F. (1997). Potassium-evoked neuronal release of serotonin in experimental chronic portal-systemic encephalopathy. Metab Brain Dis. Vol.12, No.3, (September 1997), pp 193-202.

Bergqvist, P., Wikell, C., Hjorth, S., Apelqvist, G \& Bengtsson, F. (1997). Effect of citalopram on brain serotonin release in experimental hepatic encephalopathy: implications for thymoleptic drug safety in liver insufficiency. Clin Neuropharmacol. Vol.20, No.6, (December 1997), pp 511-522.

Bismuth, M., Funakoshi, N., Cadranel, J \& Blanc, P. (2011). Hepatic encephalopathy: from pathophysiology to therapeutic management. Eur J Gastroenterol Hepatol. Vol.23, No.1, (January 2011), pp 8-22.

Bito, L., Davson, H., Levin, E., Murray, M \& Snider, N. (1966). The concentrations of free amino acids and other electrolytes in cerebrospinal fluid, in vivo dialysate of brain, and blood plasma of the dog. J Neurochem. Vol.13, No.11, (November 1966), PP 1057-67. 
Bonate, P.(1995). Animal models for studying transport across the blood-brain barrier. J Neurosci Methods. Vol.56, No.1,(January 1995), pp 1-15.

Borkowska, H., Oja, S., Oja, O., Saransaari, P., Hilgier, W \& Albrecht, J. (1999). N-methyl-Daspartate-evoked changes in the striatal extracellular levels of dopamine and its metabolites in vivo in rats with acute hepatic encephalopathy. Neurosci Lett. Vol.268, No.3, (June 1999), pp 151-154.

Boschi, G. \& Scherrmann, J. (2000). Microdialysis in mice for drug delivery research. Advanced Drug Delivery Reviews. Vol.45, No.2-3, (December 2000), pp 271-281.

Bosman, D., Deutz, N., Maas, M., van Eijk, H., Smit, J., de Haan, J., Chamuleau, R \& J- van, Gool. (1992). Amino acid release from cerebral cortex in experimental acute liver failure, studied by in vivo cerebral cortex microdialysis. J Neurochem. Vol.59, No.2, (August 1992), pp 591-599.

Canales, J., Elayadi, A., Errami, M., Llansola, M., Cauli, O \& Felipo, V. (2003). Chronic hyperammonemia alters motor and neurochemical responses to activation of group I metabotropic glutamate receptors in the nucleus accumbens in rats in vivo. Neurobiol Dis. Vol.14, No.3, (December 2003), pp 380-390.

Cash, W., McConville, P., McDermott, E., McCormick, P., Callender, M \& McDougall, NI. (2010). Current concepts in the assessment and treatment of hepatic encephalopathy. QJM. Vol.103, No.1, (November 2009), pp 9-16.

Cauli, O., Llansola, M., Erceg, S \& Felipo, V. (2006). Hypolocomotion in rats with chronic liver failure is due to increased glutamate and activation of metabotropic glutamate receptors in substantia nigra. J Hepatol. Vol.45, No.5, (November 2006), pp 654-661.

Cauli, O., Mlili, N., Llansola, M \& Felipo, V. (2007). Motor activity is modulated via different neuronal circuits in rats with chronic liver failure than in normal rats. Eur J Neurosci. Vol.25, No.7, (April 2007), pp 2112-2122.

Cauli, O., Rodrigo, R., Boix, J., Piedrafita, B., Agusti, A \& Felipo, V. (2008). Acute liver failure-induced death of rats is delayed or prevented by blocking NMDA receptors in brain. Am J Physiol Gastrointest Liver Physiol. Vol.295, No.3, (September 2008), pp G503-G511.

Cauli, O., Rodrigo, R., Piedrafita, B., Llansola, M., Mansouri, M \& Felipo, V. (2009). Neuroinflammation contributes to hypokinesia in rats with hepatic encephalopathy: ibuprofen restores its motor activity. J Neurosci Res. Vol. 87, No.6, (May 2009), pp 1369-1374.

Chung, R. \& Podolsky, D. (2005). Cirrhosis and its complications. In Kasper DL, Braunwald E, Fauci AS, et al. Harrison's Principles of Internal Medicine (16th ed). New York, NY: McGraw-Hill. pp.1858-1869.

Clement, H., Vazquez, J., Sommer, O., Heiser, P., Morawietz, H., Hopt, U., Schulz, E \&von Dobschütz, E. (2010). Lipopolysaccharide-induced radical formation in the striatum is abolished in Nox2 gp91phox-deficient mice. J Neural Transm. Vol.117, No.1, (January 2010), pp 13-22.

Corbett, R., Laptook, A., Gee, J., Garcia, D., Silmon, S \& Tollefsbol, G. (1998). Age-related differences in the effect of dichloroacetate on postischemic lactate and acid clearance measured in vivo using magnetic resonance spectroscopy and microdialysis. J Neurochem. Vol.71, No.3, (September 1998), pp 1205-1214. 
Dash, A., Haney, P. \& Garavalia, M. Development of an in vitro dissolution method using microdialysis sampling technique for implantable drug delivery systems. J Pharm Sci. Vol. 88, No.10, (October 1999), pp 1036-40.

Deshpande, G., Adachi, N., Liu, K., Motoki, A., Mitsuyo, T., Nagaro, T \& Arai, T. (2007). A Recovery of brain dopamine metabolism by branched-chain amino acids in rats with acute hepatic failure. J Neurosurg Anesthesiol. Vol.19, No.4, (October 2007), pp 243-248.

Di Chiara, G., Tanda, G. \& Carboni, E. (1996). Estimation of in-vivo neurotransmitter release by brain microdialysis: the issue of validity. Behav Pharmacol. Vol.7, No.7, (November 1996), pp 640-657.

ElMlili, N., Boix, J., Ahabrach, H., Rodrigo, R., Errami, M \& Felipo, V. (2010). Chronic hyperammonemia induces tonic activation of NMDA receptors in cerebellum. $J$ Neurochem. Vol.112, No.4, (February 2010), pp 1005-1014.

Elmquist, W. \& Sawchuk, R. (1997). Application of Microdialysis in Pharmacokinetic Studies. Pharmaceutical Research. Vol 14 No 3, (March 1997), pp 267-288.

Erceg. S., Monfort, P., Hernández-Viadel, M., Rodrigo, R., Montoliu, C \& Felipo, V. (2005). Oral administration of sildenafil restores learning ability in rats with hyperammonemia and with portacaval shunts. Hepatology. Vol.41, No.2, (February 2005), pp 299-306.

Espey, M., Kustova, Y., Sei, Y \& Basile, A. (1998). Extracellular glutamate levels are chronically elevated in the brains of LP-BM5-infected mice: a mechanism of retrovirus-induced encephalopathy. J Neurochem. Vol.71, No.5, (November 1998), pp 2079-2087.

Goetz, C. et al. (eds). , (1999). Textbook of Clinical Neurology, (1st edition), Philadelphia: W.B. Saunders Company, 1999.

Häussinger, D. (2010) Hepatic encephalopathy. Acta Gastroenterol Belg. Vol.73, No.4, (OctDec 2010), pp 457-464.

Hermenegildo, C., Monfort. P \& Felipo V. (2000). Activation of N-methyl-D-aspartate receptors in rat brain in vivo following acute ammonia intoxication: characterization by in vivo brain microdialysis. Hepatology. Vol.31, No.3, (March 2000), pp 709-715.

Hilgier, W., Wegrzynowicz, M., Ruszkiewicz, J., Oja, S., Saransaari, P \& Albrecht, J. (2010). Direct exposure to ammonia and hyperammonemia increase the extracellular accumulation and degradation of astroglia-derived glutathione in the rat prefrontal cortex. J Toxicol Sci. Vol.117, No.1, (September 2010), pp 163-168.

Hillbom, M. \& Marttila, M. (2010). Encephalopathies due to vitamin deficiency. Duodecim. Vol.126, No.18, pp 2132-2138.

Hitzman, C., Wiedmann, T., Dai, H. \& Elmquist, W. (2005). Measurement of drug release from microcarriers by microdialysis. J Pharm Sci. Vol.94, No.7, (July 2005), pp 14561466.

Hocht, C., Opezzo, J \& Taira C. (2007). Applicability of reverse microdialysis in pharmacological and toxicological studies. Journal Pharmacology and Toxicology Method. Vol.55, No.1, (January-February 2007), pp 3-15.

Hsiao, J., Ball, B., Morrison, P., Mefford, I \& Bungay, P. (1990). Effects of different semipermeable membranes on in vitro and in vivo performance of microdialysis probes. J Neurochem. Vol.54, No.4, (April 1990), pp 1449-1452. 
Hutchinson, P., Gimson, A., Al-Rawi, P., O'Connell, M., Czosnyka, M \& Menon, D. (2006), Microdialysis in the management of hepatic encephalopathy. Neurocrit Care. Vol.5, No.3, (2006), pp 202-205.

Jaitovich, A. (2003). Chapter Introducción al tráfico de sustancias a través de la membrana celular. In: Bases fisiológicas de la práctica médica. Best and Taylor $13^{\mathrm{a}} \mathrm{Ed}$, Editorial Médica Panamericana, Argentina.

Johnston, A. \& Gupta, A. (2002). Advanced monitoring in the neurology intensive care unit: microdialysis. Curr Opin Crit Care. Vol.8, No.2, (April 2002), pp 121-127.

Juhasz, G., Tarcali, J., Pungor, K \& Pungor, E. (1989). Electrochemical calibration of in vivo brain dialysis samplers. J Neurosci Methods. Vol.29, No. 2, (August 1989), pp 131-7.

Kaneko, K., Kurumaji, A., Watanabe, A., Yamada, S \& Toru, M. (1998). Changes in high K+evoked serotonin release and serotonin $2 \mathrm{~A} / 2 \mathrm{C}$ receptor binding in the frontal cortex of rats with thioacetamide-induced hepatic encephalopathy. J Neural Transm. Vol.105, No.1, (1998), pp 13-30.

Kendrick, K., Keverne, E., Chapman, C \& Baldwin, B. (1988). Microdialysis measurement of oxytocin, aspartate, gamma-aminobutyric acid and glutamate release from the olfactory bulb of the sheep during vaginocervical stimulation. Brain Res. Vol. 442, No.1, (February1988), pp171-174.

Kenneth, E., Pasas S., Cooper, J \& Malonne I. (2002). A review of membrane sampling from biological tissues with applications in pharmacokinetics, metabolism and pharmacodynamics. European Journal of Pharmaceutical Sciences. Vol.17, No.1-2, (October 2002), pp 1-12.

Langlais, P., Zhang, S., Weilersbacher, G., Hough, L \& Barke, K. (1994). Histamine-mediated neuronal death in a rat model of Wernicke's encephalopathy. J Neurosci Res. Vol.38, No.5, (August 1994), pp 565-574.

McArdle, P., Penning, D., Dexter, F \& Reynolds J. (1996). Flumazenil does not affect the increase in rat hippocampal extracellular glutamate concentration produced during thioacetamide-induced hepatic encephalopathy. Metab Brain Dis. Vol.11, No.4, (December 1996), pp 329-342.

McRee, R., Terry-Ferguson, M., Langlais, P., Chen, Y., Nalwalk, J., Blumenstock, F., \& Hough, L. (2000). Increased histamine release and granulocytes within the thalamus of a rat model of Wernicke's encephalopathy. Brain Res. Vol.858, No.2, (March 2000), pp 227-236.

Mendelowitsch, A. (2001). Microdialysis: intraoperative and posttraumatic applications in neurosurgery. Methods. Vol.23, No.1, (January 2001), pp 73-81.

Michalak \& Butterworth. (1997). Selective increases of extracellular brain concentrations of aromatic and branched-chain amino acids in relation to deterioration of neurological status in acute (ischemic) liver failure. Metab Brain Dis. Vol.12, No.4, (December 1997), pp 259-269.

Michalak, A., Rose, C \& Butterworth, R. (2010). Loss of noradrenaline transporter sites in frontal cortex of rats with acute (ischemic) liver failure. Neurochem Int. Vol.38, No.1, (January 2001), pp 25-30.

Michalak, A., Rose, C., Butterworth, J \& Butterworth, F. (1996). Neuroactive amino acids and glutamate (NMDA) receptors in frontal cortex of rats with experimental acute liver failure. Hepatology. Vol.24, No. 4, (October 1996), pp 908-913. 
Michalak, A., Rose, C., Buu, P \& Butterworth, R. (1998). Evidence for altered central noradrenergic function in experimental acute liver failure in the rat. Hepatology. Vol.27, No.2, (February 1998), pp 362-368.

Montgomery, J. \& Bajaj J. (2011). Advances in the evaluation and management of minimal hepatic encephalopathy. Curr Gastroenterol Rep. Vol.13, No.1, (October 2010), pp 2633.

Müller, M., Baumeie,r A., Ringelstein, E \& Husstedt, I. (2008). Long-term tracking of neurological complications of encephalopathy and myopathy in a patient with nephropathic cystinosis: a case report and review of the literature. J Med Case Reports. Vol.2, No.235. (July 2008).

Munoz, S. (2008). Hepatic encephalopathy. Med Clin North Am. Vol.92, No.4, (July 2008), pp 795-812.

Nabbout, R., Vezzani, A., Dulac, O \&Chiron, C. (2011). Acute encephalopathy with inflammation-mediated status epilepticus. Lancet Neurol. Vol.10, No.1, (January 2011), pp 99-108.

Ogasawara, M., Nakajima, W., Ishida, A \& Takada, G. (1999). Striatal perfusion of indomethacin attenuates dopamine increase in immature rat brain exposed to anoxia: an in vivo microdialysis study. Brain Res. Vol.842, No.2, (September 1999), pp 487-490.

Orłowska-Majdak, M. (2004). Microdialysis of the brain structures: application in behavioral research on vasopressin and oxytocin. Acta Neurobiol Exp. Vol.64, No.2, (2004), pp177-188.

Parent, M., Bush, D., Rauw, G., Master, S., Vaccarino, F. \& Baker, G. (2001). Analysis of amino acids and catecholamines, 5-hydroxytryptamine and their metabolites in brain areas in the rat using in vivo microdialysis. Methods. Vol.23, No.1, (January 2001), pp 11-20.

Pearigen, P., Gwinn, R \& Simon, R. (1996). The effects in vivo of hypoxia on brain injury. Brain Res. Vol.725. No. 2, (July 1996), pp 184-191.

Plock, N. \& Kloft, C. Microdialysis theoretical background and recent implementation in applied life-sciences. European Journal of Pharmaceutical Sciences. Vol.25, No.1, (May 2005), pp 1-24.

Prakash, R. \& Mullen, K. (2010). Mechanisms, diagnosis and management of hepatic encephalopathy. Nat Rev Gastroenterol Hepatol. Vol.7, pp 515-525.

Randolph, C., Hilsabeck, R., Kato, A., Kharbanda, P., Li, YY \& Mapelli D. (2009). Neuropsychological assessment of hepatic encephalopathy: ISHEN practice guidelines. Liver Int. Vol.29, No.5, (May 2009), pp 629-635.

Randolph, C., Tierney, M., Mohr, E \& Chase, T. (1998). The Repeatable Battery for the Assessment of Neuropsychological Status (RBANS): preliminary clinical validity. $J$ Clin Exp Neuropsychol. Vol.20, No.3, pp 310-319.

Rao, V., Audet, R \& Butterworth, R. (1995). Selective alterations of extracellular brain amino acids in relation to function in experimental portal-systemic encephalopathy: results of an in vivo microdialysis study. J Neurochem. Vol.65, No.3, (September 1995), pp 1221-1228.

Robinson, T. \& Justice, J. Jr. (1991). Microdialysis in neurosciences. ElSELVIER, Amsterdam, New York., ISBN 10: 044481194x. 
Rodrigo, R., Rodriguez-Diaz, j., Saez-Valero, J., Piedrafita, B., Suarez, I \& Felipo, V. (2007). Glutamate-induced activation of nitric oxide synthase is impaired in cerebral cortex in vivo in rats with chronic liver failure. Neurochem. Vol.102, No.1, (July 2007), pp 51-64.

Rose, C., Michalak, A., Pannunzio, M., Chatauret, N., Rambaldi, A \& Butterworth, R. (2000). Mild hypothermia delays the onset of coma and prevents brain edema and extracellular brain glutamate accumulation in rats with acute liver failure. Hepatology. Vol.31, No.4, (April 2000), pp 872-877.

Sato, T. \& Moriuchi, H. (2010). Influenza-associated encephalopathy. Nippon Rinsho. Vol.68, No.9, pp 1661-1665.

Sima, A. (2010). Encephalopathies: the emerging diabetic complications. Acta Diabetol. Vol.47, No.4, (August 2010), pp 279-293.

Sundaram, V. \& Shaikh, O. (2009) Hepatic encephalopathy: pathophysiology and emerging therapies. Med. Clin. North Am. Vol.93. No4, (July 2009), pp 819-836.

Suzuki, K., Matsuo, N., Moriguchi, T., Takeyama, N., Kitazawa, Y \& Tanaka, T. (1992). Changes in brain ECF amino acids in rats with experimentally induced hyperammonemia. Metab Brain Dis. Vol.7, No.2, (June 1992), pp 63-75.

Szerb, J. \& Redondo, I. (1993). Astrocytes and the entry of circulating ammonia into the brain: effect of fluoroacetate. Metab Brain Dis, Vol.8, No.4, (December 1993), pp 217234).

Thoresen, M., Hallström, A., Whitelaw, A., Puka-Sundvall, M., Løberg, E., Satas, S., Ungerstedt, U., Steen, P \& Hagberg, H. (1998). Lactate and pyruvate changes in the cerebral gray and white matter during posthypoxic seizures in newborn pigs. Pediatr Res. Vol.44, No.5, (November 1998), pp 746-754.

Todd, K. \& Butterworth, R. (1998). Evaluation of the role of NMDA-mediated excitotoxicity in the selective neuronal loss in experimental Wernicke encephalopathy. Exp Neurol. Vol.149, No.1, (January 1998), pp 130-138.

Todd, K. \& Butterworth, R. (2001). In vivo microdialysis in an animal model of neurological disease: thiamine deficiency (Wernicke) encephalopathy. Methods. Vol.23, No.1, (January 2001), pp 55-61.

Ungerstedt, U. \& Hallström, A. (1987). A. In vivo microdialysis-a new approach to the analysis of neurotransmitters in the brain. Life Sci. Vol.41, No.7, (August 1987), pp 861-864.

Verbeeck, R.K. (2000). Blood microdiálisis in pharmacokinetic and drug metabolism. Advanced Drug Delivery Reviews. Vol 45, No. 2-3, (December 2000), pp 217-228.

Viñas, F. (2001). Bedside invasive monitoring techniques in severe brain-injured patients. Neurol Res. Vol.23, No.2-3, (March-April 2001), pp 157-166.

Vogels, B., Karlsen, O., Mass, M., Boveé, W \& Chamuleau, R. (1997). L-ornithine vs. Lornithine-L-aspartate as a treatment for hyperammonemia-induced encephalopathy in rats. J Hepatol. Vol.26, No.1, (January 1997), pp 174-182.

Wikell, C., Apelqvist, G., Hjorth, S., Kullingsjö, J., Bergqvist, P \& Bengtsson, F. (2002). Effects on drug disposition, brain monoamines and behavior after chronic treatment with the antidepressant venlafaxine in rats with experimental hepatic encephalopathy. Eur Neuropsychopharmacol. Vol.12, No.4, (August 2002), pp 327336. 
Wikell, C., Bergqvist, P., Hjorth, S., Apelqvist, G., Björk, H \& Bengtsson, F. (1998). Brain monoamine output alterations after a single venlafaxine challenge in experimental hepatic encephalopathy. Clin Neuropharmacol. Vol.21, No.5, (Sep-Oct 1998), pp 296306.

Wikell, C., Kugelberg, F., Hjorth, S., Apelqvist, G \& Bengtsson, F. (2001). Effect of halving the dose of venlafaxine to adjust for putative pharmacokinetic and pharmacodynamic changes in an animal model of chronic hepatic encephalopathy. Clin Neuropharmacol. Vol.24, No.6, (Nov-Dec 2001), pp 324-333.

Yano, M., Adachi, N., Liu, K \& Arai, T. (2005). Flumazenil-induced improvement of the central dopaminergic system in rats with acute hepatic failure. I Neurosurg Anesthesiol. Vo.17, No.2, (April 2005), pp 69-74.

Zielińska, M., Hilgier, W., Borkowska, H., Oja, S., Saransaari, P \& Albrecht J. (2001). Lubeluzole attenuates $\mathrm{K}(+)$-evoked extracellular accumulation of taurine in the striatum of healthy rats and rats with hepatic failure. Brain Res. Vol.904, No.1, (June 2001), pp 173-176.

Zwingmann, C., Desjardins, P., Hazell, A., Chatauret, N., Michalak, A \& Butterworth, R. (2002). Reduced expression of astrocytic glycine transporter (Glyt-1) in acute liver failure. Metab Brain Dis. Vo. 17, No.4, (December 2002), pp 263-273.

Zwirner, K., Thiel C, Thiel. K., Morgalla, MH., Königsrainer, A \& Schenk, M. (2010). Extracellular brain ammonia levels in association with arterial ammonia, intracranial pressure and the use of albumin dialysis devices in pigs with acute liver failure. Metab Brain Dis, Vol.25, No.4, (December 2010), pp. 407-412. 


\title{
Portal-Systemic Encephalopathy in Emergency Treatment of Cirrhosis and Bleeding Esophageal Varices
}

\author{
Marshall J. Orloff \\ University of California, San Diego, Medical Center
}

USA

\section{Introduction}

Bleeding esophageal varices (BEV) is a common and highly lethal complication of cirrhosis of the liver (Graham \& Smith, 1981; Smith \& Graham, 1982; Burroughs et al., 1989; Bornman et al., 1994; Khan et al., 2006; Orloff et al., 1977; Mikkelsen, 1974; Terblanche et al., 1989; \& D'Amico et al., 1995) The mortality rate associated with BEV is highest during the period surrounding the episode of acute bleeding. If the varices remain untreated after recovery from a bout of acute bleeding, we (Orloff et al., 1977) and others observed a 95\% incidence of recurrent bleeding, and death within 2 to 5 years in $90 \%$ to $100 \%$ of the patients. Recurrent bleeding has been reported to develop most often within the first few days after the acute bleeding episode (Graham \& Smith, 1981; Smith \& Graham, 1982). Thus, it is clear that emergency treatment of acute bleeding is of paramount importance in the care of patients with portal hypertension and esophagogastric varices.

Because of the over-riding importance of emergency treatment of variceal bleeding, from 1958 to 2011 we conducted and reported studies of emergency therapy in patients with cirrhosis (Orloff, 1967; Orloff et al., 1980; Orloff et al., 1992); Orloff et al., 1994; Orloff et al., 1995a; Orloff et al., 2009a; Orloff et al., 2009b; Orloff et al., 2011a; Orloff et al., 2011b; Orloff et al., 2010; Orloff et al., 2011c; Orloff et al., 2011d; Orloff et al, 2011e; Orloff et al., 2011f; Orloff et al., 2011g; Orloff et al., 2011h; Orloff et al., 2011i). Our studies have been distinguished by three features that, together, make them different from other reported investigations. All patients admitted to our institution with cirrhosis and BEV ("all comers"), regardless of their condition, were included without selection; the specific emergency treatment undergoing evaluation was administered within 24 hours and usually within 8 hours of initial contact; and our studies were prospective, meaning that a welldefined protocol was consistently used and data were collected on-line. Evaluation, prevention, and treatment of portal-systemic encephalopathy (PSE) were an important part of each of our studies.

\section{The Eck fistula}

In 1877, Nikolai V. Eck (1849-1908), a 29-year-old military surgeon working in the Military Medical Academy in St. Petersburg, published a brief article describing his experiments and 
technique for creating a portacaval shunt (Eck fistula) in eight dogs (Eck, 1877). This was not only the beginning of the surgical treatment of portal hypertension but also the first vascular anastomosis. In his article, Eck stated: "I am conducting these experiments with the purpose of clarifying some physiologic problems as well as to determine whether it would be possible to treat some cases of mechanical ascites by means of forming such a fistula. I operated on 8 dogs, one recovered completely and lived in the laboratory for 2.5 months. Because of lack of attention, he ran away. I had to postpone further experiments because I was called to join the active army."

Eck's work would probably have remained unknown outside Russia had it not been for Ivan Pavlov (1849-1936), who used the procedure extensively in laboratory studies of liver physiology and named the operation the Eck fistula, giving full credit to Eck for its invention (Hahn et al., 1893; Pavlov, 1893). The landmark description by Pavlov et al. of portal-systemic encephalopathy (PSE) following feeding meat to dogs with an Eck fistula, which they called "meat intoxication," was part of the work that resulted in Pavlov's receiving the Nobel Prize in 1904 for his contributions on the physiology of digestion. Pavlov spent his entire career at the Military Medical Academy in St. Petersburg, the same institution where Eck developed the portacaval shunt. Since its original description, the Eck fistula has been used extensively for a wide range of studies in animals and humans and clinically for the treatment of the life-threatening complications of portal hypertension.

\section{Portal-Systemic Encephalopathy (PSE)}

Hepatic encephalopathy is a neuropsychiatric disorder that results from impaired liver function. It occurs in two distinct forms: (1) in acute or fulminant hepatic failure it takes the form of a neurological disorder that progresses from altered mental status to coma, generally within hours or days, and is associated with increased intracranial pressure caused by massive brain edema; (2) portal-systemic encephalopathy (PSE), which commonly occurs in cirrhosis, in which there are spontaneous or created portal-systemic shunts that permit toxins of intestinal origin to bypass the liver into the systemic circulation. Neurologically, PSE develops slowly, starting with sleep abnormalities, shortened attention span, and muscular incoordination, progressing through lethargy, ataxia, stupor, and coma. PSE is often precipitated by event such as gastrointestinal bleeding, uremia, hypokalemia, or ingestion of excessive amounts of protein. Neuropathologically, PSE is characterized by astrocytic rather than neuronal changes, and brain edema and intracranial hypertension are not found.

Since the description in 1893 of "meat intoxication" in the Eck fistula dog by the Pavlov group, animals with a portacaval shunt (PCS) (Eck fistula) have been used extensively for research studies of the pathogenesis and treatment of PSE (Hahn et al., 1893). The PCS rat has been consistently reported to have neurobehavioral abnormalities when subjected to sophisticated testing (Bengtsson et al., 1980; Tricklebank et al., 1978). One of the best animal models of chronic recurrent PSE is the dog with a congenital or surgically created PCS (Maddison et al., 1991). Animal and human studies have demonstrated that chronic liver failure and spontaneous or created PCS result in the accumulation of neurotoxic substances in the brain. Two such substances are ammonia and manganese.

Ammonia derived from colonic bacteria as well as from the deamination of glutamine in the small bowel is absorbed by passive diffusion and normally undergoes a high first-pass 
extraction by the liver (Cordoba \& Blei, 1997). In chronic liver failure, hepatic urea synthesis declines and this, in addition to portal-systemic shunting, results in increased blood ammonia concentrations. Furthermore, cirrhotic patients are hypersensitive to ammoniagenic conditions such as an oral protein load or gastrointestinal hemorrhage. An illustration of this hypersensitivity is provided by a report of studies in the 1950s in which attempts were made to treat ascites in cirrhotic patients with ion-exchange resins that absorbed sodium but released ammonium ions (Gabuzda et al., 1952). This treatment led to a significant reduction in ascitic volume but precipitated severe PSE in many of the patients treated. If present in high concentrations, ammonia has the potential to adversely affect central nervous system (CNS) function by several mechanisms, which include a direct effect of the ammonium ion on inhibitory and excitatory neurotransmission as well as inhibition of the tricarboxylic acid cycle enzyme ketoglutarate dehydrogenase, with potential impairment of brain energy metabolism (Szerb \& Butterworth, 1992; Lai \& Cooper, 1986) However, brain energy metabolism does not appear to be impaired in chronic liver failure until very late stages associated with isoelectric electroencephalography (EEG) traces (Hindfelt et al., 1977). On the other hand, increases of cerebrospinal fluid lactate have been described both in cirrhotic patients with PSE (Yao et al., 1987) and in experimental animals with chronic liver failure and ammonia-precipitated encephalopathy (Therrien et al., 1991), findings that are consistent with an inhibitory effect of ammonia on cerebral glucose oxidation.

Other effects of ammonia on cerebral function include a stimulatory effect on L-arginine uptake by brain preparations resulting in increased production of nitric oxide (Raghavendra Rao et al., 1995) and inhibition of the capacity of astrocytes to accumulate glutamate (Bender \& Norenberg, 1996; Knecht et al., 1997) a major excitatory neurotransmitter.

In 1963, we performed a study that subsequently influenced the use of PCS in the treatment of portal hypertension and variceal hemorrhage. We examined the influence of the stomal size of the portacaval anastomosis, and in turn of the blood flow rate through the anastomosis, on peripheral blood ammonia levels (Orloff et al., 1963). Induced ammoniemia was studied in two groups of dogs, one of which had large end-to-side PCS measuring at autopsy $2.0-4.2 \mathrm{~cm}$ in greatest diameter, and the other of which had small end-to-side PCS measuring $0.5-1.8 \mathrm{~cm}$ in greatest diameter. A third group of intact dogs served as controls. Ammoniemia was induced by three standardized methods that consisted of the administration by gavage of ammonium citrate $(0.5 \mathrm{~g} / \mathrm{kg})$, gavage of fresh whole blood (30 $\mathrm{mL} / \mathrm{kg}$ ) plus urease $(4 \mathrm{U} / \mathrm{mL})$, and gavage of fresh whole blood $(30 \mathrm{~mL} / \mathrm{kg})$ alone. All three techniques of ammonium loading resulted in significantly higher mean peak blood ammonia levels in the dogs with large shunts than in those with small shunts. Furthermore, in the animals with large shunts, markedly elevated blood ammonia concentrations persisted for longer periods of time. Measurements of the pressure gradients across the shunts at operation revealed a mean gradient of $31.0 \mathrm{~mm}$ saline in the small-shunt group as compared to only $3.0 \mathrm{~mm}$ in the large-shunt group. A consistent inverse relationship was demonstrated between the height of the blood ammonia level and the magnitude of the pressure gradient across the portacaval anastomosis. Application of Gorlins' hydraulic formula to these studies provided support for the presumption that the magnitude of ammoniemia was directly related to the blood flow rate through the shunt. We concluded that the stomal size of end-to-side PCS, and in turn the rate of blood flow through the shunts, have a definite influence on the concentration of ammonia in the peripheral blood of 
the dog. These results suggested the possibility of a relationship between shunt size and postshunt PSE. The results of this study served as the basis for the subsequent use of reduced-caliber PCS in the treatment of variceal bleeding (Sarfeh \& Rypins, 1994; Zervox et al., 1998).

In addition to ammonia, chronic liver failure and portal-systemic shunting result in increased blood and brain concentrations of manganese (López-Novoa, 1988; Fitzhugh \& Nelson, 1948; Dashti et al., 1989; \& Nuber et al., 1980). Manganese is neurotoxic, affecting both neuronal and astrocytic integrity. In the case of astrocytes, exposure to manganese results in altered expression of several key astrocytic proteins (Hazell et al., 1999a; Hazell et al., 1999b) and Alzheimer type II changes (Weissenborn et al., 1995).

Other toxins in addition to ammonia and manganese are known to increase in the systemic circulation in chronic liver failure. Such toxins include mercaptans, phenols, and short-chain fatty acids (Zieve et al., 1974). While there is no convincing evidence that these toxins alone cause cerebral dysfunction in chronic liver failure, they could combine with ammonia or manganese to act synergistically (Zieve, 1989).

Recently, attention has been focused on changes in brain neurotransmitter systems as the likely mediators of the neuropsychiatric manifestations of PSE in chronic liver failure. Recent studies using molecular biological approaches continue to confirm that, when liver fails, brain responds with significant alterations in gene expression. In many cases, these alterations involve genes that code for neurotransmitter-related proteins, many of which are essential for CNS function. Many of the symptoms of early PSE in chronic liver failure, such as altered personality, depression, and inverted sleep patterns, are symptoms that have classically been associated with alterations in biogenic amine function. FNA extracts of brain tissue obtained at autopsy from cirrhotic patients who died in hepatic coma have been found to show increased expression of the neuronal isoform of the monoaminemetabolizing enzyme MAO-A (Mousseau et al., 1997). This increase in MAO-A gene expression was found to be associated with increased activities of the enzyme and increased densities of catalytic sites on the enzyme protein (Mousseau et al., 1997). Moreover, studies of the same brain extracts revealed increased concentrations of homovanillic and hydroxyindoleacetic acids, the final metabolites of dopamine and serotonin, respectively (Bergeron et al., 1989). Increased concentrations of 5-hydroxyindoleacetic acid were also reported in cerebrospinal fluid from patients (Young \& Lai, 1980) and experimental animals (Bergeron et al., 1995) with chronic liver failure. On the basis of these findings, it has been suggested that altered monoaminergic function may be responsible for the early neuropsychiatric symptoms of PSE in chronic liver disease (Bergeron et al., 1995; Bergeron et al., 1990).

The "peripheral-type" benzodiazepine receptor (PTBR) is a heterooligomeric protein complex located (like MAO-A) on the outer mitochondrial membrane of the astrocyte. Increased PTBR gene expression has been reported in brain extracts from rats with PCS (Desjardins et al., 1997). This increased gene expression resulted in increased receptor sites in the brains and peripheral tissues of these animals as revealed by quantitative receptor autoradiography and the high selective PTBR ligant [ $\left.{ }^{3} \mathrm{H}\right]$ PK 11195 (Giguère et al., 1992; Raghavendra Rao et al., 1994). Increased [3H] PK 11195 binding sites were also reported in autopsied brain tissue from cirrhotic patients who died in hepatic coma (Lavoie et al., 1990). 
There is evidence to suggest that the increased expression of PTBRs in brain in chronic liver failure is the consequence of exposure to ammonia and/or manganese. The precise mechanism whereby increased expression or activation of PRBRs results in altered brain excitability characteristic of PSE has not been established. PCS in the rat results in increased gene expression of the constitutive (neuronal) isoform of nitric oxide synthase (nNOS) in brain (Raghavendra Rao et al., 1997a). Increased nNOS mRNA is accompanied by increased nNOS protein (Raghavendra Rao et al., 1997a) and by increased nNOS enzyme activities. Recent evidence suggests that, in addition to an induction in nNOS gene expression, increased nNOS activities may also result from a stimulatory effect of ammonia on Larginine uptake by neuronal preparations shown both in vitro and in vivo (Raghavendra Rao et al., 1997b). Increased production of NO as a consequence of increased nNOS activities could contribute to the alterations of cerebral perfusion observed in chronic liver disease (Raghavendra Rao et al., 1998).

The appearance of extrapyramidal symptoms, particularly rigidity, in cirrhotic patients with end-stage liver disease has prompted, by analogy with the well-established dopamine deficit in Parkinson's disease, evaluations of the dopamine system in relation to PSE. Studies in autopsied brain tissue from cirrhotic patients (Bergeron et al., 1989) and from rats with PCS (Bergeron et al., 1995) reveal several-fold increases in concentration of the dopamine metabolite homovanillic acid, a finding that could result from increased activities of monoamine oxidase reported in the same material (Mousseau et al., 1997). In another study, densities of the postsynaptic dopamine $\mathrm{D}_{2}$ receptor were significantly reduced in pallidum/putamen from cirrhotic patients (Mousseau et al., 1993) a finding that could have resulted from manganese deposition in the brains of these patients (Pomier Layrargues et al., 1995).

Strategies aimed at the prevention and treatment of PSE in chronic liver failure are of two major types, namely, ammonia-lowering strategies and approaches aimed directly at the CNS (Cordoba \& Blei, 1997; Ferenci et al., 1996). Since PSE is frequently precipitated by ammoniagenic situations such as an oral protein load or a gastrointestinal hemorrhage, various treatment modalities are aimed at the gut. Such strategies include reduction of the absorption of nitrogenous substances arising from bacterial action in the colon. Colonic cleansing reduces the luminal ammonia content and lowers blood ammonia content in cirrhotic patients (Wolpert et al., 1970). Nonabsorbable disaccharides are routinely used to decrease ammonia production in the gut. The action of the most popular substance in this class, lactulose, involves increased fecal nitrogen excretion by facilitation of the incorporation of ammonia into bacteria as well as a cathartic effect (Cordoba \& Blei, 1997). Lactulose administered orally reaches the cecum, where it is metabolized by enteric bacteria, causing a fall in $\mathrm{pH}$ (Brown et al., 1974). The dose is adjusted to produce two or three soft bowel movements daily (Cordoba \& Blei, 1997).

Antibiotics such as neomycin are also useful for lowering blood ammonia, mainly by an effect on ammonia production by intestinal bacteria. However, neomycin therapy may be associated with some toxic side effects (Cordoba \& Blei, 1997). More recently, rifaximin, a nonabsorbable derivative of the antibiotic rifamycin, has been shown to be effective in lowering blood ammonia and has proven to be of superior efficacy compared with lactulose (Bismuth et al., 2011; Munoz, 2008). Most of an orally administered dose is eliminated unchanged in the feces. Thus, it lacks significant toxicity and side effects because of minimal gastrointestinal absorption. 
Restriction of dietary protein remains a cornerstone of therapy for PSE in cirrhotic patients and has been an essential part of our treatment of patients with bleeding esophageal varices following portacaval shunt (Wolpert et al., 1970). However, long-term nitrogen restriction is potentially harmful and a positive nitrogen balance is necessary to promote liver regeneration as well as to increase the capacity of skeletal muscle to remove ammonia in the form of glutamine (Lockwood et al., 1979). Protein intake of $1 \mathrm{~g} / \mathrm{kg}$ per day may be required in order to maintain an adequate nitrogen balance (Swart et al., 1989).

An alternative strategy for the lowering of blood ammonia is the stimulation of ammonia fixation (Desjardins et al., 1997). Under normal physiological conditions, ammonia is removed by the formation of urea in periportal hepatocytes and by glutamine synthesis in perivenous hepatocytes, skeletal muscle, and brain. In cirrhosis, both urea cycle enzymes and glutamine synthetase in liver are decreased in activity. Strategies to stimulate residual urea cycle activities and/or glutamine synthesis have been tried over the past 20 years. One of the most successful agents to be used so far is L-ornithine-L-aspartate (OA). RCTs with OA demonstrate significant ammonia lowering and concomitant improvement in psychometric test scores in cirrhotic patients with PSE (Kircheis et al., 1997). Studies in experimental animals suggest that the metabolic basis for the beneficial effect of OA on blood ammonia in chronic liver failure resides in its ability to stimulate residual hepatic urea cycle function and also to promote glutamine synthesis, particularly in skeletal muscle (Rose et al., 1998).

Benzoate is also effective in reducing blood ammonia both in patients with inherited urea cycle disorders and in cirrhotic patients [64]. In a RCT with sodium benzoate versus lactulose, improvement in neuropsychiatric performance was found to be comparable using both treatments (Sushma et al., 1992).

In contrast to the multiple strategies used successfully to lower blood ammonia and improve neurological status in patients with chronic liver failure, drugs that act directly on neuronal excitability have not been widely applied in this patient group. The major reason for this is that the precise neurotransmitter changes responsible for PSE in chronic liver failure are still being elucidated. Some attempts to treat PSE in cirrhotic patients with benzodiazepine receptor antagonists and dopamine agonists have occurred, but with limited success.

Several RCTs have been performed to assess the efficacy of the benzodiazepine receptor antagonist flumazenil in cirrhotic patients with various degrees of severity of PSE [69]. Spectacular improvements in neuropsychiatric status were recorded in a subset of patients receiving flumazenil (Pomier Layrargues, 1994; Gyr et al., 1996). However, enthusiasm for this approach has been tempered by the possible confounding effects of prior exposure to benzodiazepines and the seeming lack of correlation between clinical response and blood levels of substances with benzodiazepine receptor agonist properties in these patients (Butterworth et al., 1995).

\section{Emergency portacaval shunt for bleeding esophageal varices}

Portal-systemic shunt is the only available definitive treatment for portal hypertensionrelated bleeding. Numerous studies have shown that a technically satisfactory PSS will permanently solve the problem of bleeding in the vast majority of patients. The obvious 
potential advantage of performing this procedure under emergency circumstances is that, unlike other forms of treatment, it can be expected to provide both immediate and prolonged control of hemorrhage. The question is, can cirrhotic patients tolerate an operation of this magnitude when it is performed as an emergency in the face of bleeding? To answer this question, we have conducted prospective studies of emergency PCS (EPCS) over the past 47 years, as follows: (1) an unrandomized study of 400 unselected patients who underwent EPCS; (2) a RCT of EPCS versus emergency medical therapy involving 43 patients at our Veterans Administration Hospital; (3) an unrandomized study of 94 unselected, consecutive patients with Child's class C cirrhosis; (4) a RCT of portacaval shunt versus endoscopic sclerotherapy in 518 unselected patients bleeding from gastric varices; (5) an unrandomized study in 12 patients with uncontrollable bleeding from portal hypertensive gastropathy; (6) a NIH grant supported RCT of EPCS versus emergency endoscopic sclerotherapy (EST) that enrolled 211 patients who have had more than 10 years of follow-up or until death; and (7) a NIH grant supported RCT of TIPS versus EPCS that enrolled 154 patients who have been followed up for 5-10 years (Orloff, 1967; Orloff et al., 1980; Orloff et al., 1992; Orloff et al., 1994; Orloff et al., 1995a; Orloff et al., 2009a; Orloff et al., 2009b; Orloff et al., 2011a; Orloff et al., 2011b; Orloff et al., 2010; Orloff et al., 2011c; Orloff et al., 2011d; Orloff et al, 2011e; Orloff et al., 2011f; Orloff et al., 2011g; Orloff et al., 2011h; Orloff et al., 2011i; Orloff \& Bell, 1983; Bell, et al., 1981; Orloff, 1968; Orloff, 1969; Orloff et al., 1974; Orloff et al., 1975; Orloff \& Bell, 1986; Orloff et al., 1995b; Orloff et al., 1997) . The unique features of our studies that, together, make them different from other reported investigations are as follows: (1) EPCS was undertaken within $24 \mathrm{~h}$ of initial contact of the patient with our institution in one study and within $8 \mathrm{~h}$ in the other six studies; (2) the patients were unselected, which means that all patients with bleeding varices, regardless of their condition ("all comers"), were entered in the studies and treated; (3) the studies were prospective, which means that the patients were managed according to a well-defined and consistently applied protocol, and specific data were collected at the time of diagnosis, treatment, and follow-up; and (4) the patients were followed up monthly for the first year and every 3 months thereafter for life, such that the $1-, 5-$, and 10-year follow-up rates were 100, 98, and 97\%, respectively. A total of 1,432 patients have been involved in these studies.

\section{Portal-systemic encephalopathy in our RCT of endoscopic sclerotherapy versus emergency portal shunt}

\subsection{Quantitation of PSE}

This recently published RCT is representative of all of our studies (Orloff et al., 2009a; Orloff et al., 2009b). PSE was quantitated during hospitalizations and at each clinic visit by grading 4 variously weighted components on a scale of 0 to 4: (1) mental state, (2) asterixis, (3) number connection test, and (4) arterial blood ammonia. A PSE Index was calculated according to the method of Conn et al. (Conn \& Lieberthal, 1978) in which the scores of the 4 components were added to yield a PSE Sum, and then divided by the maximum possible PSE Sum. Mental state was given a weight of 3 and the other components a weight of 1 . To increase objectivity, a senior faculty gastroenterologist who was not otherwise involved in the BEV study evaluated each patient for PSE during the clinic visits. The gastroenterologist was "blinded" in that he was not told what therapy the patient had received. 
The definition of PSE was based on any one of the following criteria: (1) classical signs of altered mental status on physical examination performed by an experienced faculty physician; (2) classical signs of altered mental status described by outside physicians, close relatives of the patient, or the patient himself; (3) a high PSE Index of 0.33 or greater. Electroencephalography was not included in the evaluation because it was considered cumbersome, costly, and impractical given the numerous programmed and unprogrammed outpatient clinic and emergency department visits made by the patients. Patients were classified as having recurrent PSE when they had 2 or more episodes of PSE after primary therapy.

\subsection{Incidence of PSE}

Patients who were discharged from the hospital and survived more than 30 days after entry in the study numbered 93 in the EST group and 89 in the EPCS group. Calculations of the incidence and manifestations of recurrent PSE are based on this population. Deaths on or before 30 days were considered indeterminate and were unrelated to PSE. Dietary protein tolerance up to $80 \mathrm{~g}$ per day was observed in all patients before discharge. A 60-g protein restriction was prescribed upon discharge. Table 1 and Fig. 1 show data on this group of patients. Patients who developed recurrent PSE after discharge from the initial hospital admission numbered 33 or $35 \%$ in the EST group and 13 or $15 \%$ in the EPCS group. The difference was highly significant with a P value of 0.001 .

\begin{tabular}{|l|l|l|l|}
\hline & $\begin{array}{l}\text { EST } \\
(\mathrm{n}=93)\end{array}$ & $\begin{array}{l}\text { EPCS } \\
(\mathrm{n}=89)\end{array}$ & $\begin{array}{l}\text { p } \\
\text { Value }\end{array}$ \\
\hline Incidence of recurrent PSE - n (\%) & $33(35)$ & $13(15)$ & $0.001^{*}$ \\
\hline Incidence of transient PSE - n (\%) & $3(3)$ & $3(3)$ & 1.0 \\
\hline Length of survival: & 133,243 & 266,169 & $<0.001^{*}$ \\
Total days & 364.8 & 728.7 & \\
Total years & 1432.7 & 2990.7 & \\
Total days per patient & 3.9 & 8.2 & $0.003^{*}$ \\
Total years per patient & & & \\
\hline Episodes of recurrent PSE & $179(1.92)$ & $94(1.06)$ & $0.003^{*}$ \\
Total episodes - n (n/person) & 0.49 & 0.13 & \\
Episodes per year of follow-up & 2.03 & 7.74 & \\
Interval between episodes (years) & & $87(0.98)$ & \\
\hline Hospital readmissions for recurrent PSE & $146(1.57)$ & 0.12 & \\
Total readmissions - n (n/ person) & 0.40 & 8.38 & \\
Readmissions per year of follow-up & 2.50 & $60(64)$ & \\
Interval between readmissions (years) & & $8(9)$ & \\
\hline Cause of recurrent PSE episodes - n (\%) & $75(42)$ & $12(13)$ & \\
Dietary protein indiscretion & $57(32)$ & $4(4)$ & \\
UGI bleeding & $18(10)$ & $11(12)$ & \\
Infection & $28(16)$ & $5(0)$ & \\
Alcoholism & $2(1)$ & $5(5)$ & \\
Uncontrolled diabetes & $7(4)$ & & \\
Hepatic failure & & \\
Other & $5(3)$ & & \\
\hline
\end{tabular}




\begin{tabular}{|c|c|c|c|}
\hline $\begin{array}{l}\text { Timing of first episode of recurrent PSE }-\mathrm{n}(\%) \\
\text { First } 6 \text { months } \\
\text { Second } 6 \text { months } \\
\text { Year-1 } \\
\text { Year-2 } \\
\text { Year-3 } \\
\text { Year-4 } \\
\text { Year-5 } \\
\text { Year }>5\end{array}$ & $\begin{array}{l}17(52) \\
6(18) \\
23(70) \\
4(12) \\
2(6) \\
0(0) \\
1(3) \\
3(9) \\
\end{array}$ & $\begin{array}{l}8(62) \\
1(8) \\
9(69) \\
3(23) \\
0(0) \\
0(0) \\
0(0) \\
1(8)\end{array}$ & 0.30 \\
\hline $\begin{array}{l}\text { Timing of all episodes of recurrent PSE }-\mathrm{n}(\%) \\
\text { First } 6 \text { months } \\
\text { Second } 6 \text { months } \\
\text { Year-1 } \\
\text { Year-2 } \\
\text { Year-3 } \\
\text { Year-4 } \\
\text { Year-5 } \\
\text { Year }>5\end{array}$ & $\begin{array}{l}24(15) \\
31(19) \\
55(34) \\
46(28) \\
18(11) \\
15(9) \\
4(2) \\
26(16) \\
\end{array}$ & $\begin{array}{l}23(24) \\
8(8) \\
31(33) \\
32(34) \\
11(12) \\
14(15) \\
1(1) \\
6(6) \\
\end{array}$ & 0.33 \\
\hline $\begin{array}{l}\text { Relationship of recurrent PSE to survival - } \\
\text { Years (median, } 95 \% \mathrm{CI} \text { ) } \\
\text { Patient with recurrent PSE: } \\
\text { Overall survival } \\
\text { Survival after first PSE } \\
\text { Patients free of recurrent PSE: } \\
\text { Overall survival } \\
\text { P value - Recurrent PSE vs. no PSE }\end{array}$ & $\begin{array}{l}2.90(1.5,4.74) \\
1.23(0.94,3.17) \\
3.26(2.00,4.34) \\
0.042^{*}\end{array}$ & $\begin{array}{l}5.18(1.26, \text { Inf }) \\
4.14(1.17, \text { Inf }) \\
10.37(6.19, \text { Inf }) \\
0.001^{*}\end{array}$ & \\
\hline $\begin{array}{l}\text { High PSE Index }-\mathrm{n}(\%) \\
\text { Patients with PSE Index } \geq 0.33 \\
\text { Patients with PSE Index } \geq 0.33 \text { who } \\
\text { had recurrent clinical PSE }\end{array}$ & $19(20)$ & $12(13)$ & $\begin{array}{l}0.24 \\
0.022^{*}\end{array}$ \\
\hline
\end{tabular}

* statistically significant difference

Table 1. Recurrent PSE in Patients Who Survived >30 Days and Were Discharged from the Hospital

The 93 early survivors in the EST group lived for a collective total of 364.8 years, while the 89 early survivors who underwent EPCS lived a collective total of 728.7 years, twice as long as the EST patients $(\mathrm{p}<0.001)$. The patients with recurrent PSE in the EST group had a collective total of 179 episodes of PSE and were readmitted to the hospital 146 times for treatment of PSE. In contrast, the patients with recurrent PSE following EPCS, experienced 94 episodes and 87 hospital readmissions. The calculated yearly frequency of PSE episodes was $0.49 / \mathrm{yr}$ in the EST group and $0.13 / \mathrm{yr}$ in the EPCS group, and the calculated hospital readmission rate was $0.40 / \mathrm{yr}$ in the EST patients and only $0.12 / \mathrm{yr}$ in the EPCS patients $(p=0.003)$. Expressed as per-patient average, patients in the EST group were hospitalized for recurrent PSE every 2.50 years, while patients who were treated by EPCS required hospitalization for recurrent PSE every 8.38 years $(\mathrm{p}=0.003)$. 


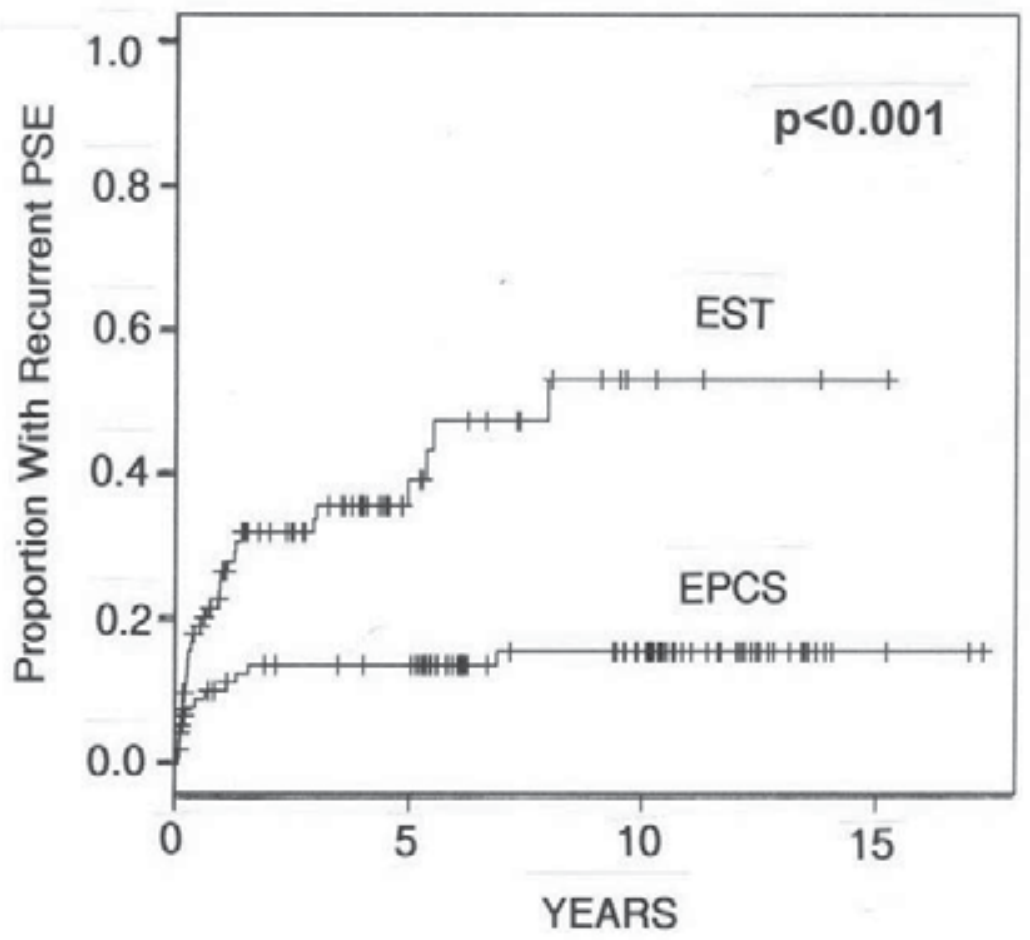

Fig. 1. Incidence and timing of recurrent PSE in patients who were randomized to EST $(n=93)$ or EPCS $(n=89)$ and survived $>30$ days and were discharged from the hospital.

\subsection{Cause of recurrent PSE}

Dietary indiscretion with regard to protein restriction was the most frequent cause of recurrent PSE in both groups more so in the patients treated by EPCS. Portal hypertensionrelated UGI bleeding, usually from esophageal varices, was the main cause of recurrent PSE in $32 \%$ of the episodes experienced in patients treated by EST, and was a contributing cause in an additional 18\%. Gastrointestinal bleeding was infrequently responsible for PSE in patients who underwent EPCS $(\mathrm{p}<0.001)$. Thus, gastrointestinal bleeding played a role in 57 episodes of PSE in the EST group, compared to 8 episodes in the EPCS group, and undoubtedly was a major factor in the higher incidence of recurrent PSE following EST. Other conditions responsible for at least $10 \%$ of PSE episodes were infection, alcoholism, and uncontrolled diabetes.

\subsection{Relationship of recurrent PSE to survival}

In the EST group, patients with recurrent PSE lived a median 2.90 years while those who remained free of PSE lived a median 3.6 years, a longer period of time. This difference was significant $(p=0.042)$ with a hazard ratio of death due to recurrent PSE of 1.60 (95\% confidence interval 1.01, 2.56). It is not possible in the EST group to determine the effect of PSE on survival because of other factors, particularly recurrent BEV, which influenced survival. 
In the EPCS group, patients who remained free of PSE lived a median 10.37 years, significantly longer than those with recurrent PSE who survived a median 5.18 years. The difference was significant $(p=0.001)$. It is tempting to attribute the longer survival to freedom from PSE, but the many factors that affect survival make it inappropriate to single out PSE as the main determinant of life or death.

The results with regard to PSE of our randomized controlled trial are contrary to conventional beliefs about both portacaval shunt and endoscopic sclerotherapy. The incidence of recurrent PSE during long-term follow-up of unselected patients treated by EPCS was only 15\%, which was significantly lower than the 35\% incidence of PSE following emergency and long-term EST. Moreover, every aspect of PSE was less severe following EPCS than after EST. The number of PSE episodes was fewer (94 vs. 179), the number of hospital readmissions for PSE was fewer (87 vs. 146), the calculated yearly frequency of PSE was lower, and the calculated per-patient average hospitalization for PSE was less frequent (once every $8.38 \mathrm{yr}$ vs. once every $2.50 \mathrm{yr}$ ) in the EPCS group than in the EST patients.

A number of factors appear to have played a role in causing PSE, but two of these factors were predominant. The first was dietary protein indiscretion. Despite concerted, repeated and often successful efforts to educate patients on limiting dietary protein intake to $60 \mathrm{~g}$ per day, dietary protein indiscretion was responsible for 75 of the 179 episodes of PSE in the EST group and 60 of the 94 episodes of PSE in the EPCS group. The second causative factor was recurrent variceal bleeding which was directly responsible for $32 \%$ of the episodes of PSE in patients treated by EST, and was a major contributing cause in an additional $18 \%$ of the episodes. In contrast, UGI bleeding at best may have been responsible for only 8 of the 94 episodes of PSE in patients who had EPCS. It appears highly likely that the higher incidence of PSE following EST compared to EPCS was due to the failure of EST to achieve long-term control of BEV. It is noteworthy that resumption of alcoholism may have played a role in only $12 \%$ of the episodes of PSE. These results demonstrate that a low incidence of PSE is possible when patients are free of recurrent UGI bleeding, abstain from alcohol, and comply with a diet of moderate protein restriction.

\section{Portal-systemic encephalopathy in our RCT of TIPS versus emergency portacaval shunt}

We have completed a RCT of emergency TIPS versus EPCS that enrolled 154 unselected consecutive patients who have been followed up for 5 to 10 years. The results of this RCT have been submitted for publication (Orloff et al., 2011h; Orloff et al., 2011j). Transjugular intrahepatic portosystemic shunt, or TIPS, is a side-to-side portacaval shunt performed radiologically by a percutaneous approach. It is the most widely used form of portal decompression and is used to control BEV when other measures, such as endoscopic treatment and pharmacologic therapy, have failed. The major shortcomings of TIPS reported in numerous studies have been a high incidence of occlusion of the shunt and the frequent occurrence of PSE.

In our RCT, regular follow-up to determine patency of the TIPS and EPCS revealed stenosis or occlusion of the TIPS in $84 \%$ of this group of patients. Patients treated by TIPS had a mean 2.1 episodes of TIPS stenosis or occlusion. Revision of the TIPS by balloon angioplasty or insertion of one or more additional stents was performed in $63 \%$ of the patients with TIPS 
malfunction. The revisions failed in $80 \%$ of the patients. The consequences of TIPS malfunction were recurrent BEV and PSE. Durability of TIPS was disappointing. In marked contrast, EPCS remained permanently patent in $97 \%$ of the patients in that groups, as a result of which there was permanent prevention of recurrent BEV and a significantly lower incidence of PSE. In the overall, $61 \%$ of the patients treated by TIPS developed recurrent PSE, compared to $21 \%$ of the patients treated by EPCS. The three-fold greater incidence of recurrent PSE in the TIPS group was highly significant $(\mathrm{p}<0.001)$.

In both of our recently reported RCTs, EPCS was a total shunt that involved a direct anastomosis between the portal vein and inferior vena cava. EPCS permanently controlled variceal bleeding, resulted in long-term survival that was substantially greater than that obtained by EST, and was followed by a relatively low rate of PSE that was less than onehalf the rate associated with EST. How do we account for the low incidence of PSE? We believe that a number of factors played an important role in the results achieved by EPCS in our study. First, and most important, EPCS was uniformly successful in preventing recurrent $\mathrm{BEV}$, a frequent cause of PSE. Second, hepatic function improved and stabilized in a substantial majority of patients as a result of both freedom from bleeding and abstinence from alcohol, which was permanent in $62 \%$ of the patients. Third, follow-up evaluation and support was rigorous, frequent and lifelong in all patients. Fourth, follow-up included regular counseling by a dietitian on restriction of dietary protein intake to $60 \mathrm{~g} /$ day, an amount that is quite ample for nutrition and is compatible with an appetizing diet. A high level of patient compliance was obtained by repeated education about the importance of moderate protein restriction. Fifth, long-term patency and function of the portacaval shunt was obtained in $98 \%$ of patients. No one of these factors alone can account for the low incidence of PSE, but together they resulted in a rate of PSE that was less than one-half the rate associated with EST.

\section{Conclusions}

In these prospective randomized controlled trials of emergency treatment of acute bleeding esophageal or gastric varices in 4 separate studies involving 628 unselected, consecutive patients with cirrhosis of all grades of severity, EPCS was followed by a 15-21\% incidence of recurrent PSE, which was significantly lower than the 35\% incidence of PSE that followed emergency and long-term EST and the 61\% incidence associated with TIPS. These results contradict the widespread belief that portal-systemic shunts are associated with a high incidence of PSE. Moreover, these results call into question the widespread practice of using portal-systemic shunt mainly or only as salvage for failure of endoscopic and pharmacologic therapy because of the belief that the superior control of variceal bleeding by surgery is offset by life-threatening PSE.

\section{Acknowledgement}

This work was supported by NIH grants A3084, A5919, AM07315, AM07511, AM1228, AM19875, AM12280, DK41920, and a grant from the Surgical Education and Research Foundation [501(c)(3)].

Registration: clinicaltrials.gov NCT00690027 


\section{References}

Bell, R.H. Jr.; Hyde, P.V.B.; Skivolocki, W.P.; Brimm, J.E., \& Orloff, M.J. (1981) Prospective study of portasystemic encephalopathy after emergency portacaval shunt for bleeding varices. American Journal of Surgery 142(1), 144-150.

Bender, A.S.; \& Norenberg, M.D. (1996) Effects of ammonia on L-glutamate uptake in cultured astrocytes. Neurochemistry Research 21(5), 567-573.

Bengtsson, F.; Nobin, A.; Falck, B.; Gage, F.H.; \& Jeppsson, B. (1980) Portacaval shunt in the rat: Selective alterations in behaviour and brain serotonin. Pharmacology Biochemistry and Behavior 24(6), 1611-1616.

Bergeron, M.; Reader, T.A.; Pomier Layrargues, G.; \& Butterworth, R.F. (1989) Monoamines and metabolites in autopsied brain tissue from cirrhotic patients with hepatic encephalopathy. Neurochemistry Research 14(9), 853-859.

Bergeron, M.; Swain, M.S.; Reader, T.A.; Grondin, L.; \& Butterworth, R.F. (1990) Effect of ammonia on brain serotonin metabolism in relation to function in the portacavalshunted rat. Journal of Neurochemistry 55(1), 222-229.

Bergeron, M.; Swain, M.S.; Reader, T.A.; \& Butterworth, R.F. (1995) Regional alterations of dopamine and its metabolites in rat brain following portacaval anastomosis. Neurochemistry Research 20(1), 79-86.

Bismuth, M.; Funakoshi, N.; Cadranel, J-F.; \& Blanc, P. (2011) Hepatic encephalopathy: from pathophysiology to therapeutic management. European Journal of Gastroenterology and Hepatology 23(1), 8-22.

Bornman, P.C.; Krige, J.E.; \& Terblanche, J. (1994) Management of esophageal varices. Lancet 343(8905), 1079-1084.

Brown, R.L.; Gibson, J.A.; Sladen, G.E.; Hicks, B.; \& Dawson, A.M. (1974) Effects of lactulose and other laxatives on ileal and colonic $\mathrm{pH}$ as measured by a radiotelemetry device. Gut 15(12), 999-1004.

Burroughs, A.K.; Mezzanotte, G.; Phillips, A.; McCormick, P.A.; \& McIntyre, N. (1989) Cirrhotics with variceal hemorrhage: the importance of the time interval between admission and the start of analysis for survival and rebleeding rates. Hepatology 9(6), 801-807.

Butterworth, R.F.; Wells, J.; \& Pomier Layrargues, G. (1995) Detection of benzodiazepines in hepatic encephalopathy: reply. Hepatology 21(2), 604-605.

Conn, H.O., \& Lieberthal, M.M. (1978) The hepatic coma syndromes and lactulose. Williams \& Wilkins, Baltimore.

Cordoba, J.; \& Blei, A.T. (1997) Treatment of hepatic encephalopathy. Am J Gastroenterol 92, 1429-1439.

D'Amico, G.; Pagliaro, L.; \& Bosch, J. (1995) The treatment of portal hypertension: a metaanalytic review. Hepatology 22(1), 332-354.

Dashti, H.; Jeppsson, B., Hägerstrand, I.; Hultberg, B.; Srinivas, U., Abdulla, M., \& Bengmark, S. (1989) Thioacetamide- and carbon tetrachloride-induced liver cirrhosis. European Surgical Research 21(2), 83-91.

Desjardins, P.; Bandeira, P.; Raghavendra Rao, V.L.; Ledoux, S.; \& Butterworth, R.F. (1997) Increased expression of the peripheral-type benzodiazepine receptor-isoquinoline carboxamide binding protein mRNA brain following portacaval anastomosis. Brain Research 758(1-2), 255-258.

Eck, N.V. (1877). Kvoprosu opereviazke vorotnoi veni: Predvaritelnoye soobschjenie (Concerning ligation of the vena porta: Preliminary notification). Voen Med Zh 130, 1-2. 
Ferenci, P.; Herneth, A.; \& Steindl, P. (1996) Newer approaches to therapy of hepatic encephalopathy. Seminars in Liver Disease 16(3), 329-338.

Fitzhugh, O.G., \& Nelson, A.A. (1948) Liver tumors in rats fed thiourea or thioacetamide. Science 108(2814), 626-628.

Gabuzda, D., Jr.; Philips, G.G., \& Davidson, C.S. (1952) Reversible toxic manifestations in 2 patients with cirrhosis of the liver given cation-exchange resins. $N$ Engl J Med 246(4), 124-130.

Giguère, J-F.; Hamel, E.; \& Butterworth, R.F. (1992) Increased densities of binding sites for the "peripheral-type" benzodiazepine receptor ligand 3H-PK 11195 in rat brain following portacaval anastomosis. Brain Research 585(1-2), 295-298.

Graham, D.Y.; \& Smith H. (1981) The course of patients after variceal hemorrhage. Gastroenterology 80(4), 800-809.

Gyr, K., Meier, R.; Häussler, J.; Boulétreau, P.; Fleig, W.E.; Gatta, A.; Holstege, A.; PomierLayrargues, G.; Schalm, S.W.; Groeneweg, M.; Scollo-Lavizzari, G.; Ventura E.; Zeneroli, M.L.; Williams, R.; Yoo, Y.; \& Amrein, R. (1996) Evaluation of the efficacy and safety of flumazenil in the treatment of portal systemic encephalopathy: a double blind, randomised, placebo controlled multicentre study. Gut 39(2), 319-324.

Hahn, M., Massen, V.N., Nenski, M., \& Pavlov, I.P. (1893). Die Eck' sche Fistel zwischen der unteren Hohlvene und der Pfortader und ihre Folgen Fur den Organismus, Arch Exp Pathol Pharmakol 32, 161-210.

Hazell, A.S.; Desjardins, P.; \& Butterworth, R.F. (1999a) Chronic exposure of rat primary astrocyte cultures to manganese results in increased binding site for 'peripheraltype' benzodiazepine receptor ligand ${ }^{3} \mathrm{H}-\mathrm{PK}$ 11195. Neuroscience Letters 271(1), 5-8.

Hazell, A.S.; Desjardins, P.; Butterworth, R.F. (1999b) Increased expression of glyceraldehyde-3-phosphate dehydrogenase in cultured astrocytes following exposure to manganese. Neurochemistry International 35(1), 11-17.

Hindfelt, B.; Plum, F.; \& Duffy, T.E. (1977) Effect of acute ammonia intoxication on cerebral metabolism in rats with portacaval shunts. Journal of Clinical Investigation 59(3), 386396.

Khan, S.; Tudur Smith, C.; Williamson, P; \& Sutton, R. (2006) Portosystemic shunts versus endoscopic therapy for variceal rebleeding in patients with cirrhosis. Cochrane Database of Systematic Reviews, Issue 1. Art. No. CD000553. doi:10.1002/14651858. CD000553. Pub 2.

Kircheis, G.; Nilius, R.; Held, C.; Berndt, H.; Buchner, M.; Görtelmeyer, R.; Hendricks, R.; Krüger B.; Kuklinski, B.; Meister, H.; Otto, H.J.; Rink, C.; Rösch, W.; Stauch, S. (1997) Therapeutic efficacy of L-ornithine-L-aspartate infusions in patients with cirrhosis and hepatic encephalopathy: Results of a placebo-controlled, double-blind study. Hepatology 25(6), 1351-1360.

Knecht, K.; Michalak, A.; Rose, C., Rothstein, J.D., Butterworth, R.F. (1997) Decreased glutamate transporter (GLT-1) expression in frontal cortex of rats with acute liver failure. Neuroscience Letters 229(3), 201-203.

Lai, J.C.K.; \& Cooper, A.J.L. (1986) Brain a-Ketoglutarate dehydrogenase complex: Kinetic properties, regional distribution and effects of inhibitors. Journal of Neurochemistry 47(5), 1376-1386.

Lavoie, J.; Pomier Layrargues, G.; Butterworth, R.F. (1990) Increased densities of peripheraltype benzodiazepine receptors in brain autopsy samples from cirrhotic patients with hepatic encephalopathy. Hepatology 11(5), 874-878. 
Lockwood A.M.; McDonald, J.M.; Rieman, R.E.; Gelbard, A.S.; Laughlin, J.S.; Duff, T.E.; Plum, F. (1979) The dynamics of ammonia metabolism in man: Effects of liver disease and hyperammonemia. Journal of Clinical Investigation 63(3), 449-640.

López-Novoa, J.M. (1988) Pathophysiological features of the carbon tetrachloride/phenobarbital model of experimental liver cirrhosis in rats. In: The Kidney in Liver Disease, Epstein, M.D., ed., pp. 309-327, Williams \& Wilkins, Baltimore.

Maddison, J.E.; Watson, W.E.J.; Dodd, P.R.; Johnston, G.A.R. (1991) Alterations in cortical $\left[{ }^{3} \mathrm{H}\right]$ kainite and $\mathrm{a}-\left[{ }^{3} \mathrm{H}\right]$ amino-3-hydroxy-5-methyl-isoxazolepropionic acid binding in a spontaneous canine model of chronic hepatic encephalopathy. Journal of Neurochemistry 56(6), 1881-1888.

Mikkelsen, W.P. (1974) Therapeutic portacaval shunt. Preliminary data on controlled trial and morbid effects of acute hyaline necrosis. Arch Surg 108(3), 302-305.

Mousseau, D.D.; Perney, P.; Pomier Layrargues, G.; Butterworth, R.F. (1993) Selective loss of pallidal dopamine $\mathrm{D}_{2}$ receptor density in hepatic encephalopathy. Neuroscience Letters 162(1-2), 192-196.

Mousseau, D.D.; Baker, G.B.; Butterworth, R.F. (1997) Increased density of catalytic sites and expression of brain monoamine oxidase $\mathrm{A}$ in humans with hepatic encephalopathy. J Neurochem 68(3), 1200-1208.

Munoz, S.J. (2008) Hepatic encephalopathy. Medical Clinics of North America 92(4), 795-812.

Nuber, R.; Teutsch, H.F.; Sasse, D. (1980) Metabolic zonation in thioacetamide-induced liver cirrhosis. Histochemistry 69(3), 277-288.

Orloff, M.J.; Wall, M.H., Hickman, E.G.; Neesby, T. (1963) Influence of stomal size of portacaval shunts on peripheral blood ammonia levels. Ann Surg 158(2), 172-181.

Orloff, M.J. (1967) Emergency portacaval shunt: A comparative study of shunt, varix ligation and nonsurgical treatment of bleeding esophageal varices unselected patients with cirrhosis. Ann Surg 166(3), 456-478.

Orloff, M.J. (1968) Emergency treatment of bleeding esophageal varices. In: The Therapy of Portal Hypertension, N.G. Markoff (ed.), George Thieme Verlag, Stuttgart, pp. 211219.

Orloff, M.J. (1969) Emergency treatment of bleeding esophageal varices in alcoholic cirrhosis. In: Biochemical and Clinical Aspects of Alcohol Metabolism, V.M. Sardesai (ed.), Charles C. Thomas, Springfield, IL, 288-297.

Orloff, M.J.; Chandler, J.G.; Charters, A.C. III; Condon, J.K.; Grambort, D.E.; Modafferi, T.R.; Levin, S.E. (1974) Emergency portacaval shunt treatment for bleeding esophageal varices. Prospective study in unselected patients with alcoholic cirrhosis. Arch Surg 108(3), 293-299.

Orloff, M.J.; Charters, A.C.; Chandler, J.G., Condon, J.K.; Grambort, D.E.; Modafferi T.R.; Levin, S.E.; Brown, N.B.; Sviokla, S.C.; Knox, D.G. (1975). Portacaval shunt as emergency procedure in unselected patients with alcoholic cirrhosis. Surg Gynecol Obstet 141(1), 59-68.

Orloff, M.J.; Duguay, L.R.; Kosta, L.D. (1977) Criteria for selection of patients for emergency portacaval shunt. Am J Surg 134(1), 146-152.

Orloff, M.J.; Bell, R.H. Jr.; Hyde, P.V.; Skivolocki, W.P. (1980) Long-term results of emergency portacaval shunt for bleeding esophageal varices in unselected patients with alcoholic cirrhosis. Ann Surg, 192(3), 325-340.

Orloff, M.J.; Bell, R.H. Jr. (1983) Improved survival of unselected cirrhotic patients with bleeding esophageal varices treated by emergency portacaval shunt. Gastroenterology, 84, 1388. 
Orloff, M.J.; Bell, R.H. Jr. (1986) Long-term survival after emergency portacaval shunting for bleeding varices in patients with alcoholic cirrhosis. Amer J Surg 151(1), 176-183.

Orloff, M.J.; Orloff, M.S.; Rambotti, M.; Girard, B. (1992) Is portal-systemic shunt worthwhile in Child's class $C$ cirrhosis? Long-term results of emergency shunt in 94 patients with bleeding varices. Ann Surg 216(3), 256-268.

Orloff, M.J.; Bell, R.H. Jr.; Orloff, M.S.; Hardison, W.G.M., Greenburg, A.G. (1994) Prospective randomized trial of emergency portacaval shunt and emergency medical therapy in unselected cirrhotic patients with bleeding varices. Hepatology $20(4, \mathrm{pt} 1), 863-872$.

Orloff, M.J.; Orloff, M.S.; Orloff, S.L.; Rambotti, M. (1995a) Three decades of experience with emergency portacaval shunt for acutely bleeding esophageal varices in 400 unselected patients with cirrhosis of the liver. Journal of the American College of Surgeons 180(3), 257-272.

Orloff, M.J.; Orloff, M.S.; Orloff, S.L.; Haynes, K.S. (1995b) Treatment of bleeding from portal hypertensive gastropathy by portacaval shunt. Hepatology 21(4), 1011-1017.

Orloff, M.J.; Orloff, M.S.; Orloff, S.L.; Girard, B. (1997) Long-term results of portacaval shunt for bleeding gastric varices in 224 patients with cirrhosis. Gastroenterology 112(4), A3194.

Orloff, M.J.; Isenberg, J.I.; Wheeler, H.O.; Haynes, K.S.; Jinich-Brook, H.; Rapier, R.; Vaida, F.; Hye, R.J. (2009a) Randomized trial of emergency endoscopic sclerotherapy versus emergency portacaval shunt for acutely bleeding esophageal varices in cirrhosis. Journal of the American College of Surgeons 209(1), 25-40.

Orloff, M.J.; Isenberg, J.I.; Wheeler, H.O.; Haynes, K.S.; Jinich-Brook, H.; Rapier, R.; Vaida F, Hye RJ. (2009b) Portal-systemic encephalopathy in a randomized controlled trial of endoscopic sclerotherapy versus emergency portacaval shunt treatment of acutely bleeding esophageal varices in cirrhosis. Ann Surg 250(4), 598-610.

Orloff, M.J.; Isenberg, J.I.; Wheeler, H.O.; Haynes, K.S.; Jinich-Brook, H.; Rapier, R.; Vaida, F.; Hye, R.J., \& Orloff, S.L. (2010) Liver transplantation in a randomized controlled trial of emergency treatment of acutely bleeding esophageal varices in cirrhosis. Transplantation Proceedings 42(10), 4101-4108.

Orloff, M.J.; Isenberg, J.I.; Wheeler, H.O.; Haynes, K.S.; Jinich-Brook, H.; Rapier, R.; Vaida, F.; Hye, R.J. (2011a) Emergency portacaval shunt versus rescue portacaval shunt in a randomized controlled trial of emergency treatment of acutely bleeding esophageal varices in cirrhosis - part 3. Journal of Gastrointestinal Surgery 14(11), 1782-1795.

Orloff, M.J.; Isenberg, J.I.; Wheeler, H.O.; Haynes, K.S.; Jinich-Brook, H.; Rapier, R.C.; Vaida, F.N.; Hye, R.J.; Orloff, S.L. (2011b) Direct costs of care in a randomized controlled trial of endoscopic sclerotherapy versus emergency portacaval shunt for bleeding esophageal varices in cirrhosis - part 4. Journal of Gastrointestinal Surgery 15(1), 3847.

Orloff, M.J.; Isenberg, J.I.; Wheeler, H.O.; Haynes, K.S.; Jinich-Brook, H.; Rapier, R.; Vaida, F.; Hye, R.J.; Orloff, S.L. (2011c) Hepatocellular carcinoma in a randomized controlled trial of emergency treatment of bleeding esophageal varices in cirrhosis. Am J Surg, in press.

Orloff, M.J.; Isenberg, J.I.; Wheeler, H.O.; Haynes, K.S.; Jinich-Brook, H.; Rapier, R.; Vaida, F.; Hye, R.J.; Orloff, S.L. (2011d) Alcoholic versus nonalcoholic cirrhosis in a randomized controlled trial of emergency treatment of acutely bleeding esophageal varices. Journal of Surgical Research; in press. 
Orloff, M.J.; Isenberg, J.I.; Wheeler, H.O.; Haynes, K.S.; Jinich-Brook, H.; Rapier, R.; Vaida, F.; Hye, R.J.; Max, W. (2011e) Economic cost of mortality and morbidity in a randomized controlled trial of emergency therapy of bleeding esophageal varices in cirrhosis. Hepatology, in press.

Orloff, M.J.; Vaida, F; Isenberg, J.I.; Wheeler, H.O.; Haynes, K.S.; Jinich-Brook, H.; Rapier, R.; Hye, R.J.; Orloff, S.L. (2011f) Child-Turcotte score versus MELD for prognosis in a randomized controlled trial of emergency treatment of bleeding esophageal varices in cirrhosis. J Hepatology, in press.

Orloff, M.J.; Isenberg, J.I.; Wheeler, H.O.; Haynes, K.S.; Jinich-Brook, H.; Rapier, R.; Vaida, F.; Hye, R.J.; Orloff, S.L. (2011g) Disability index in a randomized controlled trial of sclerotherapy versus portacaval shunt for bleeding varices in cirrhosis. Amer J Surg, submitted for publication.

Orloff, M.J.; Vaida, F.; Wheeler, H.O.; Isenberg, J.I.; Haynes, K.S.; Girard., B. (2011h) Randomized controlled trial of endoscopic therapy versus portacaval shunt treatment of bleeding gastric varices in cirrhosis. Journal of Gastrointestinal Surgery, in press.

Orloff, M.J.; Vaida, F., Roberts, A., Rose, S., Haynes, K., Hye, R.J.; Isenberg, J. (2011i) Randomized controlled trial of emergency transjugular Intrahepatic portosystemic shunt versus emergency portacaval shunt treatment of acute bleeding esophageal varices in cirrhosis. Journal of the American College of Surgery, in press.

Orloff, M.J., Vaida, F., Roberts, A.C., Rose, S.C., Haynes, K.S., Hye, R.J., \& Isenberg, J.I. (2011j). Portal-systemic encephalopathy in a randomized controlled trial of emergency transjugular intrahepatic portosystemic, shunt versus emergency portacaval shunt treatment of acute bleeding esophageal varices in cirrhosis. Submitted for publication.

Pavlov, I.P. (1893). On a modification of the Eck fistula between the portal vein and the inferior vena cava. Arch Sci Biol 2, 580-585.

Pomier Layrargues, G.; Giguère, J.F.; Lavoie, J.; Perney, P.; Gagnon, S.; D’Amour, M., Wells, J.; Butterworth, R.F. (1994) Flumazenil in cirrhotic patients in hepatic coma: a randomized double-blind placebo-controlled crossover trial. Hepatology 19(1), 3237.

Pomier Layrargues, G.; Spahr, L.; Butterworth, R.F. (1995) Increased manganese concentrations in pallidum of cirrhotic patients. Lancet 345(8951), 735.

Raghavendra Rao, V.L.; Audet, R.; Therrien, G.; Butterworth, R.F. (1994) Tissue-specific alterations of binding sites for peripheral-type benzodiazepine receptor ligand $\left[{ }^{3} \mathrm{H}\right] \mathrm{PK} 11195$ in rats following portacaval anastomosis. Digestive Disease and Science 39(5), 1055-1063.

Raghavendra Rao, V.L.; Audet, R.M.; Butterworth, R.F. (1995) Increased nitric oxide synthase activities and $\mathrm{I}-[3 \mathrm{H}]$ arginine uptake in brain following portacaval anastomosis. Journal of Neurochemistry 65(2), 677-681.

Raghavendra Rao, V.L.; Audet, R.M.; Butterworth, R.F. (1997a) Increased neuronal nitric oxide synthase expression in brain following portacaval anastomosis. Brain Research 765(1), 169-172.

Raghavendra Rao, V.L.; Audet, R.M.; Butterworth, R.F. (1997b) Portacaval shunting and hyperammonemia stimulate the uptake of $\mathrm{L}-3 \mathrm{H}$-arginine but not of $\mathrm{L}-3^{3} \mathrm{H}-$ nitroarginine into rat brain synaptosomes. Journal of Neurochemistry 68(1), 337-343.

Raghavendra Rao, V.L.; Butterworth, R.F. (1998) Neuronal nitric oxide synthase and hepatic encephalopathy. Metabolic Brain Disease 13(3), 175-189. 
Rose, C.; Michalak, A.; Pannunzio, P.; Thierrien, G.; Quack, G.; Kircheis, G.; Butterworth, R.F. (1998) L-orthinine-L-aspartate in experimental portal-systemic encephalopathy: therapeutic efficacy and mechanism of action. Metabolic Brain Disorders 13(2), 147-157.

Sarfeh, J.J.; Rypins, E.B. (1994) Partial versus total portacaval shunt in alcoholic cirrhosis. Results of a prospective, randomized clinical trial. Ann Surg 219(4), 353-361.

Smith, J.L.; Graham, D.Y. (1982) Variceal hemorrhage: a critical evaluation of survival analysis. Gastroenterology 82(5), 968-973.

Sushma, S.; Dasarathy, S.; Tandon, R.K.; Jain, S.; Gupta, S.; Bhist, M.S. (1992) Sodium benzoate in the treatment of acute hepatic encephalopathy: a double-blind randomized trial. Hepatology 16(1), 138-144.

Swart, G.R.; van den Berg, J.W.O.; van Vuure, J.K.; Rietveld, T.; Wattimena, D.L.; Frenkel, M. (1989) Minimum protein requirements in liver cirrhosis determined by nitrogen balance measurements at three levels of protein intake. Clinical Nutrition 8(6), 329336.

Szerb, J.C., Butterworth, R.F. (1992) Effect of ammonium ions on synaptic transmission in the mammalian central nervous system. Progressive Neurobiology 39(2), 135-153.

Terblanche, J.; Burroughs, A.K.; Hobbs, K.E.F. (1989) Controversies in the management of bleeding oesophageal varices. N Engl J Med 320(21), 1393-1398.

Therrien, G.; Giguère, J-F.; Butterworth, R.F. (1991) Increased cerebrospinal fluid lactate reflects deterioration of neurological status in experimental portal-systemic encephalopathy. Metabolic Brain Disorders, 6(4), 225-231.

Tricklebank, M.D.; Smart, J.L.; Bloxam, D.L., et al. (1978) Effects of chronic experimental liver dysfunction and L-tryptophan on behavior in the rat. Pharmacology Biochemisry and Behavior 9(2), 181-189.

Weissenborn, K.; Ehrenheim, C.H.; Hori, A.; Kubicka, S.; Manns, M.P. (1995) Pallidal lesions in patients with liver cirrhosis: clinical and MRI evaluation. Metabolic Brain Disorders 10(3), 219-231.

Wolpert, E.; Phillips, S.F.; Summerskill, W.H.J. (1970) Ammonia production in the human colon. Effects of cleansing, neomycin and acetohydroxamic acid. $N$ Engl J Med 283(4), 159-164.

Yao, H.; Sadoshima, S.; Fujii, K.; Kusuda, K.; Ishitsuka, T.; Tamaki, K.; Fujishima, M. (1987) Cerebrospinal fluid lactate in patients with hepatic encephalopathy. European Neurology 27(3), 182-187.

Young, S.N.; Lai, S. (1980) CNS tryptamine metabolism in hepatic coma. Journal of Neural Transmission 47(3), 153-161.

Zervox, E.E.; Goode, S.E.; \& Rosemurgy, A.S. (1998) Immediate and long-term portal hemodynamic consequences of small-diameter H-graft portacaval shunt. Journal of Surgical Research 74(1), 71-75.

Zieve, L.; Doizaki, W.M.; Zieve, J. (1974) Synergism between mercaptans and ammonia or fatty acids in the production of coma: a possible role for mercaptans in the pathogenesis of hepatic coma. Journal of Laboratory and Clinical Medicine 83(1), 16-28.

Zieve, L. (1989) Role of toxins and synergism in hepatic encephalopathy. In: Hepatic Encephalopathy: Pathophysiology and Treatment. Butterworth, R.F., \& Pomier Layrargues, G., eds, pp. 141-156. Humana Press; Clifton, NJ. 


\title{
Future Perspectives for the Treatment of Neonatal Hypoxic-Ischemic Encephalopathy
}

\author{
Pedro M. Pimentel-Coelho, Marcelo F. Santiago \\ and Rosalia Mendez-Otero \\ Instituto de Biofísica Carlos Chagas Filho, Instituto de Ciências Biomédicas \\ Universidade Federal do Rio de Janeiro \\ Brazil
}

\section{Introduction}

As described by Nelson and Leviton, "neonatal encephalopathy (NE) is a clinical defined syndrome of disturbed neurologic function in the earliest days of life in the term infant, manifested by difficulty with initiating and maintaining respiration, depression of tone and reflexes, subnormal level of consciousness, and often by seizures" (Nelson and Leviton, 1991). Although NE can be caused by several etiologies (including metabolic and genetic disorders, and infections), perinatal asphyxia is the most common cause, occurring in 30 to $60 \%$ of the cases (Kurinczuk et al., 2010).

The diagnosis of perinatal asphyxia depends on the presence of multiple markers that were compiled in three consensus statements (table 1). One of the most important criteria for the diagnosis of perinatal asphyxia is the presence of metabolic acidosis in umbilical artery blood ( $\mathrm{pH}<7,0$ and base deficit $>12 \mathrm{mmol} / \mathrm{L}$ ). In a recent meta-analysis, it was shown that $23 \%$ of the neonates with this degree o acidosis had neonatal neurologic morbidity or mortality (Graham et al., 2008). Other criteria, such as abrupt changes in fetal heart rate, a low Apgar score, imaging evidences or the presence of a sentinel event, are also important to determine the timing of asphyxia. In this regard, Cowan et al. showed that acutely evolving lesions in MRI scans were observed in $80 \%$ of the infants with NE and evidence of perinatal asphyxia, indicating that most of the lesions were acquired in the perinatal period (Cowan et al., 2008).

When perinatal asphyxia is the cause of the NE, the syndrome is called neonatal hypoxicischemic encephalopathy (HIE), occurring in 1.5 per 1000 live births (Kurinczuk et al., 2010). HIE can be classified into mild, moderate or severe encephalopathy, according to the classification of Sarnat and Sarnat. The percentage of adverse outcomes, including motor/cognitive impairment or death, is $0 \%$ for mild, $32 \%$ for moderate and almost $100 \%$ for severe HIE, in infants under 3 years of age (Pin et al., 2009). Long-term evaluations have also shown more subtle cognitive deficits and alterations in daily life behavioural functioning, even in cases of mild HIE (de Vries \& Jongmans, 2010). Moreover, HIE is the cause of cerebral palsy in at least $14 \%$ of the cases (Graham et al., 2008).

There are two patterns of injury that can be observed with MRI in HIE: 
1. The basal ganglia-thalamus pattern (BGT) affects bilaterally the deep gray nuclei and perirolandic cortex, occurring more often after an acute sentinel event, such as placental abruption, uterine rupture or umbilical cord prolapse. Hippocampus, brain stem and white matter may also be affected (de Vries \& Groenendaal, 2010). BGT is associated with cerebral palsy in $70 \%$ of the survivors, and with epilepsy in $30-40 \%$ of HIE survivors. Visual impairments and dysarthria are also common in children with HIE and BGT injury (Martinez-Biarge et al., 2010).

2. The watershed predominant pattern (WS) is the second pattern of injury and involves the white matter, particularly the vascular watershed zones (anterior-middle cerebral artery and posterior-middle cerebral artery), and also the cortex when severe (de Vries \& Groenendaal, 2010). WS is associated with cognitive deficits and epilepsy, but usually is not the cause of severe motor impairment (Martinez-Biarge et al., 2010).

Besides the imaging studies performed in human infants, most of the observations related to the mechanisms of brain damage and brain plasticity after HIE came from preclinical studies using the Rice-Vannucci animal model of HIE. The model consists of unilateral common carotid artery ligation followed by systemic hypoxia (8\% oxygen-balance nitrogen) in post-natal day 7 (P7) rats (Vannucci et al., 1999). The damage is restricted to the hemisphere ipsilateral to the common carotid artery occlusion, affecting the cerebral cortex, thalamus, striatum, hippocampus and subcortical white matter. Importantly, the HI animals develop several cognitive and motor deficits (Lubics et al., 2005).

In this book chapter, we will discuss possible new treatments for HIE, focusing on neuroprotective strategies and on cell therapies.

\section{Neuroprotective strategies for HIE}

Since, in most cases, the hypoxic-ischemic (HI) insult occurs near birth, it is feasible that neuroprotection could be achieved in the first few hours after birth. Accordingly, therapeutic hypothermia, when started within 6 hours of birth, modestly improves the neurologic outcome of full-term infants with moderate HIE and is becoming a standard therapy for this condition (Edwards et al., 2010). Besides the neurological improvement, therapeutic hypothermia was associated with a decreased injury in basal ganglia/thalamus and white matter in MRI scans (Rutherford et al., 2010), confirming the neuroprotective effect of this treatment, as observed in animal models of HIE (Gunn et al., 1997).

However, given the limited benefits of therapeutic hypothermia, new neuroprotective treatments that could reduce or prevent the long-term neurodevelopmental sequelae of children with HIE, affecting one (or a combination) of the mechanisms that contribute to secondary brain injury, are urgently needed.

The therapeutic window of hypothermia coincides with a latent phase, when cerebral energy metabolism returns to normal following perinatal asphyxia. Using phosphorus magnetic resonance spectroscopy (31P-RMS), it was showed that brain energy metabolism returns to normal levels after a successful resuscitation. After 6-24 hours, this latent phase is followed by a secondary energy failure (Lorek et al., 1994), when there is a correlation between the degree of derangement of oxidative metabolism and the neurodevelopmental outcome (Martin et al., 1996). Thereby, it has been suggested that irreversible cell death occurs with a certain delay after HIE. 


\begin{tabular}{|c|c|c|}
\hline $\begin{array}{l}\text { American Academy of } \\
\text { Paediatrics/American } \\
\text { College of Obstetrics and } \\
\text { Gynaecology (1992) }\end{array}$ & $\begin{array}{l}\text { International Cerebral } \\
\text { Palsy Task Force (1999) }\end{array}$ & $\begin{array}{l}\text { American College of } \\
\text { Obstetrics and } \\
\text { Gynaecology (2002) }\end{array}$ \\
\hline Essential criteria & Essential criteria & Essential criteria \\
\hline $\begin{array}{l}\text { Profound metabolic acidosis } \\
(\mathrm{pH}<7.0) \text { in an umbilical } \\
\text { artery sample }\end{array}$ & $\begin{array}{c}\text { Metabolic acidosis in early } \\
\text { neonatal blood sample } \\
\text { (pH<7.0 and base deficit }> \\
12 \mathrm{mmol} / \mathrm{L})\end{array}$ & $\begin{array}{c}\text { Metabolic acidosis } \\
(\mathrm{pH}<7.0 \text { and base deficit } \\
>12 \mathrm{mmol} / \mathrm{L}) \text { in umbilical } \\
\text { artery sample }\end{array}$ \\
\hline $\begin{array}{c}\text { Apgar score } \leq 3 \text { beyond } 5 \\
\text { minutes }\end{array}$ & $\begin{array}{c}\text { Moderate or severe } \\
\text { encephalopathy }\end{array}$ & $\begin{array}{c}\text { Moderate or severe } \\
\text { neonatal encephalopathy }\end{array}$ \\
\hline Neonatal encephalopathy & $\begin{array}{l}\text { Cerebral palsy: spastic } \\
\text { quadriplegia, dyskinetic, } \\
\text { or mixed }\end{array}$ & $\begin{array}{c}\text { Cerebral palsy of spastic } \\
\text { quadriplegia or dyskinetic } \\
\text { type }\end{array}$ \\
\hline \multirow[t]{7}{*}{$\begin{array}{c}\text { Multi-organ system } \\
\text { dysfunction }\end{array}$} & & $\begin{array}{c}\text { Exclusion of other } \\
\text { etiologies }\end{array}$ \\
\hline & $\begin{array}{l}\text { Criteria suggestive of } \\
\text { intrapartum timing }\end{array}$ & $\begin{array}{l}\text { Criteria suggestive of } \\
\text { intrapartum timing }\end{array}$ \\
\hline & $\begin{array}{c}\text { Sentinel event associated } \\
\text { with labor }\end{array}$ & $\begin{array}{c}\text { Sentinel event associated } \\
\text { with labor }\end{array}$ \\
\hline & $\begin{array}{l}\text { Severe fetal heart rate } \\
\text { changes }\end{array}$ & $\begin{array}{c}\text { Abrupt fetal heart rate } \\
\text { changes: bradycardia, loss } \\
\text { of variability, decelerations }\end{array}$ \\
\hline & $\begin{array}{c}\text { Apgar score }<6 \text { beyond } 5 \\
\text { min }\end{array}$ & $\begin{array}{c}\text { Apgar score } \leq 3 \text { beyond } 5 \\
\text { min }\end{array}$ \\
\hline & Multi-system involvement & Multi-system failure \\
\hline & Early imaging evidence & Early imaging evidence \\
\hline
\end{tabular}

Table 1. Consensus statements for the diagnosis of intrapartum asphyxia (Shevell, 2004; Kumar \& Paterson-Brown, 2010)

In the latent phase, although high-energy phosphate stores return to normal, many mechanisms contributing to secondary brain injury are going on, including inflammation, production of nitric oxide/reactive oxygen species, glutamate excitotoxicity and trophic factors withdrawal. All these mechanisms will result on mitochondrial permeabilization and cell death through activation of both caspase-dependent and -independent pathways (Hagberg et al., 2009).

The involvement of caspases in neonatal HI injury was demonstrated in several studies. Caspase- 3 cleavage and activation after $\mathrm{HI}$ is more pronounced in the immature than in the juvenile and adult brains (Zhu et al., 2005). However, different forms of cell death coexist in the HI immature brain. Besides the presence of classically apoptotic and classically necrotic neurons, an intermediate "continuum" form of neuronal cell death can be observed after the neonatal HI, combining biochemical characteristics of apoptosis and necrosis (Northington et al., 2007).

In this scenario, specific caspase-3 inhibition provides no or only partial neuroprotection after HI. It was shown that blockade of caspase-3 activation and cleavage of its substrates 
does not prevent necrosis-related calpain activation in the cerebral cortex (Han et al., 2003). Moreover, caspase-3 deficient mice are more vulnerable to neonatal HI brain damage, through upregulation of caspase-independent pathways (West et al., 2006).

In the same way, intracerebroventricular administration of necrostatin-1, which inhibits receptor-interacting protein (RIP)-1 kinase and programmed necrosis, decreases injury in the forebrain and thalamus after neonatal HI, despite an increase in apoptotic cell death (Northington et al., 2011).

The use of drugs or a combination of drugs to inhibit multiple targets could be a good strategy to overcome this problem. In this regard, the administration of MDL 28170, a calpain inhibitor, resulted in neuroprotection, decreasing both necrosis and apoptosis (Kawamura et al., 2005).

One of the most important signalling pathways for caspase-3 activation in the newborn brain is the intrinsic mitochondria-mediated pathway, which occurs through mitochondrial outer membrane permeabilization and translocation of cytochrome $\mathrm{C}$ to the cytosol, leading to the assembly of the apoptosome. While in the adult brain, cyclophilin D is crucial for mitochondrial outer membrane permeabilization, Bax-dependent mitochondrial permeabilization predominates in the immature brain. Indeed, cyclophilin D acts as an antiapoptotic protein after neonatal HI, in contrast with its role as a cell death mediator in the adult brain (Wang et al., 2009). Knockout of Bax (Gibson et al., 2001) or pretreatment with a cell-penetrating Bax-inhibitory peptide (Wang et al., 2009; Wang et al., 2010) protects the immature brain fom HIE, resulting in neuroprotection and functional improvement in sensorimotor and memory tests.

Other proteins of the bcl-2 protooncogene family are also involved in neonatal HI, regulating Bax-dependent mitochondrial permeabilization and cell death, such as the antiapoptotic protein Bcl- $x_{\mathrm{L}}$ (Parsadanian et al., 1998) and the pro-apoptotic proteins Bad and Bim (Ness et al., 2006). In this regard,the tumor suppressor p53 acts as a transcription factor for proteins involved in cell cycle checkpoints and growth control and for proapoptotic proteins like PUMA and Bax. Treatment with pifithrin- $\mu$ (PFT- $\mu)$, a molecule that inhibits the association of p53 with mitochondria, prevented the upregulation of p53 proapoptotic target genes and mitochondrial permeabilization, inhibiting caspase- 3 activation. These effects resulted in strong neuroprotection and long-term improvements in sensorimotor and cognitive functions after neonatal HI (Nijboer et al., 2011).

Recent studies showed that autophagy, a process in which the degradation of cellular components by the lysosomal system occurs in a tightly controlled manner, is activated in the HI brain (Zhu et al., 2006; Ginet et al., 2009). Pharmacological inhibition of autophagy before HIE increases brain injury, switching the mechanism of cell death from apoptosis to necrosis (Carloni et al., 2008). Conversely, inhibition of autophagy 4 hours after the injury has a strong neuroprotective effect (Puyal et al., 2009), suggesting a time-dependent role of autophagy after HIE.

Regional differences in the mechanisms of cell death can also be observed after neonatal HI. Neurodegeneration in the somatosensory thalamus occurs predominantly through apoptotic mechanisms mediated by Fas death receptor activation and is delayed compared with cortical and striatal neuronal death (Northington et al., 2001). Moreover, decreased brain 
injury can be observed in the cerebral cortex, striatum and thalamus, but not in the hippocampus, of neonatal HI mice lacking functional Fas death receptors, which are involved in the activation of caspase-8 (Graham et al., 2004). Regional differences in the involvement of autophagic cell death in HI brain injury were also observed. While hippocampal CA1 neurons exhibited strong apoptotic characteristics, CA3 neurons had an authophagic cell death phenotype and cortical neurons presented a mixed phenotype, combining characteristics of both apoptosis and autophagy (Ginet et al., 2009).

The temporal pattern of neuronal death is also different for each brain region and apoptotic cells can still be observed in the cerebral cortex and in the striatum over several days after the HI injury (Nakajima et al., 2000). The existence of delayed and secondary neuronal death indicates that a subpopulation of neurons might be protected even if the adequate neuroprotective treatment is initiated a few days after the HI insult. These differences also suggest that therapies aiming to block a very specific target in the cell death pathway may not be equally effective for all brain regions.

Recently, infant male sex was considered a risk factor for HIE (Wu et al., 2010) and was associated with an increased development of cerebral palsy in very preterm infants (Beaino et al., 2010). Although the biological basis of this increased risk of brain injury in male babies is not completely understood, several preclinical studies have demonstrated sex-dependent differences in the mechanisms of cell death after HIE. The nuclear enzyme poly(ADP-ribose) polymerase-1 (PARP), involved in DNA repair, is activated by HIE in both sexes, but contributes to neuronal injury, through depletion in $\mathrm{NAD}^{+}$stores, only in male pups (Hagberg et al., 2004). It was also shown that while an increased translocation of apoptosisinducing factor (AIF) occurred in the immature male brain, a stronger activation of caspase3 was observed in the female brain after HIE (Zhu et al., 2006). Similarly, under conditions of nitrosative stress in vitro, male neurons die via an AIF-mediated pathway, while a more prominent cytochrome $\mathrm{c}$ release from the mitochondria occurs in female neurons (Du et al., 2004), suggesting that intrinsic gender differences in the mechanisms of cell death may occur independently of circulating sex hormones. However, it is also possible that elevated circulating levels of dihydrotestosterone in males during the late embryonic period, persisting through the first year of life (Knickmeyer \& Baron-Cohen, 2006) could be partially responsible for these differences. Indeed, pre-treatment of female pups with testosteronepropionate increased the behavioral deficits on an acoustic processing task after HIE (Hill et al., 2011). Moreover, androgens increase the excitotoxic cell death induced by $\mathrm{GABA}_{\mathrm{A}}$ activation in the developing hippocampus (Nuñez \& McCarthy, 2008).

Taken together, these studies suggest that different neuroprotective strategies might be necessary for the treatment of boys and girls with HIE. In this regard, it was shown that the non-competitive N-methyl-D-aspartate receptor antagonist dextromethorphan protects only male pups from brain ischemia (Comi et al., 2006) and that the neuroprotective drug 2iminobiotin protects only female pups from HIE (Nijboer et al., 2007).

Neuroprotection can be obtained by targeting the vascular system. It was shown that capsaicin pre-treatment reduces brain injury after HIE. Interestingly, the treatment prevented the HI-induced loss of myogenic tone in segments of the middle cerebral artery (Khatibi et al., 2011a). 
Furthermore, modulation of the vascular effects of endothelin vasomotor peptides could also provide neuroprotection after cerebral ischemia. S-0139, an antagonist of the endothelin type A receptor reduces plasma extravasation and brain injury after stroke in adult animals. However, this effect was only observed if the drug was administered no later than 1 hour after the injury (Matsuo et al., 2001) and administration of the endothelin type A receptor antagonist ABT627 immediately after HI had no effect on brain injury after HIE (Khatibi et al., 2011b).

\section{Boosting the endogenous regenerative capacity of the neonatal brain}

\subsection{HIE effects on neurogenesis}

During the early neonatal period several alterations are taking place in the neurogenic niches of the mammalian forebrain. During the peak of embryonic cortical neurogenesis, most of the cycling neuronal precursor cells have morphological and molecular characteristics of radial glial cells (RGC; Noctor et al., 2002), expressing nestin and vimentin and presenting a radial morphology, with the cell body in the ventricular zone (VZ), a long process contacting the pial surface and an endfoot contacting the ventricular surface. RGC serve as a neural precursor, dividing in a self-renewing manner and giving rise to neurons (Anthony et al., 2004) and to intermediate progenitor cells that populate the embryonic subventricular zone (SVZ), a second site of cortical neurogenesis (Miyata et al., 2004, Noctor et al., 2004). At the end of neurogenesis, RGC generate astrocytes and ependymal cells (Spassky et al., 2005) and give rise to neural stem cells that persist in the adult SVZ (Merkle et al., 2004).

In humans, the transformation of RGC into astrocytes occurs during the second half of gestation and RGC can still be found in some regions of the fetal prosencephalon as late as in the $8^{\text {th }}$ gestational month (Ulfig et al., 1999). It was shown that HIE disrupts the radiallyoriented pattern of RGC processes in the preterm-like rat brain, correlating in time with the appearance of reactive astrocytes (Sizonenko et al., 2007). However, it remains to be investigated if HIE accelerates the differentiation of RGC into astrocytes.

During the perinatal period and throughout postnatal life, neurogenesis occurs in two neurogenic niches: in the SVZ (in the walls of the lateral ventricles) and in the subgranular layer of the hippocampal dentate gyrus. New neurons are continuously generated in the SVZ, migrating long distances through the rostral migratory stream (RMS) to the olfactory bulb, where they replace local interneurons. In the hippocampus, neuroblasts migrate from the subgranular layer to the inner granule cell layer, differentiating into dentate granule cells (Ming \& Song, 2011).

Recently, a population of interneuron precursor cells was also described in the dorsal white matter of newborn mice. These cells migrated to the overlying anterior cingulate cortex and expressed the calcium-binding protein calretinin (Riccio et al., 2011), although it is still unknown if a similar population of cortical interneurons is generated during the perinatal period in humans.

Neurogenesis is tightly controlled by the local environment. Neural stem/progenitor cells (NSPC) of the SVZ, called B1 cells, are localized in close contact with several cell types, including amplifying progenitors, neuroblasts and ependymal cells. B1 cells are also in 
contact with the cerebrospinal fluid and with blood vessels, being exposed to diffusible factors, basement membrane and extracellular matrix components, neurotransmitters and growth factors (Ihrie \& Alvarez-Buylla, 2011).

Astrocytes and microglial cells could also regulate NSPC function, especially after a HI brain injury, when these cells play an important role in the orchestration of inflammation. Indeed, all the components of the neurogenic niches are affected by $\mathrm{HI}$, including changes in the blood-brain barrier, cerebrospinal fluid and oxygen tension. As a result, an increased proliferation and migration of progenitors from the SVZ to the cerebral cortex and to the striatum are observed after HIE, for at least 5 months after the injury. However, $85 \%$ of the newly formed neurons die before maturation (Yang et al., 2007). Moreover, most of the neurons that migrate to the striatum differentiate into calretinin-positive interneurons, which represent less than $5 \%$ of the neuronal population of the striatum. GABAergic medium-sized spiny projection neurons, the most common neuronal phenotype in the striatum, were not replaced after the injured (Yang et al., 2008).

Recently, Yang and colleagues showed that Emx1-expressing NSPC of the dorsolateral SVZ give rise to calretin-positive neurons both in the intact and in the HI striatum (Wei et al., 2011). Therefore, despite the increase in neurogenesis after HIE, this effect is not accompanied by a change in the fate of the newly formed neurons, suggesting that this regenerative process is not effective enough to replace all neuronal subtypes lost after the injury. New therapies that could increase the survival of these new neurons, promoting their differentiation into the appropriate phenotypes are a promising strategy to replace lost cells in the HI brain.

Epidermal growth factor (EGF), signalling through its receptor EGFR, is one important regulator of NSPC response after HIE (Alagappan et al., 2009). Indeed, a 2-week intraventricular infusion of brain-derived neurotrophic factor (BDNF) and EGF, 5 weeks after the injury, promoted functional recovery in HI mice. This improvement in motor function occurred in conjunction with increased proliferation of NSPC in the SVZ and with an increase in the survival of newly generated striatal neurons (Im et al., 2010). Noteworthy, BDNF also has a strong neuroprotective action in the acute phase of HI brain injury (Han \& Holtzman, 2000).

Erythropoietin also represents a good example of a drug that improves neurological outcome in animal models of HIE through a combination of several mechanisms. Erythropoietin treatment reduces apoptotic cell death (Sun et al., 2004) and brain edema (Brissaud et al., 2010) and has an anti-inflammatory effect in the HI brain (Juul et al., 2009). It affects regenerative processes, promoting an increase in functional revascularization, in NSPC proliferation in the SVZ and in the number of new neurons that migrate to the striatum and to the cerebral cortex (Iwai et al., 2007). However, one study showed that erythropoietin reduced brain damage and prevented neurological deficits only in females (Fan et al., 2011), suggesting that more attention should be given to the gender-dependent effects of this treatment.

Erythropoietin has been used for the treatment of anemia in premature infants and erythropoietin treatment in newborns with HIE was shown to be safe and feasible in two recent clinical trials. The preliminary efficacy of the treatment was also reported, suggesting an improved neurodevelopmental outcome in term children with mild/moderate HIE (Zhu et al., 2009; Elmahdy et al. 2010). 
HIE also increases neurogenesis in the subgranular layer of the hippocampal dentate gyrus (Bartley et al., 2005) and this effect can be potentiated by lithium, a promising drug that combines neuroprotection with an effect on the NSPC hippocampal pool after HIE. Lithium treatment increases both proliferation and survival of progenitors in the hippocampal dentate gyrus, an effect that persists for at least 7 weeks after $\mathrm{HI}$ (Li et al., 2011). However, despite the use of lithium for the treatment of bipolar disorder in children, the possible side effects of this drug in the developing newborn brain are still unknown.

\subsection{HIE effects on gliogenesis}

HIE has an effect on glial progenitors of the SVZ, increasing the generation of astrocytes, at the expense of a reduced generation of oligodendrocytes. This effect is mediated by EGF, leukemia inhibitory factor (LIF) and transforming growth factor beta (TGF- $\beta$ ), and contributes to the formation of the glial scar (Bain et al., 2010).

In this regard, it was shown that HIE induces an increase in the number of newly born oligodendrocytes in the striatum and in the corpus callosum (Ong et al., 2005; Zaidi et al., 2004). These newly formed oligodendrocytes are generated by local oligodendrocyte progenitor cells (OPC) and the SVZ contributes minimally to the increase of OPC in the striatum (Dizon et al., 2009). Moreover, proliferation and accumulation of OPC after HIE are followed by an arrest of differentiation and maturation, resulting in a failure to initiate myelination (Segovia et al., 2008).

The importance of white matter injury in HIE was demonstrated by the observation that an abnormal magnetic resonance signal intensity in the posterior limb of the internal capsule (PLIC) is able to predict a poor neurodevelopmental outcome and the inability to walk at 2 years in term children with HIE (Edwards et al., 1998; Martinez-Biarge et al., 2011).

New therapies to avoid myelination delay in children with HIE should increase the proliferation of OPC in the SVZ, striatum and white matter, inducing the migration of these progenitors and the formation of mature oligodendrocytes.

For instance, modulators of the endocannabinoid system could be used to modulate the response of NSPC and OPC after HI. WIN55212-2, a synthetic cannabinoid receptor agonist, increases both neurogenesis and the generation of mature oligodendrocytes after HIE in rats (Férnandez-López et al., 2010). The administration of WIN55212 also has a strong neuroprotective effect in the HI brain, through activation of the cannabinoid receptors CB1 and CB2 (Férnandez-López et al., 2007). However, the clinical use of this compound might be limited by the production of psychoactive effects. In this regard, although the nonpsychoactive cannabinoid cannabidiol could be used to promote neuroprotection after HIE (Alvarez et al., 2008), it is still unknown if this compound also modulates the regenerative response of the brain.

Furthermore, besides the effect on neurogenesis, erythropoietin is also able to estimulate the generation and the maturation of new oligodendrocytes, decreasing white matter injury after HIE (Iwai et al., 2010).

As an alternative, transplantation of NSPC and/or glial progenitors could be a strategy to replace lost cells and induce myelination. 


\section{Neural stem/progenitor cell transplantation in HIE}

NSPC can be obtained from several sources, including the fetal and the adult brain, or from embryonic stem cells (ES) and induced pluripotent stem cells (iPS).

When transplanted into the HI brain, NSPC migrate long distances in direction to areas of neural damage. Even when injected in the contralateral hemisphere, NSCP are able to migrate through the interhemispheric comissures toward the damaged hemisphere (Imitola et al., 2004; Park et al., 2006a). The chemokine SDF-1, which is upregulated in the HI brain (Miller et al., 2005), regulates the migration of NSPC and is one of the main molecules involved in the recruitment of transplanted NSPC after HIE (Imitola et al., 2004).

In a recent study, when transplanted in the brain of sham-operated control rats, NSPC remained in the original site of injection. However, when injected in $\mathrm{HI}$ rats 3 days after the injury, the cells migrated around 100-125 $\mu \mathrm{m}$ /day, reaching the border of the injury in the right hemisphere within 10-12 days. NSPC proliferated in the HI brain over the first 4 weeks after transplantation and, despite a volume decrease over time, many cells survived for up to 58 weeks in the injured hemisphere (Obenaus et al., 2011).

Timing of transplantation is important for the survival of transplanted NSPC in the HI brain. While a robust engraftment can be obtained if the cells are injected in the acute/subacute phase of the injury, no engraftment is observed when the cells are injected 6 weeks after the injury (Park et al., 2006a).

The therapeutic potential of NSPC is based on at least two main mechanisms of action:

1. It is expected that NSPC should migrate to the damaged areas, differentiating into the adequate neuronal subtypes, which should extend axons and form new connections, restoring function. In this regard, it was observed that the HI brain induces the differentiation of NSPC into neurons. When transplanted in HI animals, 5\% of the donor cells differentiate into neurons, while neuronal differentiation is not observed if NSPC are injected in the intact brain (Park et al., 2006a). However, the long-term integration of these new neurons and the capacity to re-establishing neural circuitries remain to be demonstrated.

2. NSPC secrete many molecules, including neurotrophic factors and cytokines, as well as modulate the production of these factors by host cells. Therefore, NSPC transplantation could result in neuroprotection and immunomodulation and could estimulate endogenous mechanisms of brain plasticity and regeneration, through a paracrine mechanism.

Recent observations suggest that intracerebral transplantation of NSPC benefits the recovery from HIE through both mechanisms. Besides the differentiation of transplanted human NSPC into neurons and astrocytes in the HI brain, the treatment increases axonal sprouting and induces an upregulation of host endogenous genes involved in neurogenesis and neurotrophic support. As a result, an improved motor function is observed in the treated animals (Daadi et al., 2010). It was also reported that NSPC can decrease $\mathrm{HI}$ brain injury, when injected in combination with chondroitinase $A B C$, an enzyme that degrades glycosaminoglycans side chains of chondroitin sulphate proteoglicans (Sato et al., 2008). 
Genetically manipulation of NSPC could also be used to modulate the survival, the migration and/or the differentiation capacity of the injected cells. For instance, NT-3 (neurotrophin-3) overexpression in NSPC dramatically increases the neuronal differentiation of these cells when they are transplanted in the HI brain (Park et al., 2006b). FGF-2 overexpression can also enhance proliferation and migration of transplanted NSPC, increasing the number of donor NSPC-derived immature neurons in the HI brain (Dayer et al., 2007).

Within the next years, new effort should be made to define the best cell source of NSPC for transplantation in HIE. Although ES can be used to generate NSPC with high efficiency, the risk of formation of teratomas exists if undifferentiated ES persist in the transplant pool (Ben-David \& Benvenisty, 2011).

Moreover, allogeneic grafts might trigger an immune response leading to graft rejection in the adult central nervous system. The use of systemic immune suppression to avoid this response is associated with an increased risk of opportunistic infections and with an increased susceptibility to malignancies.

Alternatively, iPS-derived NSPC could be obtained after reprogramming of somatic cells from the own patient, allowing an autologous transplantation. However, it is possible that even iPSC-derived cells could elicit an immune response in syngeneic recipients (Zhao et al., 2011). Furthermore, although ES and iPSC use the same transcriptional networks for neural differentiation, iPSC are less efficient and show an increased variability (Hu et al., 2010).

Other questions regarding the safety of NSPC transplantation, such as the genetic stability of ES and iPSC in culture and the need of manufacturing consistence for the production of these cells, should be addressed in preclinical studies.

It will also be important to address questions such as the best timing, the delivery route and the number of cells needed for a greater efficiency of the therapy.

The safety and preliminary efficacy of intracerebral human NSPC transplantation was tested in children with infantile/late-infantile forms of neuronal ceroid lipofuscinosis (NCL, http://clinicaltrials.gov, identifier NCT00337636) and is currently being tested in children aged 6 months to 5 years with connatal Pelizaeus-Merzbacher disease (PMD, http://clinicaltrials.gov, identifier NCT01005004). The children will receive immunossupression for 9 months. These two studies will give important information concerning the use of a NSPC-based therapy in children and will be of a great value for the development of a possible therapy for other types of brain injury, including HIE.

Alternatively, transplantation of MSC and umbilical cord blood mononuclear cells could also be used to estimulate endogenous regenerative mechanisms, neuroprotection and immunomodulation.

\section{Mesenchymal stem/progenitor cell transplantation in HIE}

Mesenchymal stem/progenitor cells (MSC) is a cell population that can differentiate into specialized mesenchymal cells, such as osteoblasts, chondrocytes and adipocytes. MSC can be isolated from most of the tissues in the body, where they reside in perivascular niches. The International Society for Cellular Therapy proposed a set of minimal criteria to define 
MSC. Accordingly, MSC should be plastic-adherent and must express 3 cell surface molecules (CD105, CD73 and CD90), but lack expression of CD45, CD34, CD14 or CD11b, CD79alpha or CD19 and HLA-DR (Dominici et al., 2006).

MSC secrete many bioactive molecules, including growth factors and cytokines when maintained in standard culture conditions (Meirelles et al., 2009). In this regard, the conditioned medium of adipose tissue-derived MSC can reduce brain damage after HIE in rats, promoting a long-term improvement in cognition. This effect was partially abolished by neutralization of IGF-1 or BDNF, suggesting that a combination of factors may be responsible for the observed effects (Wei et al., 2009).

It was demonstrated that TNF-a increases the secretion of VEGF (vascular endothelial growth factor), HGF (hepatocyte growth factor) and IGF-1 (insulin-like growth factor-1) by MSC (Wang et al., 2006), suggesting that the paracrine effects of MSC could be modulated by inflammation. Therefore, intracerebral transplantation of MSC could be used to deliver these trophic factors in response to the local HI environment.

Indeed, when intracerebrally transplanted 3 or 10 days after the injury, bone marrowderived MSC decreased neuronal death and demyelination. When injected 3 days after HIE, MSC enhanced the regenerative capacity of the brain, as evidenced by an increase in the number of newly-formed neurons and oligodendrocytes in the hippocampus and cortex. Moreover, the treatment reduced the number of proliferating microglia (van Velthoven et al., 2010a), in accordance with the immunomodulatory properties of MSC (Uccelli et al., 2008).

Further evidences supporting the adaptation of MSC function to the environment came from a study in which it was shown that a combination of two administrations of MSC, at 3 and 10 days after HIE, was more effective in improving the performance of the animals in a sensorimotor test. Moreover, only the combined treatment induced an extensive remodelling of the corticospinal tract (van Velthoven et al., 2010c).

MSC migrate from the peripheral blood to sites of brain injury, in response to chemokines and blood vessel activation (Wang et al., 2008), indicating that MSC could be administered systemically. However, the intravenous injection of MSC is associated with the entrapment and clearance of these cells in the lungs, after the pulmonary passage (Fischer et al., 2009), suggesting that an intra-arterial injection of MSC may be necessary to permit an effective engraftment of these cells in the brain. In this regard, an intracardic injection of human bone marrow-derived MSC 3 days after HIE, reduced the sensorimotor deficits of the treated animals, although the lesion size was not reduced (Lee et al., 2010).

Recently, it was also shown that intranasally delivered MSC are able to migrate to the brain after crossing the cribiform plate. The transplanted cells could be detected in the damaged hippocampus for at least 28 days after HIE and the treatment resulted in a decreased brain injury and in a better functional outcome (van Velthoven et al., 2010b).

Ideally, autologous MSC transplantation should be performed. For this purpose, MSC could be obtained from the bone marrow, the adipose tissue, the Warthon's jelly (the connective tissue of the umbilical cord), or even from other vascularised tissues, and expanded in vitro. However, the time required to acquire the adequate number of cells for transplantation might make unfeasible the use of these cells in the acute phase of the injury. On the other 
hand, although allogeneic MSC transplantation appears to be safe and well tolerated (Koç et al., 2002), recent observations have suggested that MSC are susceptible to lysis by CD8positive T-lymphocytes and NK cells (Crop et al., 2011).

These observations suggest that MSC transplantation could be used as a promising new strategy to reduce neuronal death, promote brain plasticity and regeneration and modulate inflammation after HIE. However, it is still necessary to define the best source of MSC, the therapeutic window, the delivery route and the cell dose, before this therapy can be tested in clinical trials.

\section{Human umbilical cord blood cell (HUCBC) transplantation in HIE}

The human umbilical cord blood is a rich source of hematopoietic stem/progenitors and has been used as an alternative to bone marrow transplantation in children and adults. The mononuclear cell fraction of the umbilical cord blood also contains other cell types, including endothelial progenitors, monocytes, lymphocytes (including regulatory T-cells) and even a small percentage of MSC.

In the last years, it was demonstrated that HUCB systemic administration improves neurological function in several models of brain injury (Sanberg et al., 2011).

Intraperitoneal administration of HUCB 24 hours after HIE decreases motor (Meier et al., 2006) and sensorimotor impairments in rats. Part of this effect was attributed to a reduction of the impairments of neural processing in the primary somatosensory cortex (Geibler et al., 2011).

The homing of HUCB to the HI brain after intraperitoneal transplantation was demonstrated by Meier and colleagues. They showed that the engraftment of HUCB in the brain depends on the chemokine stromal-derived factor-1 (SDF-1) (Rosenkranz et al., 2010) and that there was no evidence of differentiation of the donor cells into neural cells (Meier et al., 2006).

However, other studies failed to find a large number of cells in the HI brain after intraperitoneal (Pimentel-Coelho et al., 2010) or intravenous transplantation (dePaula et al., 2009; Yasuhara et al., 2010). While the treatment had no effects in the neurological outcome of HI rats in one of these studies (dePaula et al., 2009), an improved performance in 2 neonatal reflexes and in a motor test was reported in the other two studies (Pimentel-Coelho et al., 2010; Yasuhara et al., 2010, respectively). HUCBC promoted these benefits through a neuroprotective effect in the striatum and an anti-inflammatory effect in the cerebral cortex (Pimentel-Coelho et al., 2010). The treatment also enhanced synaptic plasticity in the hippocampus and promoted an increase in the levels of the neurotrophic factors NGF (nerve growth factor), GDNF (glial cell-derived neurotrophic factor) and BDNF in the brains of HI animals (Yasuhara et al., 2010).

These observations suggest that HUCBC could exert a therapeutic role in HIE, even when low numbers of donor cells are found in the brain. Indeed, it was reported that HUCB transplantation promotes an immunomodulatory effect in animal models of stroke, acting in the spleen (Vendrame et al., 2006). 
Although it is still not clear how HUCBC exert these effects after HIE, it is possible that the main mechanism of action of these cells occurs through a paracrine effect. HUCBC produce and secrete several trophic factors and cytokines when freshly isolated (Fan et al., 2005) or when maintained in culture (Newman et al., 2006).

HUCBC are available for autologous transplantation in the first few hours after birth. Furthermore, autologous intravenous HUCB transplantation is safe and feasible in young children with acquired neurological disorders (Sun et al., 2010) and is currently being evaluated in children with HIE in a clinical trial (http://clinicaltrials.gov; Identifier: NCT00593242). In this clinical trial, conducted at Duke University, the safety and feasibility of autologous infusions of HUCB is being evaluated in children with HIE, up to 14 days after birth.

\section{Conclusion}

In conclusion, the feasibility of achieving neuroprotection after HIE has been demonstrated by hypothermia. However, given the limitations of this therapy, new neuroprotective strategies should be pursued. Studies using the Rice-Vannucci animal model of HIE have indicated that neuronal death occurs through several pathways and that new therapies should target multiple mechanisms of cell death.

New therapies that could modulate the endogenous regenerative response of the brain, increasing the generation, the survival and the integration of new neurons and glial cells could also offer and additional benefit after HIE. In this regard, erythropoietin treatment might have a neuroprotective effect, while stimulating neurogenesis and oligodendrogenesis. The use of erythropoietin in neonates with HIE is safe and feasible, but more studies are still necessary to evaluate the efficacy of this treatment.

Finally, cell therapies could afford multiple benefits in the HI brain. NSPC, MSC and HUCB might decrease brain injury through a combination of mechanisms, including neuroprotection, immunomodulation and, in the case of NSPC, cell replacement.

\section{References}

Alagappan D., Lazzarino D.A., Felling R.J., Balan M., Kotenko S.V., Levison S.W. (2009). Brain injury expands the numbers of neural stem cells and progenitors in the SVZ by enhancing their responsiveness to EGF. ASN Neuro, Vol.1, No.2, pp. 1759-0914

Alvarez F.J., Lafuente H., Rey-Santano M.C., Mielgo V.E., Gastiasoro E., Rueda M., Pertwee R.G., Castillo A.I., Romero J., Martinez-Orgado J. (2008). Neuroprotective effects of the nonpsychoactive cannabinoid cannabidiol in hypoxic-ischemic newborn piglets. Pediatr Research, Vol.64, No.6, (December 2006), pp. 653-658, ISSN 1530-0447

Anthony T.E., Klein C., Fishell G., Heintz N. (2004). Radial glia serve as neuronal progenitors in all regions of the central nervous system. Neuron, Vol.41, No.6, (March 2004), pp. 881-890, ISSN 0896-6273 
Bain J.M., Ziegler A., Yang Z., Levison S.W., Sen E. (2010). TGFbeta1 stimulates the overproduction of white matter astrocytes from precursors of the "brain marrow" in a rodent model of neonatal encephalopathy. PLoS One, Vol.5, No.3, pp. e9567, ISSN1932-6203

Bartley J., Soltau T., Wimborne H., Kim S., Martin-Studdard A., Hess D., Hill W., Waller J., Carroll J. (2005). BrdU-positive cells in the neonatal mouse hippocampus following hypoxic-ischemic brain injury. BMC Neuroscience, Vol.6, pp. 15, 1471-2202, ISSN1471-2202

Beaino G., Khoshnood B., Kaminski M., Pierrat V., Marret S., Matis J., Ledesert B., Thiriez G., Fresson J., Roze J.C., Zupan-Simunek V., Arnaud C., Burguet A., Larroque B., Breart G., Ancel P.Y. (2010). Predictors of cerebral palsy in very preterm infants: the EPIPAGE prospective population-based cohort study. Developmental Medicine $\mathcal{E}$ Children Neurology, Vol.52, No.6, (June 2010), pp. e119-125, ISSN 1469-8749

Ben-David U., Benvenisty N. (2011). The tumorigenicity of human embryonic and induced pluripotent stem cells. Nature Reviews Cancer, Vol.11, No.4, (April 2011), pp. 268277, ISSN 1474-1768

Brissaud O., Villega F., Pieter Konsman J., Sanchez S., Raffard G., Franconi J.M., Chateil J.F., Bouzier-Sore A.K. (2010). Short-term effect of erythropoietin on brain lesions and aquaporin-4 expression in a hypoxic-ischemic neonatal rat model assessed by magnetic resonance diffusion weighted imaging and immunohistochemistry. Pediatric Research, Vol.68, No.2, (August 2010), pp. 123-127, ISSN 1530-0447

Carloni S., Buonocore G., Balduini W. (2008). Protective role of autophagy in neonatal hypoxia-ischemia induced brain injury. Neurobiology of Disease, Vol.32, No.3, (December 2008), pp. 329-339, ISSN 1095-953X

Comi A.M., Highet B.H., Mehta P., Hana Chong T., Johnston M.V., Wilson M.A. (2006). Dextromethorphan protects male but not female mice with brain ischemia. Neuroreport, Vol.17, No.12, (August 2006), pp. 1319-1322, ISSN 0959-4965

Cowan F., Rutherford M., Groenendaal F., Eken P., Mercuri E., Bydder G.M., Meiners L.C., Dubowitz L.M., de Vries L.S. (2003). Origin and timing of brain lesions in term infants with neonatal encephalopathy. Lancet, Vol.361, No.9359, (March 2003), pp. 736-742, ISSN 0140-6736

Crop M.J., Korevaar S.S., de Kuiper R., Ijzermans J.N., van Besouw N.M., Baan C.C., Weimar W., Hoogduijn M.J. (2011). Human mesenchymal stem cells are susceptible to lysis by CD8+ T-cells and NK cells. Cell Transplantation, (March 2011), pp. 1555-3892, ISSN 0963-6897

Daadi M.M., Davis A.S., Arac A., Li Z., Maag A.L., Bhatnagar R., Jiang K., Sun G., Wu J.C., Steinberg G.K. (2010). Human neural stem cell grafts modify microglial response and enhance axonal sprouting in neonatal hypoxic-ischemic brain injury. Stroke, Vol.41, No.3, (March 2010), pp. 516-523, ISSN 1524-4628

Dayer A.G., Jenny B., Sauvain M.O., Potter G., Salmon P., Zgraggen E., Kanemitsu M., Gascon E., Sizonenko S., Trono D., Kiss J.Z. (2007). Expression of FGF-2 in neural progenitor cells enhances their potential for cellular brain repair in the rodent cortex. Brain, Vol.130, No.Pt 11, (November 2007), pp. 2962-2976, ISSN 1460-2156 
de Paula S., Vitola A.S., Greggio S., de Paula D., Mello P.B., Lubianca J.M., Xavier L.L., Fiori H.H., Dacosta J.C. (2009). Hemispheric brain injury and behavioral deficits induced by severe neonatal hypoxia-ischemia in rats are not attenuated by intravenous administration of human umbilical cord blood cells. Pediatric Research, Vol.65, No.6, (June 2009), pp. 631-635, ISSN 1530-0447

de Vries L.S., Groenendaal F. (2010). Patterns of neonatal hypoxic-ischaemic brain injury. Neuroradiology, Vol.52, No.6, (June 2010), pp. 555-566, ISSN 1432-1920

de Vries, L.S. \& Jongmans, M.J. (2010) Long-term outcome after neonatal hypoxic-ischaemic encephalopathy. Archives of disease in childhood. Fetal and neonatal edition, Vol. 95, No.3, (May 2010), pp. 220-224, ISSN: 1468-2052

Dizon M., Szele F., Kessler J.A. (2010). Hypoxia-ischemia induces an endogenous reparative response by local neural progenitors in the postnatal mouse telencephalon. Developmental Neuroscience, Vol.32, No.3, (August 2010), pp. 173-183, ISSN 1421-9859

Dominici M., Le Blanc K., Mueller I., Slaper-Cortenbach I., Marini F., Krause D., Deans R., Keating A., Prockop D., Horwitz E. (2006). Minimal criteria for defining multipotent mesenchymal stromal cells. The International Society for Cellular Therapy position statement. Cytotherapy, Vol.8, No.4, pp. 315-317, ISSN 1465-3249

Du L., Bayir H., Lai Y., Zhang X., Kochanek P.M., Watkins S.C., Graham S.H., Clark R.S. (2004). Innate gender-based proclivity in response to cytotoxicity and programmed cell death pathway. JBC, Vol.279, No.37, (September 2004), pp. 38563-38570, ISSN 0021-9258

Edwards A.D., Brocklehurst P., Gunn A.J., Halliday H., Juszczak E., Levene M., Strohm B., Thoresen M., Whitelaw A., Azzopardi D. (2010). Neurological outcomes at 18 months of age after moderate hypothermia for perinatal hypoxic ischaemic encephalopathy: synthesis and meta-analysis of trial data. BMJ, Vol.340, pp. c363, ISSN 1468-5833

Elmahdy H., El-Mashad A.R., El-Bahrawy H., El-Gohary T., El-Barbary A., Aly H. (2010). Human recombinant erythropoietin in asphyxia neonatorum: pilot trial. Pediatrics, Vol.125, No.5, (May 2010), pp. e1135-1142, ISSN 1098-4275

Elsayed M.H., Hogan T.P., Shaw P.L., Castro A.J. (1996). Use of fetal cortical grafts in hypoxic-ischemic brain injury in neonatal rats. Experimental Neurology, Vol.137, No.1, (January 1996), pp. 127-141, ISSN 0014-4886

Fan C.G., Zhang Q.J., Tang F.W., Han Z.B., Wang G.S., Han Z.C. (2005). Human umbilical cord blood cells express neurotrophic factors. Neuroscience Letters, Vol.380, No.3, (June 2005), pp. 322-325, ISSN 0304-3940

Fan X., Heijnen C.J., van der K.M., Groenendaal F., van Bel F. (2011). Beneficial effect of erythropoietin on sensorimotor function and white matter after hypoxia-ischemia in neonatal mice. Pediatric Research, Vol.69, No.1, (January 2011), pp. 56-61, ISSN 1530-0447

Fernandez-Lopez D., Pazos M.R., Tolon R.M., Moro M.A., Romero J., Lizasoain I., MartinezOrgado J. (2007). The cannabinoid agonist WIN55212 reduces brain damage in an in vivo model of hypoxic-ischemic encephalopathy in newborn rats. Pediatric Research, Vol.62, No.3, (September 2007), pp. 255-260, ISSN 0031-3998 
Fernandez-Lopez D., Pradillo J.M., Garcia-Yebenes I., Martinez-Orgado J.A., Moro M.A., Lizasoain I. (2010). The cannabinoid WIN55212-2 promotes neural repair after neonatal hypoxia-ischemia. Stroke, Vol.41, No.12, (December 2010), pp. 2956-2964, ISSN 1524-4628

Fischer U.M., Harting M.T., Jimenez F., Monzon-Posadas W.O., Xue H., Savitz S.I., Laine G.A., Cox C.S., Jr. (2009). Pulmonary passage is a major obstacle for intravenous stem cell delivery: the pulmonary first-pass effect. Stem Cells and Development, Vol.18, No.5, (June 2009), pp. 683-692, ISSN 1557-8534

Geissler M., Dinse H.R., Neuhoff S., Kreikemeier K., Meier C. (2011). Human Umbilical Cord Blood Cells Restore Brain Damage Induced Changes in Rat Somatosensory Cortex. PLoS One, Vol.6, No.6, pp. e20194, ISSN 1932-6203

Gibson M.E., Han B.H., Choi J., Knudson C.M., Korsmeyer S.J., Parsadanian M., Holtzman D.M. (2001). BAX contributes to apoptotic-like death following neonatal hypoxiaischemia: evidence for distinct apoptosis pathways. Molecular Medicine, Vol.7, No.9, (September 2001), pp. 644-655, ISSN 1076-1551

Ginet V., Puyal J., Clarke P.G., Truttmann A.C. (2009). Enhancement of autophagic flux after neonatal cerebral hypoxia-ischemia and its region-specific relationship to apoptotic mechanisms. American Journal of Pathology, Vol.175, No.5, (November 2009), pp. 1962-1974, ISSN 1525-2191

Graham E.M., Ruis K.A., Hartman A.L., Northington F.J., Fox H.E. (2008). A systematic review of the role of intrapartum hypoxia-ischemia in the causation of neonatal encephalopathy. American Journal of Obstetetrics \& Gynecology, Vol.199, No.6, (December 2008), pp. 587-595, ISSN 1097-6868

Graham E.M., Sheldon R.A., Flock D.L., Ferriero D.M., Martin L.J., O'Riordan D.P., Northington F.J. (2004). Neonatal mice lacking functional Fas death receptors are resistant to hypoxic-ischemic brain injury. Neurobiology of Disease, Vol.17, No.1, (October 2004), pp. 89-98, ISSN 0969-9961

Gunn A.J., Gunn T.R., de Haan H.H., Williams C.E., Gluckman P.D. (1997). Dramatic neuronal rescue with prolonged selective head cooling after ischemia in fetal lambs. Journal of Clinical Investigation, Vol.99, No.2, (January 1997), pp. 248-256, ISSN 0021-9738

Hagberg H., Mallard C., Rousset C.I., Xiaoyang W. (2009). Apoptotic mechanisms in the immature brain: involvement of mitochondria. Journal of Children Neurology, Vol.24, No.9, (September 2009), pp. 1141-1146, ISSN 1708-8283

Hagberg H., Wilson M.A., Matsushita H., Zhu C., Lange M., Gustavsson M., Poitras M.F., Dawson T.M., Dawson V.L., Northington F., Johnston M.V. (2004). PARP-1 gene disruption in mice preferentially protects males from perinatal brain injury. Journal of Neurochemistry, Vol.90, No.5, (September 2004), pp. 1068-1075, ISSN 0022-3042

Han B.H., Holtzman D.M. (2000). BDNF protects the neonatal brain from hypoxic-ischemic injury in vivo via the ERK pathway. Journal of Neuroscience, Vol.20, No.15, (August 2000), pp. 5775-5781, ISSN 0270-6474

Han B.H., Xu D., Choi J., Han Y., Xanthoudakis S., Roy S., Tam J., Vaillancourt J., Colucci J., Siman R., Giroux A., Robertson G.S., Zamboni R., Nicholson D.W., Holtzman D.M. (2002). Selective, reversible caspase-3 inhibitor is neuroprotective and reveals 
distinct pathways of cell death after neonatal hypoxic-ischemic brain injury. JBC, Vol.277, No.33, (August 2002), pp. 30128-30136, ISSN 0021-9258

Hill, C.A., Threlkeld, S.W. \& Fitch, R.H. (2011) Reprint of "Early testosterone modulated sex differences in behavioral outcome following neonatal hypoxia ischemia in rats". International journal of developmental neuroscience, Vol. 29, No.6, (October 2011), pp. 621-628, ISSN: 1873-474X

Hu, B.Y., Weick, J.P., Yu, J., Ma, L.X., Zhang, X.Q., Thomson, J.A. \& Zhang, S.C. (2010) Neural differentiation of human induced pluripotent stem cells follows developmental principles but with variable potency. Proceedings of the National Academy of Sciences of the United States of America, Vol. 107, No.9, (March 2010), pp. 4335-4340, ISSN: 1091-6490

Ihrie R.A., Alvarez-Buylla A. (2011). Lake-front property: a unique germinal niche by the lateral ventricles of the adult brain. Neuron, Vol.70, No.4, (May 2011), pp. 674-686, ISSN 1097-4199

Im S.H., Yu J.H., Park E.S., Lee J.E., Kim H.O., Park K.I., Kim G.W., Park C.I., Cho S.R. (2010). Induction of striatal neurogenesis enhances functional recovery in an adult animal model of neonatal hypoxic-ischemic brain injury. Neuroscience, Vol.169, No.1, (August 2010), pp. 259-268, ISSN 1873-7544

Imitola J., Raddassi K., Park K.I., Mueller F.J., Nieto M., Teng Y.D., Frenkel D., Li J., Sidman R.L., Walsh C.A., Snyder E.Y., Khoury S.J. (2004). Directed migration of neural stem cells to sites of CNS injury by the stromal cell-derived factor 1alpha/CXC chemokine receptor 4 pathway. PNAS U S A, Vol.101, No.52, (December 2004), pp. 18117-18122, ISSN 0027-8424

Iwai M., Cao G., Yin W., Stetler R.A., Liu J., Chen J. (2007). Erythropoietin promotes neuronal replacement through revascularization and neurogenesis after neonatal hypoxia/ischemia in rats. Stroke, Vol.38, No.10, (October 2007), pp. 2795-2803, ISSN 1524-4628

Iwai M., Stetler R.A., Xing J., Hu X., Gao Y., Zhang W., Chen J., Cao G. (2010). Enhanced oligodendrogenesis and recovery of neurological function by erythropoietin after neonatal hypoxic/ischemic brain injury. Stroke, Vol.41, No.5, (May 2010), pp. 10321037, ISSN 1524-4628

Jin-qiao S., Bin S., Wen-hao Z., Yi Y. (2009). Basic fibroblast growth factor stimulates the proliferation and differentiation of neural stem cells in neonatal rats after ischemic brain injury. Brain \& Development, Vol.31, No.5, (May 2009), pp. 331-340, ISSN 18727131

Juul S.E., Beyer R.P., Bammler T.K., McPherson R.J., Wilkerson J., Farin F.M. (2009). Microarray analysis of high-dose recombinant erythropoietin treatment of unilateral brain injury in neonatal mouse hippocampus. Pediatric Research, Vol.65, No.5, (May 2009), pp. 485-492, ISSN 1530-0447

Karimi-Abdolrezaee S., Eftekharpour E., Wang J., Schut D., Fehlings M.G. (2010). Synergistic effects of transplanted adult neural stem/progenitor cells, chondroitinase, and growth factors promote functional repair and plasticity of the chronically injured spinal cord. Journal of Neuroscience, Vol.30, No.5, (February 2010), pp. 1657-1676, ISSN 1529-2401 
Kawamura M., Nakajima W., Ishida A., Ohmura A., Miura S., Takada G. (2005). Calpain inhibitor MDL 28170 protects hypoxic-ischemic brain injury in neonatal rats by inhibition of both apoptosis and necrosis. Brain Research, Vol.1037, No.1-2, (March 2005), pp. 59-69, ISSN 0006-8993

Khatibi N.H., Jadhav V., Charles S., Chiu J., Buchholz J., Tang J., Zhang J.H. (2011a). Capsaicin Pre-treatment Provides Neurovascular Protection Against Neonatal Hypoxic-Ischemic Brain Injury in Rats. Acta Neurochirurgica Supplementum, Vol.111, pp. 225-230, ISSN 0065-1419

Khatibi N.H., Lee L.K., Zhou Y., Chen W., Rolland W., Fathali N., Martin R., Applegate R., Stier G., Zhang J.H. (2011b). Endothelin Receptor-A (ET(a)) Inhibition Fails to Improve Neonatal Hypoxic-Ischemic Brain Injury in Rats. Acta Neurochirurgica Supplementum, Vol.111, pp. 207-212, ISSN 0065-1419

Knickmeyer R., Baron-Cohen S. (2006). Fetal testosterone and sex differences. Early Human Development, Vol.82, No.12, (December 2006), pp. 755-760, ISSN 0378-3782

Koc O.N., Day J., Nieder M., Gerson S.L., Lazarus H.M., Krivit W. (2002). Allogeneic mesenchymal stem cell infusion for treatment of metachromatic leukodystrophy (MLD) and Hurler syndrome (MPS-IH). Bone Marrow Transplantation, Vol.30, No.4, (August 2002), pp. 215-222, ISSN 0268-3369

Kumar S., Paterson-Brown S. (2010). Obstetric aspects of hypoxic ischemic encephalopathy. Early Human Development, Vol.86, No.6, (June 2010), pp. 339-344, ISSN 1872-6232

Kurinczuk J.J., White-Koning M., Badawi N. Epidemiology of neonatal encephalopathy and hypoxic-ischaemic encephalopathy. Early Human Development, Vol. 86, No.6, (June 2010), pp. 329-338, ISSN: 1872-6232

Lee J.A., Kim B.I., Jo C.H., Choi C.W., Kim E.K., Kim H.S., Yoon K.S., Choi J.H. (2010). Mesenchymal stem-cell transplantation for hypoxic-ischemic brain injury in neonatal rat model. Pediatric Research, Vol.67, No.1, (January 2010), pp. 42-46, ISSN 1530-0447

Li H., Li Q., Du X., Sun Y., Wang X., Kroemer G., Blomgren K., Zhu C. (2011). Lithiummediated long-term neuroprotection in neonatal rat hypoxia-ischemia is associated with antiinflammatory effects and enhanced proliferation and survival of neural stem/progenitor cells. Journal of Cerebral Blood Flow and Metabolism, (May 2011), pp. 1559-7016, ISSN

Lorek A., Takei Y., Cady E.B., Wyatt J.S., Penrice J., Edwards A.D., Peebles D., Wylezinska M., Owen-Reece H., Kirkbride V., et al. (1994). Delayed ("secondary") cerebral energy failure after acute hypoxia-ischemia in the newborn piglet: continuous 48hour studies by phosphorus magnetic resonance spectroscopy. Pediatric Research, Vol.36, No.6, (December 1994), pp. 699-706, ISSN 0031-3998

Lubics A., Reglodi D., Tamas A., Kiss P., Szalai M., Szalontay L., Lengvari I. (2005). Neurological reflexes and early motor behavior in rats subjected to neonatal hypoxic-ischemic injury. Behavioural Brain Research, Vol.157, No.1, (February 2005), pp. 157-165, ISSN 0166-4328

Ma J., Wang Y., Yang J., Yang M., Chang K.A., Zhang L., Jiang F., Li Y., Zhang Z., Heo C., Suh Y.H. (2007). Treatment of hypoxic-ischemic encephalopathy in mouse by 
transplantation of embryonic stem cell-derived cells. Neurochemistry International, Vol.51, No.1, (July 2007), pp. 57-65, ISSN 0197-0186

Martin E., Buchli R., Ritter S., Schmid R., Largo R.H., Boltshauser E., Fanconi S., Duc G., Rumpel H. (1996). Diagnostic and prognostic value of cerebral 31P magnetic resonance spectroscopy in neonates with perinatal asphyxia. Pediatric Research, Vol.40, No.5, (November 1996), pp. 749-758, ISSN 0031-3998

Martinez-Biarge, M., Diez-Sebastian, J., Rutherford, M.A. \& Cowan, F.M. (2010) Outcomes after central grey matter injury in term perinatal hypoxic-ischaemic encephalopathy. Early Human Development, Vol. 86, No.11, (November 2010), pp. 675-682, ISSN: 1872-6232

Matsuo Y., Mihara S., Ninomiya M., Fujimoto M. (2001). Protective effect of endothelin type A receptor antagonist on brain edema and injury after transient middle cerebral artery occlusion in rats. Stroke, Vol.32, No.9, (September 2001), pp. 2143-2148, ISSN 1524-4628

Meier C., Middelanis J., Wasielewski B., Neuhoff S., Roth-Haerer A., Gantert M., Dinse H.R., Dermietzel R., Jensen A. (2006). Spastic paresis after perinatal brain damage in rats is reduced by human cord blood mononuclear cells. Pediatric Research, Vol.59, No.2, (February 2006), pp. 244-249, ISSN 0031-3998

Meirelles Lda S., Fontes A.M., Covas D.T., Caplan A.I. (2009). Mechanisms involved in the therapeutic properties of mesenchymal stem cells. Cytokine Growth Factor Reviews, Vol.20, No.5-6, (October-December 2009), pp. 419-427, ISSN 1879-0305

Merkle F.T., Tramontin A.D., Garcia-Verdugo J.M., Alvarez-Buylla A. (2004). Radial glia give rise to adult neural stem cells in the subventricular zone. PNAS U S A, Vol.101, No.50, (December 2004), pp. 17528-17532, ISSN 0027-8424

Miller J.T., Bartley J.H., Wimborne H.J., Walker A.L., Hess D.C., Hill W.D., Carroll J.E. (2005). The neuroblast and angioblast chemotaxic factor SDF-1 (CXCL12) expression is briefly up regulated by reactive astrocytes in brain following neonatal hypoxic-ischemic injury. BMC Neuroscience, Vol.6, pp. 63, ISSN 1471-2202

Ming G.L., Song H. (2011). Adult neurogenesis in the mammalian brain: significant answers and significant questions. Neuron, Vol.70, No.4, (May 2011), pp. 687-702, ISSN 10974199

Miyata T., Kawaguchi A., Saito K., Kawano M., Muto T., Ogawa M. (2004). Asymmetric production of surface-dividing and non-surface-dividing cortical progenitor cells. Development, Vol.131, No.13, (July 2004), pp. 3133-3145, ISSN 0950-1991

Nakajima W., Ishida A., Lange M.S., Gabrielson K.L., Wilson M.A., Martin L.J., Blue M.E., Johnston M.V. (2000). Apoptosis has a prolonged role in the neurodegeneration after hypoxic ischemia in the newborn rat. Journal of Neuroscience, Vol.20, No.21, (November 2000), pp. 7994-8004, ISSN 1529-2401

Nelson K.B. and Leviton A. (1991). How much of neonatal encephalopathy is due to birth asphyxia? American Journal of Diseases of Children, Vol.145, No.11, (November 1991), pp. 1325-1331, ISSN: 0002-922X

Ness J.M., Harvey C.A., Strasser A., Bouillet P., Klocke B.J., Roth K.A. (2006). Selective involvement of BH3-only Bcl-2 family members Bim and Bad in neonatal hypoxiaischemia. Brain Research, Vol.1099, No.1, (July 2006), pp. 150-159, ISSN 0006-8993 
Newman M.B., Willing A.E., Manresa J.J., Sanberg C.D., Sanberg P.R. (2006). Cytokines produced by cultured human umbilical cord blood (HUCB) cells: implications for brain repair. Experimental Neurology, Vol.199, No.1, (May 2006), pp. 201-208, ISSN 0014-4886

Nijboer C.H., Groenendaal F., Kavelaars A., Hagberg H.H., van Bel F., Heijnen C.J. (2007). Gender-specific neuroprotection by 2-iminobiotin after hypoxia-ischemia in the neonatal rat via a nitric oxide independent pathway. Journal of Cerebral Blood Flow and Metabolism, Vol.27, No.2, (February 2007), pp. 282-292, ISSN 0271-678X

Nijboer C.H., Heijnen C.J., van der Kooij M.A., Zijlstra J., van Velthoven C.T., Culmsee C., van Bel F., Hagberg H., Kavelaars A. (2011). Targeting the p53 pathway to protect the neonatal ischemic brain. Annals of Neurology, (March 2011), pp. 1531-8249, ISSN 0364-5134

Noctor, S.C., Flint, A.C., Weissman, T.A., Wong, W.S., Clinton, B.K. \& Kriegstein, A.R. (2002) Dividing precursor cells of the embryonic cortical ventricular zone have morphological and molecular characteristics of radial glia. The Journal of Neuroscience, Vol. 22, No.8, (April 2002), pp. 3161-3173, ISSN: 1529-2401

Noctor S.C., Martinez-Cerdeno V., Ivic L., Kriegstein A.R. (2004). Cortical neurons arise in symmetric and asymmetric division zones and migrate through specific phases. Nature Neuroscience, Vol.7, No.2, (February 2004), pp. 136-144, ISSN 1097-6256

Northington F.J., Chavez-Valdez R., Graham E.M., Razdan S., Gauda E.B., Martin L.J. (2011). Necrostatin decreases oxidative damage, inflammation, and injury after neonatal HI. Journal of Cerebral Blood Flow and Metabolism, Vol.31, No.1, (January 2011), pp. 178-189, ISSN 1559-7016

Northington F.J., Ferriero D.M., Flock D.L., Martin L.J. (2001). Delayed neurodegeneration in neonatal rat thalamus after hypoxia-ischemia is apoptosis. Journal of Neuroscience, Vol.21, No.6, (March 2001), pp. 1931-1938, ISSN 1529-2401

Northington F.J., Zelaya M.E., O'Riordan D.P., Blomgren K., Flock D.L., Hagberg H., Ferriero D.M., Martin L.J. (2007). Failure to complete apoptosis following neonatal hypoxia-ischemia manifests as "continuum" phenotype of cell death and occurs with multiple manifestations of mitochondrial dysfunction in rodent forebrain. Neuroscience, Vol.149, No.4, (November 2007), pp. 822-833, ISSN 0306-4522

Nunez J.L., McCarthy M.M. (2008). Androgens predispose males to GABAA-mediated excitotoxicity in the developing hippocampus. Experimental Neurology, Vol.210, No.2, (April 2008), pp. 699-708, ISSN 0014-4886

Obenaus A., Dilmac N., Tone B., Tian H.R., Hartman R., Digicaylioglu M., Snyder E.Y., Ashwal S. (2011). Long-term magnetic resonance imaging of stem cells in neonatal ischemic injury. Annals of Neurology, Vol.69, No.2, (February 2011), pp. 282-291, ISSN 1531-8249

Ong J., Plane J.M., Parent J.M., Silverstein F.S. (2005). Hypoxic-ischemic injury stimulates subventricular zone proliferation and neurogenesis in the neonatal rat. Pediatric Research, Vol.58, No.3, (September 2005), pp. 600-606, ISSN 0031-3998

Park K.I., Hack M.A., Ourednik J., Yandava B., Flax J.D., Stieg P.E., Gullans S., Jensen F.E., Sidman R.L., Ourednik V., Snyder E.Y. (2006). Acute injury directs the migration, proliferation, and differentiation of solid organ stem cells: evidence from the effect 
of hypoxia-ischemia in the CNS on clonal "reporter" neural stem cells. Experimental Neurology, Vol.199, No.1, (May 2006), pp. 156-178, ISSN 0014-4886

Park K.I., Himes B.T., Stieg P.E., Tessler A., Fischer I., Snyder E.Y. (2006). Neural stem cells may be uniquely suited for combined gene therapy and cell replacement: Evidence from engraftment of Neurotrophin-3-expressing stem cells in hypoxic-ischemic brain injury. Experimental Neurology, Vol.199, No.1, (May 2006), pp. 179-190, ISSN 0014-4886

Parsadanian A.S., Cheng Y., Keller-Peck C.R., Holtzman D.M., Snider W.D. (1998). Bcl-xL is an antiapoptotic regulator for postnatal CNS neurons. Journal of Neuroscience, Vol.18, No.3, (February 1998), pp. 1009-1019, ISSN 0270-6474

Pimentel-Coelho P.M., Magalhaes E.S., Lopes L.M., deAzevedo L.C., Santiago M.F., Mendez-Otero R. (2010). Human cord blood transplantation in a neonatal rat model of hypoxic-ischemic brain damage: functional outcome related to neuroprotection in the striatum. Stem Cells and Development, Vol.19, No.3, (March 2010), pp. 351-358, ISSN 1557-8534

Pin T.W., Eldridge B., Galea M.P. (2009). A review of developmental outcomes of term infants with post-asphyxia neonatal encephalopathy. European Journal of Paediatric Neurology, Vol.13, No.3, (May 2009), pp. 224-234, ISSN 1532-2130

Puyal J., Vaslin A., Mottier V., Clarke P.G. (2009). Postischemic treatment of neonatal cerebral ischemia should target autophagy. Annals of Neurology, Vol.66, No.3, (September 2009), pp. 378-389, ISSN 1531-8249

Riccio O., Murthy S., Szabo G., Vutskits L., Kiss J.Z., Vitalis T., Lebrand C., Dayer A.G. (2011). New Pool of Cortical Interneuron Precursors in the Early Postnatal Dorsal White Matter. Cerebral Cortex, (June 2011), pp. 1460-2199, ISSN 1047-3211

Rosenkranz K., Kumbruch S., Lebermann K., Marschner K., Jensen A., Dermietzel R., Meier C. (2010). The chemokine SDF-1/CXCL12 contributes to the 'homing' of umbilical cord blood cells to a hypoxic-ischemic lesion in the rat brain. Journal of Neuroscience Research, Vol.88, No.6, (May 2010), pp. 1223-1233, ISSN 1097-4547

Rutherford M., Ramenghi L.A., Edwards A.D., Brocklehurst P., Halliday H., Levene M., Strohm B., Thoresen M., Whitelaw A., Azzopardi D. (2010). Assessment of brain tissue injury after moderate hypothermia in neonates with hypoxic-ischaemic encephalopathy: a nested substudy of a randomised controlled trial. Lancet Neurology, Vol.9, No.1, (January 2010), pp. 39-45, ISSN 1474-4465

Rutherford M.A., Pennock J.M., Counsell S.J., Mercuri E., Cowan F.M., Dubowitz L.M., Edwards A.D. (1998). Abnormal magnetic resonance signal in the internal capsule predicts poor neurodevelopmental outcome in infants with hypoxic-ischemic encephalopathy. Pediatrics, Vol.102, No.2 Pt 1, (August 1998), pp. 323-328, ISSN 0031-4005

Sanberg P.R., Eve D.J., Willing A.E., Garbuzova-Davis S., Tan J., Sanberg C.D., Allickson J.G., Cruz L.E., Borlongan C.V. (2011). The treatment of neurodegenerative disorders using umbilical cord blood and menstrual blood-derived stem cells. Cell Transplantation, Vol.20, No.1, pp. 85-94, ISSN 1555-3892

Sato, Y., Nakanishi, K., Hayakawa, M., Kakizawa, H., Saito, A., Kuroda, Y., Ida, M., Tokita, Y., Aono, S., Matsui, F., Kojima, S. \& Oohira, A. (2008) Reduction of brain injury in 
neonatal hypoxic-ischemic rats by intracerebroventricular injection of neural stem/progenitor cells together with chondroitinase ABC. Reproductive Sciences, Vol. 15, No.6, (July 2008), pp. 613-620, ISSN: 1933-7205

Segovia K.N., McClure M., Moravec M., Luo N.L., Wan Y., Gong X., Riddle A., Craig A., Struve J., Sherman L.S., Back S.A. (2008). Arrested oligodendrocyte lineage maturation in chronic perinatal white matter injury. Annals of Neurology, Vol.63, No.4, (April 2008), pp. 520-530, ISSN 1531-8249

Shevell M.I. (2004). The "Bermuda triangle" of neonatal neurology: cerebral palsy, neonatal encephalopathy, and intrapartum asphyxia. Seminars in Pediatric Neurology, Vol.11, No.1, (March 2004), pp. 24-30, ISSN 1071-9091

Sizonenko S.V., Camm E.J., Dayer A., Kiss J.Z. (2008). Glial responses to neonatal hypoxicischemic injury in the rat cerebral cortex. International Journal of Developmental Neuroscience, Vol.26, No.1, (February 2008), pp. 37-45, ISSN 0736-5748

Spassky N., Merkle F.T., Flames N., Tramontin A.D., Garcia-Verdugo J.M., Alvarez-Buylla A. (2005). Adult ependymal cells are postmitotic and are derived from radial glial cells during embryogenesis. Journal of Neuroscience, Vol.25, No.1, (January 2005), pp. 10-18, ISSN 1529-2401

Sun J., Allison J., McLaughlin C., Sledge L., Waters-Pick B., Wease S., Kurtzberg J. (2010). Differences in quality between privately and publicly banked umbilical cord blood units: a pilot study of autologous cord blood infusion in children with acquired neurologic disorders. Transfusion, Vol.50, No.9, (September 2010), pp. 1980-1987, ISSN 1537-2995

Sun Y., Zhou C., Polk P., Nanda A., Zhang J.H. (2004). Mechanisms of erythropoietininduced brain protection in neonatal hypoxia-ischemia rat model. Journal of Cerebral Blood Flow and Metabolism, Vol.24, No.2, (February 2004), pp. 259-270, ISSN 0271$678 \mathrm{X}$

Uccelli A., Moretta L., Pistoia V. (2008). Mesenchymal stem cells in health and disease. Nature Reviews Immunology, Vol.8, No.9, (September 2008), pp. 726-736, ISSN 14741733

Ulfig N., Neudorfer F., Bohl J. (1999). Distribution patterns of vimentin-immunoreactive structures in the human prosencephalon during the second half of gestation. Journal of Anatomy, Vol.195 ( Pt 1), (July 1999), pp. 87-100, ISSN 0021-8782

van Velthoven C.T., Kavelaars A., van Bel F., Heijnen C.J. (2010a). Mesenchymal stem cell treatment after neonatal hypoxic-ischemic brain injury improves behavioral outcome and induces neuronal and oligodendrocyte regeneration. Brain, Behavior and Immunity, Vol.24, No.3, (March 2010), pp. 387-393, ISSN 1090-2139

van Velthoven C.T., Kavelaars A., van Bel F., Heijnen C.J. (2010b). Nasal administration of stem cells: a promising novel route to treat neonatal ischemic brain damage. Pediatric Research, Vol.68, No.5, (November 2010), pp. 419-422, ISSN 1530-0447

van Velthoven C.T., Kavelaars A., van Bel F., Heijnen C.J. (2010c). Repeated mesenchymal stem cell treatment after neonatal hypoxia-ischemia has distinct effects on formation and maturation of new neurons and oligodendrocytes leading to restoration of damage, corticospinal motor tract activity, and sensorimotor function. Journal of Neuroscience, Vol.30, No.28, (July 2010), pp. 9603-9611, ISSN 1529-2401 
Vannucci R.C., Connor J.R., Mauger D.T., Palmer C., Smith M.B., Towfighi J., Vannucci S.J. (1999). Rat model of perinatal hypoxic-ischemic brain damage. Journal of Neuroscience Research, Vol.55, No.2, (January 1999), pp. 158-163, ISSN 0360-4012

Vendrame M., Gemma C., Pennypacker K.R., Bickford P.C., Davis Sanberg C., Sanberg P.R., Willing A.E. (2006). Cord blood rescues stroke-induced changes in splenocyte phenotype and function. Experimental Neurology, Vol.199, No.1, (May 2006), pp. 191-200, ISSN 0014-4886

Wang M., Crisostomo P.R., Herring C., Meldrum K.K., Meldrum D.R. (2006). Human progenitor cells from bone marrow or adipose tissue produce VEGF, HGF, and IGF-I in response to TNF by a p38 MAPK-dependent mechanism. American Journal of Physiology Regulatory, Integrative and Comparative Physiology, Vol.291, No.4, (October 2006), pp. R880-884, ISSN 0363-6119

Wang X., Carlsson Y., Basso E., Zhu C., Rousset C.I., Rasola A., Johansson B.R., Blomgren K., Mallard C., Bernardi P., Forte M.A., Hagberg H. (2009). Developmental shift of cyclophilin D contribution to hypoxic-ischemic brain injury. Journal of Neuroscience, Vol.29, No.8, (February 2009), pp. 2588-2596, ISSN 1529-2401

Wang X., Han W., Du X., Zhu C., Carlsson Y., Mallard C., Jacotot E., Hagberg H. (2010). Neuroprotective effect of Bax-inhibiting peptide on neonatal brain injury. Stroke, Vol.41, No.9, (September 2010), pp. 2050-2055, ISSN 1524-4628

Wang Y., Cheng X., He Q., Zheng Y., Kim D.H., Whittemore S.R., Cao Q.L. (2011). Astrocytes from the contused spinal cord inhibit oligodendrocyte differentiation of adult oligodendrocyte precursor cells by increasing the expression of bone morphogenetic proteins. Journal of Neuroscience, Vol.31, No.16, (April 2011), pp. 6053-6058, ISSN 1529-2401

Wang Y., Deng Y., Zhou G.Q. (2008). SDF-1alpha/CXCR4-mediated migration of systemically transplanted bone marrow stromal cells towards ischemic brain lesion in a rat model. Brain Res, Vol.1195, (February 2008), pp. 104-112, ISSN 0006-8993

Wei B., Nie Y., Li X., Wang C., Ma T., Huang Z., Tian M., Sun C., Cai Y., You Y., Liu F., Yang Z. (2011). Emx1-expressing neural stem cells in the subventricular zone give rise to new interneurons in the ischemic injured striatum. European Journal of Neuroscience, Vol.33, No.5, (March 2011), pp. 819-830, ISSN 1460-9568

Wei X., Du Z., Zhao L., Feng D., Wei G., He Y., Tan J., Lee W.H., Hampel H., Dodel R., Johnstone B.H., March K.L., Farlow M.R., Du Y. (2009). IFATS collection: The conditioned media of adipose stromal cells protect against hypoxia-ischemiainduced brain damage in neonatal rats. Stem Cells, Vol.27, No.2, (February 2009), pp. 478-488, ISSN 1549-4918

West T., Atzeva M., Holtzman D.M. (2006). Caspase-3 deficiency during development increases vulnerability to hypoxic-ischemic injury through caspase-3-independent pathways. Neurobiology of Disease, Vol.22, No.3, (June 2006), pp. 523-537, ISSN 09699961

Wu Y.W., Pham T.N., Danielsen B., Towner D., Smith L., Johnston S.C. (2011). Nighttime delivery and risk of neonatal encephalopathy. American Journal of Obstetetrics $\mathcal{E}$ Gynecology, Vol.204, No.1, (January 2011), pp. 37 e31-36, ISSN 1097-6868 
Xiong T., Qu Y., Mu D., Ferriero D. (2011). Erythropoietin for neonatal brain injury: opportunity and challenge. International Journal of Developmental Neuroscience, (January 2011), pp. 1873-474X, ISSN 0736-5748

Yang Z., Covey M.V., Bitel C.L., Ni L., Jonakait G.M., Levison S.W. (2007). Sustained neocortical neurogenesis after neonatal hypoxic/ischemic injury. Annals of Neurology, Vol.61, No.3, (March 2007), pp. 199-208, ISSN 0364-5134

Yang Z., You Y., Levison S.W. (2008). Neonatal hypoxic/ischemic brain injury induces production of calretinin-expressing interneurons in the striatum. Journal of Comparative Neurology, Vol.511, No.1, (November 2008), pp. 19-33, ISSN 1096-9861

Yasuhara T., Hara K., Maki M., Xu L., Yu G., Ali M.M., Masuda T., Yu S.J., Bae E.K., Hayashi T., Matsukawa N., Kaneko Y., Kuzmin-Nichols N., Ellovitch S., Cruz E.L., Klasko S.K., Sanberg C.D., Sanberg P.R., Borlongan C.V. (2010). Mannitol facilitates neurotrophic factor up-regulation and behavioural recovery in neonatal hypoxicischaemic rats with human umbilical cord blood grafts. Journal of Cellular and Molecular Medicine, Vol.14, No.4, (April 2010), pp. 914-921, ISSN 1582-4934

Zaidi A.U., Bessert D.A., Ong J.E., Xu H., Barks J.D., Silverstein F.S., Skoff R.P. (2004). New oligodendrocytes are generated after neonatal hypoxic-ischemic brain injury in rodents. Glia, Vol.46, No.4, (May 2004), pp. 380-390, ISSN 0894-1491

Zhao T., Zhang Z.N., Rong Z., Xu Y. (2011). Immunogenicity of induced pluripotent stem cells. Nature, Vol.474, No.7350, (June 2011), pp. 212-215, ISSN 1476-4687

Zhu C., Kang W., Xu F., Cheng X., Zhang Z., Jia L., Ji L., Guo X., Xiong H., Simbruner G., Blomgren K., Wang X. (2009). Erythropoietin improved neurologic outcomes in newborns with hypoxic-ischemic encephalopathy. Pediatrics, Vol.124, No.2, (August 2009), pp. e218-226, ISSN 1098-4275

Zhu C., Wang X., Xu F., Bahr B.A., Shibata M., Uchiyama Y., Hagberg H., Blomgren K. (2005). The influence of age on apoptotic and other mechanisms of cell death after cerebral hypoxia-ischemia. Cell Death and Differentiation, Vol.12, No.2, (February 2005), pp. 162-176, ISSN 1350-9047

Zhu C., Xu F., Wang X., Shibata M., Uchiyama Y., Blomgren K., Hagberg H. (2006). Different apoptotic mechanisms are activated in male and female brains after neonatal hypoxia-ischaemia. Journal of Neurochemistry, Vol.96, No.4, (February 2006), pp. 1016-1027, ISSN 0022-3042 


\title{
Molecular Defects of Vitamin $B_{6}$ Metabolism Associated with Neonatal Epileptic Encephalopathy
}

\author{
Mohini S. Ghatge ${ }^{*}$, Martino L. Di Salvo ${ }^{2 *}$, Roberto Contestabile2, \\ Dorothy N. Eseonu ${ }^{3}$, Sayali Karve ${ }^{1}$, Verne Schirch ${ }^{1}$ and Martin K. Safo ${ }^{1 \dagger}$ \\ 1 Department of Medicinal Chemistry, School of Pharmacy and Institute for Structural \\ Biology and Drug Discovery, Virginia Commonwealth University, Richmond, VA, \\ ${ }^{2}$ Istituto Pasteur-Fondazione Cenci Bolognetti, \\ Dipartimento di Scienze Biochimiche, Sapienza Università di Roma, \\ ${ }^{3}$ Department of Natural and Physical Sciences, School of Mathematics, Science and \\ Technology, Virginia Union University, Richmond, VA \\ 1,3USA \\ 2Italy
}

\section{Introduction}

Neonatal epileptic encephalopathy (NEE) is a seizure disorder that occurs within hours from birth and arises from central nervous system (CNS) dysfunctions of various origins, including metabolic or inflammatory conditions, abnormalities of brain structure and cerebrovascular diseases. In some rare circumstances, NEE is refractory to conventional antiepileptic drugs (AEDs) but responds very well to treatment with vitamin $B_{6}$ in the form of either pyridoxine (PN) or pyridoxal 5'-phosphate (PLP) [1-7]. Vitamin $\mathrm{B}_{6}$ exists in all organisms in the form of six vitamers but only PLP is the biologically active form acting as a cofactor for many enzymes (Figure 1.).

Vitamin $B_{6}$-dependent NEE derives from a deficiency of cellular PLP; either due to inborn errors in enzymes, such as pyridoxine $5^{\prime}$-phosphate oxidase (PNPOx) and pyridoxal kinase (PL kinase), involved in the PLP salvage pathway or from inherited mutations in other enzymes, such as a-aminoadipic semialdehyde dehydrogenase (also known as antiquitin), involved in other metabolic pathways, which lead to the accumulation of intermediates that react with PLP, reducing its availability.

Clinical phenotypes observed in vitamin $\mathrm{B}_{6}$-dependent NEE patients may include fetal distress, hypoglycemia, acidosis, anemia, and asphyxia [2,5-7]. The health state of untreated patients may undergo progressive deterioration, which can lead to death within weeks. Surviving children are usually mentally retarded and are dependent on vitamin $\mathrm{B}_{6}$ to

*These authors equally contributed to this work

tCorrespondening Author 
control the disease. Several known cases of $\mathrm{B}_{6}$-dependent NEE, however do not or only mildly manifest some of the above clinical features, and are characterized by mild to moderate developmental delay.

This chapter will review the molecular mechanisms of how in-born errors in PNPOx or antiquitin affect PLP levels in the cell and lead to NEE. We will also review important clinical and general features associated with PLP dependent NEE, and provide some directions for clinicians to diagnose and treat or manage the disease.

\section{Vitamin $B_{6}$}

There are six $\mathrm{B}_{6}$ vitamers, including the primary forms pyridoxal $(\mathbf{P L})$, pyridoxine $(\mathbf{P N})$, and pyridoxamine (PM), and their phosphorylated derivatives PLP, pyridoxine 5'-phosphate (PNP) and pyridoxamine 5'-phosphate (PMP) (Figure. 1). Pyridoxal 5'-phosphate, the active form of vitamin $\mathrm{B}_{6}$ serves as a cofactor for over 160 vitamin $\mathrm{B}_{6}$ dependent enzymes (or PLPdependent enzymes) involved in amino acid metabolism (e.g. degradation of threonine and glycine), neurotransmitter biosynthesis, biologically active amine biosynthesis, lipid metabolism, carbohydrate breakdown, heme synthesis, nucleic acid synthesis and several other metabolic pathways $[8,9]$.<smiles>[CH]c1c(CO)cnc(C)c1O</smiles>

Pyridoxine (PN)<smiles>Cc1ncc(CO)c([CH]N)c1O</smiles>

Pyridoxamine (PM)<smiles>Cc1ncc(CO)c(C=O)c1O</smiles>

Pyridoxal (PL)<smiles>Cc1ncc(CO[P+]([O-])([O-])[O-])c(C(O)O)c1O</smiles>

Pyridoxine 5'-phosphate (PNP)<smiles>Cc1ncc(CO[P+]([O-])([O-])[O-])c(C(N)N)c1O</smiles>

Pyridoxamine 5'-phosphate (PMP)<smiles>Cc1ncc(CO[P+]([O-])([O-])[O-])c(C=O)c1O</smiles>

Pyridoxal 5'-phosphate (PLP)

Fig. 1. Structures of $B_{6}$ vitamers

\subsection{Vitamin $B_{6}$ metabolism}

Unlike microorganisms and plants, animals cannot synthesize PLP from small metabolites by a de novo pathway and require the dietary $\mathrm{B}_{6}$ vitamers, pyridoxal, pyridoxine, or pyridoxamine (Figure 1), for the synthesis of PLP via a so-called salvage pathway [10]. Enzymes which are critical for this salvage pathway are PL kinase, PNPOx and several different phosphatases (Figure 2). 
PL kinase catalyzes the phosphorylation of the $5^{\prime}$-alcohol group of PN, PM, and PL to form PNP, PMP, and PLP, respectively (Figures 1 and 2). Both PNP and PMP are subsequently converted to PLP in an FMN-dependent oxidative process catalyzed by PNPOx (Figures 2 and 3). PLP adds to newly synthesized apo- $\mathrm{B}_{6}$ enzymes converting them to the catalytically active holo- $\mathrm{B}_{6}$ enzymes. Typically, PLP is then released during protein turnover of $\mathrm{B}_{6}$ enzymes and is converted to PL by cellular phosphatases, followed by re-phosphorylation to PLP by PL kinase $[10,11]$.

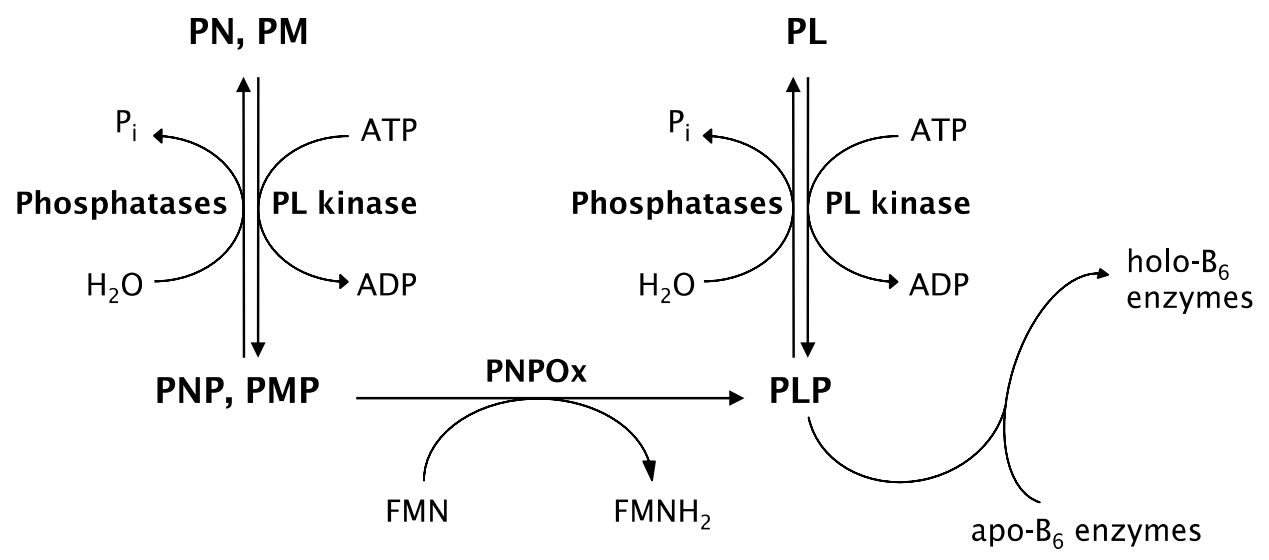

Fig. 2. The PLP salvage pathway

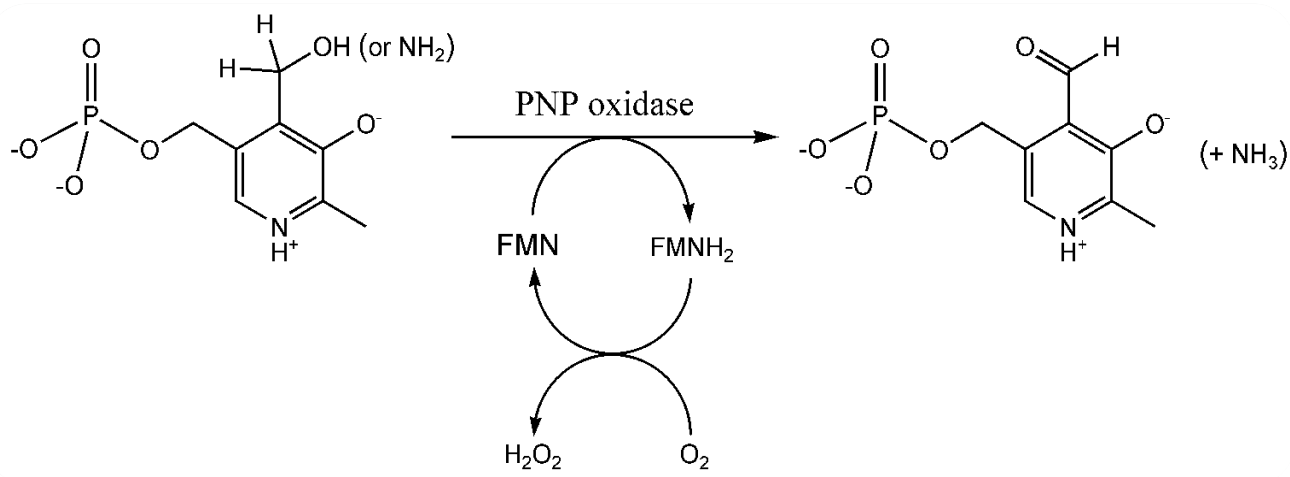

Fig. 3. Conversion of PNP (or PMP) to PLP by PNPOx

Mammals acquire $\mathrm{B}_{6}$ vitamers via intestinal absorption (Figure 4) and convert them into PLP through the salvage pathway (Figure 2). $\mathrm{B}_{6}$ vitamers are absorbed from food and from the microflora, which normally colonizes the large intestine. In animal-derived foods, vitamin $\mathrm{B}_{6}$ is mainly present as PLP, associated to glycogen phosphorylase, and in smaller amounts as PMP, while in plants it is present as PN and PN-5'-beta-D-glucoside [12]. PN-5'-beta-Dglucoside appears to have reduced availability because of the need for hydrolysis by an intestinal glucosidase [13]. In human breast milk, vitamin $B_{6}$ is present as PLP and PL [14]. 
The form of vitamin $B_{6}$ that is usually employed to fortify foods is pyridoxine hydrochloride, since this is the most commercially convenient form.

Once ingested (Figure 4), PLP, PNP and PMP are dephosphorylated by the ecto-enzyme tissue-specific intestinal phosphatase, prior to absorption from the upper small intestine by a carrier-mediated system [15]. The portal circulation delivers PM, PN and PL to the liver, where they are phosphorylated by PL kinase. PNP and PMP are then both oxidized to PLP by PNPOx. PLP must return to the blood stream in order to reach all tissues. Albuminbound PLP represents about $60 \%$ of circulating vitamin $\mathrm{B}_{6}$, with PN, PM and PL making up the remaining part [16]. While dephosphorylated $\mathrm{B}_{6}$ vitamers can enter the cells and pass the blood-brain barrier, PLP needs to be dephosphorylated again. Tissue-specific phosphatases, expressed in placenta and germ cells $[17,18]$, and the tissue-nonspecific alkaline phosphatase (TNAP) are membrane-associated enzymes responsible for this step. PL is the vitamer form that is mainly acquired by neurons; once formed by TNAP at the choroid plexus, PL is transported into the cerebrospinal fluid by an active transport mechanism and crosses the neuron plasma membranes to be re-phosphorylated in the cells [16]. In the cytoplasm, PL, $\mathrm{PN}$ and PM are converted into the $5^{\prime}$-phosphorylated vitamers by PL kinase, while PNPOx converts PNP and PMP into PLP.

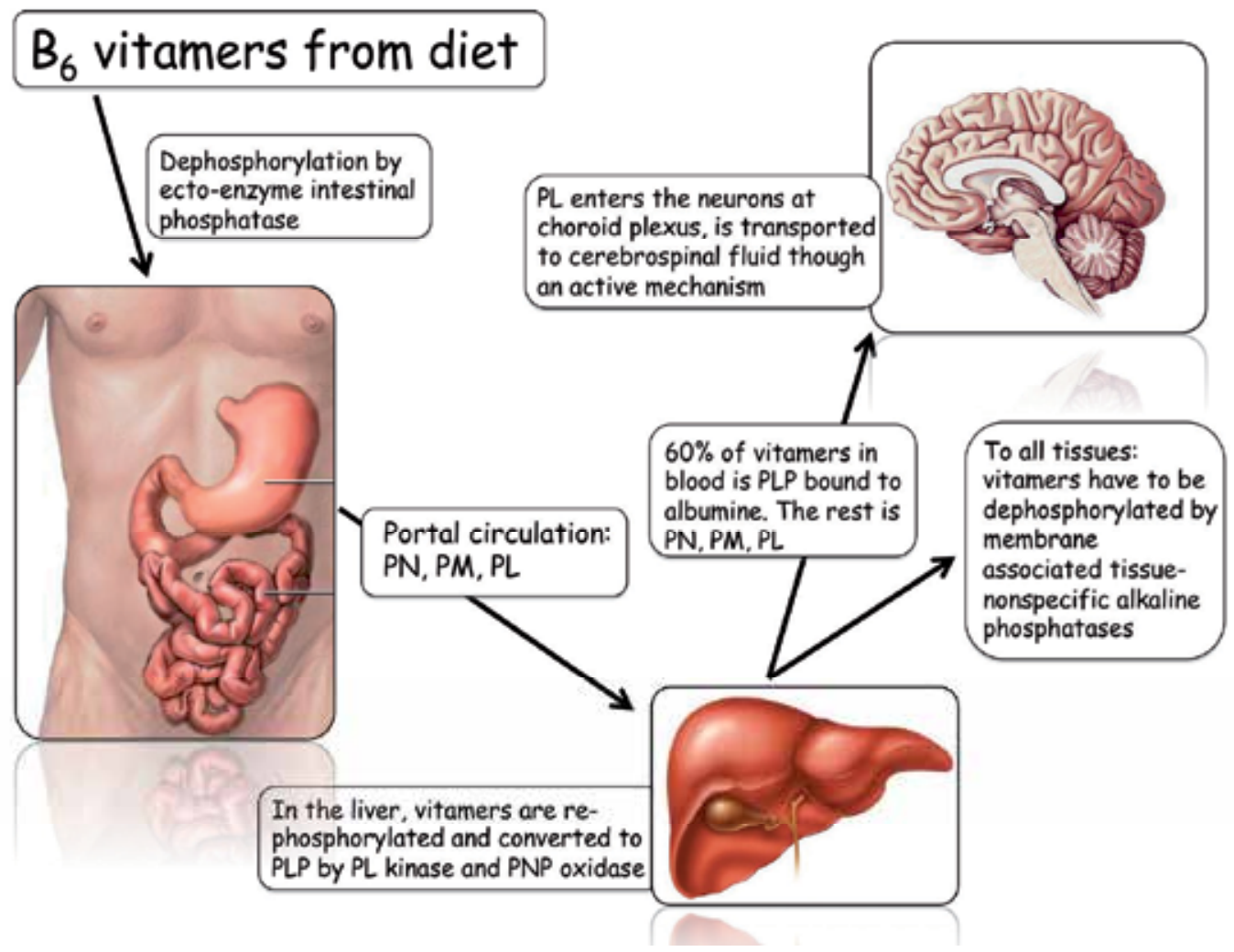

Fig. 4. Vitamin $\mathrm{B}_{6}$ transport in human organs. 
The expression of mRNA of all enzymes of the salvage pathway is ubiquitous, but is highly regulated at the level of transcription in a tissue-specific manner. While PL kinase is expressed in all tissues, although with the presence of isoforms [19], the major sites of PNPOx expression are liver, skeletal muscle and kidneys; PNPOx is also present, to a lesser extent, in the brain, in particular in the cerebral cortex [20]. Remarkably, PNPOx activity seems to be absent in liver and in neurally derived tumors [21,22]. It should be emphasized that the pool of free PLP in vivo must be maintained at a very low level in the body, to prevent toxic buildup. In fact, PLP being a very reactive aldehyde, easily combines with amines and thiols in the cell [23]. This characteristic has been related to the neurotoxic effect of excess consumption of vitamin $B_{6}$ [24], even at a dosage of 200 $\mathrm{mg} /$ day. The current recommended dietary allowance for $B_{6}$ is $1-2 \mathrm{mg} /$ day for adult individuals. People taking high doses of $\mathrm{B}_{6}$ are known to develop acute, profound and permanent sensory neuropathy, and in some instances motor neuropathy and damage of the central nervous system [25]. High doses of PLP are also found to induce convulsion in animal studies; suggested to be caused by formation of a Schiff base between PLP and a crucial lysine residue located in an extracellular loop of the GABA receptor [26]. PLP has also been reported to inhibit phenol sulfotransferase, an important detoxification enzyme [27].

One mechanism involved in PLP homeostasis is the regulation of its rate of production by PL kinase and PNPOx. Interestingly, it has been shown that both enzymes are able to bind their reaction product, PLP, and that their activity is finely regulated by it. Inhibition of PNPOx activity by the PLP product has been observed in the E. coli enzyme, with a $K_{i}$ of 8 $\mu \mathrm{M}$ [28]. Also, substrate inhibition of $E$. coli PL kinase by MgATP has been observed in the presence of either PNP or PLP [29]. A concurring mechanism for maintaining low levels of free PLP is expected to be its dephosphorylation by phosphatases. Catalytic conversion of pyridoxal to 4-pyridoxic acid by aldehyde oxidases and NAD-dependent dehydrogenases also keeps the level of PLP low [30]. These regulatory mechanisms keep the concentration of free PLP as low as $1 \mu \mathrm{M}$ in eukaryotic cells [31].

The low free PLP concentration in the cell is not believed to be sufficient to allow for spontaneous formation of holo- $\mathrm{B}_{6}$ enzymes [31,32] and raises the intriguing question of how PLP is actually delivered to the apoenzymes that use it as a cofactor. The importance of vitamin $\mathrm{B}_{6}$ in several cellular processes and in the onset of different pathologies should also be looked at from this new perspective. Studies on PNPOx and PL kinase suggest that once PLP is formed by these enzymes, it is unlikely to be released into solution, where it could be sequestered and/or dephosphorylated by phosphatases, and that it could be rather directly channeled to apo- $\mathrm{B}_{6}$ enzymes [32-34]. Failure to maintain a correct tuning between PLP biosynthesis and degradation, transport to peripheral tissues and delivery to newly formed $\mathrm{B}_{6}$ enzymes might end up in vitamin $\mathrm{B}_{6}$-associated pathogenesis. The salvage pathway is very efficient, resulting in only small amounts of vitamin $B_{6}$ being required in the diet. Vitamin $\mathrm{B}_{6}$ deficiency is rare in humans receiving a normal diet.

\subsection{PLP-dependent enzymes and brain function}

The role of PLP-dependent enzymes in brain function is vital since the synthesis of several neurotransmitters, including D-serine, D-aspartate, L-glutamate, glycine, $\gamma$-aminobutyric 
acid (GABA), serotonin, epinephrine, norepinephrine, histamine and dopamine is dependent on these enzymes. For example, branched-chain amino acid aminotransferase (BCAT), a PLP-dependent enzyme, catalyzes the transamination of essential branched-chain amino acids, such as leucine and valine, to form L-glutamate. L-Glutamate is considered to be one of the major excitatory signals in the mammalian central nervous system and is important for cognition, learning and memory. Low or abnormal levels have been implicated in Alzheimer's, Parkinson's, Huntington's, and Tourette's diseases [35-39]. LGlutamate also serves as a precursor for the formation of GABA, catalyzed by the PLPdependent enzyme glutamate decarboxylase (GAD). GABA is a major inhibitory neurotransmitter in the mammalian CNS. Low levels of GABA have been implicated in symptoms associated with epilepsy, convulsions, Parkinson's, Alzheimer's and Huntington's diseases [40-43]. The PLP-dependent enzyme DOPA decarboxylase (also known as aromatic L-amino acid decarboxylase (AAAD)) catalyzes the synthesis of dopamine via decarboxylation of L-3,4-dihydroxyphenylalanine (L-DOPA). Dopamine serves as a precursor for norepinephrine and epinephrine. AAAD also catalyzes the synthesis of serotonin via decarboxylation of L-5-hydroxytryptophan. Low levels of dopamine and serotonin have been involved in clinical disorders, including Parkinson's disease and Attention Deficit Hyperactivity Disorder (ADHD) [44,45]. The PLP-dependent enzyme serine racemase catalyzes the direct racemization of L-serine to D-serine. Endogenous D-serine is required for physiological NMDA (N-methyl D-aspartate) neurotransmission, neuronal plasticity, memory and learning [46]. Histidine decarboxylase (HDC), another PLP-dependent enzyme, is responsible for the catalytic conversion of Lhistidine to histamine. Histamine plays important roles in a number of physiological processes, among them as a neurotransmitter involved in memory $[47,48]$. Glycine is formed from L-serine by the PLP-dependent serine hydroxymethyltransferase and is degraded by the glycine cleavage system, of which one of the four protein components is glycine decarboxylase, another PLP-dependent enzyme. Glycine is also a neurotransmitter, acting as both inhibitory and excitatory functions within the CNS [49]. PLP-dependent enzymes also take part in the kynurenine oxidative pathway of tryptophan degradation, whose catabolic intermediates (kynurenic acid, 3-hydroxykynurenine and quinolinic acid) are involved in the physiological tuning of the CNS and in the etiogenesis and progression of several human neurodegenerative disorders [50,51].

It is obvious that inadequate PLP levels will result in loss of activity of $\mathrm{B}_{6}$ enzymes, leading to a significant decrease of some or several neurotransmitters. As mentioned above, PLP, as well as the other phosphorylated forms of vitamin $B_{6}$, do not easily cross the blood-brain barrier; therefore PLP must be synthesized within the brain tissues from the nonphosphorylated vitamers. Thus, the potential for PLP depletion in the brain is likely to occur with inactivation of the $\mathrm{B}_{6}$ salvage pathway enzymes [31]. Moreover, because apo- $\mathrm{B}_{6}$ enzymes in the brain compete for the available PLP, even a modest decrease in PLP availability could have more dire consequences on brain function than would be expected. Those apo- $\mathrm{B}_{6}$ enzymes that bind PLP with higher affinity may get most of the available PLP. As an example, DOPA decarboxylase is known to be drastically affected by PLP deficiency [52]. Consistently, inactivation of DOPA decarboxylase has been used in the past as a model for determining $\mathrm{B}_{6}$ status. 


\subsection{Pyridoxine 5'-phosphate oxidase (PNPOx)}

PNPOx is a flavin mononucleotide (FMN)-dependent enzyme, catalyzing the terminal step in the de novo vitamin $\mathrm{B}_{6}$ biosynthesis pathway in $E$. coli. The enzyme is also part of the salvage pathway in E. coli and mammalian cells [10,11,28,53-61]. FMN is a phosphorylated form of vitamin $B_{2}$ (riboflavin). The catalytic mechanism of PNPOx involves direct hydride transfer from the 4 ' hydroxyl group of PNP or the $4^{\prime}$ amino group of PMP to FMN to form $\mathrm{PLP}$ and $\mathrm{FMNH}_{2}$, respectively (Figure 3). Oxidized FMN is then regenerated by the transfer of two electrons to oxygen, forming hydrogen peroxide [57]. The enzyme is sluggish with a low catalytic rate constant ranging from $0.2 \mathrm{sec}^{-1}$ to $0.8 \mathrm{sec}^{-1}$ and $\mathrm{K}_{\mathrm{m}}$ values in the low micromolar range for both the substrates PNP and PMP $[28,55,56]$. The product PLP has a greater affinity than either of the substrates, suggesting that it may function as a product inhibitor of the enzyme [28,58,59].

The crystal structure of the human enzyme, as well as those from other species have been determined, all of which show a similar two-fold related dimer with two FMN molecules bound at the dimer interface making non-covalent interactions with both subunits (Figure 5) [55-57,60,61]. Mutations of key amino acid residues can affect folding of the enzyme into a form with diminished activity or no activity, can strongly influence the binding affinity of substrates, can reduce the affinity for the cofactor FMN and can affect catalytic activity. Knowing the residues involved in substrate and cofactor binding and in the catalytic mechanism is of paramount importance for understanding the function of PNPOx.

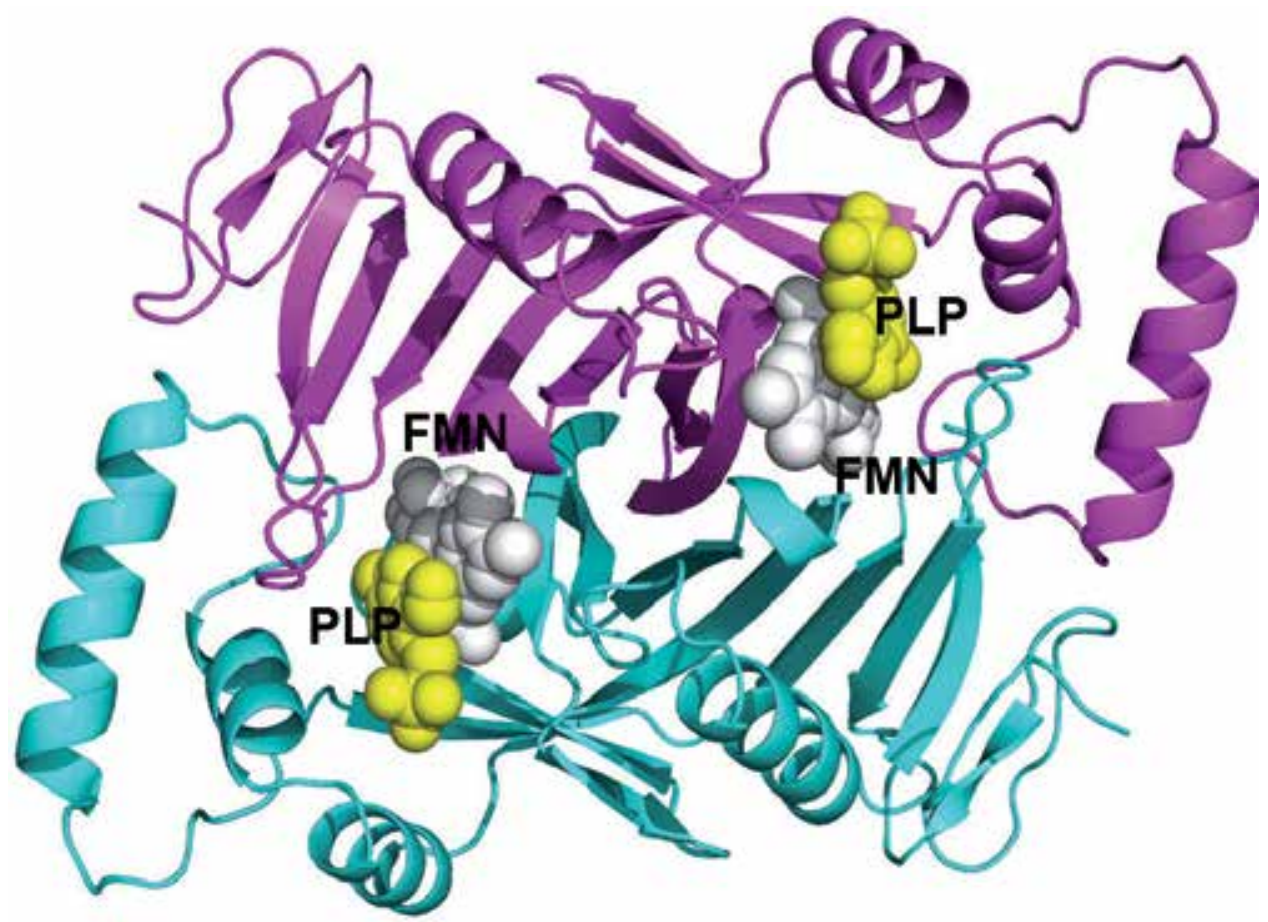

Fig. 5. Three-dimensional structure of human PNPOx complexed with the cofactor (FMN, in grey) and the product (PLP, in yellow). The two monomers forming the homodimeric structure of the protein are colored magenta and cyan. 
In the structure of human PNPOx, the isoalloxazine ring of FMN is involved in both hydrophobic and hydrogen-bond interactions with both subunits (monomers) of the protein, including Thr111, Glu174, Phe110, Leu98 and Met96 (Figure 6) [56]. The FMN ribityl group makes hydrogen-bond interactions with Met96, Gln139, Trp219, Pro261, Trp219, Lys117, Glu217, Gln174, Arg229, Arg138 and Arg141. The FMN phosphate moiety, which is located deeper into the protein structure, makes hydrogen-bond/salt-bridge interactions to Arg95, Arg141, Arg161, Ser175, Arg116, Lys117 and Arg229. The substrate PNP, as well as the product PLP, binds at the re-face of the bound FMN, where the PLP pyridine ring stacks parallel against the pyrazine and pyrimidine portions of the FMN isoalloxazine ring with extensive van der Waals contacts between the two, with the C4' of PLP and N5 of FMN separated by about $3.4 \AA$ (Figure 6). The phosphate moiety of PLP, which extends toward the surface of the binding pocket, makes salt bridges and hydrogen bond interactions to Lys100, Arg161, Arg225, Tyr157 and Ser165. The O3'-hydroxyl group of PLP makes a hydrogen bond interaction to His227, while the carbonyl oxygen on C4' makes a water-mediated interaction to Glu77. Lastly, the pyridine nitrogen makes a water-mediated hydrogen bond interaction with Trp206. Not only do the interactions described above help stabilize FMN and substrate binding, but they also ensure correct orientation of FMN and PNP for substrate oxidation. Additionally, the interactions with both FMN and the substrate/product, which are at the interface of the two subunits, help stabilize the homodimeric structure.

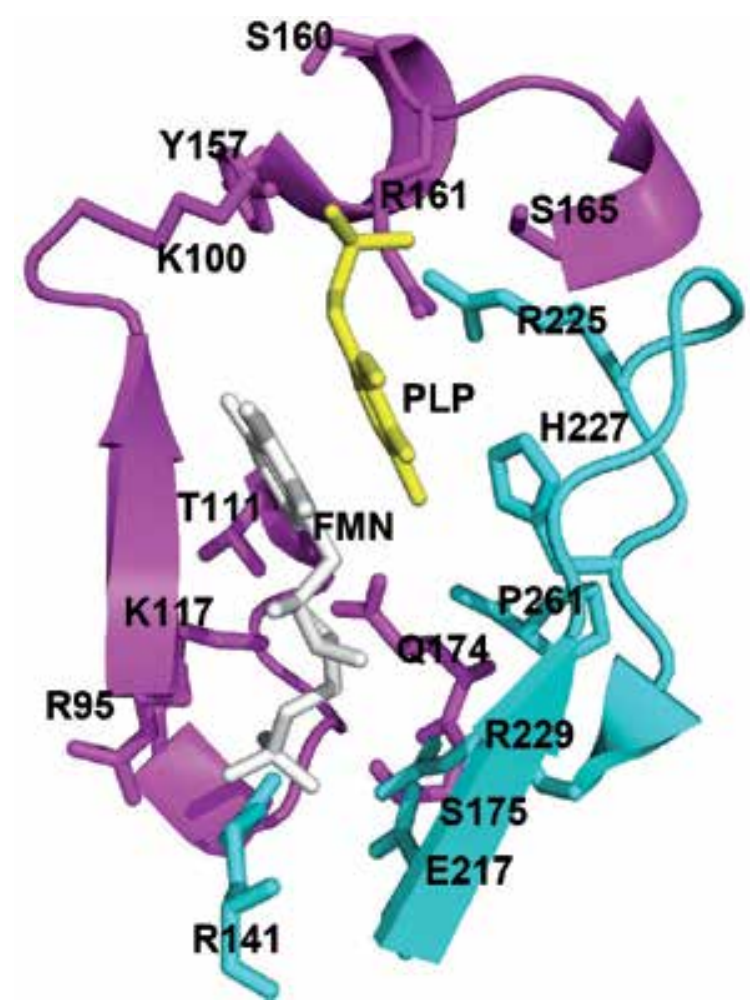

Fig. 6. The active site of human PNPOx, showing the bound PLP (yellow) at the re-face of the FMN (grey). The two monomers, forming the homodimeric structure of the protein, are colored magenta and cyan, respectively. For clarity, not all the residues interacting with the ligands are shown. 


\section{PLP deficiency and NEE}

PLP deficiency due to dietary insufficiency is rare, since most dietary sources contain vitamin $\mathrm{B}_{6}$. Nonetheless, pathogenic mutations in the PNPO gene encoding for PNPOx are known to cause a reduced or null activity of PNPOx, resulting in PLP deficiency. Disruption of the salvage pathway can also occur by pathogenic mutations in the PL kinase gene or by drug induced inhibition of PNPOx and PL kinase enzymatic activity [5,6,62-67]. Metabolites that accumulate as a consequence of inherited defects in enzymes of other metabolic pathways and drugs are also known to form complexes with PLP and reduce its availability [1,67-70]. Since PLP must be metabolized by brain cells to meet the demand of newly synthesized apo- $\mathrm{B}_{6}$ enzymes disruption of the PLP salvage pathway could lead to improper functioning of PLP-dependent enzymes in the brain, resulting in neurological disorders, such as NEE.

\subsection{PNPOx deficiency-related NEE}

The most severe form of NEE has been found to be due to mutations in PNPOx, which lead to null or decreased activity of the enzyme. Observed mainly in Turkish and Asian populations, this syndrome, called PNPOx deficiency, shows symptoms such as fetal distress, hypoglycemia, anemia, acidosis and asphyxia [2,5-7,71]. This disorder is sometimes referred to as PLP-dependent NEE as individuals with PNPO deficiency respond only to PLP or PL therapy, and not to the other forms of vitamin $\mathrm{B}_{6}$, such as PN and PM. At least seven mutations in the PNPO gene, including homozygous missense, stop codon, nonsense, splice site and frameshift mutations have been identified to cause PLP-dependent NEE [5,6,62-67]. Most patients with these mutations are born prematurely and the disease can be fatal. Surviving children are usually mentally retarded and show an abnormal dependence on PLP/PL. The fact that these patients are unresponsive to treatment with PN but responsive to PL demonstrates that the defect is in PNPOx, which is required for PN conversion to PLP. Conversion of PL to PLP is achieved by PL kinase and does not require PNPOx activity (Figure 2). However, PLP from the diet has to be converted to PL by phosphatases, and is then reconverted to PLP in the cells by PL kinase.

\subsection{Pathogenic mutations in PNPOx}

The human PNPOx gene is situated on chromosome 17q21.2 [72], and several mutations in the gene have been implicated in NEE. In a study by Mills et al., the authors sequenced the PNPOx gene in patients with NEE and identified homozygous missense (R229W, in which the arginine residue in position 229 is substituted with a tryptophan residue), splice site (IVS3-1g > a) and stop codon $(\mathrm{X} 262 \mathrm{Q})$ mutations [5]. The stop codon and splice site mutations result in null PNPOx activity. The missense mutation (in genetics, defined as a point mutation in which a single nucleotide is changed, resulting in a codon that codes for a different amino acid) was reported to lead to $\sim 70 \%$ reduction in PNPOx activity compared to the wild-type enzyme. Although significant, a $70 \%$ reduction in activity does not explain the observed drastic pathological effect in patients [5]. A later study showed the activity of the mutant enzyme to be almost null [66].

Other PNPOx, including two novel homozygous missense mutations (R95H and R95C, in which arginine residue 95 is substituted by either a histidine or a cysteine) and a nonsense 
mutation (A174X; a nonsense mutation is a point mutation in a sequence of DNA that results in a premature stop codon, or a nonsense codon in the transcribed mRNA, and in a truncated, incomplete, and usually nonfunctional protein product) have also been identified in several patients with classical symptoms of PLP-dependent NEE [5,6,62-67]. As will be discussed later, the elucidation of the crystal structure of PNPOx and the mechanism of PNP or PMP oxidation [57] have now contributed to the precise mechanism governing how some of these mutations lead to partial or complete loss of enzymatic function.

\subsection{Molecular basis of reduced enzymatic activity of PNPOx R229W and R95C mutants}

Our research group has carried out studies using site-directed mutagenesis, enzyme kinetics, X-ray crystallography and other functional studies aimed at understanding on a molecular level why PNPOx R229W and R95C mutants lead to reduced oxidase activity with the concomitant onset of NEE. The results from the R229W mutant have been published quite recently [66]. Arg229 is strictly conserved, and in the wild-type enzyme, this residue makes a short hydrogen-bond interaction with the FMN phosphate moiety (Figures 6 and 7). Arg229 also makes both intra- and inter-subunit hydrogen-bond interactions with Arg141, Ser175, Glu217 and Ala260. Another interesting observation is that the side chain of Arg229 is sandwiched tightly between the residues Arg141, Glu217, Ala260, Trp219, Gln139, and Pro261.

The R229W variant shows $\sim 850$-fold less catalytic efficiency than the wild-type enzyme due to a $\sim 192$-fold decrease in PNP affinity and $\sim 4.5$-fold decrease in the catalytic rate constant $\mathrm{k}_{\text {cat }}$. This shows that the in vitro activity of the R229 mutant enzyme is less than $0.2 \%$ of the wild-type enzyme, effectively making the activity so low that it will not function in the production of PLP from PNP. Fluorescence titration experiments also indicate an $\sim 50$-fold reduction in FMN affinity for the R229W mutant. Normally, wild-type PNPOx does not require the aadditional FMN to the assay because of its very tight binding. However, the mutant PNPOx loses even more activity unless FMN is added to the assay. Crystallographic studies showed that the substitution of the charged Arg229 residue with the larger tryptophan residue at the FMN binding site (Figure 7) has led to loss of hydrogen-bond and salt-bridge interactions with FMN. Additionally, the substitution with a bigger amino side chain has led to an alteration of the active site structure of PNPOx, resulting in loss of two critical hydrogen-bond interactions between PNP and the residues His227 and Arg225 (Figure 7). These two hydrogen-bond interactions have previously been shown to be important for substrate binding and proper orientation for catalysis by allowing the PNP ring to orient parallel to the FMN ring [57]. Expectedly, in the ternary three-dimensional structure of the mutant enzyme, the PLP and FMN ring moieties have moved significantly apart and are no longer parallel to each other (Figure 7) [66]. The C4' of the substrate and N5 of FMN are now separated by $\sim 4.5 \AA$ compared to the $\sim 3.4 \AA$ catalytic optimal position observed in the wild-type enzyme. In effect, the inability of Arg225 and His227 to make contact with the substrate has led to the destabilization of the substrate, preventing its proper orientation relative to FMN for efficient hydride transfer.

In yet another study by our group (unpublished data) with the PNPOx R95C mutant, we have also observed a 4.8 fold decrease in $\mathrm{k}_{\text {cat }}$ compared to the wild-type enzyme, and a $\sim 70$ fold increase in $\mathrm{K}_{\mathrm{m}}$, relative to the substrate PNP resulting in an $\sim 341$-fold decrease in the catalytic efficiency of the mutant enzyme compared to the wild-type enzyme. The FMN 
affinity is also reduced by $\sim 15 \%$ compared to the wild-type enzyme. Arg95 forms salt bridge/hydrogen bond interactions with the phosphate side chain of the FMN molecule utilizing two of the guanidinium group nitrogens (Figure 6), and the diminished binding of FMN to the enzyme may be a result of the loss of this interaction. In the absence of a crystal structure, we can only speculate that this mutation also leads to loss of several important interactions between the protein and PNP as evidenced by a decrease in PNP affinity, resulting in improper positioning of the substrate for optimal catalysis.

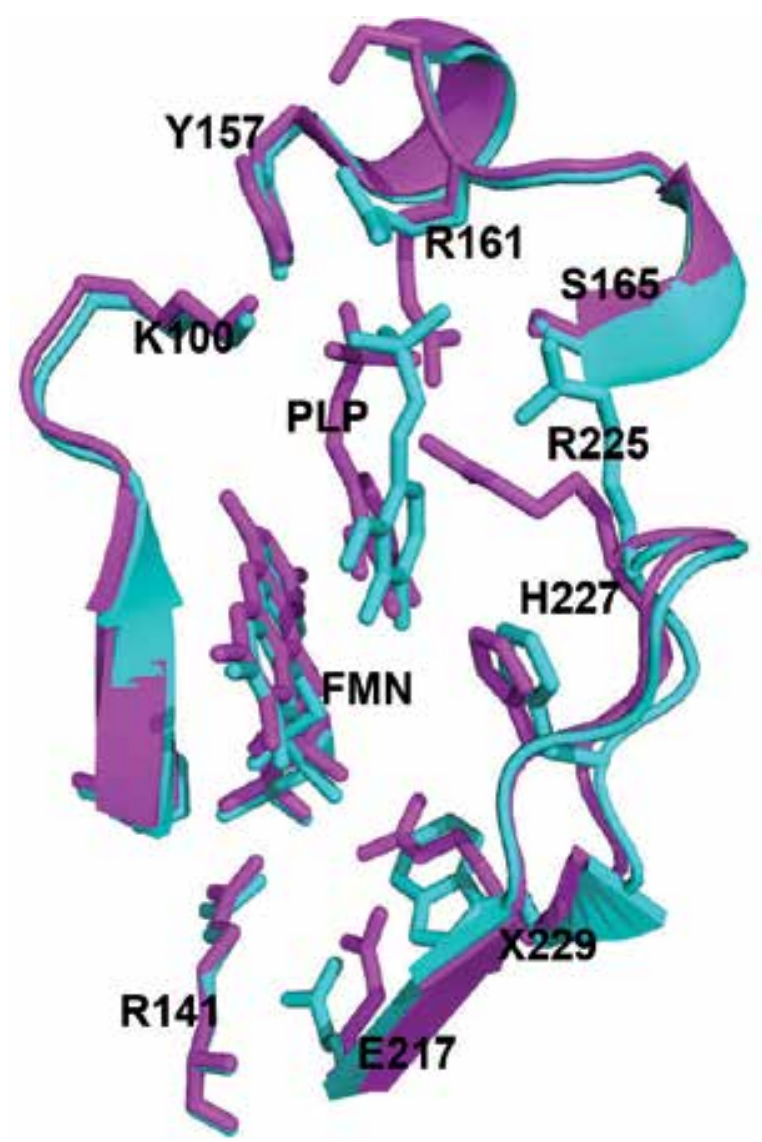

Fig. 7. Superposition of the active site of R229W PNPOx mutant (cyan) with that of wildtype PNPOx (magenta). Note the significant movements of the residues His227 and Arg225 in the R229W mutant form, compared to the wild-type, resulting in the ligand PLP moving away from its optimal catalytic position.

Neither of these mutations seems to have any significant effect on the secondary structure or thermal stability of the enzyme, even though affinity of the enzyme towards its cofactor FMN is diminished. These results provide molecular insight into how the R229W and R95C missense mutations affect PNPOx catalytic activity, and clearly explain why the treatment of NEE with PN is unsuccessful in patients with PNPOx mutants because of the inability of the enzyme to oxidize PNP (or PMP) to form PLP. The current treatment with PLP or PL is appropriate since the other enzyme involved in PLP metabolism, PL kinase, can convert PL 
to PLP. Nevertheless, it's clear that PLP made in the cell from PL is not enough, indicating that PL kinase alone and most likely PNPOx alone is not sufficient to meet the cell's requirements and both the salvage pathway enzymes are necessary to provide adequate PLP to activate the many different apo- $\mathrm{B}_{6}$ dependent enzymes. It is possible, although yet to be proven, that the use of riboflavin (vitamin $\mathrm{B}_{2}$ ) and $\mathrm{PN}$, in conjunction with PL, may offer some improvement over the current treatment protocol of PL or PLP alone due to the apparent loss of FMN from the PNPOx pathogenic variants.

\subsection{Predicting the molecular basis of reduced enzymatic activity of other PNPOx pathogenic mutants}

The precise mechanisms governing how the splice site (IVS3-1g > a), stop codon (X262Q), nonsense (A174X), and R95H missense mutations lead to partial or complete loss of PNPOx function are not known. Nevertheless, based on the crystal structure of PNPOx and the studies with the R95C and R229W mutants, several inferences can be made. In particular, it's easy to predict how the mutant $\mathrm{R} 95 \mathrm{H}$ may also affect the enzymatic activity based on the study with R95C. Although His95 may still be capable of forming hydrogen-bond interactions with the FMN phosphate, most likely the interaction would be weaker. Unlike the wild-type Arg, which makes a bidentate interaction with the FMN phosphate moiety, His can only utilize one of its nitrogens for interacting with the phosphate moiety. This mutation could also result in a decreased binding of PNP, as observed in the R229W and R95C mutants.

In the splice site (IVS3-1g > a) mutation, the residues 122-139 are absent from the enzyme. Some of these residues are involved in the formation of the FMN binding cavity and interact with the cofactor. Others contribute to either a hydrophobic patch or a network of inter-dimer hydrogenbond interactions that stabilize the tertiary and quaternary structure of the wild-type protein. Notable among these residues are Asn124, Ser128, Tyr132, Glu134, Glu139, Asn137, Trp133, Phe131 and Leu129. Therefore, these amino acids are expected to be important for a proper tertiary fold, dimer stabilization, and binding of the cofactor for consequent catalysis. It is perhaps no surprise that the splice site mutation leads to total enzyme dysfunction.

The abolition of the stop codon (X262Q) causes a C-terminal 28 amino acid extension (residues 262-289). The last residue at the C-terminus of the wild-type protein is the proline 261, and is strictly conserved in all PNP oxidases. Pro261 is tucked at the dimer interface, and is located at the re-face of the FMN and directly underneath PNP (Figure 6). It's likely that the C-terminus extension would result in at least the first five extended residues (Gln262, Leu263, Trp264, Asp265, Leu266) being accommodated at the dimer interface and more significantly occupy the substrate/product binding site permanently to inhibit substrate binding for catalysis. The extra 23 residues that extend from the active site may fold outside the dimer interface. On the other hand, if the 28-amino acid peptide extension does not fold as suggested above, it might disturb the dimer interface and result in the dissociation of the subunits into non-functional monomers. This mutation also results in a PNPOx with null activity.

\subsection{Clinical and general biochemical features of patients with PNPOx deficiency- related NEE and management of the disorder}

There are several clinical features that manifest in patients with PNPOx deficiency-related NEE or PLP-dependent NEE. These include, but are not limited to, fetal distress, convulsion, 
hypoglycemia, lactic acidosis, intractable seizures, hypoglycemia, acidosis, anemia, increased blood lactate, electroencephalogram with burst suppression pattern, and asphyxia $[5,6,62-67]$. Due to the severe deficiency of PLP, people with this disorder show reduced activity of several PLP-dependent enzymes, e.g. aromatic L-amino acid decarboxylase, ornithine aminotransferase, serine hydroxymethyltransferase, etc. As a result, cerebrospinal fluid (CSF), plasma and urine of these patients show deficient flux through PLP-dependent pathways [5,72], including lowered levels of homovanillic acid, 5-hydoxyindoleacetic acid and arginine; raised levels of 3-methoxytyrosine, L-DOPA, vanillacetate, glycine, threonine, histidine, ornithine and taurine. Most children with this disorder are born premature, progressively deteriorate and die within weeks if untreated. Surviving children are usually mentally retarded, and non-responsive to AEDs or pyridoxine, but dependent on a daily consumption of PLP to control the disease. This underscores the importance of promptly and properly diagnosing PNPOx deficiency-related NEE for correct pharmacological intervention.

In a study by Wang et al. [73], the investigators gave 94 patients who were on AEDs, but still had intractable epilepsy, intravenous PLP, that was continued for another 3 days if seizures were controlled. Eleven of the patients responded to the intravenous PLP with complete control of the seizures. PLP was then replaced with oral pyridoxine in these patients and six relapsed. For patients who had a seizure relapse, the intravenous PLP was again administered followed by a daily oral PLP. AEDs were sequentially withdrawn and the patients remained on oral PLP. Three of the patients relapsed while three remained seizure free with withdrawal of the AEDs. Clinicians can use a similar protocol to diagnose PNPOx deficiency-related NEE. It's also very important that after initial diagnosis of PLP dependency, there should be a follow-up to further confirm the diagnosis, i.e. whether the disorder is PNPOx deficiency related by carrying out a metabolic investigation, including screening the CSF for threonine and glycine levels, urine for vanillacetic acid levels and the CSF for neurotransmitters. Diagnosis should also be confirmed by mutational analysis of PNPOx genes.

Hoffmann et al., have suggested an oral PLP dose of $30 \mathrm{mg} / \mathrm{kg}$ per day in three doses plus $100 \mathrm{mg}$ i.v. pyridoxine (in a single dose), plus $3-5 \mathrm{mg} / \mathrm{kg}$ folinic acid for treating PNPOx deficiency-related NEE [6]. The investigators found no therapeutic response with pyridoxine and folinic acid when used without PLP (the latter compound discussed in some detail later). In some instances, the above recommended PLP dose may have to be increased to effectively treat the disease [6]. As noted by Wang et al., [73], it may also require concomitant use of PLP and AEDs to effectively treat the disorder. Although not yet proven, it might also be useful to administer PLP/PL in conjunction with riboflavin (vitamin $\mathrm{B}_{2}$ ), the metabolic precursor of FMN since studies showed that mutations could drastically decrease the affinity of PNPOx for the FMN cofactor [66]. Against this backdrop is the potential of neurotoxic side-effects of too much PLP.

\subsection{Pyridoxine-dependent NEE and management of the disorder}

There are several cases of NEE where patients responded to treatment with pyridoxine. These PN-dependent NEE patients rarely show the severe fetal distress, elevated threonine and glycine, PLP-dependent enzyme deficiency, and premature birth that characterize 
PNPOx deficiency-related NEE. PN-dependent NEE is an autosomal recessive disease of probably underestimated incidence, first described in 1954 [74], that is usually presented with neonatal epileptic encephalopathy. It is believed that lack of biological markers has contributed to the under diagnosis of several cases of PN-dependent NEE disorder [1].

Children with PN-dependent seizures have mutations in the ALDH7A1 gene (maps to 5 q31), which encodes for $\alpha$-aminoadipic semialdehyde dehydrogenase (also called antiquitin). Sequencing of ALDH7A1 gene from individuals with pyridoxine-dependent seizures revealed homozygous as well as compound heterozygous mutations [75]. A comprehensive review of these mutations (missense, nonsense, splice-site and small deletions) has recently been provided by Scharer et al. [70]. One of these (E399Q, a missense mutation) is of particularly high frequency, and seems to alter cofactor binding and catalysis, having a significant impact on enzyme activity.

Antiquitin functions as an aldehyde dehydrogenase within the cerebral lysine catabolism pathway by catalyzing the oxidation of a-aminoadipic semialdehyde, derived from pipecolic acid, to a-aminoadipic acid [70,75]. The abolished antiquitin activity thus results in accumulation of pipecolic acid, a-aminoadipic semialdehyde (a-AASA) and piperideine-6carboxylic acid (P6C) (Figure 8). P6C reacts with free PLP (Figure 8) forming a Knoevenagel condensation product depleting PLP, leading to an imbalance between the excitatory neurotransmitter glutamic acid and the inhibitory neurotransmitter GABA, thus providing an explanation for the observed seizures.

As noted by Gospe, et al., patients with PN-dependent NEE are usually diagnosed by administration from one to five $100 \mathrm{mg}$ doses of pyridoxine intravenously (under electroencephalography and vital-sign monitoring) during a clinical seizure, and noting prompt resolution of the clinical and electrographic features [4]. Alternatively, patients may be given $15 \mathrm{mg} / \mathrm{Kg}$ per day and noting complete control within a week [69,76]. For pyridoxine-deficient NEE, seizure relapse would occur after withdrawal of pyridoxine, but promptly controlled when re-administered. It's important that AEDs are sequentially withdrawn to confirm the diagnosis. It should be noted that, if there is no improvement with pyridoxine administration, the patient should be promptly treated with PLP. Screening for PN-dependent seizures is also possible via determination of plasma pipecolic acid and by measuring urinary or plasma aAASA and P6C levels [77,78]. Diagnosis is also confirmed by mutational analysis of the antiquitin gene. Typically, onset of such seizures is in neonatal period or shortly thereafter with irritability. Those infants need to be treated using pharmacologic doses of pyridoxine and a life-long therapy is needed. Seizures recur upon pyridoxine withdrawal, and if untreated die from status epilepticus. The children with this disorder show mild to moderate developmental delay, and do very well even with a single intravenous dose of PN to prevent seizures [69].

It's quite possible that treatment with PL/PLP in the pyridoxine deficient NEE patients could also work, and physicians should consider combining both PN and PLP/PL therapy. A lysine-free diet has also been recommended for treating this disorder. While the rationale for a lysine restricted diet is clear (Figure 8), the clinical effect on the outcome in pyridoxinedependent seizures still has to be documented before this can be universally recommended. Pyridoxine treatment does not address the potential toxicity of accumulating pipecolic acid, aAASA and P6C. 

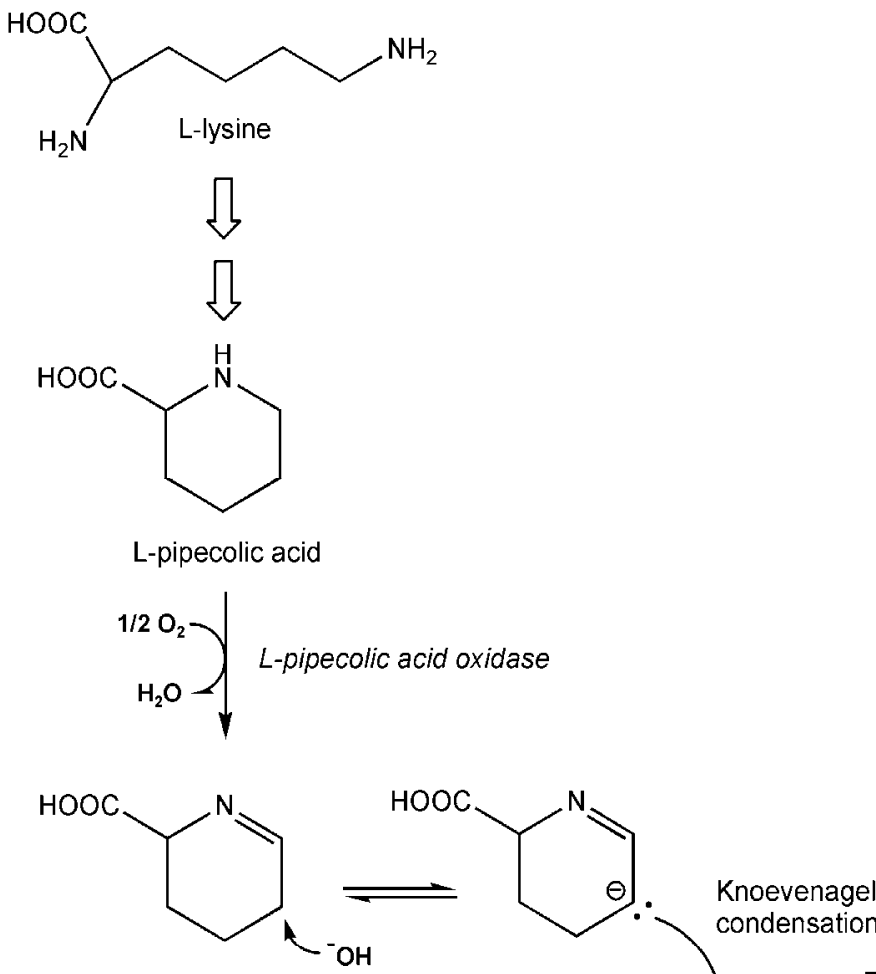

L- $\Delta^{1}$-piperideine-6-carboxylic acid<smiles>O=CC1CCCCC1O</smiles>

HOOC<smiles>CCC(N)CCCC=O</smiles>

$\alpha$-aminoadipic-semialdehyde
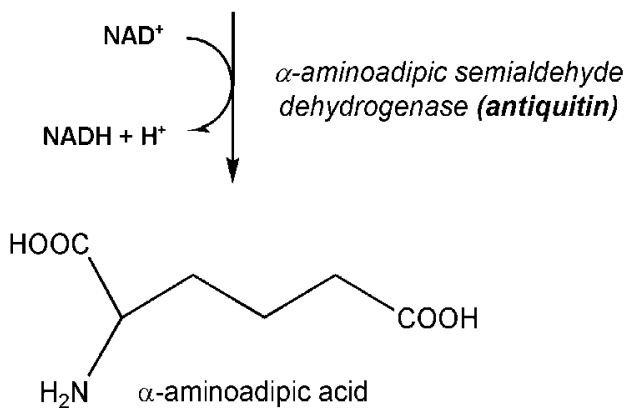

Fig. 8. The interaction of lysine degradation intermediates with pyridoxal 5'-phosphate. Pyridoxine-dependent NEE is due to a deficient activity of antiquitin. 
A few cases of neonatal epileptic encephalopathy have been reported to respond to treatment with folinic acid (5-formyl tetrahydrofolate, also known as leucovorin) [79,80]. These cases have been attributed to a distinct disorder with respect to pyridoxine-dependent epilepsy, until it was demonstrated that folinic acid-responsive patients also respond to pyridoxine, are antiquitin deficient and have mutations in the ALDH7A1 gene [80]. The response to folinic acid, which has been serendipitously discovered, is variable and is accompanied by a variable degree of responsiveness to pyridoxine. In our opinion, this observation, together with the fact that all folinic acid-responsive subjects showed the presence of two unidentified compounds in the cerebrospinal fluid, suggest that folinic acidresponsive seizures, although related to pyridoxine-dependent epilepsy, might be considered a different condition.

Another rare neonatal encephalopathy condition, the so-called hyperprolinemia type II, usually presented in childhood with fits and encephalopathy, is caused by the deficiency of the enzyme $\Delta^{1}$-pyrroline-5-carboxylate dehydrogenase, involved in proline degradation and encoded by the ALDH4A1 gene. This deficiency results in the accumulation of proline and L- $\Delta^{1}$-pyrroline-5-carboxylic acid in plasma and the consequent excessive excretion of the latter compound in urine [81]. L- $\Delta^{1}$-Pyrroline-5-carboxylic acid reacts in a Knoevenagel condensation with the aldehyde carbon of PLP, in a reaction similar to the one shown in Figure 8 for L- $\Delta^{1}$-piperideine-6-carboxylic acid [82,83]. The molecular mechanism of PLP depletion is indeed related to the one described before for the antiquitin-dependent epileptic encephalopathy. Seizures, which manifest in approximately half of the affected individuals, also respond well to pyridoxine treatment.

\section{Conclusions}

Neonatal epileptic encephalopathies are rare neurological disorders that may originate from PLP deficiency, owing to defects in the PNPOx enzyme of the vitamin $\mathrm{B}_{6}$ salvage pathway or in the antiquitin enzyme of the lysine degradation pathway. The basic metabolic faults of these two distinct causes of the disease are completely different. PNPOx defects slow down or block the conversion of PNP and PMP into PLP. Patients respond to treatment with PL or PLP but are insensitive to PN, and the disorder is usually referred to as PNPOx deficiencyrelated or PLP-dependent NEE. On the other hand, antiquitin mutations result in the accumulation of metabolic intermediates that react with PLP to form complexes that do not permit activation of apo- $\mathrm{B}_{6}$ enzymes into their active holo-forms. Patients affected by antiquitin defects can be treated with any of the $\mathrm{B}_{6}$ vitamers (PN is the most used vitamer), since their salvage pathway is functioning correctly to produce PLP. Several different pathological mutations of PNPOx and antiquitin have been characterized in detail. In particular, our research group has characterized the missense mutations R95C and R229W of PNPOx. Patients affected by this disorder present several clinical and general features that are useful for diagnosing and managing the disease.

Like NEE, several other neurological disorders, including seizures, autism, attention deficit hyperactive disorder, Alzheimer's, Down Syndrome, schizophrenia, Parkinson's, learning disability, anxiety disorders have also been associated with PLP deficiency [45,84-89]. This has inspired considerable interest in treating the symptoms of some of these disorders with vitamin $\mathrm{B}_{6}$. However, studies on the effect of $\mathrm{B}_{6}$ supplementation show conflicting results; some of the diseases have been responsive to PN or PLP/PL. For example PLP has been 
successfully used in Japan in the treatment of a range of epilepsies from infantile spasms to status epilepticus [89,90]. PN has also been shown to improve drug-induced parkinsonism and psychosis in a schizophrenic patient [88]. Other studies have shown no such beneficial effect, although most of these studies were conducted with PN and not PL or PLP. It is quite clear that therapy with PN or PM in patients with a defective PNPOx will not be successful, because this enzyme is needed to oxidize PNP and PMP into PLP after the kinase has changed them into PNP and PMP. Also, therapy with PL, PN or PM in patients with a dysfunctional PL kinase will not work, because these vitamers would have to be phosphorylated by the kinase.

It is also apparent from this review that neurological disorders, including epileptic episodes that are not responsive to conventional anticonvulsants could be due to mutations in either PNPOx, its salvage partner PL kinase or antiquitin. The genes coding for these enzymes are clearly candidates for mutational analysis in affected patients. If such an error is identified, studies could be performed to investigate the role of the mutations in the enzymatic activity and the associated phenotypes. This perhaps would point to the correct pharmacologic intervention.

\section{References}

[1] Stockler, S., Plecko, B., Gospe, S. M.Jr, Coulter-Mackie, M., Connolly, M., Van Karnebeek, C., Mercimek-Mahmutoglu, S., Hartmann, H., Scharer, G., Struijs, E., Tein, I., Jakobs, C., Clayton, P. And Van Hove, J. L. (2011) Pyridoxine Dependent Epilepsy And Antiquitin Deficiency Clinical And Molecular Characteristics And Recommendations For Diagnosis, Treatment And Follow-Up. Mol. Genet. Metab. . Doi:10.1016/J.Ymgme.2011.05.014

[2] Gospe, S. M.Jr. (2010) Neonatal Vitamin-Responsive Epileptic Encephalopathies. Chang Gung Med. J. 33, 1-12

[3] Gospe, S. M.,Jr. (2010) Pyridoxine-Dependent Epilepsy And Pyridoxine Phosphate Oxidase Deficiency: Unique Clinical Symptoms And Non-Specific Eeg Characteristics. Dev. Med. Child Neurol. 52, 602-603. Doi:10.1111/J.14698749.2010.03668.X

[4] Gospe, S. M.Jr. (2006) Pyridoxine-Dependent Seizures: New Genetic And Biochemical Clues To Help With Diagnosis And Treatment. Curr. Opin. Neurol. 19, 148-153. Doi:10.1097/01.Wco.0000218230.81301.12

[5] Mills, P. B., Surtees, R. A., Champion, M. P., Beesley, C. E., Dalton, N., Scambler, P. J., Heales, S. J., Briddon, A., Scheimberg, I., Hoffmann, G. F., Zschocke, J. And Clayton, P. T. (2005) Neonatal Epileptic Encephalopathy Caused By Mutations In The Pnpo Gene Encoding Pyridox(Am)Ine 5'-Phosphate Oxidase. Hum. Mol. Genet. 14, 1077-1086. Doi:10.1093/Hmg/Ddi120

[6] Hoffmann, G. F., Schmitt, B., Windfuhr, M., Wagner, N., Strehl, H., Bagci, S., Franz, A. R., Mills, P. B., Clayton, P. T., Baumgartner, M. R., Steinmann, B., Bast, T., Wolf, N. I. And Zschocke, J. (2007) Pyridoxal 5'-Phosphate May Be Curative In Early-Onset Epileptic Encephalopathy. J. Inherit. Metab. Dis. 30, 96-99. Doi:10.1007/S10545-0060508-4 
[7] Ormazabal, A., Oppenheim, M., Serrano, M., Garcia-Cazorla, A., Campistol, J., Ribes, A., Ruiz, A., Moreno, J., Hyland, K., Clayton, P., Heales, S. And Artuch, R. (2008) Pyridoxal 5'-Phosphate Values In Cerebrospinal Fluid: Reference Values And Diagnosis Of Pnpo Deficiency In Paediatric Patients. Mol. Genet. Metab. 94, 173177. Doi:10.1016/J.Ymgme.2008.01.004

[8] Percudani, R. And Peracchi, A. (2003) A Genomic Overview Of Pyridoxal-PhosphateDependent Enzymes. Embo Rep. 4, 850-854. Doi:10.1038/Sj.Embor.Embor914

[9] Schneider, G., Kack, H. And Lindqvist, Y. (2000) The Manifold Of Vitamin B6 Dependent Enzymes. Structure. 8, R1-6

[10] Mccormick, D. B. And Chen, H. (1999) Update On Interconversions Of Vitamin B-6 With Its Coenzyme. J. Nutr. 129, 325-327

[11] Jang, Y. M., Kim, D. W., Kang, T. C., Won, M. H., Baek, N. I., Moon, B. J., Choi, S. Y. And Kwon, O. S. (2003) Human Pyridoxal Phosphatase. Molecular Cloning, Functional Expression, And Tissue Distribution. J. Biol. Chem. 278, 50040-50046. Doi:10.1074/Jbc.M309619200

[12] Mccormick, D. B. (1989) Two Interconnected B Vitamins: Riboflavin And Pyridoxine. Physiol. Rev. 69, 1170-1198

[13] Mackey, A. D., Lieu, S. O., Carman, C. And Gregory, J. F.,3rd. (2003) Hydrolytic Activity Toward Pyridoxine-5'-Beta-D-Glucoside In Rat Intestinal Mucosa Is Not Increased By Vitamin B-6 Deficiency: Effect Of Basal Diet Composition And Pyridoxine Intake. J. Nutr. 133, 1362-1367

[14] Morrison, L. A. And Driskell, J. A. (1985) Quantities Of B6 Vitamers In Human Milk By High-Performance Liquid Chromatography. Influence Of Maternal Vitamin B6 Status. J. Chromatogr. 337, 249-258

[15] Said, H. M. (2004) Recent Advances In Carrier-Mediated Intestinal Absorption Of Water-Soluble Vitamins. Annu. Rev. Physiol. 66, 419-446. Doi:10.1146/Annurev.Physiol.66.032102.144611

[16] Surtees, R. A., Mills, P. B., And Clayton, P. (2006) Inborn Errors Affecting Vitamin B6 Metabolism, Future Medicine. 1, 615-620.

[17] Hoylaerts, M. F., Ding, L., Narisawa, S., Van Kerckhoven, S. And Millan, J. L. (2006) Mammalian Alkaline Phosphatase Catalysis Requires Active Site Structure Stabilization Via The N-Terminal Amino Acid Microenvironment. Biochemistry. 45, 9756-9766. Doi:10.1021/Bi052471+

[18] Millan, J. L. (2006) Alkaline Phosphatases : Structure, Substrate Specificity And Functional Relatedness To Other Members Of A Large Superfamily Of Enzymes. Purinergic Signal. 2, 335-341. Doi:10.1007/S11302-005-5435-6

[19] Fang, X., Zhou, Z. M., Lu, L., Yin, L. L., Li, J. M., Zhen, Y., Wang, H. And Sha, J. H. (2004) Expression Of A Novel Pyridoxal Kinase Mrna Splice Variant, Pkh-T, In Human Testis. Asian J. Androl. 6, 83-91

[20] Kang, J. H., Hong, M. L., Kim, D. W., Park, J., Kang, T. C., Won, M. H., Baek, N. I., Moon, B. J., Choi, S. Y. And Kwon, O. S. (2004) Genomic Organization, Tissue Distribution And Deletion Mutation Of Human Pyridoxine 5'-Phosphate Oxidase. Eur. J. Biochem. 271, 2452-2461. Doi:10.1111/J.1432-1033.2004.04175.X 
[21] Ngo, E. O., Lepage, G. R., Thanassi, J. W., Meisler, N. And Nutter, L. M. (1998) Absence Of Pyridoxine-5'-Phosphate Oxidase (Pnpo) Activity In Neoplastic Cells: Isolation, Characterization, And Expression Of Pnpo Cdna. Biochemistry. 37, 7741-7748. Doi:10.1021/Bi972983r

[22] Meisler, N. T. And Thanassi, J. W. (1988) Vitamin B6 Metabolism In Mca-Rh7777 Cells. Cancer Res. 48, 1080-1085

[23] Fu, T. F., Di Salvo, M. And Schirch, V. (2001) Distribution Of B6 Vitamers In Escherichia Coli As Determined By Enzymatic Assay. Anal. Biochem. 298, 314-321. Doi:10.1006/Abio.2001.5401

[24] Chung, J. Y., Choi, J. H., Hwang, C. Y. And Youn, H. Y. (2008) Pyridoxine Induced Neuropathy By Subcutaneous Administration In Dogs. J. Vet. Sci. 9, 127-131

[25] Morra, M., Philipszoon, H. D., D'andrea, G., Cananzi, A. R., L'erario, R. And Milone, F. F. (1993) Sensory And Motor Neuropathy Caused By Excessive Ingestion Of Vitamin B6: A Case Report. Funct. Neurol. 8, 429-432

[26] Salazar, P. And Tapia, R. (2001) Seizures Induced By Intracerebral Administration Of Pyridoxal-5'-Phosphate: Effect Of Gabaergic Drugs And Glutamate Receptor Antagonists. Neuropharmacology. 41, 546-553

[27] Bartzatt, R. And Beckmann, J. D. (1994) Inhibition Of Phenol Sulfotransferase By Pyridoxal Phosphate. Biochem. Pharmacol. 47, 2087-2095

[28] Zhao, G. And Winkler, M. E. (1995) Kinetic Limitation And Cellular Amount Of Pyridoxine (Pyridoxamine) 5'-Phosphate Oxidase Of Escherichia Coli K-12. J. Bacteriol. 177, 883-891

[29] Safo, M. K., Musayev, F. N., Di Salvo, M. L., Hunt, S., Claude, J. B. And Schirch, V. (2006) Crystal Structure Of Pyridoxal Kinase From The Escherichia Coli Pdxk Gene: Implications For The Classification Of Pyridoxal Kinases. J. Bacteriol. 188, 45424552. Doi:10.1128/Jb.00122-06

[30] Stanulovic, M., Jeremic, V., Leskovac, V. And Chaykin, S. (1976) New Pathway Of Conversion Of Pyridoxal To 4-Pyridoxic Acid. Enzyme. 21, 357-369

[31] Li, T. K., Lumeng, L. And Veitch, R. L. (1974) Regulation Of Pyridoxal 5'-Phosphate Metabolism In Liver. Biochem. Biophys. Res. Commun. 61, 677-684

[32] Kim, Y. T., Kwok, F. And Churchich, J. E. (1988) Interactions Of Pyridoxal Kinase And Aspartate Aminotransferase Emission Anisotropy And Compartmentation Studies. J. Biol. Chem. 263, 13712-13717

[33] Yang, E. S. And Schirch, V. (2000) Tight Binding Of Pyridoxal 5'-Phosphate To Recombinant Escherichia Coli Pyridoxine 5'-Phosphate Oxidase. Arch. Biochem. Biophys. 377, 109-114. Doi:10.1006/ Abbi.2000.1737

[34] Cheung, P. Y., Fong, C. C., Ng, K. T., Lam, W. C., Leung, Y. C., Tsang, C. W., Yang, M. And Wong, M. S. (2003) Interaction Between Pyridoxal Kinase And Pyridoxal-5Phosphate-Dependent Enzymes. J. Biochem. 134, 731-738

[35] Andre, V. M., Cepeda, C. And Levine, M. S. (2010) Dopamine And Glutamate In Huntington's Disease: A Balancing Act. Cns Neurosci. Ther. 16, 163-178. Doi:10.1111/J.1755-5949.2010.00134.X 
[36] Leckman, J. F., Bloch, M. H., Smith, M. E., Larabi, D. And Hampson, M. (2010) Neurobiological Substrates Of Tourette's Disorder. J. Child Adolesc. Psychopharmacol. 20, 237-247. Doi:10.1089/Cap.2009.0118

[37] Rupsingh, R., Borrie, M., Smith, M., Wells, J. L. And Bartha, R. (2011) Reduced Hippocampal Glutamate In Alzheimer Disease. Neurobiol. Aging. 32, 802-810. Doi:Doi: 10.1016/J.Neurobiolaging.2009.05.002

[38] Blandini, F., Porter, R. H. And Greenamyre, J. T. (1996) Glutamate And Parkinson's Disease. Mol. Neurobiol. 12, 73-94. Doi:10.1007/Bf02740748

[39] Butterworth, J., Yates, C. M. And Simpson, J. (1983) Phosphate-Activated Glutaminase In Relation To Huntington's Disease And Agonal State. J. Neurochem. 41, 440-447

[40] Pearl, P. L. And Gibson, K. M. (2004) Clinical Aspects Of The Disorders Of Gaba Metabolism In Children. Curr. Opin. Neurol. 17, 107-113

[41] Nishino, N., Fujiwara, H., Noguchi-Kuno, S. A. And Tanaka, C. (1988) Gabaa Receptor But Not Muscarinic Receptor Density Was Decreased In The Brain Of Patients With Parkinson's Disease. Jpn. J. Pharmacol. 48, 331-339

[42] Abbott, R., Pye, I. And Nahorski, S. (1982) Csf And Plasma Gaba Levels In Parkinson's Disease. J. Neurol. Neurosurg. Psychiatry. 45, 253-256

[43] Aoyagi, T., Wada, T., Nagai, M., Kojima, F., Harada, S., Takeuchi, T., Takahashi, H., Hirokawa, K. And Tsumita, T. (1990) Increased Gamma-Aminobutyrate Aminotransferase Activity In Brain Of Patients With Alzheimer's Disease. Chem. Pharm. Bull. (Tokyo). 38, 1748-1749

[44] Schrag, A. (2004) Psychiatric Aspects Of Parkinson's Disease--An Update. J. Neurol. 251, 795-804. Doi:10.1007/S00415-004-0483-3

[45] Herrmann, W., Lorenzl, S. And Obeid, R. (2007) Review Of The Role Of Hyperhomocysteinemia And B-Vitamin Deficiency In Neurological And Psychiatric Disorders--Current Evidence And Preliminary Recommendations. Fortschr Neurol. Psychiatr. 75, 515-527. Doi:10.1055/S-2007-980112

[46] Snyder, S. H. And Ferris, C. D. (2000) Novel Neurotransmitters And Their Neuropsychiatric Relevance. Am. J. Psychiatry. 157, 1738-1751

[47] Haas, H. And Panula, P. (2003) The Role Of Histamine And The Tuberomamillary Nucleus In The Nervous System. Nat. Rev. Neurosci. 4, 121-130. Doi:10.1038/Nrn1034

[48] Ohtsu, H. And Watanabe, T. (2003) New Functions Of Histamine Found In Histidine Decarboxylase Gene Knockout Mice. Biochem. Biophys. Res. Commun. 305, 443447

[49] Lopez-Corcuera, B., Geerlings, A. And Aragon, C. (2001) Glycine Neurotransmitter Transporters: An Update. Mol. Membr. Biol. 18, 13-20

[50] Stone, T. W., Mackay, G. M., Forrest, C. M., Clark, C. J. And Darlington, L. G. (2003) Tryptophan Metabolites And Brain Disorders. Clin. Chem. Lab. Med. 41, 852-859. Doi:10.1515/Cclm.2003.129

[51] Schwarcz, R. And Pellicciari, R. (2002) Manipulation Of Brain Kynurenines: Glial Targets, Neuronal Effects, And Clinical Opportunities. J. Pharmacol. Exp. Ther. 303, 1-10. Doi:10.1124/Jpet.102.034439 
[52] Rahman, M. K., Nagatsu, T., Sakurai, T., Hori, S., Abe, M. And Matsuda, M. (1982) Effect Of Pyridoxal Phosphate Deficiency On Aromatic L-Amino Acid Decarboxylase Activity With L-Dopa And L-5-Hydroxytryptophan As Substrates In Rats. Jpn. J. Pharmacol. 32, 803-811

[53] Hill, R. E., Himmeldirk, K., Kennedy, I. A., Pauloski, R. M., Sayer, B. G., Wolf, E. And Spenser, I. D. (1996) The Biogenetic Anatomy Of Vitamin B6. A 13c Nmr Investigation Of The Biosynthesis Of Pyridoxol In Escherichia Coli. J. Biol. Chem. 271, 30426-30435

[54] Di Salvo, M., Yang, E., Zhao, G., Winkler, M. E. And Schirch, V. (1998) Expression, Purification, And Characterization Of Recombinant Escherichia Coli Pyridoxine 5'Phosphate Oxidase. Protein Expr. Purif. 13, 349-356. Doi:10.1006/Prep.1998.0904

[55] Di Salvo, M. L., Safo, M. K., Musayev, F. N., Bossa, F. And Schirch, V. (2003) Structure And Mechanism Of Escherichia Coli Pyridoxine 5'-Phosphate Oxidase. Biochim. Biophys. Acta. 1647, 76-82

[56] Musayev, F. N., Di Salvo, M. L., Ko, T. P., Schirch, V. And Safo, M. K. (2003) Structure And Properties Of Recombinant Human Pyridoxine 5'-Phosphate Oxidase. Protein Sci. 12, 1455-1463

[57] Di Salvo, M. L., Ko, T. P., Musayev, F. N., Raboni, S., Schirch, V. And Safo, M. K. (2002) Active Site Structure And Stereospecificity Of Escherichia Coli Pyridoxine-5'Phosphate Oxidase. J. Mol. Biol. 315, 385-397. Doi:10.1006/Jmbi.2001.5254

[58] Kazarinoff, M. N. And Mccormick, D. B. (1975) Rabbit Liver Pyridoxamine (Pyridoxine) 5'-Phosphate Oxidase. Purification And Properties. J. Biol. Chem. 250, 3436-3442

[59] Choi, S. Y., Churchich, J. E., Zaiden, E. And Kwok, F. (1987) Brain Pyridoxine-5Phosphate Oxidase. Modulation Of Its Catalytic Activity By Reaction With Pyridoxal 5-Phosphate And Analogs. J. Biol. Chem. 262, 12013-12017

[60] Safo, M. K., Mathews, I., Musayev, F. N., Di Salvo, M. L., Thiel, D. J., Abraham, D. J. And Schirch, V. (2000) X-Ray Structure Of Escherichia Coli Pyridoxine 5'-Phosphate Oxidase Complexed With Fmn At 1.8 A Resolution. Structure. 8, 751-762

[61] Pedelacq, J. D., Rho, B. S., Kim, C. Y., Waldo, G. S., Lekin, T. P., Segelke, B. W., Rupp, B., Hung, L. W., Kim, S. I. And Terwilliger, T. C. (2006) Crystal Structure Of A Putative Pyridoxine 5'-Phosphate Oxidase (Rv2607) From Mycobacterium Tuberculosis. Proteins. 62, 563-569. Doi:10.1002/Prot.20824

[62] Martinez De Haas, M. G., Poels, P. J., De Weert, C. J., Thomas, C. M., Rooyackers, J. M. And Hoefnagels, W. H. (1997) Subnormal Vitamin B6 Levels In Theophylline Users. Ned. Tijdschr. Geneeskd. 141, 2176-2179

[63] Khayat, M., Korman, S. H., Frankel, P., Weintraub, Z., Hershckowitz, S., Sheffer, V. F., Ben Elisha, M., Wevers, R. A. And Falik-Zaccai, T. C. (2008) Pnpo Deficiency: An Under Diagnosed Inborn Error Of Pyridoxine Metabolism. Mol. Genet. Metab. 94, 431-434. Doi:10.1016/J.Ymgme.2008.04.008

[64] Bagci, S., Zschocke, J., Hoffmann, G. F., Bast, T., Klepper, J., Muller, A., Heep, A., Bartmann, P. And Franz, A. R. (2008) Pyridoxal Phosphate-Dependent Neonatal Epileptic Encephalopathy. Arch. Dis. Child. Fetal Neonatal Ed. 93, F151-2. Doi:10.1136/Adc.2006.115162 
[65] Ruiz, A., Garcia-Villoria, J., Ormazabal, A., Zschocke, J., Fiol, M., Navarro-Sastre, A., Artuch, R., Vilaseca, M. A. And Ribes, A. (2008) A New Fatal Case Of Pyridox(Am)Ine 5'-Phosphate Oxidase (Pnpo) Deficiency. Mol. Genet. Metab. 93, 216-218. Doi:10.1016/J.Ymgme.2007.10.003

[66] Musayev, F. N., Di Salvo, M. L., Saavedra, M. A., Contestabile, R., Ghatge, M. S., Haynes, A., Schirch, V. And Safo, M. K. (2009) Molecular Basis Of Reduced Pyridoxine 5'-Phosphate Oxidase Catalytic Activity In Neonatal Epileptic Encephalopathy Disorder. J. Biol. Chem. 284, 30949-30956. Doi:10.1074/Jbc.M109.038372

[67] Laine-Cessac, P., Cailleux, A., Allain, P. (1997) Mechanisms Of The Inhibition Of Human Erythrocyte Pyridoxal Kinase By Drugs. Biochem. Pharmacol. 54, 863

[68] Rankin, P. M., Harrison, S., Chong, W. K., Boyd, S. And Aylett, S. E. (2007) PyridoxineDependent Seizures: A Family Phenotype That Leads To Severe Cognitive Deficits, Regardless Of Treatment Regime. Dev. Med. Child Neurol. 49, 300-305. Doi:10.1111/J.1469-8749.2007.00300.X

[69] Baxter, P. (2003) Pyridoxine-Dependent Seizures: A Clinical And Biochemical Conundrum. Biochim. Biophys. Acta. 1647, 36-41

[70] Scharer, G., Brocker, C., Vasiliou, V., Creadon-Swindell, G., Gallagher, R. C., Spector, E. And Van Hove, J. L. (2010) The Genotypic And Phenotypic Spectrum Of Pyridoxine-Dependent Epilepsy Due To Mutations In Aldh7a1. J. Inherit. Metab. Dis. 33, 571-581. Doi:10.1007/S10545-010-9187-2

[71] Clayton, P. T., Surtees, R. A., Devile, C., Hyland, K. And Heales, S. J. (2003) Neonatal Epileptic Encephalopathy. Lancet. 361, 1614

[72] Aaltonen, J., Bjorses, P., Sandkuijl, L., Perheentupa, J. And Peltonen, L. (1994) An Autosomal Locus Causing Autoimmune Disease: Autoimmune Polyglandular Disease Type I Assigned To Chromosome 21. Nat. Genet. 8, 83-87. Doi:10.1038/Ng0994-83

[73] Wang, H. S., Kuo, M. F., Chou, M. L., Hung, P. C., Lin, K. L., Hsieh, M. Y. And Chang, M. Y. (2005) Pyridoxal Phosphate Is Better Than Pyridoxine For Controlling Idiopathic Intractable Epilepsy. Arch. Dis. Child. 90, 512-515. Doi:10.1136/Adc.2003.045963

[74] Hunt, A. D.Jr, Stokes, J.,Jr, Mccrory, W. W. And Stroud, H. H. (1954) Pyridoxine Dependency: Report Of A Case Of Intractable Convulsions In An Infant Controlled By Pyridoxine. Pediatrics. 13, 140-145

[75] Mills, P. B., Struys, E., Jakobs, C., Plecko, B., Baxter, P., Baumgartner, M., Willemsen, M. A., Omran, H., Tacke, U., Uhlenberg, B., Weschke, B. And Clayton, P. T. (2006) Mutations In Antiquitin In Individuals With Pyridoxine-Dependent Seizures. Nat. Med. 12, 307-309. Doi:10.1038/Nm1366

[76] Gospe, S. M. (2002) Pyridoxine-Dependent Seizures: Findings From Recent Studies Pose New Questions. Pediatr. Neurol. 26, 181-185

[77] Plecko, B., Hikel, C., Korenke, G. C., Schmitt, B., Baumgartner, M., Baumeister, F., Jakobs, C., Struys, E., Erwa, W. And Stockler-Ipsiroglu, S. (2005) Pipecolic Acid As A Diagnostic Marker Of Pyridoxine-Dependent Epilepsy. Neuropediatrics. 36, 200205. Doi:10.1055/S-2005-865727 
[78] Plecko, B., Hoeger, H., Jakobs, C., Struys, E., Stromberger, C., Leschnik, M., Muehl, A. And Stoeckler-Ipsiroglu, S. (2005) Pipecolic Acid Concentrations In Brain Tissue Of Nutritionally Pyridoxine-Deficient Rats. J. Inherit. Metab. Dis. 28, 689-693. Doi:10.1007/S10545-005-0071-4

[79] Hyland, K., Buist, N. R., Powell, B. R., Hoffman, G. F., Rating, D., Mcgrath, J. And Acworth, I. N. (1995) Folinic Acid Responsive Seizures: A New Syndrome? J. Inherit. Metab. Dis. 18, 177-181

[80] Gallagher, R. C., Van Hove, J. L., Scharer, G., Hyland, K., Plecko, B., Waters, P. J., Mercimek-Mahmutoglu, S., Stockler-Ipsiroglu, S., Salomons, G. S., Rosenberg, E. H., Struys, E. A. And Jakobs, C. (2009) Folinic Acid-Responsive Seizures Are Identical To Pyridoxine-Dependent Epilepsy. Ann. Neurol. 65, 550-556. Doi:10.1002/Ana.21568

[81] Walker, V., Mills, G. A., Peters, S. A. And Merton, W. L. (2000) Fits, Pyridoxine, And Hyperprolinaemia Type Ii. Arch. Dis. Child. 82, 236-237

[82] Farrant, R. D., Walker, V., Mills, G. A., Mellor, J. M. And Langley, G. J. (2001) Pyridoxal Phosphate De-Activation By Pyrroline-5-Carboxylic Acid. Increased Risk Of Vitamin B6 Deficiency And Seizures In Hyperprolinemia Type Ii. J. Biol. Chem. 276, 15107-15116. Doi:10.1074/Jbc.M010860200

[83] Walker, V., Mills, G. A., Mellor, J. M., Langley, G. J. And Farrant, R. D. (2003) A Novel Pyrroline-5-Carboxylic Acid And Acetoacetic Acid Adduct In Hyperprolinaemia Type Ii. Clin. Chim. Acta. 331, 7-17

[84] Mousain-Bosc, M., Roche, M., Polge, A., Pradal-Prat, D., Rapin, J. And Bali, J. P. (2006) Improvement Of Neurobehavioral Disorders In Children Supplemented With Magnesium-Vitamin B6. Ii. Pervasive Developmental Disorder-Autism. Magnes. Res. 19, 53-62

[85] Nogovitsina, O. R. And Levitina, E. V. (2006) Effect Of Magne-B6 On The Clinical And Biochemical Manifestations Of The Syndrome Of Attention Deficit And Hyperactivity In Children. Eksp. Klin. Farmakol. 69, 74-77

[86] Fuso, A., Nicolia, V., Cavallaro, R. A., Ricceri, L., D'anselmi, F., Coluccia, P., Calamandrei, G. And Scarpa, S. (2008) B-Vitamin Deprivation Induces Hyperhomocysteinemia And Brain S-Adenosylhomocysteine, Depletes Brain SAdenosylmethionine, And Enhances Ps1 And Bace Expression And AmyloidBeta Deposition In Mice. Mol. Cell. Neurosci. 37, 731-746. Doi:10.1016/J.Mcn.2007.12.018

[87] Adams, J. B., George, F. And Audhya, T. (2006) Abnormally High Plasma Levels Of Vitamin B6 In Children With Autism Not Taking Supplements Compared To Controls Not Taking Supplements. J. Altern. Complement. Med. 12, 59-63. Doi:10.1089/Acm.2006.12.59

[88] Sandyk, R. And Pardeshi, R. (1990) Pyridoxine Improves Drug-Induced Parkinsonism And Psychosis In A Schizophrenic Patient. Int. J. Neurosci. 52, 225-232

[89] Takuma, Y. (1998) Acth Therapy For Infantile Spasms: A Combination Therapy With High-Dose Pyridoxal Phosphate And Low-Dose Acth. Epilepsia. 39 Suppl 5, 4245 
[90] Nakagawa, E., Tanaka, T., Ohno, M., Yamano, T. And Shimada, M. (1997) Efficacy Of Pyridoxal Phosphate In Treating An Adult With Intractable Status Epilepticus. Neurology. 48, 1468-1469 


\title{
Disaccharides in the Treatment of Hepatic Encephalopathy in Patients with Cirrhosis
}

\author{
Praveen Sharma \\ Department of Hepatology, Institute of Liver and Biliary Sciences, \\ New Delhi, \\ India
}

\section{Introduction}

Hepatic encephalopathy (HE) is one of the major complications of liver cirrhosis. It has a considerable socioeconomic impact as it reduces the individual's quality of life and needs repeated admission in hospital for its treatment. HE is a complex neuropsychiatric syndrome, which may complicate acute or chronic liver failure and defined as a disturbance in central nervous system function because of hepatic insufficiency and after exclusion of other brain disease. Traditionally, HE is graded according to the West Haven criteria, which define HE grades I-IV based on the presence of specific clinical signs and symptoms and their severity $(1,2)$. The Working Party proposed a nomenclature of HE and classified into three types : type A, associated with acute liver failure; type B, associated with the existence of porto-systemic communications without intrinsic liver disease; and type $C$, associated with cirrhosis, which, depending on the manner of presentation, is classified into episodic HE (related to precipitating factors) or spontaneous (persistent HE)(1). However, patients with cirrhosis present with a continuous severity spectrum of neuropsychological symptoms ranging from entire normality (HE 0) up to obvious deficits (3). Even in minimal HE (MHE) without obvious clinical symptoms, neuropsychological and neurophysiological testing uncovers deficits which impact on the quality of life and the fitness to drive a motor vehicle (4-7).In this article we will focus mainly on treatment of HE related to cirrhosis (type-C) using disaccharides.

\section{Pathophysiology of HE and role of ammonia}

The pathogenesis of HE in cirrhosis is complex and has multiple components, including ammonia, inflammatory cytokines, benzodiazepine-like compounds, and manganese, that cause functional impairment of neuronal cells. Numerous factors have been shown to precipitate HE including infections, sedatives, gastrointestinal bleeding, dietary protein excess, diuretics, and electrolyte imbalance (8)

There is consensus that ammonia is a key toxin in HE, which may sensitize the brain to the different precipitating factors $(9,10)$. Ammonia is created primarily from nitrogenous products in the diet, bacterial metabolism of urea and proteins in the colon, and from deamination of glutamine in the small intestine by the enzyme glutaminase $(11,12)$. Due to 
hepatocellular dysfunction and portosystemic collaterals in cirrhosis, the ammonia concentration rises in the bloodstream and crosses the blood-brain barrier. Exposure to ammonia results in structural alterations in neurons manifested primarily as astrocyte swelling resembling Alzheimer type II astrocytosis $(13,14)$.In chronic liver disease, urea synthesis is impaired and the brain acts as an alternative major ammonia detoxification pathway. Astrocytes have the ability to eliminate ammonia by the synthesis of glutamine through amidation of glutamate by the enzyme glutamine synthetase (15-18). Glutamine enters the mitochondria (Trojan horse hypothesis) and is subsequently cleaved by glutaminase to ammonia and glutamate, thereby increasing intracellular ammonia levels. This causes a feed-forward loop as elevation in mitochondrial ammonia causes production of reactive nitrogen and oxygen species (RNOS) leading to further edema(19-22).Studies have also examined the role of ammonia-induced potentiation of aquaporin- 4 , a water channel expressed copiously in astrocytes(23). Astrocyte edema also causes depletion of taurine, a molecule that serves as an antioxidant and helps to buffer ammonia-induced toxicity(24).Increased ammonia levels cause abnormal cerebral blood flow and glucose metabolism. Single photon emission computed tomographic (SPECT) studies have demonstrated altered cerebral perfusion with hyperammonemia resulting in a redistribution of blood flow from the cortex to the subcortical regions, which was associated with impaired performance on neuropsychiatric testing(25). Further support for the ammonia-glutaminebrain water hypothesis has been provided by inducing hyperammonemia in patients with cirrhosis through the oral administration of an amino acid solution. An increase in brain glutamine, reduction in magnetization transfer ratio, and significant deterioration in neuropsychological function was suggestive of an increase in brain water (26).

\section{Treatment of HE}

The most important aspect of HE management is prompt recognition and treatment of precipitating factors. Fessel and colleagues demonstrated that HE is caused by reversible factors in over $80 \%$ of patients(27). These common reversible factors include constipation, infection, hypokalemia, gastrointestinal bleeding, increased protein intake, sedatives, and tranquilizers. Thus, identifying and correcting the reversible precipitating factors can be beneficial in treating most episodes of HE. Administration of various ammonia-lowering therapies such as nonabsorbable disaccharides and selected antimicrobial agents and other less utilized alternative and investigational treatment options include administration of benzodiazepine receptor antagonists, branched-chain amino acids (BCAAs) and ornithine aspartate. If infection is suspected or the patient has clinical evidence of ascites, a paracentesis should be performed with analysis of cell count, gram stain, and culture to evaluate for spontaneous bacterial peritonitis. Additional investigations for infection should be performed with blood cultures, urinalysis, or chest radiographs. Broadspectrum antibiotics should be initiated after obtaining cultures if infection is suspected, especially in patients with stage III or IV HE. Hypokalemia can be corrected with oral or intravenous potassium. Severe hyponatremia, particularly serum sodium less than $120 \mathrm{mEq} / \mathrm{L}$, should be addressed with appropriate free-water restriction(28). Vasopressin receptor antagonists may be considered for symptomatic patients with severe hyponatremia. HE precipitated by dietary protein intake or use of sedatives often resolves gradually with supportive care $(29,30)$. 


\section{Non-absorbable disaccharides and mechanism of action}

Non-absorbable disaccharides are recommended as first-line pharmacotherapy in current guidelines(2,28).

Current therapies for HE are based on ammonia lowering, with the hypothesis that the colon is the primary organ that generates ammonia $(2,28)$. Lactulose is the most commonly utilized non-absorbable disaccharide for HE. Lactulose, a synthetic disaccharide, is comprised of the monosaccharides lactose and galactose, and is available as a syrup. Doses are generally titrated to achieve two to four semi-soft stools daily, with typical doses of $20 \mathrm{~g} / 30 \mathrm{~mL}$ orally three to four times per day. A second non-absorbable disaccharide, lactitol, has also been used in the treatment of HE, but it is not currently commercially available in some countires like US(31,32). Lactitol (p-galactosido-sorbitol) is a disaccharide analog of lactulose which is neither absorbed nor broken down in the small intestine, but is extensively metabolized by colonic bacteria. It is available in a highly soluble crystalline powder form and is reported to be less sweet in taste than lactulose. Clinical trials have reported lactitol dosages of 10-12 g every 6 hours, titrated to two bowel movements daily to be effective in treatment of HE (31,33). Disaccharides (both lactulose and lactitol) remain undigested until they reach the colon, where they get metabolized by the bacteria in the colon to acetic and lactic acid. This acidification of colonic lumen inhibits bacterial ammonia production and trap ammonia as non-diffusable ammonium in the intestinal lumen.Due to their cathartic effect it facilitates a 4 -fold increase in faecal nitrogen excretion. Both effects result in reduced levels of ammonia in the colon and portal blood. (Figure-1)(34-36).

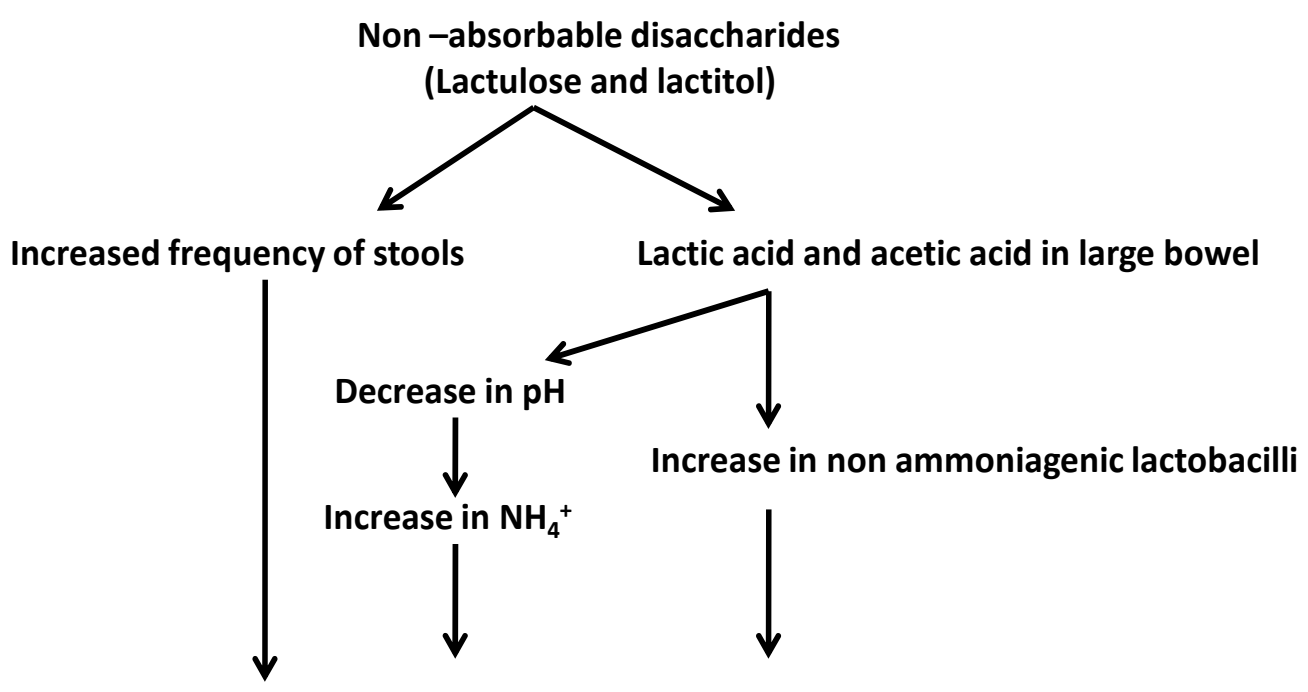

Decrease in ammonia absorption

Fig. 1. Mechanism of actions of disaccharides. 


\section{Clinical efficacy of non-absorbable disaccharides}

\subsection{Lactulose or lactitol versus placebo or no intervention}

\subsubsection{Overt hepatic encephalopathy}

The non-absorbable disaccharides have been a mainstay of therapy for HE for decades, and have been extensively studied in several small clinical trials since the late 1960s for this indication. In most of these trials patients had cirrhosis with acute, chronic, acute or chronic or minimal hepatic encephalopathy (37-44). Oral lactulose was used in majority of these studies though some had used lactitol and lactulose enemas also (40). In most of the studies the daily mean doses of lactulose ranged from $30 \mathrm{~g}$ to $80 \mathrm{~g}$ (median $50 \mathrm{~g}$ ) to obtain two to three semisoft stools per day. The median duration of treatment was 15 days (range 5 to 360 days). None of the trials followed up patients after the end of treatment. Uribe M et al (40) performed a double-blind, controlled trial to study the efficacy of acidifying enemas of lactitol and lactose versus nonacidifying tap-water enemas in 45 episodes of acute portalsystemic encephalopathy. A favorable response to treatment was obtained in $19(86 \%)$ of the patients receiving lactitol enemas and in 14 (78\%) of those receiving lactose enemas. They concluded that acidifying agents like lactose and lactitol are effective and superior to tapwater enemas for the treatment of acute nitrogenous portal-systemic encephalopathy. Study by Mas et al(45) in 53 patients with acute hepatic encephalopathy which were treated with lactitol $60 \mathrm{~g} /$ day for $5-10$ days concluded lactitol to be effective in $81 \%$ of patients. A recent meta-analysis evaluated 22 clinical trials in order to better assess the utilization of nonabsorbable disaccharides in the management of HE when compared with placebo, no intervention or antimicrobials. Compared with placebo or no intervention, lactulose and lactitol seemed to reduce the risk of no improvement of hepatic encephalopathy (relative risk $0.62,95 \%$ confidence interval 0.46 to 0.84 ). However high quality trials found no significant effect of lactulose or lactitol on the risk of no improvement $(0.92,0.42$ to 2.04), whereas low quality trials found a significant beneficial effect of lactulose or lactitol $(0.57$, 0.40 to 0.83$)(46,47)$. At the present time, however, there is a lack of sufficient evidence to thoroughly refute the use of non-absorbable disaccharides for the treatment of HE. Sharma et al(48) had shown that high baseline model for end stage liver disease(MELD), high total leukocyte count, low serum sodium, low mean arterial pressure, and presence of hepatocellular carcinoma were predictors of nonresponse to lactulose.

\section{Disaccharides in treatment of minimal hepatic encephalopathy}

Trials in patients with minimal hepatic encephalopathy (MHE) found that lactulose or lactitol significantly reduced the risk of no improvement assessed by various psychometric tests $(0.61$, 0.47 to 0.79 ). Compared with placebo or no intervention, lactulose and lactitol had no significant effect on mortality but tended to lower blood ammonia. A summary analysis of some clinical trials of non-absorbable disaccharides versus placebo/ no treatment is shown in table-1.In a recent study Prasad et al(44) found that treatment with lactulose improved both cognitive function and health-related quality of life, as measured by the Sickness Impact Profile(SIP), when compared against a 'no treatment' patient group. The mean total SIP score improved among patients in the treated group (baseline, 10.39 [95\% CI 9.36-11.43]; after 3 months, 3.77 [95\% CI 2.52-5.02]) compared with patients in the untreated group (baseline, 10.36 [95\% CI 8.98-11.73]; after 3 months, 10.39 [95\% CI 8.36-12.42]); $\mathrm{p}=0.002$. Improvement in health related quality of life (HRQOL) was related to the improvement in psychometry. Study 
by Sharma et al had shown that on univariate and multivariate analysis, serum sodium and venous ammonia were the only two parameters associated with nonresponse to lactulose(49). Reported adverse events were not serious, and all originated from the gastrointestinal tract (diarrhoea, flatulence, abdominal pain, or nausea)(46).

\begin{tabular}{|c|c|c|c|c|c|c|}
\hline Trial & \begin{tabular}{|l|}
$\begin{array}{l}\text { Study } \\
\text { design }\end{array}$ \\
\end{tabular} & Patients & No & treatment & Assessment & Efficacy \\
\hline $\begin{array}{l}\text { Simmons et } \\
\text { al (1970)(37) }\end{array}$ & Parallel & $\mathrm{AHE}+\mathrm{CHE}$ & 26 & $\begin{array}{l}\text { Lactulose } \\
\text { /glucose }\end{array}$ & $\begin{array}{l}\text { Clinical grading, } \\
\text { ammonia, stool } \\
\text { production }\end{array}$ & $\begin{array}{l}\text { Lactulose= } \\
\text { glucose }\end{array}$ \\
\hline $\begin{array}{l}\text { Rodgers et al } \\
(1973)(38)\end{array}$ & Crossever & $\mathrm{CHE}$ & 6 & \begin{tabular}{|l} 
Lactulose \\
/ sorbitol
\end{tabular} & $\begin{array}{l}\text { Clinical grading, } \\
\text { EEG, Ammonia }\end{array}$ & $\begin{array}{l}\text { Lactuose= } \\
\text { sorbitol }\end{array}$ \\
\hline $\begin{array}{l}\text { Corazza et } \\
\text { al(1982)(39) }\end{array}$ & Parallel & $\mathrm{CHE}$ & 32 & $\begin{array}{l}\text { Lactulose } \\
\text { / placebo }\end{array}$ & $\begin{array}{l}\text { Encephalopathy } \\
\text { intensity score, } \\
\text { ammonia }\end{array}$ & $\begin{array}{l}\text { Lactulose } \\
\text { better than } \\
\text { placebo } \\
\end{array}$ \\
\hline $\begin{array}{l}\text { Uribe et al } \\
(1987)(40)\end{array}$ & Parallel & AHE & 15 & $\begin{array}{l}\begin{array}{l}\text { Lactulose } \\
\text { enema }\end{array} \\
\end{array}$ & $\begin{array}{l}\text { Mortality, clinical } \\
\text { grading }\end{array}$ & $\begin{array}{l}\text { Lactulose> } \\
\text { placebo }\end{array}$ \\
\hline $\begin{array}{l}\text { Horsmans et } \\
\text { al (1997)(41) }\end{array}$ & Parallel & MHE & 14 & $\begin{array}{l}\text { Lactulose } \\
\text { / placebo }\end{array}$ & $\begin{array}{l}\text { Psychometric tests, } \\
\text { ammonia levels }\end{array}$ & $\begin{array}{l}\text { Lactulose > } \\
\text { placebo }\end{array}$ \\
\hline $\begin{array}{l}\text { Wantabe et } \\
\text { al (1997)(42) }\end{array}$ & Parallel & $\mathrm{MHE}$ & 36 & $\begin{array}{l}\text { Lactulose } \\
\text { / no treatment }\end{array}$ & $\begin{array}{l}\text { Three psychometric } \\
\text { tests, ammonia }\end{array}$ & $\begin{array}{l}\text { Lactulose > } \\
\text { placebo }\end{array}$ \\
\hline $\begin{array}{l}\text { Dhiman et al } \\
(2000)(43)\end{array}$ & Parallel & MHE & 26 & \begin{tabular}{|l|} 
Lactulose \\
/ no treatment \\
\end{tabular} & psychometric tests & $\begin{array}{l}\text { Lactulose > } \\
\text { placebo }\end{array}$ \\
\hline $\begin{array}{l}\text { Prasad et al } \\
(2007)(44)\end{array}$ & Parallel & MHE & 61 & \begin{tabular}{|l|} 
Lactulose \\
/ no treatment \\
\end{tabular} & $\begin{array}{l}\text { psychometric tests } \\
\text { and HRQOL }\end{array}$ & $\begin{array}{l}\text { Lactulose > } \\
\text { placebo }\end{array}$ \\
\hline
\end{tabular}

AHE: acute hepatic encephalopathy CHE: Chronic hepatic encephalopathy

MHE: Minimal hepatic encephalopathy EEG: Electroencephalography

Table 1. Comparison of non absorbable disaccharides and placebo or no treatment for hepatic encephalopathy.

\section{Lactulose versus lactitol for the treatment of hepatic encephalopathy}

Lactulose and lactitol both have been used for the treatment of HE and lactulose has been compared with lactitol in various studies (32,33,50-57)(Table-2).Morgan et al(32) enrolled twenty-five cirrhotic patients experiencing 28 episodes of acute hepatic encephalopathy which were randomized blindly to treatment with either lactitol $(n=15)$ or lactulose $(n=13)$. At the end of the trial, $67 \%$ of the patients in the lactitol group and $69 \%$ of the lactulose group were clinically normal. Metaanalysis by Blanc et al(56) studied the efficiency and tolerance of both compounds(lactulose and lactitol) by using a meta-analysis on the basis of published controlled trials. Analyzed parameters were the portosystemic encephalopathy index of Conn after treatment, the percentage of improved patients and the percentage of patients who had ill effects related to the treatment (flatulence, diarrhea). Bibliographical screening revealed five studies comparing the effects of lactitol and lactulose in chronic hepatic encephalopathy. Four crossover studies were done that included 48 patients and one parallel study that included 29 patients. The duration of the treatment ranged from 3 to 6 months. All studies found a similar efficiency with both drugs. However, they exhibited some discrepancies in the relative frequency of adverse reactions (flatulence). 
Meta-analysis showed no statistical differences in the portosystemic encephalopathy index after lactitol or lactulose treatment. The percentage of improved patients after lactitol or lactulose was similar. In contrast, the analysis revealed a higher frequency ( $p$ less than 0.01) of flatulence in patients treated with lactulose compared with those treated with lactitol(56). In conclusion, this meta-analysis shows no statistical difference between therapeutic effects of lactitol and lactulose, but it does show a higher frequency of flatulence with lactulose. However another metaanalysis by Camma et al (57) showed that lactitol was as effective as other disaccharides in the treatment of encephalopathy: pooled odds ratio was $0.83,95 \%$ confidence interval was 0.38-1.82. Patients experienced fewer side effects during treatment with lactitol, but the pooled odds ratio was not statistically significant. In all studies lactitol was considered more palatable $(50,55)$. Clinical effectiveness of lactitol, in long-term treatment of chronic encephalopathy, is similar to those of lactulose. It seems that lactitol has lower side effects than lactulose.

\begin{tabular}{|c|c|c|c|c|c|c|}
\hline Trial & Study design & Patients & No & $\begin{array}{l}\text { Treatment } \\
\text { duration }\end{array}$ & Assessment & Efficacy \\
\hline \begin{tabular}{|l|} 
Lanthier et \\
al (1985)(50)
\end{tabular} & Crossover & $\mathrm{CHE}$ & 5 & 6 months & $\begin{array}{l}\text { Clinical examination, } \\
\text { psychometric tests, } \\
\text { ammonia levels, EEG, } \\
\text { cerebral blood flow }\end{array}$ & $\begin{array}{l}\text { Lactulose } \\
=\text { lactitol }\end{array}$ \\
\hline \begin{tabular}{|l|} 
Morgan and \\
Hawley et \\
al $(1987)(32)$ \\
\end{tabular} & $\begin{array}{l}\text { Parallel, } \\
\text { double blind }\end{array}$ & AHE & 25 & 5 days & $\begin{array}{l}\text { Psychometric tests, } \\
\text { EEG, PSE index }\end{array}$ & $\begin{array}{l}\text { Lactulose } \\
=\text { lactitol }\end{array}$ \\
\hline $\begin{array}{l}\text { Heredia et } \\
\text { al(1987)(51) }\end{array}$ & Parallel & AHE & 40 & 5 days & $\begin{array}{l}\text { Mortality, clinical } \\
\text { grading, PSE grade, } \\
\text { adverse events }\end{array}$ & $\begin{array}{l}\text { Lactulose } \\
=\text { lactitol }\end{array}$ \\
\hline $\begin{array}{l}\text { Heredia et } \\
\text { al(1988)(34) }\end{array}$ & $\begin{array}{l}\text { Randomized, } \\
\text { crossover }\end{array}$ & $\mathrm{CHE}$ & 25 & 6months & $\begin{array}{l}\text { Psychometric tests, } \\
\text { ammonia levels, EEG, } \\
\text { PSE index }\end{array}$ & $\begin{array}{l}\text { Lactulose } \\
=\text { lactitol }\end{array}$ \\
\hline $\begin{array}{l}\text { Morgan et } \\
\text { al(1989)(52) }\end{array}$ & \begin{tabular}{|l|} 
Double-blind, \\
randomized, \\
Crossover \\
\end{tabular} & MHE & 9 & 3 months & $\begin{array}{l}\text { Psychometric tests, } \\
\text { ammonia levels, EE }\end{array}$ & $\begin{array}{l}\text { Lactulose } \\
=\text { lactitol }\end{array}$ \\
\hline $\begin{array}{l}\text { Riggio et al } \\
1989(53)\end{array}$ & Parallel & $\begin{array}{l}\mathrm{CHE}+ \\
\mathrm{MHE}\end{array}$ & 31 & 6 months & $\begin{array}{l}\text { PSE Index, new } \\
\text { episodes of HE, } \\
\text { adverse events }\end{array}$ & $\begin{array}{l}\text { Lactulose } \\
=\text { lactitol }\end{array}$ \\
\hline \begin{tabular}{|l|} 
Grandi \\
$1991(54)$ \\
\end{tabular} & Cross-over & $\mathrm{CHE}$ & 40 & - & $\begin{array}{l}\text { PSE Index, adverse } \\
\text { events }\end{array}$ & \begin{tabular}{|l|}
$\begin{array}{l}\text { Lactulose } \\
=\text { lactitol }\end{array}$ \\
\end{tabular} \\
\hline $\begin{array}{l}\text { Pai et al } \\
1995(55)\end{array}$ & Parallel & AHE & 45 & 5 days & $\begin{array}{l}\text { PSE Index, adverse } \\
\text { events }\end{array}$ & $\begin{array}{l}\text { Lactitol>la } \\
\text { ctulose }\end{array}$ \\
\hline \begin{tabular}{|l|} 
Blanc P et al \\
$1992(56)$
\end{tabular} & Metaanalysis & $\mathrm{CHE}$ & 77 & 3-6months & PSE Index & $\begin{array}{l}\text { Lactulose } \\
=\text { lactitol }\end{array}$ \\
\hline \begin{tabular}{|l|} 
Cammà C et \\
al 1993(57)
\end{tabular} & Metaanalysis & $\mathrm{CHE}$ & - & - & PSE Index & $\begin{array}{l}\text { Lactulose } \\
=\text { lactitol }\end{array}$ \\
\hline
\end{tabular}

AHE: acute hepatic encephalopathy CHE: Chronic hepatic encephalopathy

MHE: Minimal hepatic encephalopathy PSE Index: Portosystemic encephalopathy index

Table 2. Comparison of lactulose and lactitol for hepatic encephalopathy. 


\section{Comparison of lactulose and antimicrobial agents for hepatic encephalopathy}

Certain oral antibiotics can reduce urease-producing bacteria in the intestines, resulting in a decrease of ammonia production and absorption through the gastrointestinal tract. Antibiotics are used generally in patients who do not tolerate nonabsorbable disaccharides or in patients who are symptomatic on nonabsorbable disaccharides and require a second agent. Antibiotics with activity against urease-producing bacteria (e.g. neomycin, metronidazole) are effective in reducing intestinal ammonia production. However, the safety and efficacy of these antibiotics in treatment of HE have not been adequately demonstrated. Neomycin and other antimicrobials are utilized as a treatment modality in HE due to their ability to inhibit ammonia production by intestinal bacteria(58). Conn et al(58) conducted a randomized double blind clinical comparison of neomycin and lactulose in 33 cirrhotic patients with chronic portal-systemic encephalopathy. Serial assessments were made in all patients regarding mental status, asterixis, trailmaking test (TMT), electroencephalograms (EEG) and arterial ammonia levels. Both neomycin-sorbitol and lactulose were effective in the majority of patients (83 and $90 \%$, respectively. Atterbury et al (59) evaluated in double-blind, randomized study the efficacy of lactulose and compared with neomycin-sorbitol in 45 episodes of acute nitrogenous portal-systemic encephalopathy (PSE) induced by dietary protein, azotemia, or gastrointestinal hemorrhage. All patients had underlying cirrhosis, and at the time of randomization had encephalopathy of at least grade 2 severity and arterial ammonia concentrations greater than 150 microgram/100 ml. Two thirds of the patients in each group returned to normal mental status and more than $80 \%$ in each group showed at least one grade improvement in mental state. In addition, there was equivalent improvement in asterixis, in the performance of the Number Connection Test, in the electroencephalographic pattern, and in arterial ammonia concentration. The principal difference between the two groups was a greater reduction in stool $\mathrm{pH}$ after lactulose therapy than after neomycin-sorbitol therapy. Other antimicrobials, including metronidazole and vancomycin, have been studied to a more limited extent than neomycin(59-61)(Table-3). Orlandi et al (60) conducted a randomized

\begin{tabular}{|l|l|l|l|l|l|}
\hline Trial & Study design & $\begin{array}{l}\text { No of } \\
\text { patients }\end{array}$ & $\begin{array}{l}\text { Duration of } \\
\text { treatment }\end{array}$ & Assessment & Efficacy \\
\hline $\begin{array}{l}\text { Conn et } \\
\text { al(1977)(58) }\end{array}$ & $\begin{array}{l}\text { Neomycin vs } \\
\text { lactulose } \\
\text { (double-blind, } \\
\text { randomized, } \\
\text { crossover) }\end{array}$ & 29 & $\begin{array}{l}\text { 10 days } \\
\text { each } \\
\text { arm before } \\
\text { crossover }\end{array}$ & $\begin{array}{l}\text { Mental status, } \\
\text { asterixis } \\
\text { score, EEG, ammonia } \\
\text { levels, PSE index }\end{array}$ & $\begin{array}{l}\text { Neomycin = } \\
\text { lactulose }\end{array}$ \\
\hline $\begin{array}{l}\text { Atterbury et } \\
\text { al(1978)(59) }\end{array}$ & Parallel & 47 & 7 days & $\begin{array}{l}\text { Mental status, } \\
\text { asterixis } \\
\text { score, EEG, ammonia } \\
\text { levels, PSE index }\end{array}$ & $\begin{array}{l}\text { Neomycin = } \\
\text { lactulose }\end{array}$ \\
\hline $\begin{array}{l}\text { Orlandi et } \\
\text { al(1981)(60) }\end{array}$ & Single blind & 173 & 14 days & $\begin{array}{l}\text { Mental status, } \\
\text { asterixis } \\
\text { score, EEG, ammonia } \\
\text { levels, HE change }\end{array}$ & $\begin{array}{l}\text { Neomycin = } \\
\text { lactulose }\end{array}$ \\
\hline
\end{tabular}

EEG: Electroencephalography

Table 3. Comparison of lactulose and neomycin for hepatic encephalopathy. 


\begin{tabular}{|c|c|c|c|c|c|}
\hline Trial & Study design & $\begin{array}{l}\text { No of } \\
\text { patients }\end{array}$ & $\begin{array}{l}\text { Duration of } \\
\text { treatment }\end{array}$ & Assessment & Efficacy \\
\hline $\begin{array}{l}\text { Festi et } \\
\text { al(1993)(64) }\end{array}$ & $\begin{array}{l}\text { Lactulose } \\
\text { (open-label) }\end{array}$ & 21 & 21 & $\begin{array}{l}\text { Neurological } \\
\text { signs of HE, } \\
\text { asterixis score, } \\
\text { HRNB, EEG, } \\
\text { ammonia levels }\end{array}$ & $\begin{array}{l}\text { Rifaximin } \\
=\text { lactulose }\end{array}$ \\
\hline $\begin{array}{l}\text { Bucci and } \\
\text { Palmieri } \\
(1993)(68)\end{array}$ & $\begin{array}{l}\text { Lactulose } \\
\text { (double-blind, } \\
\text { double- } \\
\text { dummy) }\end{array}$ & 58 & 15 & $\begin{array}{l}\text { Neurological } \\
\text { status, asterixis } \\
\text { score, HRNB, } \\
\text { cancellation tasks, } \\
\text { EEG, ammonia } \\
\text { levels }\end{array}$ & $\begin{array}{l}\text { Rifaximin } \\
>\text { lactulose }\end{array}$ \\
\hline $\begin{array}{l}\text { Massa } \\
\text { et al(1993)(70) }\end{array}$ & $\begin{array}{l}\text { Lactulose } \\
\text { (double-blind, } \\
\text { double- } \\
\text { dummy) } \\
\end{array}$ & 40 & 15 & $\begin{array}{l}\text { HE index severity, } \\
\text { mental status, } \\
\text { cancellation tasks, } \\
\text { HRNB, EEG }\end{array}$ & $\begin{array}{l}\text { Rifaximin } \\
>\text { lactulose }\end{array}$ \\
\hline $\begin{array}{l}\text { Fera et } \\
\text { al(1993)(72) }\end{array}$ & $\begin{array}{l}\text { Lactulose } \\
\text { (double-blind, } \\
\text { double- } \\
\text { dummy) }\end{array}$ & 40 & $\begin{array}{l}\text { First } 2 \text { weeks } \\
\text { of each month } \\
\text { for } 3 \text { months }\end{array}$ & $\begin{array}{l}\text { Mental status, } \\
\text { asterixis score, } \\
\text { cancellation } \\
\text { tasks, HRNB, } \\
\text { EEG, ammonia } \\
\text { levels, PSE } \\
\text { index } \\
\end{array}$ & $\begin{array}{l}\text { Rifaximin } \\
>\text { lactulose }\end{array}$ \\
\hline $\begin{array}{l}\text { Mas et } \\
\operatorname{al}(2003)(71)\end{array}$ & $\begin{array}{l}\text { Lactitol } \\
\text { (double-blind, } \\
\text { double- } \\
\text { dummy) }\end{array}$ & 103 & 5-10 days & $\begin{array}{l}\text { Mental status, } \\
\text { asterixis score, } \\
\text { EEG, } \\
\text { ammonia levels, } \\
\text { PSE index, } \\
\text { psychometric } \\
\text { tests }\end{array}$ & $\begin{array}{l}\text { Rifaximin } \\
=\text { lactitol }\end{array}$ \\
\hline $\begin{array}{l}\text { Leevy et } \\
\text { al(2007)(73) }\end{array}$ & $\begin{array}{l}\text { Lactulose } \\
\text { (crossover) }\end{array}$ & 145 & $\begin{array}{l}>6 \text { months } \\
\text { lactulose } \\
>6 \text { months } \\
\text { rifaximin }\end{array}$ & $\begin{array}{l}\text { HE grade, } \\
\text { asterixis score }\end{array}$ & $\begin{array}{l}\text { Rifaximin } \\
>\text { lactulose }\end{array}$ \\
\hline $\begin{array}{l}\text { Paik et } \\
\text { al(2005)(74) }\end{array}$ & $\begin{array}{l}\text { Lactulose } \\
\text { (open-label) }\end{array}$ & 54 & 7 days & $\begin{array}{l}\text { Ammonia levels, } \\
\text { flapping tremor, } \\
\text { mental } \\
\text { status, HE index, } \\
\text { psychometric tests }\end{array}$ & $\begin{array}{l}\text { Rifaximin } \\
=\text { lactitol }\end{array}$ \\
\hline $\begin{array}{l}\text { Jiang Q et } \\
\text { al(2008)(76) }\end{array}$ & meta-analysis & 264 & - & - & $\begin{array}{l}\text { Rifaximin } \\
=\text { lactitol }\end{array}$ \\
\hline
\end{tabular}

Table 4. Comparison of disaccharides and rifaximin for hepatic encephalopathy. 
study in order to compare the course of HE in patients treated with neomycin plus magnesium sulfate or with lactulose. The treatment groups were similar in terms of clinical characteristics, fatalities, recovery rate from grade 1 encephalopathy, and disappearance rate of neuropsychiatric signs. Transitions from severe to grade 1 or 0 encephalopathy showed a 0.17 (NS) difference in favor of neomycin. Early therapy and evidence of precipitating factors showed a favorable prognostic significance. Ascites, hyperbilirubinemia, poor nutritional state, and hypoprothrombinemia showed bad prognostic significance.

A combination of disaccharides and antibiotics is generally used in difficult to treat patients with HE, but clinical data is limited. In a retrospective chart review of 213 cases, Mantry and colleagues showed that the number of hospitalizations and the duration of hospital stays were shortened for patients receiving combination therapy compared with those receiving lactulose monotherapy. Additional studies to further investigate the potential therapeutic benefit of combination therapy will be needed.(77,78)

\section{Disaccharides versus other therapy for hepatic encephalopathy}

Mittal et al(79) compared lactulose, probiotics, and L-ornithine L-aspartate (LOLA) in treatment of MHE and effect on health related quality of life by Sickness Impact Profile questionnaire. MHE improved significantly in all three treatment groups. Lactulose, probiotics, and LOLA significantly improve MHE and health related quality of life in patients with chronic liver disease. Similar results were found in other studies $(80,81)$ Table-5

\begin{tabular}{|l|l|l|l|l|l|l|}
\hline Trial & $\begin{array}{l}\text { Study } \\
\text { design }\end{array}$ & $\begin{array}{l}\text { No of } \\
\text { patients }\end{array}$ & $\begin{array}{l}\text { Type of } \\
\text { patient }\end{array}$ & Treatment & assessment & Efficacy \\
\hline $\begin{array}{l}\text { Loguercio et al } \\
\text { 1987(82) }\end{array}$ & Parallel & 40 & CHE & $\begin{array}{l}\text { Lactobacillus } \\
\text { SF68 / } \\
\text { lactulose }\end{array}$ & $\begin{array}{l}\text { PSE } \\
\text { parameters, } \\
\text { adverse } \\
\text { events }\end{array}$ & SF68=lactulose \\
\hline $\begin{array}{l}\text { Loguercio et al } \\
\text { 1995(83) }\end{array}$ & Parallel & 40 & CHE & $\begin{array}{l}\text { Lactobacillus } \\
\text { SF68 / } \\
\text { lactulose } \\
\text { pSE } \\
\text { parameters, } \\
\text { adverse } \\
\text { events }\end{array}$ & SF68=lactulose \\
\hline $\begin{array}{l}\text { Sushma et al } \\
\text { 1992(84) }\end{array}$ & Parallel & 74 & AHE & $\begin{array}{l}\text { Sodium } \\
\text { benzoate / } \\
\text { lactulose }\end{array}$ & $\begin{array}{l}\text { Mortality, } \\
\text { PSE } \\
\text { parameters }\end{array}$ & $\begin{array}{l}\text { Sodium } \\
\text { benzoate=lactulose }\end{array}$ \\
\hline $\begin{array}{l}\text { Fiaccadori } \\
\text { 1980(86) }\end{array}$ & Parallel & 23 & $\begin{array}{l}\text { AHE }+ \\
\text { CHE }\end{array}$ & $\begin{array}{l}\text { BCAA/BCA } \\
\text { A } \\
+ \text { lactulose / } \\
\text { lactulose }\end{array}$ & $\begin{array}{l}\text { Clinical } \\
\text { grading }\end{array}$ & $\begin{array}{l}\text { BCAA+lactulose> } \\
\text { BCAA/lactulose }\end{array}$ \\
\hline $\begin{array}{l}\text { Rossi- } \\
\text { Fanelli 1982(87) }\end{array}$ & Parallel & 40 & AHE & $\begin{array}{l}\text { BCAA / } \\
\text { lactulose }\end{array}$ & $\begin{array}{l}\text { Clinical } \\
\text { grading }\end{array}$ & BCAA=Lactulose \\
\hline
\end{tabular}

Table 5. Disaccharides versus other therapy for hepatic encephalopathy. 
Loguercio et al. (82) in 40 patients with cirrhosis on a dietary protein regimen of $1 \mathrm{~g} / \mathrm{kg}$ determined the effect on chronic hepatic encephalopathy of long-term administration of Enterococcus faecium (SF68) versus lactulose. The patients received one of the two treatments for three periods of 4 weeks, each separated by drug-free 2 -week intervals. The efficacy of treatment was assessed by arterial blood ammonia concentration, mental status, number connection (Reitan's part A) test and flash-evoked visual potentials. At the end of the third period the reduction in both blood ammonia concentrations and Reitan's test times was more enhanced in patients on SF68 than in patients on lactulose. In conclusion, SF68 is at least as useful as lactulose for the chronic treatment of chronic hepatic encephalopathy; it has no adverse effects, and treatment can be interrupted for 2 weeks without losing the beneficial effects.

Sushma et al(84) conducted a prospective randomized double-blind study to evaluate the efficacy of sodium benzoate in the treatment of acute portal-systemic encephalopathy. Seventy-four consecutive patients with cirrhosis or surgical portasystemic anastomosis and hepatic encephalopathy of less than 7 days duration were randomized to receive lactulose (dose adjusted for 2 or 3 semiformed stools/day) or sodium benzoate ( $5 \mathrm{gm}$ twice daily). Assessment of response included mental status, asterixis, arterial ammonia level, electroencephalogram and number-connection test. The incidence of side effects was similar in the two treatment groups. The cost of lactulose for one course of therapy was 30 times that of sodium benzoate. They concluded that sodium benzoate is a safe and effective alternative to lactulose in the treatment of acute portasystemic encephalopathy. However in a study by Efrati et al (85) Blood ammonia increased after the glutamine load both before (from $66 \pm 12 \mathrm{mg} / \mathrm{dl}$ to $123 \pm 34 \mathrm{mg} / \mathrm{dl}$ and $179 \pm 53 \mathrm{mg} / \mathrm{dl}$ after 30 and $60 \mathrm{~min}$, respectively; $\mathrm{p}<0.0004$ ) and after benzoate treatment (from $102 \pm 27 \mathrm{mg} / \mathrm{dl}$ to $185 \pm 49 \mathrm{mg} / \mathrm{dl}$ and $250 \pm 39$ $\mathrm{mg} / \mathrm{dl}$ after 30 and $60 \mathrm{~min}$, respectively; $\mathrm{p}<0.00001)$. The Number Connection test and the Posner's test were not altered by benzoate treatment and this study suggests a note of caution in the use of sodium benzoate in cirrhotic patients.

Rossi-Fanelli F et al(87) conducted a controlled study in two groups of 20 cirrhotic patients with deep coma in order to compare the efficacy of intravenous branched-chain amino acid solutions in $20 \%$ glucose (group A) vs. lactulose plus glucose in isocaloric amount (group B). Complete mental recovery was obtained in $70 \%$ of patients in group A and in $47 \%$ in group B. They concluded that, branched-chain amino acids are at least as effective as lactulose in deep hepatic coma. However, in a meta-analysis of eleven randomised trials (556 patients) assessing BCAA versus carbohydrates, neomycin/lactulose, or isonitrogenous control. There was no significant evidence of an effect of BCAA on improvement of hepatic encephalopathy in trials with adequate generation of the allocation sequence (RR 1.01, 95\% CI 0.84 to 1.23 , three trials), adequate allocation concealment (RR 1.09, 95\% CI 0.89 to 1.33 , five trials), or adequate double-blinding (RR 1.20, 95\% CI 0.83 to 1.73, three trials). So it was concluded that BCAA had no significant beneficial effect on patients with hepatic encephalopathy (88).

\section{Disaccharides for primary prophylaxis of hepatic encephalopathy}

Certain patients are at risk of development of HE like patients with minimal hepatic encephalopathy and those with advanced liver disease (89-91). Variceal bleed is an important precipitating factor for HE in patients with cirrhosis. In a randomized trial, (92) 
we enrolled seventy patients with acute variceal bleed into group-1(lactulose, $n=35$ ) and group-2 (no lactulose, $n=35$ ). Nineteen $(27 \%)$ patients developed HE, 5 patients $(14 \%)$ in the lactulose and 14 patients $(40 \%)$ in no lactulose, $p=0.03$. On multivariate analysis, only baseline arterial ammonia, blood requirement during hospital stay and no lactulose therapy were predictors of development of HE. Hence, lactulose was effective in preventing HE in these patients. We therefore, recommend lactulose $(30-60 \mathrm{ml} /$ day) so that patients pass two to three semi formed stools in a day in these subsets of patients.

Recently our group has shown in a randomized trial (93) involving 120(48\%) patients, receiving either lactulose $(n=60)$ or no lactulose $(n=60)$. Twenty $(19 \%)$ of 105 patients followed for 12 mo developed an episode of overt HE. Six (11\%) of 55 in the lactulose group and $14(28 \%)$ of 50 in the no lactulose group ( $p=0.02)$ developed HE. Ten $(20 \%)$ of 50 patients no lactulose group and $5(9 \%)$ of 55 patients lactulose group died, $\mathrm{p}=0.16$. On multivariate analysis, Child's score and presence of MHE at baseline was significantly associated with development of HE. Lactulose is effective in the primary prevention of HE.

\section{Disaccharides for secondary prophylaxis of hepatic encephalopathy}

Hepatic encephalopathy may be a cyclic condition in which patients develop overt HE, receive treatment, enter remission, and relapse. The emergence of HE after transjugular intrahepatic portosystemic shunt (TIPS) is of major concern for patients undergoing this procedure for refractory ascites or for prevention of variceal rebleeding. This clinical complication tends to occur within the first few days post-procedure.

Although the majority of post-TIPS HE episodes are mild and responsive to pharmacological therapy, there are some cases where intractable HE develops and hospitalization is required. There are limited data on the use of drug therapy for the prophylaxis of HE after a TIPS procedure or in patients who have recovered from an episode of HE who may benefit from pharmacological prophylaxis to prevent future recurrences. Until recently, there has not been any conclusive evidence to support routine use of pharmacological prophylaxis for this purpose. Riggio and colleagues (94) conducted the first randomized controlled trial utilizing lactitol or rifaximin as pharmacological prophylaxis of post-shunt HE. Seventy-five consecutive patients with cirrhosis undergoing a TIPS procedure were randomized to receive lactitol $60 \mathrm{~mL} /$ day, rifaximin $1200 \mathrm{mg} /$ day or no treatment. Patients in the rifaximin or no-treatment groups were allowed administration of a sorbitol enema $(120 \mathrm{~mL})$ in cases of minimal bowel movement $(<1$ bowel movement/day). Treatments were continued for 1 month post-TIPS or until the occurrence of an episode of HE. There was no significant difference in rate of HE occurrence among the three patient groups $(\mathrm{p}=0.97)$.

Two recent clinical trials have been conducted to evaluate the efficacy of lactulose or rifaximin used concomitantly with lactulose, as secondary prophylaxis of overt HE compared with placebo(95,96). Sharma and colleagues(95) conducted a single-centre, openlabel, randomized controlled trial in 125 cirrhotic patients who had recovered from at least one previous episode of HE. Patients were randomized to receive either lactulose 30$60 \mathrm{~mL} /$ day or placebo. Development of overt HE was the primary study endpoint. At the end of a median follow-up time of 14 months, significantly more patients in the placebo group (30 of 64 patients [46.8\%]) than in the lactulose group (12 of 61 patients [19.6\%]) developed HE $(p=0.001)$. 
In a recent multicenter double blind randomized clinical trial to assess the secondary prevention of HE Bass and colleagues(96), enrolled 299 cirrhotic patients with a history of at least two episodes of overt HE to receive either rifaximin (550 mg twice daily; $\mathrm{n}=140$ ) or placebo $(n=159)$ for a period of 6 months. All enrolled patients had Model for End-Stage Liver Disease (MELD) scores of $<25$. More than $90 \%$ of patients in both groups were also maintained on concomitant lactulose therapy. A significantly lower percentage of patients in the rifaximin group (22.1\%) experienced a breakthrough HE episode during the study period than in the placebo group $(45.9 \%)$, with a hazard ratio (HR) of $0.42(95 \%$ CI $0.28,0.64 ; p<0.001)$. In addition, there was a significantly reduced risk of hospitalization due to hepatic encephalopathy in the rifaximin patient group when compared with placebo; 19 patients in the rifaximin group $(13.6 \%)$ versus 36 patients in the placebo group $(22.6 \%),(p=0.01)$. No significant difference in the incidence of adverse events was found between the two groups. In a similar study by Sanyal et al(97) patients with cirrhosis in remission from HE (Conn score = 0 or 1$)$ and a documented history of recurrent HE episodes ( $\geq 2$ within 6 months of screening) were randomised to rifaximin $550 \mathrm{mg}$ twice daily $(\mathrm{N}=101)$ or placebo $(\mathrm{N}=118)$ for 6 months. Concomitant lactulose was permitted during the study. The Chronic Liver Disease Questionnaire (CLDQ) was administered every 4 weeks, and time for occurrence of $\mathrm{HE}$ breakthrough was recorded. The time-weighted averages of the overall CLDQ score and each domain score were significantly higher in the rifaximin group vs. placebo (P-values ranged from 0.0087 to 0.0436 ); and were significantly lower in patients who experienced HE breakthrough compared to those who remained in remission (P-values were $<0.0001$ )(Table-6). Hence lactulose and rifaximin for the secondary prophylaxis of $\mathrm{HE}$ is recommended.

\begin{tabular}{|l|l|l|l|l|l|}
\hline Trial & Study design & $\begin{array}{l}\text { No of } \\
\text { patients }\end{array}$ & $\begin{array}{l}\text { Duration of } \\
\text { treatment }\end{array}$ & assessment & Efficacy \\
\hline $\begin{array}{l}\text { Sharma BC } \\
\text { et al } \\
(2009)(69)\end{array}$ & $\begin{array}{l}\text { Lactulose (open- } \\
\text { label) }\end{array}$ & 140 & 14 months & $\begin{array}{l}\text { Psychometry } \\
\text { and CFF }\end{array}$ & $\begin{array}{l}\text { Lactulose> } \\
\text { no treatment }\end{array}$ \\
\hline $\begin{array}{l}\text { Bass et al } \\
(2010)(70)\end{array}$ & $\begin{array}{l}\text { Rifxaximin+lactulose } \\
\text { (randomized, } \\
\text { double-blind, } \\
\text { placebo-controlled) }\end{array}$ & 299 & 6 months & HE clinical & $\begin{array}{l}\text { Rifaximin } \\
>\text { placebo }\end{array}$ \\
\hline
\end{tabular}

Table 6. Disaccharides for secondary prophylaxis of hepatic encephalopathy.

\section{Conclusion}

Current pharmacotherapy for the management of HE is fairly limited, mainly because of the complex and relatively limited understanding of the pathophysiology of the disorder. Although the evidence of ammonia is robust, in everyday clinical practice a consistent correlation between the concentration of ammonia in the blood and the manifest symptoms of $\mathrm{HE}$ is not observed. More recently the synergistic role of inflammation and infection in modulating the cerebral effects of ammonia has been shown to be important. The most commonly utilized pharmacological agents include the non-absorbable disaccharides lactulose and lactitol, and the antimicrobial agent rifaximin. Lactulose and lactitol, the synthetic compounds of lactose, are poorly absorbed from the small intestine, and both are almost exclusively metabolized by the bacterial flora of the colon. These disaccharides 
shorten the small bowel transit time mechanically and lower the $\mathrm{pH}$ of the bowel, both by their osmotic effects and by the acidic $\mathrm{pH}$ of their metabolites hence diminish the absorption of ammonia. Lactulose and lactitol are the classical therapy of choice for the treatment of hepatic encephalopathy. Recent literature has questioned the clinical efficacy of disaccharides in improving morbidity and mortality in patients with HE and, although antimicrobial agents such rifaximin have had an established role in the treatment of low grade (West Haven grade 1 and 2) encephalopathy, its use in high grade HE (grade 3 and 4) needs more data. Till we have more definitive agents non-absorbable disaccharides lactulose still continues to be the first line therapy for the prevention, treatment and secondary prophylaxis of hepatic encephalopathy.

\section{References}

[1] Ferenci P, Lockwood A, Mullen K, Tarter R, Weissenborn K, Blei AT. Hepatic encephalopathy- definition, nomenclature, diagnosis, and quantification: final report of the working party at the 11th World Congresses of Gastroenterology, Vienna, 1998. Hepatology 2002;35:716-721

[2] Riordan SM, Williams R. Treatment of hepatic encephalopathy. N Engl J Med 1997;337:473- 479.

[3] Bajaj JS, Wade JB, Sanyal AJ. Spectrum of neurocognitive impairment in cirrhosis: Implications for the assessment of hepatic encephalopathy Hepatology. 2009 Dec;50(6):2014-21

[4] Bajaj JS, Hafeezullah M, Hoffmann RG, Saeian K. Minimal hepatic encephalopathy: a vehicle for accidents and traffic violations. Am J Gastroenterol 2007;102:1903-9

[5] Groeneweg M, Quero JC, De Bruijn I, Hartmann IJC, Essink-bot MI, Hop WCJ et al. Subclinical hepatic encephalopathy impairs daily functioning. HEPATOLOGY 1998;28:45-9

[6] Bajaj JS, Stein AC, Dubinsky RM. What Is Driving the Legal Interest in Hepatic Encephalopathy? Clin Gastroenterol Hepatol. 2010 Nov 9

[7] Bajaj JS, Saeian K, Schubert CM, Hafeezullah M, Franco J et al Minimal hepatic encephalopathy is associated with motor vehicle crashes: the reality beyond the driving test Hepatology. 2009 Oct;50(4):1175-83

[8] Haussinger D, Schliess F. Pathogenetic mechanisms of hepatic encephalopathy. Gut 2008;57:1156-65

[9] Bismuth M, Funakoshi N, Cadranel JF, Blanc P. Hepatic encephalopathy: from pathophysiology to therapeutic management Eur J Gastroenterol Hepatol. 2011 Jan;23(1):8-22

[10] Albrecht J, Zielińska M, Norenberg MD Glutamine as a mediator of ammonia neurotoxicity: A critical appraisal. Biochem Pharmacol. 2010 Nov 1;80(9):1303-8

[11] Gerber T, Schomerus H. Hepatic encephalopathy in liver cirrhosis: pathogenesis, diagnosis and management. Drugs 2000;60:1353-70

[12] Lockwood A, Yap E, Wong W. Cerebral ammonia metabolism in patients with severe liver disease and minimal hepatic encephalopathy. J Cereb Blood Flow Metab 1991;11:337-341.

[13] Gerber T, Schomerus H. Hepatic encephalopathy in liver cirrhosis: pathogenesis, diagnosis and management. Drugs 2000;60:1353-70 
[14] Butterworth RF. Complications of cirrhosis III. Hepatic encephalopathy. J Hepatol 2000;32:171-80

[15] Pilbeam CM, Anderson RM, Bhathal PS. The brain in experimental portalsystemic encephalopathy. I. Morphological changes in three animal models. J Pathol 1983;140:331-45

[16] Albrecht J, Norenberg MD. Glutamine: a Trojan horse in ammonia neurotoxicity. Hepatology 2006;44:788-94.

[17] Shawcross DL, Olde Damink SW, Butterworth RF, Jalan R. Ammonia and hepatic encephalopathy: the more things change, the more they remain the same Metab Brain Dis. 2005 Sep;20(3):169-79

[18] Ha"ussinger D, Kircheis G, Fischer R, et al. Hepatic encephalopathy in chronic liver desease: a clinical manifestation of astrocyte swelling and low grade cerebral edema. J Hepatol 2000;32:1035-8.

[19] Tanigami H, Rebel A, Martin LJ, et al. Effect of glutamine synthetase inhibition on astrocyte swelling and altered astroglial protein expression during hyperammonemia in rats. Neuroscience 2005;131:437-49.

[20] Cordoba J, Alonso J, Rovira A, Jacas C, Sanpedro F, Castells L, et al. The development of low-grade cerebral oedema in cirrhosis is supported by the evolution of $1 \mathrm{H}$ magnetic resonance abnormalities after liver transplantation.J Hepatol 2001;35:598604.

[21] Schliess F, Foster N, Gorg B, et al. Hypoosmotic swelling increases protein tyrosine nitration in cultured rat astrocytes. Glia 2004;47:21-9.

[22] Murthy CR, Rama Rao KV, Bai G, et al. Ammonia-induced production of free radicals in primary cultures of rat astrocytes. J Neurosci Res 2001;66:282-8.

[23] Jayakumar AR, Rama Rao KV, Schousboe A, et al. Glutamine-induced free radical production in cultured astrocytes. Glia 2004;46:296-301.

[24] Rama Rao KV, Chen M, Simard JM, et al. Suppression of ammonia-induced astrocyte swelling by cyclosporin A. J Neurosci Res 2003;74:891-7.

[25] Rama Rao KV, Norenberg MD. Aquaporin-4 in hepatic encephalopathy. Metab Brain Dis 2007;22:265-75.

[26] Chepkova AN, Sergeeva OA, Haas HL. Taurine rescues hippocampal long-term potentiation from ammonia-induced impairment. Neurobiol Dis 2006;23:512-21.

[27] Weissenborn K, Bokemeyer M, Ahl B, et al. Functional imaging of the brain in patients with liver cirrhosis. Metab Brain Dis 2004;19:269-80.

[28] Balata S, Olde Damink S, Ferguson K, Marshall I, Hayes P, Deutz N, et al. Induced hyperammonemia alters neuropsychology, brain MR spectroscopy and magnetization transfer in cirrhosis. HEPATOLOGY 2003;37:931-939.

[29] Fessel JN, Conn HO. An analysis of the causes and prevention of hepatic coma. Gastroenterology. 1972;62:191.

[30] Blei AT, Cordoba J. Hepatic encephalopathy. Am J Gastroenterol 2001; 96: 1968-76

[31] Schrier RW, Gross P, Gheorghiade M, et al. Tolvaptan, a selective oral vasopressin V2receptor antagonist, for hyponatremia. N Engl J Med 2006;355: 2099-112

[32] Sundaram V, Shaikh OS. Hepatic encephalopathy: pathophysiology and emerging therapies. Med Clin North Am. 2009 Jul;93(4):819-36.

[33] Riggio O, Balducci G, Ariosto F, et al. Lactitol in prevention of recurrent episodes of hepatic encephalopathy in cirrhotic patients with portal-systemic shunt. Dig Dis Sci 1989; 34 (6): 823-9 
[34] Morgan MY, Hawley KE. Lactitol vs. lactulose in the treatment of acute hepatic encephalopathy in cirrhotic patients: a double-blind, randomized trial. Hepatology 1987; 7 (6): 1278-84

[35] Heredia D, Tere's J, Orteu N, et al. Lactitol vs. lactulose in the treatment of chronic recurrent portal-systemic encephalopathy. J Hepatol 1988; 7: 106-10

[36] Cordo' ba J, Blei AT. Treatment of hepatic encephalopathy. Am J Gastroenterol 1997; 92 (9): 1429-39

[37] Clausen MR, Mortensen PB. Lactulose, disaccharides and colonic flora: clinical consequences. Drugs 1997; 53 (6): 930-42

[38] Eroglu Y, Byrne W. Hepatic encephalopathy. Emerg Med Clin N Am 2009; 27: 401-14

[39] Simmons F, Goldstein H, Boyle JD. A controlled clinical trial of lactulose in hepatic encephalopathy. Gastroenterology 1970;59:827-32.

[40] Rodgers JB Jr, Kiley JE, Balint JA. Comparison of results of long-term treatment of chronic hepatic encephalopathy with lactulose and sorbitol. Am J Gastroenterol 1973;60:459-65.

[41] Corazza GR, Tacconi C, Zoli G, Somarolli M, D'Ambro A, Bernardi M. Use of pyridoxine-alpha-ketoglutarate $(\mathrm{PAK})$ in hepatic encephalopathy. Int $\mathrm{J}$ Clin Pharmacol Res 1982;2:7-13.

[42] Uribe M, Campollo O, Vargas F, Ravelli GP, Mundo F, Zapata L. Acidifying enemas (lactitol and lactose) vs. nonacidifying enemas (tap water) to treat acute portalsystemic encephalopathy: a double-blind, randomized clinical trial. Hepatology 1987;7:639-43.

[43] Horsmans Y, Solbreux PM, Daenens C, et al. Lactulose improves psychometric testing in cirrhotic patients with subclinical encephalopathy. Aliment Pharmacol Ther 1997; 11 (1): 165-70

[44] Watanabe A, Sakai T, Sato S, et al. Clinical efficacy of lactulose in cirrhotic patients with and without subclinical hepatic encephalopathy. Hepatology 1997; 26 (6): 1410-4

[45] Dhiman RK, Sawhney MS, Chawla YK, et al. Efficacy of lactulose in cirrhotic patients with subclinical hepatic encephalopathy. Dig Dis Sci 2000; 45 (8): 1549-52

[46] Prasad S, Dhiman RK, Duseja A, et al. Lactulose improves cognitive functions and health-related quality of life in patients with cirrhosis who have minimal hepatic encephalopathy. Hepatology 2007; 45 (3): 549-59

[47] Mas A, Rodés J, Sunyer L, Rodrigo L, Planas R, Vargas V, et al Comparison of rifaximin and lactitol in the treatment of acute hepatic encephalopathy: results of a randomized, double-blind, double-dummy, controlled clinical trial J Hepatol. 2003 Jan;38(1):51-8

[48] Als-Nielsen B, Gluud LL, Gluud C. Non-absorbable disaccharides for hepatic encephalopathy: systematic review of randomised trials. BMJ. 2004 May 1;328(7447):1046

[49] Als-Nielsen B, Gluud LL, Gluud C. Nonabsorbable disaccharides for hepatic encephalopathy Cochrane Database Syst Rev. 2004;(2):CD003044

[50] Sharma P, Sharma BC, Sarin SK. Predictors of nonresponse to lactulose in patients with cirrhosis and hepatic encephalopathy Eur J Gastroenterol Hepatol. 2010 May;22(5):526-31

[51] Sharma P, Sharma BC, Sarin SK Predictors of nonresponse to lactulose for minimal hepatic encephalopathy in patients with cirrhosis Liver Int. 2009 Oct;29(9):1365-71

[52] Lanthier PL, Morgan MY. Lactitol in the treatment of chronic hepatic encephalopathy: an open comparison with lactulose. Gut 1985; 26: 415-20 
[53] Heredia D, Terés J, Orteu N, Rodés J Lactitol vs. lactulose in the treatment of chronic recurrent portal-systemic encephalopathy. J Hepatol. 1988 Aug;7(1):106-10

[54] Morgan MY, Alonso M, Stanger LC. Lactitol and lactulose for the treatment of subclinical hepatic encephalopathy in cirrhotic patients. A randomised, cross-over study. J Hepatol. 1989 Mar;8(2):208-17

[55] Riggio O, Balducci G, Ariosto F, Merli M, Pieche U, Pinto G et al Lactitol in prevention of recurrent episodes of hepatic encephalopathy in cirrhotic patients with portalsystemic shunt. Dig Dis Sci. 1989 Jun;34(6):823-9

[56] Grandi M, Sacchetti C, Pederzoli S, Celani MF A clinical comparative study of crystalline pure lactulose and powder pure lactitol in portasystemic encephalopathy of cirrhotic patients Minerva Gastroenterol Dietol. 1991 OctDec;37(4):225-30

[57] Pai CH, Huang YS, Jeng WC, Chan CY, Lee SD Treatment of porto-systemic encephalopathy with lactitol verus lactulose: a randomized controlled study. Zhonghua Yi Xue Za Zhi (Taipei). 1995 Jan;55(1):31-6

[58] Blanc P, Daures JP, Rouillon JM, Peray P, Pierrugues R et al Lactitol or lactulose in the treatment of chronic hepatic encephalopathy: results of a meta-analysis. Hepatology. 1992 Feb;15(2):222-8.

[59] Cammà C, Fiorello F, Tinè F, Marchesini G, Fabbri A et al Lactitol in treatment of chronic hepatic encephalopathy. A meta-analysis. Dig Dis Sci. 1993 May;38(5):91622.

[60] Conn HO, Leevy CM, Vlacevic ZR, Rodgers JB, Maddrey WC, Seef L. Comparison of lactulose and neomycin in the treatment of chronic portal-systemic encephalopathy. A double blind controlled trial. Gastroenterology 1977;72:573-83.

[61] Atterbury CE, Maddrey WC, Conn HO. Neomycin-sorbitol and lactulose in the treatment of acute portal-systemic encephalopathy. A controlled, double-blind clinical trial. Am J Dig Dis 1978;23:398-406

[62] Orlandi F, Freddara U, Candelaresi MT, Morettini A, Corazza GR, Di Simone A. Comparison between neomycin and lactulose in 173 patients with hepatic encephalopathy: a randomized clinical study. Dig Dis Sci 1981;26:498-506.

[63] Blanc P, Couderc M, Peray P, Liautard J, Larrey D, Michel H, et al. Lactitol versus vancomycin in the treatment of acute hepatic encephalopathy: a double blind, randomized trial [abstract]. Gut 1993;34:46.

[64] Maclayton DO, Eaton-Maxwell A. Rifaximin for treatment of hepatic encephalopathy Ann Pharmacother. 2009 Jan;43(1):77-84

[65] Mullen K, Prakash R Rifaximin for the treatment of hepatic encephalopathy. Expert Rev Gastroenterol Hepatol. 2010 Dec;4(6):665-77

[66] Festi D, Mazzella G, Orsini M et al. Rifaximin in the treatment of chronic hepatic encephalopathy: results of a multicenter study of efficacy and safety. Curr Ther Res. 1993,54(5), 598-609.

[67] Palmer M. The antibiotic rifaximin improves hepatic encephalopathy symptoms in patients with cirrhosis due to hepatitis C virus. Pract Gastroenterol.2007, 31(2), 72 76

[68] Puxeddu A, Quartini M, Massimetti A, Ferrieri A. Rifaximin in the treatment of chronic hepatic encephalopathy. Curr Med Res Opin.1995, 13(5), 274-281

[69] Sama C, Morselli-Labate AM, Pianta P, Lambertini L, Berardi S, Martini G. Clinical effects of rifaximin in patients with hepatic encephalopathy intolerant or 
nonresponsive to previous lactulose treatment: an open-label, pilot study. Curr Ther Res.2004, 65(5), 413-422

[70] Bucci L, Palmieri GC. Double-blind, double-dummy comparison between treatment with rifaximin and lactulose in patients with medium to severe degree hepatic encephalopathy. Curr Med Res Opin. 1993,13(2), 109-118

[71] Loguercio C, Federico A, De Girolamo V, Ferrieri A, Del Vicchio BD. Cyclic treatment of chronic hepatic encephalopathy with rifaximin. Results of a double-blind clinical study. Minerva Gastroenterol Dietol 2003;49:53-62

[72] Massa P, Vallerino E, Dodero M. Treatment of hepatic encephalopathy with rifaximin: double blind, double dummy study versus lactulose. Eur J Clin Res.1993; 4, 7-18

[73] Mas A, Rodes J, Sunyer L, Rodrigo L, Planas R, Vargas V. Comparison of rifaximin and lactitol in the treatment of acute hepatic encephalopathy: results of a randomized, double-blind, double-dummy, controlled clinical trial. J Hepatol 2003;38:51-8.

[74] Fera G, Agostinacchio F, Nigro M, et al. Rifaximin in the treatment of hepatic encephalopathy. Eur J Clin Res 1993;4: 57-66

[75] Leevy CB, Phillips JA. Hospitalizations during the use of rifaximin versus lactulose for the treatment of hepatic encephalopathy. Dig Dis Sci 2007; 52: 737-41

[76] Paik YH, Lee KS, Han KH, et al. Comparison of rifaximin and lactulose for the treatment of hepatic encephalopathy: a prospective randomized study. Yonsei Med J 2005; 46 (3):399-407

[77] Leevy CB, Phillips JA. Hospitalizations during the use of rifaximin versus lactulose for the treatment of hepatic encephalopathy Dig Dis Sci. 2007 Mar;52(3):737-41

[78] Jiang Q, Jiang $\mathrm{XH}$, Zheng $\mathrm{MH}$, Jiang LM, Chen YP, Wang L. Rifaximin versus nonabsorbable disaccharides in the management of hepatic encephalopathy: a meta-analysis Eur J Gastroenterol Hepatol. 2008 Nov;20(11):1064-70

[79] Mantry PS, Munsaf S. Does the addition of rifaximin to lactulose reduce the severity of hepatic encephalopathy? A single-center experience [abstract] Hepatology. 2008;48:517A, \#472.

[80] Mantry PS, Munsaf S. Comparison of tolerability of adjunctive rifaximin treatment versus lactulose monotherapy for hepatic encephalopathy [abstract] Hepatology. 2008;48:518A, \#473.

[81] Mittal VV, Sharma BC, Sharma P, Sarin SK. A randomized controlled trial comparing lactulose, probiotics, and L-ornithine L-aspartate in treatment of minimal hepatic encephalopathy Eur J Gastroenterol Hepatol. 2011 Aug;23(8):725-32

[82] Sharma P, Sharma BC, Puri V, Sarin SK. An open-label randomized controlled trial of lactulose and probiotics in the treatment of minimal hepatic encephalopathy Eur J Gastroenterol Hepatol. 2008 Jun;20(6):506-11

[83] Malaguarnera M, Gargante MP, Malaguarnera G, Bifidobacterium combined with fructo-oligosaccharide versus lactulose in the treatment of patients with hepatic encephalopathy Eur J Gastroenterol Hepatol. 2010 Feb;22(2):199-206)

[84] Loguercio C, Del Vecchio Blanco C, Coltorti M. Enterococcus lactic acid bacteria strain SF68 and lactulose in hepatic encephalopathy: a controlled study. J Int Med Res. 1987 Nov-Dec;15(6):335-43

[85] Loguercio C, Abbiati R, Rinaldi M, Romano A, Del Vecchio Blanco C et al Long-term effects of Enterococcus faecium SF68 versus lactulose in the treatment of patients with cirrhosis and grade 1-2 hepatic encephalopathy. J Hepatol. 1995 Jul;23(1):39-46 
[86] Sushma S, Dasarathy S, Tandon RK, Jain S, Gupta S, Bhist MS Sodium benzoate in the treatment of acute hepatic encephalopathy: a double-blind randomized trial. Hepatology. 1992 Jul;16(1):138-44

[87] Efrati C, Masini A, Merli M, Valeriano V, Riggio O Effect of Sodium Benzoate on Blood Ammonia Response to Oral Glutamine Challenge in Cirrhotic Patients: A Note of Caution Am J Gastroenterol 2000;95:3574 -3578

[88] Fiaccadori F, Ghinelli F, Pelosi G, Sacchini D, Vaona GL, Zeneroli ML et al Selective amono acid solutions in hepatic encephalopathy treatment (a preliminary report). Ric Clin Lab. 1980 Apr-Jun;10(2):411-22

[89] Rossi-Fanelli F, Riggio O, Cangiano C, Cascino A, De Conciliis D, Merli M et al Branched-chain amino acids vs lactulose in the treatment of hepatic coma: a controlled study. Dig Dis Sci. 1982 Oct;27(10):929-35

[90] Als-Nielsen B, Koretz RL, Kjaergard LL, Gluud C. Branched-chain amino acids for hepatic encephalopathy Cochrane Database Syst Rev. 2003;(2):CD001939

[91] Romero-Gomez M, Boza F, Garcia-Valdecasas MS, García E, Aguilar-Reina J. Subclinical hepatic encephalopathy predicts the development of overt hepatic encephalopathy. Am J Gastroenterol 2001;96:2718-2723

[92] Das A, Dhiman RK, Saraswat VA, Verma M, Naik SR. Prevalence and natural history of subclinical hepatic encephalopathy in cirrhosis. J Gastroenterol Hepatol 2001;16:531 535.

[93] Hartmann IJ, Groeneweg M, Quero JC, Beijeman SJ, de Man RA, Hop WC et al. The prognostic significance of subclinical hepatic encephalopathy. Am J Gastroenterol 2000; 95: 2029-34.

[94] Sharma P, Agrawal A, Sharma BC, Sarin SK Prophylaxis of Hepatic Encephalopathy in Acute Variceal Bleed: A Randomized Controlled Trial of Lactulose versus no Lactulose J Gastroenterol Hepatol. 2010

[95] Sharma P, Agrawal A, Sharma BC, Sarin SK. Primary prophylaxis of hepatic encephalopathy in patients with cirrhosis: an open labeled randomized controlled trial of lactulose versus no lactulose. Indian J Gastroenterol 2010; 29 (Suppl 1): A8

[96] Riggio O, Masini A, Efrati C, et al. Pharmacological prophylaxis of hepatic encephalopathy after transjugular intrahepatic portosystemic shunt: a randomized controlled study. J Hepatol 2005; 42: 674-9

[97] Sharma BC, Sharma P, Agrawal A, et al. Secondary prophylaxis of hepatic encephalopathy: an open-label randomized controlled trial of lactulose versus placebo. Gastroenterology 2009; 137: 885-91

[98] Bass NM, Mullen KD, Sanyal A, et al. Rifaximin treatment in hepatic encephalopathy. N Engl J Med 2010; 362 (12): 1071-81

[99] Sanyal A, Younossi ZM, Bass NM, Mullen KD, Poordad F, Brown RS, et al Randomised clinical trial: rifaximin improves health-related quality of life in cirrhotic patients with hepatic encephalopathy - a double-blind placebo-controlled study Aliment Pharmacol Ther. 2011 


\title{
Dopaminergic Dysfunction in Experimental Hepatic Encephalopathy
}

\author{
Isabel Suárez ${ }^{1}$, Guillermo Bodega ${ }^{1}$ and Benjamín Fernández ${ }^{2}$ \\ 1 Department of Cell Biology \& Genetics, Faculty of Biology, Alcalá University, Madrid \\ ${ }^{2}$ Department of Cell Biology, Faculty of Biology, Complutense University, Madrid
}

Spain

\section{Introduction}

Hepatic encephalopathy (HE) is a complex neuropsychiatric syndrome that appears as a consequence of acute or chronic liver dysfunction. Ammonia is considered central to its pathogenesis, but the factors contributing to the neurological dysfunction the disorder entails remain unclear (Butterworth et al., 2002). In humans with HE, it has been suggested that the basal ganglia may be selectively affected (Spahr et al., 2002), and lesions detected by magnetic resonance in the basal ganglia of such patients (Spahr et al., 2000; Burkhard et al., 2003) have been associated with movement disorders (Layrargues, 2001; Spahr et al., 2002; Weissenborn \& Kolbe, 1998). The mechanisms by which liver failure leads to abnormal motor function are not clear, but the symptoms described in HE patients (Spahr et al., 2002) are similar to those of Parkinson's disease (PD).

In patients with chronic $\mathrm{HE}$, the neurological symptoms resemble those observed in chronic hyperammonaemia in the rat resulting from portacaval anastomosis (PCA) - PCA is used as an experimental model of HE since it induces chronic hyperammonaemia (Audet \& Butterworth, 1998; Bodega et al., 1991). Rats exposed to PCA show motor deficits (Cauli et al., 2006; Martin, 1986; Steindl et al., 1996) which might be associated with degeneration and/or dysfunction of the dopaminergic system. Since it is well known that motor deficits in PD are due to the dysfunction of the nigrostriatal system, the aim of this chapter is to analyse whether its dysfunction occurs in the PCA model of chronic HE, and to discuss what its consequences might be.

\section{The nigrostriatal system}

The nigrostriatal pathway projects from the substantia nigra (SN) to the basal ganglia and is involved in motor control (Smith and Bolam, 1990). The SN is the brain region in which the main dopaminergic (DAergic) pathway supplies dopamine (DA) to the striatum. The DAergic neurons of the midbrain have traditionally been described as those nerve cells positioned in the mesencephalon that possess the ability to synthesise, package, release, and reuptake the neurotransmitter dopamine. In the normal brain, DAergic midbrain neurons in the SN pars compacta (SNc) synthesise dopamine, which is immediately taken up and stored in synaptic vesicles. Under normal conditions, the efficient sequestration of 
dopamine by these vesicles provides a major means of protecting DAergic neurons from the harmful effects of dopamine oxidation. Striatal delivery of DA by midbrain DAergic SNc neurons is vital for motor control. Dysfunctional DAergic neurotransmission between the $\mathrm{SNc}$ and the dorsal striatum causes several prominent movement disorders, such as that seen in PD. In patients with this disease, the DAergic projections to the striatum deteriorate, and the decline in DAergic modulation of the basal ganglia leads to muscle control problems (Gauthier \& Sourkes, 1982).

Tyrosine hydroxylase (TH) is the rate-limiting enzyme in dopamine synthesis, making it a major marker of DAergic neurons. In the production of catecholamines, L-tyrosine is converted to L-dopamine by TH. TH expression is regulated by many factors, e.g., growth factors, hormones and ion-channels, which can induce changes in nigrostriatal DAergic neurotransmission. A positive relationship between the activity of adult SNc neurons and the expression of TH has recently been reported (Aumann et al., 2011).

There is increasing evidence of functional diversity as well as plasticity within the population of DAergic midbrain neurons; indeed, this diversity may extend to the molecular level (Korotkova et al., 2004). Further, DAergic midbrain neurons are not homogeneously affected by neurodegenerative diseases, but rather show differences in their relative vulnerability, especially with respect to cell death. The DAergic neurons in the SNc deteriorate selectively in PD and those that project to the dorsolateral striatum are substantially more vulnerable (Damier et al., 1999). Often no symptoms appear until approximately $60 \%$ of the DAergic cells in the SNc have died (Gaig \& Tolosa, 2009).

To date, no studies have described the neurotoxic effects of hyperammonaemia on nigral DAergic cells in vivo, and it is unsure whether the down-regulation of TH expression leads to the disturbance of DAergic neurotransmission in chronic hyperammonaemia following PCA. Our group has shown that PCA leads to a reduction in the number of $\mathrm{TH}$ immunoreactive neurons in the SN (Fig. 1), as well as causing a reduction in TH expression in the TH-positive neurons of this area. The loss of TH-positive neurons might be attributable to their sensitivity to high circulating ammonia concentrations, which are induced by PCA (Bodega et al., 1991).

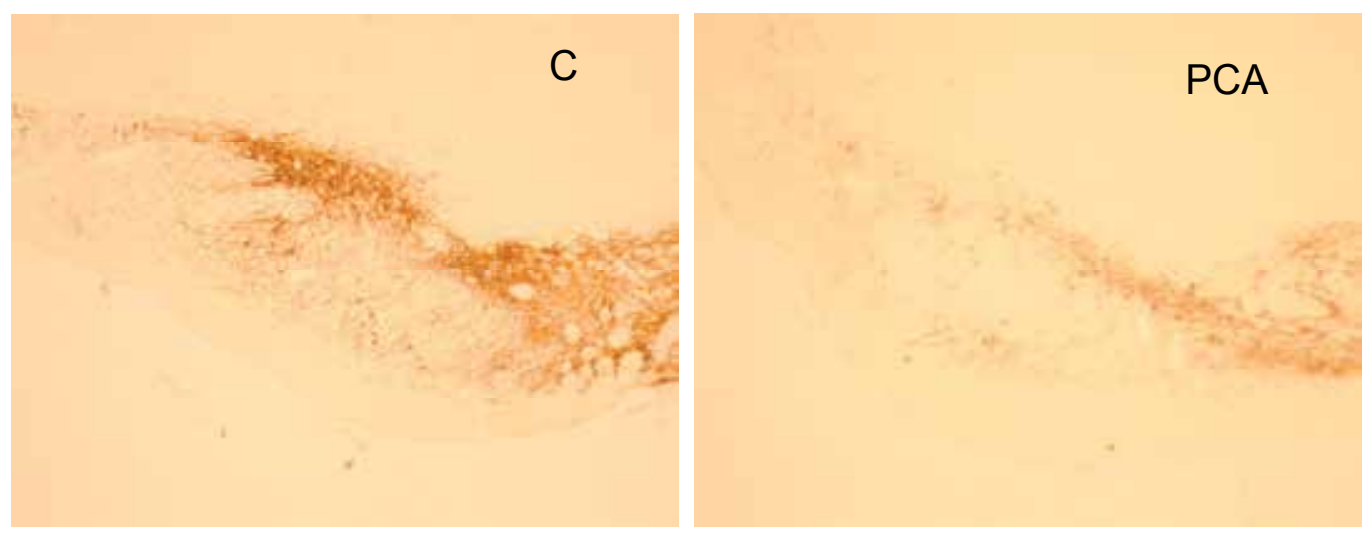

Fig. 1. TH immunoreactivity in the substantia nigra of control (C) and PCA-exposed rats (PCA). Note the reduction in TH expression in PCA rats, as well as in the number of TH+ neurons. 
It is well known that ammonia plays an important role in the pathophysiology of HE. Certainly, an increase in the ammonia concentration can affect numerous cellular processes that might contribute towards the neurological deficits associated with the disease, and it has been proposed that the ammonia levels in blood correlate with the severity of HE symptoms (Ong et al., 2003). Since PCA leads to a reduction in TH immunoreactivity in the SN and the dorsal striatum, a specific susceptibility of nigral DAergic neurons to hyperammonaemic conditions during PCA exposure might be inferred. There is a possible diversity of response within the DAergic SNc population since functionally and biochemically distinct sub-populations of SNc DAergic neurons have been described (Hajos \& Greenfield, 1993; Korotkova et al., 2004; Liss \& Roeper, 2008). This phenomenon of differential vulnerability of DA neurons towards degeneration is well documented in PD; some of the DA cell groups in the midbrain are particularly affected by neurodegenerative process while others are relatively spared of its effects (Damier et al., 1999). Since a relationship between $\mathrm{TH}$ expression and activity in adult $\mathrm{SNc}$ neurons has been described (Aumann et al., 2011), it seems reasonable to conclude that an increase in ammonia levels in PCA and/or hyperammonaemia can lead to reduced TH expression in SNc cells, and that this might contribute towards the vulnerability of DAergic cells - and therefore to their reduced activity. In fact, the presence of acid-sensitive ion channels has been described in DAergic neurons, which are also sensitive to ammonium ions (Pidoplichko \& Dani, 2006). Recently, it has been shown that ammonium chloride treatment reduces the number of $\mathrm{TH}$ positive DAergic neurons in the medaka fish, causing the selective loss of DAergic neurons and the appearance of Lewy body-like inclusions (Matsui et al., 2010). Together, these observations suggest DAergic neurons to be particularly sensitive to increased levels of ammonia, as occurs following PCA.

There is very good evidence that the loss of TH-immunoreactivity in the $\mathrm{SN}$ is a good marker for the death of nigral DAergic neurons in neurodegenerative diseases. Although it is generally assumed that neuronal cell death is minimal in liver failure, recent studies have shown severe neuronal dysfunction and neuronal cell death to occur in end-stage liver failure (Butterworth, 2007). To date, no selective loss of DAergic neurons has been described in PCA, although, as mentioned above, a reduced number of TH-positive neurons are seen in PCA exposed rats.

In addition to the reduced number of TH-positive neurons in the $\mathrm{SN}$, TH-positive nerve terminals within the striatum are significantly reduced in PCA-treated rats compared to controls; this might suggest dysfunctional neurotransmission. The smaller number of $\mathrm{TH}-$ positive neurons might in turn affect the innervation of the striatum, which agrees with the fact that TH staining in the striatum shows lower expression in PCA-exposed rats than in controls. These differences suggest an imbalance in the proper functioning of the striatum and might underlie the behavioural deficits observed in long-term PCA-exposed rats.

The presence of extrapyramidal signs and symptoms in patients with end-stage chronic liver failure might suggest dysfunctional DAergic neurotransmission between the SNc and the dorsal striatum (the nigrostriatal pathway). The striatal content of dopamine is reduced in encephalopathic animals after bile duct ligation, which is consistent with results observed in patients with cirrhosis (Galvez-Gastélum et al., 2011). This could be due to dopamineaccelerated metabolism and/or a reduction in $\mathrm{TH}$. 


\subsection{Dopamine and glutamate interactions in the nigrostriatal system}

There is evidence that the brain dopamine system is under glutamatergic regulatory influence. Nigral DAergic neurons possess glutamate receptors (Chatha et al., 2000; Testa et al., 1994) and receive glutamatergic input from the neurons of the subthalamic nucleus (Groenewegen \& Berendse, 1990; Kita \& Kitai, 1987). These DAergic neurons degenerate when exposed to excitotoxic glutamate (Alexi et al., 2000; Tapia et al., 1999). In vitro studies have shown that DAergic neurons are preferentially affected by glutamate toxicity. DAergic neurons exposed to glutamate for $24 \mathrm{~h}$ were more vulnerable than non-DAergic neurons exposed to chronic glutamate-induced toxicity (Izumi et al., 2009), leading to selective DAergic neuronal death.

The glutamatergic modulation of DA release in SN DAergic neurons appears to be associated with increased DA release in the striatum (Morari et al., 1998). The rat dorsal striatum receives DAergic projections from the $\mathrm{SN}$ and glutamatergic projections from the cortex. Glutamatergic receptors are located on DAergic terminals and dopamine receptors on glutamatergic presynaptic endings. This anatomic arrangement implies that dopamine and glutamate may act in concert to regulate the activity of striatal neurons (Kulagina et al., 2001). Through the activation of ionotropic glutamate receptors, glutamate regulates the basal extracellular dopamine concentration in the striatum (Borland \& Michael, 2004; Kulagina et al., 2001). In contrast, the inhibitory role of DA in the modulation of glutamate release is well established and in the striatum DA acts via the activation of dopamine D2 receptors. It would seem that the overstimulation of glutamate receptors on nigral DAergic neurons may be involved in the progression of neurodegenerative diseases. It is known that the balance between glutamate and dopamine is disturbed when DAergic nigrostriatal neurons degenerate in the course of PD (Starr, 1995). However, it is not clear to what extent dopamine and glutamate interact with each other in hyperammonaemic situations. The densities of postsynaptic dopamine D2 receptors are reduced in the pallidum of HE patients (Mousseau et al., 1993) and it has recently been described that the binding site densities of dopamine receptors (D1 and D2) are down-regulated in the putamen (Palomero-Gallagher et al., 2006). These alterations of dopamine D2 receptor sites are indicative of DAergic synaptic dysfunction (Watanabe et al., 2008). This suggests that the nature of the dopamine-glutamate interaction varies depending on the receptor subtype involved and the experimental conditions employed.

Under hyperammonaemic conditions, high extracellular concentrations of glutamate can cause neurodegeneration by excessive stimulation of the post-synaptic glutamate receptors. Glutamate receptors are reduced in PCA-treated rats (Suárez et al., 1997). In addition, the increased extracellular brain concentrations of glutamate in experimental HE are due, in part, to the failure of astrocytic glutamate transporters (Knecht et al., 1997; Suárez et al., 2000). Their down-regulation has important consequences on the amount of extracellular glutamate since astrocytes are the brain cells that metabolise ammonia and glutamate via glutamine synthetase (GS). Thus, the activity of the enzyme glutamine synthetase, the glutamine/glutamate cycle and the brain's capacity to eliminate toxic substances, all influence the toxicity of increased ammonium levels. 


\section{Astrogliosis in the nigrostriatal system}

In response to almost any kind of CNS injury, astrocytes change their appearance (at different times post-injury) and undergo a characteristic hypertrophy of their processes, a condition known as astrogliosis. One of the hallmarks of this phenomenon is the up-regulation of the intermediate filament protein glial fibrillary acidic protein (GFAP). Increased GFAP expression is also seen in neurodegenerative diseases (Eng \& Ghirnikar, 1994; Johansson et al., 2007). Both astrogliosis and increased GFAP in the SN in neurodegenerative diseases are associated with the reduction of $\mathrm{TH}$ and the progressive degeneration of DAergic neurons (McGeer \& McGeer, 2008). In experimental models of PD, DAergic cells show reduced TH immunoreactivity, whereas reactive astrocytes show increased GFAP reactivity (Gomide et al., 2005; Reinhard et al., 1988). It has recently been postulated that glial cells are responsible for the progression of PD since more astrocytes are affected over the course of the disease (Halliday \& Stevens, 2011).

The importance of astrocytes in neurodegenerative diseases, particularly $\mathrm{HE}$, is well accepted (Butterworth, 2003; Norenberg, 1998). As mentioned above, astrocytes metabolise ammonia and glutamate through GS (review in Suárez et al., 2002). The principal neuropathological finding in HE is modified astrocyte morphology, which has been used as a marker of HE. Changes in astrocytes include nuclear enlargement, chromatin peripheral margination and prominent nucleoli; these changes are found mainly in the basal ganglia of patients dying of the disease (Norenberg, 1981).

It is well known that astrocytes perform a number of important functions including the regulation of the extracellular concentration of neurotransmitters, the promotion of synapse formation, and the promotion of neuronal survival. GFAP expression in the brain has been studied in both experimental and human chronic liver failure, in which it is reported reduced or increased depending on the brain region in question (Norenberg, 1987; Sobel et al., 1981; Suárez et al., 1998). The basis for these regional brain differences remains unknown. Although it is not known precisely why basal ganglia have a high affinity for ammonia, astrocytes show increased GFAP immunoreactivity in the striatum during the prolonged elevation of ammonia following PCA (Suárez et al., 2009).

Interactions have been reported between DAergic neurons and mesencephalic astrocytes in vitro, and it has been postulated that mesencephalic astrocytes may exert a morphogenetic effect on DAergic neurons (Denis-Donini et al., 1984). We showed astrocyte activation in the nigrostriatal pathway, which parallels the PCA-induced loss of DAergic neurons and terminals after PCA. The normal function of astrocytes was compromised following PCA, as evidenced by the increased GFAP expression in the SN, which was accompanied by neuronal dysfunction. Also, astrocytes in the striatum of PCA-exposed rats expressed increased GFAP immunoreactivity compared to controls (Fig. 2). These data reinforce the results of neuropathologic and molecular studies which indicate that HE in both acute and chronic liver failure is primarily a disorder of astroglial cells, and add support to the idea that HE is a classic example of a primary gliopathy (Butterworth, 2010; Norenberg, 1987). 

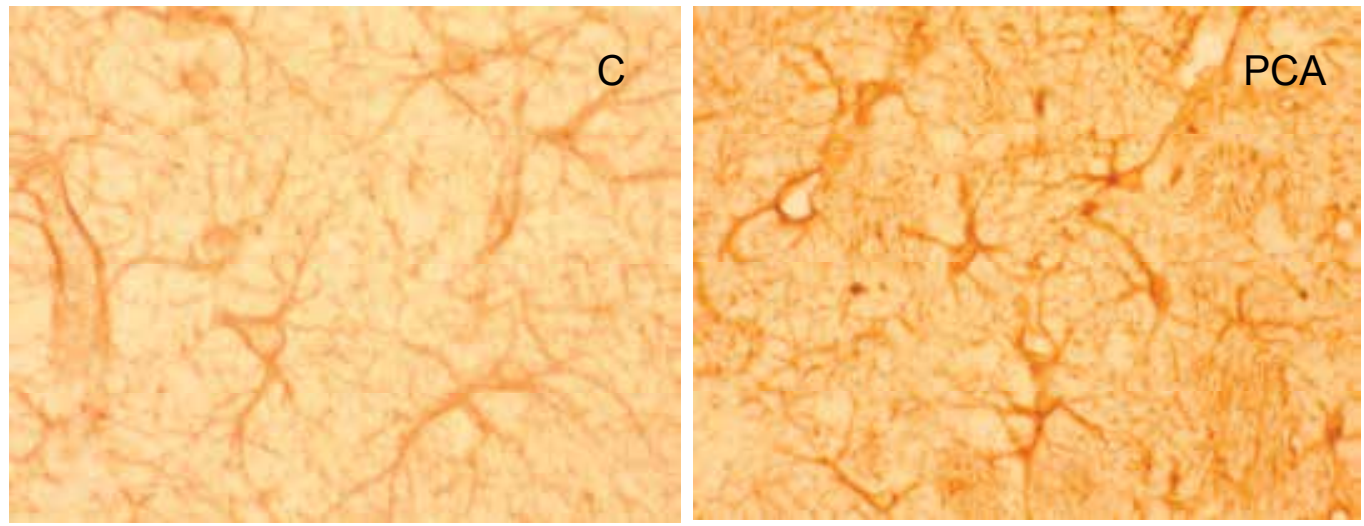

Fig. 2. GFAP immunoreactivity in the striatum of control (C) and PCA-exposed rats (PCA). Note the increase in GFAP expression in PCA rats.

\section{4. a-synuclein in the nigrostriatal system}

a-synuclein is a normally soluble, neuronal protein found in the synaptic terminals (Maroteaux \& Sheller, 1991; Sidhu et al., 2004). There is growing evidence that it is an important regulator of DAergic transmission; certainly, it participates in the life cycle of dopamine from its synthesis through to its storage, release and reuptake (Al-Wandi et al., 2010; Chadchankar et al., 2011; Liu et al., 2008; Shidu et al., 2004; Yavich et al., 2004). However, very little is known about the mechanisms, signalling pathways or transcriptional elements that regulate $\alpha$-synuclein expression. The overexpression of a-synuclein may, however, lead to degenerative processes (Goedert, 2001; Kirik et al., 2002; Lee \& Trojanowski, 2006). It can inhibit TH expression (Lo Bianco et al., 2002; Niu et al., 2009; Yu et al., 2004) and TH activity (Luo et al., 2007; Peng et al., 2005; Perez et al., 2002) in DAergic cells, and impair dopamine release (Nemari et al., 2010; Pelkonen et al., 2010). Indeed, studies on transgenic animals and gene-transfected DAergic cells have shown that overexpression is accompanied by the degeneration and even death of these cells as well as the formation of a-synuclein-positive intracellular inclusions (Lo Bianco et al., 2002; Masliah et al., 2000; Xu et al., 2002; Yamada et al., 2004). In fact, the abnormal aggregation of a-synuclein is thought to cause neurodegeneration (Cookson, 2009; Dawson \& Dawson, 2003; Ma et al., 2003; Tofaris \& Spillantini, 2005). Once aggregation begins the normal physiological functions regulated by this protein can be severely compromised (Shidu et al., 2004). a-synuclein has been shown to form aggregates or insoluble fibrils finally leading to pathological deposits such as those seen in PD, in dementia with Lewy bodies (DLB), and in MSA (Duda et al., 2000; Goedert, 2001; Spillantini et al., 1998; Trojanowski \& Lee, 2001; Wakabayashi et al., 2007). Experimental PD models show increased asynuclein expression and aggregation as well as DAergic neuron degeneration/loss (Cannon \& Greenamyre, 2010; Chesselet, 2008; Khodr et al., 2011), accompanied by motor deficit (Khodr et al., 2011).

The pathological involvement of a-synuclein in HE has never before been investigated. In vitro studies have shown that cultured astrocytes exposed to ammonia produce a-synuclein mRNA (Bodega et al., 2006). Our group studied the modifications of a-synuclein 
conformation in PCA-treated rats and found that PCA clearly affected a-synuclein expression in the cerebellum (Suárez et al., 2010). It has been postulated that the structure of a-synuclein is extremely sensitive to its environment, and studies with the SH-SY5Y human DAergic cell line have shown that ammonium chloride treatment can induce the formation of a-synuclein inclusions (Matsui et al., 2010). This is in agreement with our other observations that indicate that chronically high ammonia levels in PCA-exposed rats induce a-synuclein expression in the nigrostriatal pathway. Although the precise mechanisms of the upregulation of a-synuclein are unknown, previous evidence (Lo Bianco et al., 2002; Gomide et al., 2005; Luo et al., 2007; Niu et al., 2009; Peng et al., 2005; Perez et al., 2002) suggests that the overexpression of a-synuclein in the nigrostriatal system may contribute to the reduced $\mathrm{TH}$ expression in PCA-exposed rats and to the degeneration of DAergic neurons. DAergic neurons might be more vulnerable to chronic hyperammonaemia in the presence of a-synuclein, supporting the idea of the latter's importance in the pathogenesis of HE. The vulnerability of SN neurons in which a-synuclein is overexpressed has been associated with the loss of DAergic neurons in a rat model of PD (Yamada et al., 2004).

a-synuclein has been reported to accumulate in the neuronal cytoplasm and processes in the brains of patients with PD and DLB (Polymeropoulos et al., 1997; Zarranz et al., 2004), and recently it has been shown that most aggregates are located at the presynapses in the form of very small deposits (Schulz-Schaeffer, 2010). This would impair synaptic plasticity in the basal ganglia prior to neurodegeneration (Kurz et al., 2010).

Although a-synuclein is not normally expressed by glial cells in the adult brain, asynuclein-immunoreactive inclusions have been reported in these cells in patients with PD, DLB and MSA (Duda et al., 2000; Piao et al., 2000; Spillantini et al., 1998; Wakabayashi et al., 1998, 2000). Exposure to extracellular a-synuclein aggregates can lead to astroglial activation; studies in vitro have shown that a-synuclein treatment can directly cause GFAP reactivity in human astrocytes (Koob et al., 2010), and experimental in vivo studies with asynuclein mutant mice have indicated that the number of GFAP-positive astrocytes increases in the brainstem (Gu et al., 2010).

Astrocytes, however, are not known to synthesize a-synuclein (Mori et al., 2002). It has been suggested that a-synuclein is released from neurons into the extracellular space (Borghi et al., 2000; Lee et al., 2005) and accumulates there - something seen in the brains of patients with PD (Lee, 2008). In PD, the altered a-synuclein molecule can, however, be taken up and accumulated by astrocytes (Braak et al., 2007; Lee et al., 2010), leading to the progression of the disease (Halliday \& Stevens, 2011). In PCA-treated rats, the increase in a-synuclein expression is concomitant with the increase in GFAP expression in the striatum (Suárez et al., 2009). It is possible that astroglial activation is caused by increased a-synuclein production associated with the markedly elevated ammonia levels observed in PCA-treated animals.

\section{Nitric oxide in the nigrostriatal system}

Nitric oxide (NO) has been implicated in the pathogenesis of several CNS diseases. It is well known that increased extracellular glutamate leads to an increased inflow of $\mathrm{Ca}^{2+}$ into postsynaptic neurons, the activation of nitric oxide synthases (NOS), and the formation of NO. 
Nitric oxide is a key modulator of neuronal activity in the dorsal striatum and is thought to play an important role in different complex processes, including the control of motor function (West et al., 2002). In the striatum, NOS, the enzyme involved in the synthesis of $\mathrm{NO}$, has been selectively found in a scattered population of interneurons (Vincent \& Kimura, 1992). The level of neuronal NOS (nNOS) is increased in neurodegenerative diseases in which disorders of the basal ganglia have been described (Aguilera et al., 2007; Pérez-Severiano et al., 2002). Increased NO (via nNOS activity) can be harmful to surrounding cells in neurodegenerative processes mediated by glutamate. Striatal nNOSproducing neurons are particularly vulnerable to glutamate (Calabresi et al., 2000; Mitchell et al., 1999); they receive asymmetric synapses from glutamatergic afferents (Vuillet et al., 1989) and express glutamate receptors (Kawaguchi, 1997), which play a primary role in stimulating nNOS activity. The glutamate receptor activation of nNOS-producing neurons induces NO formation in the striatum (Bogdanov \& Wurtman, 1997) and, in turn, NO increases glutamate release (Trabace \& Kendrick, 2000).

In addition to glutamatergic inputs, striatal nNOS-expressing interneurons are innervated by DAergic terminals (Fujiyama \& Masuko, 1996; Hidaka \& Totterdell, 2001). Striatal NOS interneurons express dopamine D1/5 receptors (Rivera et al., 2002), and dopamine D1 receptor activation stimulates striatal NO synthesis. In addition, the glutamatergic activation of the NMDA receptors stimulates nNOS activity and NO production (Garthwaite, 2008) in a manner likely to be modulated by reciprocal dopamine D1-NMDA glutamate receptor interactions; the latter play a critical role in regulating striatal nNOS interneuron activity (Hoque et al., 2010). Further, in vitro and in vivo studies have shown that NO modulates dopamine and glutamate release in the striatum (Bogdanov \& Wurtman, 1997; Hanbauer et al., 1992; Lin et al., 1995; Sandor et al., 1995; Shibata et al., 1996). These findings indicate that interactions between striatal DAergic, glutamatergic, and nitrergic systems play a significant role in the regulation of striatal function (Hoque et al., 2010; West et al., 2002).

In recent years, it has been suggested that nitrosative stress is involved in the pathophysiological cascade in HE (Bemeur et al., 2010); nitrosative stress is potentially lethal to neurons and occurs in the brain in both acute and chronic liver failure (Larsen et al., 2001; Schliess et al., 2002). NOS activity is increased in the brains of portacaval shunted rats (Rao et al. 1995). The number of nNOS-positive neurons increases in the striatum of PCA-treated rats (Fig. 3), and the astrocytes of the striatum express nNOS as well as inducible NOS (iNOS) (Suárez et al., 2009). The induction of iNOS in astrocytes may contribute to neuronal damage in chronic neurodegenerative disorders since glial cells can synthesise large amounts of NO (Dawson \& Dawson, 1998). Certainly, iNOS stimulates DAergic neurodegeneration in an animal model of PD (Liberatore et al., 1999). Thus, the induction of iNOS in reactive astrocytes may generate toxic levels of NO, contributing to the induction of neuronal damage associated with chronic hyperammonaemia in PCA-exposed rats/patients with HE.

The NO produced by activated astrocytes can be harmful to neurons since it reacts with superoxide to generate peroxynitrite (Catania, 2001), a molecule that contributes to cell death. Nitrotyrosine (NT) is a relatively stable marker for peroxynitrite production (Gow et al., 1996) and has been proposed a valuable indicator of pathological levels of NO and peroxynitrite (Beckman, 1996). When our group analysed nitrotyrosine expression in PCArats showing NT expression in the nigrostriatal pathway, a significant increase was 
observed in NT immunoreactivity in some of the cell bodies in the SN. NT expression in the astroglial cells occurred in the upper part of the striatum and colocalised with GFAP (Suárez et al., 2009). Therefore, the DAergic system might be particularly susceptible to NO neurotoxicity, which may contribute to the dysfunction of DAergic neurons after PCA exposure.
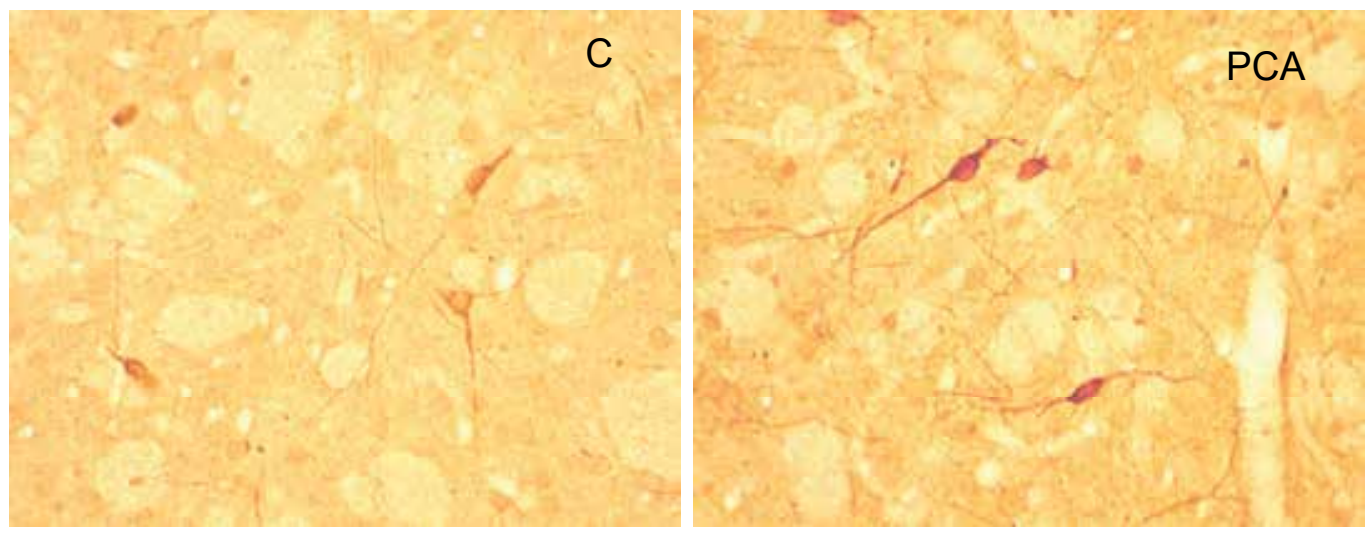

Fig. 3. nNOS immunoreactivity in the striatum. In controls (C), nNOS-immunoreactive neurons were homogeneously distributed in the striatum. In PCA exposed brains, the number of nNOS+ neurons was increased and immunolabeled neurons presented intensified immunoreactivity and thickened processes.

Although NO plays a crucial role in the integration of glutamate and DA transmission, the role of $\mathrm{NO}$ in modulating neurotransmitter interaction in the striatum following PCA and/or hyperammonaemia remains somewhat unclear. The involvement of $\mathrm{NO}$ in $\mathrm{HE}$ pathogenesis is supported by the observations that nNOS and iNOS expressions are upregulated in experimental HE. This evidence strongly supports the hypothesis that the excessive production of NO in the DAergic pathway contributes towards the neuronal dysfunction observed after PCA. Experimental evidence for cerebral nitrosative stress in experimental models of HE involving acute or chronic liver failure suggests it is involved in the pathophysiological cascade responsible for the disease (Bemeur et al., 2010). In patients with HE, NO levels correlate with the presence and severity of HE (Papadopoulos et al., 2010).

\section{Conclusions}

This work analyses whether chronic hyperammonaemia affects the activity of SN neurons and the regulation of TH expression following PCA. The neurones and astrocytes in the nigrostriatal system show different responses to PCA; neuronal TH decreases and astroglial GFAP increases in both the SN and the striatum. This may be related to different cellular susceptibilities to increased ammonia levels in both types of cell, which contributes to the progress of DAergic neurodegeneration. Since the DAergic neurons in the SN that project to the dorsolateral striatum are substantially more vulnerable to hyperammonaemic conditions, the dysfunction and/or loss of SN DAergic neurons following PCA might be attributed to the overexpression of a-synuclein as well as to the activation of nearby 
astroglia. As in PD, dysfunctional DAergic neurotransmission between the $\mathrm{SNc}$ and the dorsal striatum (the nigrostriatal pathway) might be the cause of the movement disorders observed in PCA-exposed rats as well as in HE patients. Different elements can initiate a cascade of events in the cell body, inducing DAergic neuron degeneration and ultimately HE. These events may include a-synuclein overexpression, astroglial activation and nitrosative stress.

In summary, chronic moderate hyperammonaemia, similar to that seen in HE patients, induces DAergic dysfunction in DA neurons via a reduction in $\mathrm{TH}$ immunoreactivity, astroglial activation via an increase in GFAP, the overexpression of a-synuclein in DAergic cells, and increased nNOS, iNOS and NT expression in the nigrostriatal system. These changes underlie the harmful effects of chronic hyperammonaemia on motor and cognitive function.

\section{References}

Aguilera P, Chánez-Cárdenas ME, Floriano-Sánchez E, Barrera D, Santamaría A, SánchezGonzález DJ, Pérez-Severiano F, Pedraza-Chaverrí J \& Maldonado Jiménez PD. (2007). Time-related changes in constitutive and inducible nitric oxide synthases in the rat striatum in a model of Huntington's disease. NeuroToxicology 28:1200-1207

Alexi T, Borlongan CV, Faull LM, Williams CE, Clark RG, Gluckman PD \& Hughes PE. (2000). Neuroprotective strategies for basal ganglia degeneration: Parkinson's and Huntington's diseases. Prog Neurobiol 60:409-470

Al-Wandi A, Ninkina N, Millership S, Williamson SJM, Jones PA \& Buchman VL. (2010). Absence of a-synuclein affects dopamine metabolism and synaptic markers in the striatum of ageing mice. Neurobiol Aging 31:796-804.

Audet RM \& Butterworth RF. (1998). Portacaval anastomosis results in more widespread alterations of cerebral metabolism in old versus young adult rats: Implications for post-shunt encephalopathy. Metab Brain Dis 13:69-78

Aumann TD, Egan K, Lim J, Boon WC, Bye CR, Chua HK, Baban N, Parish CL, Bobrovskaya L, Dickson P \& Horne MK. (2011). Neuronal activity regulates expression of tyrosine hydroxylase in adult mouse substantia nigra pars compacta neurons. $J$ Neurochem 116:646-58

Beckman JS. (1996). Oxidative damage and tyrosine nitration from peroxynitrite. Chem Res Toxicol 9:836-844

Bemeur C, Desjardins P \& Butterworth RF. (2010). Evidenc for oxidative/nitrosative stress in the pathogenesis of hepatic encephalopathy. Metab Brain Dis 25:3-9.

Bodega G, Suárez I, Arilla E, Rubio M \& Fernández B. (1991). Heterogeneous astroglial response in the rat spinal cord to long-term portacaval shunt. An immunohistochemical study. Glia 4:400-407

Bodega G, Suárez I, López-Fernández LA, Almonacid L, Zaballos A \& Fernández B. (2006). Posible implication of cilliary neurotrophic factor (CNTF) and beta-synuclein in the ammonia effect on cultured rat astroglial cells: a study using DNA and protein microarrays. Neurochem Int 48:729-38

Bogdanov MB \& Wurtman RJ. (1997). Possible involvement of nitric oxide in NMDAinduced glutamate release in the rat striatum: An in vivo microdialysis study. Neurosci Lett 221:197-201 
Borghi R, Marchese R, Negro A, Marinelli L, Forloni G, Zaccheo D, Abbruzzese G \& Tabaton M. (2000). Full length alpha-synuclein is present in cerebrospinal fluid from Parkinson's disease and normal subjects. Neurosci Lett 287:65-67

Borland LM \& Michael AC. (2004). Voltammetric study of the control of striatal dopamine release by glutamate. J Neurochem 91:220-229

Braak H, Sastre M \& Tredici KD. (2007). Development of a-synuclein immunoreactive astrocytes in the forebrain parallels stages of intraneuronal pathology in sporadic Parkinson's disease. Acta Neuropathol 114:231-241

Burkhard PR, Delavelle J, Pasquier RD \& Spahr L. (2003). Chronic parkinsonism associated with cirrhosis. Arch Neurol 60:521-528

Butterworth RF. (2002). Pathophysiology of hepatic encephalopathy: a new look at ammonia. Metab Brain Dis 17:221-227

Butterworth RF. (2003). Pathogenesis of hepatic encephalopathy: new insights from neuroimaging and molecular studies. J Hepatol 39:278-285

Butterworth RF. (2007). Neuronal cell death in hepatic encephalopathy. Metab Brain Dis 22:309-320

Butterworth RF. (2010). Altered glial-neuronal crosstalk: Cornerstone in the pathogenesis of hepatic encephalopathy. Neurochem Int 57:383-388

Calabresi P, Centonze D, Gubellini P, Marfia GA, Pisani A, Sancesario G \& Bernardi G. (2000). Synaptic transmission in the striatum: from plasticity to neurodegeneration. Prog Neurobiol 61:231-265

Cannon JR \& Greenamyre JT. (2010). Neurotoxic in vivo models of Parkinson's disease recent advances. Prog Brain Res 184:17-33

Catania MV, Aronica E, Yankaya B \& Troost D. (2001). Increased expression of neuronal nitric oxide synthase spliced variants in reactive astrocytes of amyotrophic lateral sclerosis human spinal cord. J Neurosci 21:RC148

Cauli O, Llansola M, Erceg S \& Felipo V. (2006). Hypolocomotion in rats with chronic liver failure is due to increased glutamate and activation of metabotropic glutamate receptors in substantia nigra. J Hepathol 45:654-661

Chadchankar H, Ihalainen J, Tanila H \& Yavich L. (2011). Decreased reuptake of dopamine in the dorsal striatum in the absence of alpha-synuclein. Brain Res 1382:37-44

Chatha BT, Bernard V, Streit P \& Bolam JP. (2000). Synaptic localization of ionotropic glutamate receptors in the rat substantia nigra. Neuroscience 101:1037-1051

Chesselet MF. (2008). In vivo alpha-synuclein overexpression in rodents: a useful model of Parkinson's disease? Exp Neurol 209:22-27

Cookson MR. (2009). Alpha-synuclein and neuronal cell death. Mol Neurodegeneration 4:4-9

Damier P, Hirsch EC, Agid Y \& Graybiel AM. (1999). The substantia nigra of the human brain: II. Patterns of loss of dopamine-containing neurons in Parkinson's disease. Brain 122:1437-1448

Dawson DL \& Dawson TM. (1998). Nitric oxide in neurodegeneration. Prog Brain Res 118:215-229

Dawson TM \& Dawson VL. (2003). Molecular pathways of neurodegeneration in Parkinson's disease. Science 302:819-822

Denis-Donini S, Glowinski J \& Prochiantz A. (1984). Glial heterogeneity may define the three-dimensional shape of mouse mesencephalic dopaminergic neurones. Nature 307:641-643 
Duda JE, Lee VM \& Trojanowski JQ. (2000). Neuropathology of synuclein aggregates. J Neurosci Res 61:121-7

Eng LF \& Ghirnikar RS. (1994). GFAP and astrogliosis. Brain Pathol 4:229-237

Fujiyama F \& Masuko S. (1996). Association of dopaminergic terminals and neurons releasing nitric oxide in the rat striatum: An electron microscopic study using NADPH-Diaphorase histochemistry and tyrosine hydroxylase immunohistochemistry. Brain Res Bull 40:121-127

Gaig C \& Tolosa E. (2009). When does Parkinson's disease begin?. Mov Disord 24, Suppl 2:S656- 664

Gálvez-Gastélum FJ, Garcia-Bañuelos JJ, Beas-Zárate C, Segura-Flores A, Gonzalez H, Chaparro-Huerta V, Salazar-Montes A, Sandoval-Rodriguez AS, Bueno-Topete M, Lucano-Landeros S, Medina-Preciado D, Gonzalez-Garcia I \& ArmendárizBorunda J. (2011). Combinatorial gene therapy induces regression of hepatic encephalopathy. Gene Ther 18:88-94

Garthwaite J. (2008). Concepts of neural nitric oxide-mediated transmission. Eur J Neurosci 27:2783-2802

Gauthier S \& Sourkes TL. (1982). Anatomical and biochemical basis of the extrapyramidal disorders. Prog. Neuro-psychopharmacol Biol Psychiatry 6:595-599

Goedert M. (2001). Alpha-synuclein and neurodegenerative diseases. Nat Rev Neurosci 2:492-501.

Gomide V, Bibancos T \& Chadi G. (2005). Dopamine cell morphology and glial cell hypertrophy and process branching in the nigrostriatal system after striatal 6OHDA analyzed by specific stereological tools. Int J Neurosci 115:557-582

Groenewegen HJ \& Beredse HW. (1990). Connections of the subthalamic nucleus with ventral striatopallidal parts of the basal ganglia in the rat. J Comp Neurol 294:607-22

Gow A, Duran D, Thom SR \& Ischiropoulos H. (1996). Carbon dioxide enhancement of peroxynitrite-mediated protein tyrosine nitration. Arch Biochem Biophys 333:42-48

Gu X-L, Long C-X, Sun L, Xie Ch, Lin X \& Cai H. (2010). Astrocytic expression of Parkinson's disease-related A53T a-synuclein causes neurodegeneration in mice. Mol Brain 3:12

Hajos M \& Greenfield SA. (1993). Topographic heterogeneity of substantia nigra neurons: diversity in intrinsic membrane properties and synaptic inputs. Neuroscience 55:919934

Halliday GM \& Stevens CH. (2011). Glia: Initiators and progressors of pathology in Parkinson's disease. Mov Disord 26:6-17

Hidaka S \& Totterdell S. (2001). Ultrastructural features of the nitric oxide synthasecontaining interneurons in the nucleus accumbens and their relationship with tyrosine hydroxylase-containing terminals. J Comp Neurol 431:139-154

Hanbauer I, Wink D, Osawa Y, Edelman GM \& Gally JA. (1992). Role of nitric oxide in NMDA-evoked release of [3H]-dopamine from striatal slices. NeuroReport 3:409-412

Hoque KE, Indorkar RP, Sammut S \& West AR. (2010). Impact of dopamine-glutamate interactions on striatal neuronal nitric oxide synthase activity. Psychopharmacol 207:571-581

Izumi Y, Yamamoto N, Matsuo T, Wakita S, Takeuchi H, Kume T, Katsuki H, Sawada H \& Akaike A. (2009). Vulnerability to glutamate toxicity of dopaminergic neurons is dependent on endogenous dopamine and MAPK activation. J Neurochem 110:745-755 
Johansson A, Engler H, Blomquist G, Scott B, Wall A, Aquilonius S-M, Langstrom B \& Askmark H. (2007). Evidence for astrocytosis in ALS demonstrated by [11C](L)deprenyl-D2 PET. J Neurol Sci 255:17-22

Kawaguchi Y. (1997). Neostriatal cell subtypes and their functional roles. Neurosci Res 27:1-8

Kita H \& Kitai ST. (1987). Efferent projections of the subthalamic nucleus in the rat: light and electron microscopic analysis with the PHA-L method. J Comp Neurol 260:435-52

Khodr CE, Sapru MK, Pedapati J, Han Y, West NC, Kells AP, Bankiewicz KS \& Bohn MC. (2011). An alpha-synuclein AAV gene silencing vector ameliorates a behavioral deficit in a rat model of Parkinson's disease, but displays toxicity in dopamine neurons. Brain Res 1395:94-107

Knecht K, Michalak A, Rose C, Rothstein JD \& Butterworth RF. (1997). Decreased glutamate transporter (GLT-1) expression in frontal cortex of rats with acute liver failure. Neurosci Lett 229:201-203

Kirik D, Rosenblad C, Burger C, Lundberg C, Johansen TE, Muzyczka N, Mandel RJ \& Bjorklund A. (2002). Parkinson-like neurodegeneration induced by targeted overexpression of alpha-synuclein in the nigrostriatal system. J Neurosci 22:27802791

Koob AO, Paulino AD \& Masliah E. (2010). GFAP reactivity, apolipoprotein E redistribution and cholesterol reduction in human astrocytes treated with a-synuclein. Neurosci Lett 469:11-14

Korotkova TM, Ponomarenko AA, Brown RE \& Haas HL. (2004). Functional diversity of ventral midbrain dopamine and GABAergic neurons. Mol Neurobiol 29:243-259

Kulagina NV, Zigmond MJ \& Michael AC. (2001). Glutamate regulates the spontaneous and evoked release of dopamine in the rat striatum. Neuroscience 102:121-128

Kurz A, Double KL, Lastres-Becker I, Tozzi A, Tantucci M, Bockhart V, Bonin M, GarcíaArencibia M, Nuber S, Schlaudraff F, Liss B, Fernández-Ruiz J, Gerlach M, Wüllner U, Lüddens H, Calabresi P, Auburger G \& Gispert S. (2010). A53T-Alpha-synuclein overexpression impairs dopamine signaling and striatal synaptic plasticity in old mice. Plos ONE 5:e11464

Larsen FS, Gottstein J \& Blei A. (2001). Cerebral hyperemia and nitric oxide synthase in rats with ammonia-induced brain edema. J Hepatol 34 :548-554

Layrargues GP. (2001). Movement Dysfunction and Hepatic Encephalopathy. Metab Brain Dis 16:27-35

Lee HJ, Patel S \& Lee SJ. (2005). Intravesicular localization and exocytosis of alpha-synuclein and its aggregates. J Neurosci 25:6016-6024

Lee SJ. (2008). Origins and effects of extracellular a-synuclein: Implications in Parkinson's disease. J Mol Neurosci 34:17-22

Lee VM \& Trojanowski JQ. (2006). Mechanisms of Parkinson's disease linked to pathological alpha-synuclein: new targets for drug discovery. Neuron 52:33-38

Lee HJ, Suk JE, Patrick C, Bae EJ, Cho JH, Hwang D, Masliah E \& Lee SJ. (2010). Direct transfer of alpha-synuclein from neuron to astroglia causes inflammatory responses in synucleinopathies. J Biol Chem 285:9262-9272

Liberatore GT, Jackson-Lewis V, Vukosavic S, Mandir AS, Vila M, McAuliffe WG, Dawson VL, Dawson TM \& Przedborski S. (1999). Inducible nitric oxide synthase stimulates dopaminergic neurodegeneration in the MPTP model of Parkinson disease. Nat Med 5:1403-1409 
Lin AM-Y, Kao L-S \& Chai CY. (1995). Involvement of nitric oxide in dopaminergic transmission in rat striatum: An in vivo electrochemical study. J Neurochem 65:2034 $-2049$

Liss B \& Roeper J. (2008). Individual dopamine midbrain neurons: functional diversity and flexibility in health and disease. Brain Res Rev 58:314-321

Liu D, Jin L, Wang H, Zhao H, Zhao C, Duan C, Lu L, Wu B, Yu S, Chan P, Li Y \& Yang H. (2008). Silencing alpha-synuclein gene expression enhances tyrosine hydroxylase activity in MN9D cells. Neurochem Res 33:1401-1409

Lo Bianco C, Ridet JL, Schneider BL, Deglon N \& Aebischer P. (2002). Alphasynucleinopathy and selective dopaminergic neuron loss in a rat lentiviral-based model of Parkinson's disease. Proc Natl Acad Sci USA 99:10813-10818

Luo C, Rajput AH, Akhtar S \& Rajput A. (2007). a-synuclein and tyrosine hydroxylase expression in acute rotenone toxicity. Int J Mol Med 19:517-521

Ma QL, Chan P, Yoshii M \& Uéda K. (2003). Alpha-synuclein aggregation and neurodegenerative diseases. J Alzheimer Dis 5:139-148

Maroteaux L \& Scheller RH. (1991). The rat brain synucleins: family of proteins transiently associated with neuronal membrane. Brain Res Mol Brain Res 11:335-343

Martin JR. (1986). Discordant motor activity levels of portacaval-shunted rats in runway and swim tests. Behav Neurosci 100:427-437

Masliah E, Rockenstein E, Veinbergs I, Mallory M, Hashimoto M, Takeda A, Sagara Y, Sisk A \& Mucke L. (2000). Dopaminergic Loss and Inclusion Body Formation in aSynuclein Mice: Implications for Neurodegenerative Disorders. Science 287:12651269

Matsui H, Ito H, Taniguchi Y, Takeda S \& Takahashi R. (2010). Ammonium chloride and tunicamycin are novel toxins for dopaminergic neurons and induce Parkinson's disease-like phenotypes in medaka fish. J Neurochem 115:1150-1160

McGeer PL \& McGeer EG. (2008). Glial reactions in Parkinson's disease. Mov Disord 15:474483

Mitchell IJ, Cooper AJ \& Griffiths MR. (1999). The selective vulnerability of striatopallidal neurons. Progress Neurobiol 59:691-719

Morari M, Marti M, Sbrenna S, Fuxe K, Bianchi C \& Beani L. (1998). Reciprocal dopamineglutamate modulation of release in the basal ganglia. Neurochem Int 33:383-397

Mori F, Tanji K, Yoshimoto M, Takahashi H \& Wakabayashi K. (2002). Demostration of asynuclein immunoreactivity in neuronal and glial cytoplasm in normal brain tissue using proteinase K and formic acid pretreatment. Exp Neurol 176:98-104

Mousseau DD, Perney P, Pomier Layrargues G \& Butterworth RF. (1993). Selective loss of pallidal dopamine D2 receptor density in hepatic encephalopathy. Neurosci Lett 162:192-196

Nemari VM, Lu W, Berge V, Nakamura K, Onoa B, Lee MK, Chaundry FA, Nicoll RA \& Edwards RH. (2010). Increased expression of a-synuclein reduces neurotransmitter release by inhibiting synaptic vesicle reclustering after endocytosis. Neuron 65:66-79

Niu Ch, Mei J, Pan Q \& Fu X. (2009). Nigral Degeneration with Inclusion Body Formation and Behavioral Changes in Rats after Proteasomal Inhibition. Stereotact Funct Neurosurg 87:69-81

Norenberg MD. (1981). The astrocyte in liver disease. In: Advances in Cellular Neurobiology, S. Fedoroff and L. Hertz, (Eds.), Vol. 2, pp. 303-352, Academic Press, New York 
Norenberg MD. (1987). The role of astrocytes in hepatic encephalopathy. Neurochem Pathol 6:13-33

Norenberg MD. (1998). Astroglial dysfunction in hepatic encephalopathy. Metab Brain Dis 13:319-335

Ong JP, Aggarwal A, Krieger D, Easley KA, Karafa MT, Van Lente F, Arroliga AC \& Mullen KD. (2003). Correlation between Ammonia Levels and the Severity of Hepatic Encephalopathy. Am J Med 114:188 -193

Palomero-Gallagher N, Reifenberger G, Kostopoulos G, Kircheis G, Häussinger D \& Zilles K. (2006). Neurotransmitter receptor alterations in hepatic encephalopathy. In: Hepatic Encephalopathy and Nitrogen Metabolism. Häussinger D, Kircheis G, Schliess F, (Eds.), pp 255-272, Dordrecht, Springer

Papadopoulos N, Soultati A, Goritsas C, Lazaropoulou C, Achimastos A, Adamopoulos A \& Dourakis SP. (2010). Nitric oxide, ammonia, and CRP levels in cirrhotic patients with hepatic encephalopathy: is there a connection?. J Clin Gastroenterol 44:713-719

Pelkonen A, Hiltunen M, Kiianmaa K \& Yavich L. (2010). Stimulated dopamine overflow and alpha-synuclein expresion in the nucleus accumbens core distinguish rats bred for differential ethanol preference. J Neurochem 114:1168-1176

Peng X-M, Tehranian R, Dietrich P, Stefanis L \& Perez RG. (2005). Alpha-synuclein activation of protein phosphatase $2 \mathrm{~A}$ reduces tyrosine hydroxylase phosphorylation in dopaminergic cells. J Cell Sci 118:3523-3530

Pérez-Severiano F, Escalante B, Vergara P, Rios C \& Segovia J. (2002). Age-dependent changes in nitric oxide synthase activity and protein expression in striata of mice transgenic for the Huntington's disease mutation. Brain Res 951:36-42

Perez RG, Waymire JC, Lin E, Liu JJ, Guo F \& Zigmond MJ. (2002). A role for alphasynuclein in the regulation of dopamine biosynthesis. J Neurosci 22:3090-3099

Piao YS, Wakawayashi K, Hayashi S, Yoshimoto M \& Takahashi H. (2000). Aggregation of a-synuclein/NACP in the neuronal and glial cells in diffuse Lewy body disease: a survey of six patients. Clin Neuropathol 19:163-169

Pidoplichko VI \& Dani JA. (2006). Acid-sensitive ionic channels in midbrain dopamine neurons are sensitive to ammonium, which may contribute to hyperammonemia damage. Proc Natl Acad Sci USA 103:11376-11380

Polymeropoulos MH, Lavedan C, Leroy E, Ide SE, Dehejia A, Dutra A, Pike B, Root H, Rubenstein J, Boyer R, Stenroos ES, Chandrasekharappa S, Athanassiadou A, Papapetropoulos T, Johnson WG, Lazzarini AM, Duvoisin RC, Di Iorio G, Golbe LI \& Nussbaum RL. (1997). Mutation in the alpha-synuclein gene identified in families with Parkinson's disease. Science 276:2045-2047

Rao VLR, Audet RM \& Butterworth RF. (1995). Increased nitric oxide synthase activities and $\mathrm{L}-[3 \mathrm{H}]$ arginine uptake in brain following portacaval anastomosis. J Neurochem 65:677-678

Reinhard JF,Jr, Miller DB \& O'Callaghan JP. (1988). The neurotoxicant MPTP (1-methyl-4phenyl-1,2,3,6-tetrahydropyridine) increases glial fibrillary acidic protein and decreases dopamine levels of the mouse striatum: evidence for glial response to injury. Neurosci Lett 95:246-251

Rivera A, Alberti I, Martín AB, Narvaez JA, Calle A \& Moratalla R. (2002). Molecular phenotype of rat striatal neurons expressing the dopamine D5 receptor subtype. Eur J Neurosci 16:2049-2058 
Sandor NT, Brassai A, Puskas A \& Lendvai B. (1995). Role of nitric oxide in modulating neurotransmitter release from rat striatum. Brain Res Bull 36:483- 486

Schliess F, Görg B, Fischer R, Desjardins P, Bidmon HJ, Herrmann A, Butterworth RF, Zilles K \& Häussinger D. (2002). Ammonia induces MK-801-sensitive nitration and phosphorylation of protein tyrosine residues in rat astrocytes. FASEB J 16:739-741

Schulz-Schaeffer WJ. (2010). The synaptic pathology of a-synuclein aggregation in dementia with Lewy bodies, Parkinson's disease and Parkinson's disease dementia. Acta Neuropathol 120:131-143

Shibata M, Araki N, Ohta K, Hamada J, Shimizu K \& Fukuuchi Y. (1996). Nitric oxide regulates NMDA-induced dopamine release in rat striatum. NeuroReport 7:605- 608

Sidhu A, Wersinger C \& Vernier P. (2004). Does alpha-synuclein modulate dopaminergic synaptic content and tone at the synapse? FASEB J 18:637-647

Smith AD \& Bolam JP. (1990). The neural network of the basal ganglia as revealed by the study of synaptic connections of identified neurones. Trends Neurosci 13:259-265

Sobel RA, De Armond SJ, Forno LS \& Eng LF. (1981). Glial fibrillary acidic protein in hepatic encephalopathy: An immunohistochemical study. J Neuropathol Exp Neurol 40:625 632

Spahr L, Burkhard PR, Grötzsch H \& Hadengue A. (2002). Clinical significance of basal ganglia alterations at brain MRI and $1 \mathrm{H}$ MRS in cirrhosis and role in the pathogenesis of hepatic encephalopathy. Metab Brain Dis 17:399-413

Spahr L, Vingerhoets F, Lazeyras F, Delavelle J, DuPasquier R, Giostra E, Mentha G, Terrier F \& Hadengue A. (2000). Magnetic resonance imaging and proton spectroscopic alterations correlate with parkinsonian signs in patients with cirrhosis. Gastroenterology 119:774-781

Spillantini MG, Crowwther RA, Jakes R, Hasegawa M \& Goedert M. (1998). a-Synuclein in filamentous inclusions of Lewy bodies from Parkinson's disease and dementia with Lewy bodies. Proc Natl Acad Sci USA 95:6469-6473

Starr MS. (1995). Glutamate/dopamine D1/D2 balance in the basal ganglia and its relevance to Parkinson's disease. Synapse 19:264-293

Steindl PE, Coy DL, Finn B, Zee PC \& Blei AT. (1996). A low-protein diet ameliorates disrupted diurnal locomotor activity in rats after portacaval anastomosis. Am J Physiol 271:G555-G560

Suárez I, Bodega G \& Fernández B. (1997). Modulation of AMPA receptor subunits GluR1 and GluR2/3 in the rat cerebellum in experimental hepatic encephalopathy model. Brain Res 778:346-353

Suárez I, Bodega G \& Fernández B. (1998). Changes in glial fibrillary acidic protein immunoreactivity in response to experimental hepatic encephalopathy in the rat hippocampus. Rest Neurol Neurosci 12:1-6

Suárez I, Bodega G \& Fernández B. (2000). Modulation of glutamate transporters (GLAST, GLT-1 and EAAC1) in the rat cerebellum following portocaval anastomosis. Brain Res 859:293-302

Suárez I, Bodega G \& Fernández B. (2002). Glutamine synthetase in brain: effect of ammonia. Neurochem Int 41:123-142

Suárez I, Bodega G \& Fernández B. (2010). Upregulation of a-synuclein in the rat cerebellum in experimental hepatic encephalopathy. Neuropathol Appl Neurobiol 36:422-435 
Suárez I, Bodega G, Rubio M \& Fernández B. (2009). Induction of NOS and nitrotyrosine expression in the rat striatum following experimental hepatic encephalopathy. Metab Brain Dis 24:395-408

Tapia R, Medina-Ceja L \& Pena F. (1999). On the relationship between extracellular glutamate, hyperexcitation and neurodegeneration, in vivo. Neurochem Int 14:23-31

Testa CM, Catania MV \& Young AB. (1994). Anatomical distribution of metabotropic glutamate receptors in mammalian brain. In: The metabotropic glutamate receptors. Conn PJ, Patel PJ (Eds.), pp 99-123, Humana Press, Totowa, New Jersey

Tofaris GK \& Spillantini MG. (2005). Alpha-synuclein dysfunction in Lewy body diseases. Mov Disord 12:S37-44

Trojanowski JQ \& Lee VM. (2001). Parkinson's disease and related neurodegenerative synucleinopathies linked to progressive accumulations of synuclein aggregates in brain. Parkinsonism Relat Disord 7:247-251

Trabace L \& Kendrick KM. (2000). Nitric oxide can differentially modulate striatal neurotransmitter concentrations via soluble guanylate cyclase and peroxynitrite formation. J Neurochem 75:1664-1674

Vincent SR \& Kimura H. (1992). Histochemical mapping of nitric oxide synthase in the rat brain. Neuroscience 46:755-784

Vuillet J, Kerkerian L, Kachidian P, Bosler O \& Nieoullon A. (1989). Ultrastructural correlates of functional relationships between nigral dopaminergic or cortical afferent fibers and neuropeptide Y-containing neurons in the rat striatum. Neurosci Lett 100:99-104

Wakabayashi K, Hayashi S, Yoshimoto M, Kudo H \& Takahashi H. (2000). NACP/alphasynuclein-positive filamentous inclusions in astrocytes and oligodendrocytes of Parkinson's disease brains. Acta Neuropathol 99:14-20

Wakabayashi K, Tanji K, Mori F \& Takahashi H. (2007). The Lewy body in Parkinson's disease: molecules implicated in the formation and degradation of alpha-synuclein aggregates. Neuropathology 27:494-506

Wakabayashi K, Yoshimoto M, Tsuji S \& Takahashi H. (1998). Alpha-synuclein immunoreactivity in glial cytoplasmic inclusions in multiple system atrophy. Neurosci Lett 249:180-182

Watanabe Y, Kato A, Sawara K, Butterworth RF, Sasaki T, Terasaki K, Sera K \& Suzuki K. (2008). Selective alterations of brain dopamine D2 receptor binding in cirrhotic patients: results of a 11C-N-methylspiperone PET study. Metab Brain Dis 23:265-274

Weissenborn K \& Kolbe H. (1998). The basal ganglia and portal-systemic encephalopathy. Metab Brain Dis 13:261-272

West AR, Galloway MP \& Grace AA. (2002). Regulation of striatal dopamine neurotransmission by nitric oxide: Effector pathways and signaling mechanisms. Synapse 44:227-245

Xu J, Kao SY, Lee FJ, Song W, Jin LW \& Yankner BA. (2002). Dopamine-dependent neurotoxicity of alpha-synuclein: a mechanism for selective neurodegeneration in Parkinson disease. Nat Med 8:600-606

Yamada M, Iwatsubo T, Mizuno Y \& Mochizuki H. (2004). Overexpression of a-synuclein in rat substantia nigra results in loss of dopaminergic neurons, phosphorylation of asynuclein and activation of caspase-9: resemblance to pathogenetic changes in Parkinson's disease. J Neurochem 91:451-461 
Yavich L, Tanila H, Vepsalainen S \& Jakala P. (2004). Role of alpha-synuclein in presynaptic dopamine recruitment. J Neurosci 24:11165-11170

Yu S, Zuo X, Li Y, Zhang Ch, Zhang YA, Uéda K \& Chan P. (2004). Inhibition of tyrosine hydroxylase expression in a-synuclein-transfected dopaminergic neuronal cells. Neurosci Lett 367:34-39

Zarranz JJ, Alegre J, Gomez-Esteban JC, Lezcano E, Ros R, Ampuero I, Vidal L, Hoenicka J, Rodriguez O, Atares B, Llorens V, Gomez Tortosa E, del Ser T, Munoz DG \& de Yebenes JG. (2004). The new mutation, E46K, of alpha-synuclein causes Parkinson and Lewy body dementia. Ann Neurol 55:164-173 


\title{
Wernicke's Encephalopathy
}

\author{
Radu Tanasescu ${ }^{1,2}$ et al.* \\ ${ }^{1}$ Carol Davila University of Medicine and Pharmacy, Bucharest, \\ ${ }^{2}$ Department of Neurology, Colentina Clinical Hospital, Bucharest \\ Romania
}

\section{Introduction}

Wernicke's Encephalopathy (WE) is an underdiagnosed, potentially fatal, acute or subacute neurologic disorder caused by the impairment of thiamine (vitamin B1) -dependent enzymatic activity in susceptible brain cells. The biologically active form of thiamine (TH), thiamine diphosphate (THDP), serves as a cofactor for several apoenzymes involved mainly in the carbohydrate metabolism. Except for very rare cases, WE occurs in the presence of TH deficiency, which is directly related to at least two other clinical entities: neurological beriberi and cardiovascular beriberi. The preferential expression of one (or more) of these entities may be the consequence of genetic polymorphism of genes encoding $\mathrm{TH}$ transporters. The topography of WE brain lesions is highly specific, typically the periventricular and periaqueductal grey areas being symmetrically involved. In the majority of cases the early so-called 'biochemical lesions' are completely reversed if TH is promptly supplied. However, if the THDP dependent enzymatic activity is not restored, irreversible structural damage and eventually exitus may occur (Donnino, Vega et al. 2007; Hazell and Butterworth 2009; Thorarinsson, Olafsson et al. 2011).

Carl Wernicke was the first to describe the clinical and neuropathological characteristics of the encephalopathy that currently, according to the ICD-10 (WHO 2010), bears his name. The first case compatible with WE was reported in 1822 by James Jackson. In the following years Samuel Wilks (1868) and Charles Gayet (1875) encountered other similar cases. In 1881, Carl Wernicke published the cases of three patients - two alcohol abusing men, and a young woman with persistent vomiting - that died within two weeks after the acute onset of the nowadays considered classical triad - i.e. stance and/or gait ataxia, ocular motility sings and mental-status changes - accompanied by similar fundoscopic modifications. Neuropathological examinations were conducted. Wernicke considered that they all had the same disease he named 'polioencephalitis haemorrhagica superioris' (Pearce 2008;

\footnotetext{
*Laura Dumitrescu ${ }^{2}$, Carmen Dragos ${ }^{3}$, Dimela Luca ${ }^{2}$, Alexandra Oprisan ${ }^{1,2}$, Catalina Coclitu, Oana Simionescu², Lorena Cojocaru², Marius Stan², Andreea Carasca²,

Andreea Gitman ${ }^{2}$, Adela Chiru ${ }^{2}$ and Marina Ticmeanu ${ }^{1,2}$

${ }^{1}$ Carol Davila University of Medicine and Pharmacy, Bucharest, Romania

${ }^{2}$ Department of Neurology, Colentina Clinical Hospital, Bucharest Romania

${ }^{3}$ Department of Radiology, Coltea Clinical Hospital, Bucharest, Romania

${ }^{4}$ Department of Neurology, University Emergency Hospital, Bucharest, Romania
} 
Thorarinsson, Olafsson et al. 2011). During the late 1880's, Korsakoff published three comprehensive series of cases presenting the characteristic features of the amnestic syndrome that currently bears his name (i.e. Korsakoff syndrome/'psychosis', KS), commonly preceded or accompanied by a clinical picture highly suggestive of WE. He considered that all these patients had the same disease he named 'polyneuritic psychosis'. The KS results from the bilateral dysfunction of the limbic system. As expected considering the distribution of WE lesions, KS frequently accompanies WE. The association between WE and KS was noticed and in this respect acute or sequelar WE with prominent KS and/or post-WE KS are denominated by some Wernicke-Korsakoff syndrome (WKS). The etiology and treatment of WE remained unknown until up to almost the half of the XXth century. Beginning with Strauss (1935), Campbell (1940), Russell (1940) and Phillips (1952), who established that TH deficiency is directly related to the development of WE, and with Williams and Cline (1936), who published the first correct TH chemical formula and synthesis pathway, remarkable progress has been made in understanding the spectrum of diseases caused by TH deficiency (Phillips, Victor et al. 1952; Donnino, Vega et al. 2007). Currently, WE is a highly and easily treatable disease frequently associated with alcoholism and/or malnutrition related TH deficiency. The diagnosis remains mainly clinical as none of the modern diagnostic tools has adequate sensitivity and/or specificity. The classical triad is encountered in less than a third of cases and may be completely absent. The clinical picture may vary from the classical signs to hypotension or coma. Unfortunately, the misconception that WE is a rare and stereotypic disease occurring only in malnourished alcoholics is still found in clinical practice, frequently leading to the delay or even failure of diagnosis (Tanasescu 2009).

The following chapter provides a thorough overview of WE, with updates on the recent epidemiological and etiophysiopathological data. A brief reference to the possibility of effective prophylaxis is made. The diagnosis and treatment are discussed. The relationship with other TH deficiency diseases, the particularities of alcohol versus non-alcohol related cases and the correlations with KS are underlined. Considering the deleterious consequences of untreated WE, the importance of maintaining a high index of suspicion for diagnosis and a low threshold for parenteral $\mathrm{TH}$ administration - as recommended by the 2010 EFNS guideline - is advocated (Galvin, Brathen et al. 2010).

\section{Epidemiology}

WE epidemiological data are scarce and mostly based on necroptic class IV studies conducted in the developed countries. However the literature is abundant in case reports and to a lesser extent in small retrospective clinical studies. WE occurs throughout the world, but, even if considering the probable report bias, appears to have uneven geographic distribution (Galvin, Brathen et al. 2010).

According to the retrospective clinical studies available, the prevalence of WE is lower than $0.13 \%$. Nevertheless, necropsies of the general population reveal brain lesions consistent with WE in 0.4 to $2.8 \%$ of the cases (average $1.3 \%$ ), the highest rates being reported in West Australia during the 1970's (Harper 1979; Harper 1983; Harper, Giles et al. 1986). In prior alcohol abusers the prevalence of neuropathology confirmed WE is reported to be around $12 \%$ and raises up to $30 \%$ in those associating cerebellar atrophy. If considering only those 
with alcohol related deaths, WE prevalence may reach 59\% (Victor, Adams et al. 1971; Thomson and Marshall 2006). Other populations with significantly higher WE prevalence were identified, necroptic changes compatible with WE being found in approximately $10 \%$ of the AIDS patients and in $6 \%$ of those that underwent bone marrow transplant (Butterworth, Gaudreau et al. 1991; Boldorini, Vago et al. 1992; Donnino, Vega et al. 2007). The correct diagnosis of WE is made prior to necropsy in only up to $25 \%$ of the adult cases (values as low as $1 \%$ being reported!), and in approximately $40 \%$ of the pediatric cases). Up to $70 \%$ of the alcohol related cases and almost $95 \%$ of the non alcohol related cases are not diagnosed prior to death (Victor, Adams et al. 1971; Harper, Giles et al. 1986; Harper, Gold et al. 1989; Torvik 1991; Vasconcelos, Silva et al. 1999). AIDS patients seem to be the category most often misdiagnosed (Butterworth, Gaudreau et al. 1991). Considering the discrepancy between the prevalence of the clinic versus necroptic diagnosis $(0.4-2.8 \%$ versus $0.04-0.13 \%)$, WE appears to be underdiagnosed during lifetime (Victor, Adams et al. 1971). Since necropsy studies may be biased towards preferentially identifying the more severe cases, the prevalence of WE may be even higher than the one predicted by the necroptic studies (Galvin, Brathen et al. 2010). However, some have suggested that the histopathological changes may precede the clinical onset of disease, and thus the necropsies of the general population may also identify mild subclinical cases (Caine, Halliday et al. 1997). It was estimated that 13 to $35 \%$ of alcoholics and up to $1.5 \%$ of the non-alcoholics develop WE (Harper, Rodriguez et al. 1988). The incidence and prevalence of WE are considered to be at least 10 times higher in alcoholics than in non-alcoholics (Harper 2006). In order to have a clearer picture, the 2010 EFNS guideline recommends performing necropsies with detailed neuropathological examination in all patients dying from diseases suggestive of WE (Galvin, Brathen et al. 2010).

Besides exceptionally rare cases, all WE patients have TH deficiency. According to the available data, TH deficiency is not uncommon in the developed countries. In the UK, approximately $20 \%$ of the patients admitted to the emergency departments had $\mathrm{TH}$ deficiency (Jamieson, Obeid et al. 1999). In the USA, 8 to $31 \%$ of the elderly living at home and 23 to $40 \%$ of those in nursing homes had TH deficiency (Harper 2006). In Canada, TH deficiency was found in almost $13 \%$ of the critically ill children (Fattal-Valevski 2011). During the 1992-1993 Cuban neuropathy epidemic the local prevalence of TH deficiency ranged from $30 \%$ in some regions to $70 \%$ in others (Macias-Matos, Rodriguez-Ojea et al. 1996). Among chronic alcoholics, 25 to $80 \%$ may have a certain degree of $\mathrm{TH}$ deficiency (Caine, Halliday et al. 1997). Several studies on AIDS patients found TH deficiency in 10 to 23\% of the cases (Davtyan and Vinters 1987; Foresti and Confalonieri 1987; Hutchin 1987). According to the WHO's report on TH (WHO 1999), in South-East Asia TH deficiency has a high prevalence, while in Africa and Central and North America it has a low prevalence. Chronic ethanol consumption and/or malnutrition have a significant impact on the incidence and prevalence of WE, up to $90 \%$ of the WE patients having TH deficiency in this context (Antunez, Estruch et al. 1998). Several important TH deficiency epidemics have been recorded by the modern history. At the beginning of the XXth century the introduction of the large scale use of cheap polished rice in urban South-East Asia led to several great outbreaks of TH deficiency associated with beriberi. During the last two decades of the XXth century TH deficiency epidemics were recorded in Thailand, Guinea, Djibouti, East Ethiopia and Nepal, especially among political refugees (WHO 1999). In 2003 a TH deficiency 
outbreak affecting infants fed with a soy milk formula with no detectible $\mathrm{TH}$ content emerged in Israel (Fattal-Valevski, Kesler et al. 2005). To the best of our knowledge, WErelated epidemiologic data from the developing or underdeveloped countries are not available. Approximately $90 \%$ of the WE cases occurring in the developed countries are alcohol related (Thomson 2000). A correlation between the per capita alcohol consumption and the prevalence of WE could not be established (Torvik 1991). The male to female ratios range from 1.7:1 to 3:1 in necroptic studies, and were reported to be 5:1 in a large clinical study (Victor, Adams et al. 1971; Harper 1979; Victor 1989; Rolland and Truswell 1998). WE may affect individuals of any age but appears to have a higher prevalence during the fifth decade of life (Vasconcelos, Silva et al. 1999). A trend towards an increased incidence and prevalence of WE has recently been observed in USA, UK and Japan, possibly related to the increased number of bariatric surgery interventions and the persistent shortages in intravenous vitamins, to the restriction of parenteral $\mathrm{TH}$ administration due to fear of anaphylaxis (leading to the routine prophylaxis in hospitalized alcoholics with per os instead of parenteral thiamine) and respectively to the restriction of parenteral vitamins supplementation by a healthcare policy (Ramayya and Jauhar 1997; Hahn, Berquist et al. 1998; Shikata, Mizutani et al. 2000; Feeney and Connor 2008). Several regional socioeconomic and cultural particularities along with local health care related factors appear to have important influences on the prevalence and prognosis of WE. Considering the important burden that untreated WE puts on health care systems worldwide (Galvin, Brathen et al. 2010), further epidemiological studies are needed in order to better define the populations at risk and to identify efficient prophylactic approaches.

\section{Etiology}

WE is caused by the disruption of the THDP dependent enzymatic activity in susceptible brain cells, commonly secondary to $\mathrm{TH}$ deficiency. This correlates directly with three pathogenic entities: WE, cardiovascular beriberi, and neuropathic beriberi. Recently, two other conditions that seem to be directly related to TH deficiency have been described: African (Nigerian) seasonal ataxia and gastrointestinal beriberi (Adamolekun and Ndububa 1994; Nishimune, Watanabe et al. 2000; Donnino 2004). TH deficiency seems also to be involved in other diseases like Strachan syndrome (i.e. polyneuropathy, optic neuropathy, orogenital ulcerations), 'tobacco-alcohol amblyopia', tropical ataxic neuropathy (i.e. sensory neuropathy, optic neuropathy, sensoneural deafness), Marchiafava-Bignami disease, subacute 'alcoholic' cerebellar degeneration and epidemic spastic paraparesis (konzo). TH competitive antagonists and/or impaired TH to THDP conversion may lead to WE even in the presence of normal TH blood levels. Impaired apoenzyme activation due to magnesium $(\mathrm{Mg})$ deficiency, and/or decreased activity of the THDP dependent enzymes may condition the degree of susceptibility to borderline low levels of THDP. Due to physiologic particularities not all tissues are equally susceptible, the nervous system and the cardiac muscle being the most vulnerable. The pattern of cellular susceptibility may be influenced by genetic and environmental factors, including the nutritional and hormonal status, and seems not to be homogenously represented among individuals, thus possibly explaining the preferential expression of one (or more) of the potential pathologic entities related to $\mathrm{TH}$ deficiency (Zhao, Gao et al. 2002). 


\subsection{Thiamine}

$\mathrm{TH}$ is a water soluble heat labile quaternary ammonia compound, containing an aminopyrimidine ring linked by a methylene bridge to a thiazole ring. It is synthesized by different biochemical reactions in fungi, bacteria, plants and some protozoa, but not in humans (Fattal-Valevski 2011). In the human body it is found as unphosporilated TH (i.e. free $\mathrm{TH}$ ) and as phosphorilated derivates: TH monophospate (THMP), TH diphospate (THDP, aka TH pyrophosphate), and TH triphospate (THTP). Intracellularly, free $\mathrm{TH}$ is converted by thiaminpyrophosphokinase into THDP, in a process requiring $\mathrm{Mg}$ as cofactor. Three plasma membrane bidirectional transporters for $\mathrm{TH}$ and $\mathrm{TH}$ derivates have been described: TH transporter 1 (THTR1) encoded by the SCL19A2 gene (location 1q23.3), TH transporter 2 (THTR2) encoded by the SCL19A3 gene (location 2q37) and reduced folate carrier transporter 1 (RFC1) encoded by the SCL19A1 gene (location 21q22.3). A mitochondria membrane transporter for THDP (i.e. mitochondria membrane THDP transporter) encoded by the SCL25A19 has also been described. THTR1 and THTR2 transport free TH. THTR1 seems to be highly expressed in skeletal and heart muscle and to lesser degrees in placenta, liver, kidneys, small intestine and lungs. THTR2 seems to be highly express in the placenta, kidneys, liver and thalamus and also in the small intestine. RFC1 is mainly a folate transporter, but also transports THDP and THMP. Considering that THDP is found exclusively intracellularly, RFC1 transports THDP only from the intracellular to the extracellular space (where THDP is rapidly converted to free TH). In the presence of low free TH and THMP plasma levels the cells that highly express RFC1 on their membranes have an overall negative TH balance, exporting THDP without importing free TH or THMP. RFC1 is highly expressed on the apical brush border of the choroid plexus. The pattern of distribution of the $\mathrm{TH}$ and $\mathrm{TH}$ derivates transporters may play a significant role in establishing and maintaining the tissue distribution of TH (Boulware, Subramanian et al. 2003; Subramanian, Marchant et al. 2003; Said, Balamurugan et al. 2004; Miyajima and Kono 2010). The intestinal absorption of TH occurs mainly in the proximal small intestine by an active saturable mechanism and probably also by passive diffusion. At the intestinal brush border $\mathrm{TH}$ is mainly found in its free form. $\mathrm{TH}$ absorption is enhanced by $\mathrm{TH}$ deficiency and reduced by thyroid hormones, ethanol exposure, low temperature and $\mathrm{TH}$ analogs. TH absorption may also be reduced in those with diabetes mellitus or advanced aged. At low concentration $(<2$ microM/liter) $\mathrm{TH}$ absorption is an active, rate-limited process, involving the high affinity THTR2 and, to a lesser extent, THTR1. At high intestinal TH concentration (5-50 microM/liter) TH seems to be absorbed through passive diffusion. It has been reported that under physiological circumstances, even when large TH quantities are administered, no more than 4.5 to $5 \mathrm{mg}$ can be absorbed from a single oral dose. $\mathrm{TH}$ has restricted distribution. Up to $90 \%$ of the circulating $\mathrm{TH}$ is found in the red cells (mostly as THDP), the rest being found in the other blood cells and in plasma, mainly bound by proteins, as free TH or THMP (Dudeja, Tyagi et al. 2001; Martin, Singleton et al. 2003). Under physiologic circumstances, TH is excreted renally. In the presence of high plasmatic $\mathrm{TH}$ concentration rapid renal excretion as free $\mathrm{TH}$ occurs. After the intravenous administration of $50 \mathrm{mg}$ of $\mathrm{TH}$ hydrochloride the plasma half-time is about 96 minutes. Thus, for correcting $\mathrm{TH}$ deficiency the administration of parenteral $\mathrm{TH}$ in many smaller doses rather than in an equivalent single dose seems justified (Donnino; Boulware, Subramanian et al. 2003). The blood brain barrier (BBB) allows the passage of free $\mathrm{TH}$ and THMP through both active and passive mechanisms. Active passage occurs at low $\mathrm{TH}$ 
serum concentrations. At high serum concentrations, free TH passes the BBB passively, driven by the existing concentration gradient. The intravenous administration of $\mathrm{TH}$ provides a superior concentration gradient that facilitates passive diffusion (Thomson, Cook et al. 2002). Several natural and synthetic TH structural analogues having different pharmacological profiles exist. Pyrithiamine and oxythiamine act as competitive antagonists. Pyrithiamine passes the BBB and thus is useful for inducing TH deficiency encephalopathy, the experimental model of WE. TH hydrochloride and TH mononitrate are water soluble $\mathrm{TH}$ salts that act as $\mathrm{TH}$ agonists and have similar bioavailability, distribution and excretion with $\mathrm{TH}$. Allithiamine is a naturally occurring lipophylic $\mathrm{TH}$ analogue resulting from the enzymatic conversion of $\mathrm{TH}$ in the freshly crushed bulbs of garlic and other alli plants. Thiaminetetrahydrofufuryl disulfide is a synthetic analogue of allithiamine. Prosultiamine and sulbutiamine are lipid-soluble synthetic $\mathrm{TH}$ analogues. All these lipophylic $\mathrm{TH}$ derivates have better bioavailability and BBB penetrability than $\mathrm{TH}$. Benfotiamine is a synthetic $\mathrm{TH}$ analogue that has better bioavailability and cellular penetrability than TH, but does not pass the BBB (Baker and Frank 1976; Kitamori and Itokawa 1993). TH may have a structural role as part of the cellular membranes, and may be involved in the synaptic transmission, cellular differentiation, axonal growth, myelinogenesis and regulation of brain development during fetal and early postnatal life. To the best of our knowledge THMP and THTP have no clearly identified metabolic or structural roles (Makarchikov, Lakaye et al. 2003). THDP serves as a cofactor for several apoenzymes involved in the carbohydrate metabolism: apo-alpha-ketoglutarate dehydrogenase (aKGDH), apo-pyruvate dehydrogenase (PDH) and apo-transketolase (TK). $\mathrm{Mg}$ is the second cofactor required by these apoenzymes, especially by apo-TK. aKGDH and $\mathrm{PDH}$ are mitochondrial enzymes important for the tricarboxylic acid cycle (TAC, i.e. Krebs cycle), though the latter is not part of it. TK is a cytosolic enzyme involved in the nonoxidative phase of the pentose-phosphate pathway (PPP or hexose monophosphat shunt). It has been shown that TH deficiency inhibits the expression of the genes encoding TK and PDH (Pekovich, Martin et al. 1998; Donnino, Vega et al. 2007). Under physiologic circumstances, almost $30 \%$ of the brain glucose is metabolized to pyruvate. In the absence of a functional PDH complex and Krebs cycle pyruvate is reduced to lactate (Ishii, Sarai et al. 1979). The human body has $\mathrm{TH}$ deposits ranging from 25 to $50 \mathrm{mg}$, commonly corresponding to the amount of $\mathrm{TH}$ required for 18 to 42 days. Most of the $\mathrm{TH}$ is stored in the liver as THDP. Food sources of TH are cereals, beans, nuts, brown (unpolished) rice and meat. Polished (white) rice, highly purified cereals and excessively cooked food may contain no $\mathrm{TH}$. The daily $\mathrm{TH}$ requirements for a healthy adult may range from 1 to $2 \mathrm{mg}$ and depend on the carbohydrate intake and on several metabolic factors. According to the current literature, the TH intake should be of at least $0.33 \mathrm{mg}$ per $1000 \mathrm{kcal}$, ideally $0.5 \mathrm{mg}$ per $1000 \mathrm{kcal}$, but no less than $1 \mathrm{mg}$ per day. Some recommend daily intakes above $1.1 \mathrm{mg}$ for adult women and $1.2 \mathrm{mg}$ for adult men, even if the corresponding caloric intake is lower. A balanced diet usually contains the recommended quantity. No upper tolerability limit intake has been established for $\mathrm{TH}$ and to the best of our knowledge no cases of oral $\mathrm{TH}$ toxicity have been described (WHO 1999; Thomson and Marshall 2006; Sechi and Serra 2007; Fattal-Valevski 2011).

The TH content of pharmacological or biological samples may be measured directly by several methods including spectrophotometry, spectrofluorometry, various techniques of high performance liquid chromatography (HPLC), capillary electrophoresis and voltametry. 
The classical method used to assess the human THDP status is the estimation of the effect of THDP on the erythrocyte TK activity (ETKA). A low ETKA with more than $25 \%$ increase after THDP adding confirms THDP erythrocyte deficit, and though indirect, has good sensibility, specificity and reproducibility in estimating the whole blood total $\mathrm{TH}$ and the erythrocyte THDP levels. However, since it is laborious, it has been replaced by the direct measurement of the total TH (free TH and its phosphatesters) or of THDP in the whole blood using various techniques of HPLC. The whole blood THDP levels correlate well with erythrocyte THDP levels providing the correction with the haemoglobin level is made. The measurement of whole blood THDP was suggested to be the most suitable method for use in clinical practice. (Lee, Ong et al. 1991; Tallaksen, Bell et al. 1993; Herve, Beyne et al. 1994). In the apparently healthy human adults the THDP and total TH blood levels range within nanomoles per liter levels, specific values depending upon the technique used. In animal studies the lethal blood TH level ranges from 7.2 to $10 \mathrm{mg} / \mathrm{dl}$. Death occurs due to respiratory failure. If respiratory support is provided blood levels as high as $36.9 \mathrm{mg} / \mathrm{dl}$ are tolerated (Smith, Foa et al. 1947; Galvin, Brathen et al. 2010).

\subsection{Predisposing factors}

TH deficiency is the predisposing factor most frequently associated with WE. TH deficiency is the consequence of one or more of the following mechanisms: inadequate dietary intake (absolute or relative), impaired intestinal absorption, impaired storage, excessive elimination and/or increased metabolic requirements. Impaired $\mathrm{TH}$ intestinal absorption may occur due to gastrointestinal diseases, protein-caloric malnutrition (decrease in the active TH absorption) and/or ingestion of certain substances (e.g. 'anti-TH factors', antacids, phenytoin, cephalosporins, tetracycline). Impaired TH storage may occur due to chronic liver disease. Excessive renal elimination may occur due to renal disease, use of certain drugs and/or impaired TH storage. In those already marginally deficient WE may be precipitated by an event that rapidly increases the metabolic requirements of TH. In most of the cases, TH deficiency may be traced back to improper diet. Regardless of the cause, unbalanced nutrition persisting for more than 14 to 21 days, or even less in those already marginally deficient or with higher demands, may lead to TH deficiency. In healthy adults, intakes of less than $0.2 \mathrm{mg}$ per $1000 \mathrm{kcal}$ or of less than $0.66 \mathrm{mg}$ per day lasting for several weeks lead to clinically manifest TH deficiency. Diets rich in the so-called 'anti-TH factors' (i.e. 'thiaminases' and dietary 'TH antagonists') may result in $\mathrm{TH}$ deficiency. The thiaminases are heat labile enzymes that disintegrate $\mathrm{TH}$ (found in raw or fermented fish, shellfish, ferns and certain bacteria) or reduce its intrinsic activity (found in certain bacteria). The dietary 'TH antagonists' are heat stable non-enzymatic substances that interfere with the intestinal absorption of $\mathrm{TH}$, including polyphenols (e.g. caffeic acid, chlorogenic acid, tannic acid, tartaric acid, citric acid, ascorbic acid which are found in tea, coffee, betel nuts, red currants), flavonoids (quercetin and rutin, found mainly in fruits), haemin (found in animal tissues) and sulphites in high amounts (WHO 1999; Thomson and Marshall 2006; Fattal-Valevski 2011). Gender may influence the risk of developing WE, possibly because of genetic differences but also because of gender-related environmental factors. No definite race predisposition has been described, but a population-specific susceptibility has been reported: it seems that Asians with TH deficiency are prone to cardiovascular beriberi, while Europeans with TH deficiency are more likely develop neurological beriberi and/or WE (Sechi and Serra 2007). 
Chronic ethanol abuse is the condition most frequently associated to WE. Alcoholics may have higher $\mathrm{TH}$ demands, $\mathrm{TH}$ being necessary for the metabolism of ethanol. They frequently have $\mathrm{TH}$ intake below $0.29 \mathrm{mg}$ per $1000 \mathrm{kcal}$ and associate $\mathrm{Mg}$ depletion (Thomson 2000). They may have impaired TH absorption secondary to ethanol-induced intestinal mucosa damage, impaired transmembrane transport due to folate or other B vitamins deficiency, decreased intestinal ATP-ase activity and reduced expression of the THTR1 and THTR2 encoding genes (Hoyumpa 1980; Subramanya, Subramanian et al. 2010). The type of alcoholic beverage consumed may have an influence (Lemos, Azevedo et al. 2005). TH malabsorption seems to be reversible providing ethanol consumption stops (Bujanda 2000). Considering that not all alcoholics with similar nutritional status develop $\mathrm{WE}$, it may be speculated that other environmental and/or genetic factors may interfere (Mukherjee, Svoronos et al. 1987). Physiological hypercatabolic states like infancy, pregnancy and lactation may predispose to TH deficiency. A particular situation is that of hyperemesis gravidarium. It has been reported that vomiting persisting for more than three weeks and elevated transaminase levels highly correlate with the occurrence of WE in pregnant women (Rotman, Hassin et al. 1994 ). The infants fed by TH deficient mother or by TH deficient milk formula develop TH deficiency. Pathological hypercatabolic states may predispose to $\mathrm{TH}$ deficiency not only because of increase TH requirements but also because they are frequently associated with improper nutrition, impaired intestinal absorption, persistent vomiting and use of drugs that may interfere with TH utilization (Otsuka, Tada et al. 1997; Sechi and Serra 2007). In children, malignancy has been reported to be the condition most frequently associated to WE (Vasconcelos, Silva et al. 1999). Gastrointestinal surgery that removes or by-passes the parts of the gastrointestinal system involved in TH absorption is an important predisposing factor for TH deficiency. Bariatric surgery has been identified as predisposing factor in a significant number of the recently reported WE cases. Persistent vomiting may lead to TH deficiency if adequate parenteral supplementation is not provided (Singh and Kumar 2007). Chronic liver disease (occurring in up to half of the alcoholics developing WE) may lead to TH deficiency due to impaired storage. Hemodialysis and peritoneal dialysis have been reported to increase TH elimination (Sun, Yang et al. 2006; Ueda, Utsunomiya et al. 2007). Uremic encephalopathy may cause impaired cerebral TK activity and thus may predispose to WE (Brouns and De Deyn 2004). High doses of intravenous glucose may precipitate WE in marginally TH deficient individuals. Refeeding, hyperalimentation and total parenteral nutrition without adequate $\mathrm{TH}$ supplementation may also precipitate iatrogenic WE (Watson, Walker et al. 1981). Drugs such as nitroglycerin and tolazamide may may play a role in the development of WE in susceptible individuals (Sechi and Serra 2007). The chronic use of metronidazole may predispose to WE due to its conversion into a TH analogue that acts as a TH competitive antagonist (Alston and Abeles 1987). The chemotherapeutic drugs 5-fluorouracil, cisplatin, erbulozole and ifosfamide seem to interfere with TH pharmacokinetics, predisposing to WE (Van Belle, Distelmans et al. 1993; Kondo, Fujiwara et al. 1996; Hamadani and Awan 2006; Cho, Chang et al. 2009). WE occurring during the chronic use of tolazamide, a sulfonylurea blood glucose lowering drug that might increase the intracellular demands of $\mathrm{TH}$, has been reported (Kwee and Nakada 1983).

Several genetic factors seem to predispose to the development of WE. The occurrence of WE is more frequently encountered in both monozygotic twins than in both heterozygote twins (Martin, Singleton et al. 2003), but to the best of our knowledge, no significant family 
aggregation has been reported. The function and/or upregulation of the receptors responsible for the intestinal and renal uptake of $\mathrm{TH}$ may be genetically impaired in some individuals who develop WE. Some reported that the presence of a genetically conditioned low affinity TK may predispose to WE (Mukherjee, Svoronos et al. 1987). Since no differences in the nucleotide sequence of the encoding gene or in the amino acid sequence were identified, it has been proposed that the biochemical difference in the activity of TK may be caused by posttranscriptional changes or by differences in the three-dimensional conformation (McCool, Plonk et al. 1993). Another possible genetic factor predisposing to WE is the mutation of the X-linked transketolase-like 1 gene (Coy, Dubel et al. 1996). Genetic variants of the enzymes involved in the metabolism of ethanol may also predispose to WE (Sechi and Serra 2007). Mutations in an untranslated regulatory region of SLC19A2 gene (also involved in TH-responsive megaloblastic anemia) seem to be involved in the genetic predisposition to WE (Guerrini, Thomson et al. 2005). These genetic defects might explain the inability of certain individuals to cope with borderline-low TH deficiency. A WE-like phenotype caused by defects of the SLC19A3 gene (typically involved in childhood onset biotin-responsive basal ganglia disease) has been reported in two Japanese brothers. Both of them were compound heterozygote for the K44E and E320Q mutations. These mutations were not found in 192 ethnically matched controls (Kono, Miyajima et al. 2009).

In conclusion, one or more genetic mutations occurring in the same individual probably cause subtle alterations in the neuroglial and/or neuronal TH transporter systems and/or in the activity of THDP dependent enzymes, that in the presence of absolute or relative THDP deficiency and/or Mg deficit lead to the development of WE (Thomson and Marshall 2006).

\section{Neuropathology}

The anatomical pathology of WE is well described, mostly due to the large number of necroptic studies performed. The macroscopic and microscopic characteristics depend on the stage and severity of the disease (Sechi and Serra 2007). WE may coexist with typical hepatic encephalopathy findings, the neuropathologic differential diagnosis being sometimes difficult (Caine, Halliday et al. 1997). To the best of our knowledge, WE electronic microscopy data are not available. Gross findings consist of bilateral symmetrical grayish discoloration, congestion and recent petechial hemorrhages involving the periaqueductal grey matter, mamillary bodies, and medial thalamus. The most frequent lesion observed (up to $75 \%$ of the cases) is spongy or granular brown-grayish discoloration of the thalamus (Victor, Adams et al. 1971). The presence of punctuate hemorrhages in the mamillary bodies is highly specific (Thorarinsson, Olafsson et al. 2011). Larger hemorrhages (up to $8 \mathrm{~mm}$ in diameter) found in the vicinity of the third and the fourth ventricle have been reported in at least two cases with otherwise typical histopathological and clinical presentation (Rosenblum and Feigin 1965; Vortmeyer, Hagel et al. 1992). Rarely, discoloration may be observed in the reticular formation of the midbrain, corpora quadrigemina and in the cortex. The cerebellum may show atrophy of the vermis. Typically the brain has normal weight, though in chronic alcoholics significant atrophy may exist. Its surface has normal appearance. The lateral ventricles may sometimes be dilated, most likely secondary to chronic alcohol abuse-related atrophy (Victor, Adams et al. 1971; Harper 1979). Atrophy of the corpus callosum has been reported in both alcohol and non alcohol 
related WE. The extent and the location of callosal atrophy seems to vary in relationship with alcohol consumption (Lee, Jung et al. 2005). In a significant number of cases gross examination alone does not reveal any lesions (Harper 1979; Donnino, Vega et al. 2007).

Microscopically, the typical aspect of the WE lesions consists of symmetric microhemorrhagic and/or necrotic lesions and microglia proliferation affecting symmetrically the cerebral midline regions, mainly the thalamus, mamillary bodies, periaqueductal region, hypothalamus, cerebellar vermis, proximity of the third ventricle and the floor of the fourth ventricle (Victor, Adams et al. 1971; Fattal-Valevski 2011). The distribution of the lesions is highly localized. The medial dorsal thalamic nucleus and the mamillary bodies are affected in virtually all patients (Victor, Adams et al. 1971). The locus ceruleus, oculomotor and vestibular nuclei and the medial aspect of the thalamus are also frequently involved. In the most severe cases extensive necrosis of the affected areas is observed. In the mild cases only loss of myelin and to a lesser degree of neuronal bodies is noticed. The number of astrocytes and macrophages is commonly increased. Focal microhemorrhages are sometimes found. Macrophages containing hemosiderin (thus indicating previous hemorrhage) may be encountered. Sometimes lesions consisting of patchy or diffuse neuronal loss and Alzheimer type II astrocytic proliferation (typically seen in hepatic encephalopathy) are found in the hippocampus, fornix, septal regions and cerebral cortex. The acute WE lesions are characterized by vascular congestion, petechial hemorrhages and astrocytes swelling affecting mainly the brainstem and the thalamus. The older lesions are characterized by demyelination, gliosis, edema and loss of neuropils in spite of the relative preservation of neurons (Sechi and Serra 2007). The capillaries are dilated and are surrounded by edema and microhemorrhages. Some have observed capillary proliferation, while others did not. Cerebellar vermis lesions compatible with those found in the alcoholic cerebellar degeneration - i.e. selective loss of Purkinje cells - are found in about half of the cases (Thorarinsson, Olafsson et al. 2011). Edema, microhemorrhages and possible necrosis involving the optic nerve may rarely be found ( $\mathrm{Li}$ and Rucker 2010). The chronic lesions usually affect the mamillary bodies and the dorsomedial thalamic nuclei. Atrophy of the mamillary bodies is highly specific for the sequelae of WE, being found even by macroscopic examination in the majority of the cases. Widening of the third and fourth ventricle and of the aqueduct is also observed in the late and sequelar stages. Microscopically there is proliferation of astrocytes, tissue destruction and gliosis, while the capillary endothelium is normal and microhemorrhages are absent (Donnino, Vega et al. 2007; Thorarinsson, Olafsson et al. 2011). Swelling, disruption and hyperplasia of the choroid plexus has been reported in AIDS patients with WE (Boldorini, Vago et al. 1992). Frequently the peripheral nerves have identical aspect with that seen in beriberi, i.e. distal demyelination. The spinal cord may be affected, a decrease in the anterior horn cells and sometimes involvement of the anterior and posterior roots being encountered (Sechi and Serra 2007).

\section{Physiopatogeny}

In spite of the fact that an animal model is easily designable the physiopathological pathways that lead to WE are incompletely understood. The disruption of the THDP dependent enzymatic activity in WE susceptible individuals results in highly localized specific metabolic dysfunction corresponding to the so-called reversible 'biochemical 
lesions'. Providing the disruption is not promptly restored, the 'biochemical lesions' are replaced by irreversible structural damage (i.e. necrosis). The typical lesions are located symmetrically in the periventricular and periaqueductal grey areas. The clinical picture is highly correlated with the topography of the lesions (Hazell and Butterworth 2009). If the THDP dependent enzymatic activity is resumed WE is cured (with or without sequelae). Otherwise, exitus commonly occurs (Sechi and Serra 2007). WE develops rapidly, being usually induced by severe short-term TH deficiency. Persistent or recurring mild THDP deficiency may lead to a chronic evolution (Thomson and Marshall 2006). The reason for the specific selective topographic distribution of the WE lesions is still a matter of debate. A high cellular specificity seems to exist, the astrocytes being the most susceptible to THDP deficiency. The degree of activity reduction seems to be different for each THDP-dependent enzyme and strongly related to the cell's type. Intuitively, one may assume that the most affected brain regions are those with higher metabolic demands, and thus with higher TH requirements. Nevertheless, the cortex is most often spared (Butterworth, Kril et al. 1993; Hazell 2009). Some have proposed that the periventricular areas are affected to a greater degree due to the parenchyma consequences of the high CSF glutamate levels (Nixon 2008). Others have observed that the occurrence of adult neurogenesis may be one of the main differences between the affected regions and the cortical areas, rendering the former more susceptible (Zhao, Pan et al. 2009). Pre and post-transcriptional regulation of the genes encoding the THDP-dependent apoenzymes may possibly be involved (Hazell 2009; Hazell and Butterworth 2009).

A chronologic sequence of the physiopathological changes encountered in WE has been proposed. Accordingly, after about 4 days of THDP deficiency the activity of the astrocytic aKGDH decreases resulting in cytotoxic edema. After 7 to 10 days a decrease in the activity of the astrocytic TK occurs. The astrocytic dysfunction leads to the increase in extracellular glutamate levels (resulting in excitocytotoxicity), accumulation of free radicals and cytokines and loss of the osmotic gradients. Endothelial cell dysfunction resulting in increase nitric oxide (NO) production occurs. The BBB is disrupted and glial and neuronal vasogenic edema appears. After about 14 days focal lactic acidosis, neuronal DNA fragmentation and neuronal necrosis occur (Sechi and Serra 2007). According to a recent in vivo animal imagistic study the first observable consequence of TH deficiency may be the dysfunction the choroid plexus leading to blood-CSF barrier alteration (Nixon, Jordan et al. 2008). The earliest biochemical change reported in experimental WE animal models consists of decreased astrocyte aKGDH activity and the first histopathological finding observed is exclusive neuroglial damage (Butterworth 1986). The THDP deficiency has a profound effect on the functional integrity of the astrocytes. One of the consequences of the decreased aKGDH activity is the impairment of the Krebs cycle leading to cellular energetic failure. Increase oxidative stress and lactate production occur, the latter leading to focal acidosis. Increased lactic acid levels are observed in the areas which subsequently develop histological lesions and the magnetic resonance spectroscopy studies demonstrate a characteristic lesional lactate peak (Butterworth 1989; Pannunzio, Hazell et al. 2000; Donnino, Vega et al. 2007; Sullivan and Pfefferbaum 2009). The disturbed function of the astrocytic membrane results in the alterations of the ionic and osmotic gradients, and thus, in cytotoxic edema. The impairment of the astrocyte function along with the subsequent endothelial dysfunction causes BBB dysfunction that leads to vasogenic edema (Hazell and Butterworth 2009). The endothelial dysfunction causes increased production of NO and 
cytokines, the former exacerbating the BBB dysfunction (Sechi and Serra 2007). The astrocyte dysfunction leads to increased extracellular glutamate levels mainly due to the suboptimal astrocyte uptake, the oxidative stress leading to the downregulation of the astrocytic glutamate 1 (GLT1) and glutamate-aspartate transporters (Langlais and Zhang 1993; Danbolt 2001; Hazell, Rao et al. 2001; Nixon 2008; Hazell and Butterworth 2009). The accumulating extracellular glutamate leads to N-methyl D-aspartate receptor (NMDA-R) mediated excitocytotoxicity resulting in neuronal loss (Todd and Butterworth 1998). Several studies have provided strong evidence for the presence of excitotoxic mediated cell death in TH deficient brains (Hazell, Butterworth et al. 1993; Langlais and Zhang 1993). The glutamate neuronal overstimulation leads to the accumulation of high extracellular potassium $(\mathrm{K})$ levels. The elevated $\mathrm{K}$ is uptaked from the extracellular space by the astrocytes which consequently swell even more via osmosis. This leads to the further impairment of the astrocytes function which become unable to adequately buffer the accumulating extracellular glutamate and $\mathrm{K}$, with deleterious consequences on the surrounding neurons (Kimelberg, Goderie et al. 1990; Kimelberg, Rutledge et al. 1995). The excess of glutamate is also removed from the interstitial fluid by passive diffusion in the CSF followed by choroid plexus active clearance. Decreased aKGDH activity in the ependymal and endothelial cells of the choroid plexus may lead to impairment of the energy dependent processes, and thus impairment of the CSF glutamate clearance. Therefore, as already mentioned, the glutamate levels may be additionally increased in the periventricular areas. The excessive presence of CSF glutamate may lead to further impairment of the choroid plexus activity (Nixon 2008). Increased aquaporin 4 (AQP4) gene expression has also been reported to occur in astrocytes, possibly being induced by the local lactic acidosis. This causes upregulation of the AQP4 membrane water channels which facilitate the astrocytic edema (Morishima, Aoyama et al. 2008; Hazell 2009; Hazell and Butterworth 2009). The loss of the aKGDH activity may also lead to a decrease in the GABA levels. The decreased GABA levels may exacerbate the glutamate mediated excitotoxic brain injury (Heroux and Butterworth 1988). It has been reported that WE patients may have increased neuronal peroxidase activity and decreased superoxide dismutase activity (Slekar, Kosman et al. 1996). The presence of oxidative stress appears to be associated with selective neurodegeneration (Calingasan, Chun et al. 1999). The pathogenic role of the oxidative stress in WE is supported by the neuroprotective effects of selegiline on the THDP deficiency induced brain injury (Slekar, Kosman et al. 1996; Hazell and Butterworth 2009). The production of NO increases rapidly in the TH deprived brain, especially in the medial thalamus, possibly as a consequence of aKGDH activity impairment. Elevated NO levels may have physiopathological significance exacerbating the oxidative stress (Fattal-Valevski 2011). The decreased activity of the global nitric oxide synthase (NOS), reported by some to selectively affect the thalamus and cerebellum of TH deficient animals, may be a marker of the neuronal loss (Rao, Mousseau et al. 1996). Due to the impairment of the PPP secondary to THDP deficiency the local reducing activity decreases and ribose production diminishes.

This results in increased oxidative stress and respectively in impaired nucleotide, nucleic acids, coenzymes and polysaccharides synthesis (Slekar, Kosman et al. 1996). In the absence of adequate PDH activity, pyruvate cannot be converted to acetyl-CoA, thus rendering oxidative phosphorylation inefficient. This exacerbates the already present lactic acidosis. It has been reported that in the presence of TH deficiency the susceptible and non-susceptible brain regions exhibit a significant upregulation in inflammatory genes transcription (Hazell and Butterworth 2009). The expression of the cyclooxigenase-2 (cox-2) seems to be 
selectively increased in the susceptible brain areas of the animals with symptomatic $\mathrm{TH}$ deficiency. The increased expression of cox-2 is accompanied by an increase in prostaglandin E2 (PGE2) levels which is not observed in the presimptomatic stages. The administration of the cox-2 inhibitor nimensulide decreases PGE2 levels but leads to the exacerbation of the neuronal injury, suggesting that PGE2 may exert a neuroprotective role. The differences in the expression of the inflammatory related genes in the different brain regions may be one of the factors leading to the selective brain vulnerability $(\mathrm{Gu}$, Desjardins et al. 2008). If the $\mathrm{TH}$ deficiency persists for more than two weeks DNA fragmentation triggering apoptosis occurs in the thalamic neurons (Pannunzio, Hazell et al. 2000). In a study on cultured neuroblastoma cells $\mathrm{TH}$ deficiency resulted in the accumulation of glutamate due to aKGDH reduced activity. Overt signs of necrosis (i.e. condensed chromatin, decreased oxygen consumption, and uncoupled mitochondria with disorganized cristae) were observed. The normalization of the TH levels resulted in the reversal of all changes, mitochondrial morphology being recovered within an hour (Bettendorff, Goessens et al. 1997). This suggests that the slowing of the Krebs cycle is the main cause of the biochemical lesions induced by TH deficiency. In vivo, the clinical improvement following $\mathrm{TH}$ administration corresponds to the improvement in the PDH activity (Thomson and Marshall 2006). This suggests that in vivo the impairment in PDH activity has great consequences, possibly by its effect on the Krebs cycle. It was proposed that the impairment of the PDH activity may lead to the impairment of acetylcholine synthesis, though several studies failed to confirm this hypothesis (Heinrich, Stadler et al. 1973). However, three case reports suggest that WE patients may benefit from the administration of the acetylcholinesterase inhibitor donepezil (Thomson and Marshall 2006). The possible role of other neurotransmitters in the physiopathology of WE has been speculated. Some have reported that changes in GABA, glutamate, and aspartate levels may occur, as their production requires proper glucose metabolism (Hazell, Butterworth et al. 1993). Others reported no impairment in these neurotransmitters levels (Fattal-Valevski 2011). Decreased levels of the serotonin metabolite 5-hydroxyindoleacetic acid where found in the CSF of WE patients, while some studies found that cerebellar extracellular serotonin levels were increased due to decreased cellular uptake (Plaitakis, Van Woert, et al. 1978).

It has been observed that alcohol related WE is more frequently associated with lesions with typical topography, irreversible brain damage and KS. Several possible physiopathological explanations have been proposed. The alcoholics may have recurrent episodes of mild TH deficiency that may render the brain's affected areas more vulnerable to future injury and/or may have persistent subclinical TH deficiency leading to a chronic evolution. In a study on rhesus monkeys the recurrent stereotypic rapid variation of the $\mathrm{TH}$ levels ranging from very low to very high led to the progressive worsening of the $\mathrm{TH}$ deficiency-induced symptoms, in spite of their complete resolution after $\mathrm{TH}$ administration (Thomson and Marshall 2006). It seems that the length of a single TH deprivation period and not their number determines the severity of the induced structural brain damage. The effects are cumulative, the symptoms of TH deprivation appearing sooner with each episode (Witt 1985; Ciccia and Langlais 2000). Some suggested that chronic alcohol exposure and/or TH deficiency may render certain brain areas (that are not typically affected in alcoholics but are often affected in non alcoholics) more resistant to TH deficiency. It has been proposed that large and repeated fluctuations in TH levels may impair the capacity of the brain to cope with low $\mathrm{TH}$ levels and in this respect some have suggested that the administration of high 
$\mathrm{TH}$ doses in alcoholic patients with asymptomatic TH deficiency may be deleterious providing a lifestyle change does not occur. Chronic ethanol exposure blocks the NMDA-R at the glutamate site. This results in the upregulation of the glutamate receptors. Alcohol withdrawal may increase $\mathrm{TH}$ requirements and may exacerbate the glutamate mediated excitocytotoxic injury, the abundant extracellular glutamate acting on an upredgulated NMDA-R population which is no longer blocked by ethanol. A preliminary clinical study suggests that the NMDA antagonist memantine might be beneficial in WE (Harper 2006). It has been suggested that ethanol may accelerate the cerebral metabolism of $\mathrm{TH}$ and that chronic alcohol exposure may reduce the TK's affinity for THDP (Laforenza, Patrini et al. 1990). Alcoholics may frequently have other vitamin and mineral deficiencies that may contribute to the WE particularities (Ihara, Ito et al. 1999). Since pyridoxine and riboflavin are necessary for the conversion of glutamate to GABA, WE alcoholics may have an additional increase of the extracellular glutamate levels (Thomson, Cook et al. 2002). Recurrent seizures and/or head trauma are more frequent in alcoholics and may subtlety alter the brain's capacity to withstand additional metabolic injury. Previous episodes of hepatic encephalopathy may render the brain more susceptible to TH deficiency (Caine, Halliday et al. 1997).

\section{Clinical presentation and anatomo-clinical correlations}

The classical hallmark of WE consists of ocular motility signs, stance and/or gait ataxia and mental status changes having acute or subacute onset. The absence of all these signs may occur in approximately $16 \%$ of the cases, though usually at least one of them appears at some point in the course of the disease. When present, the stance and/or gait ataxia usually precedes the onset of the other symptoms. The ocular motility signs have been reported in 29 to $90 \%$ of the cases, ataxia has been reported 23 to $70 \%$ and mental status changes have been reported in 82 to 90\% (Harper, Giles et al. 1986; Victor 1989; Ogershok, Rahman et al. 2002). The symptoms may develop simultaneously or in a succeeding manner, over a shorter or longer period of time. In a significant number of patients, typical fundoscopic findings are present (Doss, Mahad et al. 2003). Other less specific WE manifestations include anorexia, vomiting, hypotension, orthostatic hypotension, hypothermia, hyperthermia, miosis, urine retention, tachycardia, dyspnea, visual disturbances and sensitive ataxia (Victor 1989; Donnino, Vega et al. 2007). Except for the motor ocular and vestibular nerves nuclei, WE is not commonly responsible of cranial nerve involvement. However, bilateral peripheral facial paresis has been reported. Also, dysphagia and dysphonia in association with vagus nerve degeneration have been reported in severely malnourished WE patients (Novak and Victor 1974). The presence of the Babinsky reflex has been very rarely observed. Signs and symptoms consistent with polyneuropathy are present in up to $82 \%$ of WE patients, and usually have acute or subacute onset, closely preceding the onset of WE. Commonly the clinical signs are typical for peripheral motor and sensitive involvement, the lower extremities being significantly more frequently and severely affected than the upper extremities. Concomitant autonomic neuropathy is rare; it leads to orthostatic hypotension if the sympathetic system is affected and to urinary retention if the parasympathetic system is affected. Overt cardiovascular beriberi only rarely coexists, but mild forms have been reported. The cardiovascular involvement caused by TH deficiency is responsible of either high output cardiac failure, with pulmonary and peripheral edema, or, less often, of low 
output cardiac failure with concomitant hypotension and acidosis. The latter may have dramatic hyperacute evolution, i.e. the so-called shoshin beriberi (Thorarinsson, Olafsson et al. 2011). Gastrointestinal beriberi, resulting in abdominal pain and other digestive symptoms, may rarely coexist with WE, especially in children (Donnino 2004). The so-called subclinical TH deficiency states may be responsible of nonspecific symptoms such as recurrent headaches, irritability, abdominal discomfort and, in children, decline in growth rate. Late manifestations of untreated WE include KS (mamillo-thalamic involvement), spastic paresis (frontal cortex and/or pyramidal tracts involvement), hyperthermia unresponsive to antipyretics (anterior hypothalamus involvement), and increased muscular tone with nuchal rigidity and chorea (basal ganglia and/or mesopontine tegmental involvement) (Ogershok, Rahman et al. 2002; Donnino 2004; Harper 2006; Sechi and Serra 2007). Myoclonus has been reported in several neuropathologically confirmed WE cases, clinically diagnosed as Creutzfeldt-Jakob disease (Bertrand, Brandel et al. 2009).

The ocular motility signs encountered in WE are the consequence of lesions involving the oculomotor, abducens and vestibular nuclei, the pontine tegmentum and the internuclear fibers (Victor 1989; Ogershok, Rahman et al. 2002; Harper 2006; Sechi and Serra 2007). Typically they consist of nystagmus, bilateral lateral rectus muscle palsy and impairment of conjugate gaze. However, these three ocular signs are only rarely seen together. The nystagmus is the most common ocular finding, being reported in 10 to $85 \%$ of the patients (Harper, Giles et al. 1986; Ogershok, Rahman et al. 2002). Gaze evoked horizontal bilateral nystagmus is the type of nystagmus most frequently observed. Vertical nystagmus evoked by upward gaze may coexist, but the presence of isolated vertical or rotator nystagmus is unusual. Lateral rectus palsy is the most frequent ophtalmoplegia, occurring in 4 to $54 \%$ of WE cases. It is virtually always bilateral, but not necessarily symmetric. It is associated with diplopia and internal strabismus (Ogershok, Rahman et al. 2002). Other ocular muscles palsies may rarely occur. Conjugate gaze palsy of variable intensity may be found in up to $44 \%$ of the patients. Vertical gaze palsy occurs only exceptionally, isolated paralysis of downward gaze being seldom reported (Harper 2006). The ocular motility signs show the most rapid reversibility, ophtalmoplegia disappearing even as soon as six hours after intravenous $\mathrm{TH}$ administration. Commonly, complete recovery occurs in less than 7 days. In up to $60 \%$ of the cases the resolution of the ophtalmoplegia was reported to occur in less than a day. This may be used as clinical diagnostic test, supporting the WE diagnosis (Victor 1989; Sechi and Serra 2007). The nystagmus recovers more slowly and was reported to persist for at least 2 years in $60 \%$ of the treated patients (Thorarinsson, Olafsson et al. 2011). Pupillary dysfunction, ptosis, impaired convergence and internuclear ophtalmoplegia are very rarely found. The pupils are often spared except in the presence of hypothermia or in the final stages when they may be anisocoric, miotic and non-reacting (Victor 1989; Ogershok, Rahman et al. 2002; Harper 2006; Sechi and Serra 2007). Fundoscopic findings comprising of retinal hemorrhages, papillary edema or disc pallor are commonly seen in WE patients and have been reported even by Wernicke himself. Some have proposed that these are caused by a TH deficiency optic neuropathy associated with WE. Usually these fundoscopic changes are not associated with symptoms. Rarely disturbances of vision consisting of decreased visual acuity, scotomas and even bilateral blindness have been reported (Doss, Mahad et al. 2003; Surges, Beck et al. 2007). 
The ataxic syndrome encountered in WE is the consequence of propriocerebellar and vestibulocerebellar dysfunction. Most frequently, the WE cerebellar lesions are located in the superior vermis. However, central vestibular ataxia and disequilibrium due to central vestibular dysfunction and peripheral proprioceptive ataxia due to polyneuropathy may coexist. Typically variable degrees of ataxia of stance and gait, ranging from barely noticeable tandem walking impairment to inability to walk or stand, are found. Since $\mathrm{TH}$ deficiency usually does not lead to cerebellar hemispheric lesions, limb ataxia and other cerebellar signs are only exceptionally seen (Victor 1989; Ogershok, Rahman et al. 2002; Harper 2006). However, in acute settings mild limb ataxia might be present. WE patients may associate alcoholic cerebellar degeneration that frequently causes limb ataxia (Antunez, Estruch et al. 1998). Ataxic speech has rarely been reported in WE patients. The vestibular dysfunction is very common in acute WE, the ice-water caloric testing and the vestibuloocular reflex showing impairment in virtually all cases. It has been proposed that the vestibular dysfunction may be responsible of the postural imbalance of WE patients. Usually vertigo and/or hearing loss are not present (Doss, Mahad et al. 2003). Commonly the cerebellar and vestibular dysfunctions begin to improve after 2 to 6 days. In a significant number of cases the gait disturbances persist indefinitely (Sechi and Serra 2007).

The mental status changes encountered in WE patients are probably the consequence of the bilateral thalamic, mamillary bodies and rarely head of the caudate nuclei lesions. The connections between the left-sided anterior thalamus and the ipsilateral mamillary body, documented by functional MRI (fMRI) studies, have been reported to be impaired in those with WE, and to a lesser degree in alcoholics without WE (Kim, Ku et al. 2009). The critical pattern of the lesions that results in KS has not been identified, but is agreed upon that bilateral involvement of the limbic circuits is necessary. Some proposed that the concomitant bilateral involvement of the hippocampal (medial limbic) and amygdaloid (basolateral limbic) circuits results in KS. According to the necroptic and imagistic studies, KS is associated with lesions involving the anterior, mediodorsal and/or midline thalamus, mamillary bodies and/or the mamillo-thalamic tracts. All these may result in the disruption of the above mentioned circuits (Mishkin 1978; Mair, Warrington et al. 1979; Kopelman 1995). The fMRI based studies on patients with acute or persistent anterograde amnesia due to WE revealed absence of hippocampal activation during encoding or retrieving tasks in spite of lack of MRI evidence for medial temporal structural damage, and in the presence of fMRI proof of intact perception, attention and judgment (Caulo, Van Hecke et al. 2005) and positron emission tomography (PET) studies reported the presence of significant glucose hypometabolism in the limbic circuits, bilateral thalamic nuclei, mesial prefrontal cortices and fronto-temporo-parietal cortices (Joyce, Rio et al. 1994; Aupee, Desgranges et al. 2001; Fellgiebel, Scheurich et al. 2003). The mental status changes may range from apathy, hypoprosexia, disorientation, confusion, mild hypomnesia, anterograde and retrograde amnesia and confabulations (typically spontaneous) to hallucinations and behavioral disturbances. Altered states of consciousness ranging from somnolence and stupor to coma also occur, the latter usually being related to bilateral thalamic or head of caudate nuclei lesions. Stupor and coma may be the presenting symptoms more frequently than expected, being reported in up to $10 \%$ of the cases (Lana-Peixoto, Dos Santos et al. 1992; Thomson, Cook et al. 2008). Untreated comatose patients usually die after several days (Harper, Giles et al. 1986; Victor 1989). Confusion seems to be the most frequent symptom, being reported in up to $82 \%$ of the cases (Harper, Giles et al. 1986; Victor 1989; Antunez, Estruch et al. 1998). KS 
comprises of anterograde amnesia affecting the episodic memory and in variable degree the semantic memory (the working memory is intact and so is the secondary implicit memory!), retrograde temporally graded amnesia and confabulations (spontaneous in the early stages, latterly provoked by memory challenges) occurring in the absence of other significant cognitive impairments (language, visuo-perceptual functioning, problem solving, and judgment intact!), usually in the absence of insight (Yoneoka, Takeda et al. 2004; Kopelman, Thomson et al. 2009). Some of the mental status changes may be caused by the comorbid conditions (e.g. delirium tremens). In WE alone, agitation may rarely be seen. Seizures may occur, most likely in relationship with the comorbid conditions (e.g. alcohol withdrawal), though cortical lesion may rarely be encountered. Some have proposed that the seizure threshold may be lowered due to the presence of excessive glutamatergic stimulation. In a significant number of cases the mental status changes are only partially reversible, KS being a frequent sequel, especially in alcoholics. The mental status changes begin to improve after a few days to a week of treatment, the confusional syndrome being the first to recover. Sequels may occur, especially in those with KS. WE patients have increased risk of sudden death. The risk of sudden death is not necessarily related to $\mathrm{WE}$, the cardiovascular dysfunction due to TH deficiency or comorbid conditions (e.g. alcoholic cardiomyopathy) possibly being responsible (Harper 2006; Thomson and Marshall 2006; Sechi and Serra 2007).

Particularities of the clinical presentations of WE in alcoholics, non alcoholics and other selected sub-groups have been reported. However, no typical syndromes have been identified and part of the differences may be related with the associated conditions. The classical triad and KS occur more frequently in alcoholics. The polyneuropathy, seizures and sudden death are also encountered more often in alcoholics. Typically alcoholics with WE are malnourished and underweight, though overweight beer drinkers are also prone to WE. The onset of WE in alcoholics is usually subacute and the evolution might be chronic in those with recurrent mild, subclinical TH deficiency episodes (Harper, Giles et al. 1986; Victor 1989; Harper 2006). Most of the cases of necropsy confirmed WE presenting with coma were reported in alcoholic patients (Thomson, Cook et al. 2008). The non alcoholrelated WE usually has acute onset. Neurologic disturbances reversible after $\mathrm{TH}$ administration have been reported to occur in obese patients after 14 to 34 days of therapeutic fasting. The first symptoms where atypical and consisted of drowsiness, dizziness, apathy, psychosis and visual disturbances (Ogershok, Rahman et al. 2002). Individuals that underwent bariatric surgery have been reported to develop WE in 2 to 4 weeks after the procedure, those with persistent vomiting appearing to be more susceptible. Cases occurring as late as 12 to 24 weeks have been reported. The clinical picture was atypical. In those with non-bariatric gastrointestinal surgery WE develops in 2 to 8 weeks after the procedure, but cases occurring as early as 2 weeks or as late as 20 years have been reported. The risk of developing WE was reported to be higher in those with weight loss greater than 7 kilograms per month (Foster, Falah et al. 2005; Dallal 2006; Serra, Sechi et al. 2007; Aasheim 2008). Patients with severe hyperemesis gravidarium were reported to develop WE after $7.7+/-2.8$ weeks of persistent vomiting. The clinical triad was present in half of the cases. The resolution of symptoms was slow in spite of adequate treatment and sequelae commonly occurred. In almost half of the cases the pregnancy was loss (Chiossi, Neri et al. 2006). Hunger strikers were reported to develop symptoms consistent with WE (nystagmus and axial ataxia) after about 6 weeks of fasting. The clinical triad occurred in almost a quarter of patients. Peripheral nervous system involvement occurred, but was less 
prominent than the central nervous system involvement (Basoglu, Yetimalar et al. 2006). War prisoners on a TH deficient diet developed anorexia, nausea, vomiting, diplopia, insomnia, anxiety, hypoprosexia, memory impairment, confusion, confabulation, hallucination and eventually coma (De and Lennox 1947). In infants the onset is usually hyperacute and exitus may rapidly occur. The first noted symptoms are constipation, vomiting, restlessness, followed by aphonia, absence of deep tendon reflexes, metabolic acidosis, meningeal irritation, convulsions and heart failure (Fattal-Valevski, Kesler et al. 2005).

\section{Imagistic findings}

Brain imagistic investigations are required in virtually all patients presenting with a clinical picture suggestive of WE. The imagistic evaluation methods used in the current clinical practice are the brain X-ray computed axial tomography (CT) and the magnetic resonance imaging (MRI), the latter providing more information. Other structural and functional imagistic modalities have minute relevance for the management of WE patients, are not widely available and are expensive, therefore being mainly reserved as research tools (Sechi and Serra 2007). They will not be discussed. The MRI techniques commonly used for the assessment of WE are T1, T2 and T2 based modalities (e.g. late echo T2 and FLAIR), accompanied or not by contrast administration (i.e. gadolinium). Less frequently used, but very informative providing the correlation with the apparent diffusion coefficient (ADC) is made, is the diffusion weighted imaging (DWI). The typical WE lesions have high T2 and FLAIR signal (hyperintensity) and normal or low T1 signal (iso- or hypointensity). The FLAIR acquisition technique eliminates the hyperintense T2 signal of the CSF and therefore even small periventricular and periaqueductal high signal lesions become obvious. The contrast enhancement is the imagistic expression of BBB disruption (Zuccoli, Gallucci et al. 2007; Sullivan and Pfefferbaum 2009). The DWI is an acquisition technique in which the signal from unbound water is suppressed, thus areas with high concentrations of unbound water appearing as hypointense, while areas with low concentration of unbound water appear as hyperintense (i.e. restricted diffusion areas). The echo-planar DWI technique allows very short examination times, making its use practical even in acute settings. The typical WE lesions appear hyperintense on DWI, as if diffusion were restricted. When the DWI hyperintensity corresponds to a T2 hyperintensity, the DWI finding may be the consequence of the so-called 'T2 hyperintensity shine through effect' and may not truly represent restricted diffusion. The presence of diffusion restriction is confirmed by confronting the correspondent values of the ADC, which should be low, and the ADC map, which should show hypointensity. In the case of 'T2 hyperintensity shine through effect' the ADC value is normal or high and the ADC map shows iso- or hyperintensity. In the very early stages the WE lesions have true restricted diffusion, translating the presence of cytotoxic edema in the absence of vasogenic edema. Considering that the DWI is very sensitive for cytotoxic edema, this MRI technique might be of great use for the very early identification of WE lesions. Late, atrophic WE lesions also show restricted diffusion (Halavaara, Brander et al. 2003; Lapergue, Klein et al. 2006; Unlu, Cakir et al. 2006; Zuccoli, Santa Cruz et al. 2009). The most frequent conventional MRI findings in active WE are bilateral, symmetric, contrast enhancing or not, T2, late echo T2 and/or FLAIR hyperintensity of the mamillary bodies, anterior and medial nuclei of the thalamus, dorsomedial thalamus, periaqueductal grey matter periventricular grey matter, inferior and 
superior colliculi, caudate nuclei, midbrain and cerebellum (Schroth, Wichmann et al. 1991; $\mathrm{Chu}$, Kang et al. 2002; Zhong, Jin et al. 2005; Unlu, Cakir et al. 2006; Zuccoli, Gallucci et al. 2007; Zuccoli, Cravo et al. 2011). The characteristics of the MRI lesions change along the course of the disease. The earliest imagistic changes are probably those reflecting cytotoxic edema, followed by contrast enhancement reflecting BBB disruption, and byT2 and FLAIR signal changes reflecting the presence of vasogenic edema. In most of the cases the mamillary bodies appear to be the first structure involved. Bilateral mamillary body restricted diffusion followed by contrast enhancement and by FLAIR and T2 signal changes might be the chronology of the earliest MRI findings, though, as recently argued by one author, choroid plexus contrast enhancement may precede the other MRI changes. Providing TH is supplied, the MRI findings may disappear as early as two days. In the early stages the typically injured areas show restricted diffusion. Restricted diffusion of the corpus callosum's splenium has also been reported (Loh, Watson et al. 2005). The atrophy of the mamillary bodies, thalamus and cerebellar vermis may appear as soon as one week. The regions that frequently show contrast enhancement are the mamillary bodies, followed by the tectal plate, thalamus and periaqueductal grey matter. MRI signal changes involving the fornix, dorsal medulla oblongata, central pons, globus pallidus, putamen, frontal and/or parietal cortex, splenium, dentate nuclei, red nuclei and cranial nerve nuclei have rarely been reported (Park, Kim et al. 2001; Weidauer, Nichtweiss et al. 2003; Zuccoli, Gallucci et al. 2007; Nixon, Jordan et al. 2008; Sullivan and Pfefferbaum 2009; Zuccoli; Zuccoli, Cravo et al. 2011). WE cortical lesions comprising of linear more or less symmetric FLAIR and/or T2 hyperintensities have also been described. A case of WE related obstructive hydrocephalus in the presence of otherwise typical MRI findings has been reported (Doss, Mahad et al. 2003). To the best of our knowledge, the imagistic characteristics of WE-related optic neuropathy have not been presented. The imagistic sequelae of WE are scarcely described. Atrophy of the mamillary bodies, cerebellar vermis and less often of the thalamus (corresponding with third ventricular enlargement) has been reported. These are frequently found in alcoholics and correlate with the persistence of clinical findings. MRI evidence of mamillary bodies, pons, thalami, cerebellar hemisphere, superior vermis and hippocampal atrophy has been documented in KS patients with prior alcohol related WE. Except for hippocampal atrophy which, as reported by some, seems to occur only in KS patients, the other brain volume changes are also found in different degrees in alcoholics without KS and/or history of WE (Park, Kim et al. 2001). The typical conventional MRI findings described above are more commonly found in alcohol related WE. The bilateral mamillary body contrast enhancement, in association or not with incidental or subsequent mamillary body atrophy, shows a positive statistical correlation with alcohol consumption. Cortical, frontal lobe and cerebellar (especially vermian) atrophy are frequent CT and MRI findings in chronic alcohol abusers. These changes have higher incidence in alcoholics with concomitant WE than in alcoholics without WE. The MRI findings that may be related to chronic alcohol consumption and/or to prior WE episodes (e.g. mamillary body atrophy and possibly cerebellar atrophy) are also more frequently encountered in alcoholics. Besides the enlargement of the lateral ventricles, probably secondary to alcohol related cerebral atrophy, the enlargement of the third ventricle may be seen on the CT and MRI of alcoholic WE patients (Gallucci, Bozzao et al. 1990; Antunez, Estruch et al. 1998). Atypical MRI findings comprising of characteristic signal changes in the cerebellum, cranial nerves nuclei (especially abducens, facial, vestibular 
and hypoglossal), red nuclei, dentate nuclei, caudate nuclei, splenium and cerebral cortex, that coexist or not with the above mentioned typical MRI findings are more frequently encountered in non alcohol related WE (Zhong, Jin et al. 2005; Liu, Fuh et al. 2006; Fei, Zhong et al. 2008; Zuccoli and Motti 2008). Commonly the pediatric WE patients have similar MRI findings as adult patients, but symmetric basal ganglia alterations with bilateral involvement of the putamen has been reported (Fattal-Valevski, Kesler et al. 2005; Zuccoli, Siddiqui et al. 2010).

A

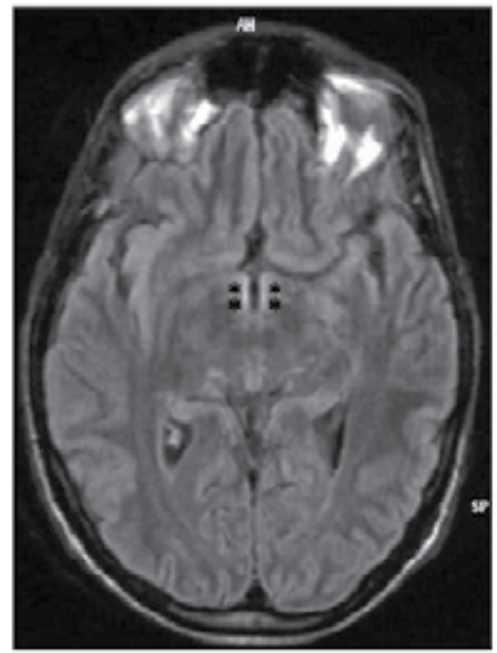

$\mathrm{C}$

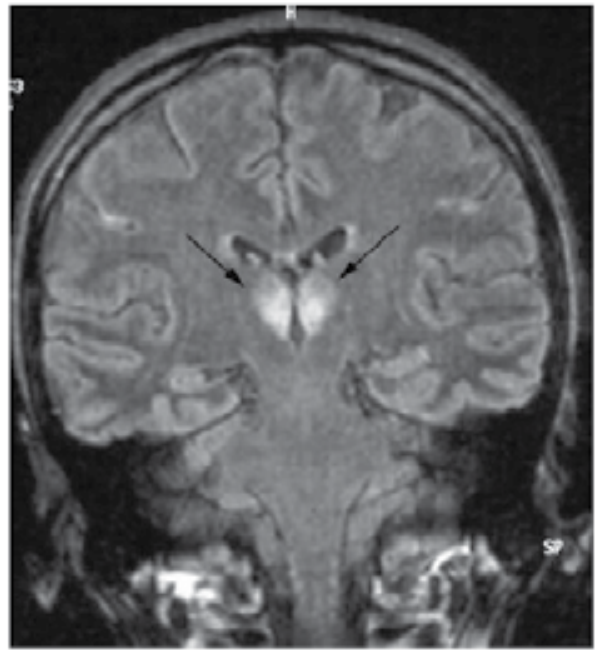

B

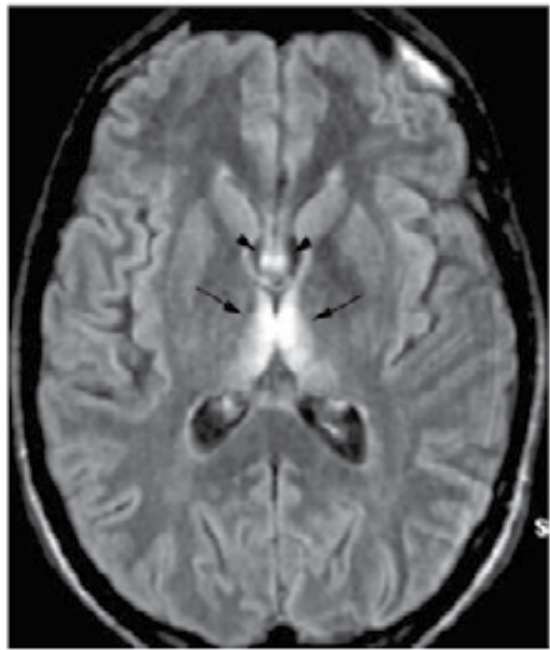

D

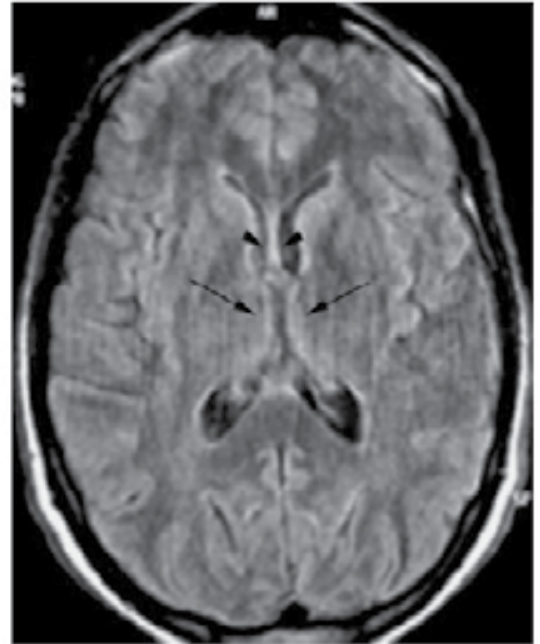

Fig. 1. 'Images from axial and coronal FLAIR sequence of the WK subject. Seven-day MRI study demonstrates signal abnormalities of the mammillary bodies (A) (stars), the medial aspect of the thalami (B, C) (arrows) and the fornix (B) (arrowheads). Five months later signal changes have almost completely disappeared (D) (arrows and arrowheads)' - With the permission of M. Caulo, Fig 2 from (Caulo, Van Hecke et al. 2005). 


\section{Diagnosis}

In spite of the significant advances in imagistic and laboratory assessments WE remains mainly a clinical diagnosis. The paraclinical workup, comprising of brain MRI, blood TH assessments, routine blood tests and sometimes ancillary investigations, improves the accuracy of the diagnosis and is mandatory for the identification of potential comorbidities. The diagnosis is highly supported by the favorable response to parenteral $\mathrm{TH}$ administration, but is not excluded by the lack of it (Harper, Giles et al. 1986; Thomson, Cook et al. 2008). WE may present with a wide spectrum of nonspecific findings. Classically, the diagnosis requires the presence of the clinical triad consisting of ocular motility signs, stance and/or gait ataxia and mental status changes, having acute or subacute onset. However, the classic triad has been reported in less than a third of the adult WE cases (up to $16 \%$ in necroptic retrospective studies!) and in $20 \%$ of the pediatric cases (Harper, Giles et al. 1986; Victor 1989; Vasconcelos, Silva et al. 1999). Based on the data available in the literature regarding neuropathological proven WE cases, the prevalence of the classical triad is estimated to be $8.2 \%$ (Galvin, Brathen et al. 2010). The WE diagnosis may easily be overlooked, the 2010 EFNS guideline recommending the maintenance of a high index of suspicion for WE. 'Operational clinical criteria for the classification of the chronic alcoholics and the identification of $W^{\prime}$ that take into account dietary deficiencies as predisposing factor for WE were proposed in 1997 by Caine. The intended purpose of these criteria was to accurately differentiate between alcoholics with WE (active and sequelae) and alcoholics without WE and between alcoholics with WE and alcoholics with hepatic encephalopathy. Since their intended applicability concerned only the alcoholic population they were not tested for reproducibility and variability in non alcoholics. According to these criteria the accurate ante-mortem identification of WE patients requires the presence of two of the following: dietary deficiencies (defined as: 'undernutrition, vitamin deficiency'), eye signs (defined as: 'ophtalmoplegia, nystagmus, gaze palsy'), cerebellar signs (defined as: 'unsteady, ataxia, cerebellar dysfunction') and either altered mental status (defined as: 'disoriented, confuse, comatose, digit span abnormal') or mild memory impairment (defined as: 'mild-moderate memory problems, confabulating'). These criteria accurately differentiate between alcoholics with and without WE (though they do not differentiate between active WE and sequelae!) and also between alcoholics with WE and hepatic encephalopathy. Their use was estimated to increase the sensitivity (but not the specificity!) of the diagnosis of WE from $31 \%$ when using the classic triad to $100 \%$ (Caine, Halliday et al. 1997). Criteria for accurately identifying WE patients in the 'accident and emergency department' were proposed in 2001 by the UK Royal College of Physicians. According to their guideline, all patient presenting with any evidence of chronic alcohol use and any of the following: acute confusion, decreased consciousness level, ataxia, ophtalmoplegia, memory disturbance, hypothermia and hypotension and all patients presenting with acute alcohol intoxication or with delirium tremens should be presumed of having WE (Thomson, Cook et al. 2002). Though these criteria may accurately identify alcoholic patients with WE they are not applicable in the non alcoholic population. Since no better alternative exists, the 2010 EFNS guideline recommends the use of the 'operational clinical criteria for the classification of the chronic alcoholics and the identification of WE' proposed by Caine in 1997 for help in the diagnosis of WE in both alcoholics and non alcoholics (Galvin, Brathen et al. 2010). According to the 2010 EFNS guideline, brain imagistic investigations and measurement of 
the total blood TH level should be included in the paraclinical workup of all those in which WE is suspected. The brain CT has low sensitivity (i.e. 13\%) for the detection of WE lesions, but nevertheless it is of great utility in acute settings, the purpose of the imagistic assessments being not only to offer additional information supporting the diagnosis of WE, but to exclude other pathologies that might be responsible for the clinical presentation. The MRI offers better sensitivity than the CT for both acute and sequelar WE lesions. The sensitivity varies accordingly to the power of the magnet and to the technique of acquisition used. The estimated sensitivity for the conventional MRI techniques (i.e. T1, T2, FLAIR, paramagnetic contrast) is just above $50 \%$, values of $53 \%$ and $58 \%$ being reported. Though it does not rule out WE, the MRI is probably the best method of confirming the diagnosis of WE, its specificity being estimated to be as high as 93\% (Antunez, Estruch et al. 1998; Weidauer, Nichtweiss et al. 2003). The presence of low TH blood levels has low specificity for WE. The venous blood necessary for the laboratory assessments should be collected prior to $\mathrm{TH}$ administration (since is highly influenced by the recent $\mathrm{TH}$ intake) and kept away from light. The presence of low total TH or THDP levels is neither necessary nor sufficient for the diagnosis of WE. The recommended laboratory assays are the measure the total TH or THDP in the whole blood through various methods of HPLC (Talwar, Davidson et al. 2000; Thomson and Marshall 2006) The measurement of the 24 hours urine TH levels has little utility in the diagnosis of WE. The level of TH in the urine collected in the following 4 hours after the administration of $1 \mathrm{mg}$ intravenous TH may be used to estimate the tissue THDP state, but due to practical difficulties is not commonly used in clinical practice (Tallaksen, Bohmer et al. 1991; Tallaksen, Bell et al. 1993). Routine laboratory investigations are mandatory for the differential diagnosis of WE and for the identification of associated disorders. The routine laboratory investigations include the assessment of blood glucose levels, serum electrolytes, acute-phase reactants, liver, renal and hematologic status. Toxicology panels may be required in those presenting with acute confusion syndromes. The performance of additional testes (e.g. blood ammonia, thyroid tests, blood lipase, blood B12 levels, viral markers, immune tests) should be adapted to each patient. WE patients may show laboratory signs of dehydration and may have abnormal liver tests, hypomagnesaemia and anaemia (Traviesa 1974; Sechi and Serra 2007). The blood levels of lactic acid and pyruvate may be increased due to TH deficiency. Their measurement may be used for estimating the activity status of the THDP dependent enzymes, but they are neither specific nor sensible for TH deficiency or WE. Some have suggested that WE patients may associate lactic acidosis with or without respiratory alkalosis, leading to a characteristic acid-base pattern. In infants and in other selected susceptible patients the assessment of the acid-base equilibrium is required (Donnino, Miller et al. 2007). Lumbar puncture may be required especially in those associating hyperthermia. Commonly the routine analysis of the CSF of WE patients shows no particularities. In the later stages of WE mild protein elevation may occur (Victor 1989). The presence of protein 14-3-3 has been reported in several patients with WE (Michowitz, Copel et al. 2005; Bertrand, Brandel et al. 2009). CSF pleocytosis or protein levels above 100 $\mathrm{mg} / \mathrm{dl}$ should suggest an alternative or a coexistent disorder. The assessment of the electroencephalogram (EEG) may be required especially in those in which epileptic partial complex status is suspected. The EEG activity may be dominated by nonspecific diffuse slow waves in about half of WE patients (Victor 1989; Sechi and Serra 2007). Considering that the WE patients may associate cardiac pathology related or not to TH deficiency (e.g. alcoholic dilatated cardiomyopathy) and considering that infections may precipitate WE, 
an electrocardiogram and chest radiography should be routinely included in the paraclinical workup. WE patients may associate other encephalopathies (hepatic encephalopathy!), as well as brain unrelated diseases. The required workup should be adapted to each patient. It should be kept in mind that these comorbidities may predispose to WE and may correlate with the atypical presentations. Considering that WE patients commonly associate polyneuropathy and that WE may mimic Miller Fischer syndrome, nerve conduction studies may be required (Harper, Giles et al. 1986; Sechi and Serra 2007).

\section{Differentials}

The diferential diagnosis of WE is vast, comprising mainly of acute or subacute toxic, nutritional and metabolic encephalopaties, encephalitis and meningoencephalitis, and particular strokes. In certain cases diferentiating betwen acute or chronic WE and WE sequelae (including KS!) may be difficult. KS may have other etiologies than WE, and these shoud also be considered for the differential diagnosis. Among the acute or subacute toxic, nutritional and metabolic encephalopaties we emphasize acute alcohol intoxication, delirium tremens, acute Marchiafava-Bignami disease, subacute alcoholic cerebellar degeneration, acute alcoholic pellagra, hepatic encephalopaty, metronidazole encephalopaty. All of these may coexist with WE (Arbelaez, Pajon et al. 2003; Zuccoli, Pipitone et al. 2008; Thorarinsson, Olafsson et al. 2011). In certain clinical settings hypoglicemic coma, hypoxic or hypercapnic encephalopaty, hypertensive encephalopaty, severe hypophosphatemia, methyl bromide intoxication and sepsis asociated encephalopaty should be taken into account. Though it appears earlier and does not asociate the ocular motility signs characteristic for WE, pancreatic encephalopaty may be considered as differential diagnosis in those with severe acute pancreatitis (Sun, Yang et al. 2006; Thorarinsson, Olafsson et al. 2011). Variant Creuzefeld-Jakob disease and Fabry's disease may resemble WE. Inflamatory diseases of the brain (infectious, autoimmune or disimmune), especially those with perimidline tropism, may present with clinical pictures compatible with WE. Among these we emphasize viral encephalitis and meningoencephalitis (influenza A, West Nile, Japanes, Murray, citomegalovirus, Herpes simplex), tuberculose meningoencephalitis, paraneoplastic cerebelitis, ventriculoencephalitis, acute disseminated encephalomyelitis, limbic encephalitis, brainstem encephalitis and primary or secondary cerebral vasculitides (Brechtelsbauer, Urbach et al. 1997; Torgovnick, Arsura et al. 2000; Chung, Kim et al. 2003; Bertrand, Brandel et al. 2009). Pontine or extrapontine mielinolysis, adult onset Leigh disease and other mitochondrial encephalopaties may be included in the diferential diagnosis of WE. In certain selected cases, the recently described 'chronic lymphocitic inflamation with pontine perivascular enhancement responsive to steroids' syndrome (CLIPPERS) (Pittock, Debruyne et al. 2010) and 'cerebellar ataxia, neuropathy and vestibular areflexia syndrome' (CANVAS) (Szmulewicz, Waterston et al. 2011) may also be taken into account. The clinical picture of Miller-Fischer syndrome, African seasonal ataxia and tropical ataxic polineuropathy may resemble WE. Percheron artery thrombosis or embolia, basilar artery trombosis and deep cerebral veins thrombosis may also be included in the differential diagnosis of WE. Thalamic and mamillothalamic tract infarcts occuring in individuals with previous strategicaly localised lesions may mimic WE (Bogousslavsky, Regli et al. 1988; Ghika-Schmid and Bogousslavsky 2000). Brain neoplasy involving the perimidline regions (e.g. primary cerebral lymphoma, bilateral thalamic glioma) may 
sometimes be considered. In children WE may mimik meningitis. Regarding atypical WE presentations, the differential diagnosis should be adapted for each situation. Optic neuritis should be considered in those presentig with optic nerve inolvement. Leber hereditary optic neuropathy precipitated by TH deficiency that also resulted in WE has been reported, and should be considered in WE patients with optic nerve involvement unresponsive to $\mathrm{TH}$ administration (Li and Rucker 2010).

\section{Treatment}

WE is a medical emergency. Its etiopathogenic treatment implies the restoration of adequate brain TH levels. As detailed further on, this is optimally accomplished by high dose parenteral $\mathrm{TH}$ administration. The correction of coexisting hypomagnesaemia (by intravenous $\mathrm{Mg}$ sulphate administration in doses titrated against $\mathrm{Mg}$ serum levels) is mandatory. The reinstitution of a balanced diet and the elimination of the associated modifiable predisposing factors are also required. Scientific evidences regarding the optimal dose, frequency, route and duration of $\mathrm{TH}$ administration for WE treatment are currently lacking. The management of the WE patients is commonly conducted according to the empirical recommendations provided by the local guidelines. Since any delay in $\mathrm{TH}$ administration may result in permanent neurologic sequelae or death, parenteral $\mathrm{TH}$ administration should be started as soon as possible. The decision of initiating parenteral TH administration should be taken on clinical grounds and should never be delayed until the paraclinical workup is completed and/or the results are available (Thomson and Marshall 2006; Sechi and Serra 2007; Galvin, Brathen et al. 2010; Thorarinsson, Olafsson et al. 2011). The identification and treatment of WE comorbidities is mandatory. Supportive and symptomatic therapy should be provided. These should be adapted to each patient, and will not be detailed in the present chapter. Considering that carbohydrate load may precipitate or aggravate WE, glucose should never be administered prior to TH (! hypoglycaemic coma or seizures). To the best of our knowledge, scientifically rigorous studies assessing the optimal TH posology for the treatment of WE have not been conducted. However, the results of several uncontrolled studies on adults are available. It is commonly accepted that a rapid restoration of brain $\mathrm{TH}$ levels is more beneficial than a slower restoration, therefore frequent high dose intravenous administration being required. Low parenteral $\mathrm{TH}$ doses and the oral route of administration are inefficient for the treatment of WE (Thomson, Cook et al. 2002; Galvin, Brathen et al. 2010). It was reported that WE patients treated with parenteral $\mathrm{TH}$ doses of 50 or $100 \mathrm{mg}$ per day fully recovered in only up to $16 \%$ of the cases, while death occurred in up to $20 \%$ (Victor 1989; Thomson, Cook et al. 2002). The recommended $\mathrm{TH}$ doses range from 100 to $1500 \mathrm{mg}$ per day (Thomson, Cook et al. 2002; Sechi and Serra 2007). Within this range, the administration of higher rather than lower TH doses is favoured. The therapeutic scheme should be constantly adapted to the patient's evolution according to the best clinical judgement of the treating physician. Differences in the therapeutic response of alcoholics and non alcoholics have been reported, alcoholics requiring higher doses and for extended periods of time, some advising $500 \mathrm{mg}$ thrice a day as the minimum dose. Non alcoholics with WE may have adequate therapeutic response providing only 100 to $200 \mathrm{mg}$ of parenteral TH are supplied. According to the 2010 EFNS recommendations both alcoholics and non alcoholics should receive a minimum of $200 \mathrm{mg}$ thrice a day. In hyperemesis gravidarium related WE cases, patients requiring significantly higher TH doses have been reported (Thomson, Cook et al. 2002; Galvin, Brathen et al. 
2010). To the best of our knowledge, the pediatric parenteral $\mathrm{TH}$ doses have not been established. According to the current literature the TH deficient infants were treated with 50 mg of intramuscular TH hydrochloride for 14 days (Fattal-Valevski, Kesler et al. 2005). The opinions regarding the adequate treatment duration vary from a few days of intravenous high dose $\mathrm{TH}$ (followed by another few days of intramuscular lower doses) to the continuation of high dose intravenous $\mathrm{TH}$ administration until there is no further clinical improvement. The latter appears to be the most reasonable and is recommended by the 2010 EFNS guideline. Secondary prophylaxis with oral TH (30 mg twice a day) is advocated by some. Some suggest that in the absence of clinical improvement $\mathrm{TH}$ administration should be stopped after 2 or 3 days. Since certain patients may require prolonged treatment with doses above $500 \mathrm{mg}$ thrice a day, the decision to stop $\mathrm{TH}$ treatment in those that seem not to respond should be taken with caution (Sechi and Serra 2007; Paparrigopoulos, Tzavellas et al. 2010). Concomitant parenteral administration of other vitamins, especially of B complex and ascorbic acid, is advocated by some. Niacin should be administered whenever signs suggestive of pellagra exist. The available pharmaceutical preparates for parenteral administration contain variable quantities of $\mathrm{TH}$ hydrochloride alone or in combination with other vitamins, especially B complex. Parenteral TH hydrochloride is available for subcutaneous, intramuscular and intravenous injections. Lipid-soluble $\mathrm{TH}$ analogues preparates are not widely available (except for benfotiamine, which does not pass the BBB). Oral TH hydrochloride administration has very good tolerability, side effects being extremely rarely reported, and overdoses being virtually impossible (Baker and Frank 1976; Wrenn, Murphy et al. 1989; Kitamori and Itokawa 1993). Intravenous thiamine is not contraindicated in pregnant or lactating women. According to the current literature, the only serious and potentially life threatening side effects of $\mathrm{TH}$ administration are anaphylactic and anaphylactoid reactions (Thomson, Cook et al. 2002; Harper 2006). These have been reported to occur during or in the few hours following $\mathrm{TH}$ administration. They are only exceptionally encountered in relation with oral $\mathrm{TH}$ administration and most frequently encountered in relation with bolus intravenous $\mathrm{TH}$ administration (Thomson, Cook et al. 2002; Galvin, Brathen et al. 2010), but even so, parenteral TH administration has a very high safety profile. The global estimated incidence of the serious adverse reactions following parenteral administration of multivitamin pharmacological products containing $\mathrm{TH}$ was reported to be 1 in every 5 million intramuscular injections and 1 in every million intravenous injections, therefore lower than that of penicillin or streptokinase. Arguments for the existence of TH induced anaphylaxis have been provided in a few case reports, but it may be speculated that anaphylaxis occurs less frequently than anaphylactoid reactions. Sensitivity skin test prior to $\mathrm{TH}$ administration is not necessary. In order to minimise the risk of anaphylactoid reactions without minimising the benefits of intravenous administration, it is recommended that $\mathrm{TH}$ is administered over at least 10 minutes by intravenous infusion. Ideally, when administering parenteral $\mathrm{TH}$, rapid access to cardiopulmonary resuscitation facilities should be available (Luskin and Luskin 1996; Harper 2006; Galvin, Brathen et al. 2010). According to the 2010 EFNS guideline TH should be given to all patients with suspected or manifest WE, before any carbohydrate, intravenously (diluted in $100 \mathrm{ml}$ of normal saline or glucose solution; 30 minutes infusion) or intramuscularly, in doses of at least $200 \mathrm{mg}$ thrice a day, even in the absence of resuscitation facilities, until there is no further clinical improvement (level $C$ evidence). The early restoration of an adequate diet is advised (Galvin, Brathen et al. 2010). 


\section{Red flags for WE, red flags for TH}

In order to prevent irreversible brain damage and death, $\mathrm{TH}$ should be administered as soon as possible to all WE patients. However, even when best clinical management is provided, the diagnosis of WE may be overlooked. Evidence based recommendations for the identification of patients that should receive parenteral $\mathrm{TH}$ are not available, but, as discussed, criteria for the accurate identification of WE in alcoholics have been proposed in 1997 by Cain and in 2002 by the UK Royal College of Physicians. Since parenteral TH administration is reasonably cheap and safe, overtreatment is preferred, some advising that parenteral TH should be routinely administered until neurologic assessments are made and paraclinical data are available to all patients prone to $\mathrm{TH}$ deficiency presenting in the emergencies departments (even in the absence of other reasonable arguments for WE), and the 2010 EFNS guideline recommending that a low threshold for parenteral TH administration is maintained (Ogershok, Rahman et al. 2002; Thomson and Marshall 2006; Galvin, Brathen et al. 2010; Thorarinsson, Olafsson et al. 2011). Red flags for WE and for TH administration include prior history of WE, presence of any of the classical triad signs, chronic ethanol abuse, acute alcohol intoxication, delirium tremens, altered mental status, altered consciousness, hypothermia, hyperthermia, edema, tachycardia, hypotension, cardiac failure and lactic acidosis, persistent emesis, impaired nutrition (prolonged fasting, malnourishment, eating disorders and other psychiatric disorders, exclusive parenteral nutrition, infants fed by nutritionally impaired women, neglected pediatric, geriatric and disabled persons), recent significant weight loss, low body mass index (BMI), history of gastrointestinal surgery, consumptive diseases (including malignancies -especially digestive tract and in children acute leukemia, AIDS, malaria, tuberculosis, meningoencephalitis, thyreotoxicosis), chronic liver disease, chronic dialysis, hepatic and uremic encephalopathy, pregnancy and lactation (Lana-Peixoto, Dos Santos et al. 1992; Antunez, Estruch et al. 1998; Ihara, Ito et al. 1999; Vasconcelos, Silva et al. 1999; Bleggi-Torres, de Medeiros et al. 2000; Donnino, Vega et al. 2007; Sechi and Serra 2007). Special consideration regarding parenteral $\mathrm{TH}$ administration should receive all those on chronic diuretic drugs presenting for cardiac failure (Khan and Garg 2011).

\section{Prognosis}

The majority of the WE cases are cured (with or without sequelae) providing prompt and adequate treatment is administered. In the absence of adequate therapy, WE has high mortality and morbidity, resulting in death in up to $24 \%$ of the cases, and in permanent disability in 75 to $85 \%$ of the survivors (Thomson, Cook et al. 2002; Donnino, Vega et al. 2007; Thorarinsson, Olafsson et al. 2011). Alcoholics have worst prognosis than non alcoholics. Death or persistent sequelae have also been reported to occur in non alcoholic patients, especially in those with hyperemesis gravidarium (Sechi and Serra 2007). Those with fewer MRI lesion, and with lesions restricted to the periaqueductal grey area and thalami seem to have a good prognosis, while those with cortical MRI lesions seem to have a worse prognosis (Varnet, De Seze et al. 2002; Zuccoli, Santa Cruz et al. 2009). The most serious sequelae are gait disturbances and KS. Gait disturbances were reported to persist in $61 \%$ of the survivors. When present, KS was reported to persist for at least two years in 56 to $84 \%$ of the cases. Up to $26 \%$ of those with KS are severely disabled (Victor 1989; Caine, 
Halliday et al. 1997). In certain cases, KS related to alcohol consumption and TH deficiency may appear in the absence of an overt episode of WE or after an interval of time of apparent health, probably due to a precipitating factor that alters the equilibrium of a susceptible brain (Harper, Giles et al. 1986; Galvin, Brathen et al. 2010). Several authors denominate the sequelae of WE 'chronic WE', though they not the consequence of persistent TH deficiency (Harper, Giles et al. 1986; Kopelman, Thomson et al. 2009; Thorarinsson, Olafsson et al. 2011). A true chronic form of WE, consequence of the cumulative effects of several mild, subclinical, more or less reversible episodes of TH deficiency, might exist, especially in alcoholics (Galvin, Brathen et al. 2010).

\section{Prophylaxis}

Currently there are no evidence based recommendations for the primary or secondary prophylaxis of WE, but attempts for $\mathrm{TH}$ deficiency prophylaxis have been made either by fortifying food or alcoholic beverages with $\mathrm{TH}$ or by administering oral or parenteral $\mathrm{TH}$ to selected populations considered at risk. Their efficacy has not been thoroughly evaluated in controlled studies. The fortification of food with TH (commonly flour and rice, but also margarine, soft drinks and other products) is inexpensive and technically feasible. Empirical primary prophylactic approaches are currently implemented in several regions. The fortification of food with $\mathrm{TH}$ is legally mandatory in certain areas (i.e. Australia, UK, Denmark, Canada, Chile) and optional but reglemented by law in others (i.e. Switzerland, Brazil, Yemen). Several countries have the minimum content of flour TH established by law (WHO 1999). Commonly, TH mononitrate is used for the fortification of flower, rice and soft drinks. The supplementation of alcoholic beverages (beer and vine) is feasible, and has been proposed as it better targets those that take most of their caloric intake from alcoholic beverages. However, experimental data suggests that alcohol consumption and/or malnourishment lead to impaired $\mathrm{TH}$ and hydro-soluble $\mathrm{TH}$ analogues absorption and/or utilization, and therefore these prophylactic approaches may result in limited benefits. The clinical retrospective studies conducted in Australia 5 years after the legally imposed bread fortification with TH reported a 39\% decrease in the incidence of WE and a 15\% decrease in the prevalence of WE (acute and chronic cases reported as WE, Korsakoff psychosis, WKS) that maintained at similar levels since the first years, while the necroptic studies reported a decrease in the prevalence of WE from $2.1 \%$ to $1.1 \%$ (Rolland and Truswell 1998). The oral prophylactic administration of high doses of TH hydrochloride was shown not to prevent WE in malnourished obese patients on therapeutic fasting and in hunger strikers. In this respect it is recommended that in certain selected population the prophylaxis of WE should be made by parenteral TH administration. All alcoholics and malnourished individuals admitted to hospitals should receive 200 to $250 \mathrm{mg}$ of parenteral $\mathrm{TH}$ hydrochloride per day for 3 to 5 days. $200 \mathrm{mg}$ of parenteral TH should be administered prior to glucose infusion to all those prone to $\mathrm{TH}$ deficiency and in comatose and status epilepticus patients. Per os TH supplementation (at least $15 \mathrm{mg}$ per day, recommended $30 \mathrm{mg}$ twice a day) is advised for the secondary prophylaxis of WE. Patients receiving drugs that may deplete the $\mathrm{TH}$ stores or undergoing dialysis should also receive per os TH supplementation.(Thomson, Cook et al. 2002; Galvin, Brathen et al. 2010). 


\section{Summary}

WE Is a potentially fatal but highly and easily treatable disease occurring throughout the world. It is commonly (but not exclusively!) encountered in TH deficient alcoholic and/or malnourished susceptible individuals. The topography of the lesions is highly specific, commonly the perimidline regions being symmetrically involved. The classical clinical hallmark (sometimes completely absent!) consists of ocular motility signs, stance and/or gait ataxia and mental status changes having acute or subacute onset. No paraclinical investigation can accurately exclude the diagnosis of WE, however, the imagistic and laboratory workup is useful for supporting the diagnosis and excluding comorbidities. Though the necessity of prompt parenteral $\mathrm{TH}$ administration in all WE patients is unanimously accepted, evidence based recommendations for its therapeutic management are not available. The administration of high dose parenteral $\mathrm{TH}$ (recommended at least 200 mg thrice a day; NB! correction of hypomagnesaemia) should be initiated as soon as possible in all patients with suspected WE. Considering the deleterious medical and economical consequences of untreated $\mathrm{WE}$, and that parenteral $\mathrm{TH}$ is reasonably safe and cheap and has no contraindication except prior allergic reaction, high dose parenteral $\mathrm{TH}$ should be administered to all those presenting to the emergency departments with $\mathrm{TH}$ deficiency predisposing factors, ethanol intoxication or delirium tremens. The maintenance of a high index of suspicion for WE and a low threshold for parenteral TH administration is advocated (Thomson, Cook et al. 2002; Sechi and Serra 2007; Galvin, Brathen et al. 2010).

\section{References}

Aasheim, E. T. (2008). "Wernicke encephalopathy after bariatric surgery: a systematic review." Ann Surg 248(5): 714-720.

Adamolekun, B. (2010). "Etiology of Konzo, epidemic spastic paraparesis associated with cyanogenic glycosides in cassava: role of thiamine deficiency?" J Neurol Sci 296(12): 30-33.

Adamolekun, B. and F. R. Ibikunle (1994). "Investigation of an epidemic of seasonal ataxia in Ikare, western Nigeria." Acta Neurol Scand 90(5): 309-311.

Adamolekun, B. and D. A. Ndububa (1994). "Epidemiology and clinical presentation of a seasonal ataxia in western Nigeria." J Neurol Sci 124(1): 95-98.

Alston, T. A. and R. H. Abeles (1987). "Enzymatic conversion of the antibiotic metronidazole to an analog of thiamine." Arch Biochem Biophys 257(2): 357-362.

Antunez, E., R. Estruch, et al. (1998). "Usefulness of CT and MR imaging in the diagnosis of acute Wernicke's encephalopathy." AJR Am J Roentgenol 171(4): 1131-1137.

Arbelaez, A., A. Pajon, et al. (2003). "Acute Marchiafava-Bignami disease: MR findings in two patients." AJNR Am J Neuroradiol 24(10): 1955-1957.

Aupee, A. M., B. Desgranges, et al. (2001). "Voxel-based mapping of brain hypometabolism in permanent amnesia with PET." Neuroimage 13(6 Pt 1): 1164-1173.

Baker, H. and O. Frank (1976). "Absorption, utilization and clinical effectiveness of allithiamines compared to water-soluble thiamines." J Nutr Sci Vitaminol (Tokyo) 22 SUPPL: 63-68.

Basoglu, M., Y. Yetimalar, et al. (2006). "Neurological complications of prolonged hunger strike." Eur J Neurol 13(10): 1089-1097. 
Bertrand, A., J. P. Brandel, et al. (2009). "Wernicke encephalopathy and Creutzfeldt-Jakob disease." J Neurol 256(6): 904-909.

Bettendorff, L., G. Goessens, et al. (1997). "Reversibility of thiamine deficiency-induced partial necrosis and mitochondrial uncoupling by addition of thiamine to neuroblastoma cell suspensions." Mol Cell Biochem 174(1-2): 121-124.

Bleggi-Torres, L. F., B. C. de Medeiros, et al. (2000). "Neuropathological findings after bone marrow transplantation: an autopsy study of 180 cases." Bone Marrow Transplant 25(3): 301-307.

Bogousslavsky, J., F. Regli, et al. (1988). "Thalamic infarcts: clinical syndromes, etiology, and prognosis." Neurology 38(6): 837-848.

Boldorini, R., L. Vago, et al. (1992). "Wernicke's encephalopathy: occurrence and pathological aspects in a series of 400 AIDS patients." Acta Biomed Ateneo Parmense 63(1-2): 43-49.

Boulware, M. J., V. S. Subramanian, et al. (2003). "Polarized expression of members of the solute carrier SLC19A gene family of water-soluble multivitamin transporters: implications for physiological function." Biochem J 376(Pt 1): 43-48.

Brechtelsbauer, D. L., H. Urbach, et al. (1997). "Cytomegalovirus encephalitis and primary cerebral lymphoma mimicking Wernicke's encephalopathy." Neuroradiology 39(1): 19-22.

Brouns, R. and P. P. De Deyn (2004). "Neurological complications in renal failure: a review." Clin Neurol Neurosurg 107(1): 1-16.

Bujanda, L. (2000). "The effects of alcohol consumption upon the gastrointestinal tract." Am J Gastroenterol 95(12): 3374-3382.

Butterworth, R. F. (1986). "Cerebral thiamine-dependent enzyme changes in experimental Wernicke's encephalopathy." Metab Brain Dis 1(3): 165-175.

Butterworth, R. F. (1989). "Effects of thiamine deficiency on brain metabolism: implications for the pathogenesis of the Wernicke-Korsakoff syndrome." Alcohol Alcohol 24(4): 271-279.

Butterworth, R. F. (1993). "Pathophysiologic mechanisms responsible for the reversible (thiamine-responsive) and irreversible (thiamine non-responsive) neurological symptoms of Wernicke's encephalopathy." Drug Alcohol Rev 12(3): 315-322.

Butterworth, R. F., C. Gaudreau, et al. (1991). "Thiamine deficiency and Wernicke's encephalopathy in AIDS." Metab Brain Dis 6(4): 207-212.

Butterworth, R. F., J. J. Kril, et al. (1993). "Thiamine-dependent enzyme changes in the brains of alcoholics: relationship to the Wernicke-Korsakoff syndrome." Alcohol Clin Exp Res 17(5): 1084-1088.

Caine, D., G. M. Halliday, et al. (1997). "Operational criteria for the classification of chronic alcoholics: identification of Wernicke's encephalopathy." J Neurol Neurosurg Psychiatry 62(1): 51-60.

Calingasan, N. Y., W. J. Chun, et al. (1999). "Oxidative stress is associated with regionspecific neuronal death during thiamine deficiency." J Neuropathol Exp Neurol 58(9): 946-958.

Calingasan, N. Y. and G. E. Gibson (2000). "Vascular endothelium is a site of free radical production and inflammation in areas of neuronal loss in thiamine-deficient brain." Ann N Y Acad Sci 903: 353-356. 
Caulo, M., J. Van Hecke, et al. (2005). "Functional MRI study of diencephalic amnesia in Wernicke-Korsakoff syndrome." Brain 128(Pt 7): 1584-1594.

Chiossi, G., I. Neri, et al. (2006). "Hyperemesis gravidarum complicated by Wernicke encephalopathy: background, case report, and review of the literature." Obstet Gynecol Surv 61(4): 255-268.

Cho, I. J., H. J. Chang, et al. (2009). "A case of Wernicke's encephalopathy following fluorouracil-based chemotherapy." J Korean Med Sci 24(4): 747-750.

Chu, K., D. W. Kang, et al. (2002). "Diffusion-weighted imaging abnormalities in wernicke encephalopathy: reversible cytotoxic edema?" Arch Neurol 59(1): 123-127.

Chung, S. P., S. W. Kim, et al. (2003). "Magnetic resonance imaging as a diagnostic adjunct to Wernicke encephalopathy in the ED." Am J Emerg Med 21(6): 497-502.

Ciccia, R. M. and P. J. Langlais (2000). "An examination of the synergistic interaction of ethanol and thiamine deficiency in the development of neurological signs and longterm cognitive and memory impairments." Alcohol Clin Exp Res 24(5): 622-634.

Coy, J. F., S. Dubel, et al. (1996). "Molecular cloning of tissue-specific transcripts of a transketolase-related gene: implications for the evolution of new vertebrate genes." Genomics 32(3): 309-316.

Dallal, R. M. (2006). "Wernicke encephalopathy after bariatric surgery: losing more than just weight." Neurology 66(11): 1786.

Danbolt, N. C. (2001). "Glutamate uptake." Prog Neurobiol 65(1): 1-105.

Davtyan, D. G. and H. V. Vinters (1987). "Wernicke's encephalopathy in AIDS patient treated with zidovudine." Lancet 1(8538): 919-920.

De, W. H. and B. Lennox (1947). "Cerebral beriberi (Wernicke's encephalopathy); review of 52 cases in a Singapore prisoner-of-war hospital." Lancet 1(6436): 11-17.

Donnino, M. (2004). "Gastrointestinal beriberi: a previously unrecognized syndrome." Ann Intern Med 141(11): 898-899.

Donnino, M. W., J. Miller, et al. (2007). "Distinctive acid-base pattern in Wernicke's encephalopathy." Ann Emerg Med 50(6): 722-725.

Donnino, M. W., J. Vega, et al. (2007). "Myths and misconceptions of Wernicke's encephalopathy: what every emergency physician should know." Ann Emerg Med 50(6): 715-721.

Doss, A., D. Mahad, et al. (2003). "Wernicke encephalopathy: unusual findings in nonalcoholic patients." J Comput Assist Tomogr 27(2): 235-240.

Dudeja, P. K., S. Tyagi, et al. (2001). "Mechanism of thiamine uptake by human jejunal brush-border membrane vesicles." Am J Physiol Cell Physiol 281(3): C786-792.

Fattal-Valevski, A. (2011). "Thiamine (Vitamin B1)." Journal of Evidence-Based Complementary\&Alternative Medicine I(16): 12-20.

Fattal-Valevski, A., A. Kesler, et al. (2005). "Outbreak of life-threatening thiamine deficiency in infants in Israel caused by a defective soy-based formula." Pediatrics 115(2): e233-238.

Feeney, G. F. and J. P. Connor (2008). "Wernicke-Korsakoff syndrome (WKS) in Australia: no room for complacency." Drug Alcohol Rev 27(4): 388-392.

Fehily, L. (1944). "Human-milk Intoxication due to B1 Avitaminosis." Br Med J 2(4374): 590592.

Fei, G. Q., C. Zhong, et al. (2008). "Clinical characteristics and MR imaging features of nonalcoholic Wernicke encephalopathy." AJNR Am J Neuroradiol 29(1): 164-169. 
Fellgiebel, A., A. Scheurich, et al. (2003). "Persistence of disturbed thalamic glucose metabolism in a case of Wernicke-Korsakoff syndrome." Psychiatry Res 124(2): 105112.

Flink, E. B. (1978). "Role of magnesium depletion in Wernicke-Korsakoff syndrome." N Engl J Med 298(13): 743-744.

Foresti, V. and F. Confalonieri (1987). "Wernicke's encephalopathy in AIDS." Lancet 1(8548): 1499.

Foster, D., M. Falah, et al. (2005). "Wernicke encephalopathy after bariatric surgery: losing more than just weight." Neurology 65(12): 1987; discussion 1847.

Fournier, H. and R. F. Butterworth (1989). "Effects of maternal thiamine deficiency on the development of thiamine-dependent enzymes in regions of the rat brain." Neurochem Int 15(4): 439-444.

Gallucci, M., A. Bozzao, et al. (1990). "Wernicke encephalopathy: MR findings in five patients." AJNR Am J Neuroradiol 11(5): 887-892.

Galvin, R., G. Brathen, et al. (2010). "EFNS guidelines for diagnosis, therapy and prevention of Wernicke encephalopathy." Eur J Neurol 17(12): 1408-1418.

Ghika-Schmid, F. and J. Bogousslavsky (2000). "The acute behavioral syndrome of anterior thalamic infarction: a prospective study of 12 cases." Ann Neurol 48(2): 220-227.

Guerrini, I., A. D. Thomson, et al. (2005). "Direct genomic PCR sequencing of the high affinity thiamine transporter (SLC19A2) gene identifies three genetic variants in Wernicke Korsakoff syndrome (WKS)." Am J Med Genet B Neuropsychiatr Genet 137B(1): 17-19.

Hahn, J. S., W. Berquist, et al. (1998). "Wernicke encephalopathy and beriberi during total parenteral nutrition attributable to multivitamin infusion shortage." Pediatrics 101(1): E10.

Halavaara, J., A. Brander, et al. (2003). "Wernicke's encephalopathy: is diffusion-weighted MRI useful?" Neuroradiology 45(8): 519-523.

Hamadani, M. and F. Awan (2006). "Role of thiamine in managing ifosfamide-induced encephalopathy." J Oncol Pharm Pract 12(4): 237-239.

Harata, N. and Y. Iwasaki (1995). "Evidence for early blood-brain barrier breakdown in experimental thiamine deficiency in the mouse." Metab Brain Dis 10(2): 159-174.

Harper, C. (1979). "Wernicke's encephalopathy: a more common disease than realised. A neuropathological study of 51 cases." J Neurol Neurosurg Psychiatry 42(3): 226-231.

Harper, C. (1983). "The incidence of Wernicke's encephalopathy in Australia--a neuropathological study of 131 cases." J Neurol Neurosurg Psychiatry 46(7): 593598.

Harper, C. (2006). "Thiamine (vitamin B1) deficiency and associated brain damage is still common throughout the world and prevention is simple and safe!" Eur J Neurol 13(10): 1078-1082.

Harper, C. (2009). "The neuropathology of alcohol-related brain damage." Alcohol Alcohol 44(2): 136-140.

Harper, C., J. Gold, et al. (1989). "The prevalence of the Wernicke-Korsakoff syndrome in Sydney, Australia: a prospective necropsy study." J Neurol Neurosurg Psychiatry 52(2): 282-285.

Harper, C., M. Rodriguez, et al. (1988). "The Wernicke-Korsakoff syndrome in Sydney--a prospective necropsy study." Med J Aust 149(11-12): 718, 720. 
Harper, C. G., M. Giles, et al. (1986). "Clinical signs in the Wernicke-Korsakoff complex: a retrospective analysis of 131 cases diagnosed at necropsy." J Neurol Neurosurg Psychiatry 49(4): 341-345.

Harper, C. G. and J. J. Kril (1990). "Neuropathology of alcoholism." Alcohol Alcohol 25(2-3): 207-216.

Hazell, A. S. (2009). "Astrocytes are a major target in thiamine deficiency and Wernicke's encephalopathy." Neurochem Int 55(1-3): 129-135.

Hazell, A. S. and R. F. Butterworth (2009). "Update of cell damage mechanisms in thiamine deficiency: focus on oxidative stress, excitotoxicity and inflammation." Alcohol Alcohol 44(2): 141-147.

Hazell, A. S., R. F. Butterworth, et al. (1993). "Cerebral vulnerability is associated with selective increase in extracellular glutamate concentration in experimental thiamine deficiency." J Neurochem 61(3): 1155.

Hazell, A. S., K. V. Rao, et al. (2001). "Selective down-regulation of the astrocyte glutamate transporters GLT-1 and GLAST within the medial thalamus in experimental Wernicke's encephalopathy." J Neurochem 78(3): 560-568.

Heinrich, C. P., H. Stadler, et al. (1973). "The effect of thiamine deficiency on the acetylcoenzyme A and acetylcholine levels in the rat brain." J Neurochem 21(5): 1273-1281.

Heroux, M. and R. F. Butterworth (1988). "Reversible alterations of cerebral gammaaminobutyric acid in pyrithiamine-treated rats: implications for the pathogenesis of Wernicke's encephalopathy." J Neurochem 51(4): 1221-1226.

Herve, C., P. Beyne, et al. (1994). "Determination of thiamine and its phosphate esters in human erythrocytes by high-performance liquid chromatography with isocratic elution." J Chromatogr B Biomed Appl 653(2): 217-220.

Hoyumpa, A. M., Jr. (1980). "Mechanisms of thiamin deficiency in chronic alcoholism." Am J Clin Nutr 33(12): 2750-2761.

Hutchin, K. C. (1987). "Thiamine deficiency, Wernicke's encephalopathy, and AIDS." Lancet 1(8543): 1200.

Ihara, M., T. Ito, et al. (1999). "Wernicke's encephalopathy associated with hemodialysis: report of two cases and review of the literature." Clin Neurol Neurosurg 101(2): 118-121.

Indraccolo, U., G. Gentile, et al. (2005). "Thiamine deficiency and beriberi features in a patient with hyperemesis gravidarum." Nutrition 21(9): 967-968.

Ishii, K., K. Sarai, et al. (1979). "Concentrations of thiamine and its phosphate esters in rat tissues determined by high-performance liquid chromatography." J Nutr Sci Vitaminol (Tokyo) 25(6): 517-523.

Jamieson, C. P., O. A. Obeid, et al. (1999). "The thiamin, riboflavin and pyridoxine status of patients on emergency admission to hospital." Clin Nutr 18(2): 87-91.

Joyce, E. M., D. E. Rio, et al. (1994). "Decreased cingulate and precuneate glucose utilization in alcoholic Korsakoff's syndrome." Psychiatry Res 54(3): 225-239.

Khan, A. and P. Garg (2011). "Acute decompensated heart failure secondary to thiamine deficiency: often a missed diagnosis." Clin Med 11(2): 203; author reply 203-204.

Kim, E., J. Ku, et al. (2009). "Mammillothalamic functional connectivity and memory function in Wernicke's encephalopathy." Brain 132(Pt 2): 369-376. 
Kimelberg, H. K., S. K. Goderie, et al. (1990). "Swelling-induced release of glutamate, aspartate, and taurine from astrocyte cultures." J Neurosci 10(5): 1583-1591.

Kimelberg, H. K., E. Rutledge, et al. (1995). "Astrocytic swelling due to hypotonic or high K+ medium causes inhibition of glutamate and aspartate uptake and increases their release." J Cereb Blood Flow Metab 15(3): 409-416.

Kitamori, N. and Y. Itokawa (1993). "Pharmacokinetics of thiamin after oral administration of thiamin tetrahydrofurfuryl disulfide to humans." J Nutr Sci Vitaminol (Tokyo) 39(5): 465-472.

Kondo, K., M. Fujiwara, et al. (1996). "Severe acute metabolic acidosis and Wernicke's encephalopathy following chemotherapy with 5-fluorouracil and cisplatin: case report and review of the literature." Jpn J Clin Oncol 26(4): 234-236.

Kono, S., H. Miyajima, et al. (2009). "Mutations in a thiamine-transporter gene and Wernicke's-like encephalopathy." N Engl J Med 360(17): 1792-1794.

Kopelman, M. D. (1995). "The Korsakoff syndrome." Br J Psychiatry 166(2): 154-173.

Kopelman, M. D., A. D. Thomson, et al. (2009). "The Korsakoff syndrome: clinical aspects, psychology and treatment." Alcohol Alcohol 44(2): 148-154.

Laforenza, U., C. Patrini, et al. (1990). "Effects of acute and chronic ethanol administration on thiamine metabolizing enzymes in some brain areas and in other organs of the rat." Alcohol Alcohol 25(6): 591-603.

Lana-Peixoto, M. A., E. C. Dos Santos, et al. (1992). "Coma and death in unrecognized Wernicke's encephalopathy. An autopsy study." Arq Neuropsiquiatr 50(3): 329-333.

Langlais, P. J. and S. X. Zhang (1993). "Extracellular glutamate is increased in thalamus during thiamine deficiency-induced lesions and is blocked by MK-801." J Neurochem 61(6): 2175-2182.

Lapergue, B., I. Klein, et al. (2006). "Diffusion weighted imaging of cerebellar lesions in Wernicke's encephalopathy." J Neuroradiol 33(2): 126-128.

Lee, B. L., H. Y. Ong, et al. (1991). "Determination of thiamine and its phosphate esters by gradient-elution high-performance liquid chromatography." J Chromatogr 567(1): 71-80.

Lee, S. T., Y. M. Jung, et al. (2005). "Corpus callosum atrophy in Wernicke's encephalopathy." J Neuroimaging 15(4): 367-372.

Leevy, C. M., L. Cardi, et al. (1965). "Incidence and significance of hypovitaminemia in a randomly selected municipal hospital population." Am J Clin Nutr 17(4): 259-271.

Lemos, C., I. Azevedo, et al. (2005). "Effect of red wine on the intestinal absorption of thiamine and folate in the rat: comparison with the effect of ethanol alone." Alcohol Clin Exp Res 29(4): 664-671.

Li, J. M. and J. C. Rucker (2010). "Irreversible optic neuropathy in wernicke encephalopathy and leber hereditary optic neuropathy." J Neuroophthalmol 30(1): 49-53.

Liu, Y. T., J. L. Fuh, et al. (2006). "Correlation of magnetic resonance images with neuropathology in acute Wernicke's encephalopathy." Clin Neurol Neurosurg 108(7): 682-687.

Loh, Y., W. D. Watson, et al. (2005). "Restricted diffusion of the splenium in acute Wernicke's encephalopathy." J Neuroimaging 15(4): 373-375.

Luskin, A. T. and S. S. Luskin (1996). "Anaphylaxis and Anaphylactoid Reactions: Diagnosis and Management." Am J Ther 3(7): 515-520. 
Macias-Matos, C., A. Rodriguez-Ojea, et al. (1996). "Biochemical evidence of thiamine depletion during the Cuban neuropathy epidemic, 1992-1993." Am J Clin Nutr 64(3): 347-353.

Mair, W. G., E. K. Warrington, et al. (1979). "Memory disorder in Korsakoff's psychosis: a neuropathological and neuropsychological investigation of two cases." Brain 102(4): 749-783.

Makarchikov, A. F., B. Lakaye, et al. (2003). "Thiamine triphosphate and thiamine triphosphatase activities: from bacteria to mammals." Cell Mol Life Sci 60(7): 14771488.

Martin, P. R., C. K. Singleton, et al. (2003). "The role of thiamine deficiency in alcoholic brain disease." Alcohol Res Health 27(2): 134-142.

McCool, B. A., S. G. Plonk, et al. (1993). "Cloning of human transketolase cDNAs and comparison of the nucleotide sequence of the coding region in Wernicke-Korsakoff and non-Wernicke-Korsakoff individuals." J Biol Chem 268(2): 1397-1404.

Michowitz, Y., L. Copel, et al. (2005). "Non-alcoholic Wernicke's encephalopathy - unusual clinical findings." Eur J Intern Med 16(6): 443-444.

Miyajima, H. and S. Kono (2010). "Familial Wernicke's-like encephalopathy." Rinsho Shinkeigaku 50(11): 855-857.

Morishima, T., M. Aoyama, et al. (2008). "Lactic acid increases aquaporin 4 expression on the cell membrane of cultured rat astrocytes." Neurosci Res 61(1): 18-26.

Mukherjee, A. B., S. Svoronos, et al. (1987). "Transketolase abnormality in cultured fibroblasts from familial chronic alcoholic men and their male offspring." J Clin Invest 79(4): 1039-1043.

Nishimune, T., Y. Watanabe, et al. (2000). "Thiamin is decomposed due to Anaphe spp. entomophagy in seasonal ataxia patients in Nigeria." J Nutr 130(6): 1625-1628.

Nixon, P. F. (2008). "Glutamate export at the choroid plexus in health, thiamin deficiency, and ethanol intoxication: review and hypothesis." Alcohol Clin Exp Res 32(8): 13391349.

Nixon, P. F., L. Jordan, et al. (2008). "Choroid plexus dysfunction: the initial event in the pathogenesis of Wernicke's encephalopathy and ethanol intoxication." Alcohol Clin Exp Res 32(8): 1513-1523.

Novak, D. J. and M. Victor (1974). "The vagus and sympathetic nerves in alcoholic polyneuropathy." Arch Neurol 30(4): 273-284.

Ogershok, P. R., A. Rahman, et al. (2002). "Wernicke encephalopathy in nonalcoholic patients." Am J Med Sci 323(2): 107-111.

Osuntokun, B. O., A. Aladetoyinbo, et al. (1985). "Vitamin B nutrition in the Nigerian tropical ataxic neuropathy." J Neurol Neurosurg Psychiatry 48(2): 154-156.

Otsuka, F., K. Tada, et al. (1997). "Gestational thyrotoxicosis manifesting as wernicke encephalopathy: a case report." Endocr J 44(3): 447-452.

Pacal, L., J. Tomandl, et al. (2011). "Role of thiamine status and genetic variability in transketolase and other pentose phosphate cycle enzymes in the progression of diabetic nephropathy." Nephrol Dial Transplant 26(4): 1229-1236.

Pannunzio, P., A. S. Hazell, et al. (2000). "Thiamine deficiency results in metabolic acidosis and energy failure in cerebellar granule cells: an in vitro model for the study of cell death mechanisms in Wernicke's encephalopathy." J Neurosci Res 62(2): 286-292. 
Paparrigopoulos, T., E. Tzavellas, et al. (2010). "Complete recovery from undertreated Wernicke-Korsakoff syndrome following aggressive thiamine treatment." In Vivo 24(2): 231-233.

Park, S. H., M. Kim, et al. (2001). "Magnetic resonance reflects the pathological evolution of Wernicke encephalopathy." J Neuroimaging 11(4): 406-411.

Pearce, J. M. (2008). "Wernicke-Korsakoff encephalopathy." Eur Neurol 59(1-2): 101-104.

Pekovich, S. R., P. R. Martin, et al. (1998). "Thiamine deficiency decreases steady-state transketolase and pyruvate dehydrogenase but not alpha-ketoglutarate dehydrogenase mRNA levels in three human cell types." J Nutr 128(4): 683-687.

Phillips, G. B., M. Victor, et al. (1952). "A study of the nutritional defect in Wernicke's syndrome; the effect of a purified diet, thiamine, and other vitamins on the clinical manifestations." J Clin Invest 31(10): 859.

Pittock, S. J., J. Debruyne, et al. (2010). "Chronic lymphocytic inflammation with pontine perivascular enhancement responsive to steroids (CLIPPERS)." Brain 133(9): 26262634.

Ramayya, A. and P. Jauhar (1997). "Increasing incidence of Korsakoff's psychosis in the east end of Glasgow." Alcohol Alcohol 32(3): 281-285.

Rao, V. L., D. D. Mousseau, et al. (1996). "Nitric oxide synthase activities are selectively decreased in vulnerable brain regions in thiamine deficiency." Neurosci Lett 208(1): $17-20$.

Rolland, S. and A. S. Truswell (1998). "Wernicke-Korsakoff syndrome in Sydney hospitals after 6 years of thiamin enrichment of bread." Public Health Nutr 1(2): 117-122.

Rosenblum, W. I. and I. Feigin (1965). "The hemorrhagic component of Wernicke's encephalopathy." Arch Neurol 13(6): 627-632.

Rotman, P., D. Hassin, et al. (1994). "Wernicke's encephalopathy in hyperemesis gravidarum: association with abnormal liver function." Isr J Med Sci 30(3): 225-228.

Said, H. M., K. Balamurugan, et al. (2004). "Expression and functional contribution of hTHTR-2 in thiamin absorption in human intestine." Am J Physiol Gastrointest Liver Physiol 286(3): G491-498.

Schroth, G., W. Wichmann, et al. (1991). "Blood-brain-barrier disruption in acute Wernicke encephalopathy: MR findings." J Comput Assist Tomogr 15(6): 1059-1061.

Sechi, G. and A. Serra (2007). "Wernicke's encephalopathy: new clinical settings and recent advances in diagnosis and management." Lancet Neurol 6(5): 442-455.

Serra, A., G. Sechi, et al. (2007). "Wernicke encephalopathy after obesity surgery: a systematic review." Neurology 69(6): 615; author reply 615-616.

Shikata, E., T. Mizutani, et al. (2000). "'Iatrogenic' Wernicke's encephalopathy in Japan." Eur Neurol 44(3): 156-161.

Singh, S. and A. Kumar (2007). "Wernicke encephalopathy after obesity surgery: a systematic review." Neurology 68(11): 807-811.

Slekar, K. H., D. J. Kosman, et al. (1996). "The yeast copper/zinc superoxide dismutase and the pentose phosphate pathway play overlapping roles in oxidative stress protection." J Biol Chem 271(46): 28831-28836.

Smith, J. A., P. P. Foa, et al. (1947). "Some toxic effects of thiamine." Fed Proc 6(1 Pt 2): 204.

Subramanian, V. S., J. S. Marchant, et al. (2003). "Cell biology of the human thiamine transporter-1 (hTHTR1). Intracellular trafficking and membrane targeting mechanisms." J Biol Chem 278(6): 3976-3984. 
Subramanian, V. S., J. S. Marchant, et al. (2006). "Targeting and trafficking of the human thiamine transporter-2 in epithelial cells." J Biol Chem 281(8): 5233-5245.

Subramanya, S. B., V. S. Subramanian, et al. (2010). "Chronic alcohol consumption and intestinal thiamin absorption: effects on physiological and molecular parameters of the uptake process." Am J Physiol Gastrointest Liver Physiol 299(1): G23-31.

Sullivan, E. V. and A. Pfefferbaum (2009). "Neuroimaging of the Wernicke-Korsakoff syndrome." Alcohol Alcohol 44(2): 155-165.

Sun, G. H., Y. S. Yang, et al. (2006). "Pancreatic encephalopathy and Wernicke encephalopathy in association with acute pancreatitis: a clinical study." World J Gastroenterol 12(26): 4224-4227.

Surges, R., S. Beck, et al. (2007). "Sudden bilateral blindness in Wernicke's encephalopathy: case report and review of the literature." J Neurol Sci 260(1-2): 261-264.

Szmulewicz, D. J., J. A. Waterston, et al. (2011). "Sensory neuropathy as part of the cerebellar ataxia neuropathy vestibular areflexia syndrome." Neurology 76(22): 1903-1910

Tallaksen, C. M., H. Bell, et al. (1993). "Thiamin and thiamin phosphate ester deficiency assessed by high performance liquid chromatography in four clinical cases of Wernicke encephalopathy." Alcohol Clin Exp Res 17(3): 712-716.

Tallaksen, C. M., T. Bohmer, et al. (1991). "Concomitant determination of thiamin and its phosphate esters in human blood and serum by high-performance liquid chromatography." J Chromatogr 564(1): 127-136.

Tallaksen, C. M., A. Sande, et al. (1993). "Kinetics of thiamin and thiamin phosphate esters in human blood, plasma and urine after $50 \mathrm{mg}$ intravenously or orally." Eur J Clin Pharmacol 44(1): 73-78.

Talwar, D., H. Davidson, et al. (2000). "Vitamin B(1) status assessed by direct measurement of thiamin pyrophosphate in erythrocytes or whole blood by HPLC: comparison with erythrocyte transketolase activation assay." Clin Chem 46(5): 704-710.

Tanasescu, R. (2009). "Wernicke's Encephalopathy In General Neurological Practice: Short Considerations On The Need For Revision (I)." Romanian Journal of Neurology VIII(3): 3.

Thomson, A. D. (2000). "Mechanisms of vitamin deficiency in chronic alcohol misusers and the development of the Wernicke-Korsakoff syndrome." Alcohol Alcohol Suppl 35(1): 2-7.

Thomson, A. D., C. C. Cook, et al. (2008). "Wernicke's encephalopathy: 'Plus ca change, plus c'est la meme chose'." Alcohol Alcohol 43(2): 180-186.

Thomson, A. D., C. C. Cook, et al. (2002). "The Royal College of Physicians report on alcohol: guidelines for managing Wernicke's encephalopathy in the accident and Emergency Department." Alcohol Alcohol 37(6): 513-521.

Thomson, A. D., C. C. Cook, et al. (2008). "Wernicke's encephalopathy revisited. Translation of the case history section of the original manuscript by Carl Wernicke 'Lehrbuch der Gehirnkrankheiten fur Aerzte and Studirende' (1881) with a commentary." Alcohol Alcohol 43(2): 174-179.

Thomson, A. D. and E. J. Marshall (2006). "The natural history and pathophysiology of Wernicke's Encephalopathy and Korsakoff's Psychosis." Alcohol Alcohol 41(2): 151158.

Thomson, A. D. and E. J. Marshall (2006). "The treatment of patients at risk of developing Wernicke's encephalopathy in the community." Alcohol Alcohol 41(2): 159-167. 
Thorarinsson, B. L., E. Olafsson, et al. (2011). "[Wernicke's encephalopathy in chronic alcoholics]." Laeknabladid 97(1): 21-29.

Todd, K. G. and R. F. Butterworth (1998). "Evaluation of the role of NMDA-mediated excitotoxicity in the selective neuronal loss in experimental Wernicke encephalopathy." Exp Neurol 149(1): 130-138.

Todd, K. G., A. S. Hazell, et al. (1999). "Alcohol-thiamine interactions: an update on the pathogenesis of Wernicke encephalopathy." Addict Biol 4(3): 261-272.

Torgovnick, J., E. L. Arsura, et al. (2000). "Cytomegalovirus ventriculoencephalitis presenting as a Wernicke's encephalopathy-like syndrome." Neurology 55(12): 1910-1913.

Torvik, A. (1991). "Wernicke's encephalopathy--prevalence and clinical spectrum." Alcohol Alcohol Suppl 1: 381-384.

Traviesa, D. C. (1974). "Magnesium deficiency: a possible cause of thiamine refractoriness in Wernicke-Korsakoff encephalopathy." J Neurol Neurosurg Psychiatry 37(8): 959962.

Ueda, Y., H. Utsunomiya, et al. (2007). "[Wernicke encephalopathy in a chronic peritoneal dialysis patient--correlation between diffusion MR and pathological findings]." No To Hattatsu 39(3): 210-213.

Unlu, E., B. Cakir, et al. (2006). "MRI findings of Wernicke encephalopathy revisited due to hunger strike." Eur J Radiol 57(1): 43-53.

Van Belle, S. J., W. Distelmans, et al. (1993). "Phase I trial of erbulozole (R55104)." Anticancer Res 13(6B): 2389-2391.

Varnet, O., J. De Seze, et al. (2002). "[Wernicke-Korsakoff syndrome: diagnostic contribution of magnetic resonance imaging]." Rev Neurol (Paris) 158(12): 1181-1185.

Vasconcelos, M. M., K. P. Silva, et al. (1999). "Early diagnosis of pediatric Wernicke's encephalopathy." Pediatr Neurol 20(4): 289-294.

Victor, M., R. D. Adams, et al. (1971). "The Wernicke-Korsakoff syndrome. A clinical and pathological study of 245 patients, 82 with post-mortem examinations." Contemp Neurol Ser 7: 1-206.

Victor, M., Adams, K. M., and Collins, G. H. (1989). "The Wernicke-Korsakoff Syndrome and Related Disorders due to Alcoholism and Malnutrition."

Vortmeyer, A. O., C. Hagel, et al. (1992). "Haemorrhagic thiamine deficient encephalopathy following prolonged parenteral nutrition." J Neurol Neurosurg Psychiatry 55(9): 826-829.

Watanabe, I. (1978). "Pyrithiamine-induced acute thiamine-deficient encephalopathy in the mouse." Exp Mol Pathol 28(3): 381-394.

Watson, A. J., J. F. Walker, et al. (1981). "Acute Wernickes encephalopathy precipitated by glucose loading." Ir J Med Sci 150(10): 301-303.

Weidauer, S., M. Nichtweiss, et al. (2003). "Wernicke encephalopathy: MR findings and clinical presentation." Eur Radiol 13(5): 1001-1009.

WHO (1999). "Thiamine deficiency and its prevention and control in major emergencies". http://www.who.int/nutrition/publications/emergencies/WHO_NHD_99.13/en /index.html

WHO (version 2010). "International Statistical Classification of Diseases and Related Health Problems 10th Revision". http://apps.who.int/classifications/icd10/browse/2010/en 
Winston, A. P., C. P. Jamieson, et al. (2000). "Prevalence of thiamin deficiency in anorexia nervosa." Int J Eat Disord 28(4): 451-454.

Witt, E. D. (1985). "Neuroanatomical consequences of thiamine deficiency: a comparative analysis." Alcohol Alcohol 20(2): 201-221.

Wood, B., J. Currie, et al. (1986). "Wernicke's encephalopathy in a metropolitan hospital. A prospective study of incidence, characteristics and outcome." Med J Aust 144(1): 1216.

Wrenn, K. D., F. Murphy, et al. (1989). "A toxicity study of parenteral thiamine hydrochloride." Ann Emerg Med 18(8): 867-870.

Yoneoka, Y., N. Takeda, et al. (2004). "Acute Korsakoff syndrome following mammillothalamic tract infarction." AJNR Am J Neuroradiol 25(6): 964-968.

Zhao, R., F. Gao, et al. (2002). "Reduced folate carrier transports thiamine monophosphate: an alternative route for thiamine delivery into mammalian cells." Am J Physiol Cell Physiol 282(6): C1512-1517.

Zhao, Y., X. Pan, et al. (2009). "Decreased transketolase activity contributes to impaired hippocampal neurogenesis induced by thiamine deficiency." J Neurochem 111(2): 537-546.

Zhong, C., L. Jin, et al. (2005). "MR Imaging of nonalcoholic Wernicke encephalopathy: a follow-up study." AJNR Am J Neuroradiol 26(9): 2301-2305.

Zuccoli, G., I. Cravo, et al. (2011). "Basal Ganglia involvement in wernicke encephalopathy: report of 2 cases." AJNR Am J Neuroradiol 32(7): E129-131.

Zuccoli, G., M. Gallucci, et al. (2007). "Wernicke encephalopathy: MR findings at clinical presentation in twenty-six alcoholic and nonalcoholic patients." AJNR Am J Neuroradiol 28(7): 1328-1331.

Zuccoli, G. and L. Motti (2008). "Atypical Wernicke's encephalopathy showing lesions in the cranial nerve nuclei and cerebellum." J Neuroimaging 18(2): 194-197.

Zuccoli, G., N. Pipitone, et al. (2008). "Metronidazole-induced and Wernicke encephalopathy: two different entities sharing the same metabolic pathway?" AJNR Am J Neuroradiol 29(9): E84; author reply.

Zuccoli, G., D. Santa Cruz, et al. (2009). "MR imaging findings in 56 patients with Wernicke encephalopathy: nonalcoholics may differ from alcoholics." AJNR Am J Neuroradiol 30(1): 171-176.

Zuccoli, G., N. Siddiqui, et al. (2010). "Neuroimaging findings in pediatric Wernicke encephalopathy: a review." Neuroradiology 52(6): 523-529. 


\title{
L-carnitine in Hyperammonemia and Hepatic Encephalopathy
}

\author{
Jane Missler and Claudia Zwingmann \\ Centre de Recherche, de l'Université de Montréal (CHUM), Hôpital Saint-Luc \\ Montreal, Quebec, \\ Canada
}

\section{Introduction}

L-carnitine is suggested as a potential therapeutic agent in metabolic encephalopathies, such as ammonia-precipitated hepatic encephalopathy (HE). In humans, ammonia is produced mainly by the metabolism of proteins and amino acids. Most of the ammonia is detoxified by the urea cycle which is located in the liver. Thus, disturbances of the urea cycle in the liver lead to higher ammonia levels in blood. In humans, hyperammonemia (HA) arises mainly from liver diseases and is in most cases associated with HE. There are currently only a few therapeutic strategies, and outcome expectations for patients with HE are poor. Lcarnitine and its acyl-derivates are suggested as potential and low-cost therapeutic agents. In animal models, L-carnitine has been shown to counteract neurotoxic effects of ammonia, increase energy metabolism and decrease mortality. A protective effect against ammoniaprecipitated HE has also been observed in cirrhotic patients. A review of the literature offers in vitro and in vivo studies, as well as human trials with promising results. This chapter provides a comprehensive summary and a summarized discussion of the literature data concerning the effects of L-carnitine, with special emphasis on hyperammonemic syndromes and HE.

L-carnitine is essential for the transfer of long-chain fatty acids from the cytoplasm to the inner mitochondrial membrane, thereby facilitating mitochondrial energy metabolism. By virtue of their metabolic functions and neurophysiological roles, L-carnitine and its acetylated derivates are suggested as a therapeutic agent in several neurological disorders, including HE. In the brain, they have important roles in cerebral bioenergetics and neuroprotection through a variety of mechanisms including their antioxidant properties by modulation and promotion of synaptic neurotransmission. However, questions remain as to its systemic vs. cerebral effects, its relative effects on astrocytes and neurons, and its clinical use.

High-resolution Nuclear Magnetic Resonance (NMR) approaches using stable isotopes are now yielding large amounts of detailed information on cerebral metabolic compartmentalization and neuronal-glia interactions (Zwingmann \& Leibfritz, 2003). In order to investigate the effect of L-carnitine on energy- and fatty acid metabolism, ${ }^{1} \mathrm{H}$ - and ${ }^{13} \mathrm{C}-\mathrm{NMR}$ spectroscopy was used to measure metabolic pathways in brain and muscle 
following administration of [U-13C]glucose to rats with mild $\mathrm{HE}$ and in rats with ammoniaprecipitated encephalopathy. As a model of mild $\mathrm{HE}$, we investigated rats four weeks after an end-to-side portacaval anastomosis (PCA). PCA in the rat resulted in a broad spectrum of neurobehavioral changes, such as an $80 \%$ impaired locomotor activity, which was largely prevented by L-carnitine. Concomitantly, L-carnitine improved mitochondrial energy metabolism in brain and muscle. In ammonia-precipitated encephalopathy, L-carnitine considerably delayed the rats' time to coma, concomitantly with a prevention of ammoniainduced increased lactate synthesis in the brain. These results demonstrate that in HE, Lcarnitine acts both in the brain and in the muscle not by improving ammonia removal but by improving mitochondrial metabolism. These mechanisms explain L-carnitine's therapeutic benefit in the prevention of mild HE and ammonia-precipitated encephalopathy in cirrhotic patients. Since it is a low-cost agent with few side effects, further clinical trials could be promising in evaluating the broader use of L-carnitine and derivatives in patients with HE.

\section{Hyperammonemia}

The gut bacterial flora are responsible for the production of ammonia, the end-product of bacterial protein metabolism. The proteins that are metabolized by bacteria originate from exogenous alimentary sources (e.g. meat) or from endogenous sources (e.g. gastro-intestinal hemorrhage) (Hoyumpa et al., 1979). After ammonia is generated, ammonia can be detoxified by the formation of amino acids in the liver or in extrahepatic organs. One important mechanism is the synthesis of glutamine from glutamate and ammonia in the muscle (Fig. 1). The glutamine derived from muscle can be used as an energy substrate in the gut and is also transported to the kidney, where it is hydrolyzed to glutamate and ammonia. In the kidney, ammonia is detoxified by urea synthesis, which is released by the urine. Portal vein ammonia is present at a very high concentration. It is transported through the portal circulation to the liver, where it is normally converted to urea through the urea cycle and subsequently released by the kidney (McDermott, 1957). In liver diseases, the normal ammonia detoxification mechanisms may be compromised, and excess ammonia enters the brain. Furthermore, as it can occur in chronic cirrhosis or construction of a portacaval shunt, the shunting of the blood passing the liver will greatly increase systemic ammonia concentrations, which may then enter the central nervous system (CNS) (Butterworth et al., 1987; Lockwood et al., 1979). Since the brain lacks an effective urea cycle, basically the only mechanism to detoxify ammonia is conversion to glutamine through glutamine synthetase (GS). In the CNS, GS is localized almost exclusively in the astrocytes (Norenberg \& Martinez-Hernandez, 1979).

The metabolic conversion system that leads to the production of urea is highly efficient, and under healthy conditions it is unlikely to become hyperammonemic. HA is not a true disease; it is a sign that specific abnormalities, e.g. urea cycle disorders and liver diseases, that cause blood ammonia levels to become elevated may be present.

HA is considered a key factor in HE, which is defined as a spectrum of neuropsychiatric abnormalities in patients with liver dysfunction, after other known brain disease has been ruled out. In the brain, high ammonia levels modify several amino acid pathways and neurotransmitter systems, as well as cerebral energy metabolism, nitric oxide synthesis, antioxidant systems, mitochondrial permeability transition and signal transduction 
pathways. HE due to both chronic liver failure (CLF) and acute liver failure (ALF) is manifested by neuropathological changes that primarily affect astrocytes. The main pathological manifestation in CLF and chronic HA is the appearance of Alzheimer type II astrocytosis (Norenberg, 1977, 1987). In HA and HE due to ALF, death most frequently results from brainstem herniation due to increased intracranial pressure as a result of a progressive increase of brain water content (brain edema) due to astrocyte swelling (Blei, 2001; Butterworth, 1998; Chung et al., 2001; Cordoba \& Blei, 1995; Larsen et al., 2001). Among other mechanisms, ammonia-induced disturbances in the synthesis and degradation of the neuronal neurotransmitter amino acid glutamate lead to changes in its brain levels as well as in its reuptake by astrocytes (Butterworth, 2002; Michalak et al., 1996). The resulting excitotoxic levels in the cerebrospinal fluids (CSF) can lead to an overactivation of glutamatergic receptors on the postsynaptic neuron.

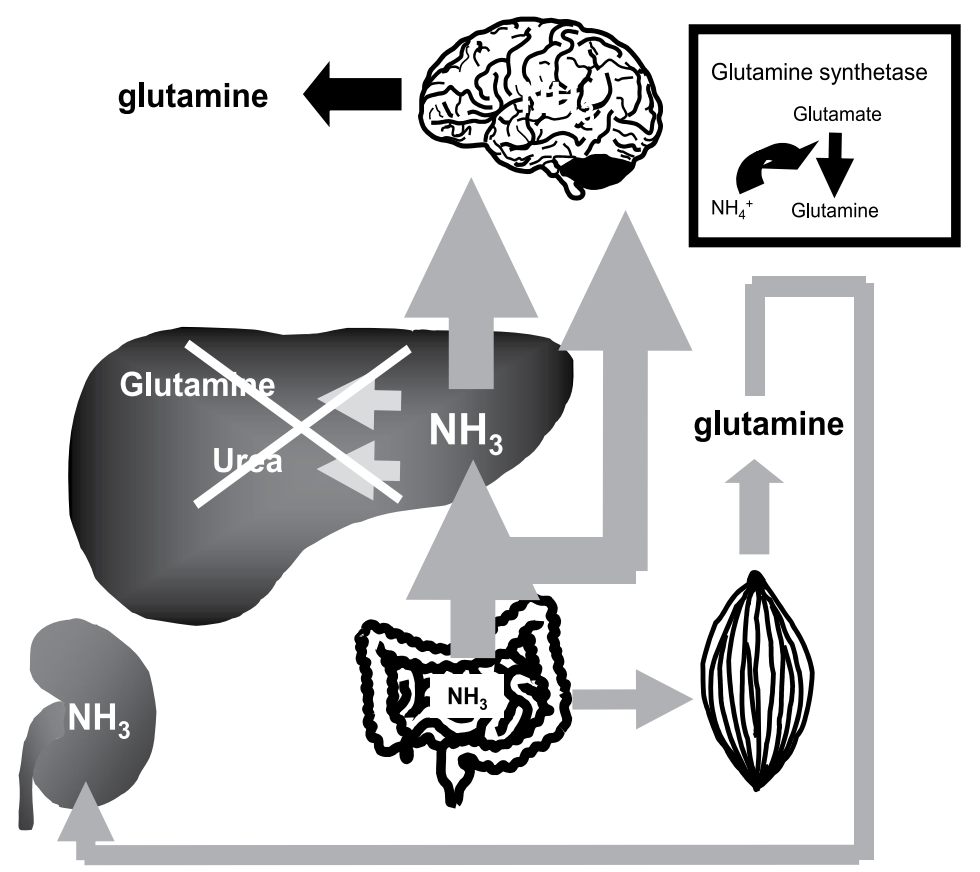

Fig. 1. Development of hyperammonemia. The production of ammonia in the gastrointestinal tract occurs as a result of the action of bacteria on nitrogenous substances, as well as gastrointestinal bleeding. After ammonia is generated, the principal fate is incorporation into glutamine in extrahepatic organs, such as the muscle. The glutamine derived from muscle acts as an energy source for the gut and at the same time releases ammonia for urea synthesis in the kidney. Portal vein ammonia, on the other hand, which is present at a much higher concentration, is largely detoxified as urea in the liver and released by the kidney. When the liver fails due to dysfunction or shunting of the portal venous blood, large amounts of ammonia enter the brain. Basically the only way to detoxify ammonia in the brain is by synthesis of glutamine. 
The neuronal N-methyl-D-aspartate (NMDA) receptor is a glutamatergic ionotropic receptor, and elevated ammonia levels lead to an excessive activation of this receptor (Cauli et al., 2008; Monfort et al., 2002). This induces an increased expression of the neuronal nitric oxide synthase (nNOS) producing nitric oxide (NO). NO reduces the activity of antioxidant enzymes, leading to increased formation of superoxides and therefore to oxidative stress (Kosenko et al., 1998, 1999). NO can also interact with superoxide anions to form peroxynitrite, which is a powerful nitration reagent. It nitrates tyrosine residues in proteins, including enzymes such as GS (Rose \& Felipo, 2005), resulting in an inhibition of their activity. Another consequence of oxidative stress is the activation of mitogen-activated protein kinases (MAPKs). The kinases are key substances of intracellular signaling cascades, which control a number of cellular responses to extracellular impulses. Increased ammonia levels lead to phosphorylation of these enzymes, and recent studies suggest the involvement of MAPK phosphorylation in the mechanisms of ammonia-induced astrocyte dysfunction and cell swelling (Jayakumar et al., 2006).

The energy metabolism of the CNS is affected by ammonia in different ways. Increased ammonia levels have been suggested to inhibit the tricarboxylic acid (TCA) cycle enzyme aketoglutarate dehydrogenase (Lai \& Cooper, 1986). More recently, a direct effect of ammonia on neuronal pyruvate dehydrogenase (PDH) has been proposed by NMR studies (Chatauret et al, 2003; Zwingmann et al., 2003). PDH is a mitochondrial matrix multienzyme complex that provides the link between glycolysis and the TCA cycle by catalyzing the conversion of pyruvate into acetyl-CoA. If $\mathrm{PDH}$, and subsequently mitochondrial energy production, is compromised, a switch to glycolysis may in part compensate by cytosolic ATP production. As a result, and to maintain reduced equivalents needed for glycolytic conversion of glucose, lactate dehydrogenase (LDH) converts pyruvate to lactate which accumulates (Kala \& Hertz, 2005; Zwingmann et al., 2003, 2007; Zwingmann \& Butterworth, 2005). On the other hand, the stimulation of the glycolytic enzyme phosphofructokinase by ammonia (Ratnakumari \& Murthy, 1993) also leads to elevated lactate levels. Consistent with these possibilities, brain lactate concentrations have been consistently found to be increased in brain and extracellular fluid of various animal models of acute HA (Bates et al., 1989; Deutz et al., 1988; Holmin et al., 1983; Michalak et al., 1996; Peeling et al., 1993), as well as in patients with ALF (Tofteng \& Larsen, 2002). Previous studies have shown that, like ammonia, lactate exposure to cultured astrocytes also results in significant cell swelling (Staub et al., 1990).

While ammonia may impair mitochondrial glucose oxidation, some of the neuropathological manifestations in hyperammonemic states in liver failure may be mediated by the accumulation of astrocytic glutamine as a result of ammonia detoxification (Norenberg et al., 2007). While the osmotic effect of glutamine might not be the primary cause of astrocyte swelling, its accumulation has been shown to have secondary effects on astrocyte function, energy metabolism and neurotransmitter homeostasis. For example, glutamine, as well as ammonia itself, can induce the mitochondrial permeability transition (Rama Rao et al., 2003). The increased permeability can lead to elevated levels of osmolytes in the astrocytes and subsequent to a collapse of the mitochondrial inner membrane potential, resulting in mitochondrial dysfunction and enhanced free radical production (Norenberg et al., 2007). Increased levels of both glutamine and lactate, as well as changes in membrane permeability, can contribute to an enhanced water influx into the astrocytes and 
thereby to swelling of the cells (Bachmann, 2002; Chatauret et al., 2003; Norenberg et al., 1998; Rama Rao et al., 2003). Specifically in acute disease states, astrocyte swelling is a major cause of cerebral edema and intracranial hypertension, leading to encephalopathy, coma and death.

\section{Hepatic encephalopathy}

HE is a clinical manifestation due to CLF or ALF. HE is classified according to the West Haven Criteria. These define HE grade I to IV, based on the presence of specific clinical signs and symptoms and their intenseness (Ferenci et al., 1998). Manifest encephalopathy is characterized by neuropsychiatric symptoms ranging from mild personality changes and impairment of consciousness, attention span and orientation (grade I) to deep coma (grade IV). It is diagnosed by clinical signs, neuropsychiatric evaluations and neuroimaging methods. Patients with minimal HE show no significant symptoms of brain dysfunction, but it can have a far-reaching impact on quality of life, ability to function in daily life and progression to overt hepatic encephalopathy. For example, many patients with minimal HE show an impaired ability to drive a motor vehicle (Wein et al., 2004). The diagnosis of minimal HE is primarily carried out by the flicker test (Kircheis et al., 2002a). The critical flicker frequency (CFF) analysis is an objective and reproducible assay of HE severity. It defines the frequency at which light pulses are perceived as fused or flickering light. The technique shows little dependence upon age, education or training and is even able to detect minimal encephalopathy.

HA is considered to be a key factor in both forms of liver failure, but its relation to neurological damage in HE is poorly understood. Furthermore, there are other precipitating factors in HE, such as elevated manganese, hyponatraemia, inflammatory cytokines and benzodiazepine-type sedatives (Cordoba \& Minguez, 2008; Haeussinger \& Schliess, 2008). HE occurs mostly as a result of a chronic disease. The current epidemic of hepatitis $C$, for example, emphasizes the importance of this clinical problem, particularly with regard to the extremely high mortality rate of $80-90 \%$ (Schiodt et al., 1999). However, HE development also appears acutely in previously healthy individuals due to viral hepatitis or overexposure to hepatotoxins like acetaminophen. While the chronic form of HE progresses slowly, rapid deterioration in consciousness level and death usually occur in the acute form of HE.

\subsection{Therapeutic means in Hepatic encephalopathy}

There are different therapeutic means of treating patients with HE. One possibility is a liver support device and liver transplantation, a definitive therapeutic strategy that improves outcomes in patients. However, there are several drugs being examined in research and clinical trials that could benefit patients with HE. Most of them tend to reduce the production and absorption of ammonia from the colon (Bass, 2007; Morgan et al., 2007). Furthermore, non-absorbable disaccharides inhibit bacterial ammonia production in the colon and retard ammonia as non-diffusable ammonium in the intestinal colon (Cammà et al., 1993). But in many cases, studies approving the efficacy and safety of the drugs are limited (Bass, 2007). Antibiotics are administered to reduce the quantity of enteric bacteria which produce ammonia. For example, rifaximin seems to be more effective than other antibiotics and is inexpensive (Leevy \& Phillips, 2007). 
Also, substances modifying ammonia metabolism are considered as therapeutic means in HE. Commonly used pharmacologic agents to treat HA are sodium phenylacetate and sodium benzoate, which can serve as alternatives to urea for nitrogen excretion. Phenylacetate conjugates with glutamine, a major detoxification product of ammonia, to form phenylacetylglutamine, which is excreted by the kidney. Sodium benzoate, on the other hand, reduces ammonia levels in blood because it is metabolized to hippurate by conjugation with glycine, which is rapidly excreted by the kidney (Morgan et al., 2007).

A primary liver function is the regulation of amino acid supply to peripheral tissues. In HE, an increase of aromatic amino acids compared to branched-chain amino acids has been observed, which could be modified by the intake of branched-chain amino acids (Bass, 2007). Also L-Ornithine-L-Aspartate lowers ammonia levels in blood as it provides substrates for the metabolic conversion of ammonia to urea and glutamine (Kircheis et al., $2002 b$ ). Another means of treating HA is restriction of dietary protein (a source of ammonia), while caloric intake is provided by glucose and fat. Less investigated to date are L-carnitine and its derivatives, which are suggested to increase fat metabolism and have been suggested to decrease ammonia concentrations in blood by raising metabolic energy production (Bass, 2007).

\section{L-carnitine}

L-carnitine (L-4-N-trimethylammonium-3-hydroxybutyric acid) is known to be involved in long-chain fatty acid metabolism. The quatenary amine is synthesized in the liver and kidney from lysine and methionine (Bremer, 1983; Steiber et al., 2004). However, the major part of the daily requirement is covered by dietary intake. For example, food of animal origin, particularly red meat, contains significant amounts of L-carnitine (Steiber et al., 2004).

L-carnitine is essential for the transfer of long-chain fatty acids from the cytoplasm to the inner mitochondrial membrane for their oxidation (Fig. 2). Fatty acids are released from adipose tissue into the plasma. There, they are bound to albumin and carried to muscles, where a membrane transporter yields them into the cytoplasm. In the cytoplasm the fatty acids are activated by conjugation to Coenzyme A by means of Coenzyme A synthase. The acyl group from acyl-CoA is then transferred to the inner aspect of the outer mitochondrial membrane via the L-carnitne palmitoyl transferase I (CPTI) where it is linked to L-carnitine. The acylcarnitine is subsequently translocated across the inner mitochondrial membrane by the carnitine-acylcarnitine translocase (CACT). In the mitochondrial matrix acylcarnitine is reconverted to acyl-CoA and L-carnitine through carnitine palmitoyl transferase II (CPTII). The released L-carnitine is returned to the cytoplasm via carnitine palmitoyltransferase II, while the acyl-CoA can be metabolized through $\beta$-oxidation to acetyl-CoA (Bremer, 1983; Luppa, 2004; Virmani \& Binienda, 2004). The $\beta$-oxidation of fatty acids is important because it provides acetyl-CoA, a substrate for the TCA cycle. Thus, decreased intramitochondrial transport of fatty acids due to carnitine deficieny can lead to an inhibition of mitochondrial pyruvate oxidation in the TCA cycle. Since the reaction of the carnitine palmitoyl transferase is reversible, it is possible to release acetylcarnitine from the mitochondria into the plasma. Therefore, L-carnitine acts as a buffer for the acetyl-CoA/CoASH ratio (Luppa, 2004; Steiber et al., 2004) and activates the mitochondrial respiration rate. 


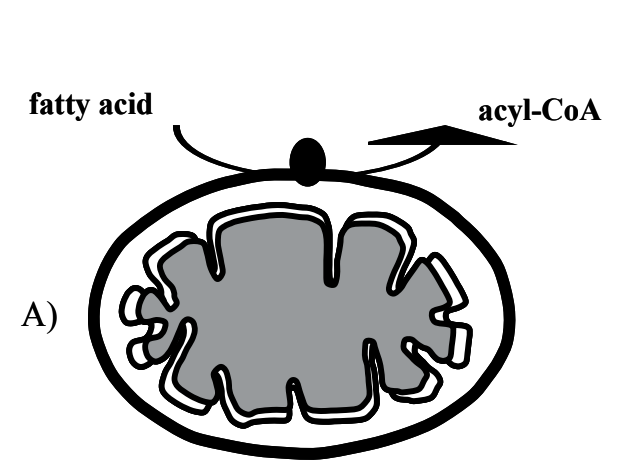

acyl-CoA + carnitine

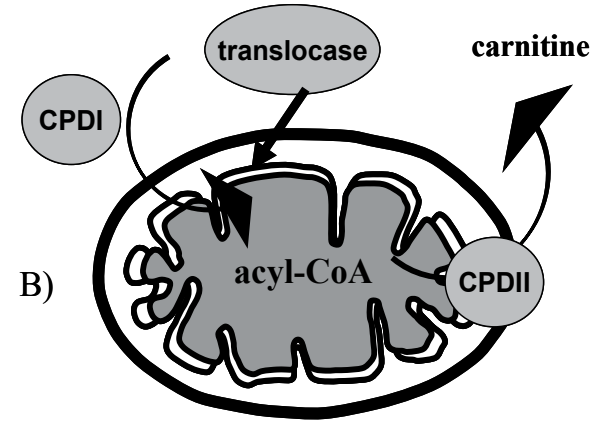

Fig. 2. Suggested action of L-carnitine. L-carnitine can facilitate the entry of fatty acids into the mitochondria. These fatty acids can be used to produce energy. A) Fatty acids must first be activated in the outer mitochondrial membrane before they can be used. Acetyl-CoA synthetase activates the fatty acid and transforms it to acyl-CoA. B) Activated fatty acids in the form of acyl-CoA are carried across the mitochondrial membrane by L-carnitine. L-carnitine is recycled back. In the mitochondria, acyl-CoA undergoes $\beta$-oxidation to produce acetyl-CoA, which is used for mitochondrial energy production in the TCA cycle.

\subsection{L-cartinine in the CNS}

Tissue that copes with its energy demands by fatty acid oxidation, i.e. liver, muscles, kidney and heart, contains high levels of L-carnitine. Significant amounts of L-carnitine are also localized in the brain. Although the main energy production in the brain occurs by mitochondrial glucose oxidation, it has been observed that at least part of the cerebral energy production from acetyl-CoA occurs via fatty acid oxidation, most notably in astrocytes (Ebert et al., 2003). Another major task of L-carnitine in the brain is the transfer of acetyl-CoA from the mitochondria into the cytoplasm (see above). By this means acetyl-CoA also provides acetyl groups for the synthesis of the neurotransmitter acetylcholine. In addition, L-carnitine and its acylderivates can change the activity of certain proteins such as cysteine aspartic acid specific proteases (caspases) (Mutomba et al., 2000). They are also able to modify membrane fluidity and surface charge and therefore alter the activity of several enzymes and transporters that are located in the membrane (Nałecz et al., 2004; Virmani \& Binienda, 2004). L-carnitine and its acylderivates may also increase the synthesis of phospholipids, which are required for membrane formation and integrity, and thereby modulate neuronal activity (Virmani \& Binienda, 2004).

There are two known forms of L-carnitine deficiency, the primary systemic and the secondary deficiency. Primary systemic L-carnitine deficiency is caused by several defects in the biosynthesis and transport of L-carnitine. This leads to increased excretion of L-carnitine and decreased L-carnitine accumulation in tissues (Longo et al., 2006, Vielhaber et al., 2004). Secondary L-carnitine deficiency occurs due to genetically defined disturbances in fatty acid oxidation and amino acid homeostasis. Several medical conditions, such as cirrhosis and other metabolic disorders, lead to secondary L-carnitine deficiency, as well as hemodialysis or kidney failure (Fornasini et al., 2007, Virmani \& Binienda, 2004). Both disorders induce the same symptoms, like periodic encephalopathies which often come along with 
hypoglycemia and hypoketonia. Other symptoms are hypotonia, weakness, episodes of coma, seizures, neuropathy and anemia.

\subsubsection{Pharmaceutical use of L-carnitine in brain disorders}

By virtue of their metabolic functions and neurophysiological roles, L-carnitine and its acetylated derivates are suggested as a therapeutic agent in several neurological disorders. Among others, the effects of L-carnitine and its derivates have been investigated in Alzheimer's disease, chronic fatigue syndrome, Parkinson's disease, and neuropathic pain, as well as in valproic acid intoxication associated with CNS depression and raised ammonia levels without hepatotoxicity (Beal, 2003; Chan et al., 2007; Kuratsune et al., 2002; Pittler 7 Ernst, 2008; Silva et al., 2008). Fatty acid metabolism plays a major role in brain energy metabolism since acetyl-CoA enters the mitochondrial TCA cycle via PDH in both astrocytes and neurons (Fig. 3).

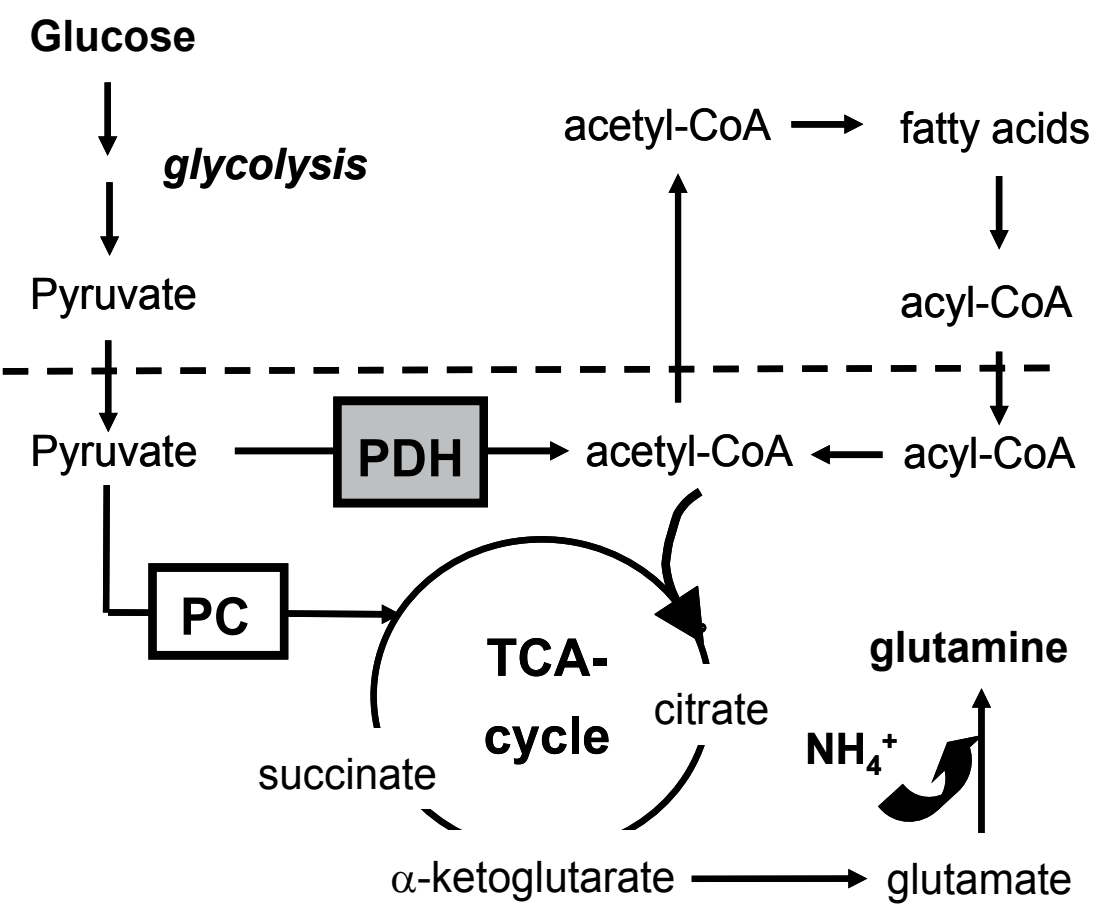

Fig. 3. Formation of acetyl-CoA from glucose and fatty acids. Glycolysis transforms glucose into pyruvate. Pyruvate may enter the TCA cycle via the anaplerotic pathway (PC; EC 6.4.1.1) or the oxidative pathway (PDH; EC 1.2.4.1) through acetyl-CoA. Acetyl-Coa can also be formed from fatty acids after the entry of acyl-CoA from the cytosol into the mitochondria, a process which is facilitated by L-carnitine.

In cerebral diseases, where mitochondrial dysfunction, impaired energy metabolism and oxidative stress play a decisive role for their development, such as in Parkinson's disease, Lcarnitine has been shown to improve overall metabolic activity by yet unknown mechanism 
(Beal, 2003, Zhang et al., 2008). Also, in vitro models have shown significant improvements of mitochondrial biogenesis when L-carnitine is given in combination with other mitochondrial nutrients, such as R-a-Lipoic acid (Zhang et al., 2008).

In the brain, both L-carnitine and acetyl-L-carnitine play further important roles in cerebral bioenergetics and neuroprotection through a variety of mechanisms, including their antioxidant properties through the modulation and promotion of synaptic neurotransmission (Inazu \& Matsumiya, 2008). Alzheimer's disease and chronic fatigue syndrome occur mainly in the elderly. L-carnitine and its derivates can restore changes in membrane phospholipid metabolism found in subjects with Alzheimer's disease (Montgomery et al., 2003; Pettegrew et al., 2000). Furthermore, it is able to enhance quality of life in patients with Alzheimer's disease and chronic fatigue syndrome because of its cholinergic function and stimulation of cellular energy production (Malaguarnera et al., 2007, 2008; Pettegrew et al., 2000). Acetyl-L-carnitine has also been successfully applied as a pharmacological agent for the treatment of chronic degenerative diseases of the senile brain and for slowing down the progression of mental deterioration in Alzheimer's disease. The mechanisms may involve both the cholinergic neuronal transmission activity of acetyl-Lcarnitine and its ability to enhance neuronal metabolism in mitochondria (Inazu \& Matsumiya, 2008). Neuropathic pain has several causes, such as diabetes polyneuropathy, HIV, or chemotherapeutic agents. In these cases, acetyl-L-carnitine has been shown to have an analgesic effect due to its cholinergic action. Moreover, it is able to improve nerve function by activating several receptors via acetylation (Chiecho et al., 2007, Sima, 2007). Valproic acid is a broad-spectrum antiepileptic drug which is known to reduce L-carnitine level, and can lead to HA. It has been suggested that L-carnitine may decrease ammonia levels by binding to valproic acid (Chan et al., 2007, Lheureux et al., 2005, Wadzinski et al., 2007).

\subsubsection{L-carnitine in experimental hyperammonemia}

HA can be induced in animal models by several approaches. Major common methods are to inject neurotoxic amounts of ammonium acetate or to feed the animals a high ammonia diet. Several studies have demonstrated that L-carnitine can prevent acute ammonia toxicity and decrease its effects on the brain. O'Connor et al. (1984 a, 1984 b) injected L-carnitine in mice $30 \mathrm{~min}$ before administration of a lethal dose of ammonia acetate. They demonstrated that Lcarnitine reduces mortality (100\% survival with $12 \mathrm{mmol} / \mathrm{kg}$ body weight) and prevents symptoms of ammonia toxicity. Survival and beneficial effects depended on the dose of Lcarnitine. From these data, it was suggested that the protective effect of L-carnitine is based on significant improvements in the cellular redox state and mitochondrial energy metabolism. The concomitant decrease in blood and brain ammonia concentrations was proposed to be due to activation of the urea cycle by L-carnitine via carbamyl phosphate synthetase. I Matsuoka \& Igisu (1993a) compared the effects of L-carnitine, D-carnitine and acetyl-L-carnitine in the therapy of acute HA in mice. Marked alterations were observed in brain energy metabolites after ammonia injection, which were suppressed by all three substances together with reduced blood and brain ammonia levels and a reduction in the frequency of seizures. Interestingly, acetyl-L-carnitine better conserved adenonsinetriphosphate (ATP) in the brain, while it reduced ammonia levels in the blood and brain less markedly. These results suggest that the protective actions of L-carnitine and its derivates are systemic, and that the effect of acetyl-L-carnitine differs from that of L-carnitine. In a 
previous study, the authors injected mice with sublethal doses of ammonium acetate to rule out the effects of seizures on brain energy metabolism (Matsuoka and Igisu, 1993b). These data further showed that L-carnitine improves brain energy metabolism in experimental HA in a systemic manner and not by suppression of seizures. Another study investigated the effects of different L-carnitine doses on cerebral and hepatic energy metabolites in hyperammonemic sparse-fur (spf) mutant mice with X-linked ornithine transcarbamylase deficiency, a model of congenital chronic HA. It was shown that L-carnitine is able to activate the fatty acid oxidation during sodium benzoate treatment (Ratnakumari et al., 1993b). Sodium benzoate reduces ammonia levels through hippurate formation. A possible side effect is decreased levels of acetyl-CoA due to formation of benzoyl-CoA, while Lcarnitine was able to restore the availability of free CoA and thereby could reduce ammonia levels and improve energy metabolism. Subsequently, Ratnakumari et al. (1995) studied the effect of acetyl-L-carnitine on the development of cerebral cholinergic parameters in the same mouse model of HA. Cholinergic deficiency in hyperammonemic mice was significantly improved by treatment with acetyl-L-carnitine. The reason for the restoration could be an L-carnitine induced elevation of nerve growth factor levels and activation of choline acetyltransferase (Ratnakumari et al., 1995). In the study by Rao and Qureshi (1999), HA led to an excessive release of glutamate, which in turn resulted in upregulation of the NMDA receptor. The cellular reuptake of glutamate depends on sufficient availability of ATP levels. The results of this study demonstrated that acetyl-L-carnitine modulates the binding sites of the NMDA receptor, while enhanced ATP production via the TCA cycle, activated by acetyl-L-carnitine, might have contributed to stimulated uptake of glutamate from the extracellular fluid.

A model of ammonia-precipitated encephalopathy is the portacaval-shunted rat receiving ammonium acetate (Belanger and Butterworth, 2005). In this model, Therrien et al. (1997) examined the effect of L-carnitine $(16 \mathrm{mmol} / \mathrm{kg}$, intraperitoneally), given 1 hour before ammonium acetate $(8.5 \mathrm{mmol} / \mathrm{kg}$, subcutaneously) to portacaval shunted rats. Hyperammonemic rats manifested severe encephalopathy progressing through loss of righting reflex to coma, and the survival rate was $5 \%$. Similar to the study by $\mathrm{O}^{\prime \prime}$ Connor et al. (1984), none of the L-carnitine treated animals died or showed neurological impairments. However, L-carnitine pretreatment did not substantially reduce plasma ammonia levels or increase CSF or brain glutamine levels in these rats, while it reduced CSF ammonia. Interestingly, L-carnitine reduced ammonia-induced increases in CSF lactate and alanine, suggesting an improvement of cerebral mitochondrial energy metabolism. According to the studies mentioned above, L-carnitine exerts protective effects against cerebral energy distortions and neuronal dysfunction in HE.

\subsection{L-carnitine in in vitro ammonia toxicity}

In addition to experimental animals with HA, studies were performed in hippocampal slices and cultured neurons to evaluate some mechanistic aspects of the effect of L-carnitine. Many of the cerebral effects of ammonia have been explained by extracellular glutamate accumulation and NMDA receptor activation by both ammonia or glutamate. In primary neurons, for example, it has been demonstrated that L-carnitine prevented glutamate neurotoxicity in a dose-dependent manner similar to that required to prevent ammonia toxicity in animals, and decreased the affinity of glutamate for the NMDA receptor (Felipo et al., 1994). In a study by Llansola et al. (2002), treatment of primary neurons in culture with 
$1 \mathrm{mM}$ glutamate caused cell death of $80 \%$. In this system glutamate neurotoxicity was mainly mediated by activation of NMDA receptors. The addition of $1 \mathrm{mM}$ L-carnitine 15 min before glutamate prevented neuronal death caused by glutamate. Furthermore, Lcarnitine increased the binding affinity of glutamate for metabotropic glutamate receptors, which was reversed if metabotropic glutamate receptors were blocked with specific antagonists (Llansola et al., 2002). Further studies in primary cultured neurons also indicated that L-carnitine interferes with NMDA-induced, NO mediated activation of MAP kinase at a step subsequent to NO formation (Llansola \& Felipo, 2002). However, as mentioned above, recent studies suggest that MAPK phosphorylation is also involved in the mechanisms of ammonia-induced astrocyte dysfunction and cell swelling (Jayakumar et al., 2006). In rat hippocampal slices, L-carnitine was able to reverse some of the inhibitory effects of ammonia on respiratory energy metabolism and mitochondrial function (Izumi et al., 2005). In these studies, mitochondrial function was measured using a mitochondrial dehydrogenase assay (Fig. 4). Incubation of the slices with $1 \mathrm{mM}$ ammonia significantly reduced the production of the formazan end product. This reduction in enzymatic activity was overcome by co-administration of $1 \mathrm{mM}$ L-carnitine, while L-carnitine itself did not facilitate formazane formation.

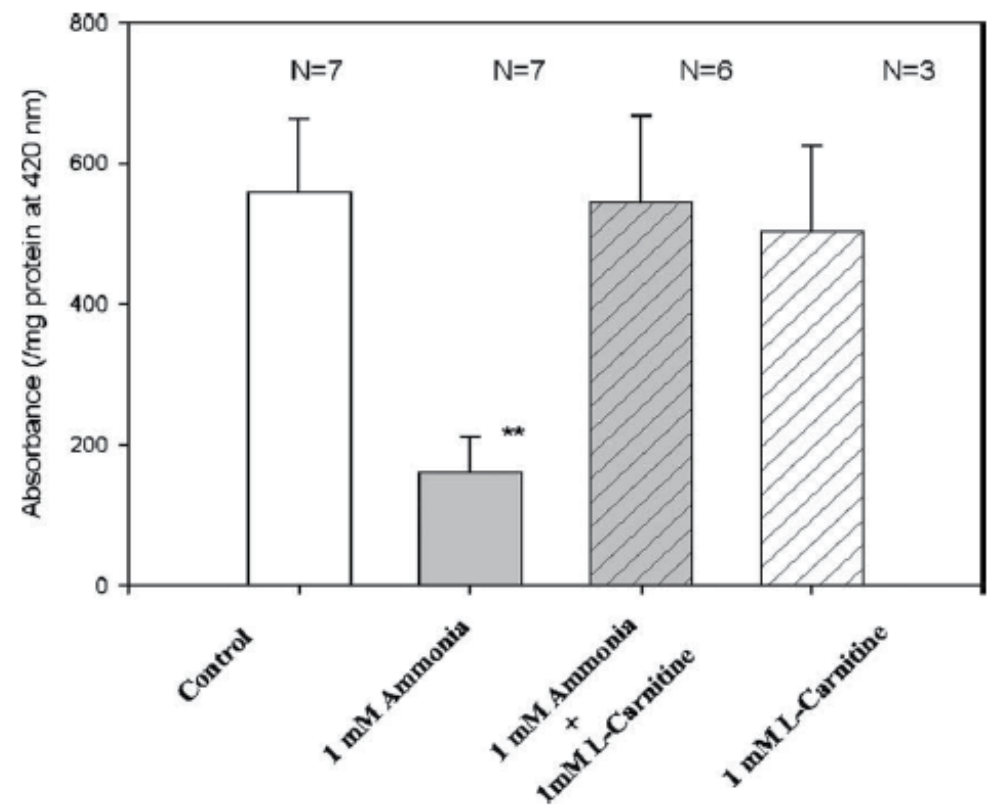

Fig. 4. (from Izumi et al., 2005). Effects of ammonia and L-carnitine on mitochondrial function. The WST-1 mitochondrial dehydrogenase assay (optical density measurements at $420 \mathrm{~nm}$ ) was performed $1 \mathrm{~h}$ after pre-incubation with ammonia in the absence and presence of L-carnitine. $\mathrm{P}^{* *}<0.01$ by paired Student's $\mathrm{t}$ test.

Similarly, DL-APV, an antagonist of NMDA receptors, diminished the effects of ammonia on reduced ATP levels and inhibition of long-term potentiation. These in vitro data suggest that L-carnitine and NMDA receptor antagonists have the potential to preserve neuronal 
function during HA, confirming results obtained in vivo in hyperammonemic animals (see above). Minana et al. (2002) compared the in vivo and in vitro effects of different trimethylamine-containing compounds on ammonia toxicity in mice and against glutamate and NMDA toxicity in primary cultures of neurons (Tables 1 and 2).

\begin{tabular}{|llll|}
\hline & & & Survival \\
\cline { 3 - 4 } Compound & Dose & Survivors/Injected & \\
& & & \\
& nmol/kg & & \\
None & & $17 / 142$ & 12 \\
L-carnitine & 16 & $24 / 28$ & 86 \\
D-carnitine & 16 & $21 / 31$ & 68 \\
Betaine & 16 & $12 / 20$ & 60 \\
TMAO & 8 & $32 / 50$ & 64 \\
Choline & 1.6 & $43 / 65$ & 56 \\
Acetylcholine & 0.28 & $17 / 30$ & 71 \\
Acetylcholine & 0.55 & $63 / 88$ & 56 \\
Carbachol & 0.0028 & $14 / 30$ & 76 \\
Carbachol & 0.0055 & $23 / 49$ & 80 \\
Acetylcarnitine & 6 & $41 / 54$ & \\
Isovalerylcarnitine & 4 & $17 / 21$ & \\
\hline
\end{tabular}

Table 1. (adapted from Minana et al., 2002). Protective effect of different compounds against acute ammonia toxicity. Groups of mice were injected i.p. with the indicated doses of each compound. Fifteen minutes later, the mice were injected i.p. with $14 \mathrm{mmol} / \mathrm{kg}$ of ammonium acetate. All values are statistically different from mice injected only with ammonia.

\subsubsection{L-carnitine in human hepatic encephalopathy}

As mentioned above, experimental HA is not a model of human liver disease and rather serves to investigate the neurotoxicity of acute or chronic ammonia exposure or to study specific aspects of liver failure (Belanger \& Butterworth, 2005). Therefore, very few studies that investigate the effects of L-carnitine in appropriate animal models of HE have been published. Clinical studies in humans with HE have only been performed by a few groups to date. Nevertheless, in initial clinical studies (del Olmo et al., 1990), the effect of L-carnitine on HA in patients with cirrhosis, subjected to a rectal ammonium overload test, was investigated. On comparing 40 cirrhotic patients given L-carnitine with 40 control cirrhotics given a placebo, no significant differences were observed in ammonium levels. However, on studying the patients with the greatest liver involvement, those given L-carnitine showed smaller elevations in ammonia and better responses to psychometric tests than those receiving the placebo. These data provided the first evidence on the effect of L-carnitine on ammonia clearance and pointed to the importance of further investigations. 


\begin{tabular}{|c|c|c|c|c|c|c|}
\hline \multirow{2}{*}{ Compound } & \multirow{2}{*}{ Dose } & Gluta & late & \multicolumn{3}{|c|}{ NMDA } \\
\hline & & \multicolumn{5}{|c|}{ Surviving neurons } \\
\hline None & & $20 \pm$ & 8 & 28 & \pm & 7 \\
\hline Carnitine & $0.5 \mathrm{mM}$ & $70 \pm$ & 8 & 47 & \pm & 8 \\
\hline Betaine & $1 \mathrm{mM}$ & $69 \pm$ & 7 & 46 & \pm & 5 \\
\hline TMAO & $1 \mathrm{mM}$ & $75 \pm$ & 4 & 64 & \pm & 12 \\
\hline Choline & $1 \mathrm{mM}$ & $65 \pm$ & 5 & 41 & \pm & 5 \\
\hline Acetylcholine & $1 \mathrm{mM}$ & $68 \pm$ & 4 & 23 & \pm & 11 NS \\
\hline Carbachol & $1 \mathrm{mM}$ & $73 \pm$ & 3 & 40 & \pm & 6 \\
\hline Acetylcarnitine & $1 \mu \mathrm{M}$ & $69 \pm$ & 3 & 48 & \pm & 8 \\
\hline Trans-ACPD & $0.1 \mathrm{mM}$ & $84 \pm$ & 6 & 65 & \pm & 5 \\
\hline MK-801 & $20 \mathrm{nM}$ & $80 \pm$ & 5 & 83 & \pm & 6 \\
\hline
\end{tabular}

Table 2. (adapted from Minana et al., 2002). Effects of trimethylamine-containing compounds on neuronal death induced by glutamate or NMDA. Neurons were incubated with these compounds for $15 \mathrm{~min}$ and then $1 \mathrm{mM}$ glutamate or $0.5 \mathrm{mM}$ NMDA was added to the plates. Neuronal survival was determined $4 \mathrm{~h}$ later by staining with fluorescein diacetate and propidium iodide.

Based on previous experiments in animal models, such as the rat model of ammoniaprecipitated encephalopathy (Therrien et al., 1997), in a clinical randomized placebocontrolled study, 120 patients with moderate HE due to cirrhosis were treated for 60 days with L-carnitine (2 g twice a day) (Malaguarnera et al., 2003). The study demonstrated a protective effect of L-carnitine in ammonia-precipitated encephalopathy in these patients after 30 days, which was more pronounced after 60 days of treatment. Concomitantly, a significant therapeutic effect of L-carnitine was observed in a psychometric test. Also, in 50 patients infected with HCV and development of chronic hepatitis, the effect of L-carnitine on interferon (IFN)-alpha-induced fatigue was evaluated (Neri et al., 2003). Fatigue is a common complaint in patients with liver disease, and can be induced also by IFN. Patients treated with IFN and L-carnitine show a marked and early significant reduction of fatigue levels compared to untreated patients. It has been suggested from these data, taking into account the severity of this symptom in this study, that L-carnitine could be used as an energetic substrate in IFN-treated patients, leading to a better response to this side effect. However, the mechanisms by which L-carnitine might exert a protective effect based on energy metabolism still remain to be investigated.

In 2005, Malaguarnera et al. continued to work with these data and evaluated the influence of L-carnitine on mental conditions and ammonia effects on patients with minimal or moderate HE due to different liver diseases, in a randomized, placebo-controlled study. The patients were randomized into an L-carnitine receiving group ( $2 \mathrm{~g}$ twice daily for 90 days) and a placebo-receiving group in a double-blind test. The results showed a protective effect of L-carnitine against ammonia-precipitated encephalopathy in cirrhotic patients, together with a significant decrease in ammonia fasting serum levels. After 30 days of treatment, 
ammonia serum levels were significantly decreased, with an ongoing trend after 60 and 90 days. Psychometric tests also clarified a significant therapeutic effect.

Based on data suggesting a protective effect of L-carnitine on HA-induced neuronal dysfunction, eighteen cirrhotic patients with $\mathrm{HE}$ and HA received a single dose of $0.5 \mathrm{~g}$ acetyl-L-carnitine to investigate its effects on neuronal function (Siciliano et al., 2006). The effects of acute acetyl-L-carnitine administration were studied by pattern-reversal visualevoked potentials (VEP) to evaluate neuronal function. The results showed a rapid effect of a single dose of acetyl-L-carnitine. It was suggested that it transiently improves neuronal function in cirrhotic patients with persistent HE and HA. However, there was no change in plasma ammonia levels. In contrast to most animal studies, these results suggest a direct effect of acetyl-L-carnitine on the CNS with as yet unknown mechanisms.

In another evaluation, Malaguarnera et al. (2006) investigated the efficacy of acetyl-Lcarnitine in the treatment of hepatic coma in a randomized placebo-controlled study. The patients received either acetyl-L-carnitine or a placebo ( $4 \mathrm{~g}$ intravenously over 3 hours, once a day for 3 days). A significant improvement in neurological symptoms and decrease in EEG grade, together with a reduction of ammonia plasma levels, were obvious at 1 to 4 hours after treatment and remained until 24 hours after. An extended study by Malaguarnera et al. (2008) evaluated the cognitive function of 125 cirrhotic patients with minimal HE after treatment with acetyl-L-carnitine ( $2 \mathrm{~g}$ twice a day for 90 days). These results showed a considerable improvement in neuropsychological functions and a significant reduction in serum ammonium levels, further confirming neuronal improvements after L-carnitine treatment.

\subsubsection{Preliminary results on metabolic alterations using multinuclear NMR spectroscopy}

High-resolution Nuclear Magnetic Resonance (NMR) approaches using stable isotopes are now yielding large amounts of detailed information on cerebral metabolic compartmentalization and neuronal-glia interactions (Zwingmann \& Leibfritz, 2003). In order to investigate the effect of L-carnitine on energy metabolism, ${ }^{1} \mathrm{H}$ - and ${ }^{13} \mathrm{C}-\mathrm{NMR}$ spectroscopy was used to measure metabolic pathways in brain and muscle following administration of [U-13C]glucose $(500 \mathrm{mg} / \mathrm{kg}$; i.p.) to rats with mild $\mathrm{HE}$ and in rats with ammonia-precipitated encephalopathy. As a model of mild HE, we investigated rats four weeks after an end-to-side portacaval anastomosis (PCA). During the last 7 days, rats with PCA and controls were administered daily either L-Carnitine $(0.8 \mathrm{mmol} / \mathrm{kg}$; i.p. $)$ or saline. After administration of [U-13C]glucose for 15 to 60 minutes, the rats were sacrificed. The frozen tissue samples of brain and muscle were powdered over liquid nitrogen and homogenized in perchloric acid (PCA) at $0^{\circ} \mathrm{C}$. Homogenates were centrifuged, the supernatants neutralized, precipitated $\mathrm{KClO}_{4}$ sedimented, and the supernatants lyophilized. The lyophilized samples were dissolved in $600 \mu \mathrm{D} 2 \mathrm{O}$, centrifuged and neutralized $(\mathrm{pH}=$ 6.8). The pellet after the first centrifugation step was homogenized in water and lyophilized. The lyophilized samples were dissolved in $800 \mu \mathrm{CDCl} / \mathrm{CD} 3 \mathrm{OD}$ to obtain the fatty acid components. All animals received humane care according to the criteria outlined in the "Guide for the Care and Use of Laboratory Animals" prepared by the National Academy of Sciences and published by the National Institutes of Health (NIH publication 6-23 (revised), 1985). 
As a neurobehavioral measure, locomotor activities were measured using a computerized Auto-Track system (Columbus Instruments, $\mathrm{OH}$ ), consisting of a 15-by-15 infrared beam array with an interbeam distance of $2.4 \mathrm{~cm}$ along the $X$ and $Y$ axis. Data were collected every $0.1 \mathrm{~s}$ and the activity was categorized as ambulatory, stereotypic, or resting. The animal activity meter was installed in a quiet isolated room with $12 \mathrm{~h}$ light/12 h dark cycles. One hour after HAL, the animals were placed individually in the auto-track system and data acquisition was started for a period of $24 \mathrm{hr}$. PCA in the rat resulted in a broad spectrum of neurobehavioral changes, such as an $80 \%$ impaired locomotor activity, which was largely prevented by L-carnitine (Fig. 5).
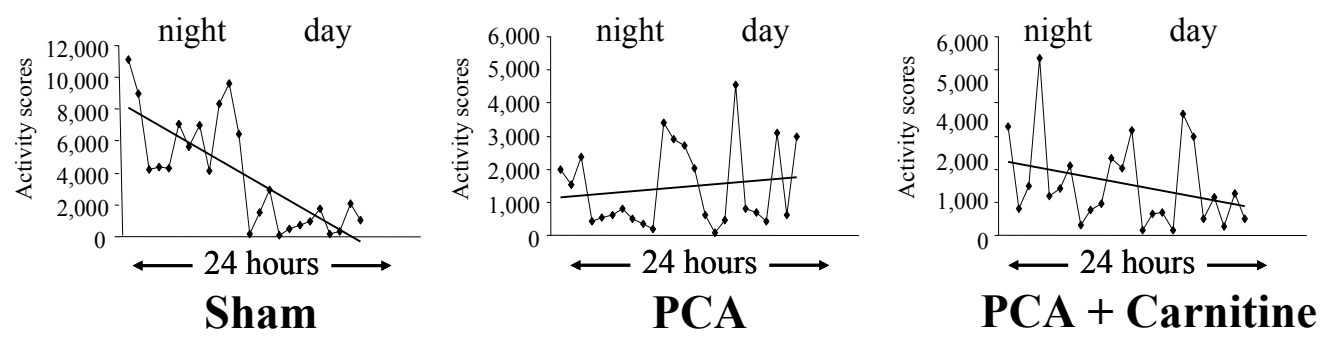

Fig. 5. Night and day locomotor activity in rats with PCA with or without treatment with Lcarnitine.

Stable isotope spectroscopic methods make it possible to measure the output of interrelated pathways concurrently and determine several atoms (e.g. ${ }^{1} \mathrm{H}$ and ${ }^{13} \mathrm{C}$ ) without transformation or isolation processes (Zwingmann \& Leibfritz, 2003). The metabolic pathways by which the ${ }^{13} \mathrm{C}$-label from $[1-13 \mathrm{C}]$ glucose is transformed into various metabolites are presented in simplified form in Fig. 6. For a more detailed description of the metabolic pathways from ${ }^{13} \mathrm{C}$-labelled glucose isotopomers, see Zwingmann \& Leibfritz (2003). Briefly, via the glycolytic pathway, $[1-13 \mathrm{C}]$ glucose is transformed into [3-13C]pyruvate, $\left[3-{ }^{13} \mathrm{C}\right]$ alanine and $\left[3-{ }^{13} \mathrm{C}\right]$ lactate. $\left[3-{ }^{13} \mathrm{C}\right]$ pyruvate may enter the TCA cycle via the anaplerotic pathway (pyruvate carboxylase, PC (or malic enzyme (ME)) or the oxidative pathway (pyruvate dehydrogenase, $\mathrm{PDH}$ ). Via PC, glutamate and glutamine are labelled at C-2. In the oxidative pathway, pyruvate enters the cycle as [2-13C]acetyl-CoA, and glutamate and glutamine are mono-labelled at C-4.

The data show that PCA in rats resulted in a decreased flux through PDH, a key enzyme for mitochondrial metabolism, which is primarily present in the neurons. Concomitant treatment with L-carnitine eliminated this effect over the levels of sham-operated controls (Fig. 7).

Another group of rats received ammonium acetate $(3.8 \mathrm{mmol} / \mathrm{kg}$; i.p.) four weeks after PCA to precipitate encephalopathy. L-carnitine was concomitantly administered in a single dose $(1.6 \mathrm{mmol} / \mathrm{kg}$; i.p.). In rats with ammonia-precipitated encephalopathy, treatment with Lcarnitine delayed the time to the onset of coma (Fig. 8). Animals in which both righting ability and corneal reflex were affected were considered to be in coma. Rats with ammoniaprecipitated encephalopathy and rats treated with L-carnitine were sacrificed at the same time points. 


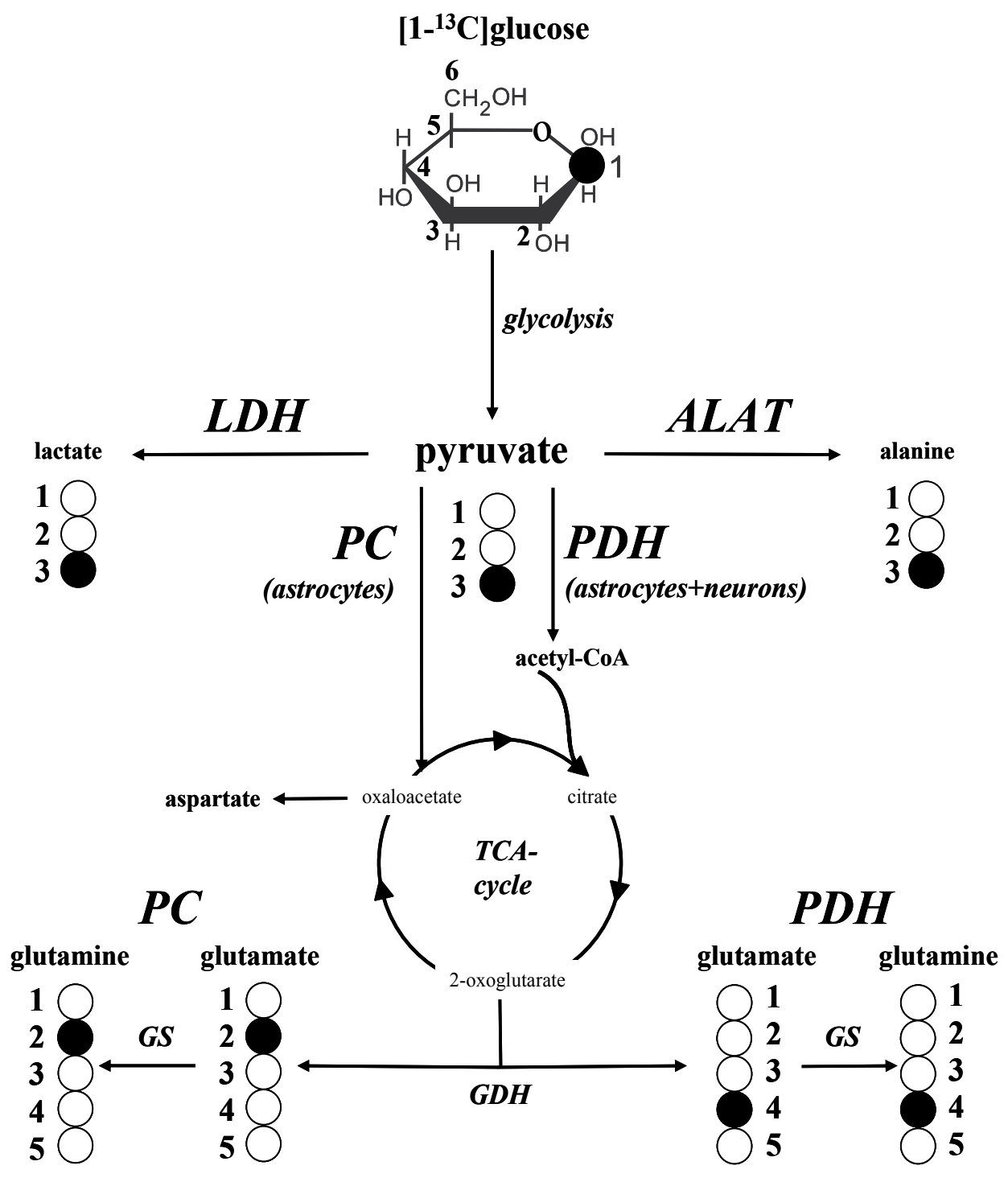

Fig. 6. Labelling of metabolites from [1-13C]glucose. Label distribution in glycolytic and TCA cycle intermediates during metabolism of [1-13C]glucose. A single turn of the TCA cycle from pyruvate via PC (pyruvate carboxylase) or PDH (pyruvate dehydrogenase) to 2oxoglutarate and subsequently glutamate and glutamine is considered. A description of the pathways leading to the different isotopomers is provided in the text. $\mathrm{LDH}$ : lactate dehydrogenase; ALAT: alanine aminotransferase; GDH: glutamate dehydrogenase; GS: glutamine synthetase. 


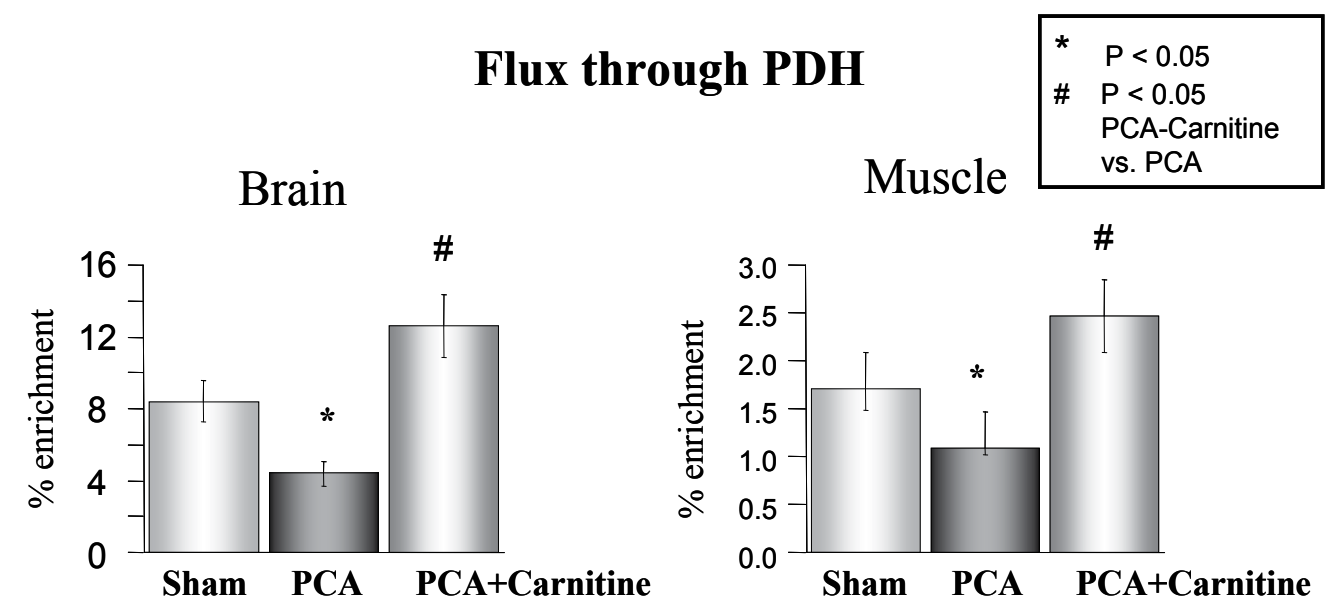

Fig. 7. Effect of L-carnitine on mitochondrial metabolism in rats with mild HE. [U-13C]glucose flux through the Krebs cycle was measured by the de novo synthesis of glutamate through PDH 12 hours after the last administration of L-carnitine or saline.

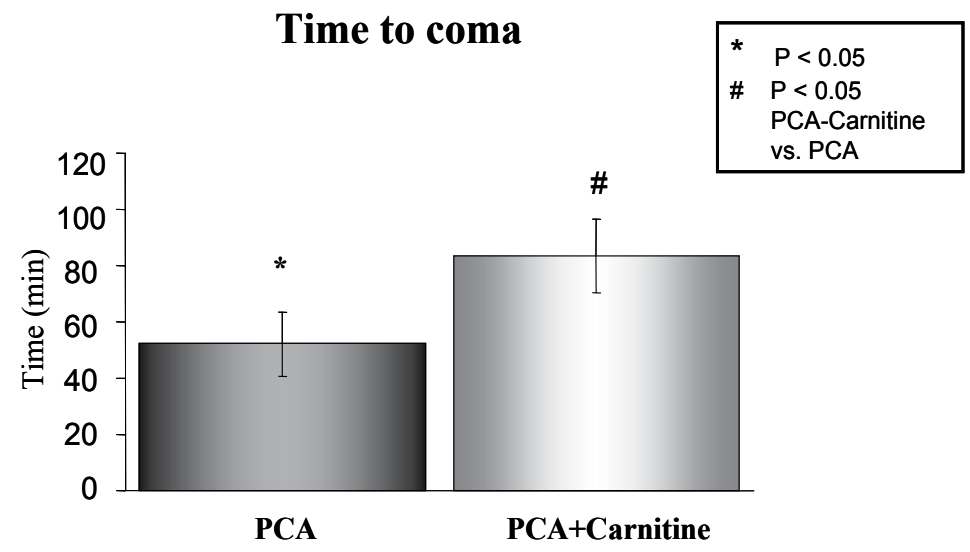

Fig. 8. Time to the onset of coma in rats with ammonia-precipitated encephalopathy.

Elevated brain lactate is a phenomenon which is characteristic of both human and experimental HE. As an indirect measurement of mitochondrial Krebs cycle metabolism, we measured the de novo synthesis of lactate from ${ }^{13} \mathrm{C}$-labelled glucose in rats with ammoniaprecipitated encephalopathy without and with treatment with L-carnitine (Fig. 9). The data showed that treatment with L-carnitine significantly eliminated the rise in brain lactate in these animals.

These preliminary data demonstrate that in chronic HE, L-carnitine acts on both brain and muscle by improving mitochondrial metabolism. These data further demonstrate that Lcarnitine prevents increased lactate synthesis in ammonia-precipitated encephalopathy, which parallels a significant increase in the time to coma. 


\section{Lactate synthesis}

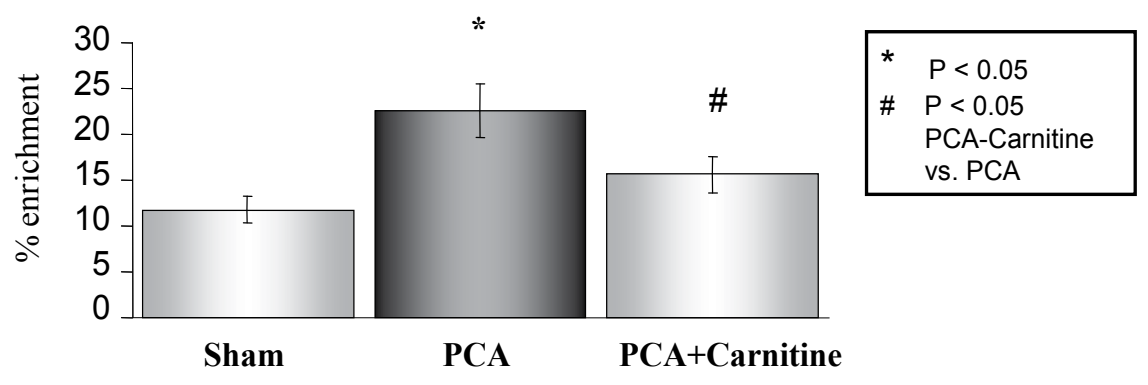

Fig. 9. Effect of L-carnitine on glycolytic lactate synthesis in rats with ammonia-precipitated encephalopathy. [U-13C] glucose flux through glycolysis was measured by the de novo synthesis of lactate.

\section{Concluding remarks}

To date, few studies have investigated the effect of L-carnitine and its derivates in human hyperammonemic syndromes and HE. However, in hyperammonemic animal models and ammonia-exposed cultured neurons L-carnitine has been shown to counteract some of the neurotoxic effects of ammonia. These data clearly show that L-carnitine is able to reduce ammonia levels, increase energy metabolism and decrease mortality. These studies further prove that HA is a key factor in HE. A protective effect of L-carnitine against disordered mental function and ammonia-precipitated encephalopathy was also observed in cirrhotic patients with HE, together with lowered circulating ammonia levels. These clinical trials are promising as they clarify that L-carnitine can reduce ammonia levels and improve patient performance in HE. The molecular mechanisms of the action of L-carnitine have not yet been fully elucidated, although the results suggest an effect on neuronal function in HE.

HE is a disorder that morphologically primarily affects the astrocytes (Norenberg, 1998). The dysfunction of astrocytes includes deficits in their ability to take up glutamate from the extracellular space, which may lead to abnormal glutamatergic neurotransmission. However, a series of studies has also demonstrated that ammonia directly causes disorders in neuronal function and neurotransmitter homeostasis by acting on glutamatergic receptors and by synthesis of neurotransmitters, i.e. glutamate and GABA. Furthermore, in recent studies an inhibition of neuronal energy metabolism in the TCA cycle has been proposed. Animal and cell culture studies have indicated that ammonia impairs neuronal function via altered metabolism and ultimately NMDA receptor activation, and that L-carnitine and NMDA receptor antagonists have the potential to preserve neuronal function during HA. Lcarnitine and its derivates increase energy metabolism via the TCA cycle by activation of fatty acid oxidation. They are able to change the activities of several enzymes involved in fatty acid metabolism, increase the synthesis of phospholipids and provide acetyl groups for the synthesis of acetylcholine. Furthermore, they can modify membrane fluidity and surface charge, which lead to altered activity of membrane transporter and enzymes. Since the brain can sustain part of its energy metabolism by fatty acid catabolism, the role of astrocytes and their energy metabolism in L-carnitine therapy needs further experimental clarification. 
The treatment of HA is uncertain and mostly directed to reducing the level of circulating ammonia. There is currently no therapy designed to counteract the molecular effects of ammonia. A further question remains as to whether the protective effects of L-carnitine against the toxicity of ammonia are systemic. In most studies, L-carnitine reduces circulating ammonia levels, suggesting a systemic effect of L-carnitine. For example, L-carnitine has been shown to have direct effects on the liver in patients with steatosis (Romano et al., 2008), and Benzerrouk and Qureshi (2001) demonstrated that acetyl-L-carnitine has a modulating effect on several hepatic mitochondrial matrix and inner membrane proteins that are modified by HA in the spf mutant mouse. On the other hand, L-carnitine seems to have direct effects on cerebral metabolism in valproic acid toxicity in the absence of hepatocellular dysfunction (Sztajnkrycer, 2002).

Another question to be clarified is why the effects of L-carnitine differ from its derivative acetyl-L-carnitine. When compared with L-carnitine, for example, acetyl-L-carnitine better preserved ATP in the brain of hyperammonemic mice, while it lowered ammonia in the blood and brain less markedly. One reason for this apparent discrepancy may lie in the use of the acetyl residue by the brain. This, on the other hand, points to an additional effect of this L-carnitine derivative on astrocytic energy metabolism, since acetate is taken up selectively by astrocytes while neurons do not use acetate (Waniewski and Martin, 1998; Zwingmann \& Leibfritz, 2003). On the other hand, there could be a close correlation between changes in astrocytic energy metabolism and neuronal (dys)function. In particular, L-carnitine exerts its effects in part by changes in NMDA receptor activation. Ammonia and extracellular glutamate cause an overactivation of this receptor, which in turn produces $\mathrm{NO}$ resulting in nitration-mediated inhibition of proteins such as the ammonia-detoxifying glutamine synthetase in the astrocytes. Improved energy metabolism in the astrocytes might counteract this deficit as well as a possible effect of ammonia on the astrocytic TCA cycle (Chatauret et al., 2003). The molecular and cell-specific energetic effects of L-carnitine and acetyl-L-carnitine therefore require further clarification.

Taken together, these results show that L-carnitine and its analogues do have the potential to suppress the neurotoxicity of ammonia. The fact that the action of acetyl-L-carnitine may differ from that of L-carnitine suggests that the classical function of L-carnitine is not the sole mechanism underlying the suppression of the neurotoxicity of ammonia (Matsuoka \& Igisu, 1993). However, further investigations are required to clarify the molecular mechanisms that lead to the protective effects of L-carnitine and its derivates in both experimental HA and human HE. Other studies may also optimize the dosage and time of administration of L-carnitine. Analysis of selected L-carnitine trials compared to currently accepted therapies suggests that L-acyl-carnitine is promising as a safe and effective treatment for HE, and further trials of this drug are warranted (Shores \& Keeffe, 2008). Since it is a low-cost agent with few side effects, further clinical trials could prove to be promising in evaluating the broader use of L-carnitine and derivatives in patients with minimal or ammonia-precipitated HE.

\section{Acknowledgements}

This work was funded by the Canadian Instituts for Health Research (CIHR). Dr. Zwingmann is a recipient of supporting awards from the Quebec Ministry of Education, the Programme québécois de bourses d'excellence, the Deutsche Forschungsgemeinschaft 
(German Research Foundation, DFG) and the Fonds de Recherche en santé du Quebec (FRSQ).

\section{References}

Bachmann, C. (2002). Mechanisms of hyperammonemia. Clin Chem Lab Med, Vol. 40, pp. 653662

Bass, N. M. (2007), Review article: the current pharmacological therapies for hepatic encephalopathy. Aliment Pharmacol Ther, Vol. 25, Suppl.1, pp. 23-31

Bates, T.E., Williams, S.R. Kauppinen, R.A., \& Gadian, D.G. (1989). Observation of cerebral metabolites in an animal model of acute liver failure in vivo: a ${ }^{1} \mathrm{H}$ and ${ }^{31} \mathrm{P}$ nuclear magnetic resonance study. J Neurochem, Vol. 53, pp. 102-110

Beal, M. F. (2003), Bioenergetic approaches for neuroprotection in Parkinson's disease. Ann Neuro., Vol. 53, Suppl 3, pp. S39-S47

Belanger, M., \& Butterworth, R.F. (2005). Acute liver failure: a critical appraisal of available animal models. Metab. Brain Dis, Vol 20, pp. 409-423

Benzerrouk R., \& Qureshi I. A. (2001). Hepatic mitochondrial proteins in congenitally hyperammonemic spf mice: effect of acetyl-L-carnitine. Biosci Biotechno. Biochem, Vol. 65, pp. 495-500

Blei, A.T. (2001), Pathophysiology of brain edema in fulminant hepatic failure, revisited. Metab Brain Dis, Vol. 16, pp. 85-94

Bremer, J. (1983), Carnitine-metabolism and functions. Physiol Rev, Vol 63, pp. 1420-1480

Butterworth, R.F., Giguere, J.F., Michaud, J., Lavoie, J., \& Layrargues, G.P. (1987). Ammonia: key factor in the pathogenesis of hepatic encephalopathy. Neurochem Pathol, Vol 6, pp. $1-12$

Butterworth, R.F. (1998). Effects of hyperammonaemia on brain function. J Inherit Metab Dis, Vol. 21 Suppl 1, pp. 6-20

Butterworth, R. F. (2002). Glutamate transporters in hyperammonemia. Neurochem Int Vol. 41, pp. $81-85$

Cammà, C., Fiorello, F., Tinè, F., Marchesini, G., Fabbri, A., \& Pagliaro, L. (1993). Lactitol in treatment of chronic hepatic encephalopathy. Dig Dis Sci, Vol. 38, pp. 916-922

Cauli, O., Rodrigo, R., Boix, J., Piedrafita, B., Agusti, A., \& Felipo, V. (2008). Acute liver failure-induced death of rats is delayed or prevented by blocking NMDA receptors in brain. Am J Physiol Gastrointest Liver Physio., Vol. 295, pp. G503-511

Chan, Y. C., Tse, M. L., \& Lau, F. L. (2007). Two cases of valproic acid poisoning treated with L-carnitine. Hum Exp Toxicol, Vol. 26, pp. 967-969

Chatauret, N., Zwingmann, C., Rose, C., Leibfritz, D., \& Butterworth, R.-F. (2003). Effects of hypothermia on brain glucose metabolism in acute liver failure: a H/C-nuclear magnetic resonance study. Gastroenterology, Vol. 125, pp. 815-824

Chiechio, S., Copani, A., Gereau, R. W. 4th, \& Nicoletti, F. (2007). Acetyl-L-carnitine in neuropathic pain: experimental data. CNS Drugs, Vol. 21 Suppl 1, pp. 31-38.

Chung, C., Gottstein, J., \& Blei, A.T. (2001), Indomethacin prevents the development of experimental ammonia-induced brain edema in rats after portacaval anastomosis. Hepatology, Vol. 34, pp. 249-254

Cordoba, J., \& Blei, A.T. (1995). Cerebral edema and intracranial pressure monitoring. Liver Transpl Surg, Vol. 1, pp. 187-194 
Cordoba, M. D., \& Minguez, M. D. (2008). Hepatic encephalopathy. Semin Liver Dis, Vol. 28, pp. $70-80$

Del Olmo, J.A., Castillo, M., Rodrigo, J.M., Aparisi, L., Serra, M.A., Wassel, A., \& Bixquert, M. (1990). Effect of L-carnitine upon ammonia tolerance test in cirrhotic patients. Adv Exp Med Biol, Vol. 272, pp. 197-208

Deutz, N.E.P., De Graaf, A.A., De Haan, J.G., Bovée, W.M.M.J., \& Chamuleau, R.A.F.M., (1988). In vivo brain ${ }^{1} \mathrm{H}-\mathrm{NMR}$ spectroscopy ( $\left.{ }^{1} \mathrm{H}-\mathrm{NMRS}\right)$ during acute hepatic encephalopathy (HE). In: Soeters PB, Wilson JHP, Meijer AJ and Holm E, eds. Advances in Ammonia Metabolism and Hepatic Encephalopathy. Chap. 57. Amsterdam: Excerpta Media, 1988; pp. 439-446

Ebert, D., Haller, R. G., \& Walton, M. E. (2003). Energy contribution of octanoate to intact brain metabolism measured by ${ }^{13} \mathrm{C}$ nuclear magnetic resonance. J Neurosci, Vol. 23, pp. 5928-5935

Felipo, V., Minana, M.D., Cabedo, H., \& Grisolia, S. (1994). L-carnitine increases the affinity of glutamate for quisqualate receptors and prevents glutamate neurotoxicity. Neurochem Res, Vol. 19, pp. 373-377

Ferenci, P., Lockwood, A., Mullen, K., Tarter, R., Weissenborn, K., \& Blei, A. T. (2002). Hepatic encephalopathy - definition, nomenclature, diagnosis, and quantification: final report of the working party at the 11th World Congresses of Gastroenterology, Vienna, 1998. Hepatology, Vol. 35, pp. 716-721

Fornasini, G., Upton, R. N., \& Evans, A. M. (2007). A pharmacokinetic model for L-carnitine in patients receiving haemodialysis. Br J Clin Pharmacol, Vol. 64, pp. 335-345

Häussinger, D., \& Schliess, F. (2008). Pathogenic mechanisms of hepatic encephalopathy. Gut, Vol. 57, pp. 1156-1165

Holmin, T., Agardh, C.-D. Alinder, G., Herlin, P., \& Hultberg B. (1983). The influence of total hepatectomy on cerebral energy state, ammonia-related amino acids of the brain and plasma amino acids in the rat. Eur J Clin Invest, Vol. 13, pp. 215-220

Hoyumpa, A.M. Jr., Desmond, P.V., Avant, G.R., \& Roberts, R.K. (1979). Hepatic encephalopathy. Gastroenterology, Vol. 76, pp. 184-195

Inazu, M., Matsumiya, T. (2008). [Physiological functions of carnitine and carnitine transporters in the central nervous system]. Nihon Shinkei Seishin Yakurigaku Yasshi, Vol. 28, pp. 113-120

Izumi, Y., Izumi, M., Matsukawa, M., Funatsu, M., \& Zorumski, C.F. Ammonia-mediated LTP inhibition: effects of NMDA receptor antagonists and L-carnitine. Neurobiol Dis, Vol. 20, pp. 615-624

Jayakumar, A.R., Panickar, K.S., Murthy, Ch.R., \& Norenberg, M.D. (2006). Oxidative stress and mitogen-activated protein kinase phosphorylation mediate ammonia-induced cell swelling and glutamate uptake inhibition in cultured astrocytes. J Neurosci, Vol. 26, pp. 4774-4784

Kala, G., \& Hertz, L. (2005). Ammonia effects on pyruvate/lactate production in astrocytes - interaction with glutamate. Neurochem Int, Vol. 47, pp. 4-12

Kircheis, G., Wettstein, M., Timmermann, L., Schnitzler, A., \& Häussinger, D. (2002a). Critical flicker frequency for quantification of low-grade hepatic encephalopathy. Hepatology, Vol. 35, pp. 357-366 
Kircheis, G., Wettstein, M., Dahl, S., \& Häussinger, D. (2002b). Clinical efficacy of Lornithine-L-aspartate in the management of hepatic encephalopathy. Metab Brain Dis, Vol. 17, pp. 453-462

Kosenko, E., Kaminsky, Y., Lopata, O., Muravyov, N., Kaminsky, A., Hermenegildo, C., \& Felipo V. (1998). Nitroarginine, an inhibitor of nitric oxide synthase, prevents changes in superoxide radical and antioxidant enzymes. Metab Brain Dis, Vol. 13, pp. 29-41

Kosenko, E., Kaminski, Y, Lopata, O., Muravyov, N., \& Felipo V. (1999). Blocking NMDA receptors prevents the oxidative stress induced by acute ammonia intoxication. Free Radic Bio. Med, Vol. 26, pp. 1369-374.

Kuratsune, H., Yamaguti, K., Lindh, G., Evengard, B., Hagberg, G., Matsumura, K., Iwase, M., Onoe, H., Takahashi, M., Machii, T., Kanakura, Y., Kitani, T., Langstrom, B., \& Watanabe, Y. (2002). Brain regions involved in fatigue sensation: reduced acetylcarnitine uptake into the brain. Neuroimage, Vol. 17, pp. 1256-1265

Lai, J. C., \& Cooper, A. J. (1986). Brain alpha-ketoglutarate dehydrogenase complex: kinetic properties, regional distribution, and effects of inhibitors. J Neurochem, Vol. 47, pp. 1376-1386

Larsen, F.S., Gottstein, J., \& Blei A.T. (2001). Cerebral hyperemia and nitric oxide synthase in rats with ammonia-induced brain edema. J Hepatol, Vol. 34, pp. 548-554

Leevy, C. B., \& Phillips J. A. (2007). Hospitalizations during the use of rixafimin versus lactulose for the treatment of hepatic encephalopathy. Dig Dis Sci, Vol. 52, pp. 737741

Lheureux, P. E., Penaloza, A., Zahir, S., \& Gris, M. (2005). Science review: carnitine in the treatment of valproic acid-induced toxicity - what ist he evidence? Crit Care, Vol. 9, ppp. 431-440

Llansola, M., Erceg, S., Hernandez-Viadel, M., \& Felipo, V. (2002). Prevention of ammonia and glutamate neurotoxicity by carnitine: molecular mechanisms. Metab Brain Dis, Vol. 17, pp. 389-397

Llansola, M., \& Felipo V. (2002). Carnitine prevents NMDA receptor-mediated activation of MAP-kinase and phosphorylation of microtubule-associated protein 2 in cerebellar neurons in culture. Brain Res, Vol. 947, pp. 50-56.

Lockwood, A.H., McDonald, J.M., Reiman, R.E., Gelbard, A.S., Laughlin, J.S., Duffy, T.E., \& Plum, F. (1979). The dynamics of ammonia metabolism in man. Effects of liver disease and hyperammonemia. J Clin Invest, Vol. 63, pp. 449-460

Longo, N., Amat di San Filippo, C., \& Pasquali, M. (2006). Disorders of carnitine transport and the carnitine cycle. Am J Med Genet C Semin Med Genet, Vol. 142C, pp. 77-85

Luppa, D. (2004). Beteiligung von L-Carnitin an der Regulation des Fett- und Kohlenhydratstoffwechsels. Klinische Sportmedizin, Vol. 5, pp. 25-34.

Malaguarnera, M., Pistone, G., Astuto, M., Dell'Arte, S., Finocchiaro, G., Lo Giudice, E., \& Pennisi, G. (2003). L-Carnitine in the treatment of mild or moderate hepatic encephalopathy. Dig Dis Sci, Vol. 21, pp. 271-275

Malaguarnera, M., Pistone, G., Elvira, R., Leotta, C., Scarpello, L., \& Liborio, R. (2005). Effects of L-carnitine in patients with hepatic encephalopathy. World J Gastroentero., Vol. 11, pp. 7197-7207 
Malaguarnera, M., Pistone, G., Astuto, M., Vecchio, I., Raffaele, R., Lo Giudice, E., \& Rampello, L. (2006). Effects of L-acetylcarnitine an cirrhotic patients with hepatic coma: randomized double-blind, placebo-controlled trial. Dig Dis Sci, Vol. 51, pp. 2242-2247

Malaguarnera, M., Cammalleri, L., Gargante, M. P., Vacante, M., Colonna, V., \& Motta, M. (2007). L-carnitine treatment reduces severity of physical and mental fatigue and increases cognitive functions in centenarians: a randomized and controlled clinical trial. Am J Clin Nutr, Vol. 86, pp. 1738-1744

Malaguarnera, M., Gargante, M. P., Cristaldi, E., Vacante, M., Risino, C., Cammalleri, L., Pennisi, G., \& Rampello, L. (2008). Acetyl-L-carnitine treatment in minimal hepatic encephalopathy Dig Dis Sci, Vol. 53, pp. 3018-25

Malaguarnera, M., Gargante, M. P., Cristaldi, E., Colonna, V., Messano, M., Koverech, A., Neri, S., Vacante, M., Cammalleri, L., \& Motta M. (2008). Acetyl L-carnitine (ALC) treatment in elderly patients with fatigue. Arch Gerontol Geriatr, Vol. 46, pp. 181-190

Matsuoka, M., \& Igisu, H. (1993a). Comparison of the effects of L-carnitine, D-carnitine and acetyl-L-carnitine on the neurotoxicity of ammonia. Biochem Pharmacol, Vol. 46, pp. 159-164

Matsuoka, M., \& Igisu, H. (1993b). Effects of L and D-carnitine on brain energy metabolites in mice given sublethal doses of ammonium acetate. Pharmacol Toxicol, Vol. 72, pp. 145-147

McDermott, W.V. Jr. (1957). Metabolism and toxicity of ammonia. N Engl J Med, Vol. 257, pp. 1076-1081

Michalak, A., Rose, C., Butterworth, J., \& Butterworth, R. F. (1996). Neuroactive amino acids and glutamate (NMDA) receptors in frontal cortex of rats with experimental acute liver failure. Hepatology., Vol. 24, pp. 908-913.

Minana, M.D., Hermenegildo, C., Llansola, M., Montoliu, C., Grisolia, S., \& Felipo, V. (1998). Carnitine and choline derivatives containing a trimethylamine group prevent ammonia toxicity in mice and glutamate toxicity in primary cultures of neurons. J Pharm Exp Ther, Vol. 279, pp. 194-199.

Monfort, P., Kosenko, E., Erceg, S., Canales, J.J., \& Felipo, V. (2002). Molecular mechanism of acute ammonia toxicity: role of NMDA receptors. Neurochem Int, Vol. 41, pp. 95-102

Montgomery, S. A., Thal, L. J., \& Amrein, R. (2003). Meta-analysis of double blind randomized controlled clinical trials of acetyl-L-carnitine versus placebo in the treatment of mild codnitive impairment and mild Alzheimer's disease. Int Clin Psychopharmacol, Vol. 18, pp. 61-71

Morgan, M. Y., Blei, A., Grüngreiff, K., Jalan, R., Kircheis, G., Marchesini, G., Riggio, O., \& Weissenborn K. (2007). The treatment of hepatic encephalopathy. Metab Brain Res, Vol. 22, pp. 389-405

Mutomba, M. C., Yuan, H., Konyavko, M., Adachi, S., Yokoyama, C. B., Esser, V., McGarry, J. D., Babior, B. M., \& Gottlieb, R. A. (2000). Regulation of the activity of caspases by L-Carnitine and palmitoylcarnitine. FEBS Lett, Vol. 478, pp. 19-25

Nałecz, K. A., Miecz, D., Berezowski, V., \& Cecchelli, R. (2004). Carnitine: transport and physiological funcions in the brain. Mol Aspects Med, Vol. 25, pp. 551-567

Neri, S., Pistone, G., Saraceno, B., Pennisi, G., Luca, S., \& Malaguarnera, M. (2003). Lcarnitine decreases severity and type of fatigue induced by interferon-alpha in the treatment of patients with hepatitis C. Neuropsychobiology, Vol. 47, pp. 94-97 
Norenberg, M.D. (1977). A light and electron microscopic study of experimental portalsystemic (ammonia) encephalopathy. Progression and reversal of the disorder. Lab Invest, Vol. 36, pp. 618-627

Norenberg, M.D, \& Martinez-Hernandez, A. (1979). Fine structural localization of glutamine synthetase in astrocytes of rat brain. Brain Res, Vol. 161, pp. 303-310

Norenberg, M.D. (1987). The role of astrocytes in hepatic encephalopathy. Neurochem Pathol, Vol. 6, pp. 13-33

Norenberg, M. D. (1998). Astroglial dysfunction in hepatic encephalopathy. Metab Brain Dis, Vol. 13, pp. 319-335

Norenberg, M.D., Jayakumar, A.R., Rama Rao, K.V., \& Panickar, K.S. (2007). New concepts in the mechanism of ammonia-induced astrocyte swelling. Metab Brain Dis, Vol. 22, pp. 219-234

O'Connor, J. E., Costell, M., \& Grisola, S. (1984). Protective effect of L-carnitine on hyperammonemia. FEBS Lett, Vol. 166, pp. 331-334

O'Connor, J. E., Costell, M., \& Grisola, S. (1984a). Prevention of ammonia toxicity by Lcarnitine: metabolic changes in brain. Neurochem Res, Vol. 9, pp. 563-570

Peeling, J., Shoemaker, L., Gauthier, T., Benarroch, A., Sutherland, G.-R., \& Minuk, G.-Y. (1993). Cerebral metabolic and histological effects of thioacetamide-induced liver failure. Am J Physiol, Vol. 265, pp. G572-G578

Pettegrew, J. W., Levine, J., \& McClure, R. J. (2000). Acetyl-L-carnitine physical-chemical, metabolic, and therapeutic properties relevance for its mode of action in Alzheimer's disease and geriatric depression. Mol Psychiatry, Vol. 5, pp. 616-632

Pittler, M.H., \& Ernst E. (2008). Complementary therapies for neuropathic and neuralgic pain: systematic review. Clin J Pain, Vol. 24, pp. 731-733

Rama Rao, K.V., Jayakumar, A.R., \& Norenberg, M.D. (2003). Ammonia neurotoxicity: role of the mitochondrial permeability transition. Metab Brain Dis, Vol. 18, pp. 113-127

Rao K. V., \& Qureshi, I. A. (1999). Reduction in the MK-801 binding sites of the NMDA subtype of glutamate receptor in a mouse model of congenital hyperammonemia: prevention by L-carnitine. Neuropharmacology, Vol. 38, pp. 383-394

Ratnakumari, L., \& Murthy, Ch.R.K. (1993a). Response of rat cerebral glycolytic enzymes to hyperammoniemic states. Neurosci Lett, Vol. 161, pp. 37-40

Ratnakumari, L., Qureshi, I. A., \& Butterworth, R. F. (1993b). Effect of L-carnitine on cerebral and hepatic energy metabolites in congenitally hyperammonemic sparse-fur mice and its role during benzoate therapy. Metabolism, Vol. 42, pp. 1039-1046

Ratnakumari, L., Qureshi, I. A., Maysinger, D., \& Butterworth R. F. (1995). Developmental deficiency of the cholinergic system in congenitally hyperammonemic spf mice: effect of acetyl-L-carnitine. J Pharmacol Exp Ther, Vol. 274, pp. 437-443

Romano, M., Vacante, M., Cristaldi, E., Colonna, V., Gargante, M.P., Cammalleri, L., \& Malaguarnera, M. (2008). L-carnitine treatment reduces steatosis in patients with chronic hepatitis C treated with alpha-interferon and ribavirin. Dig Dis Sci, Vol. 53, pp. 1114-1121

Rose, C., \& Felipo, V. (2005). Limited capacity for ammonia removal by brain in chronic liver failure: potential role of nitric oxide. Metab Brain Dis, Vol. 20, pp. 275-283

Schiodt, F. V., Atillasoy, E., \& Shakil, A. O., et al. (1999). Etiology and outcome for 295 patients with acute liver failure in the united states. Liver Transpl Surg, Vol. 5, pp. 29-34 
Shores, N.J., \& Keeffe, E.B. Is oral L-acyl-carnitine an effective therapy for hepatic encephalopathy? Review of the literature. Dig Dis Sci, Vol. 53, pp. 2330-2333

Silva, M.F., Aires, C.C., Luis, P.B., Ruiter, J.P., Ijlist, L., Duran, M., Wanders, R.J., \& Travares de Almeida, I. (2008). Valproic acid metabolism and its effects on mitochondrial fatty acid oxidation: A review. J Inherit Metab Dis, Vol. 4, [Epub ahead of print]

Siciliano, M., Annicchiarico, B. E., Lucchese, F., \& Bombardieri, G. (2006). Effects of a single, short intravenous dose of acetyl-L-carnitine on pattern-reversal visual-evoked potentials in cirrhotic patients with hepatic encephalopathy. Clin Exp Pharmacol Physiol, Vol. 33, pp. 76-80

Sima, A. A. (2007). Acetyl-L-carnitine in diabetic polyneuropathy: experimental and clinical data. CNS Drugs, Vol. 21, Suppl 1, pp. 13-23

Staub, F., Baethmann, A., Peters, J., Weigt, H., \& Kempski, O. (1990). Effects of lactacidosis on glial cell volume and viability. J Cereb Blood Flow Metab, Vol. 10, pp. 866-876

Steiber, A., Kerner, J., \& Hoppel, C. L. (2004). Carnitine: a nutritional, biosynthetic, and functional perspective. Mol. Aspects Med, Vol. 25, pp. 455-473

Sztajnkrycer, M.D. (2002). Valproic acid toxicity: overview and management. J Toxicol Clin Toxicol, Vol. 40, pp. 789-801

Therrien, G., Rose, C., Butterworth, J., \& Butterworth, R. F. (1997). Protective effect of Lcarnitine in ammonia-precipitated encephalopathy in the portacaval shunted rat. Hepatology, Vol. 25, pp. 551-556

Vielhaber, S., Feistner, H., Weis, J., Kreuder, J., Sailer, M., Schröder, J. M., \& Kunz, W. S. (2004). Primary carnitine deficiency: adult onset lipid storage myopathy with a mild clinical course. J Clin Neurosci, Vol. 11, pp. 919-924

Virmani, A., \& Binienda, Z. (2004). Role of carnitine esters in brain neuropathology. Mol Aspects Med, Vol. 25, pp. 533-549

Wadzinski, J., Franks, R., Roane, D., \& Bayard, M. (2007). Valproate-associated hyperammonemic encephalopathy. J A. Board Fam Med, Vol. 20, pp. 499-502

Waniewski, R.A., \& Martin, D.L. (1998). Preferential utilization of acetate by astrocytes is attributable to transport. J Neurosci, Vol. 18, pp. 5225-5233

Wein, C., Koch, H., Popp, B., Oehler, G., Schauder, P. (2004). Minimal hepatic encephalopathy impairs fitness to drive. Hepatology, Vol. 39, pp. 739-745

Tofteng, F., \& Larsen, F.S. (2002). Monitoring extracellular concentrations of lactate, glutamate, and glycerol by in vivo microdialysis in the brain during liver transplantation in acute liver failure. Liver Transp., Vol. 8, pp. 302-305

Zhang, H., Jia, H., Liu, J., Ao, N., Yan, B., Shen, W., Wang, X., Li, X., Luo, C., \& Liu, J. (2010). Combined R-alpha-lipoic acid and acetyl-L-carnitine exerts efficient preventative effects in a cellular model of Parkinson's disease. J Cell Mol Med, Vol. 14, pp. 215-25

Zwingmann, C., Chatauret, N., Leibfritz, D., \& Butterworth, R.F. (2003). Selective increase of brain lactate synthesis in experimental acute liver failure: results of a [H-C] nuclear magnetic resonance study. Hepatology, Vol. 37, pp. 420-428

Zwingmann, C., \& Leibfritz, D. (2003). Regulation of glial metabolism studied by ${ }^{13 C-N M R . ~}$ NMR Biomed, Vol. 16, pp. 370-399

Zwingmann, C, \& Butterworth, R.F. (2005). An update on the role of brain glutamine synthesis and its relation to cell-specific energy metabolism in the hyperammonemic brain: Further studies using NMR spectroscopy. Neurochem Int, Vol. 143, pp. 19-30 
Zwingmann, C. (2007). Nuclear magnetic resonance studies of energy metabolism and glutamine shunt in hepatic encephalopathy and hyperammonemia. J Neurosci Res, Vol. 15, pp. 3429-3442 


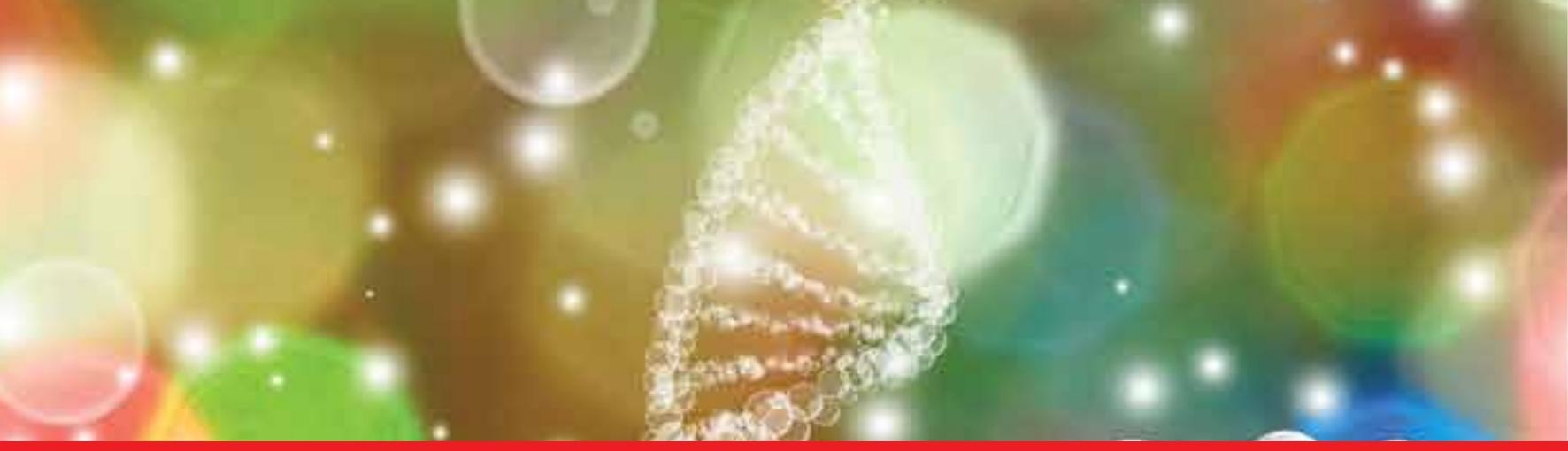

\section{Edited by Radu Tanasescu}

The book project "Miscellanea on Encephalopathies-a second look" aims to cover some of the important aspects regarding metabolic, hypoxic, neoplasm- and drug-related encephalopathies, by transmitting valuable information filtered through the real life clinical and research experience of the authors.

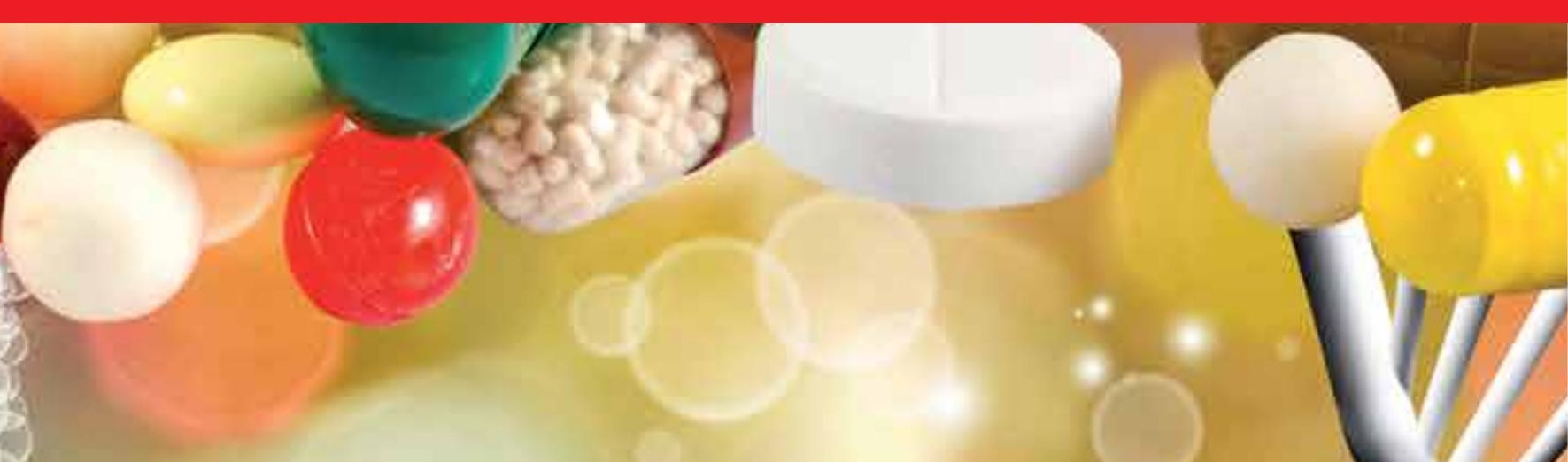

